\title{
Synthese und Anwendung eines radioaktiven \\ Photoaffinitätslabels zur Wirkortbestimmung von \\ Naturstoffen \\ sowie \\ Beiträge zur Totalsynthese des Collinolactons
}

\author{
Dissertation \\ zur Erlangung des Doktorgrades \\ der Mathematisch-Naturwissenschaftlichen Fakultäten \\ der Georg-August-Universität zu Göttingen
}

vorgelegt von

Tobias Bender

aus Halberstadt

Göttingen 2008 
D7

Referent: $\quad$ Prof. Dr. A. de Meijere

Korreferent: Prof. Dr. A. Zeeck

Tag der mündlichen Prüfung: 14. Oktober 2008 
Die vorliegende Arbeit wurde am Institut für Organische und Biomolekulare Chemie der Georg-August-Universität Göttingen in der Zeit von Mai 2005 bis Oktober 2008 unter Anleitung von Prof. Dr. A. de Meijere durchgeführt.

Herrn Prof. Dr. Paultheo von Zezschwitz und Herrn Prof. Dr. A. de Meijere danke ich für die interessante Themenstellung, das stete Interesse am Fortgang dieser Arbeit sowie für viele wertvolle Diskussionen und Anregungen. 

Für meine Familie 

Für Annett 

Die Tragödie der Wissenschaft - das Erschlagen einer schönen Hypothese durch eine hässliche Tatsache.

[There is no sadder sight in the world (of science) than to see a beautiful theory killed by a brutal fact.]

Thomas Henry Huxley (1825-95) 



\section{INHALTSVERZEICHNIS}

A.1. Methoden zur Wirkortbestimmung ......................................................... - 2 -

A.1.1 Zum Prinzip des Photoaffinitätslabelings .......................................................... - 2 -

A.1.2 Photoaktivierbare funktionelle Gruppen ..................................................... - 4 -

A.1.3 Identifizierung der markierten Proteinfragmente ............................................. - 5 -

A.2 Wirkortbestimmung von V-ATPase-Inhibitoren ..................................... - 6 -

A.2.1 Die V-ATPase-Inhibitoren Concanamycin A und Bafilomycin $\mathrm{A}_{1}$.................. - 7 -

A.2.2 Stand der Arbeiten zur Bestimmung des Wirkortes der V-ATPase-Inhibitoren

Bafilomycin und Concanamycin ................................................................... 8 -

A.3 Das Collinolacton - ein neuer Naturstoff aus Streptomyces sp. ............... - 10 -

A.3.1 Derivate des Collinolactons und deren Biologische Wirkung ${ }^{[36]}$.................... - 11 -

A.3.2 Biosynthese des Collinolactons ................................................................. - 12 -

A.3.3 Retrosynthetische Analyse und Stand der Arbeiten zum Collinolacton........... - 13 -

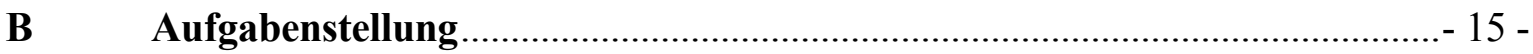

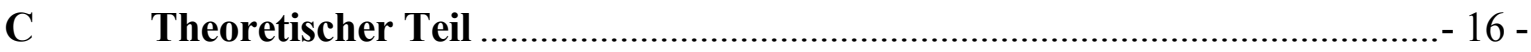

I. Hauptteil I - Synthese eines radioaktiven Photoaffinitätslabels .................. - 16 -

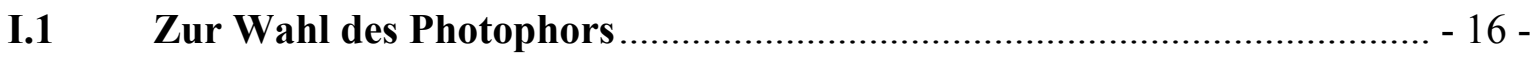

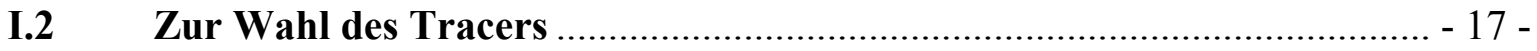

I.3 Synthese des Photoaffinitätslabels ............................................................. - 19 -

I.3.1 Synthese des Photoaffinitätslabels aus 1,4-Dibrombenzol.............................. - 20 -

I.3.2 Versuche zur direkten Carboxylierung des $p$-Bromdiazirins ........................... - 21 -

I.3.3 Darstellung des zweifach silylierten Diaziridins .......................................... - 24 -

I.3.4 Umsetzung des aus dem silylierten Diaziridin gewonnen Lithiumorganyls mit verschiedenen Elektrophilen..................................................................... - 25 -

I.3.5 Carboxylierung des lithiierten Diaziridins ................................................... - 26 -

I.3.6 Oxidation des Diaziridins zum Diazirin ........................................................ - 29 - 
I.4 Kupplung des Photoaffinitätslabels an die V-ATPase Inhibitoren $-31-$

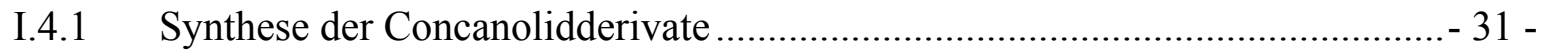

I.4.2 Synthese der Bafilomycinderivate ........................................................... 34 -

I.4.3 Synthese verschiedener Kendomycinderivate und deren Kupplung mit dem Photoaffinitätslabel. $-35-$

I.4.3.1. Synthese des Kendomycin-Acetonids und dessen Kupplung mit dem

Photoaffinitätslabel. $-36-$

I.4.3.2. Synthese des Diazirinyl-Kendomycin-Methylacetals $-38-$

I.4.4 Synthese des Diazirinyl-Apicularens $-40-$

I.5 Ergebnisse der Photoaffinitätsstudien mit den markierten Naturstoffen..- 41 -

I.5.1 Ergebnisse der Untersuchungen mit den Concanolid- und den Bafilomycinderivaten .......................................................................... 41 -

I.5.2 Ergebnisse der Untersuchungen mit den Kendomycinderivaten ...................... 42 -

I.5.3 Ergebnisse der Untersuchungen mit den Apicularenen .................................... 44 -

II. Hauptteil II - Zur Totalsynthese des Collinolactons ….................................. 45 -

II.1 Vorüberlegungen zur geplanten Totalsynthese des Collinolactons ............ - 45 -

II.2 Die Intramolekulare Diels-AIder Reaktion................................................ 46 -

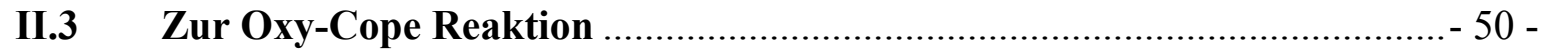

II.3.1 Oxy-Cope-Reaktion in der Synthese des Collinolactons .................................. 51 -

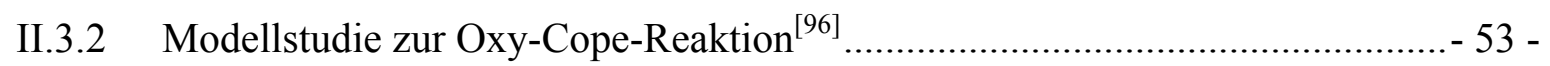

II.4 Abschluss der Synthese und Baeyer-Villiger Oxidation ............................. 56 -

II.5 Praktische Arbeiten zum Collinolacton ....................................................... 57 -

II.5.1 Versuche zur stereoselektiven Synthese des Trienons über die Nagao-Aldol-

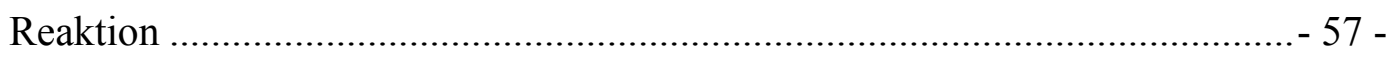

II.5.1.1 Versuche zur Darstellung des Aldehyds für die Nagao-Aldol-Reaktion ..........- 58 -

II.5.2 Synthese des IMDA-Edukts ausgehend von Acetessigsäureethylester.............- 63 -

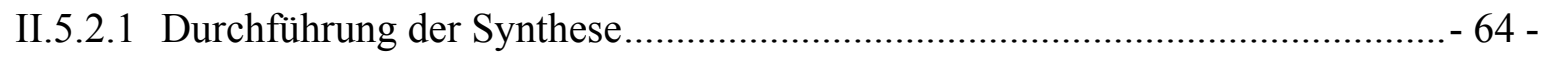

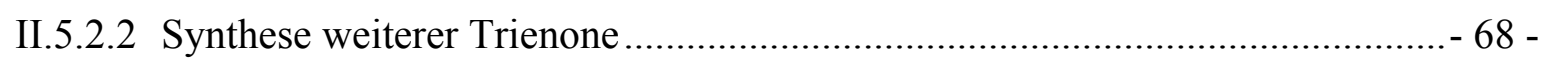

II.5.3 Versuche zur Darstellung der 5E/7E Diensäureester ...................................... 72 -

II.5.3.1 Versuche zur Isomerisierung der 5E/7Z Diensäureester zu den 5E/7E-

Verbindungen 
II.5.4 Versuche zur intramolekularen Diels-Alder-Reaktion der Trienone mit Enoletherfunktion

II.5.5 Synthese und IMDA-Reaktionen der Trienone mit unsubstituiertem

Dien $-84-$

II.5.5.1 Zusammenfassung der Synthesen der Trienone mit unsubstituiertem Dien .... - 84 -

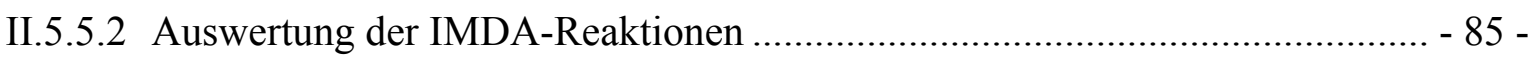

II.5.5.3 Aufklärung der relativen Konfiguration der Hauptprodukte der IMDA-

Reaktion $-89-$

II.5.6 Isomerisierungsversuch zur Darstellung des trans-Decalons. $-92-$

II.5.7 Versuche zur Einführung des $\alpha, \beta$-ungesättigten Ketons an C-6 $-93-$

II.5.7.1 Entschützung des TMS-Alkins $-93-$

II.5.7.2 Versuche zur Dioxolanbildung $-94-$

II.5.7.3 Versuche zur allylischen Oxidation. $-96-$

II.6 Alternative Syntheseroute basierend auf den bisherigen Ergebnissen $-98-$

D Zusammenfassung. $100-$

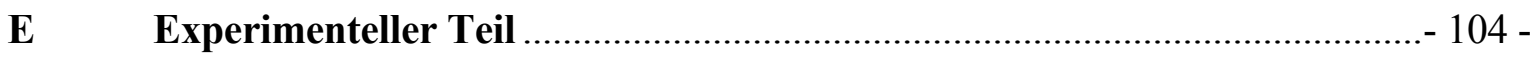

E.1 Material und Methoden ....................................................................... 104 -

E.2 Arbeitsvorschriften Hauptteil I ………................................................... 107 -

E.2.1 Synthese des Photoaffinitätslabels .............................................................. - 107 -

E.2.2 Kupplung des Photoaffinitätslabels an die Naturstoffe .................................. - 116 -

E.3 Arbeitsvorschriften Hauptteil II ............................................................ - 124 -

E.3.1 Versuche zur Synthese des Edukts für die Nagao-Aldol-Reaktion................. - 124 -

E.3.2 Synthese der IMDA-Edukte ausgehend von Acetessigsäureethylester.......... - 132 -

E.3.3 Versuche zur Darstellung des (5E,7E)-Diensäureethylesters......................... - 138 -

E.3.4 Intramolekularen Diels-Alder Reaktion .................................................... - 156 -

E.3.5 Versuche zur Einführung des $\alpha, \beta$-ungesättigten Ketons an C-6 .................... - 160 -

E.3.5.1 Abspaltung der TMS-Schutzgruppe am Alkin ............................................ - 160 -

E.3.5.2 Versuche zur Dioxolanbildung.................................................................. - 162 -

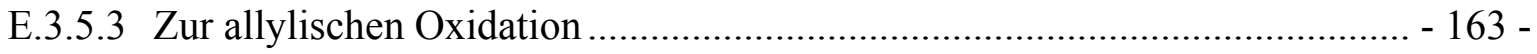


G Spektrenanhang 


\section{ABKÜRZUNGSVERZEICHNIS}

AAV

Add.

APT

Ar

$\mathrm{Ph}-\mathrm{H}$

$n$-BuLi

$t$-BuLi

COSY

DIBAH

DC

DCC

DCM

DIPA

DMAP

DMP

DMPU

DMSO

EDCI

EE

Essigester

Ether

eq

Hex

c-Hex

HMPA

HSQC

HWE

$\mathrm{IC}_{50}$

IMDA

KHMDS

$\mathrm{MOMCl}$
Allgemeine Arbeitsvorschrift

Additiv

Attached Proton Test

Aromatisch

Benzol

n-Butyllithium

tert-Butyllithium

Correlation spectroscopy

Diisobutylaluminiumhydrid

Dünnschichtchromatographie

Dicyclohexylcarbodiimid

Dichlormethan

Diisopropylamin

4-Dimethylaminopyridin

Dess-Martin-Reagenz

1,3-Dimethyltetrahydropyrimidin-2(1H)-on

Dimethylsulfoxid

$N$-(3-Dimethylaminopropyl)- $N$-ethylcarbodiimid

Essigsäureethylester

Essigsäureethylester

Diethylether

Äquivalent(e)

Hexan

Cyclohexan

Hexamethylphosphorsäuretriamid

Heteronuclear Single Quantum Coherence

HORNER-WADSWORTH-EMMONS

Konzentration eines Wirkstoffes, bei der 50\% der ursprünglichen Enzymaktivität vorliegt.

Intramolekulare Diels-Alder

Kaliumhexamethyldisilazid

Chlormethylmethylether 


\begin{tabular}{|c|c|}
\hline Rkt. & Reaktion \\
\hline n.b. & nicht bestimmt \\
\hline $\mathrm{Np}$ & Naphthalin \\
\hline NOE & Nuclear Overhauser Effect \\
\hline NOESY & Nuclear Overhauser Enhancement Spectroscopy \\
\hline NS & Niederschlag \\
\hline p.a. & pro analysi \\
\hline PG & Schutzgruppe \\
\hline PPTS & Pyridinium- $p$-toluolsulfonat \\
\hline Pent. & Pentan \\
\hline quant. & quantitative Ausbeute \\
\hline RT & Raumtemperatur \\
\hline SAR & Structure-Activity-Relationship \\
\hline TBDMS & tert-Butyldimethylsilyl \\
\hline TBDMSCl & tert-Butyldimethylsilylchlorid \\
\hline ТВНР & tert-Butylhydroperoxid \\
\hline TCAI & Trichloracetimidat \\
\hline TFA & Trifluoressigsäure \\
\hline THF & Tetrahydrofuran \\
\hline TMSOTf & Trimethylsilyltrifluormethansulfonat \\
\hline Tol & Toluol \\
\hline$p$ TsOH & para-Toluolsulfonsäure \\
\hline $\mathrm{TsCl}$ & para-Toluolsulfonsäurechlorid \\
\hline LA & Lewis Säure \\
\hline LM & Lösungsmittel \\
\hline Oxm & Oxidationsmittel \\
\hline
\end{tabular}






\section{A Einleitung}

Die Suche nach neuartigen Substanzen mit einer bestimmten biologischen Wirkung und die anschließende Weiterentwicklung zu einem marktfähigen Medikament bedürfen eines immensen wissenschaftlichen Aufwands und sind mit entsprechend hohen Kosten verbunden. Vom ersten „Hit“, also dem Finden eines biologisch aktiven Stoffes in einem High-Through-Put-Screening, bis zur Markteinführung des fertigen Medikaments können bis zu 15 Jahre vergehen und die Kosten können leicht mehrere hundert Millionen Euro betragen. Von den biologisch aktiven Substanzen schaffen es statistisch gesehen nur etwa $2 \%$ der mühevoll isolierten und in ersten Schritten weiterentwickelten Leitstrukturen auf den Markt. ${ }^{[1]}$ Es lag also auf der Hand, dass insbesondere Pharmaunternehmen große Anstrengungen unternehmen würden, um die Erfolgsaussichten von vielversprechenden Wirkstoffen zu erhöhen. Ab Mitte der 1990er Jahre versprach die targetbasierte Suche nach neuen biologisch wirksamen Substanzen zusammen mit aus der kombinatorischen Synthese gewonnenen Substanzbibliotheken eine Revolution in der Wirkstoffsuche. ${ }^{[2]}$ Die bis dahin erfolgreiche, aber doch aufwendige Suche nach biologisch aktiven Sekundärmetaboliten aus natürlichen Quellen wie Pflanzen, Bakterien, Pilzen etc. schien überflüssig zu werden. Nach dem Motto „make 'em all, let the assay sort 'em out"“ ${ }^{[3]}$ wurden mit der kombinatorischen Synthese Millionen von Verbindungen synthetisiert in dem Glauben, dass da schon für jede Krankheit „die richtige“ Substanz enthalten sein würde. Aus mehreren Gründen, wie z.B. beschränkte strukturelle Diversität der Substanzbibliotheken und eine oftmals schlechte in vivo-Aktivität der gefundenen Substanzen, konnte die kombinatorische Synthese den hohen Anforderungen nicht annähernd gerecht werden. ${ }^{[2]}$ Mit der Erkenntnis, dass sich der steinige Weg zu erfolgreichen Medikamenten auf diese Weise nicht würde abkürzen lassen, rücken nun die biologisch aktiven Naturstoffe wieder stärker ins Blickfeld. Der moderne (Naturstoff-) Chemiker hat auf dem Weg zum „perfekten Wirkstoff“, so es diesen denn gibt, eine ganze Reihe von zentralen Aufgaben zu bewältigen. Nach Isolierung und Strukturaufklärung folgt in der Regel die Totalsynthese der Verbindung, um zum Einen ausreichend Material für weitere biologische Tests bereitzustellen und zum Anderen eine möglichst große Vielzahl an Derivaten zu produzieren, um eine optimale Wirkung zu erzielen oder eine Verbindung z.B. oral bioverfügbar zu machen. Für die richtige Auswahl der zu synthetisierenden Derivate ist es von großem Vorteil, möglichst genaue Vorstellungen von Wirkort und -mechanismus einer biologisch aktiven Substanz zu haben. 


\title{
A.1. Methoden zur Wirkortbestimmung
}

\begin{tabular}{|l|c|c|}
\hline 3D-Strukturaufklärung & Chemische Methoden & Genetische Methoden \\
\hline Kristallstruktur etc. & (Photo)affinitätslabeling etc. & Zielorientierte Mutagenese etc. \\
\hline
\end{tabular}

\section{Molekulare Erkennungsmechanismen von Ligand-Biomolekülwechs elwirkungen}

\begin{abstract}
Abbildung 1: Methoden zur Wirkortbestimmung.
\end{abstract}
Für die Untersuchung der Wechselwirkungen kleiner bioaktiver Moleküle mit Proteinen stehen mehrere Strategien zur Verfügung (Abbildung 1). ${ }^{[4]}$ Sind das Zielprotein und der Wirkstoff in ausreichenden Mengen vorhanden, so bieten 3D-Strukturaufklärungsmethoden wie NMR-Untersuchungen oder zum Beispiel (Co)-Kristallstrukturen von Wirkstoff und Protein einen direkten Zugang zu den dreidimensionalen Strukturen der Zielproteine auf atomarem Level. Bei der Strukturaufklärung mittels genetischer Methoden werden durch Deletionen oder Mutationen die natürlichen Strukturen der Proteine verändert. In Abhängigkeit von den Auswirkungen auf die Wirksamkeit der Moleküle kann auf die Struktur der Bindungstasche geschlossen werden. Chemische Methoden hingegen ermöglichen die direkte Identifizierung von Zielproteinen in Proteingemischen durch das affinitätsbasierte Einbringen eines Tags in die Proteinstruktur. Ein Beispiel ist das Visualisieren von Biomolekülen mit Hilfe eines Fluorophors in Zellkompartimenten.

\section{A.1.1 Zum Prinzip des Photoaffinitätslabelings}

Das Prinzip der Photoaffinitätsmarkierung wurde erstmals 1962 von Westheimer und Mitarbeitern $^{[5]}$ beschrieben und hat seitdem breite Anwendung gefunden. ${ }^{[6 a]}$ In Abb. 2 ist eine schematische Darstellung des Verlaufs einer Photoaffinitäts-markierung wiedergegeben. Ein biologisch aktiver Ligand, welcher mit einer photoaktivierbaren Spezies (hier eine Diazirinylbenzoesäure) versehen ist, wird zum Target (einem beliebigen Protein) gegeben. Nach Bildung des Target-Ligand-Komplexes wird durch Bestrahlen mit Licht einer bestimmten Wellenlänge aus dem Photoaffinitätslabel die aktive Spezies (Carbene, Nitrene etc.) freigesetzt, welche unverzüglich mit umliegenden Aminosäuren 
eine kovalente, irreversible Bindung ausbildet. Nach Verdau und Aufreinigung der einzelnen Proteinbestandteile können schließlich die markierten Aminosäuren mit unten näher beschriebenen Methoden identifiziert werden.

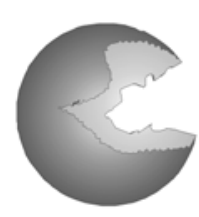

Target

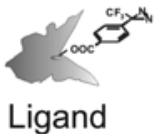

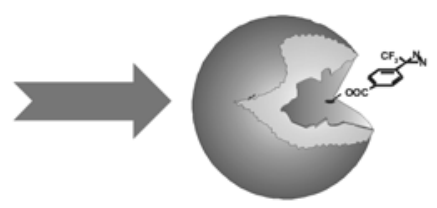

Target-LigandKomplex

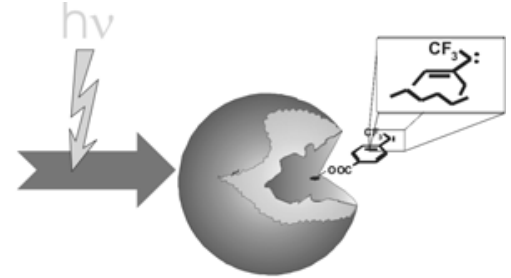

Aktiver Ligand (Carben)

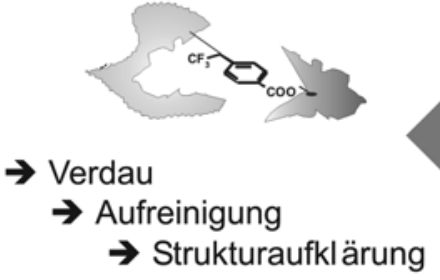

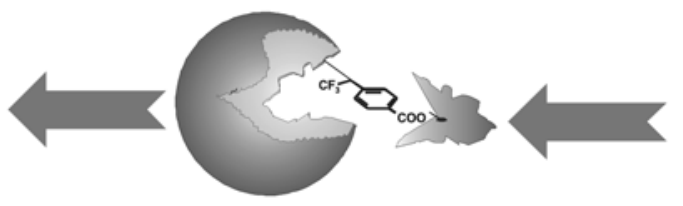

Irreversible Bindung

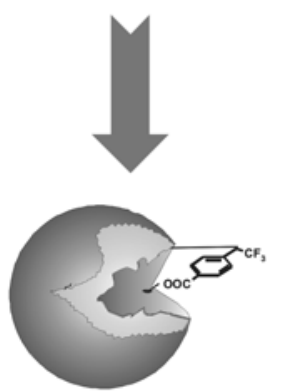

Kovalente Bindung

Abbildung 2: Schematische Darstellung des Prinzips der Photoaffinitätsmarkierung.

Das photoaktivierbare Molekül sollte dabei möglichst die folgenden Eigenschaften besitzen: Eine hohe Stabilität des Vorläufers vor der Aktivierung sowie eine sehr hohe Reaktivität der photolytisch freigesetzten reaktiven Spezies, um eine schnelle Reaktion auch mit nicht aktivierten C-H Bindungen der umliegenden Aminosäuren zu gewährleisten. Diese ist notwendig, um unspezifische Markierungen abseits der eigentlichen Bindungsstelle $\mathrm{zu}$ vermeiden. Zusätzlich sollten Nebenprodukte chemisch inert und das Label als Ganzes möglichst klein sein, um zu starke sterische Wechselwirkungen zwischen Target und Ligand zu vermeiden, welche zum vollständigen Verlust der biologischen Aktivität führen können. Um Schäden am zu untersuchenden biologischen System zu vermeiden, sollten die zur Aktivierung verwendeten Wellenlängen bei $\lambda \geq 300 \mathrm{~nm}$ liegen. 


\section{A.1.2 Photoaktivierbare funktionelle Gruppen}

Schema 1 zeigt die drei wesentlichen photoreaktiven funktionellen Gruppen, welche erfolgreich in der Photoaffinitätsmarkierung eingesetzt werden. Alle drei Gruppen besitzen spezifische Vor- sowie Nachteile.

Schema 1: Verschiedene Molekülgruppen mit photoaktiven Gruppen.<smiles>O=C(c1ccccc1)c1ccccc1</smiles>

Benzophenon<smiles>C=C(OS(=O)(=O)O)c1ccccc1</smiles><smiles>Nc1ccccc1</smiles>

Arylazid<smiles>[Y][R6](=O)(O)OC</smiles><smiles>[N]c1ccccc1</smiles><smiles>FC(F)(F)C1(c2ccccc2)N=N1</smiles>

Aryldiazirin $360 \mathrm{~nm}$<smiles>FC(F)(F)[C]c1ccccc1</smiles>

Benzophenone werden bei Wellenlängen von etwa $350 \mathrm{~nm}$ angeregt. Im Gegensatz zu den Aziden und den Diazirinen kommt es hier nicht zur Dissoziation, sondern es bildet sich ein reaktiver angeregter Triplett-Zustand, welcher mit $\mathrm{C}-\mathrm{H}$ Bindungen umliegender Aminosäuren reagieren kann. Liegt keine C-H Bindung mit passender Geometrie vor, so relaxiert das angeregte Benzophenon wieder in den Grundzustand. ${ }^{[7]}$ Dadurch sind mehrere Aktivierungszyklen möglich, so dass ein höherer Markierungsgrad erreicht werden kann, was die Benzophenone prinzipiell zu sehr guten Photoaffinitäslabeln macht. Die für die Insertion benötigte hohe Spezifität bezüglich der räumlichen Anordnung des zu abstrahierenden Protons verhindert jedoch manchmal eine effektive Markierung. ${ }^{[8]}$ Ein weiteres Problem beim Einsatz von Benzophenonen sind die verhältnismäßig langen Aktivierungszeiten, wodurch es zu Schäden am zu untersuchenden System kommen kann. Phenylazide sind wohl aufgrund der leichten Synthetisierbarkeit und hohen Stabilität vor der Aktivierung die am weitesten verbreiteten Photaffinitätslabel, ${ }^{[6 b]}$ benötigen jedoch zur Aktivierung Licht mit Wellenlängen $\leq 280 \mathrm{~nm}$. Diese Strahlungsintensitäten können jedoch zu Schäden am zu untersuchenden System und bei lebenden Zellen letztlich zum Absterben führen. ${ }^{[4]}$ Außerdem weisen die Azide im Vergleich zu den Aryldiazirinen noch weitere Schwächen auf: 1) Die Reaktivität der aktiven Spezies der Azide, der „Nitrene“, ist deutlich geringer als die der Carbene als aktiver Spezies der Diazirine. 2) Eine Markierung mit Nitrenen führt zu weniger stabilen Stickstoff-haltigen Bindungen, wohingegen man mit 
Carbenen stabilere Kohlenstoff-basierte Bindungen erhält. 3) Kurzlebige SingulettPhenylnitrene lagern sich schnell $\mathrm{zu}$ deutlich stabileren elektrophilen Ketiminen um, welche nicht mit unaktivierten C-H-Bindungen reagieren können. ${ }^{[6 f]}$ Betrachtet man nur die photochemischen Eigenschaften, so erfüllen die 3-Phenyl-3-(trifluormethyl)diazirine am ehesten sämtliche der oben genannten Kriterien bezüglich Stabilität und Reaktivität. ${ }^{[6 \mathrm{~d}]}$ Bei Wellenlängen um $360 \mathrm{~nm}$ bilden die Diazirine hochreaktive Carbene, welche schnell und unspezifisch auch mit nicht aktivierten C-H-Bindungen reagieren. Sie sind außerdem nicht nur bei Lagerung in der Dunkelheit, sondern auch gegenüber vielen Reaktionsbedingungen stabil. Trotzdem sind die Diazirine in der Literatur verhältnismäßig selten vertreten, was sicherlich auch mit der im Vergleich zu Aziden und Benzophenonen deutlich aufwendigeren Synthese zusammenhängt.

\section{A.1.3 Identifizierung der markierten Proteinfragmente}

Für die Identifizierung der markierten Proteinbausteine muss das Photophor eine leicht detektierbare Gruppe tragen. Hierfür stehen verschiedene Möglichkeiten zur Auswahl, unter anderem diese: radioaktive Label, Biotin-Seitenketten, magnetische Mikrosphären oder fluoreszierende Gruppen. ${ }^{[6 \mathrm{~h}]}$

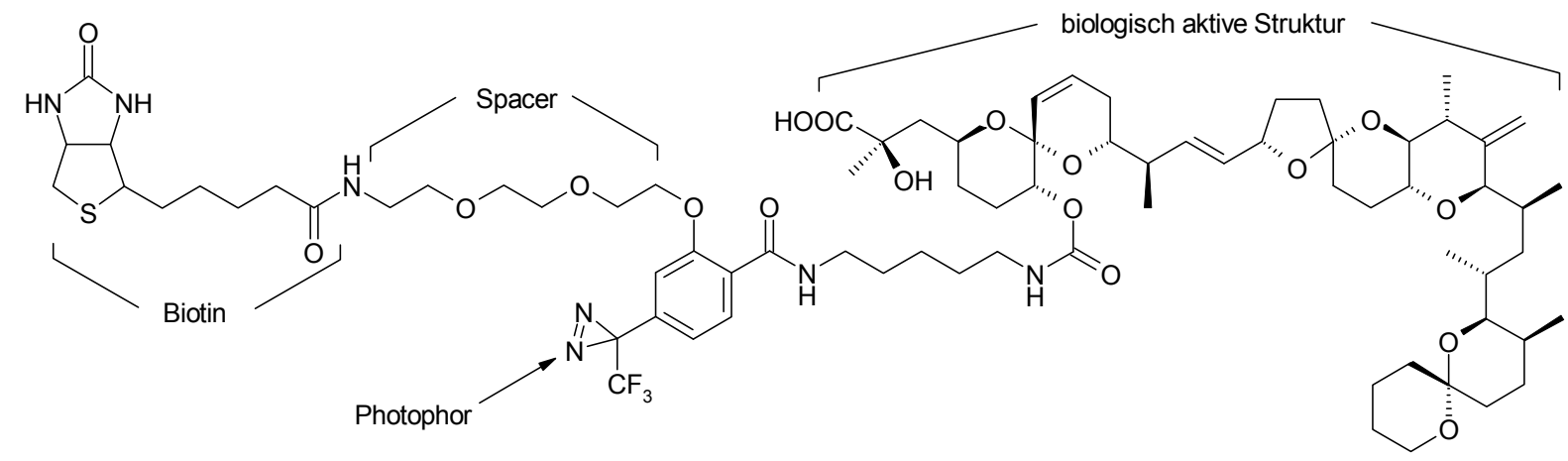

Abbildung 3: Biologisch aktives Molekül mit Photoaffinitätslabel und Biotinseitenkette.

Besonders die Biotin/Avidin-Methode hat sich bei der Untersuchung verschiedenster biologischer Systeme bewährt. Abbildung 3 zeigt ein solches Molekül, welches zur Untersuchung zellulärer Signalwege verwendet wurde. ${ }^{[6 e]}$ Der biologisch aktive Teil des Moleküls bindet zunächst an das Zielprotein, anschließend wird durch Bestrahlung mit Licht einer bestimmten Wellenlänge aus dem Photophor die aktive Spezies frei gesetzt. 
Die Seitenkette mit dem Spacer und dem Biotinmolekül ragt dabei aus der Bindungstasche des Proteins heraus, so dass es später mit höherer Affinität an die Avidinmatrix binden kann. Dabei kann es manchmal sogar zu Problemen bei der Abspaltung der markierten Fragmente kommen. Die Detektion erfolgt in der Regel über Chemilumineszenz mit einer Empfindlichkeit, die vergleichbar ist mit radioaktiven Labeln. Es wurde jedoch berichtet, dass aufgrund der großen Veränderungen, bzw. Substituenten am aktiven Molekül sterische Wechselwirkungen mit dem Target an Bedeutung gewinnen. Dies kann die Bildung eines Ligand-Rezeptor Komplexes behindern und somit zum teilweisen oder vollständigen Verlust der biologischen Aktivität führen. ${ }^{[9]}$ Um dies effektiv zu verhindern, kann alternativ ein radioaktives Label verwendet werden, welches direkt in die Struktur des Photoaffinitätslabels implementiert wird.

\section{A.2 Wirkortbestimmung von V-ATPase-Inhibitoren}

Für den Transport von Ionen entgegen eines elektrochemischen Gradientens bedarf es spezieller membranständiger Proteine. Verläuft der Transport unter Hydrolyse von ATP, spricht man von einem primären Transport, welcher von sogenannten IonentransportATPasen realisiert wird. Diese lassen sich nach PEDERSEN \& CARAFOLI ${ }^{[10]}$ in P-, F- und VATPasen einteilen, welche sich in Struktur und Funktion voneinander unterscheiden. ${ }^{[1]}$ Die P-Typ ATPasen sind die strukturell einfachsten Vertreter, zu ihnen gehören die $\mathrm{Na}^{+} / \mathrm{K}^{+}$- sowie die $\mathrm{Ca}^{2+}$-ATPasen. Die strukturell deutlich komplexeren ATPasen vom FTyp sind an der inneren Mitochondrienmembran, in der Thylakoidmembran von Chloroplasten und in der Plasmamembran von Bakterien lokalisiert, wo sie als ATP-Synthasen fungieren.

V-ATPasen wurden erstmalig aus der Vacuolenmembran der Hefe isoliert. ${ }^{[10,11]}$ In Abbildung $4^{[12]}$ ist der grundlegende Aufbau der V-ATPasen aus Hefe-Zellen dargestellt. Sie bestehen aus 13 Untereinheiten, welche sich auf zwei Domänen $\left(\mathrm{V}_{0}\right.$ und $\mathrm{V}_{1}$ ) verteilen. Vacuolare ATPasen (V-ATPasen) kommen in nahezu allen eukaryotischen Zellen vor und haben Einfluss auf viele essentielle Funktionen,

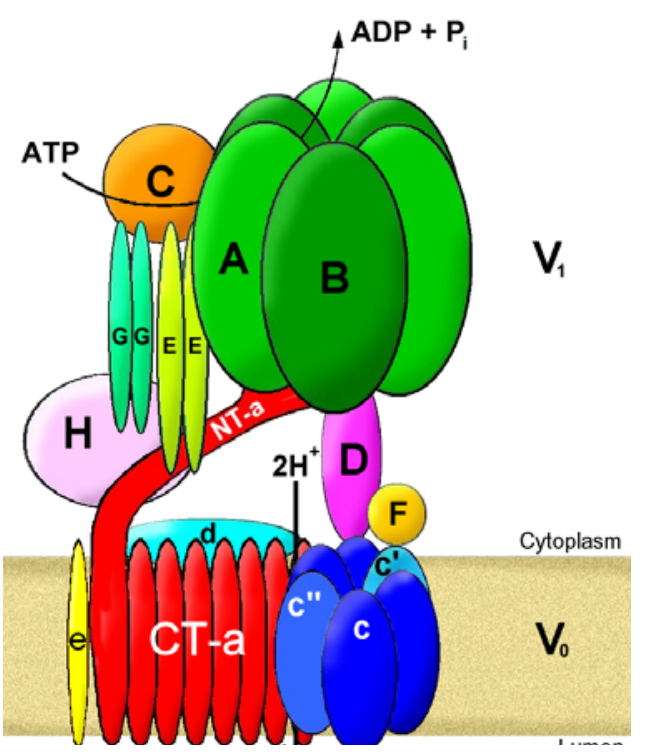

Abbildung 4: Darstellung einer V-ATPase. 
wie z.B. Knochenabbau, Säureausscheidung, Rezeptor-vermittelte Endozytose sowie die Zellteilung. ${ }^{[13]}$ Eine Fehlfunktion der V-ATPasen wird mit einer ganzen Reihe von Krankheiten wie z.B. Osteoporose, ${ }^{[14]}$ Krebs, ${ }^{[15]}$ Taubheit oder der Unfruchtbarkeit beim Mann in Verbindung gebracht. Insbesondere aufgrund des Einflusses der V-ATPasen auf die Auflösung der Knochenmatrix durch Osteoklasten und die Entwicklung von invasiven Tumoren stellen sie ein vielversprechendes pharmakologisches Target dar. ${ }^{[16,17]}$ Dies macht eine genaue Bestimmung des Wirkortes bzw. -mechanismus von V-ATPase-Inhibitoren sehr interessant.

\section{A.2.1 Die V-ATPase-Inhibitoren Concanamycin A und Bafilomycin $\mathbf{A}_{1}$}

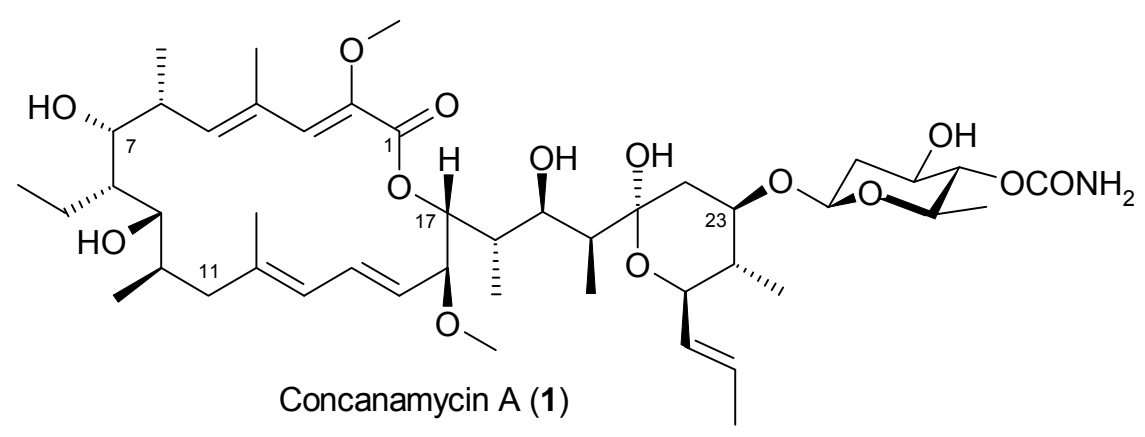

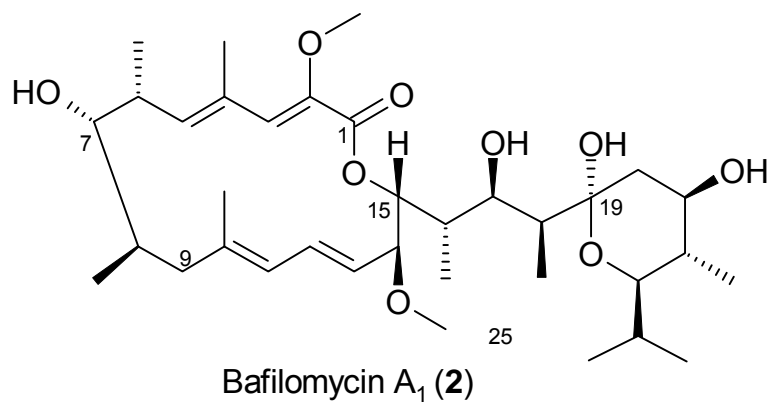

Abbildung 5: Strukturen der antibiotischen Plecomakrolide 1 und 2.

Abbildung 5 zeigt die Plecomakrolide ${ }^{[18]}$ (von griech.: $\pi \lambda \varepsilon \kappa \omega=$,ich falte“) Concanamycin $A$ (1) und Bafilomycin $A_{1}$ (2). Beide Verbindungen zeigen ein sehr breites biologisches Wirkspektrum von antifungischer über antiparasitärer bis hin zu antitumoraler Aktivität. ${ }^{[19]}$ Neben diesen schon länger bekannten Wirkungen erweitert sich das Wirkspektrum ständig, als aktuellste Beispiele seien hier die durch Bafilomycin $\mathrm{A}_{1}$ (2) beeinflusste Kaliumionenaufnahme in Mitochondrien ${ }^{[20]}$ und für Concanamycin A (1) die Hemmung der Lipopolysaccharid induzierten NO-Synthese ${ }^{[21]}$ genannt. Die jedoch wohl bemerkenswerteste Wirkung beider Moleküle ist die Fähigkeit, schon in nanomolaren 
Konzentrationen $\left(\mathrm{IC}_{50} \sim 10 \mathrm{nM}\right)$ selektiv die V-ATPasen hemmen zu können. ${ }^{[22,23,24]}$ Hingegen werden ATPasen vom P-Typ erst in mikromolarer Konzentration gehemmt und die Aktivität von ATPasen des F-Typs bleibt von Concanamycin A (1) und Bafilomycin $\mathrm{A}_{1}$ (2) gänzlich unbeeinflusst. ${ }^{[25]}$ Die hohe Selektivität, mit der $\mathbf{1}$ und $\mathbf{2}$ schon in Konzentrationen von $0.1-5.0 \mathrm{nM}$ alle bisher bekannten, aus eukaryotischen Zellen isolierten V-ATPasen hemmen können, machten sie interessant als biochemische Werkzeuge zur Untersuchung der Funktion der V-ATPasen. ${ }^{[26]}$ Eine genaue Aufklärung von Wirkort und Wirkmechanismus könnte helfen, Medikamente für Krankheiten zu entwickeln, welche mit einer Fehlbzw. Überfunktion der V-ATPasen in Verbindung gebracht werden. ${ }^{[27]}$

\section{A.2.2 Stand der Arbeiten zur Bestimmung des Wirkortes der V-ATPase-Inhibitoren Bafilomycin und Concanamycin}

Schema 2: Bisher für PAL-Studien verwendete Concanamycinderivate.
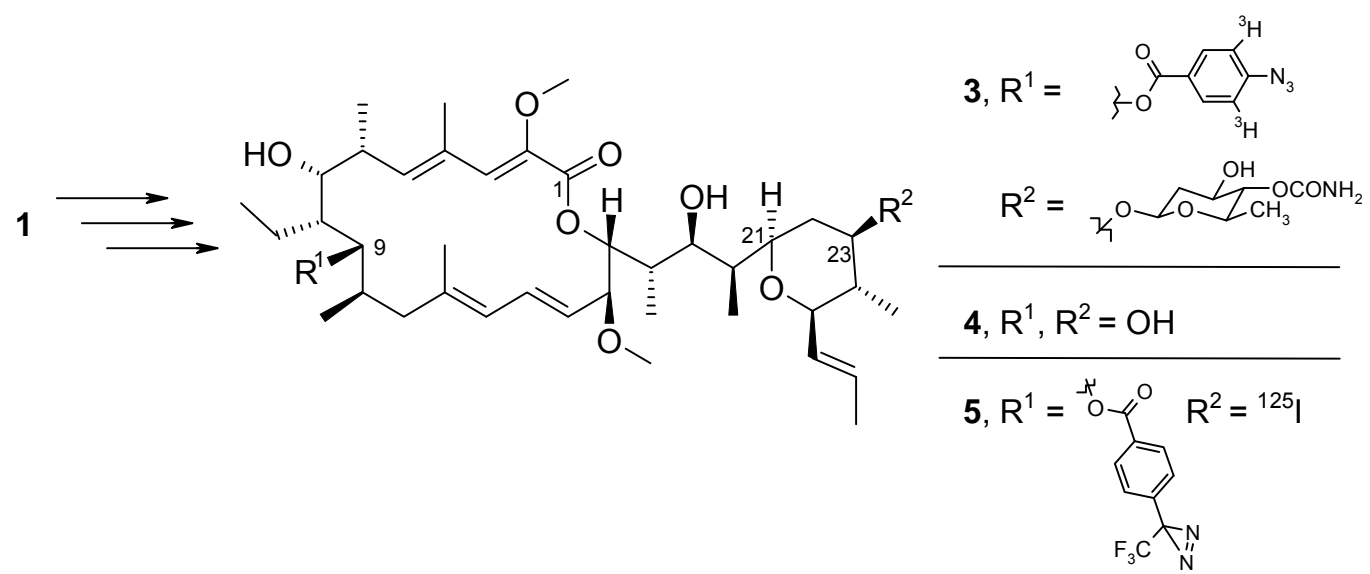

Das erste Concanamycin-Derivat, welches in Photoaffinitätsstudien verwendet wurde, war

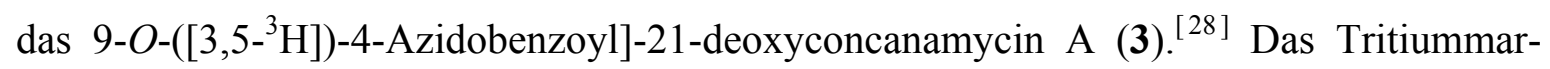
kierte Derivat konnte zwar erfolgreich für Affinitätsstudien mit P-Typ-ATPasen eingesetzt werden, lieferte jedoch keine auswertbaren Ergebnisse bei der Untersuchung der Wirkung an V-ATPasen. Ein möglicher Grund hierfür war die mit $10 \mathrm{mCi} / \mathrm{mmol}$ recht geringe spezifische Radioaktivität und die verhältnismäßig schlechte Nachweisbarkeit von Tritium. ${ }^{[30]}$ Weitere photoaffinitätsbasierte Studien gingen nicht direkt von Concanamycin A (1) aus, sondern vom in Schema 2 gezeigten, stabileren dehydroxylierten und deglycolysierten Concanamycin A - Aglycon (4). ${ }^{[29]}$ Dieses wurde in das Derivat 5 überführt, welches neben einer Diazirin-Gruppe als Photophor auch ${ }^{125} \mathrm{I}$ als radioaktiven 
Marker trug. ${ }^{[30]}$ Anschließende Photoaffinitätsstudien mit dieser Verbindung ${ }^{[31]}$ sowie genetische Studien von Bowman und BowmAN ${ }^{[27,32]}$ lieferten Hinweise darauf, dass die Bindungsstellen beider Plecomacrolide in der c-Untereinheit der V-ATPasen zu finden sind. Die Wirkung beider Verbindungen ist somit wahrscheinlich darin begründet, dass sie die Protonentranslokation durch die c-Untereinheit mittels Blockade der Rotation derselbigen unterbinden. Die konkrete Bindungsstelle innerhalb der c-Untereinheit (c, $c^{\prime}$, $\mathrm{c}^{\prime \prime}$ ), genauso wie die Frage, ob Bafilomycin und Concanamycin an identischen Bindungsstellen binden, konnte jedoch bisher noch nicht genau geklärt werden. ${ }^{[27]}$

Es zeigte sich nämlich, dass die Hemmwirkung von Derivat $\mathbf{5}$ auf die V-Typ ATPase isoliert aus Manduca sexta um drei Größenordnungen geringer war als die des Naturstoffes 1, was die Aufklärung der präzisen Bindungsstelle erschwerte. Der Grund für den Aktivitätsverlust könnte in der Einführung von hydrophoben Substituenten sowohl an C-9 als auch an C-23 des Tetrahydropyran-Ringes begründet sein. Unterstützt wird diese Annahme davon, dass das 21-Deoxyconcanolid A (4) selbst, genauso wie Derivate, welche zwar an C-23 aber nicht an C-9 modifiziert wurden, keine signifikante Verringerung der Aktivität zeigten. ${ }^{[33]}$ Dementsprechend zeigte das nur an C-21 mit einem Dibromdiazirinyllabel substituierte Bafilomycin-Derivat 6 (Abbildung 6) einen deutlich geringeren Aktivitätsverlust als das zweifach substituierte Concanolid 5. ${ }^{[34]}$

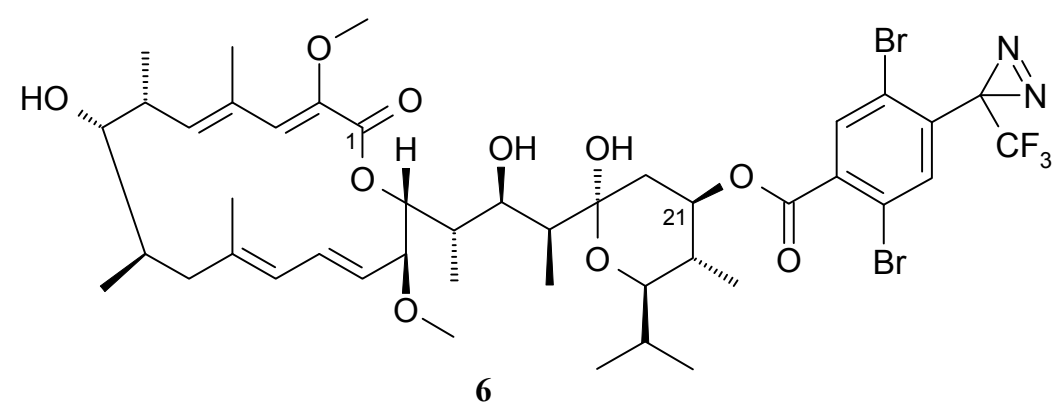

Abbildung 6: Für Photoaffinitätsstudien verwendetes Bafilomycinderivat. ${ }^{[34]}$

Allerdings gab es mit 6 Probleme, die markierten Proteinbestandteile zu identifizieren, da die zwei Bromsubstituenten keine einfache Identifizierung mittels massenspektroskopischer Untersuchungen ermöglichten. 


\section{A.3 Das Collinolacton - ein neuer Naturstoff aus Streptomyces sp.}

Das Collinolacton (7) wurde erstmals in der Göttinger Arbeitsgruppe um Prof. ZEECK aus den Streptomyceten Tü 1892 und Gö 40/10 isoliert. Im Rahmen der Doktorarbeit von H. J. SCHIEWE entstand ein erster Strukturvorschlag, welcher in Abbildung 7 dargestellt ist. ${ }^{[35]}$ Aufgrund eines doppelten Signalsatzes sowohl im ${ }^{1} \mathrm{H}$ - als auch im ${ }^{13} \mathrm{C}-\mathrm{NMR}$-Spektrum ging SCHIEWE davon aus, dass das Collinolacton als E/Z-Isomerengemisch der nicht-konjugierten Doppelbindung zwischen C-14 und C-15 vorliegt. Aus den NMR-Spektren ließ sich ein Verhältnis zwischen $7_{E}$ und $7_{Z}$ von etwa 4:1 ableiten. Im Rahmen ihrer Doktorarbeit untersuchte L. HOFFMANN oben genannte Strukturhypothese für das Collinolacton (7) noch einmal eingehend. ${ }^{[36]}$

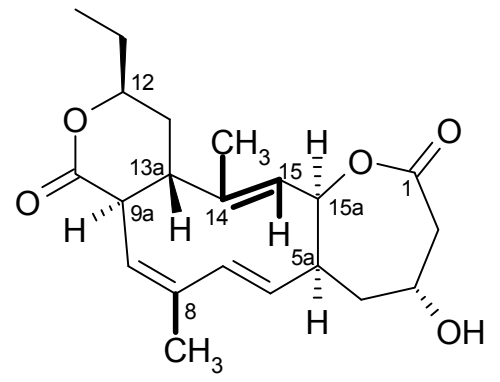

$7_{E}$

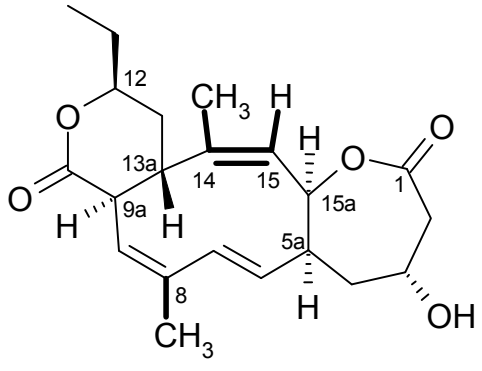

$7_{Z}$

Abbildung 7: Ursprünglich angenommene E/Z-Isomere des Collinolactons (7).

Erste Hinweise darauf, dass das Collinolacton (7) nicht als E/Z-Isomerengemisch vorliegt, lieferten die intensiven, jedoch erfolglosen Versuche, beide Verbindungen chromatographisch voneinander zu trennen (HPLC etc).

Schema 3: Struktur der beiden Rotamere des Collinolactons (7).
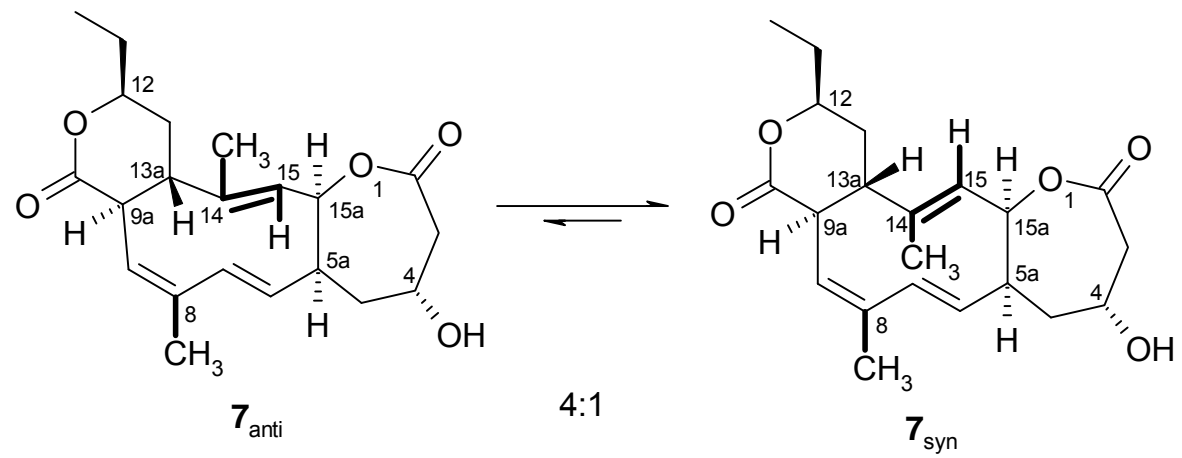

Nach eingehender Untersuchung des Problems mit modernen spektroskopischen Methoden (1D/2D-NOESY), quantenchemischen Berechnungen sowie Hoch- und Tieftemperatur 
NMR-Messungen ergab sich, dass der doppelte Signalsatz des Collinolactons (7) auf eine dynamische Rotationsisomerie des Doppelbindungsfragments C14/C15 um die Bindungen 13a-14 und 15a-15 zurückzuführen ist (Schema 3).

\section{A.3.1 Derivate des Collinolactons und deren Biologische Wirkung ${ }^{[36]}$}

In ersten Bioaktivitätstests im Rahmen der Doktorarbeit von H. J. ScHIEWE zeigte das Collinolacton (7) zytostatische und zytotoxische Eigenschaften, diese konnten jedoch in weiterführenden Tests nicht bestätigt werden. Um diesen Befund näher zu untersuchen, wurden verschiedene Derivate von 7 hergestellt, eine Auswahl ist in Abbildung 8 gezeigt. Es ist bemerkenswert, dass für den Naturstoff 7 bisher keine biologische Wirkung gefunden werden konnte, die an der 4-Position veresterten Derivate 8-10 jedoch sowohl eine potente zytostatische und zytotoxische Aktivität gegen verschiedene Krebszellinien zeigen, als auch eine besonders auffällige Wirkung auf das Mikrotubulinetzwerk des Spindelapparates der untersuchten Zellen.
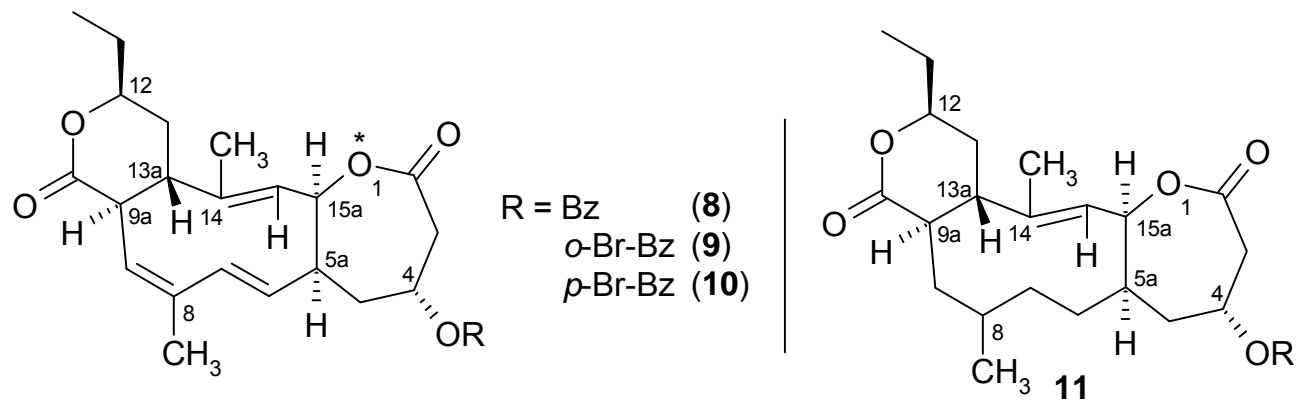

Abbildung 8: Semisynthetische Derivate des Collinolactons (7).

So zeigten die mit Derivaten 8-10 behandelten Zellen statt des normalen bipolaren Spindelapparates häufig eine monoastrale Halbspindelbildung. Der genaue Wirkmechanismus ist noch nicht geklärt. Es gibt aber Hinweise darauf, dass die Wirkung der Derivate auf eine Interaktion mit dem Enzym Kinesin Eg5 zurückzuführen ist. Kinesine sind Enzyme, welche an zellbiologischen Vorgängen wie der Organisation und Funktion der Motorproteine und dem Langstreckentransport von Vesikeln und Organellen beteiligt sind. Das Derivat 11 wurde durch Hydrierung des Naturstoffes 7 an $\mathrm{Pd} / \mathrm{C}$ unter $\mathrm{H}_{2}$-Atmosphäre gewonnen. Es besitzt zwar keine bekannte biologische Wirkung, es ist jedoch interessant, dass hier die Doppelbindungen des konjugierten Diens eine höhere Reaktivität, als die isolierte Doppelbindung aufweisen, was vermutlich auf sterische Effekte zurückzuführen ist. 


\section{A.3.2 Biosynthese des Collinolactons}

Schema 4: Postulierte Biosynthese für das Collinolacton (7).
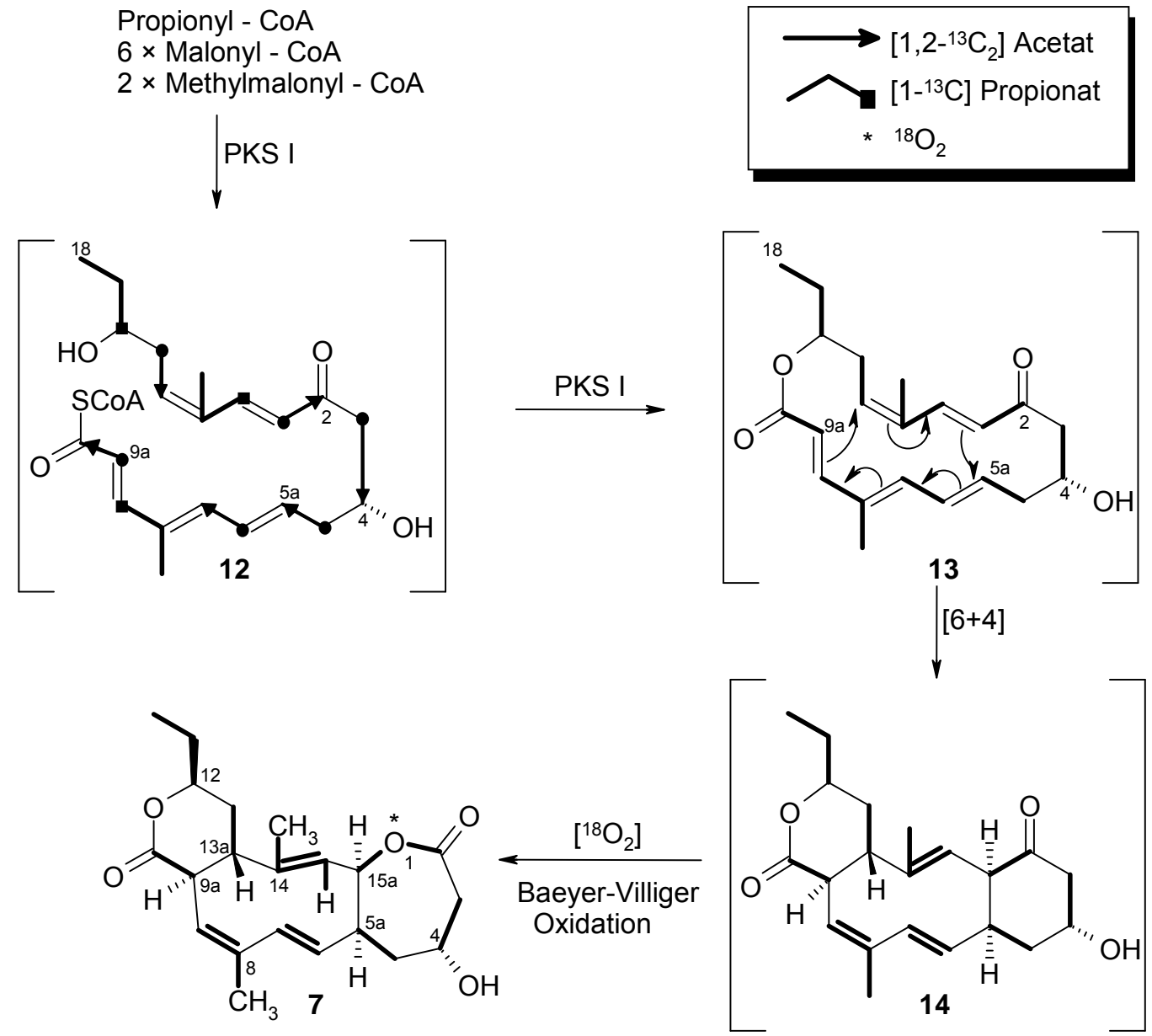

Im Rahmen der Doktorarbeit von L. HOFFMANN wurde auf Grundlage der Ergebnisse aus Fütterungsversuchen mit isotopenmarkierten Vorläufern eine Hypothese zur Biosynthese des Collinolactons (7) erarbeitet, die Resultate sind in Schema 4 dargestellt. Hiernach wird zunächst durch eine Polyketidsynthase vom Typ I das Nonaketid 12 aufgebaut, welches aus sechs Acetat- und drei Propionat-Einheiten besteht. Unter enzymatischer Katalyse bildet sich das Makrolacton 13, welches bei Vorliegen einer günstigen Ausrichtung der Doppelbindungen über eine postulierte [6+4]-Cycloaddition zum Collinoketon 14 reagiert. Ein sicheres Indiz für die als letzten Schritt der Biosynthese postulierte Baeyer-Villiger Oxidation ist der Einbau von ${ }^{18} \mathrm{O}$ in das siebengliedrige Lacton bei Fermentation unter ${ }^{18} \mathrm{O}_{2}$ Atmosphäre. Den endgültigen Beweis jedoch würde die Isolierung des Collinoketons 14 liefern, was durch Zugabe von Enzyminhibitoren möglich sein sollte, bisher jedoch erfolglos blieb. Ein synthetischer Zugang zum Collinoketon und entsprechende Fütterungsexperimente könnten hier den indirekten Nachweis der abschließenden Oxidation liefern. 


\section{A.3.3 Retrosynthetische Analyse und Stand der Arbeiten zum Collinolacton}

Das Collinolacton (7) wurde von P. vON ZEZSCHWITZ ${ }^{[37]}$ auf Grundlage der aus Biosynthese und Derivatisierungen gewonnenen Erkenntnisse (s.o.) retrosynthetisch analysiert. Das Ergebnis ist in Schema 5 dargestellt. Die angestrebte Synthese sollte, ähnlich der postulierten Biosynthese des Collinolactons (7), von einer Baeyer-Villiger-Oxidation des Collinoketons (14) abgeschlossen werden.

Schema 5: Retrosynthetische Analyse des Collinolactons (7). Schlüsselschritte der Synthese sind: A) eine anionische Oxy-Cope-Reaktion und B) eine intramolekulare Diels-Alder Reaktion.
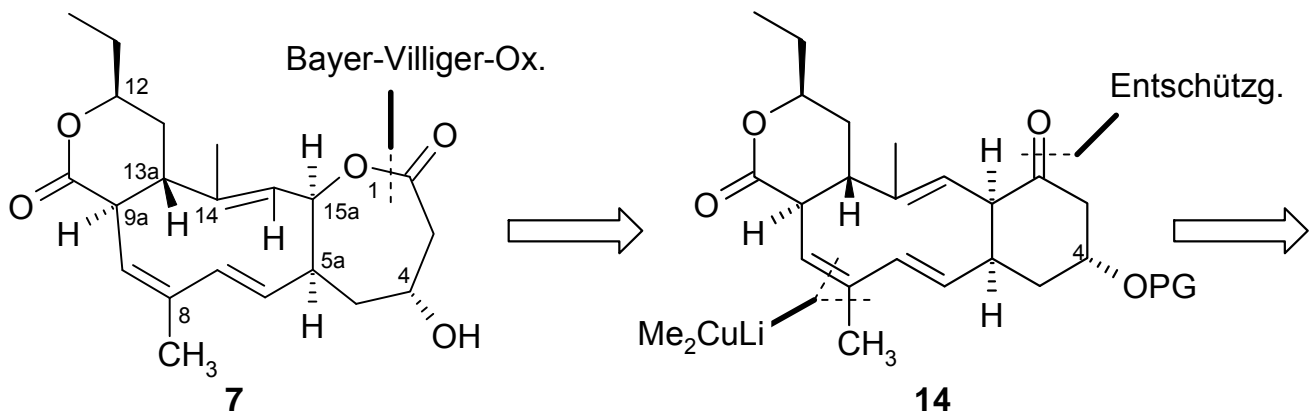

Lactonisierg.

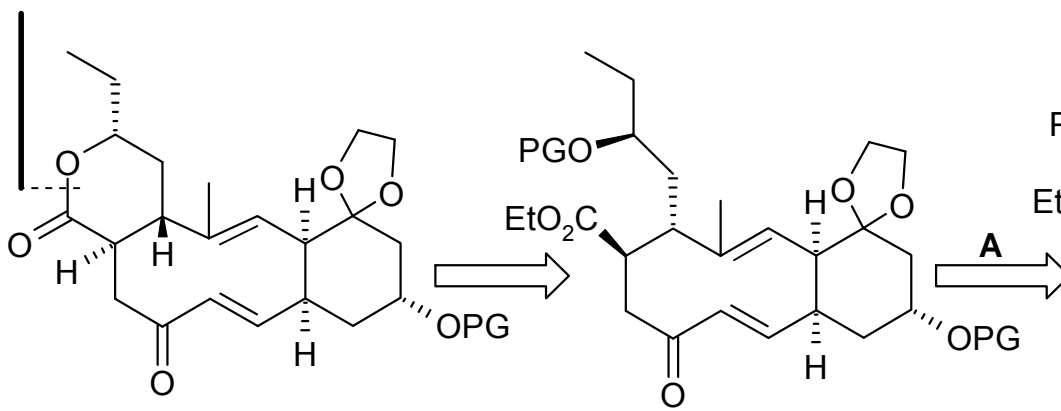

15

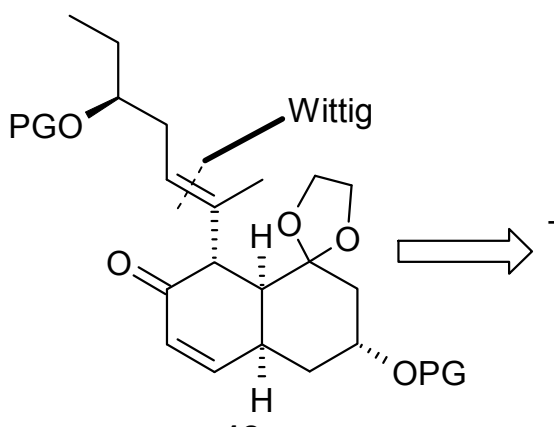

18

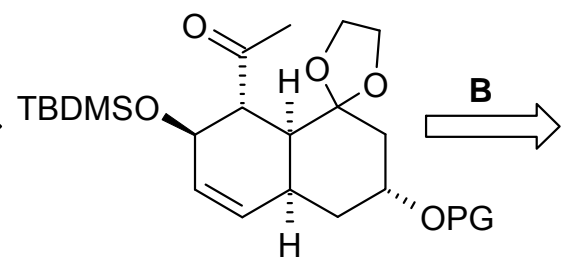

19

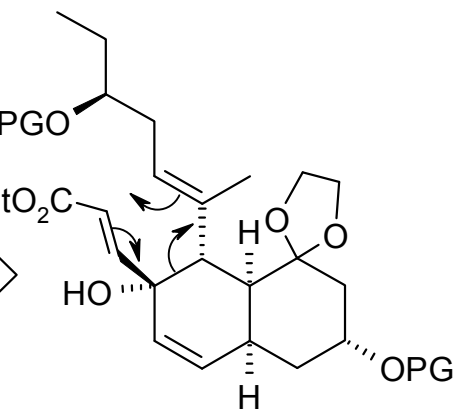

17

Durch diesen biomimtischen Ansatz könnte eine ausreichende Menge des Ketons 14 für etwaige Fütterungsexperimente (in vivo) oder Enzymtests (in vitro) zur Verfügung gestellt 
werden, was die Rolle der Baeyer-Villiger Oxidation als finalen Schritt der Biosynthese bestätigen könnte. Das entsprechende Keton 14 sollte aus 15 durch Einführen der dritten Doppelbindung und Entschützung der Ketofunktion an C-2 gewonnen werden. Die nächsten retrosynthetischen Schritte umfassten die Lactonspaltung, was $\mathbf{1 6}$ ergäbe und die als einen Schlüsselschritt anzusehende anionische Oxy-Cope Umlagerung, mit deren Hilfe der zehngliedrige Ring aufgebaut werden sollte. Die Seitenketten in $\mathbf{1 7}$ sollten sukzessiv durch nukleophile Addition an 18, bzw. Wittig-Reaktion mit 19 eingeführt werden, welches sich wiederum aus der zweiten Schlüsselreaktion der Synthese, nämlich einer intramolekularen Diels-Alder Reaktion (IMDA) des Triens 20 ergeben würde. Durch die Diels-Alder Reaktion sollen vier der sechs Stereozentren des Zielmoleküls aufgebaut werden. Details der Syntheseplanung, einschließlich mechanistischer Überlegungen, werden in Abschnitt II des Hauptteils näher betrachtet. Die angestrebte Totalsynthese sollte innerhalb von ca. 25 linearen Stufen selektiv alle sechs Stereozentren aufbauen, sowie einen Zugang zum zehngliedrigen Makrozyklus mit den drei Doppelbindungen in definierter E/Z-Konfiguration liefern.

Startpunkt der synthetischen Arbeiten von H. SHOJAEI ${ }^{[38]}$ waren das geschützte Z-Butendiol 21 und 2,5-Dimethylfuran (25), (Schema 6). Während sich Keton 24 problemlos in 4 Stufen und mit 60\% Gesamtausbeute herstellen ließ, gab es bereits bei der Synthese des Aldehydes 23 unerwartete Probleme. So lieferten weder die direkte Reduktion des entsprechenden Esters 22 mit DIBAH, noch der Umweg über den entsprechenden Alkohol mit anschließender Oxidation zuverlässig Aldehyd 23. ${ }^{[38]}$

Schema 6: Synthese des IMDA-Vorläufers 20.

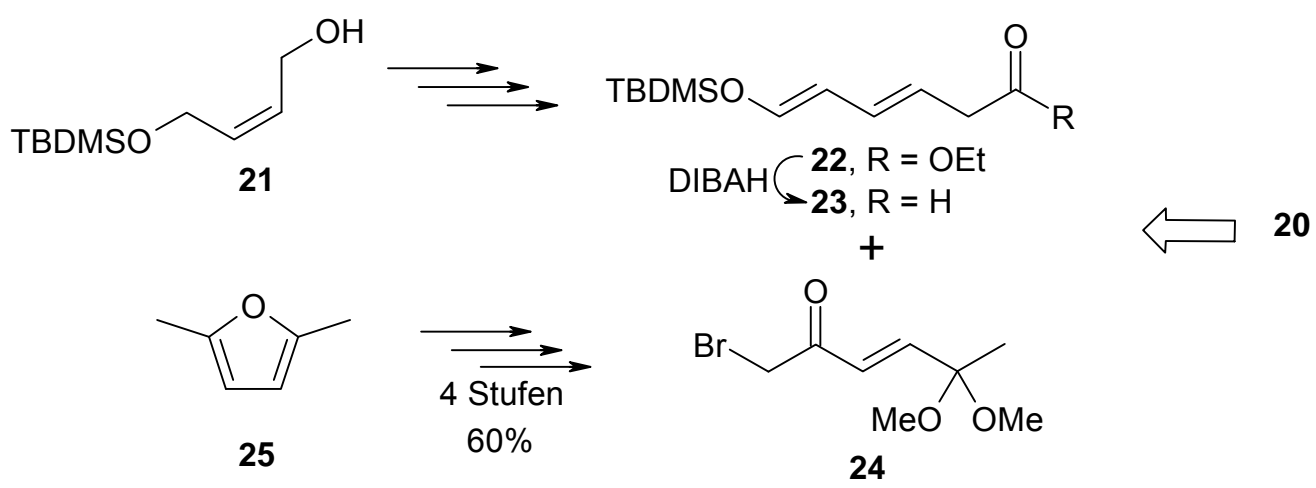




\section{B Aufgabenstellung}

Für vertiefende Photoaffinitässtudien mit den Plecomacroliden Concanamycin (1) und Bafilomycin $\mathrm{A}_{1}$ (2) sollte auf Grundlage des bekannten Photoaffinitätslabels 4-(3Trifluormethyl-3H-diazirin-3-yl)benzoesäure (48) eine Strategie zur Synthese eines radioaktiv markierten Photoaffinitätslabels erarbeitet werden. Dabei sollte ein Zugang gefunden werden, der eine möglichst späte Einführung des radioaktiven Markers ermöglicht, um so die Zahl der Reaktionen mit strahlendem Material möglichst gering zu halten. Außerdem sollte das radioaktive Label möglichst in die Grundstruktur der Verbindung integriert werden, um so eine möglichst „schlanke“ Verbindung zu erhalten, wodurch unerwünschte sterische Wechselwirkungen zwischen markiertem Makrolid und dem Zielprotein verhindern werden sollten. Nach erfolgreicher Synthese des Photoaffinitätslabels sollten zunächst „kalte“ Vorversuche, d.h. mit nicht-radioaktivem Material, zur Funktionalisierung der Naturstoffe durchgeführt werden, wofür ausreichend Material des Labels zur Verfügung gestellt werden musste. Dies wiederum setzte eine optimierte und wenn möglich diversitätsorientierte Synthesestrategie voraus. Nach Abschluss der Vorversuche sollten die entsprechenden radioaktiven Verbindungen hergestellt und den Kooperationspartnern $^{\dagger}$ für die Wirkortbestimmungen zur Verfügung gestellt werden. Bei Erfolg der Versuche mit den Plecomacroliden 1 und $\mathbf{2}$ sollten noch weitere Naturstoffe mit dem radioaktiven Label versehen werden.

Nach Abschluss der synthetischen Arbeiten zur Wirkortbestimmung der V-ATPase Inhibitoren sollte die Totalsynthese des Collinolactons (7) weiterverfolgt werden. Dabei stand zunächst die Synthese des Triens 20, dem Vorläufer des ersten Schlüsselschrittes, der intramolekularen Diels-Alder Reaktion, im Vordergrund. Besonderes Augenmerk lag dabei zunächst auf der bereits als schwierig erkannten Synthese des Aldehyds 23. Bei Bedarf sollte die Synthesestrategie für den IMDA-Vorläufer angepasst werden. Letztlich sollte es der gewählte Zugang es ermöglichen, möglichst schnell größere Mengen des Diels-AlderProduktes zu synthetisieren. Nach erfolgreicher Synthese des Triens sollte eine genaue Untersuchung der Diels-Alder Reaktion durchgeführt werden und ggf. die nächsten Syntheseschritte in Angriff genommen werden. Durch Modellstudien zur anionischen OxyCope Reaktion sollten Rückschlüsse auf die Durchführbarkeit der gewählten Synthesestrategie und insbesondere die Stereoselektivität der Reaktion gewonnen werden.

\footnotetext{
${ }^{\dagger}$ Prof. Dr. H. Wieczorek, Dr. M. Huss, Tierphysiologie, FB Biologie, Universität Osnabrück.
} 


\section{Theoretischer Teil}

\section{Hauptteil I - Synthese eines radioaktiven Photoaffinitätslabels}

Aufgrund der unter A.1.3 bereits erwähnten potentiellen Probleme bei der Verwendung der Biotin/Avidin Methode und den Ergebnissen von ZEECK et. al. mit dem radioaktiv markierten Concanolid 5 wurde die Synthese eines sterisch möglichst anspruchslosen Photoaffinitätslabels angestrebt, um einem Aktivitätsverlust durch sterische Wechselwirkungen der $\mathrm{zu}$ untersuchenden Biomoleküle mit dem Zielprotein entgegenzuwirken. Als Photophor sollte eine Trifluormethyldiazirinyl-Gruppe dienen und zur Identifizierung der markierten Proteinbestandteile sollte das Label einen radioaktiven Tracer enthalten.

\section{I.1 Zur Wahl des Photophors}

Diazirine wurden zuerst von SMITH und KNOWLES ${ }^{[39]}$ beschrieben und besitzen von allen photolytisch aktivierbaren Verbindungen die wohl besten photochemischen Eigenschaften für die Photoaffinitätsmarkierung. ${ }^{[6 a]}$ In Schema 7 sind die wesentlichen Reaktionswege gezeigt, über die ein Trifluormethyldiazirin nach Bestrahlung mit Licht (Wellenlänge $>350 \mathrm{~nm}$ ) abreagieren kann.

Schema 7: Reaktionsweg des Trifluormethyldiazirins 26 nach photochemischer Anregung.<smiles>FC(F)(F)C1(c2ccccc2)N=N1</smiles>

26<smiles>COC(c1ccccc1)C(F)(F)F</smiles>

29

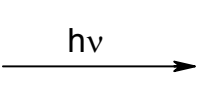

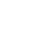

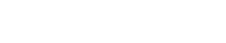

$26 a$
$-N_{2}$

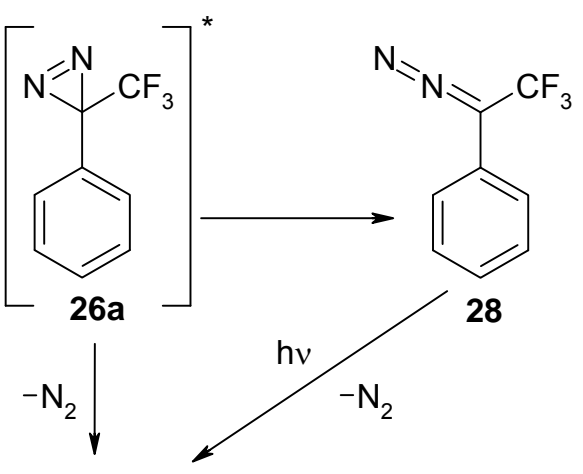<smiles>FC(F)(F)c1ccccc1</smiles>

27

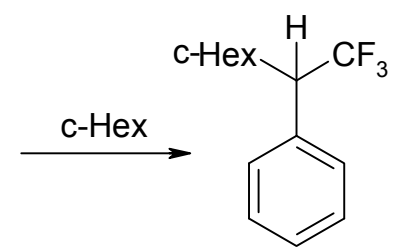

30

Nach der Aktivierung des Trifluormethyldiazirins 26 können sich aus der aktivierten Spezies 26a entweder das gewünschte Carben 27 oder die Diazoverbindung 28 bilden. 
Diazoverbindungen sind stark alkylierende Verbindungen und werden für die meisten Dunkelreaktionen, also unerwünschte Nebenreaktionen in Abwesenheit von Licht während der Verwendung von Diazirinen, verantwortlich gemacht. Um diese unerwünschten Nebenreaktionen zu unterbinden, synthetisierte BRUNNER 1980 die 3-Trifluormethyldiazirine. ${ }^{[40]}$ Diese können zwar immer noch die Diazoverbindungen generieren, durch den starken Elektronenzug der $\mathrm{CF}_{3}$-Gruppe sind diese allerdings derart stabilisiert, dass keine Nebenreaktionen stattfinden. Nach erneuter Aktivierung durch ein Photon bildet auch die Diazoverbindung das gewünschte Carben 27, welches in Abwesenheit anderer Reaktionen je nach Lösungsmittel zu den Produkten 29 oder 30 reagiert. ${ }^{[6 a]}$

\section{I.2 Zur Wahl des Tracers}

Abbildung 9 zeigt eine Auswahl literaturbekannter Photoaffinitätslabel mit radioaktivem Tracer. Das von BRUNNER et al. beschriebene Photoaffinitätslabel 31 ${ }^{[41]}$ trägt ${ }^{125} \mathrm{I}$ als Tracer und wurde über einen Halogeneinbau durch Stannylierung mit Hexabutyldistannan und anschließender Umsetzung mit $\mathrm{Na}^{125}$ I hergestellt. Die Vorteile dieses Isotopes liegen in der hohen spezifischen Aktivität des ${ }^{125} \mathrm{I}$, was den Nachweis markierter Proteinbausteine erleichtert, und im verhältnismäßig niedrigen Preis des $\mathrm{Na}^{125} \mathrm{I}$. Dem gegenüber stehen jedoch die hohe Toxizität der in der Synthese verwendeten Organozinn- und Organothaliumverbindungen, sowie die mit 60 Tagen geringe Halbwertzeit des ${ }^{125}$ I-Isotopes.

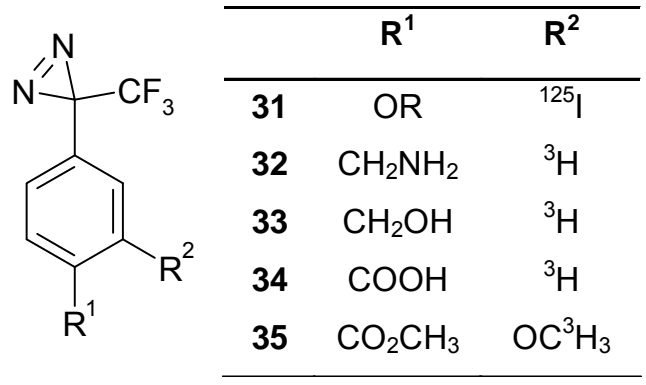

Abbildung 9: Beispiele für radioaktiv markierte Photoaffinitätslabel.

Die geringe Halbwertzeit hätte für die geplante zeitlich versetzte Funktionalisierung verschiedener Naturstoffe eine Wiederholung der aufwendigen Synthese des Labels erforderlich gemacht, was nicht praktikabel gewesen wäre.

Bei den Verbindungen 32-34, ${ }^{[42]}$ sowie $\mathbf{3 5}^{[43]}$ wurde Tritium als Radiomarker verwendet. Mit einer Halbwertzeit von 12.3 Jahren besitzt Tritium sowohl eine ausreichend lange 
Halbwertzeit als auch eine hohe spezifische Radioaktivität. Bei beiden beschriebenen Synthesen wurde jedoch hochtoxisches Thallium(III)-trifluoracetat verwendet. Ein weiterer potentieller Nachteil des Tritiums ist die manchmal beobachtete schwierige Nachweisbarkeit aufgrund der geringen Energie der emittierten $\beta$-Strahlung. ${ }^{[44]}$

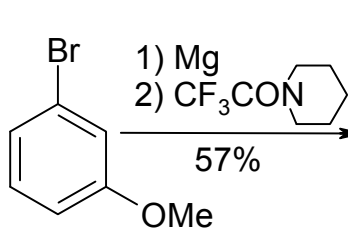

36<smiles>CCN(C)Cc1cccc(OC)c1</smiles>

40<smiles>COc1cccc(C(=O)C(F)(F)F)c1O</smiles>

37<smiles>COc1cccc(/C(=N\O)C(F)(F)F)c1</smiles>

38<smiles>COc1cccc(/C(=N\OS(N)(=O)=O)C(F)(F)F)c1</smiles>

39<smiles>COC(=O)C(c1c(OC)cccc1C1(C(F)(F)F)N=N1)C(F)(F)F</smiles>

41<smiles>[R]Oc1cc(C2(C(F)(F)F)N=N2)ccc1C(C)=O</smiles>

Schema 8: Synthese des ${ }^{14} \mathrm{C}$-markierten Photoaffinitätslabels 42a nach HATANAKA et al.

Das Kohlenstoff-14-Isotop besitzt als $\beta$-Strahler sehr gute Strahlungseigenschaften, eine hohe spezifische Aktivität und eine Halbwertzeit von mehr 5000 Jahren, es ist somit ideal für biologische Untersuchungen. Die Synthese des ${ }^{14} \mathrm{C}$-markierten Photoaffinitätslabels 42a ist in Schema 8 gezeigt. ${ }^{[43]}$ Zunächst erfolgte der Aufbau des Diazirins: Ausgehend von 3Bromanisol (36) gelangt man über eine Grignard-Reaktion zu Keton 37, welches mit Hydroxylamin zu Oxim 38 reagiert. Tosylierung und anschließende Umsetzung des Reaktionsproduktes 39 mit Ammoniak ergibt das Diaziridin 40, welches in Anwesenheit von Triethylamin mit tert-Butylhypochlorit zum Diazirin 41 oxidiert wird. Das so erhaltene Produkt wurde mit Thallium(III)-trifluoracetat umgesetzt und anschließend Pd-katalysiert in den Ester 42 überführt. Dieser wurde, nicht sehr atomökonomisch, zunächst mit $\mathrm{PBr}_{3} \mathrm{zu} 43$ entschützt und anschließend mit ${ }^{14} \mathrm{C}$-Methyliodid zu 42a umgesetzt. In einer achtstufigen Synthese unter Verwendung von hochtoxischem Thallium und Kohlenmonoxid erhält man das radioaktive Label 42a in $9 \%$ Ausbeute mit einer ausreichend hohen spezifischen Aktivität von $54.1 \mathrm{mCi} / \mathrm{mmol}$. Neben der doch recht umständlichen Synthese und der Verwendung hochtoxischer Reagenzien sind weitere Nachteile die Verwendung von Methyliodid als eher teurer ${ }^{14} \mathrm{C}$-Quelle und die ebenfalls kostenintensive Aufreinigung der 
Produkte mittels präparativer HPLC. ${ }^{[45]}$ Eine Recherche ergab, dass eine der preisgünstigsten ${ }^{14} \mathrm{C}$-Quellen $\mathrm{Ba}^{14} \mathrm{CO}_{3}$ ist. Da für die Kupplung des Photoaffinitätslabels an die Naturstoffe 1 und 2 eine Carbonsäurefunktion notwendig war, bot sich eine Synthesestrategie an, welche als zentralen Schritt eine Carboxylierung mit ${ }^{14} \mathrm{CO}_{2}$ beinhaltet.

\section{I.3 Synthese des Photoaffinitätslabels}

Aus den oben beschriebenen Vorüberlegungen ergab sich Diazirinylcarbonsäure 48, welche schon erfolgreich für Untersuchungen an dem Concanolid 4 eingesetzt wurde, ${ }^{[30]}$ als ideales Photoaffinitätslabel. In der Literatur sind bereits mehrere Synthesen für Diazirin 48 beschrieben worden (Schema 9 und Schema 10). Alle Synthesen nutzten ein Bromid als Ausgangspunkt für die Trifluormethyldiazirinyl-Gruppe, welche über die, erstmals von BRUNNER beschriebenen, fünf Standardumsetzungen a) Grignard-Reaktion, b) Oximbildung, c) Tosylierung, d) Diaziridinbildung und e) Oxidation zum Diazirin synthetisiert wird. ${ }^{[40]}$

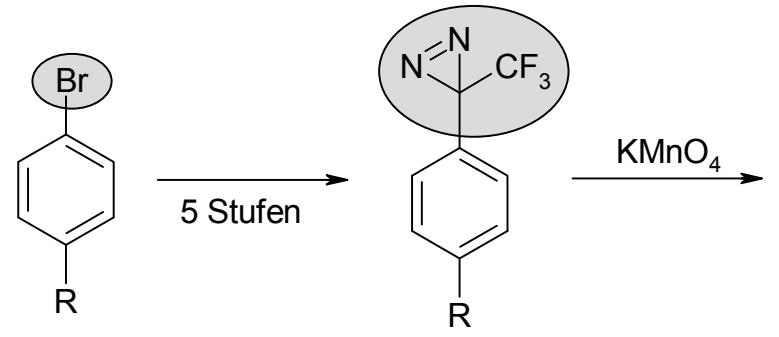
44, $\mathrm{R}=\mathrm{CH}_{3}$
45, $=\mathrm{CH}_{2} \mathrm{OTBDMS}$
46, $\mathrm{R}=\mathrm{CH}_{3}$
47, $=\mathrm{CH}_{2} \mathrm{OH}$
$58 \%$
$72 \%$

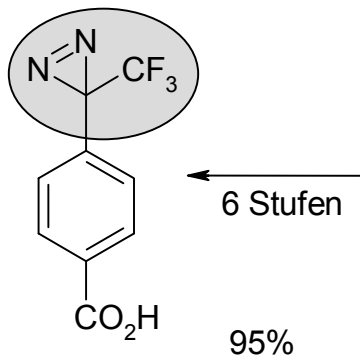

48

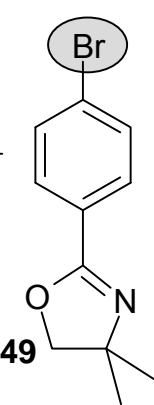

Schema 9: Verschiedene Synthesestrategien zur Synthese des Labels 48.

Zur Einführung der Säurefunktion wurden entweder eine Methylgruppe (wie in 44 bzw. 46) oder ein Alkohol (wie in 45 bzw. 47) als Vorläufer verwendet, welche dann im letzten Schritt der Synthese mit Kaliumpermanganat zur Säure oxidiert wurden. ${ }^{[42,46 a]}$ In einer weiteren Synthese diente ein Oxazolin $\mathbf{4 9}^{[46 b]}$ als Schutzgruppe, aus der im letzten Schritt die Säurefunktion freigesetzt wurde. Diese Ansätze lieferten zwar teilweise sehr gute Ausbeuten, besitzen aber nicht die Möglichkeit, ein ${ }^{14} \mathrm{C}$-markiertes Kohlenstoffatom in die Grundstruktur zu implementieren. Aldehyd 52, ein nützlicher Vorläufer zur Synthese verschiedener Photoaffinitätslabel, wurde durch eine Friedel-Crafts Acylierung des Diazirins 51 synthetisiert, welcher wiederum in Methanol mit Caro's Säure zu Ester 53 oxidiert werden konnte (Schema 10). ${ }^{[4]}$ Obwohl auch hier beide Reaktionen mit guter 
Ausbeute verlaufen, ist es problematisch, dass der verwendete Dichlormethylmethylether als stark kanzerogen gilt.

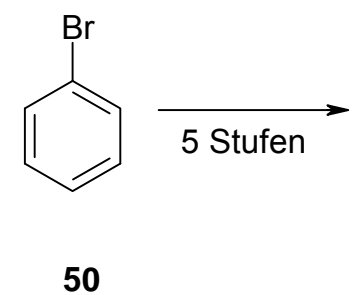<smiles>FC(F)(F)C1(c2ccccc2)N=N1</smiles>

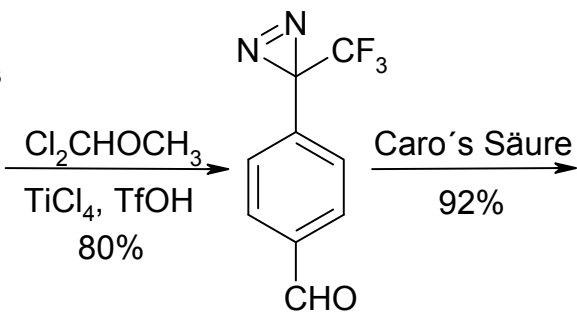

52<smiles>CC(=O)c1ccc(C2(C(F)(F)F)N=N2)cc1</smiles>

53

Schema 10: Synthese des Diazirinylbenzoesäureesters 53 nach HATANAKA et al. ${ }^{[47]}$

Zusammenfassend ist festzuhalten, dass zu Beginn dieser Arbeiten keine der publizierten Synthesen die Möglichkeit bot, zu einem späten Zeitpunkt der Synthese des Diazirins eine Carboxylierung durchzuführen. Um dieses Ziel zu ermöglichen, sollte in der anvisierten Synthese sowohl für die Diazirinyl- als auch für die Carbonsäurefunktion ein Bromid als Vorläufer verwendet werden. Die vielfältigen Reaktionsmöglichkeiten des Bromids sollten einen einfachen Zugang nicht nur zu Carbonsäure 48, sondern auch noch zu einer breiten Palette weiterer nützlicher Verbindungen ermöglichen.

\section{I.3.1 Synthese des Photoaffinitätslabels aus 1,4-Dibrombenzol}

Die Synthese startete ausgehend vom preisgünstigen 1,4-Dibrombenzol (54), welches durch Grignard-Reaktion der entsprechenden Mg-Verbindung mit Trifluoressigsäureethylester in das Keton $\mathbf{5 5}$ überführt wurde. ${ }^{[48]}$ Nach bereits beschriebener Reaktionsfolge wurde 55 zunächst in das Oxim 56, dann in Tosylat 57 und schließlich in das Diaziridin 58 überführt (Schema 11). Alle Umsetzungen, auch die in einem Autoklaven in flüssigem Ammoniak durchgeführte Umsetzung des Tosylats zum Diaziridin, sind problemlos in Multigrammmaßstäben (30-40 g Tosylat) durchführbar. Das Diaziridin 58 wurde anschließend problemlos mit tert-Butylhypochlorit $(t$-BuOCl) als Oxidationsmittel in das Diazirin 59 überführt. ${ }^{[46 \mathrm{~b}]}$ In späteren Versuchen zeigte sich, dass die Oxidation mit Iod als Oxidationsmittel in Gegenwart von Triethylamin eine einfachere Versuchsdurchführung bei gleich guten oder besseren Ausbeuten ermöglichte. ${ }^{[49]}$ 
Schema 11: Synthese des $p$-Bromdiazirins 60.<smiles>Brc1ccc(Br)cc1</smiles>

1) $\mathrm{Mg}, \mathrm{Et}_{2} \mathrm{O}$ $\underset{-78{ }^{\circ} \mathrm{C} \rightarrow \mathrm{rt}}{\stackrel{\mathrm{CF}_{3} \mathrm{CO}_{2} \mathrm{Et}}{\longrightarrow}}$ $16 \mathrm{~h}$

54<smiles>O=C(c1ccc(Br)cc1)C(F)F</smiles>

55

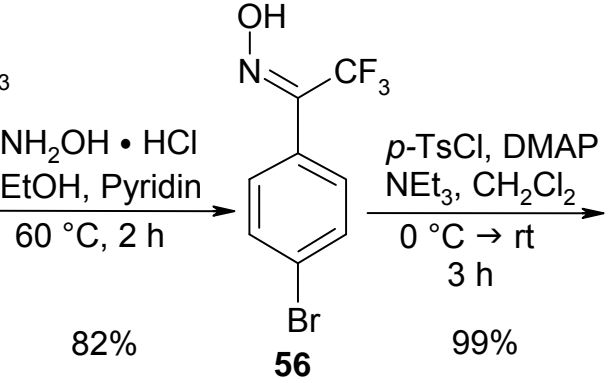

56

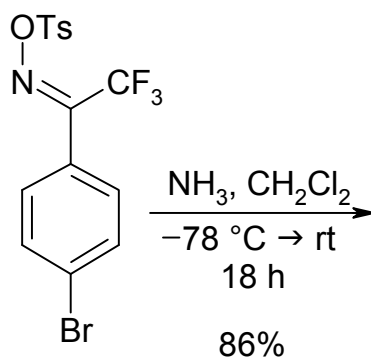

57<smiles>ClC1(c2ccc(Br)cc2)NN1</smiles>

58
$t-\mathrm{BuOCl}, \mathrm{NEt}_{3}$ $\underset{\mathrm{EtOH}, t-\mathrm{BuOH}}{0{ }^{\circ} \mathrm{C}, 2 \mathrm{~h}}$ $86 \%$<smiles>FC(F)(F)C1(c2ccc(Br)cc2)N=N1</smiles>

59

\section{I.3.2 Versuche zur direkten Carboxylierung des $p$-Bromdiazirins}

Nach der erfolgreichen Synthese des Diazirins 59 folgten Versuche zum Lithium-HalogenAustausch und der anschließenden Carboxylierung (Schema 12). Obwohl sich die Trifluormethyldiazirinyl-Gruppe unter einer ganzen Reihe von Reaktionsbedingungen, so zum Beispiel in Gegenwart von starken Basen oder starken Säuren, als stabil erwiesen hat, war der Versuch des Li-Halogenaustausches zu 61 mit $n$-BuLi nicht erfolgreich. Auch Versuche mit sec- und tert-Butyllithium, sowie unter verschiedenen Reaktionsbedingungen führten nicht zur Zielverbindung 48 (Tabelle 1). Eine Analyse der erhaltenen Reaktionsgemische zeigte, dass die Diazirin-Gruppe unter den Lithiierungsbedingungen ${ }^{[50]}$ nicht stabil ist. Bei Verwendung von $n$-Butyllithium kommt es zwar teilweise zu einer Umsetzung des Edukts, allerdings bildet sich nicht die gewünschte Titelverbindung, sondern das $n$-Butyladdukt 62. Im Gegensatz dazu konnten bei der Verwendung von sec-bzw. tert-Butyllithium in den sehr komplexen Reaktionsgemischen nur die Carboxylierungsprodukte der jeweils eingesetzten Li-Organyle identifiziert werden. Auch wenn bisher keine vergleichbaren Additionen von Li-Organylen an die Diazirinyl-Gruppe beschrieben sind, beobachteten SAMMELSON und CASIDA $^{[51]}$ beim Versuch der Ortholithiierung des Diazirins 60<smiles>FC(F)(F)c1cc(Cl)c(-n2cc(C3(C(F)(F)F)N=N3)cn2)c(Cl)c1</smiles>

60 
ebenfalls nur die Zersetzung des Eduktes, führten dies jedoch nicht explizit auf die Instabilität des Diazirins zurück.

Schema 12: Versuche zur direkten Lithiierung des Bromids 59 mit anschließender Carboxylierung waren nicht erfolgreich.

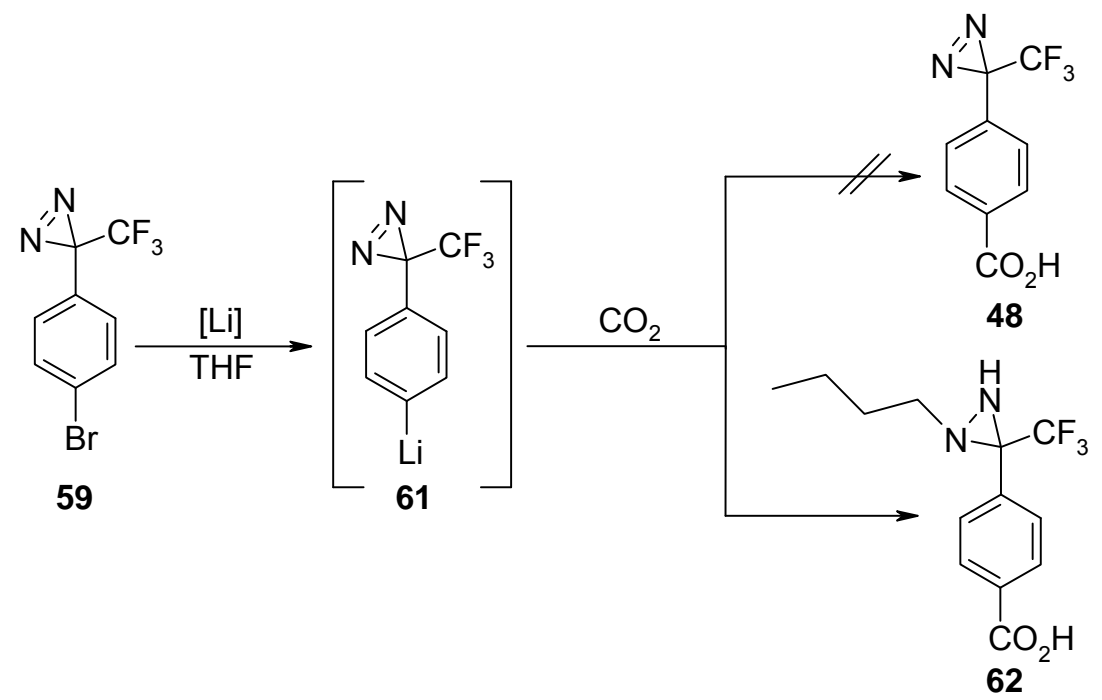

Tabelle 1

\begin{tabular}{ccccccc}
\hline $\mathbf{N r}$ & $\mathbf{T}\left[{ }^{\circ} \mathbf{C}\right]$ & $\mathbf{t}[\mathbf{h}]$ & {$[\mathbf{L i}]$} & $\mathbf{e q}$ & Add. & Hauptprodukt \\
\hline 1 & $-95 \rightarrow-30$ & 11 & $n$-BuLi & 1.1 & - & $\mathbf{6 2}$ \\
2 & -85 & 4.0 & $t$-BuLi & 2.1 & - & - \\
3 & $-85 \rightarrow \mathrm{RT}$ & 12 & $s e c-\mathrm{BuLi}$ & 1.0 & - & Isovaleriansäure \\
4 & $-90 \rightarrow \mathrm{RT}$ & 2.5 & $t$-BuLi & 1.0 & - & Pivalinsäure \\
5 & -90 & 0.5 & $s e c-\mathrm{BuLi}$ & 1.0 & $\mathrm{HMPA}$ & - \\
\hline
\end{tabular}

Da sich das Diazirin somit in Gegenwart der nukleophilen Li-Organyle als instabil erwies, wurde versucht, das entsprechende Grignard-Reagenz des Bromids 59 herzustellen (Schema 13). Die ersten Versuche zur Metallierung wurden nach Standardprotokoll ${ }^{[171 b]}$ mit Magnesiumspänen in THF oder Ether und längerem Erhitzen unter Rückfluss durchgeführt (Tabelle 2, Einträge 1 und 2). Anschließend wurde $\mathrm{CO}_{2}$, generiert aus Trockeneis, in das Reaktionsgemisch geleitet. Unter diesen Bedingungen konnte aus den Reaktionsgemischen ausschließlich Edukt isoliert werden.

Beim Versuch mit iso-Propylmagnesiumbromid (Eintrag 3) konnte als Produkt nur isoButansäure isoliert werden, was darauf schließen lässt, dass hier ebenfalls keine Ummetallierung stattfand. Um doch noch das gewünschte Grignard-Reagenz von $\mathbf{5 9}$ zu 
erzeugen, wurden die folgenden Versuche (Einträge 4-7) mit hochreaktiven RIEKE ${ }^{[52]} \mathrm{Mg}$ Spezies durchgeführt. Diese erhält man durch in situ Reduktion von $\mathrm{MgCl}_{2}$ mit elementarem Kalium als eine schwarze Suspension sehr fein verteilten Magnesiums, wodurch eine immens große Reaktionsoberfläche entsteht. Mit Hilfe der so erhaltenen, hoch reaktiven Magnesiumspezies ist es sogar möglich, 1,4-Dibrombenzol in das DiGrignard-Reagenz zu überführen. Trotz der erwiesenen Reaktivität der Magnesiumspezies konnten bei keinem der Versuche Hinweise auf eine erfolgreiche oxidative Addition gefunden werden. Um die Reaktivität des erzeugten Rieke-Magnesiums zu überprüfen, wurde $p$-Bromanisol zum Reaktionsgemisch gegeben und anschließend $\mathrm{CO}_{2}$ durch die Lösung geleitet. Auch bei diesem Versuch konnten weder die zu erwartende Carbonsäure noch das Hydrolyseprodukt isoliert werden.

Schema 13: Verbindung 59 ließ sich nicht in ihre Grignard-Verbindung überführen.

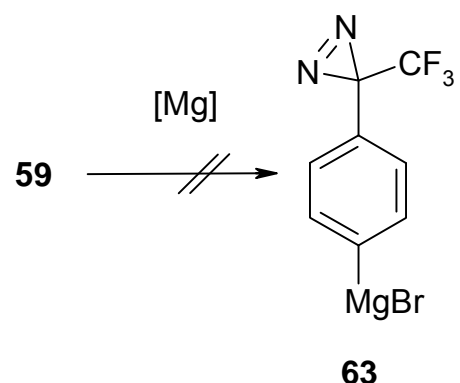

Tabelle 2

\begin{tabular}{ccccccc}
\hline $\mathbf{N r}$ & $\mathbf{T}\left[{ }^{\circ} \mathbf{C}\right]$ & $\mathbf{t}[\mathbf{h}]$ & Add. & eq & $\mathbf{L M}$ & Hauptprodukt \\
\hline 1 & Rückfluss & 3 & $\mathrm{Mg}$ & 4.0 & $\mathrm{Et} 2 \mathrm{O}$ & Edukt \\
2 & Rückfluss & 4 & $\mathrm{Mg}$ & 4.0 & $\mathrm{THF}$ & Edukt \\
3 & $-30 \rightarrow \mathrm{RT}$ & 3 & $i-\mathrm{PrMgBr}$ & 2.0 & $\mathrm{THF}$ & $i s o$-Butansäure \\
4 & $-110 \rightarrow \mathrm{RT}$ & 16 & Rieke- $\mathrm{Mg}^{\mathrm{a}}$ & 2.0 & $\mathrm{THF}$ & Edukt \\
5 & -78 & 1 & Rieke- $\mathrm{Mg}^{\mathrm{a}}$ & 4.0 & $\mathrm{THF}$ & $\begin{array}{c}\text { Zugabe von } p \text {-Br- } \\
\text { Anisol - keine Rkt.) }\end{array}$ \\
6 & -110 & 16 & Rieke- $\mathrm{Mg}^{\mathrm{b}}$ & 2.0 & $\mathrm{THF}$ & Edukt \\
7 & $-78 \rightarrow \mathrm{RT}$ & 1 & Rieke- $\mathrm{Mg}^{\mathrm{a}}$ & 4.0 & $\mathrm{THF}$ & Edukt \\
\hline a) Generiert aus $\mathrm{MgCl}_{2}, \mathrm{KI}$ und Kalium; b) Generiert aus $\mathrm{MgCl}_{2}$ und K. &
\end{tabular}

Wahrscheinlich wird die oxidative Addition durch Koordination der Stickstoffatome des Diazirins an die aktivierte Metalloberfläche und die daraus resultierende Desaktivierung des Magnesiums verhindert. Diese Beobachtung wurde auch schon von KNochel und Mitarbeitern für verschiedene Cyanide beschrieben. ${ }^{[53]}$ Aufgrund der erfolglosen Versuche 
zur Carboxylierung, bzw. zum Halogen-Metallaustausch mit Diazirin 60 wurde eine Strategie ausgehend von der stabileren Vorstufe, dem Diaziridin 59, entwickelt.

\section{I.3.3 Darstellung des zweifach silylierten Diaziridins}

Vor der Lithiierung war eine Schützung der beiden aziden Protonen der Diaziridin-Gruppe notwendig. Die gewählte Schutzgruppe sollte dabei zwei Bedingungen erfüllen: Zum Einen sollte sie eine leichte Synthese des zweifach geschützten Diaziridins ermöglichen und zum Anderen sollte schon während der wässrigen Aufarbeitung eine vollständige Entschützung stattfinden. Diese Bedingungen schien die Trimethylsilyl-Schutzgruppe am ehesten zu erfüllen. Um die geeigneten Reaktionsbedingungen $\mathrm{zu}$ ermitteln, wurden zunächst zwei orientierende Versuche in NMR-Röhrchen durchgeführt. Bei beiden Reaktionen wurden 5 eq Triethylamin als Base und $\mathrm{CDCl}_{3}$ als Lösungsmittel verwendet, als Silylierungsreagenz dienten TMSCl bzw. TMSOTf. Der Reaktionsfortschritt wurde mittels ${ }^{1}$ H-NMR-Spektroskopie im Abstand von 60-90 min verfolgt. Bei Verwendung von TMSCl konnte auch nach $16 \mathrm{~h}$ bei RT kein vollständiger Umsatz festgestellt werden. Im Gegensatz dazu zeigte sich bei der Reaktion mit TMSOTf nach 3.5 h kein Edukt mehr.

Schema 14: Bis-TMS-geschützte Diazirine 65, 66 und 67.
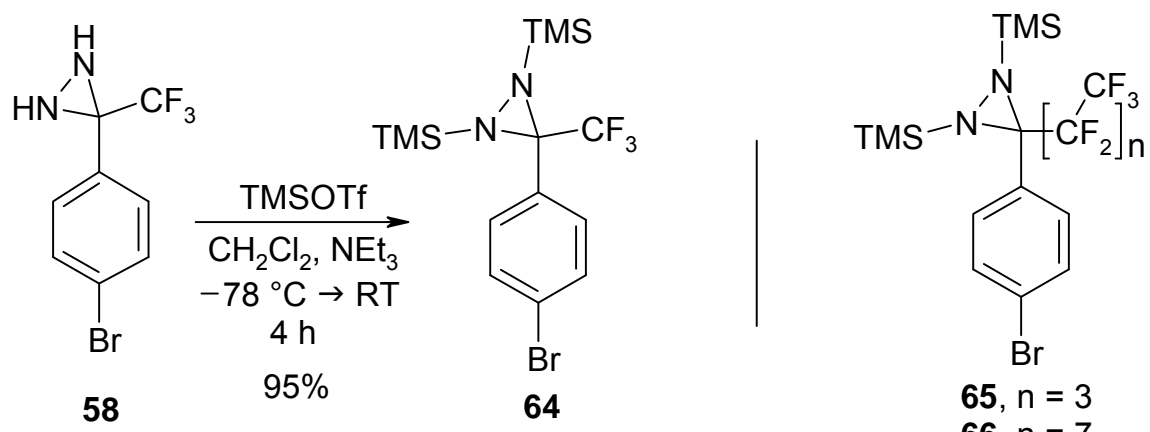

$65, \mathrm{n}=3$

66, $\mathrm{n}=7$

Die TMS-Schützung des Diaziridins 58 wurde unter besonders sorgfältigem Ausschluss von Wasser durchgeführt, da das Bis-TMS-Diaziridin 64 als sehr instabil gegenüber Wasserspuren anzusehen war. Die Reaktion wurde in Dichlormethan durchgeführt, als Base diente ein Überschuss Triethylamin (Schema 14). Der Reaktionsverlauf wurde per ${ }^{1} \mathrm{H}-\mathrm{NMR}$ Spektroskopie verfolgt, wofür $0.1 \mathrm{~mL}$ Proben aus der Reaktionsmischung entnommen und im Vakuum konzentriert wurden. Bei unvollständigem Umsatz wurden noch 0.5 eq TMSOTf zugesetzt; war der Umsatz vollständig, wurde das Dichlormethan 
unter vermindertem Druck entfernt und der gelbe, ölige Rückstand mehrmals mit Hexan extrahiert und das Lösungsmittel im Vakuum entfernt. Dieser Vorgang wurde so oft wiederholt, bis nach Entfernen des Lösungsmittels ein farbloser Feststoff zurückblieb. Diese Aufarbeitung lieferte das geschützte Produkt in guter Reinheit und in Ausbeuten von ca. $80 \%$. Bei Verwendung von Benzol im ersten Extraktionsschritt konnte die Ausbeute noch deutlich gesteigert werden (95\%), was wohl auf die bessere Löslichkeit der Produkte in Benzol und somit geringere Verluste während der ersten Extraktion zurückzuführen ist. Das Bis-TMS-Diaziridin 64 zeigte bei Lagerung bei $-20^{\circ} \mathrm{C}$ nach bis zu zehn Tagen keinerlei Zersetzungserscheinungen.

Aktuelle Versuche zur Synthese der perfluorierten Verbindungen $\mathbf{6 5}$ und $\mathbf{6 6}$ deuten darauf hin, dass die Bis-TMS-Diaziridinyl-Gruppe in Abwesenheit von Säuren gegenüber Wasser recht stabil ist und problemlos eine wässrige Aufarbeitung überstehen kann. Sollte sich diese Erkenntnis auch für $\mathbf{6 4}$ bestätigen lassen, würde dies den Zugang zur Carbonsäure $\mathbf{4 8}$ noch einmal erheblich vereinfachen.

\section{I.3.4 Umsetzung des aus dem silylierten Diaziridin gewonnen Lithiumorganyls mit verschiedenen Elektrophilen}

Nach der erfolgreichen Synthese des Bis-TMS-Aziridins 64 wurde zunächst die Stabilität der Verbindung unter den Reaktionsbedingungen der Lithiierung überprüft. Hierzu wurde eine kleine Probe des geschützten Aziridins 64 in THF gelöst und bei $-78^{\circ} \mathrm{C}$ mit $n$-Butyllithium versetzt. Nach $3 \mathrm{~h}$ Rühren bei $-78^{\circ} \mathrm{C}$ wurde das Reaktionsgemisch mit 10 eq Deuteriumoxid versetzt. Standardmäßige, wässrige Aufarbeitung ergab das deuterierte Reaktionsprodukt in zufriedenstellender Reinheit und quantitativer Ausbeute. Um die Reaktivität des Li-Organyls $68 \mathrm{zu}$ untersuchen, wurde es mit verschiedenen Elektrophilen zur Reaktion gebracht (Schema 15). Die Umsetzungen des Li-Organyls mit Benzaldehyd und Aceton lieferten nach Chromatographie erwartungsgemäß die Alkohole $\mathbf{7 0}$ und $\mathbf{7 1}$ in zufrieden stellenden Ausbeuten. Die Reaktion mit Formaldehyd gestaltete sich etwas schwieriger. Zunächst wurde gasförmiger Formaldehyd, welcher durch Erhitzen von Paraformaldehyd gewonnen wurde, über einen Teflonschlauch direkt in die Reaktionslösung mit dem Li-Organyl geleitet. 
Schema 15: Umsetzung des Li-Organyls 68 mit verschiedenen Elektrophilen.

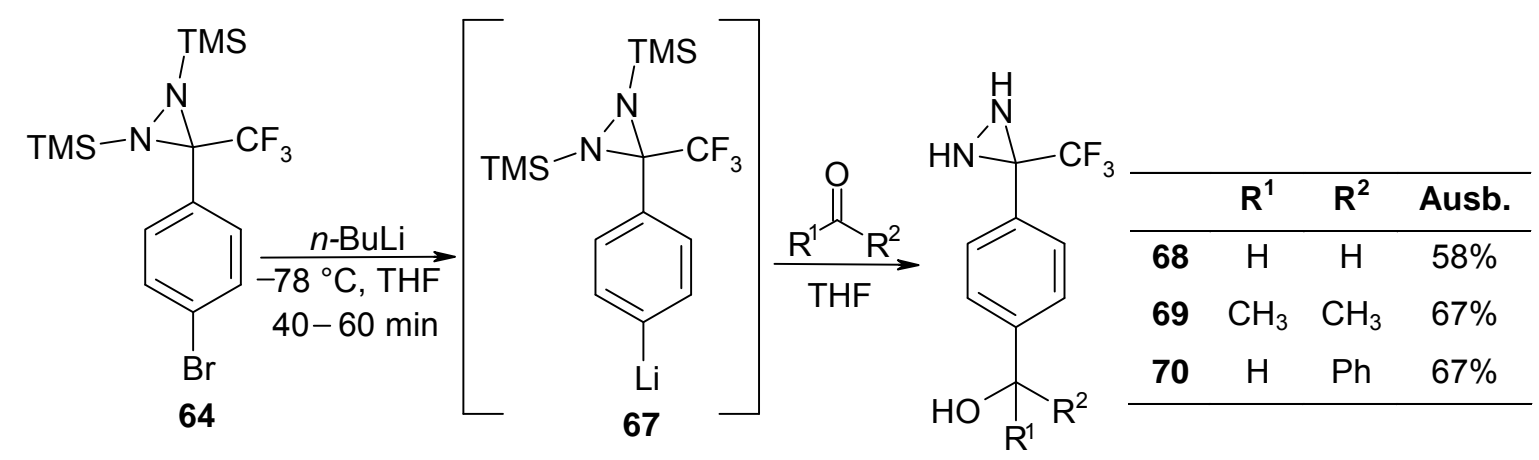

Das eingeleitete Formaldehyd-Gas polymerisierte jedoch sofort und aus dem resultierenden polymeren Reaktionsgemisch konnte kein Produkt isoliert werden. Das Problem der sofortigen Polymerisation konnte durch Verwendung einer nach SCHLOSSER frisch hergestellten Formaldehyd-Lösung umgangen werden. ${ }^{[54]}$ Dabei wird aus einer Paraformaldehyd-Suspension in THF und katalytischen Mengen Bortrifluorid-Etherat, azeotrop eine THF-Lösung abdestilliert, welche monomeren Formaldehyd in einer Konzentration von etwa $0.7 \mathrm{~mol} / \mathrm{L}$ enthält. Die Lösung kann, Lagerung bei $-78{ }^{\circ} \mathrm{C}$ vorausgesetzt, etwa drei Tage lang eingesetzt werden. Mit dieser Formaldehydlösung konnte $\mathbf{6 8}$ in 58\% Ausbeute isoliert werden. Nachdem durch diese Versuche Stabilität und Reaktivität des LiOrganyls 67 bewiesen waren, konnte die Carboxylierung in Angriff genommen werden.

\section{I.3.5 Carboxylierung des lithiierten Diaziridins}

Schema 16: Synthese der Diaziridine 71 und 71a.
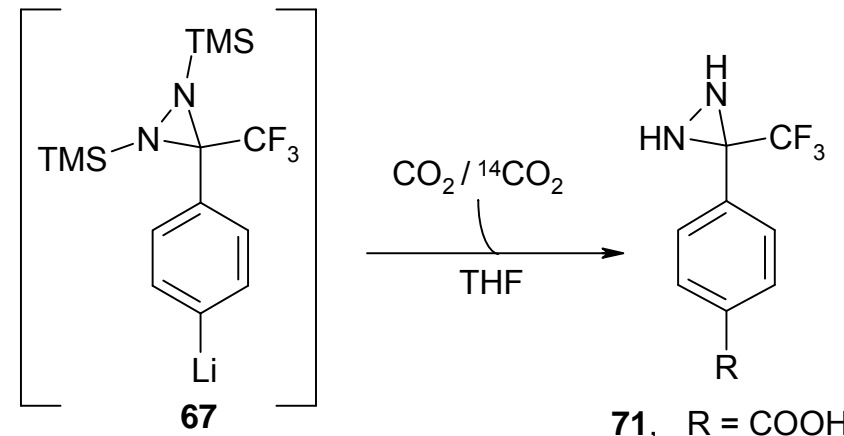

71, $\mathrm{R}=\mathrm{COOH}$

71a, $\mathrm{R}={ }^{14} \mathrm{COOH}$

Um ausreichend Material für die parallel verlaufenden Versuche zur Derivatisierung der verschiedenen Naturstoffe bereitstellen zu können (siehe Kap I.4), wurde zunächst versucht, die Ausbeute der Carboxylierung bezüglich des geschützten Diaziridins 64 zu optimieren. Einträge 1-3 in Tabelle 3 zeigen eine exemplarische Auswahl an Reaktionen 
aus dieser Versuchsreihe. Das geschützte Diaziridin 64 wurde in THF gelöst, auf $-78{ }^{\circ} \mathrm{C}$ gekühlt und mit $n$-Butyllithium versetzt. Nach $30-45$ min Rühren bei $-78{ }^{\circ} \mathrm{C}$ wurde über einen Teflonschlauch für ca. 30 min gasförmiges $\mathrm{CO}_{2}$ in die Reaktionslösung eingeleitet, welches in einem separaten Kolben aus Trockeneis freigesetzt wurde. Bei der Versuchsauswertung zeigte sich, dass die Ausbeuten teils erheblich variierten, dies ist wahrscheinlich weniger auf die unterschiedlichen Reaktionsbedingungen, sondern eher auf Probleme in der Trocknung des $\mathrm{CO}_{2}$-Gasstroms zurückzuführen. Versuche mit $\mathrm{CaCl}_{2}$, $\mathrm{KOH}$ oder Blaugel als Trockenmittel lieferten nicht zufriedenstellende oder nicht reproduzierbare Ausbeuten. Durch Überleiten des $\mathrm{CO}_{2}$-Stroms über aktiviertes Molekularsieb ( $\left.3 \AA, \mathrm{t}>5 \mathrm{~h}, \mathrm{~T}>250{ }^{\circ} \mathrm{C}, \mathrm{p}<0.1 \mathrm{mbar}\right)$ und Verwendung von frisch generiertem TMS-Aziridin 64 konnte die Ausbeute auf 89\% gesteigert werden. Für die Carboxylierung von 64 mit aus radioaktivem $\mathrm{Ba}^{14} \mathrm{CO}_{3}$ freigesetztem ${ }^{14} \mathrm{CO}_{2}$ war es notwendig, den Reaktionsaufbau so anzupassen, dass eine möglichst hohe Ausbeute bezüglich des eingesetzten Bariumcarbonats erzielt werden konnte. Als besonders schwierig gestaltete sich dabei, das aus dem Bariumcarbonat generierte $\mathrm{CO}_{2}$ zuverlässig und möglichst quantitativ in die Reaktionslösung einzubringen, welche das Li-Organyl 67 enthielt.

Tabelle 3: Versuchsreihe zur Carboxylierung von 64.

\begin{tabular}{ccccccc}
\hline Nr. & eq (64) & eq $(\mathbf{n B u L i})$ & Zeit $[\mathbf{h}]$ & $\mathbf{T}_{\mathbf{1}}\left[{ }^{\circ} \mathbf{C}\right]$ & $\mathbf{T}_{\mathbf{2}}\left[{ }^{\circ} \mathbf{C}\right]$ & $\mathbf{A}[\%]$ \\
\hline $\mathbf{1}$ & 1.00 & 1.10 & 16 & - & $-78 \rightarrow \mathrm{RT}$ & $35^{\mathrm{a}}$ \\
$\mathbf{2}$ & 1.00 & 1.00 & 4.0 & - & $-95 \rightarrow-30$ & $40^{\mathrm{a}}$ \\
$\mathbf{3}$ & 1.00 & 1.05 & 16 & - & -78 & $89^{\mathrm{a}}$ \\
$\mathbf{4}^{\mathrm{c}}$ & 4.01 & 4.00 & 0.5 & -196 & $-95 \rightarrow \mathrm{RT}$ & $50^{\mathrm{b}}$ \\
$\mathbf{5}^{\mathrm{c}}$ & 2.00 & 1.95 & 1.0 & -120 & $-120 \rightarrow \mathrm{RT}$ & $20^{\mathrm{b}}$ \\
$\mathbf{6}$ & 2.45 & 2.45 & 2.0 & -196 & $-196 \rightarrow \mathrm{RT}$ & $22^{\mathrm{b}}$ \\
$\mathbf{7}$ & 2.51 & 2.50 & 6.0 & -196 & $-120 \rightarrow \mathrm{RT}$ & $26^{\mathrm{b}}$ \\
$\mathbf{8}$ & 2.48 & 2.32 & 5.0 & - & $-78 \rightarrow \mathrm{RT}$ & $72^{\mathrm{b}}$ \\
$\mathbf{9}^{\mathbf{d}}$ & 3.30 & 3.14 & 5.0 & - & $-78 \rightarrow \mathrm{RT}$ & $78^{\mathrm{b}}$ \\
\hline
\end{tabular}

a) Ausbeute bezogen auf Bis-TMS-Diaziridin 64; b) Ausbeute bezogen auf $\mathrm{BaCO}_{3}$; c) Trocknung des $\mathrm{CO}_{2}$-Stroms mit Trockenrohr; d) $\mathrm{Ba}^{14} \mathrm{CO}_{3}$ verwendet; $\mathrm{T}_{1}$ Ausfriertemperatur für $\mathrm{CO}_{2} ; \mathrm{T}_{2}$ Reaktionstemperatur.

In den ersten Versuchen wurde das durch konz. Schwefelsäure freigesetzte $\mathrm{CO}_{2}$ mit einem Teflonschlauch in einen Schlenkkolben geleitet, wo es bei Temperaturen von-196, bzw. $-120{ }^{\circ} \mathrm{C}$ einkondensiert wurde. Danach wurde, per Spritze oder Teflonschlauch, die LiOrganyl-Lösung in den Kolben gegeben. Ein Problem hierbei war, dass sich das Li- 
Organyl beim Transfer in den Schlenkkolben, welcher das ausgefrorene $\mathrm{CO}_{2}$ enthielt, zu stark erwärmte. Die Folge war eine Verfärbung der Reaktionslösung zu einem kräftigen Dunkelblau oder -grün, was auf Zersetzungsreaktionen schließen ließ. Diese Annahme wurde durch die mäßigen Ausbeuten bestätigt (Einträge 4-7). Deshalb wurde das aus dem Bariumcarbonat freigesetzte $\mathrm{CO}_{2}$ in den folgenden Versuchen mit einem Teflonschlauch direkt in die Reaktionslösung geleitet. Zur Optimierung der Ausbeute wurden verschiedene Versuchsanordnungen bezüglich $\mathrm{CO}_{2}$-Trocknung (konz. $\mathrm{H}_{2} \mathrm{SO}_{4}, \mathrm{CaCl}_{2}$, Blaugel, Kältebad), Gaseinleitung (Schläuche, Septen, Fritten etc.), Temperatur bei Gaseinleitung ( -100 bis $78^{\circ} \mathrm{C}$ ) sowie Reaktionstemperatur und -zeit getestet.

Als Ergebnis dieser Versuche ergab sich für die Umsetzungen mit $\mathrm{Ba}^{14} \mathrm{CO}_{3}$ der in Abbildung 10 gezeigte Versuchsaufbau. Die in der Abbildung gezeigte Anordnung entspricht exakt dem Versuchsaufbau für die Reaktionen mit radioaktivem Material. Für die Umsetzungen mit ,kaltem“ $\mathrm{BaCO}_{3}$ diente als Vorratsgefäß (4) ein $10 \mathrm{~mL}$ Einhalskolben, welcher durch ein Septum verschlossen war.

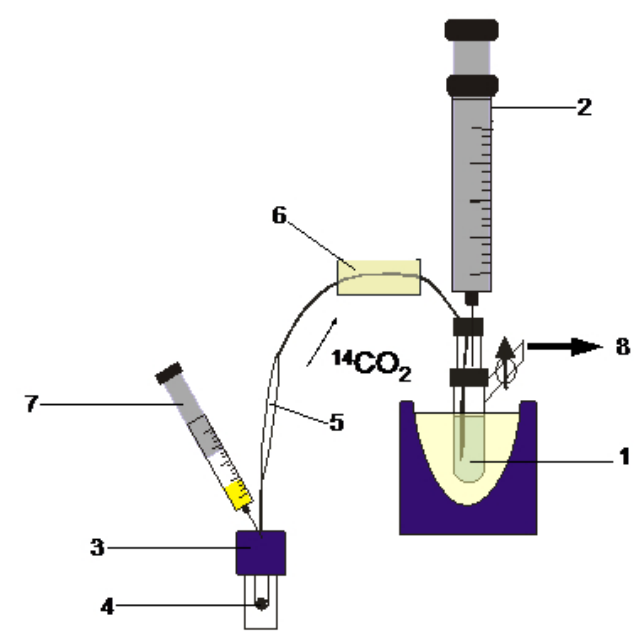

1 - Li-Aryl in THF bei $-78^{\circ} \mathrm{C}$

2 - Kolbenprober als Puffervolumen für ${ }^{14} \mathrm{CO}_{2}$

3 - gelochte Plastikkappe

4 - Glasgefäß mit $\mathrm{Ba}^{14} \mathrm{CO}_{3}$

5 - Puffervolumen

6 - Kühlbad bei $-78{ }^{\circ} \mathrm{C}$

7 - Spritze mit konz. $\mathrm{H}_{2} \mathrm{SO}_{4}$

8 - Natronkalk für überschüssiges ${ }^{14} \mathrm{CO}_{2}$

Abbildung 10: Experimenteller Aufbau der Carboxylierung mit radioaktivem $\mathrm{Ba}^{14} \mathrm{CO}_{3}$.

Das geschützte Aziridin 64 wurde in einem Schlenkrohr (1) vorgelegt, auf $-78^{\circ} \mathrm{C}$ gekühlt und mit $n$-BuLi versetzt. An das Schlenkrohr, welches durch ein Septum verschlossen war, wurde ein Kolbenprober (2) angeschlossen, welcher als Puffervolumen diente. Die Verschlusskappe (3) des Glasgefäßes (4), welches das $\mathrm{Ba}^{14} \mathrm{CO}_{3}$ enthielt, wurde durchbohrt und zwischen Deckel und Glasflasche wurde ein zweites Gummiseptum eingefügt. Über einen Teflonschlauch wurde das Vorratsgefäß (4) mit dem Reaktionskolben verbunden. Um zu verhindern dass Säurespuren durch den Teflonschlauch in das Reaktionsgefäß gelangten, wurde ein Puffervolumen (beidseitig ausgezogene Glaspipette, 5) eingefügt und 
außerdem ein ca. $10 \mathrm{~cm}$ langes Teilstück auf $-78^{\circ} \mathrm{C}$ gekühlt (6). Mit einer Einwegspritze (7) wurden zwei Tropfen konz. Schwefelsäure zum $\mathrm{Ba}^{14} \mathrm{CO}_{3}$ gegeben und so die Carboxylierung gestartet. Nach dem Ende der Gasentwicklung wurde vorsichtig die restliche Säure (insgesamt $2 \mathrm{~mL}$ ) zugegeben und 10-15 min gerührt. Als die Gasentwicklung beendet war, wurde der Teflonschlauch mit einer Gefäßklemme verschlossen und das Reaktionsgemisch weitere $5 \mathrm{~h}$ bei $-78^{\circ} \mathrm{C}$ gerührt. Die Reaktion wurde durch Zugabe von Wasser beendet. Um ein Entweichen von nicht reagiertem ${ }^{14} \mathrm{CO}_{2}$ zu verhindern, wurde die Apparatur mit Stickstoff gespült und der Gasstrom über Natronkalk geleitet. Nach wässriger Aufarbeitung erhielt man das carboxylierte Produkt, welches für weitere Umsetzungen ausreichend rein war (siehe Experimentellen Teil). Die guten Ausbeuten an Diaziridincarbonsäure 71 in der nicht-radioaktiven Testreaktion (72\%, Eintrag 8, Tabelle 3) konnten durch Verwendung eines größeren Überschusses an LiOrganyl 67 (3.3 eq) bei der „heißen“ Reaktion sogar noch einmal leicht auf 78\% des freigesetzten ${ }^{14} \mathrm{CO}_{2}$ gesteigert werden. So konnten aus $50 \mathrm{mCi}(0.91 \mathrm{mmol}) \mathrm{Ba}^{14} \mathrm{CO}_{3}$ $165 \mathrm{mg}$ des ${ }^{14} \mathrm{C}$-Diaziridins 71a gewonnen werden.

\section{I.3.6 Oxidation des Diaziridins zum Diazirin}

Für die Oxidation des Diaziridins 71 zum Diazirin 48 (Schema 17) wurden verschiedene Oxidationsmittel getestet (Tabelle 4). Oxidationsversuche mit tert-Butylhypochlorit, ${ }^{[46 b]}$ Braunstein ${ }^{[55]}$ und $\mathrm{Ag}_{2} \mathrm{O}^{[56]}$ lieferten keinen Umsatz und es wurde jeweils nur das verwendete Edukt zurückgewonnen (Einträge 1-3). Warum die Oxidation mit tert-Butylhypochlorit, welches ja für das Diaziridin 58 schon gute Ergebnisse geliefert hatte, hier keinen Umsatz lieferte, ist unklar. Eventuell war das eingesetzte Hypochlorit nicht mehr reaktiv genug. Unabhängig davon konnte die Oxidation mit Iod als Oxidationsmittel in Gegenwart von Triethylamin realisiert werden. Die zunächst geringe Ausbeute (Eintrag 4) ist auf Verluste während der chromatographischen Aufreinigung zurückzuführen. Nach Optimierung der Reaktionsbedingungen (Eintrag 5) konnte in einer einfachen Reaktionsdurchführung zuverlässig und in annähernd quantitativer Ausbeute das Oxidationsprodukt, Diazirin 48 isoliert werden. Dazu wurde das Diaziridin $\mathbf{7 1}$ in Methanol gelöst und mit Triehthylamin versetzt. 
Schema 17: Oxidation der Diaziridine 71 und 71a zu den entsprechenden Diazirinen.

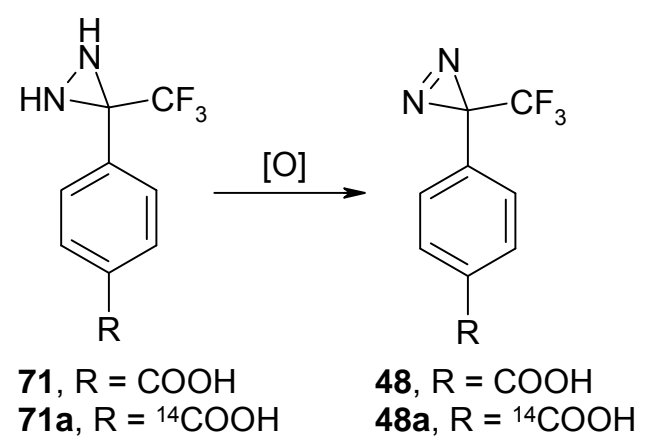

Tabelle 4: Variation der Oxidationsmittel und Reaktionsbedingungen.

\begin{tabular}{cccccccc}
\hline $\mathbf{N r}$ & $\mathbf{E}$ & {$[\mathbf{O}]$} & Zeit $[\mathbf{h}]$ & $\mathbf{L m}$ & $\mathbf{B}$ & $\mathbf{T}\left[{ }^{\circ} \mathbf{C}\right]$ & Beob. \\
\hline 1 & $\mathbf{7 1}$ & $t$ - $\mathrm{BuOCl}$ & 4.5 & $\mathrm{MeOH}$ & $\mathrm{NaHCO}_{3}$ & $-78 \rightarrow \mathrm{RT}$ & Edukt \\
2 & $\mathbf{7 1}$ & $\mathrm{MnO}_{2}$ & 2.0 & $\mathrm{THF}$ & $\mathrm{NEt}_{3}$ & $0 \rightarrow \mathrm{RT}$ & Edukt \\
3 & $\mathbf{7 1}$ & $\mathrm{Ag}_{2} \mathrm{O}$ & 5.0 & $\mathrm{EtOH}$ & $\mathrm{NEt}_{3}$ & $\mathrm{RT}$ & Edukt \\
4 & $\mathbf{7 1}$ & $\mathrm{I}_{2}$ & 3.5 & $\mathrm{MeOH}$ & $\mathrm{NEt}_{3}$ & $\mathrm{RT}$ & $32 \%{ }^{\mathrm{a}}$ \\
5 & $\mathbf{7 1}$ & $\mathrm{I}_{2}$ & 0.5 & $\mathrm{MeOH}$ & $\mathrm{NEt}_{3}$ & $\mathrm{RT}$ & $99 \%{ }^{\mathrm{b}}$ \\
6 & $\mathbf{7 1 a}$ & $\mathrm{I}_{2}$ & 0.5 & $\mathrm{MeOH}$ & $\mathrm{NEt}_{3}$ & $\mathrm{RT}$ & $83 \%{ }^{\mathrm{b}}$ \\
\hline
\end{tabular}

a) Chromatographische Reinigung; b) Basische Extraktion.

Dann wurde solange eine methanolische Iodlösung zugetropft, bis eine rot-braune Färbung bestehen blieb und anschließend noch 20-30 min unter Lichtausschluss bei Raumtemperatur gerührt. Nach basischer Extraktion, Ansäuern, Reextraktion mit Diethylether, Trocknen über Natriumsulfat und Entfernen des Lösungsmittels war Diazirin 48 rein genug für weitere Umsetzungen. Auf eine chromatographische Reinigung konnte hierbei verzichtet werden, dies war insbesondere für die Reaktionsdurchführung mit dem radioaktiven Diazirin 48a (Eintrag 6) ein wichtiger Vorteil, da dadurch die Mengen des zu entsorgenden radioaktiven Abfalls reduziert werden konnten. Die spezifische Radioaktivität des Diazirins 48 a wurde zu $44.11 \mathrm{mCi} / \mathrm{mmol}$ bestimmt und das Produkt wurde bei $-18{ }^{\circ} \mathrm{C}$ in einer Dichlormethanlösung der Konzentration $4 \mathrm{mCi} / \mathrm{mL}$ gelagert. Die gute Haltbarkeit der Photoaffinitätlabel 48 bzw. 48a wurde dadurch unter Beweis gestellt, dass mehr als ein Jahr nach der Synthese beider Verbindungen im Rahmen einer Kooperation mit dem HKI Braunschweig der selektive V-ATPase Inhibitor Archazolid ${ }^{[57]}$ 72 erfolgreich mit dem Azirin 48a für Photoaffinitätsstudien derivatisiert werden konnte (Schema 18). ${ }^{[58]}$ Die spezifischen Aktivitäten betrugen exzellente $78.0 \mathrm{mCi} / \mathrm{mmol}$ für $\mathbf{7 4}$ bzw. $147.6 \mathrm{mCi} / \mathrm{mmol}$ für das zweifach substituierte Derivat $\mathbf{7 3}$. 
Schema 18: Kupplung des Photoaffinitätslabels 48a an das Archazolid (72).<smiles>[R2]O[C@H](c1csc([C@H](CC(C)C)OC(=O)NC)n1)[C@H](C)/C=C/C=C(\C)[C@@H](OC)[C@@H](C)[C@@H](O)/C=C/C(C)=C/C(C)=C\[C@H](C)[C@@H](O)/C=C(\C)C/C=C(\C)C(=O)O</smiles>

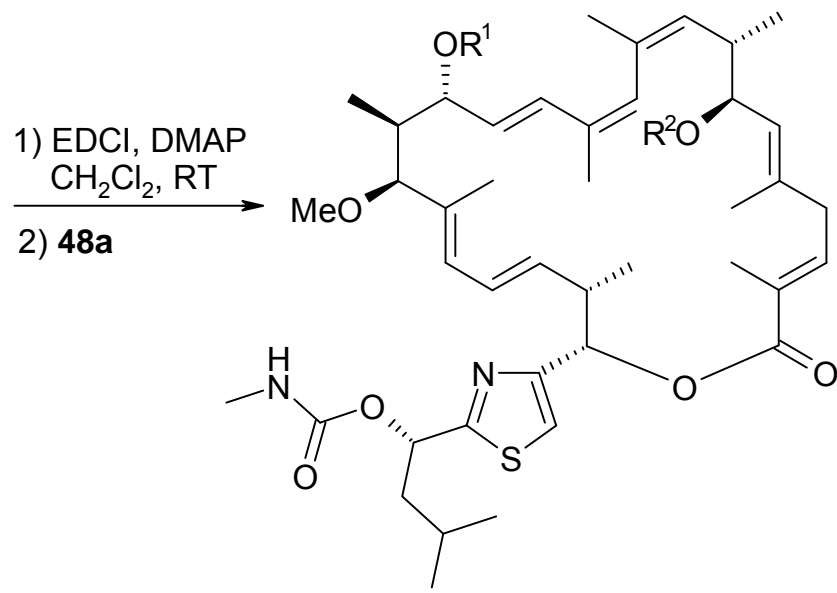

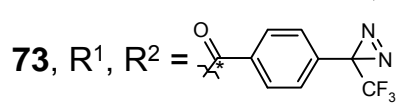

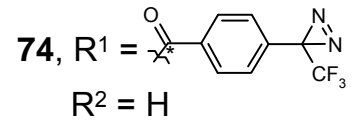

\section{I.4 Kupplung des Photoaffinitätslabels an die V-ATPase Inhibitoren}

Alle Naturstoffe, ausgenommen Apicularen (85), wurden von der Arbeitsgruppe S. GROND zur Verfügung gestellt. Das Apicularen (85) wurde im Rahmen einer Kooperation vom Helmholtzzentrum für Infektionsforschung in Braunschweig geliefert. Die Reaktionen erfolgten unter Lichtausschluss und alle Manipulationen mit radioaktivem Material wurden, unter Berücksichtigung entsprechender Sicherheitsmaßnahmen, im Sicherheitsbereich des Isotopenlabors des Physikalisch-Chemischen Institutes der Universität Göttingen durchgeführt.

\section{I.4.1 Synthese der Concanolidderivate}

Auf Grundlage der in der Einleitung diskutierten SAR-Studien sollte das Concanolid A (4) an der 23-OH Gruppe funktionalisiert werden, wodurch eine ausreichend hohe biologische Aktivität des derivatisierten Naturstoffes gewährleistet werden sollte. Glücklicherweise hatten vorangegangene Untersuchungen bereits gezeigt, dass die sekundäre Hydroxyfunktion an C-23 in Veresterungen eine deutlich höhere Reaktivität besitzt als jene an C-7, C-19 und C-9. ${ }^{[30,31]}$ Die verringerte Reaktivität aller Hydroxyfunktionen außer der außerhalb des Makrolactons liegenden 23-OH, ist wohl auf eine Stabilisierung durch intramolekulare Wasserstoffbrücken (19-OH) sowie auf eine Vorzugskonformation 
zurückzuführen, in der die betreffenden Hydroxyfunktionen sterisch abgeschirmt sind (7$\mathrm{OH}, 9-\mathrm{OH}) \cdot{ }^{[30,17 \mathrm{a}]}$ Dadurch sollte eine gezielte Funktionalisierung an 23-OH auch ohne intermediäre Schützung möglich sein.

Schema 19: Derivatisierung des Concanolids 4.

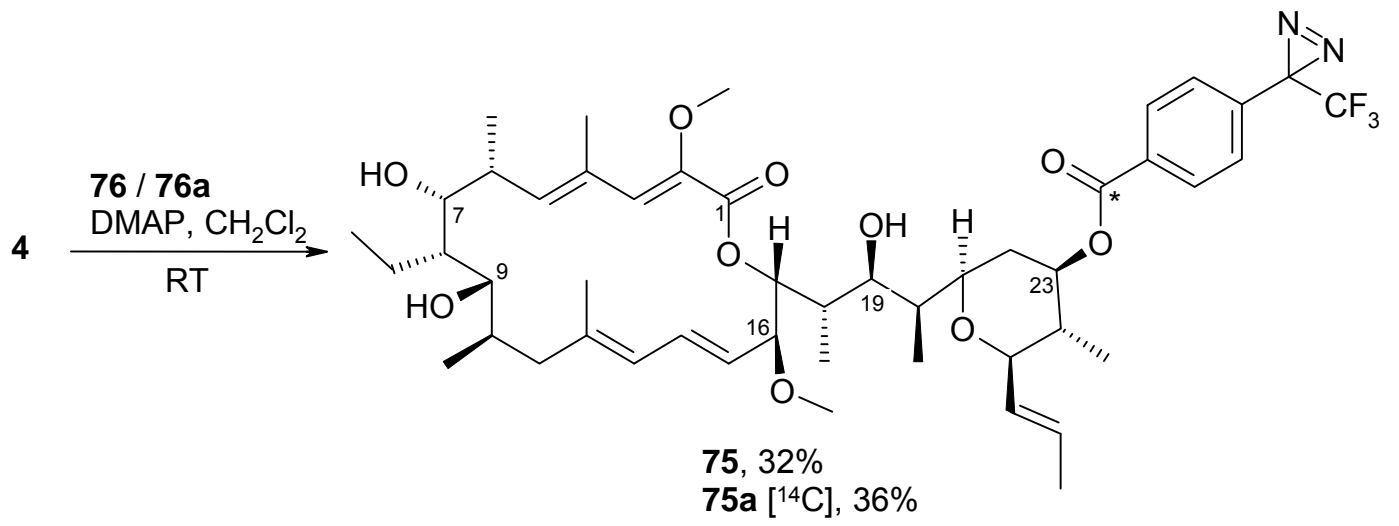

Um die Stabilität des Concanolids gegenüber den für die Veresterung verwendeten Reaktionsbedingungen zu überprüfen, wurde zunächst eine Probe $(\sim 1 \mathrm{mg})$ in Dichlormethan gelöst und mit jeweils einer Spatelspitze $N$-(3-Dimethylaminopropyl)- $N$ ethylcarbodiimid (EDCI), N,N-Dimethylaminopyridin (DMAP) und Diazirin 48 versetzt. Dann wurde bei RT gerührt und der Reaktionsfortschritt mittels Dünnschichtchromatographie verfolgt. Nach $2 \mathrm{~h}$ Rühren war auf den Dünnschichtchromatogrammen kein definierter Spot mehr zu erkennen. Da Concanolid 4 unter diesen Reaktionsbedingungen nicht stabil zu sein schien, wurde eine zweite Testreaktion mit Dicyclohexylcarbodiimid (DCC) als Wasserfänger durchgeführt. Das Ergebnis war jedoch das gleiche wie mit EDCI.

Schema 20: Herstellung der Säurechloride 76 und 76a.<smiles>[R]c1ccc(C2(c3ccc([R])cc3C(F)(F)F)N=N2)cc1</smiles>
$48, \mathrm{R}=\mathrm{COOH}$
76, $\mathrm{R}=\mathrm{COCl}$
48a, $\mathrm{R}={ }^{14} \mathrm{COOH}$
$76 \mathrm{a}, \mathrm{R}={ }^{14} \mathrm{COCl}$

Deshalb wurde das reaktivere Säurechlorid 76 des Diazirins 48 synthetisiert, welches nicht den Einsatz von EDCI oder DCC erfordert (Schema 20). Für die Synthese der Säurechloride 76 und 76a erwies sich Thionylchlorid als das Reagenz der Wahl. ${ }^{[59]}$ Damit 
konnte, anders als mit Oxalylchlorid, nach $15 \mathrm{~h}$ Rühren bei RT vollständiger Umsatz zu den Säurechloriden 76 und 76a erzielt werden. Dabei war die Versuchsdurchführung denkbar einfach: Die Carbonsäuren wurden in Thionylchlorid gelöst und man ließ über Nacht und unter Lichtausschluss bei RT rühren. Beim Entfernen des überschüssigen Thionylchlorids unter verringertem Druck musste jedoch sehr sorgfältig auf den Druck geachtet werden. So zeigte sich, dass sich die Säurechloride 76 und 76a schon bei Drücken $<10$ mbar langsam verflüchtigen. Ist der Druck jedoch zu hoch gewählt, bleiben Säurespuren zurück, welche zu Problemen in den Reaktionen mit den teils sehr empfindlichen Naturstoffen führen können (siehe unten). Bei einem Druck von 40 mbar konnte das überschüssige Thionylchlorid zuverlässig entfernt und die Säurechloride $\mathbf{7 6}$ und 76a in annähernd quantitativen Ausbeuten isoliert werden. Nach der erfolgreichen Synthese der Säurechloride konnte nun die Derivatisierung des Concanolids $\mathbf{4}$ durchgeführt werden. Die Ergebnisse der Versuche sind in Tabelle 5 zusammengefasst.

Tabelle 5

\begin{tabular}{|c|c|c|c|c|c|c|c|}
\hline \multirow{2}{*}{$\mathrm{Nr}$} & \multicolumn{2}{|c|}{ PAL } & \multirow{2}{*}{$\begin{array}{c}\text { DMAP } \\
\text { [eq] }\end{array}$} & \multirow{2}{*}{$\mathbf{L M}$} & \multirow{2}{*}{$\mathbf{t}[\mathbf{h}]$} & \multicolumn{2}{|c|}{ Ausbeute [\%] } \\
\hline & & eq & & & & 75 (a) & 4 \\
\hline 1 & 76 & 10 & 12 & DCM & 20 & - & 11 \\
\hline 2 & 76 & 2.9 & 3.5 & DCM & 15 & - & - \\
\hline 3 & 76 & 1.8 & 5.3 & $\mathrm{DCM} / \mathrm{NEt}_{3}$ & 15 & 32 & 38 \\
\hline 4 & $76 a$ & 4.9 & 6.3 & $\mathrm{DCM} / \mathrm{NEt}_{3}$ & 7 & 36 & 31 \\
\hline
\end{tabular}

Aus den ersten Versuchen (Einträge 1 und 2) konnte kein Zielprodukt isoliert werden, weil in der Benzoylchloridlösung wahrscheinlich noch Säurespuren enthalten waren. Die isolierten Zersetzungsprodukte konnten zwar nicht eindeutig identifiziert werden, allerdings wurden im Versuch zu Eintrag 2 zwei Verbindungen isoliert, welche im ${ }^{1} \mathrm{H}$ NMR Spektrum kein Signal für die 16-OMe Gruppe aufwiesen. Dies lässt auf eine Säurekatalysierte Abspaltung von Methanol schließen. Durch eine sorgfältigere Entfernung des überschüssigen Thionylchlorids sowie die Verwendung von Triethylamin als Lösungsmittelbestandteil bzw. eines Überschusses an DMAP (Eintrag 4) wurden diese Probleme überwunden und die Zielprodukte 75 und 75a erfolgreich synthetisiert. Die derivatisierten Naturstoffe konnten nach Säulenchromatographie an Kieselgel in 32\% Ausbeute für 75 bzw. 36\% für 75a (52\% Ausbeute bezogen auf 69\% Umsatz) isoliert werden. Außer den Zielprodukten wurde bei beiden Umsetzungen jeweils etwa ein Drittel 
des eingesetzten Naturstoffes reisoliert. Die zurückgewonnenen Edukte waren rein genug für weitere Umsetzungen. Die spezifische Aktivität des radioaktiven Derivates 75a wurde mit einem Flüssigkeitsszintillations-messgerät zu $40 \mathrm{mCi} / \mathrm{mmol}$ bestimmt.

\section{I.4.2 Synthese der Bafilomycinderivate}

Die Derivatisierung des Macrolids 2 wurde nach einem Standardprotokoll ${ }^{[34]}$ durch eine EDCI-vermittelte Veresterung in Gegenwart von DMAP erreicht, wodurch das Produkt in Ausbeuten $>40 \%$ isoliert werden konnte (Tabelle 6). Trotz Zugabe größerer Mengen des Diazirins 48 und EDCI sowie verlängerter Reaktionszeiten (Eintrag 1) konnte kein vollständiger Umsatz erzielt werden. Auch die Verwendung größerer Mengen DMAP führte nicht zu einer besseren Ausbeute (Eintrag 2).

Schema 21: Herstellung der Bafilomycinderivate 77 und 77a.<smiles>[Y20][Z10]([H])([H])Cl</smiles>

Tabelle 6

\begin{tabular}{|c|c|c|c|c|c|c|c|c|}
\hline \multirow{2}{*}{$\mathbf{N r}$} & \multicolumn{2}{|c|}{ PAL } & \multirow{2}{*}{$\begin{array}{c}\text { DMAP } \\
{[\mathrm{eq}]}\end{array}$} & \multirow{2}{*}{$\begin{array}{c}\text { EDCI } \\
{[\text { eq] }}\end{array}$} & \multirow{2}{*}{ LM } & \multirow{2}{*}{$\mathbf{t}[\mathbf{h}]$} & \multicolumn{2}{|c|}{ Ausbeute [\%] } \\
\hline & & [eq] & & & & & (75) & (2) \\
\hline 1 & 48 & 2.90 & kat. & $>10$ & DCM & 50 & 42 & 34 \\
\hline 2 & 48 & 2.08 & 2.43 & 2.20 & DCM & 16 & 41 & 22 \\
\hline 3 & 76 & 2.14 & 4.91 & - & DCM/Pyr & 18 & - & - \\
\hline 4 & 76 & 4.50 & kat. & - & DCM/Pyr & 12 & - & - \\
\hline 5 & $48 \mathbf{a}$ & 2.00 & 2.05 & 2.05 & DCM & 16 & 41 & 31 \\
\hline
\end{tabular}

Um doch noch vollständigen Umsatz zu erreichen, wurde Bafilomycin 2 mit dem Säurechlorid 76 zur Reaktion gebracht. Aus diesen Versuchen (Einträge 3 und 4) konnten jedoch nur nicht identifizierbare Zersetzungsprodukte isoliert werden. Die Reaktion mit 
dem radioaktiven Diazirin 48a lieferte das Zielprodukt 77a in 41\% Ausbeute, wobei auch hier 31\% des eingesetzten Naturstoffes reisoliert werden konnten (59\% Ausbeute bezogen auf 69\% Umsatz). Die Reinigung des Rohproduktes erfolgte über eine kurze Kieselgelsäule, eine aufwendige und kostenintensive HPLC-Trennung war nicht notwendig. Das Zielprodukt 77a besaß eine spezifische Aktivität von $34 \mathrm{mCi} / \mathrm{mmol}$.

\section{I.4.3 Synthese verschiedener Kendomycinderivate und deren Kupplung mit dem Photoaffinitätslabel}

Das in Abbildung 11 gezeigte Macrolid Kendomycin (78) wurde erstmals in einem Japanischen Patent von 1996 beschrieben und später in der Arbeitsgruppe ZEECK aus dem Stamm Streptomyces violaceoruber isoliert. ${ }^{[60]}$ Neben einer bemerkenswerten antibiotischen und cytotoxischen Aktivität besitzt $\mathbf{7 8}$ auch eine Hemmwirkung gegenüber V-ATPasen, was es für die im Folgenden beschriebenen Photoaffinitätsstudien interessant machte. ${ }^{[61]}$ In einer Reihe von Vorversuchen mit dem Kendomycin zeigte sich, dass die direkte Veresterung des Naturstoffes mit EDCI bzw. DCC und DMAP nicht möglich war, da das Kendomycin in Gegenwart von DMAP nicht stabil zu sein schien: Wurde zu einer Lösung von $\mathbf{7 8}$ in Dichlormethan DMAP zugesetzt, verfärbte sich die ursprünglich gelbe Reaktionslösung sofort tiefblau und die anschließende Reaktion mit EDCI oder DCC und Diazirin 48 führte zu keinen isolierbaren Verbindungen.

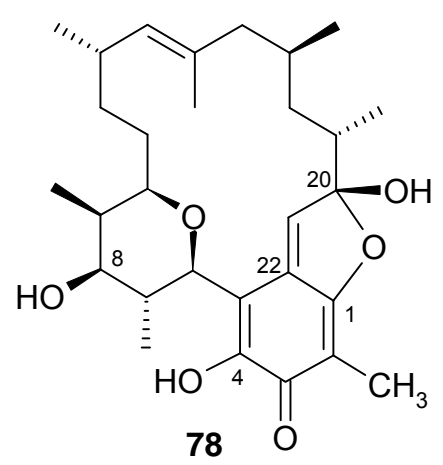

Abbildung 11: Das Kendomycin 78, ein verhältnismäßig neuer Vertreter der V-ATPaseInhibitoren.

Bei späteren Versuchen in der Arbeitsgruppe GROND wurde diese Beobachtung näher untersucht, jedoch konnte aus den blauen Reaktionslösungen nach Aufarbeitung nur das eingesetzte Edukt 78 isoliert werden. Dies lässt auf eine reversible Deprotonierung des 
Kendomycins (78) und eine daraus entstehende blaue, anionische Verbindung schließen. Die Tatsache, dass dieses Phänomen mit anderen Basen nicht (z.B. NEt ${ }_{3}$ ) oder sehr viel schwächer (z.B. Pyridin) zu beobachten ist, könnte auch auf nicht-kovalente Wechselwirkungen zwischen den $\pi$-Elektronen des DMAP und denen des Kendomycins zurückzuführen sein. Aufgrund der Tatsache, dass sich das Kendomycin (78) nicht direkt funktionalisieren ließ, wurden zwei Derivate hergestellt, die gegenüber den Reaktionsbedingungen der Veresterung stabil waren.

\section{I.4.3.1. Synthese des Kendomycin-Acetonids und dessen Kupplung mit dem Photoaffinitätslabel}

Das Acetonid $\mathbf{8 1}$ wurde analog der von H. BODE publizierten Synthesevorschrift hergestellt. ${ }^{[62]}$ Dabei wird das Kendomycin (78) bei RT in Aceton gelöst und mit einem Überschuss an $\mathrm{FeCl}_{3}$ versetzt. Nach Aufarbeitung und Chromatographie erhielt man Acetonid 81 in mäßigen Ausbeuten von bis zu 25\%. Ein möglicher Mechanismus für diese Reaktion ist in Schema 22 dargestellt. Dabei kommt es zuerst zu einem nukleophilen Angriff der Hydroxylfunktion an C-20 auf ein Acetonmolekül. Das so gewonnene Intermediat 77 bildet über einen intramolekularen nukleophilen Angriff auf C-21 das aromatische 1,2-Diol 78, welches durch Oxidation, wahrscheinlich durch Luftsauerstoff, das Chinon 79 bildet. $^{[63]}$ Die Stereokonfiguration des Hauptisomers wurde durch H. BoDE mittels moderner spektroskopischer Methoden bestätigt. Aus den Untersuchungen der Rohprodukte der Acetonidbildung lässt sich schließen, dass noch weitere Diastereomere gebildet werden. Diese Minderkomponenten (ca. 10\% des Rohproduktes) konnten jedoch chromatographisch nicht sauber aufgereinigt werden, weshalb die Bestimmung der Konfiguration dieser Isomere nicht möglich war. Versuche, die Ausbeute der Acetonidbildung durch Variation der Reaktionstemperatur und der verwendeten Äquivalente Eisen(III)-Chlorid zu erhöhen, waren nicht erfolgreich. Für die Photoaffinitätsstudien wurde die Hydroxyfunktion an C-8 mit Azirin 48 verestert (Schema 23). Die Ergebnisse dieser Experimente sind in Tabelle 7 zusammengefasst. 
Schema 22: Möglicher Mechanismus der Acetonidbildung aus Kendomycin (78).

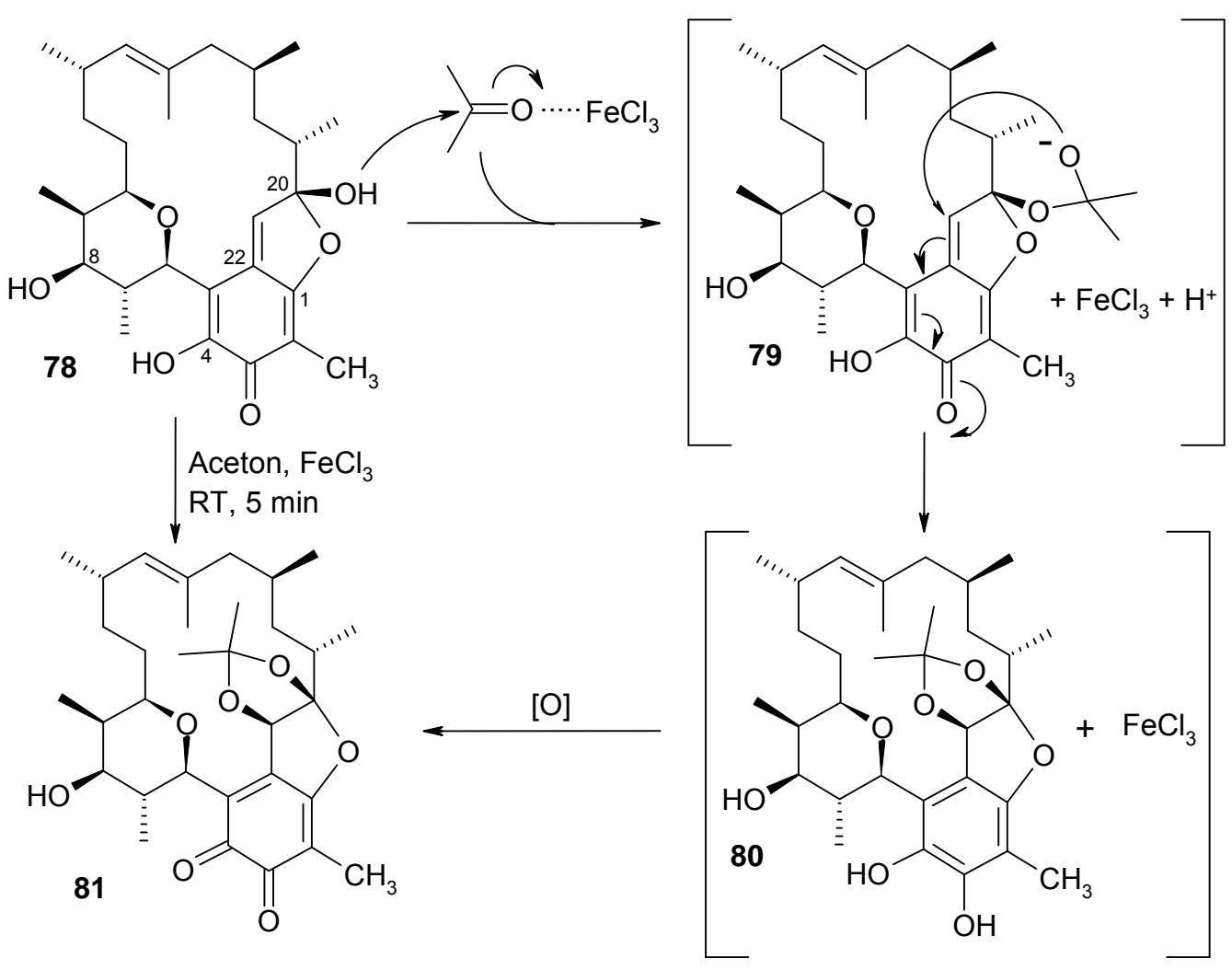

Schema 23: Kupplung der Säurechloride 76 und 76a an das Kendomycin-Acetonid 81.

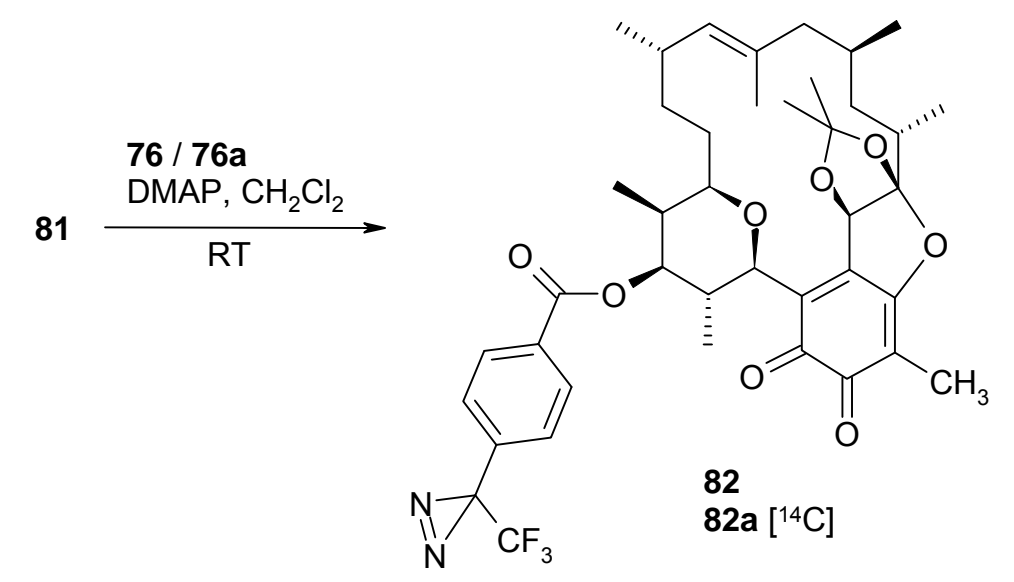

Tabelle 7

\begin{tabular}{|c|c|c|c|c|c|c|}
\hline \multirow{2}{*}{$\mathbf{N r}$} & \multicolumn{2}{|c|}{ PAL } & \multirow{2}{*}{$\begin{array}{c}\text { DMAP } \\
\text { [eq] }\end{array}$} & \multirow{2}{*}{ LM } & \multirow{2}{*}{$\mathbf{t}$} & \multirow{2}{*}{$\begin{array}{c}\text { A } \\
{[\%]}\end{array}$} \\
\hline & & [eq] & & & & \\
\hline 1 & 76 & 2.5 & 2.0 & $\mathrm{DCM} / \mathrm{Pyr}$ & $6.0 \mathrm{~h}$ & - \\
\hline 2 & 76 & 2.5 & 2.8 & DCM & $20 \mathrm{~min}$ & - \\
\hline 3 & 76 & 4.5 & 5.0 & $\mathrm{DCM} / \mathrm{NEt}_{3}$ & $3.5 \mathrm{~h}$ & 14 \\
\hline 4 & 76 & 1.5 & 13 & DCM & $20 \mathrm{~min}$ & 49 \\
\hline 5 & $76 a$ & 2.1 & 15 & DCM & $25 \mathrm{~min}$ & 37 \\
\hline
\end{tabular}


Die Versuche zeigten, dass sich zu lange Reaktionszeiten (Eintrag 1) sowie ein zu geringer Überschuss an DMAP (Eintrag 2) nachteilig auf die Ausbeuten auswirken. Dies ließ darauf schließen, dass 81 unter den Reaktionsbedingungen nur mäßig stabil war. Dementsprechend konnte die beste Ausbeute (49\%) mit einem großen Überschuss DMAP (13 eq) und einer Reaktionszeit von nur 20 min (Eintrag 4) erzielt werden. Nach dem Ende der Reaktion wurde das Reaktionsgemisch direkt auf eine präparative DC-Platte aufgetragen. Durch die kräftige, rote Eigenfarbe war die Detektion des Produktes sehr einfach, die ja aufgrund der Diazirinfunktion nicht unter UV-Licht erfolgen konnte. Bei der Umsetzung des Kendomycin-Acetonids 81 mit dem radioaktiven Label 76a konnte die Zielverbindung 82a in $37 \%$ Ausbeute und mit einer spezifischen Aktivität von $42.3 \mathrm{mCi} / \mathrm{mmol}$ isoliert werden.

\section{I.4.3.2. Synthese des Diazirinyl-Kendomycin-Methylacetals}

Zunächst erfolgte die Säure-katalysierte Synthese des Kendomycin-Methylacetals 83 (Schema 24, Tabelle 8). Dazu wurde Kendomycin (78) in Methanol gelöst und mit katalytischen Mengen $p$-Toluolsulfonsäure versetzt. Nach einer Reaktionszeit von drei Tagen war der Umsatz vollständig. Die Aufreinigung per Kieselgelsäule lieferte $\mathbf{8 3}$ in einer mäßigen Ausbeute von 48\% (Eintrag 4), was darauf schließen ließ, dass es gegenüber Kieselgel nur leidlich stabil war. Die Aufreinigung durch direktes Auftragen des Reaktionsgemisches auf eine Sephadex-LH 20 Säule (MeOH, LH-20) lieferte 83 in quantitativer Ausbeute als gelben, amorphen Feststoff. Dabei ist bemerkenswert, dass dieses als diastereomerenreine Verbindung gebildet wurde. Obwohl die Konfiguration des Acetals 83 nicht einwandfrei spektroskopisch bewiesen wurde, ist es sehr wahrscheinlich, dass es in der abgebildeten Konfiguration vorliegt. Diese entspricht der Konfiguration des Naturstoffes, von dem bisher keine Diastereomere beschrieben wurden. Es ist also recht wahrscheinlich, dass die untere Seite des Kendomycins durch eine entsprechende Faltung des Moleküls sterisch so abgeschirmt ist, dass von der Unterseite kein nukleophiler Angriff auf C-20 erfolgen kann. Für die Kupplung des Kendomycin-Me-Acetals 83 mit Diazirin 48 (Tabelle 9) wurde DCC als Wasserfänger verwendet, da sich $\mathbf{8 3}$ in Gegenwart von EDCI zersetzte. Nach Größenausschlusschromatographie an Sephadex LH-20 mit Methanol als Eluent erhielt man zwei Fraktionen. Die erste Fraktion enthielt neben dem Produkt 84 größere Mengen eines weißen Feststoffes, welcher sich später als Hydrolyseprodukt $(N, N-$ Dicyclohexylharnstoff) des DCC herausstellte. Durch Zugabe von Pentan und anschließende Filtration konnte diese Verunreinigung leicht abgetrennt werden. Nach Entfernen des 
Pentans erhielt man so die Zielverbindung 84 in 48\% Ausbeute. Das Acetal 84 ist, genau wie Naturstoff 78, ein gelber, amorpher Feststoff. Die zweite Fraktion enthielt ein Gemisch aus Edukt (ca. 32\%) und dem Methylester des Azirins 48.

Schema 24: Herstellung des Me-Acetals 83, sowie Kupplung mit den Photoaffinitätslabeln 48 und $48 a$.

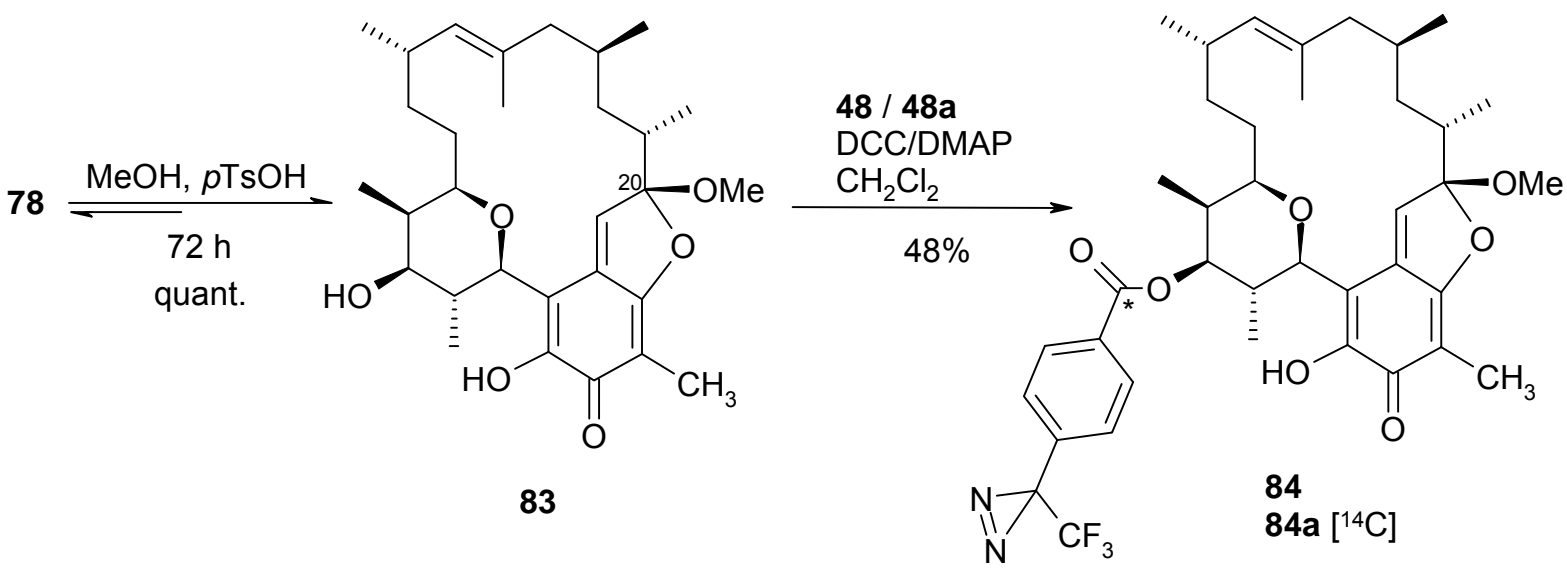

Tabelle 8: Herstellung des Me-Acetals 83.

\begin{tabular}{ccccc}
\hline $\mathbf{N r}$ & Kat. & $\mathbf{t}[\mathbf{h}]$ & $\mathbf{A}[\%]$ & Bemerk. \\
\hline 1 & $\mathrm{CeCl}_{3}$ & 24 & - & Edukt \\
2 & $\mathrm{CSA}$ & 12 & - & Edukt \\
3 & $\mathrm{PPTs}$ & 12 & - & Edukt \\
4 & $p$-TsOH & 72 & 48 & KG-Säule \\
5 & $p$-TsOH & 72 & quant. & LH-20 \\
\hline
\end{tabular}

Tabelle 9: Kupplung der Photoaffinitätslabel 48 und 48a an das Me-Acetal 83.

\begin{tabular}{ccccccccc}
\hline & \multicolumn{2}{c}{ PAL } & DMAP & & & $\mathbf{t}$ & $\mathbf{A}$ & Bemerk. \\
\cline { 2 - 4 } $\mathbf{N r}$ & & {$[\mathbf{e q}]$} & {$[\mathbf{e q}]$} & & {$[\mathbf{e q}]$} & {$[\mathbf{h}]$} & {$[\%]$} & \\
\hline 1 & $\mathbf{4 8}$ & 2.0 & 2.0 & EDCI & 2.0 & 5.0 & - & Zersetz. \\
2 & $\mathbf{4 8}$ & 1.2 & 8.0 & EDCI & 2.0 & 2.5 & - & Zersetz. \\
3 & $\mathbf{4 8}$ & 3.0 & 3.5 & DCC & 1.5 & 24 & - & Testrkt. \\
4 & $\mathbf{4 8}$ & 2.0 & 2.3 & DCC & 2.6 & 16 & 48 & LH-20 \\
5 & $\mathbf{4 8 a}$ & 2.0 & 2.3 & DCC & 2.6 & 16 & - & LH-20 \\
\hline
\end{tabular}


Die Umsetzung mit dem radioaktiven Azirin 48a wurde nach fast identischen Reaktionsbedingungen durchgeführt, lieferte jedoch aus unbekannten Gründen nicht die Zielverbindung 82a, sondern nur nicht identifizierbare Zersetzungsprodukte.

\section{I.4.4 Synthese des Diazirinyl-Apicularens}

Das antibiotische und cytotoxische Apicularen A (85) wurde erstmals von HöFLE et al. aus verschiedenen Spezies von Myxobacterien isoliert (Schema 25). ${ }^{[64]}$ Aufgrund seiner bemerkenswerten biologischen Wirkung zog das Apicularen (85) schnell das Interesse verschiedener Arbeitsgruppen auf sich. Als Ergebnis standen neben einer ganzen Reihe an Totalsynthesen $^{[65]}$ auch Untersuchungen auf eine Hemmwirkung von 85 gegenüber V-ATPasen. Als Ergebnis dieser Versuche zeigte sich, dass das Macrolacton, in vergleichbaren Konzentrationen wie Bafilomycin und Concanamycin, selektiv die V-ATPasen hemmt. ${ }^{[66]}$ In ersten Untersuchungen zur Bindungsstelle des Apicularens 85 an der V-ATPase zeigte sich, dass es das radioaktive ${ }^{125}$ I-markierte Concanolid-Derivat 5 nicht aus dessen Bindungsstelle verdrängen kann. Dies ist ein Hinweis darauf, dass Apicularen 85 an einer anderen Stelle bindet als die Plecomacrolide 1 und 2. Für Untersuchungen zur genauen Bindungsstelle des Apicularens an der V-ATPase wurde das Diazirinylderivat 86 synthetisiert. Die Veresterung erfolgte in Dichlormethan unter Standardbedingungen ${ }^{[34]}$ mit EDCI und DMAP. Wie erwartet zeigte die Hydroxylgruppe an C-3 unter den Reaktionsbedingungen eine höhere Reaktivität als die sekundäre Hydroxyfunktion an C-11 und so konnte die Zielverbindung 86 nach säulenchromatographischer Reinigung in $60 \%$ Ausbeute erhalten werden.

Schema 25: Derivatisierung des Apicularen A (83).

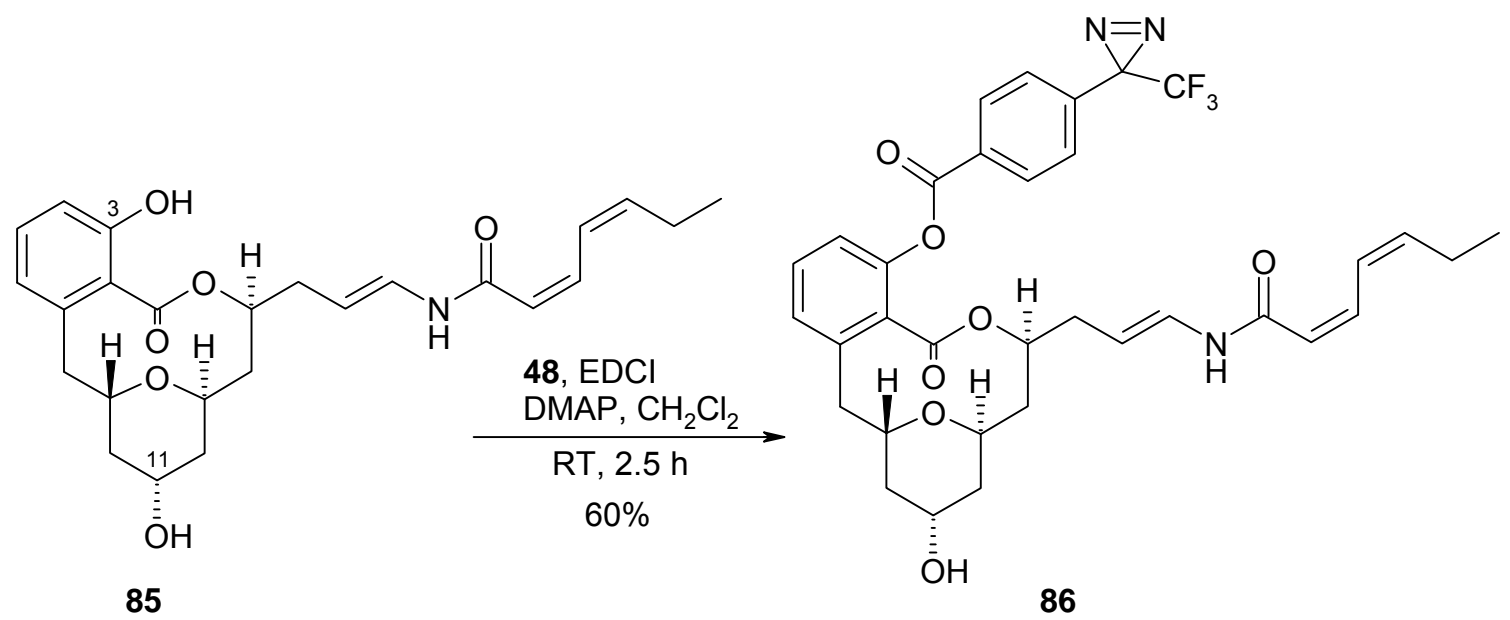




\section{I.5 Ergebnisse der Photoaffinitätsstudien mit den markierten Naturstoffen}

Die biologischen Untersuchungen mit den derivatisierten Naturstoffen wurden von Dr. M. HuSS (Universität Osnabrück) durchgeführt.

\section{I.5.1 Ergebnisse der Untersuchungen mit den Concanolid- und den Bafilomycinderivaten}

Die Ergebnisse der V-ATPase-Untersuchungen mit Concanamycin A (1) und dessen Derivaten 4 und 75, Bafilomycin (2) sowie Diazirinyl-Bafilomycin (77) sind in Abbildung 12 dargestellt. In der Reihe der Concanamycinderivate war das Concanolid A (4) die aktivste Verbindung, gefolgt vom Naturstoff Concanamycin A (1). Das Diazirinyl-Concanolid A (75) zeigte eine nur unwesentlich geringere Aktivität als der Naturstoff 1, was eine substantielle Verbesserung zum ${ }^{125}$ I-Concanolid (5) darstellt, dessen Aktivität im Vergleich zum Naturstoff um etwa Faktor 1000 schlechter war. ${ }^{[30]}$

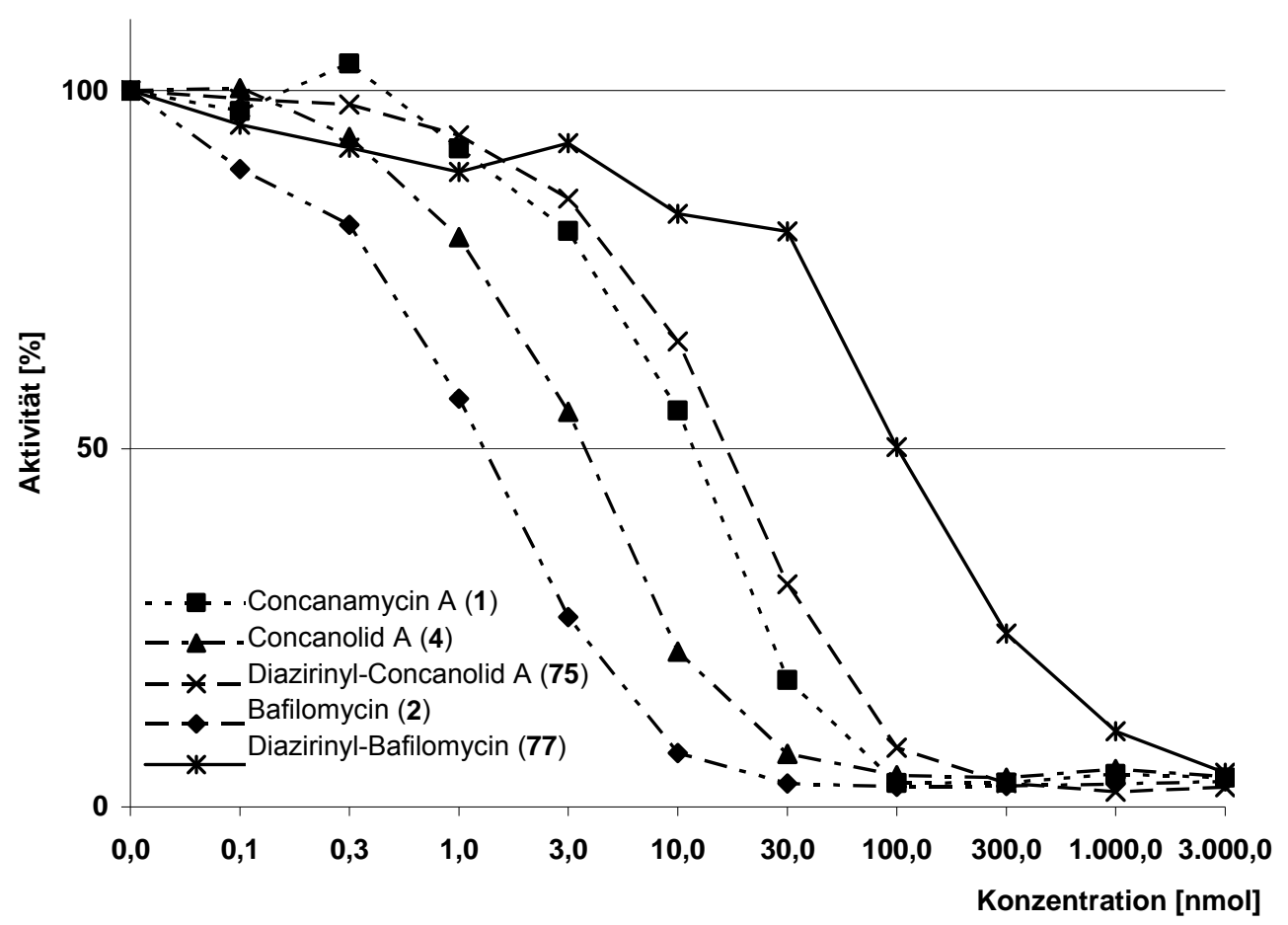

Abbildung 12: Die PAL-Derivate 75 und 77 zeigen eine gute Hemmwirkung gegen die VATPase. 


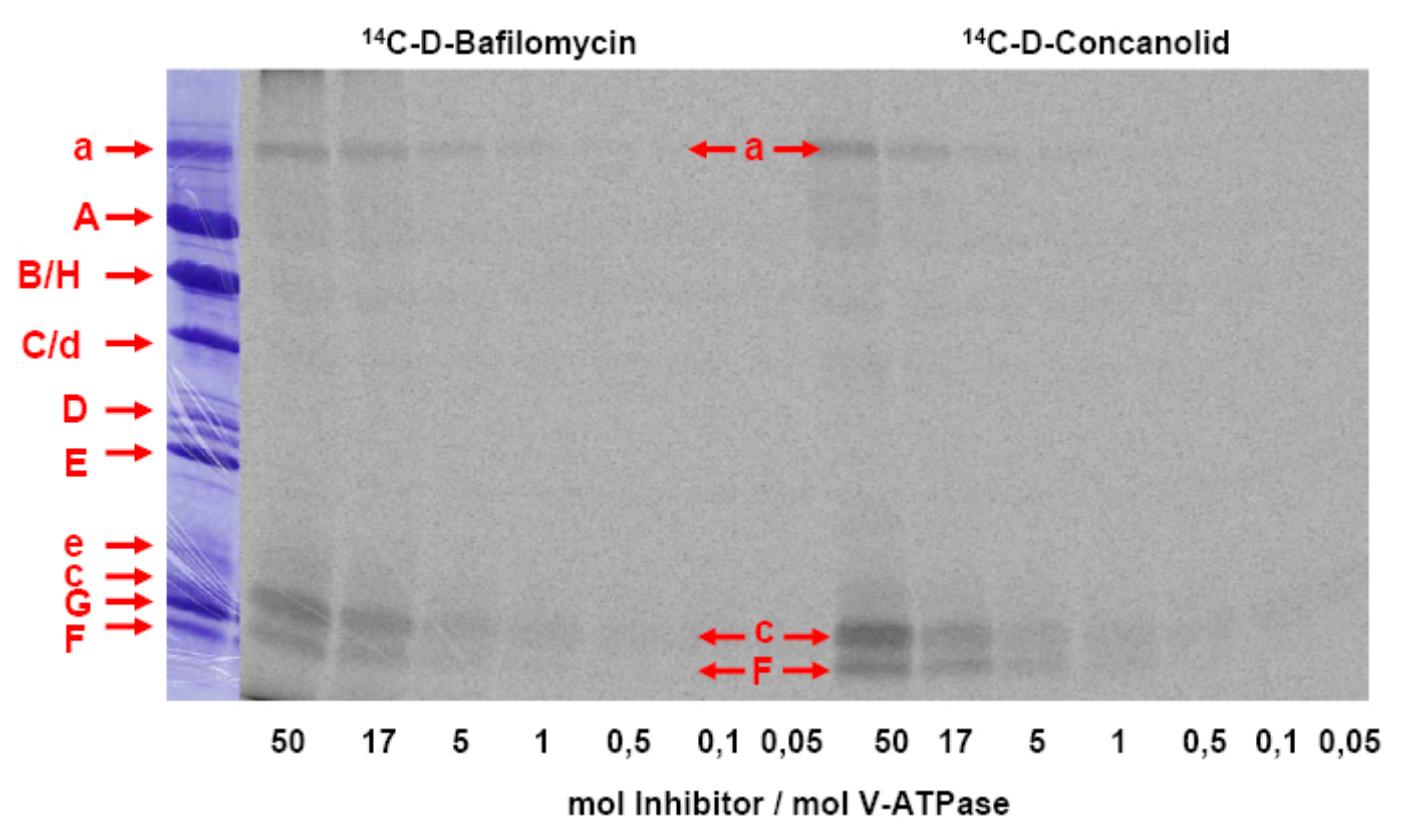

Abbildung 13: Autoradiogram der ${ }^{14} \mathrm{C}$-markierten Plecomacrolide 75a und 77a. Die radioaktiven Markierungen in den Untereinheiten a, c und F sind gut zu erkennen.

Nachdem die Wirksamkeit der diazirinylierten Derivate nachgewiesen war, konnten die Photoaffinitätsstudien mit den radioaktiv markierten Verbindungen durchgeführt werden. Abbildung 13 zeigt das Autoradiogramm der ${ }^{14}$ C-markierten Plecomacrolide 75a und 77a. Beide Verbindungen zeigten eindeutige Markierungen in den Untereinheiten c, a und F der $\mathrm{V}_{0}$-Domäne der ATPase. Es ist also davon auszugehen, dass der Molekülteil um C-23 des Concanolides 4 bzw. C-21 des Bafilomycins (2) in der Schnittstelle zwischen diesen drei Einheiten liegen. Zieht man noch die früheren Ergebnisse des ${ }^{125}$ I-Derivates 5 hinzu, wo Markierungen ausschließlich in der c-Untereinheit des $\mathrm{V}_{0}$-Komplexes gefunden wurden, so ergibt sich ein noch genaueres Bild, in dem der westliche Molekülteil um C-9 (Concanamycin) bzw. C-7 (Bafilomycin) tief in der c-Untereinheit liegt, währenddessen der östliche Molekülteil (C-23 bzw C-21) in einer Lücke zwischen c,a- und F-Untereinheit, daraus herausragt.

\section{I.5.2 Ergebnisse der Untersuchungen mit den Kendomycinderivaten}

Die Hemmwirkung der Kendomycine gegenüber der V-ATPase ist mit $\mathrm{IC}_{50}$-Werten von 1$10 \mu \mathrm{mol}$ etwa um den Faktor 1000 schlechter als die Wirksamkeit der Plecomacrolide 1 und 2. Das Diazirinyl-Me-Kendomycin 84 zeigte in den Untersuchungen an der V-ATPase aus Meduca sexta von allen Kendomycinen die geringste Aktivität (Abbildung 14) und als einziges Derivat überhaupt eine geringere Aktivität als der Naturstoff 76. 


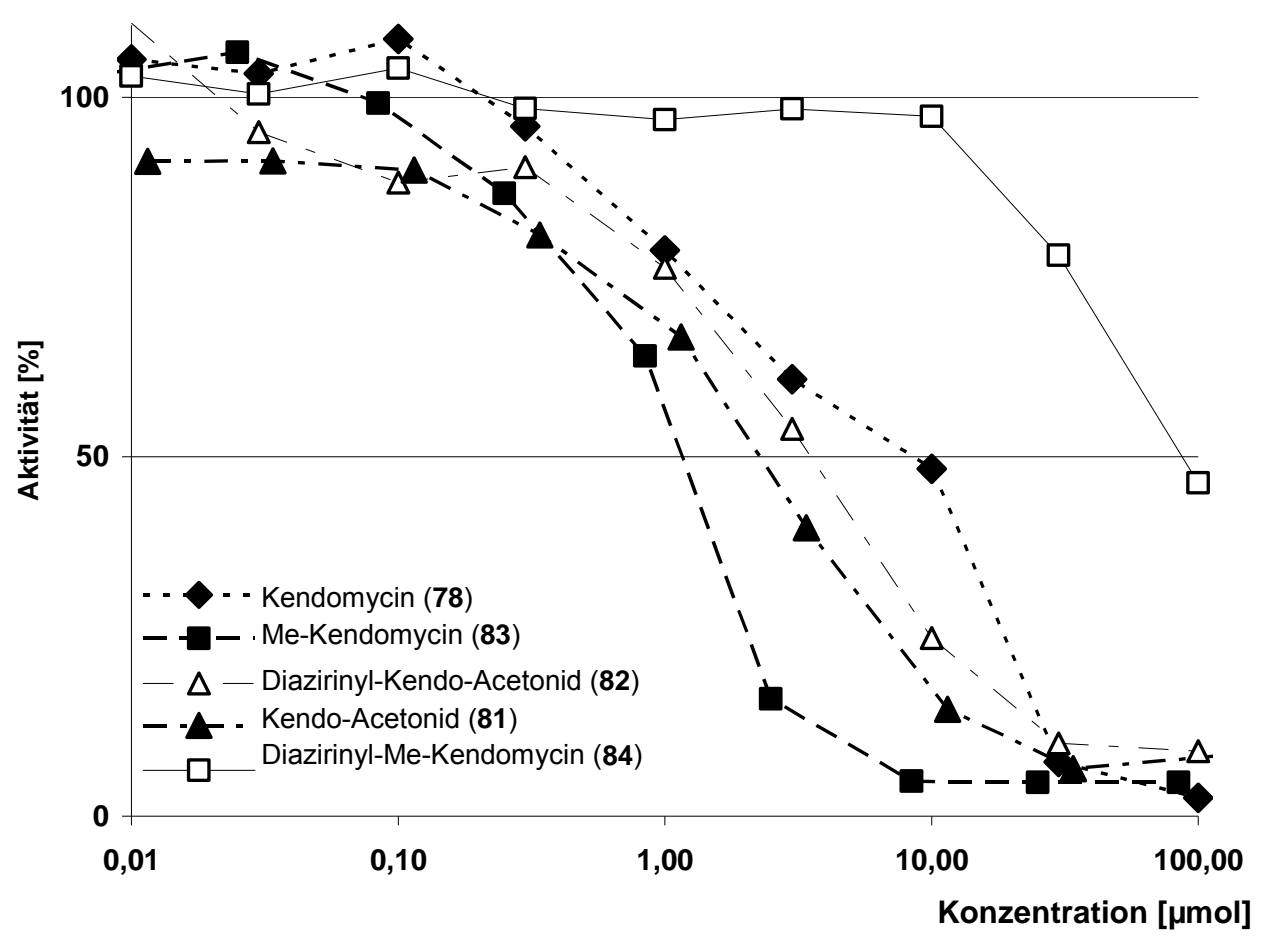

Abbildung 14: Ergebnisse der V-ATPase Hemmung mit den Kendomycinderivaten.

Die im Vergleich mit dem Naturstoff $\mathbf{7 8}$ leicht bessere Hemmwirkung der Derivate 81-83 könnte unter Umständen auf eine höhere Stabilität der Verbindungen unter den gewählten Untersuchungsbedingungen zurückzuführen sein. Um Hinweise darauf zu erhalten, ob das Kendomycin (78) und dessen Derivate im $\mathrm{V}_{1}$ oder im $\mathrm{V}_{0}$-Teil der V-ATPasen wirken, wurde die Aktivität des $\mathrm{V}_{1} \mathrm{~V}_{0}$-Holoenzyms und des löslichen $\mathrm{V}_{1}$-Komplexes in Abhängigkeit von $\mathrm{Mg}^{2+}$ und $\mathrm{Ca}^{2+}$ untersucht (Tabelle 10). Bei der Hydrolyse von Mg-ATP durch das Holoenzym werden (in vivo) Protonen transloziert, bei Ca-ATP dagegen nicht. Hier wird also entkoppelt hydrolysiert. Zum Vergleich: Die Mg-ATPase Aktivität ist durch die im $\mathrm{V}_{0}$-Komplex wirksamen Inhibitoren Bafilomycin (2) oder Concanamycin A (1) inhibierbar, die Ca-ATPase Aktivität jedoch nicht. Dies ist also ein Hinweis, dass es sich bei Kendomycin um einen $V_{1}$ Inhibitor handelt. Dies zeigt sich auch bei den Versuchen mit dem löslichen $\mathrm{V}_{1}$-Komplex, allerdings verhalten sich die Derivate hier zum Teil sehr unterschiedlich. $\mathrm{Zu}$ beachten ist, dass der $\mathrm{V}_{1}$-Komplex nur in Gegenwart von $25 \%$ Methanol eine Mg-ATPase Aktivität hat. Bei Untersuchungen mit den anderen ATPasen zeigte sich, dass bis auf 84 alle Kendomycinderivate dazu in der Lage waren, die PATPase zu inhibieren, jedoch keines der Derivate eine signifikante Wirkung auf FATPasen besitzt (Tabelle 10). 
Tabelle 10: $\mathrm{IC}_{50}$-Werte für die Kendomycine in verschiedenen Versuchsassays.

\begin{tabular}{|c|c|c|c|c|c|c|}
\hline \multirow[t]{2}{*}{ Inhibitor } & \multicolumn{2}{|c|}{$\begin{array}{c}\mathrm{V}_{1} \mathrm{~V}_{\mathrm{o}} \\
\text { Holoenzyme } \\
(M . \text { sexta })\end{array}$} & \multicolumn{2}{|c|}{$\begin{array}{c}\mathrm{V}_{1} \text { complex } \\
(M . \text { sexta })\end{array}$} & \multirow{2}{*}{$\begin{array}{c}\begin{array}{c}\text { P-ATPase } \\
\text { (pig kidney) }\end{array} \\
\mathrm{Mg}^{2+}\end{array}$} & \multirow{2}{*}{ 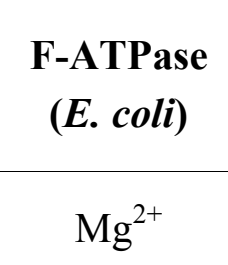 } \\
\hline & $\mathrm{Mg}^{2+}$ & $\mathrm{Ca}^{2+}$ & $\mathrm{Mg}^{2+}$ & $\mathrm{Ca}^{2+}$ & & \\
\hline Kendomycin (78) & 10 & 100 & 50 & 100 & 3 & $\begin{aligned} & 70 \% \\
\mathrm{c}= & 100 \mu \mathrm{mol}\end{aligned}$ \\
\hline Me-Kendomycin (83) & 1 & 3 & 3 & 10 & 10 & $*$ \\
\hline $\begin{array}{l}\text { Diazirinyl-Me- } \\
\text { Kendomycin (84) }\end{array}$ & 100 & n.b. & n.b. & n.b. & n.b. & $*$ \\
\hline $\begin{array}{c}\text { Kendomycin Acetonid } \\
\text { (81) }\end{array}$ & 3 & 10 & 10 & 33 & 10 & * \\
\hline $\begin{array}{c}\text { Diazirinyl-Kendomycin } \\
\text { Acetonid (82) }\end{array}$ & 3 & $>500$ & 100 & $>500$ & 10 & $*$ \\
\hline
\end{tabular}

* Keine Hemmwirkung bis $\mathrm{c}=100 \mu \mathrm{mol}$.

\section{I.5.3 Ergebnisse der Untersuchungen mit den Apicularenen}

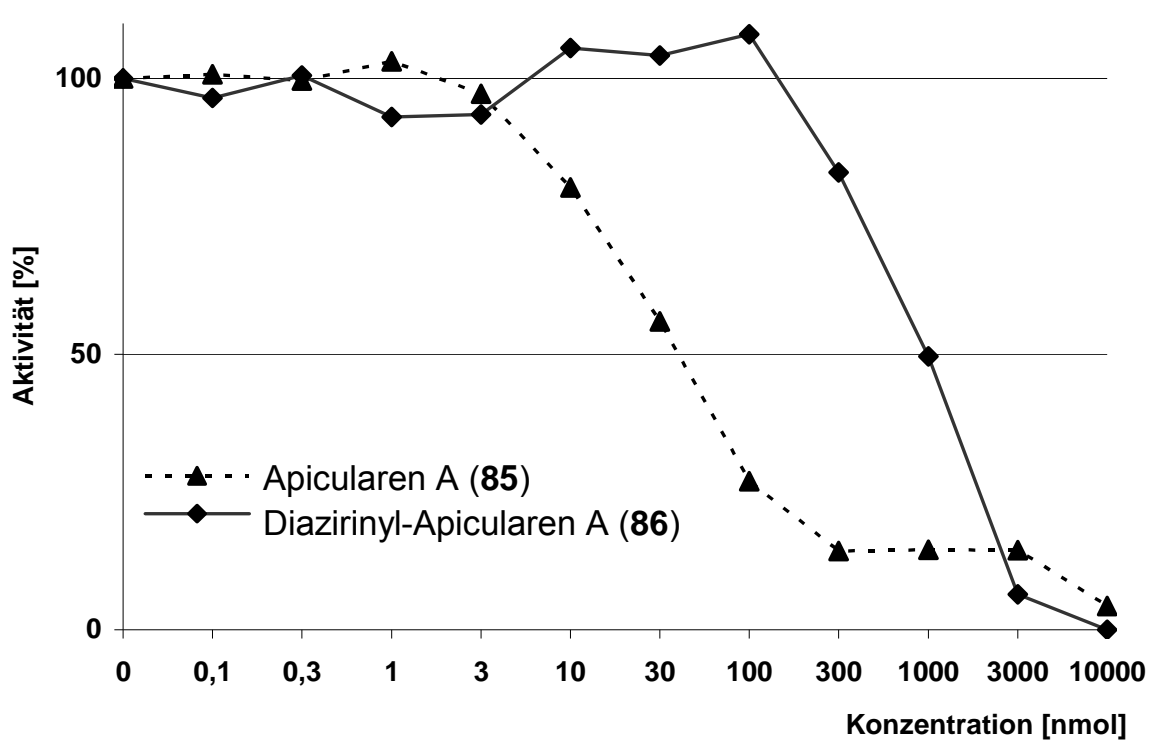

Abbildung 15: Ergebnisse der V-ATPase-Hemmung der Apicularene 85 und 86.

Das Diazirinyl-Apicularen A (86) zeigte im Vergleich zum Naturstoff 85 eine um den Faktor 30 geringere Wirksamkeit, was jedoch für die Photoaffinitätsstudien immer noch völlig ausreichend ist. Die Synthese des entsprechenden ${ }^{14} \mathrm{C}$-Diazirinyl-Apicularens und fortführende Untersuchungen zum Wirkort sind Gegenstand aktueller Arbeiten. 


\section{Hauptteil II - Zur Totalsynthese des Collinolactons}

\section{II.1 Vorüberlegungen zur geplanten Totalsynthese des Collinolactons}

Wie bereits in der Einleitung erwähnt wurde, postulierte L. HOFFMANN als zentralen Schritt der Biosynthese des Collinolactons (7) eine [6+4]-Cycloaddition, welche ausgehend vom Pentaen 13 das Collinoketon (14) ergibt. ${ }^{[36]}$ Auch wenn eine enzymatisch katalysierte, konzertierte [6+4]-Cycloaddition nicht auszuschließen ist, muss doch festgestellt werden, dass bis heute in der Literatur nur wenige synthetische und keine biosynthetischen Beispiele für diese pericyclische Reaktion beschrieben wurden. So gibt es neben theoretischen Überlegungen zur Durchführbarkeit der [6+4]-Cycloaddition, ${ }^{[67]}$ einige praktische Anwendungen. ${ }^{[68]}$ Als Beispiele seien die Synthese der Azulene ${ }^{[69]}$ oder die $\operatorname{Cr}(0)$-katalysierte Synthese eines Fragmentes des Streptovaricin $\mathrm{D}^{[70]}$ genannt. Aufgrund der geringen Ähnlichkeiten der hier verwendeten Edukte und Reaktionsbedingungen können diese jedoch kaum die Annahme stützen, dass das Collinoketon (14) durch eine [6+4]-Cycloaddition gebildet wird. Alternativ könnte es auch aus der in Schema 26 dargestellten Reaktionssequenz hervorgehen.

Schema 26: Synthese des Collinoketons (14) über eine intramolekulare [4+2]-Cycloaddition (A) und anschließende Cope-Umlagerung (B).

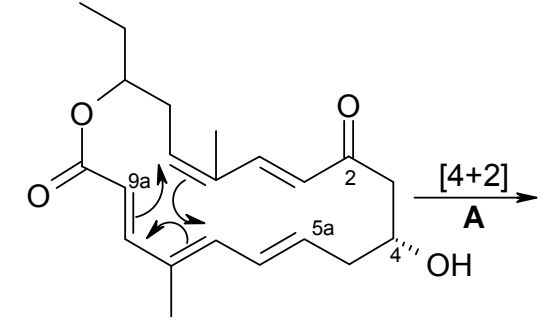

13

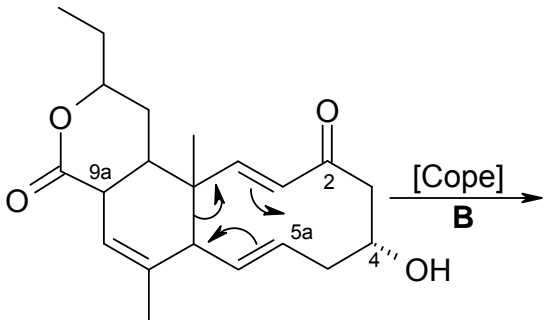

87

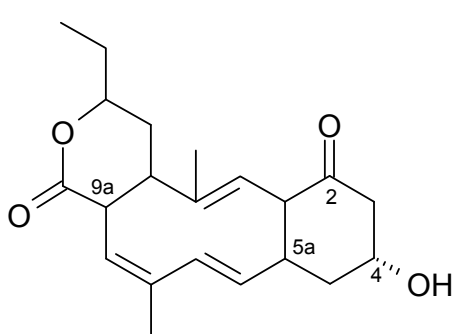

14

Über eine intramolekulare [4+2]-Cycloaddition wird zunächst das trizyklische Intermediat 87 gebildet, welches über eine Cope-Umlagerung schließlich das Collinoketon (14) ergibt. Gestützt wird diese Hypothese dadurch, dass Diels-Alder Reaktionen schon öfter als Bestandteil von Biosynthesen beschrieben wurden $^{[71]}$ und auch enzymatisch katalysierte Cope-Umlagerungen ${ }^{[72]}$ bekannt sind. In der Naturstoffsynthese fand eine Reaktionssequenz aus einer intramolekularen Diels-Alder Reaktion gefolgt von einer Cope-Umlagerung in der Darstellung der Halenaquinone bereits Anwendung. ${ }^{[73]}$ Auf Grundlage dieser Überlegungen wurde für das Collinolacton (7) die bereits vorgestellte biomimetische Totalsynthese mit der intramolekularen Diels-Alder-Reaktion, der Oxy-Cope-Reaktion und der Baeyer-Villiger 
Oxidation als Schlüsselschritte entwickelt. Die Oxy-Cope-Variante wurde gewählt, weil diese eine höhere Triebkraft besitzt und somit unter besonders milden Reaktionsbedingungen durchgeführt werden kann. Außerdem konnte auf Erfahrungen mit diesem Reaktionstyp aufgebaut werden. ${ }^{[74]}$

\section{II.2 Die Intramolekulare Diels-Alder Reaktion}

Schema 27: Von OTTO DiELS und KNUT ALDER 1928 erstmals beschriebene und heute nach ihnen benannte [4+2]-Cycloaddition. ${ }^{[75]}$

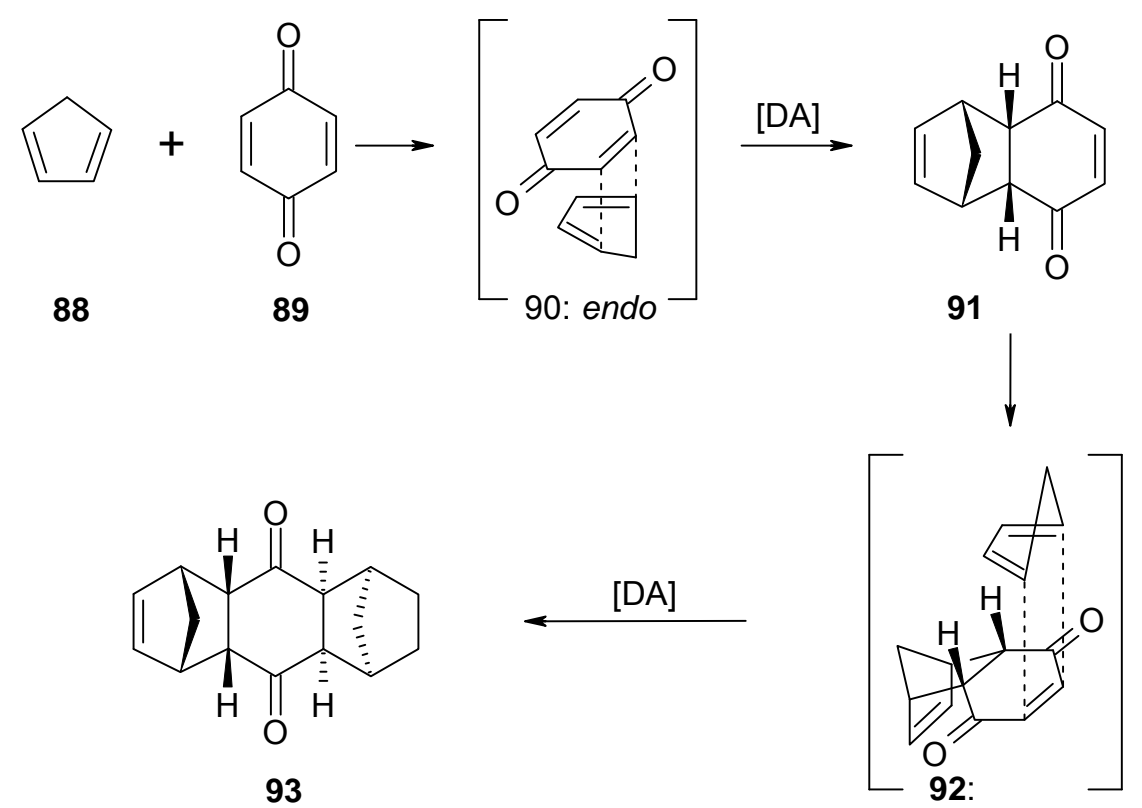

Als Professor OtTo Diels und sein Student KNUT ALDER 1928 erstmals die [4+2]Cycloaddition beschrieben (Schema 27), erkannten sie bereits damals die immense Bedeutung, die diese Reaktion für die Organische Chemie im Allgemeinen und die Totalsynthese von Naturstoffen im Speziellen haben würde. ${ }^{[75]}$ Seit der Vergabe des Nobelpreises für die Entdeckung der heute als „Diels-Alder“-Reaktion bekannten Umsetzung im Jahre 1950 hat sich diese zu einem der wertvollsten Werkzeuge in der Naturstoffsynthese entwickelt. ${ }^{\left[{ }^{76]}\right.}$ Dies ist unter anderem darauf zurückzuführen, dass bei Verwendung stereogener Edukte die Stereoselektivität oft gut vorhersagbar ist. Dies erlaubt in nur einem Reaktionsschritt den gezielten Aufbau mehrerer Stereozentren aus achiralen Verbindungen. So sollte auch bei der Totalsynthese des Collinolactons (7) die Hälfte der sechs Stereozentren des Zielmoleküls in einer intramolekularen Diels-Alder-Reaktion diastereoselektiv aufgebaut werden (Schema 28). 
Schema 28: Diastereoselektive Synthese des Decalons 94 über eine intramolekulare DielsAlder-Reaktion.
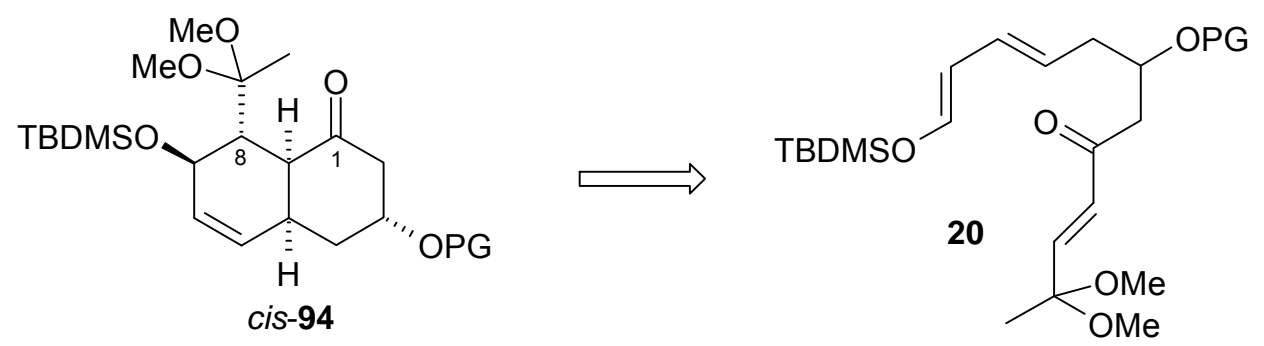

Die cis/trans-Selektivität der Ringverknüpfung in intramolekularen Diels-AlderReaktionen von 1,7,9-Decatrienonen wie 20 und ähnlichen Verbindungen war bereits Gegenstand sowohl praktischer als auch theoretischer Arbeiten. In letzteren wurden auch die Energien der möglichen Übergangszustände berechnet. ${ }^{[77]}$ Bei diesen Untersuchungen zeigte sich, dass die Substituenten an Dien und Dienophil einen entscheidenden Einfluss auf die Diastereoselektivität haben. Dabei haben die Substituenten an C-10 des Diens einen geringeren Einfluss auf das beobachtete Diastereomerenverhältnis der Produkte als die Substituenten an C-1 des Dienophils. So kann eine Änderung der Konfiguration $(E$ zu $Z)$ an C-1 des Dienophils sogar eine fast völlige Umkehr der Diastereoselektivität verursachen. ${ }^{[78]}$ Andererseits zeigte sich, dass bei Verwendung eines Gemisches aus Trienonen mit $7 E / 9 E$ und $7 E / 9 Z$-konfiguriertem Dien, ausschließlich jene Trienone mit $E / E$-Konfiguration eine Diels-Alder-Reaktion eingehen konnten. Die Trienone mit $Z / E$ Konfiguration wurden unverändert zurückgewonnen. ${ }^{[79]}$ Neben den Substituenten am $\pi$ System des Trienons besitzen auch die Substituenten am aliphatischen Henkel einen entscheidenden Einfluss auf das Ergebnis der IMDA-Reaktion. ${ }^{[77,79,80]}$ Nimmt man für den Übergangszustand des Triens in der IMDA-Reaktion eine quasiäquatoriale Stellung des Substituenten $\mathrm{R}^{2}$ an, so ergeben sich die in Schema 29 gezeigten vier möglichen Übergangszustände. Das Diastereomer cis-a, welches über den endo-Sessel Überganszustand gebildet wird, führt dabei zum Decalon 94 mit der gewünschten Stereokonfiguration. Ergebnisse aus Versuchen mit ähnlichen Trienonen zeigten eine Bevorzugung des endo-Übergangszustandes und somit der Decalone mit der gewünschten cis-Verknüpfung der beiden Ringe (Tabelle 11, Eintrag 1). ${ }^{[80]}$ Sterisch anspruchsvolle Substituenten $\mathrm{R}^{3}$ können jedoch eine Absenkung dieser Selektivität verursachen. ${ }^{[79,81]}$ Deshalb erschien es sinnvoll, anstatt des sterisch anspruchsvolleren Dimethylacetals eine „schlankere“ Ethinylfunktion als Substituent an C-8 im Zielmolekül 94 zu verwenden. 
Schema 29: Mögliche Übergangszustände der Intramolekularen Diels-Alder-Reaktion.

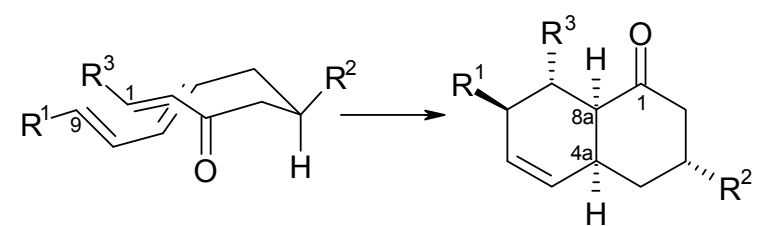

endo-Sessel

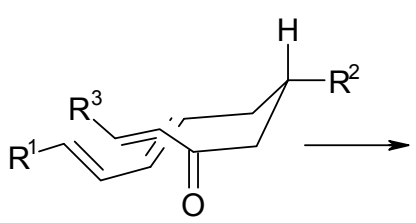

endo-Boot cis-a<smiles>[R]C1CC(=O)[C@]2(C)C([R])C([R])C=C[C@H]2C1</smiles>

cis- $\boldsymbol{b}$<smiles>[R]C/C=C(\CC(=O)CC)CC([R])CC</smiles>

exo-Sessel

trans-a

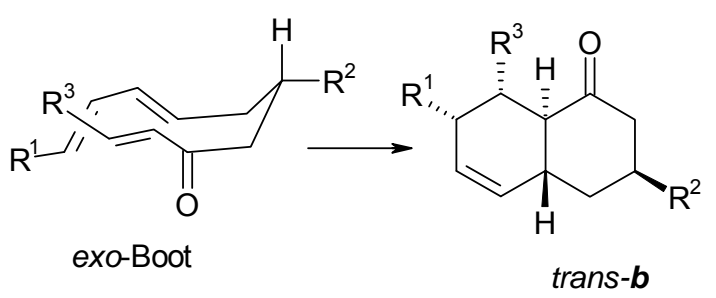

Schema 30: Optimierte Zielstrukturen des Decalons 96 und des entsprechenden Vorläufers 95.

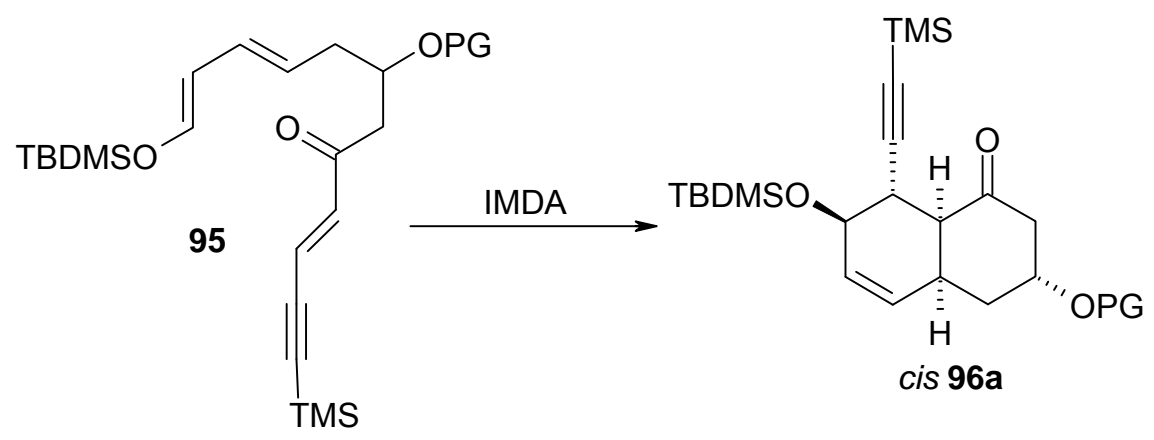

Somit ergab sich Trienon 95 als neuer Vorläufer für die IMDA-Reaktion, welche das entsprechende Decalon 96a liefern sollte (Schema 30). Eine Erhöhung der endoSelektivität kann durch Zusatz verschiedener Lewissäuren erreicht werden. So beobachtete man für die Substrate 97 und 98, entgegen den Erwartungen, in rein thermischen Reaktionen $^{[82]}$ keine nennenswerte Bevorzugung der endo-Produkte cis-a und cis-b (Tabelle 11, Eintrag 2). Durch Verwendung der Lewissäuren $\mathrm{BF}_{3} \cdot \mathrm{OEt}_{2}$ bzw. $\mathrm{SnCl}_{4}$ (Einträge 3 und 4), welche an die Carbonylfunktion koordinieren und so die sterischen Wechselwirkungen im ÜZ beeinflussen, konnte die endo-Selektivität deutlich gesteigert werden. ${ }^{[83]}$ Alle bisher diskutierten Überlegungen deuteten also darauf hin, dass die angestrebte intramolekulare Diels-Alder-Reaktion mit Trienon 95 bevorzugt über einen der beiden endo-Übergangszustände verlaufen und somit Decalon 96a mit der gewünschten cis-Verknüpfung ergeben sollte. Andererseits zeigten die Ergebnisse der Reaktion mit Trienon 98 (Tabelle 11) sowie weitere Berechnungen, dass bei der rein thermischen 
Durchführung vergleichbarer Diels-Alder-Reaktionen die Substrate bevorzugt über den endo-Boot Übergangszustand abreagierten. ${ }^{[80,84]}$ Dieser Reaktionsweg würde jedoch für 95 nicht das gewünschte Diastereomer cis-a (96a), sondern Isomer cis-b (96b) als Hauptprodukt ergeben. Dieses Problem konnte vor Beginn der Arbeiten nicht abschließend gelöst werden, da die genauen sterischen Verhältnisse in den Übergangszuständen des Trienons 95 nicht vorhersagbar waren. Wäre es jedoch nicht möglich gewesen, das gewünschte Isomer cis-a (96) als Hauptprodukt zu erhalten, hätte man über eine Umkehrung der Konfiguration an C-3 des Decalons durch eine Mitsunobu-Reaktion eine einfache Möglichkeit gehabt, um cis-b in cis-a umzuwandeln. ${ }^{\left[{ }^{85]}\right.}$

Tabelle 11: Beispiele für IMDA-Reaktionen mit verschiedenen 1,7,9-Decatrien-3-onen.<smiles>CC=CC=CCC(F)CC(=O)C=CC</smiles>
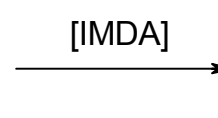<smiles>[R]C1CC(=O)[C@H]2[C@@H](C)[C@@H](C)C=C[C@H]2C1</smiles>

… $\mathrm{R}$ cis-a

$-\mathrm{R}$ cis-b<smiles>[R]C1CC(=O)[C@H]2[C@@H](C)[C@@H](C)C=C[C@H]2C1</smiles>

...' R trans-a

- R trans-b

\begin{tabular}{cccccccccccc}
\hline Nr. & & $\mathbf{R}$ & $\begin{array}{c}\text { cis- } \\
\mathbf{a}\end{array}$ & $\begin{array}{c}\text { cis- } \\
\mathbf{b}\end{array}$ & $\begin{array}{c}\text { trans- } \\
\boldsymbol{a}\end{array}$ & $\begin{array}{c}\text { trans- } \\
\mathbf{b}\end{array}$ & $\mathbf{T}\left[{ }^{\circ} \mathbf{C}\right]$ & $\mathbf{B e m}$. & $\mathbf{e q}$ & $\begin{array}{c}\mathbf{A} \\
{[\mathbf{\%}]}\end{array}$ \\
\hline 1 & $\mathbf{9 7}$ & $\mathrm{Me}$ & \multicolumn{2}{c}{99} & & 1 & & 110 & - & - & 78 \\
2 & $\mathbf{9 8}$ & $\mathrm{CO}_{2} \mathrm{Me}$ & 30 & 23 & 36 & 11 & 110 & - & - & 81 \\
3 & $\mathbf{9 8}$ & $\mathrm{CO}_{2} \mathrm{Me}$ & 18 & 73 & 9 & - & $-10 \rightarrow \mathrm{RT}$ & $\mathrm{BF}_{3} \cdot \mathrm{OEt}_{2}$ & 1.4 & 90 \\
4 & $\mathbf{9 8}$ & $\mathrm{CO}_{2} \mathrm{Me}$ & - & 71 & - & 29 & $-78 \rightarrow-40$ & $\mathrm{SnCl}_{4}$ & 2.0 & 46 \\
\hline
\end{tabular}




\section{II.3 Zur Oxy-Cope Reaktion}

Sigmatrope Reaktionen, wie die Oxy-Cope-Umlagerung, gehören zu den Standardmethoden der Organischen Chemie und fanden bereits eingehend Verwendung. ${ }^{[86]}$ Die ursprünglich thermisch induzierte Oxy-Cope-Reaktion, ${ }^{[87]}$ welche erst bei Temperaturen von deutlich über $200{ }^{\circ} \mathrm{C}$ stattfindet, war zunächst aufgrund der harschen Reaktionsbedingungen auf robuste Substrate beschränkt.

Schema 31: Themische versus anionische Oxy-Cope-Umlagerung zu Ketonen. ${ }^{[88]}$

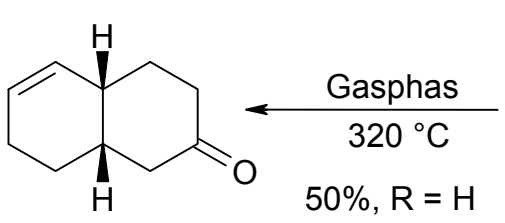

99<smiles>[R]C12C=CC(CC1)CC2(O)C=C</smiles>

100

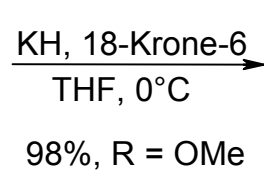

$98 \%, \mathrm{R}=\mathrm{OMe}$<smiles>COC1=C[C@H]2CCC(=O)C[C@H]2CC1</smiles>

101

Die signifikante Erhöhung der Reaktionsgeschwindigkeit in der anionischen Variante der Oxy-Cope Reaktion wurde erstmals 1975 von EvANS und GOLOB beschrieben (Schema 31). ${ }^{[88]}$ Als wahrscheinlichste Ursache für die höhere Reaktionsgeschwindigkeit gilt die, durch den Elektronenschub des Oxyanions 103 hervorgerufene Schwächung der Bindung zwischen C-3 und C-4. ${ }^{[86 a]}$ Damit lässt sich auch die Erhöhung der Reaktivität bei Zugabe von Kronenethern zum Reaktionsgemisch erklären: Durch die Komplexierung des Kations wird die Basizität des nun „nackteren“ Oxyanions erhöht, wodurch wiederum die Bindung zwischen C-3 und C-4 stärker geschwächt wird (Schema 32).

Schema 32: Ablauf der anionischen Oxy-Cope-Umlagerung.<smiles>C=CCC(O)C=C</smiles>

102<smiles></smiles>

103<smiles>CC=C[TeH]</smiles><smiles>C=CCCCC</smiles>

104<smiles>Oc1ccccc1</smiles>

105

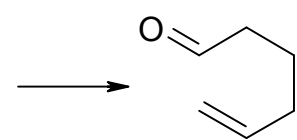

106

Dieses für das Oxyanion schlüssige Modell liefert jedoch keine Erklärung für die mangelnde Reaktionsbeschleunigung bei der Umsetzung von $\mathrm{NH}^{-}$substituierten Verbindungen. Wahrscheinlich verlaufen diese Umlagerungen über, erstmals von SWAMINATHAN ${ }^{[89]}$ postulierte, anionische Strukturen wie 108 (Schema 33). ${ }^{[90]}$ Die höhere Stabilität des entsprechenden Acroleinimins im Vergleich zum Aldehyd könnte auch die höhere Tendenz der Stickstoffanaloga zur konkurrierenden Dissoziation (Retro-En-Reaktion) erklären, die bei den Oxoanaloga wie 107 nur bei hohen Temperaturen beobachtet wird. ${ }^{\text {[91] }}$ 
Schema 33: Die Retro-En-Reaktion steht bei hohen Temperaturen in Konkurrenz zur OxyCope-Reaktion. ${ }^{[91]}$

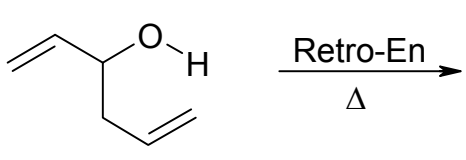

107

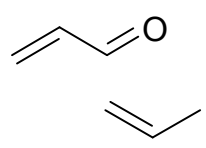

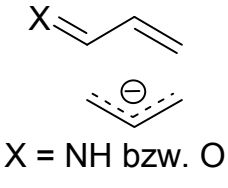

108

Entscheidend für den Erfolg der Oxy-Cope-Umlagerung in der organischen Synthese war der oft sehr gute Transfer stereochemischer Information aus chiralen Edukten in die jeweiligen Umlagerungsprodukte. Diese Beobachtung ist auf hochgeordnete Übergangszustände zurückzuführen. ${ }^{[89 b]}$ Aus energetischen Gründen sind in Abwesenheit übergeordneter sterischer, elektronischer und/oder struktureller Effekte sesselförmige Übergangszustände bevorzugt. $^{[89 \mathrm{a}]}$ Dennoch sind bootförmige Übergangszustände energetisch zugänglich und müssen in die Überlegungen miteinbezogen werden. ${ }^{[92]}$

\section{II.3.1 Oxy-Cope-Reaktion in der Synthese des Collinolactons}

Die Ketofunktion des bicyclischen Produkts cis-96, welches aus der intramolekularen DielsAlder-Reaktion hervorgeht, sollte anschließend als Dioxolan geschützt und die TMS-Gruppe am Alkin selektiv über das Silberacetylid abgespalten werden (Schema 34). Das so erhaltene Dioxolan 109 wäre dann bereit für die Einführung der oberen Seitenkette durch Carboaluminierung, Alanatbildung und Abfangen desselben mit Butenoxid. Die Verwendung enantiomeren-reinen Butenoxids sollte erst nach Optimierung der Reaktionsbedingungen erfolgen und somit den selektiven Aufbau des Stereozentrums an C12 des Collinolactons ermöglichen. Nach Schützung der im vorigen Schritt geformten sekundären Hydroxyfunktion als MOM-Ether ergibt sich der Tricyclus 110, welcher durch Abspaltung der TBDMS-Gruppe mit Tetrabutylammoniumfluorid und anschließende Oxidation (Swern, Dess-Martin etc.) in das $\alpha, \beta$-ungesättigte Keton 111 überführt wird. Durch Addition des lithiierten Orthoesters 112 wird die zweite Seitenkette eingeführt und man erhält so das Edukt für die anschließende Oxy-Cope-Reaktion. Aus sterischen Gründen ist zwar eine trans-Addition von 112 an das $\alpha, \beta$-ungesättigte Keton 111 zu erwarten, sollte jedoch dennoch das cis-Isomer von 112 gebildet werden, könnte sich aus diesem trotzdem das Isomer mit der gewünschten trans-Konfiguration bilden. So berichteten CLIVE et al., ${ }^{\text {[93] }}$ dass sich aus einem Substrat mit entsprechenden cis-ständigen Seitenketten, entgegen der Erwartungen, ein Produkt mit trans-Konfiguration der neu geformten Doppelbindung ergab. 
Dies lässt auf eine Bevorzugung einer axialen Stellung des Oxyanions schließen, welches normalerweise eine äquatoriale Position bevorzugt. ${ }^{[94]}$

Schema 34: Geplante Synthese des Intermediats 113.
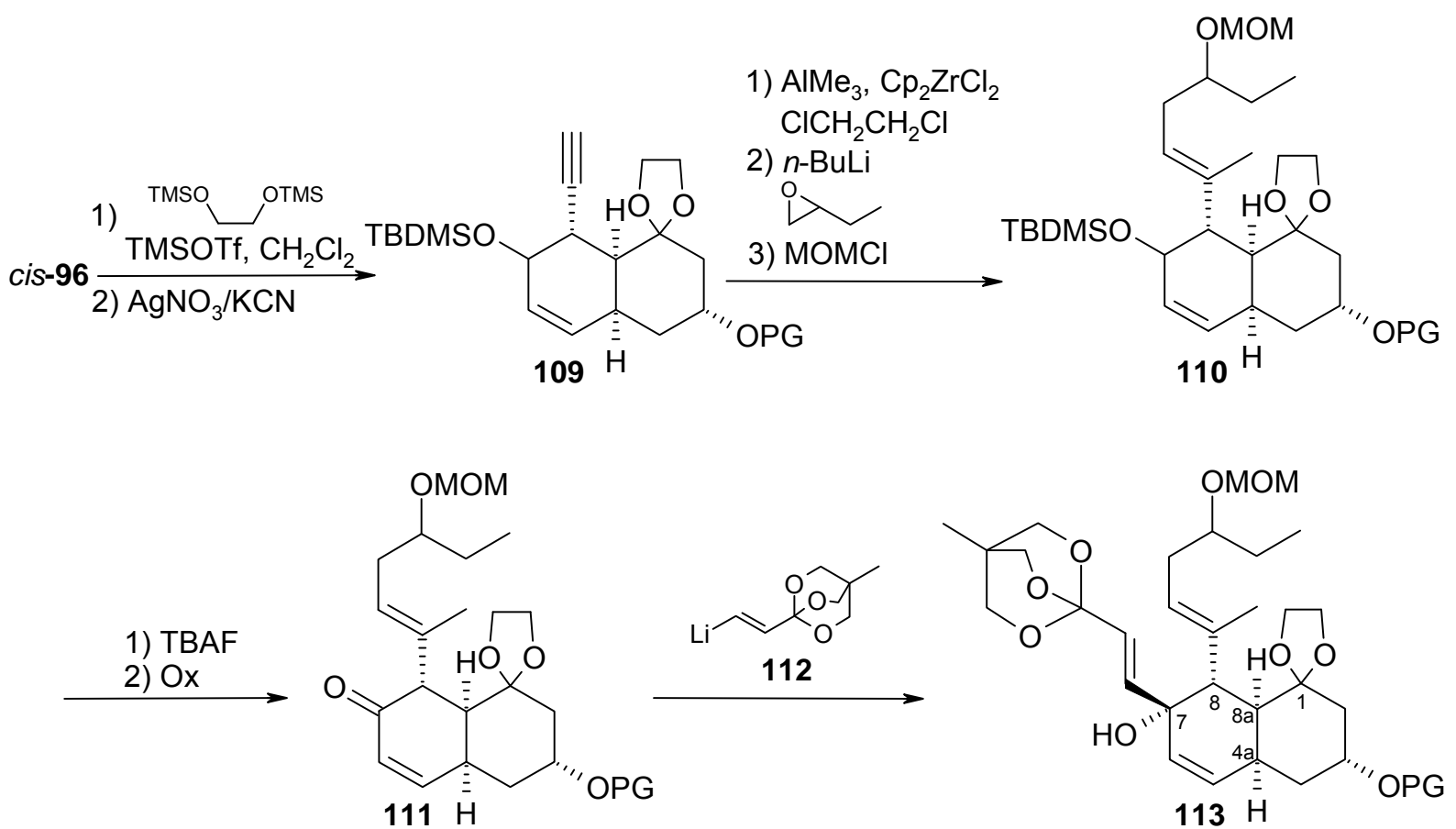

Die Oxy-Cope-Reaktion von 113 wird wahrscheinlich über die in Schema 35 gezeigten Übergangszustände verlaufen. Betrachtet man dreidimensionale Modelle von 113, so ist wohl trotz der bereits erwähnten Präferenz des Oxyanions für eine äquatoriale Position für 113 eine axiale Stellung im Übergangszustand anzunehmen. Wenn das Anion nämlich äquatorial liegt, stehen die Seitenketten anti-periplanar am Ring und können somit keinen sechsgliedrigen Übergangszustand bilden. Aus dem sesselförmigen Übergangszustand 113sessel sollte das gewünschte Produkt 114E mit trans-Stellung der Substituenten an C9a und C-13a sowie E-Konfiguration der trisubstituierten Doppelbindung hervorgehen. Aus dem energetisch ungünstigeren, bootförmigen Übergangszustand 113 boot würde sich das Produkt $114 Z$ mit cis-ständigen Substituenten an C-9a und C-13a sowie Zkonfigurierter Doppelbindung bilden. Da es schwierig ist, anhand von Modellen eine genaue Aussage über die Stereoselektivität der Oxy-Cope-Reaktion zu treffen, wurde im Rahmen dieser Dissertation eine Modellstudie zur Oxy-Cope-Reaktion durchgeführt. An einem einfachen, sechsgliedrigen System sollten so erste Erkenntnisse über Stereoselektivität, Reaktivität und Reaktionsbedingungen gewonnen werden. Die praktischen Arbeiten wurden durch Dipl.-Chem. EVELYN ZEILER im Rahmen einer Diplomarbeit durchgeführt. ${ }^{[95]}$ 
Schema 35: Wahrscheinliche Übergangszustände der Oxy-Cope-Reaktion von 113.

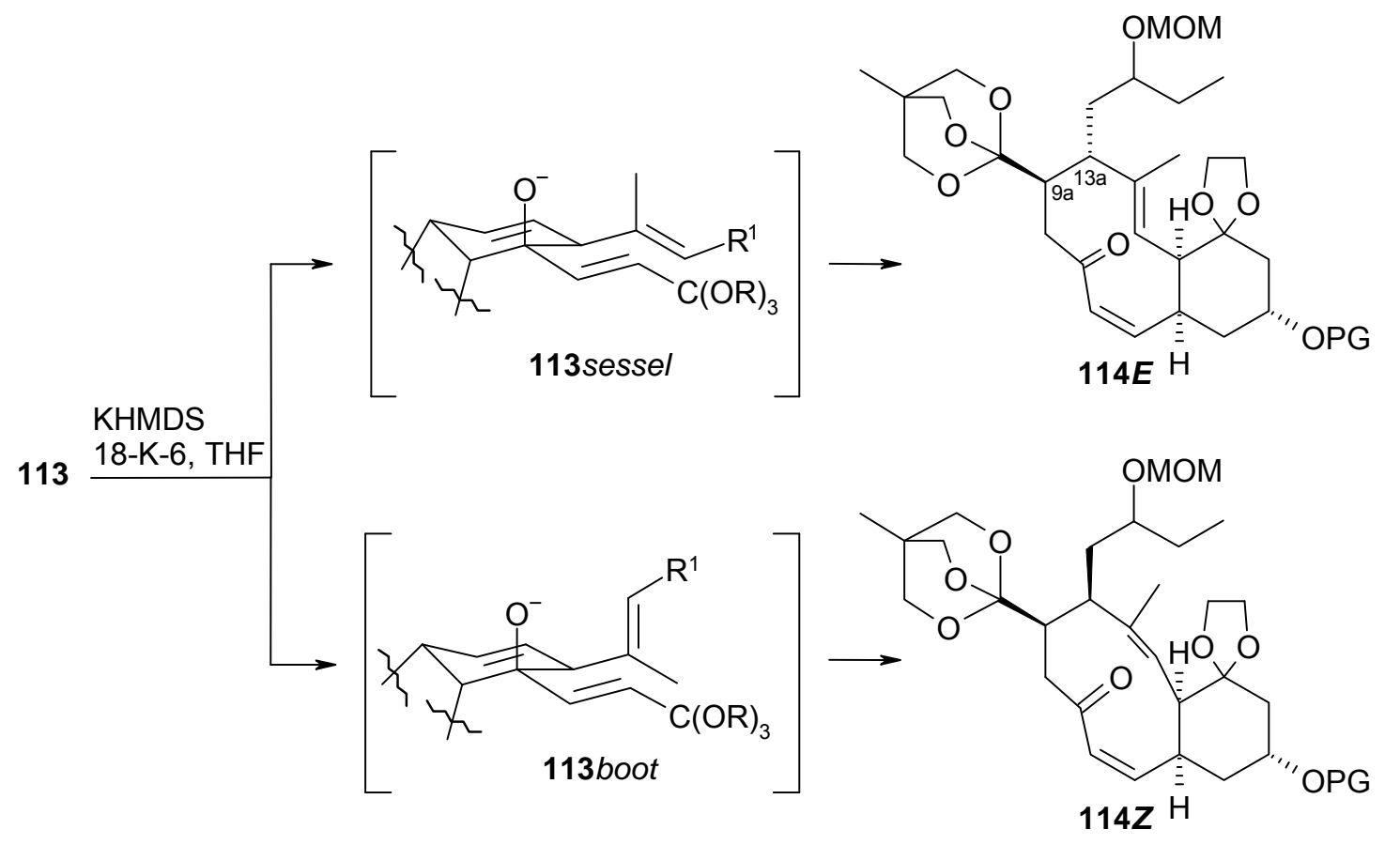

\section{II.3.2 Modellstudie zur Oxy-Cope-Reaktion ${ }^{[95]}$}

Synthese des Oxy-Cope-Vorläufers: Ausgangspunkt der Synthese war racemisches Cyclohexenoxid 115, welches mit Lithiumacetylid zum Alkin 116 umgesetzt wurde. Schützung der entstandenen Alkoholfunktion mit TBDMSCl ergab Silylether 117, welcher mit Trimethylaluminium und Zirconocendichlorid carboaluminiert wurde (Schema 36). ${ }^{[96]}$ Durch Zugabe von $n$-BuLi wurde das Alan ins reaktivere Alanat 118 überführt, wodurch die Reaktionszeit deutlich verringert werden kann. ${ }^{[97]}$ Durch Abfangen des Alanats mit Butenoxid erhielt man $\mathbf{1 1 9}$ in der abgebildeten Konfiguration. Aufgrund der langen Reaktionszeiten von mind. 2.5 Tagen wurde eine von WIPF et al. erstmals beschriebene Variante der Carboaluminierung getestet. ${ }^{[98]}$ Hierbei werden etwa 0.5 Äquivalente Wasser (bezogen auf $\mathrm{AlMe}_{3}$ ) zum Alan gegeben, wodurch sich oxo-verbrückte Zirkonocen-AlanDimere bilden, welche die Carboaluminierung sehr effektiv katalysieren. Bei der Umsetzung von $117 \mathrm{zu} 119$ wurde ebenfalls eine entsprechende Beschleunigung der Reaktion beobachtet. Die Umsetzung war in der Regel innerhalb von ca. 9 Stunden vollständig, allerdings waren die isolierten Ausbeuten etwas geringer (52\% vs. 64\%). Der sekundäre Alkohol in 119 wurde mit Chlormethylmethylether geschützt und anschließend die TBDMSSchutzguppe mit TBAF entfernt, dadurch erhielt man Cyclohexanol 120 in einer guten Ausbeute von 90\%. Anschließende Oxidation unter Swern-Bedingungen ergab das Keton 
121 in 84\%. Die Addition des lithiierten Orthoesters 112 ergab ein Produktgemisch aus dem trans-Produkt 122 und einer nicht genau identifizierten Minderkomponente, wobei es sich wahrscheinlich um das cis-Isomer handelte (Verhältnis 7:3). Der Vorläufer der Oxy-CopeReaktion 122 konnte durch Umkristallisation aus einem Pentan/Ether-Gemisch abgetrennt und dadurch in einer befriedigenden Ausbeute von 53\% isoliert werden.

Schema 36: Modellstudie zur Oxy-Cope-Reaktion in der Synthese des Collinolactons. Alle Verbindungen lagen racemisch vor.

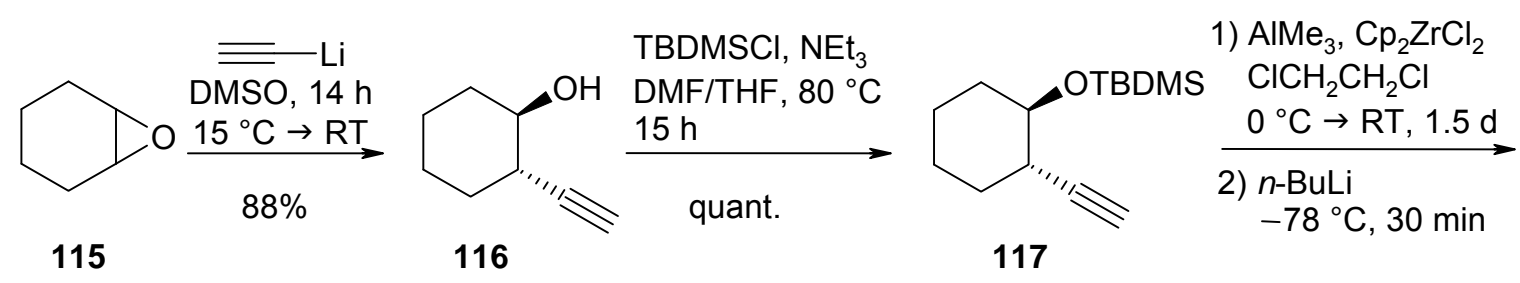

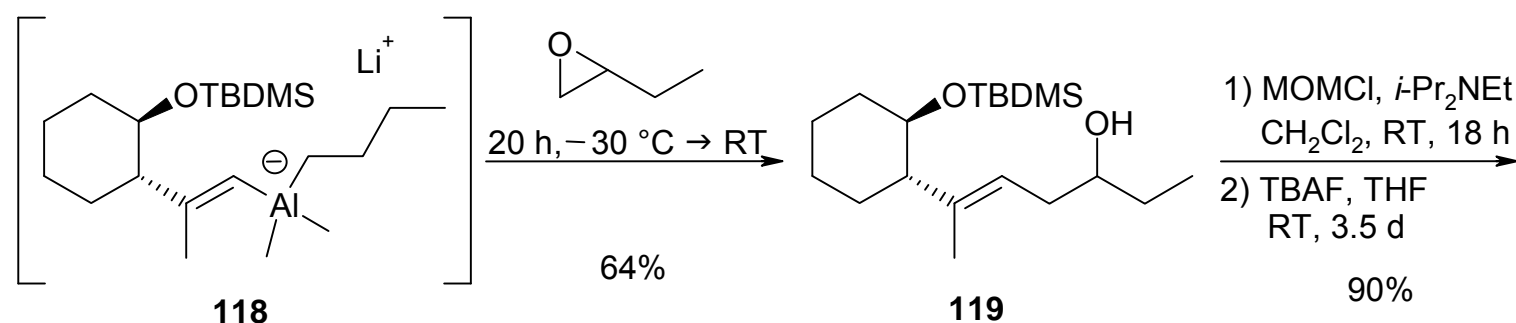<smiles>CCC(CC=C(C)[C@H]1CCCC[C@@H]1O)OC</smiles>

120<smiles>CCC(CC=C(C)[C@H]1CCCC(=O)C1=O)OC</smiles>

121
1) $\mathrm{MOMCl}, i-\mathrm{Pr}_{2} \mathrm{NEt}$

$\underset{\mathrm{CH}}{\mathrm{CH}_{2} \mathrm{Cl}_{2}, \mathrm{RT}, 18 \mathrm{~h}}$ RT, $3.5 \mathrm{~d}$

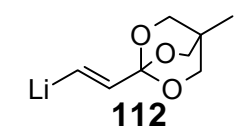
$53 \%$<smiles>CCC(C[C@H](C(C)=O)C1CCCCCC(=O)C1)OC</smiles>

Ergebnisse der Oxy-Cope-Reaktion: Die Oxy-Cope-Reaktion von 122 wurde mit Kaliumhexamethyldisilazid (2.0 eq) als Base und Kaliumspezifischem Kronenether (18-K-6, 1.5 eq) in siedendem THF durchgeführt. Bei niedrigeren Temperaturen oder Verwendung von Kaliumhydrid als Base wurde auch nach längerer Reaktionszeit kein Umsatz beobachtet. Das Rohprodukt der Reaktion enthielt neben der Hauptkomponente 123 Spuren eines zweiten Diastereomers, wobei es sich wahrscheinlich um das Isomer mit cis-ständigen 
Substituenten handelte. Die Hauptkomponente 123 konnte nach Säulenchromatographie in $59 \%$ Ausbeute isoliert werden. Erwartungsgemäß zeigte sich also, dass die Oxy-CopeReaktion mit 122 bevorzugt über den sesselförmigen Übergangszustand verläuft. Für die Umsetzungen mit dem deutlich komplexeren Collinolactonvorläufer 113 ergibt sich zunächst einmal die zufriedenstellende Erkenntnis, dass die Oxy-Cope-Reaktion mit der geplanten Konfiguration und Konstitution der Seitenketten möglich ist. Es wäre jedoch wünschenswert, schonendere Reaktionsbedingungen identifizieren zu können, da es sonst sicher schwierig wird, gute Ausbeuten zu erzielen.

Schema 37: Versuche zur Lactonisierung des Oxy-Cope-Produktes 123.<smiles>CCC1C[C@H]2C(=O)C[C@H](C(=O)OC2=O)[C@@H](C(C)=O)C1</smiles>

124<smiles>[R]#[Y]C(=O)OCC(C)(CO)CO</smiles><smiles>[R]#[V]CC(O)CC</smiles>

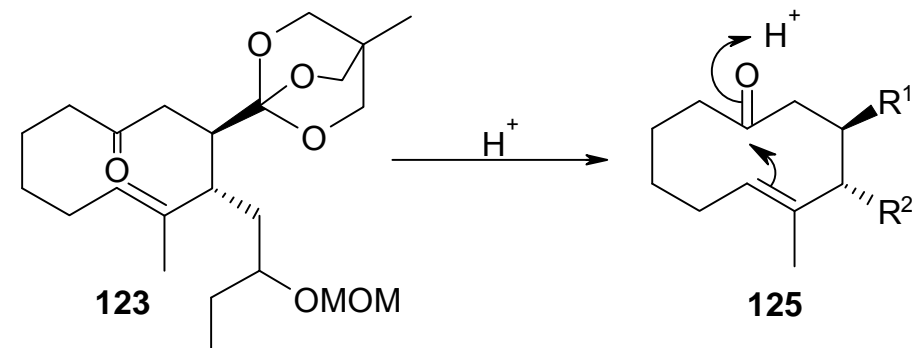<smiles>CC1([Tl])CCCCC1</smiles><smiles>[R]C1[C+](C)C2CCCCC2(O)C[C@H]1[R]</smiles>

126

Ergebnisse der Lactonisierungsversuche: Laut Syntheseplanung für das Collinolacton sollte nach der Oxy-Cope-Reaktion das sechsgliedrige Lacton aus der Nord-West-Hälfte gebildet werden. Um Erkenntnisse über den Reaktionsverlauf $\mathrm{zu}$ gewinnen, wurden mit dem Modellsubstrat 123 entsprechende Versuche durchgeführt (Schema 37). Trotz des Screenings verschiedener Säuren und Lösungsmittel konnte das Lacton 124 nicht isoliert werden. Stattdessen ergaben sich zwei Probleme: Zum Einen erwies sich der Orthoester in 123 bzw. dessen primäres Zersetzungsprodukt, das Diol, als unerwartet stabil und zum Anderen wurde ein transannularer Ringschluss zwischen C-1 und C-6 des zehngliedrigen Rings beobachtet, wodurch sich das Decalin 127 bildete. In der Literatur sind bereits diverse solcher Ringschlussreaktionen im Anschluss an eine Oxy-Cope-Reaktion beschrieben worden ${ }^{[86 \mathrm{a}]}$ Um dieses Problem zu umgehen, müsste in der Synthese des Collinolactons das Keton zuerst in die 3. Doppelbindung überführt werden, wodurch womöglich ein Substrat entstehen würde, was stabil genug für die Lactonisierung wäre. 


\section{II.4 Abschluss der Synthese und Baeyer-Villiger Oxidation}

Der nächste Schritt in der Totalsynthese des Collinolactons wird die Isomerisierung der Zkonfigurierten Doppelbindung zwischen C-5 und C-6 in 114 sein (Schema 38). Ein geeignetes Reaktionsprotokoll für diese Reaktion wurde von STILL et al. bereits für die Isomerisierung eines ähnlichen Cyclodecadienons 132 genutzt und verläuft über einen 1,4Additions/Eliminierungs-Mechanismus und anschließende Oxidation (Schema 39). ${ }^{[99]}$

Schema 38: Geplanter weiterer Verlauf der Synthese des Collinolactons 7.
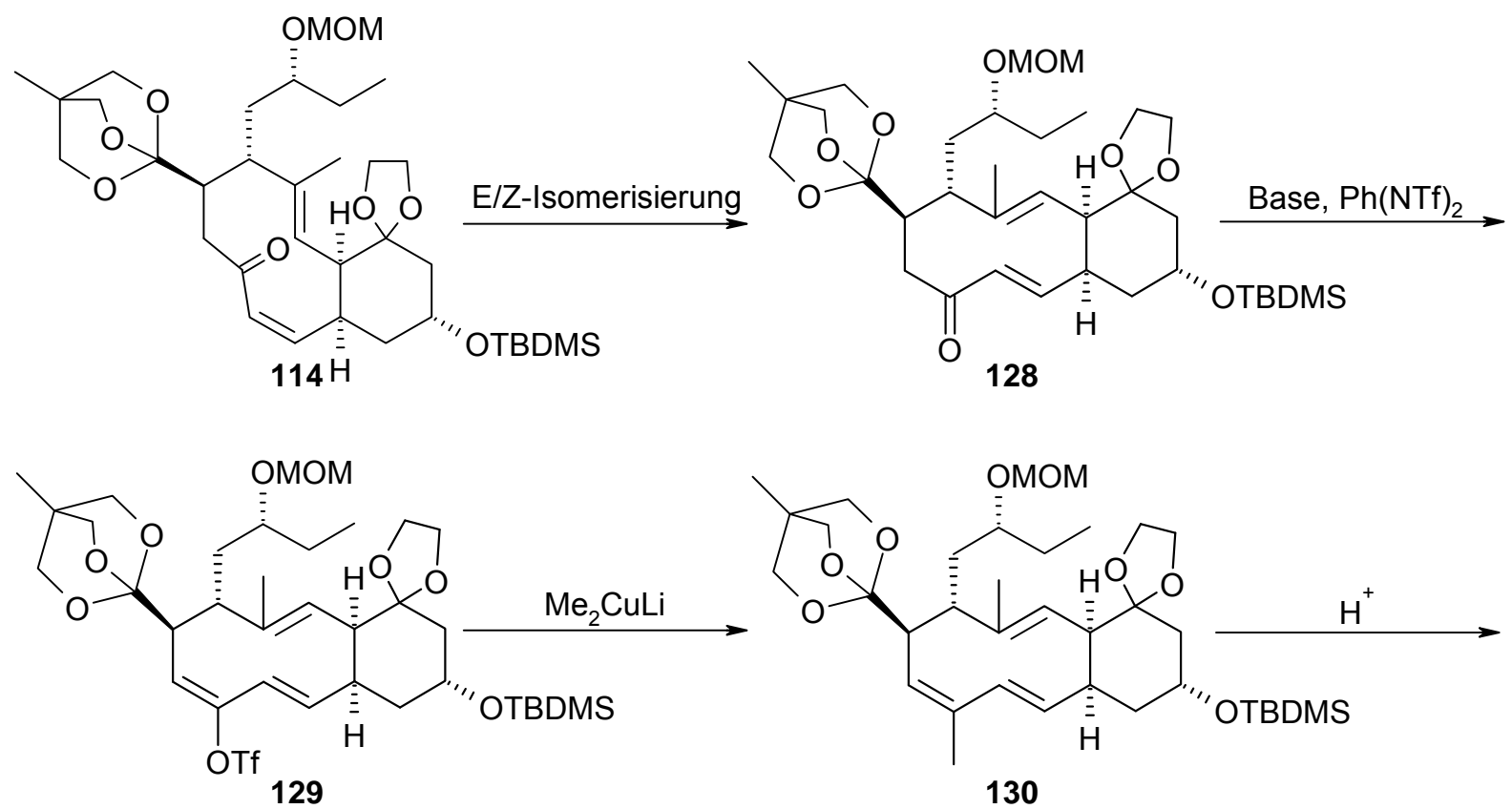

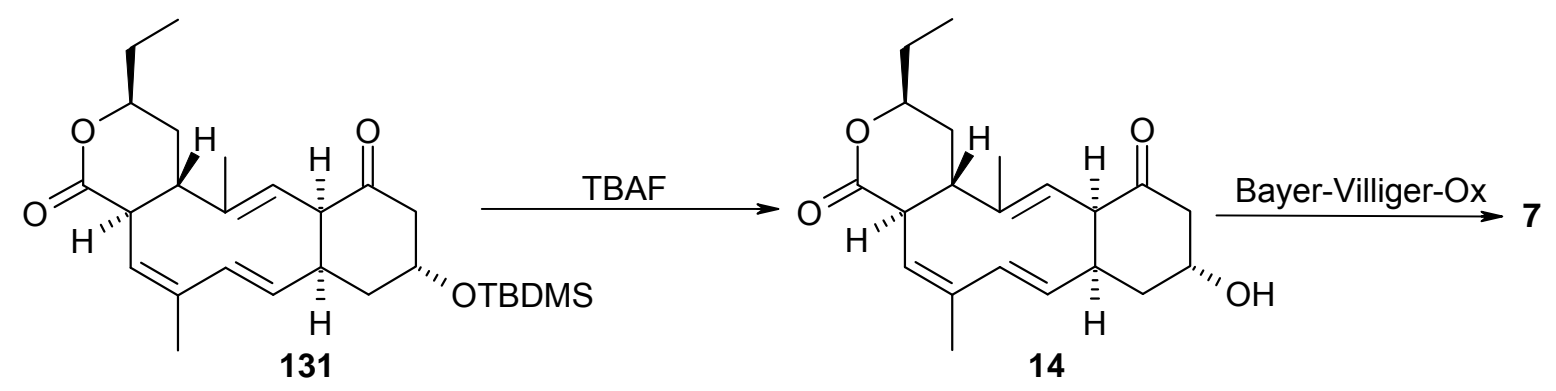

Nach erfolgreicher Isomerisierung zu 128 muss die dritte Doppelbindung eingeführt werden. Dies sollte selektiv über Enolisierung, Abfangen des entsprechenden Enolats als Triflat und anschließende Umsetzung mit einem Gilman-Cuprat gelingen. ${ }^{[100]}$ Das so erhaltene Trien 130 sollte nun stabil genug sein, um unter mild sauren Bedingungen in einem Reaktionsschritt sowohl die Lactonisierung als auch die Spaltung des Dioxolans in 130 einzugehen. Das daraus hervorgehende Lacton 131 wird schließlich durch Spaltung des Silylethers in das Collinoketon 14 überführt. 
Schema 39: Doppelbindungsisomerisierung nach Still et al. ${ }^{[99]}$<smiles>CC1=CC(=O)CCCC(C)=CCC1</smiles>

132<smiles>COC1=C2C(=CCC1)CCCC2(C)[SnH3]</smiles>

133<smiles>COC(=O)CCCC1=CCCC(C)=C1</smiles>

134

Das Collinoketon 14 wird abschließend durch eine Baeyer-Villiger-Oxidation in das Zielprodukt Collinolacton (7) überführt. Im Idealfall wird diese enzymatisch durch die bis dahin im AK GROND isolierte Baeyer-Villiger-Oxygenase durchgeführt. Sollte dies bis dahin nicht gelungen sein, stehen verschiedene enzymatische ${ }^{[101]}$ oder chemische Methoden ${ }^{[102]}$ zur Baeyer-Villiger-Oxidation in Gegenwart von Doppelbindungen zur Verfügung.

\section{II.5 Praktische Arbeiten zum Collinolacton}

\section{II.5.1 Versuche zur stereoselektiven Synthese des Trienons über Nagao-Aldol- Reaktion}

Während die von EvANS erstmals als chirale Auxiliare ${ }^{[103]}$ benutzten $\alpha$-substituierten Oxazolidinone in der Regel mit hoher Stereoselektivität die entsprechenden syn-Aldol Produkte ergeben, zeigen deren einfache Acetylanaloga eine kaum nennenswerte Diastereoselektivität. ${ }^{[104]}$ Um dieses hinreichend bekannte Problem $\mathrm{zu}$ umgehen, sollte bei der Synthese von 137 das von NAGAO et al. eingeführte

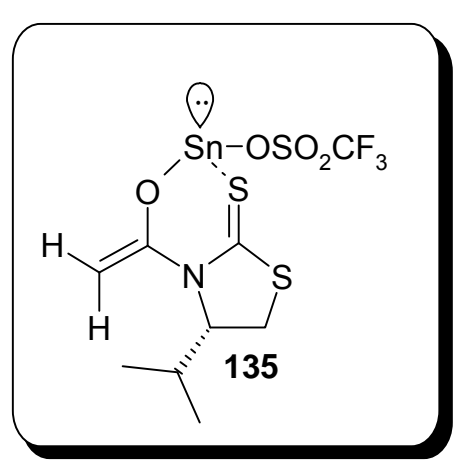
Schwefelanalogon verwendet werden. So sollte Trienon 140 ausgehend von Z-Buten-1,4diol (136) synthetisiert werden. Schlüsselschritt dieser diastereoselektiven Synthese war eine Auxiliar-gesteuerte Aldolreaktion des Aldehyds 23 mit dem Zinnenolat 135 (Schema 40). Denn mit diesem können, im Gegensatz zu den Sauerstoffanaloga, auch mit den Acetylenolaten sehr gute Diastereoselektivitäten erreicht werden. ${ }^{[105]}$ Nach erfolgreicher Synthese des enantiomerenreinen Alkohols 137, sollte das Auxiliar in situ in das entsprechende Weinreb-Amid überführt werden. ${ }^{[106]}$ Schützung der Hydroxylfunktion und Umsetzung mit der lithiierten Verbindung 139 ergäbe die Ausgangsverbindung 140 als Edukt für die IMDA-Reaktion. 
Schema 40: Geplante Syntheseroute zu Trienon 138.

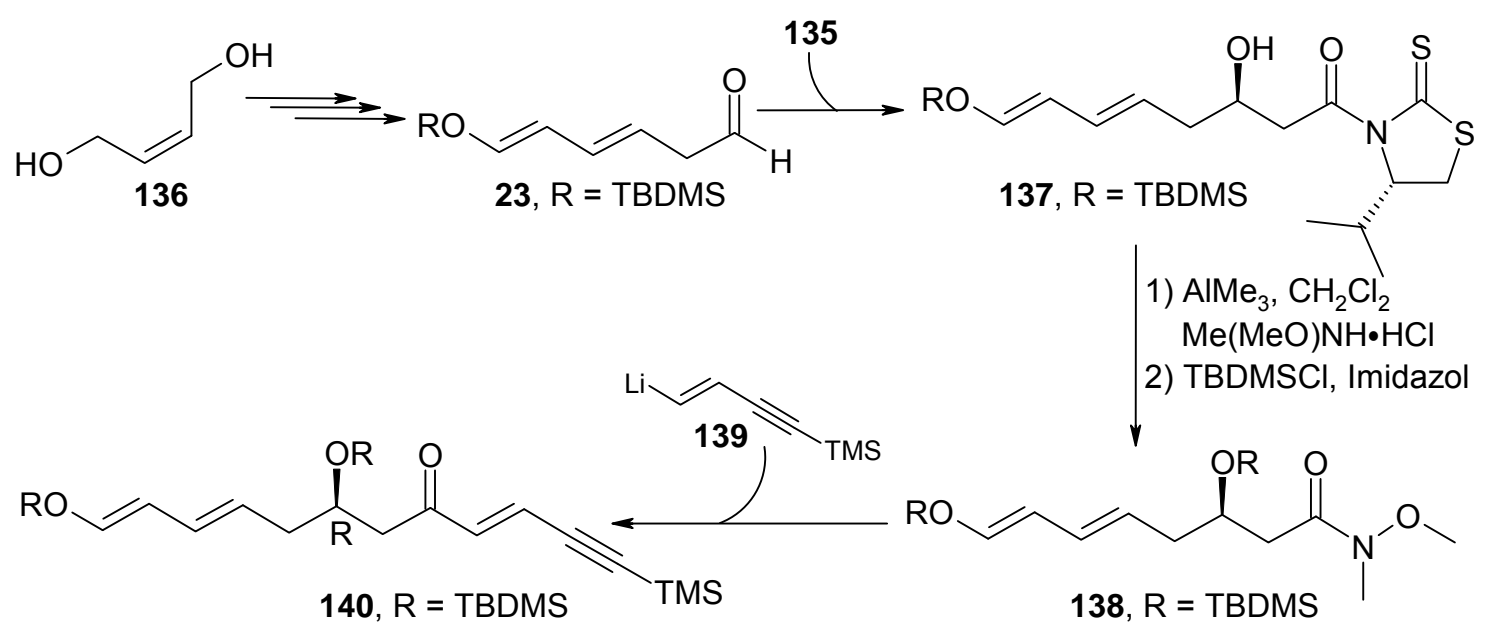

\section{II.5.1.1 Versuche zur Darstellung des Aldehyds für die Nagao-Aldol-Reaktion}

Synthese der Ester 22, 142 und 149: Die Synthese des dekonjugierten Esters 22 ist in Schema 41 dargestellt. Danach erfolgte zunächst die Silylierung einer der beiden Hydroxygruppen in Z-1,4-Butendiol (136) mit TBDMSCl. Dies ergab den Monosilylether 21, welcher durch eine Parikh-Doering-Oxidation mit Pyridin-Schwefeltrioxid Komplex selektiv zum Aldehyd 141 oxidiert wurde. ${ }^{[107]}$ Durch Verwendung des $\mathrm{SO}_{3}$-Komplexes und nicht Oxalylchlorids als Oxidationsmittel konnte die von H. SHOJAEI beobachtete Ausbeute von 69\% auf 84\% gesteigert werden. Eine HWE-Reaktion des so erhaltenen Aldehyds 141 mit Triethylphosphonoacetat und Natriumethanolat als Base ergab den konjugierten Ester 136 in 87\% Ausbeute. ${ }^{[108]}$ Die Dekonjugation des Esters 142 wurde unter kinetischen Bedingungen bei $-78^{\circ} \mathrm{C}$ in Gegenwart von 1,3-Dimethyl-3,4,5,6-tetrahydro-2(1H)pyrimidinon (DMPU) durchgeführt und ergab nach chromatographischer Reinigung den dekonjugierten Ester $\mathbf{2 2}_{Z}$ mit der abgebildeten 3E/5Z-Konfiguration in 51\% Ausbeute. Die mäßige Ausbeute ist auf stabilitätsbedingte Verluste während der Reinigung von $\mathbf{2 2}_{Z}$ an Kieselgel zurückzuführen. Um ausreichend Material für die weitere Synthese bereitstellen zu können, wurde ein weiterer synthetischer Zugang zum konjugierten Ester $\mathbf{2 2}_{Z}$ erarbeitet. 
Schema 41: Optimierte Synthese des dekonjugierten Esters $\mathbf{2 2}_{Z}$.

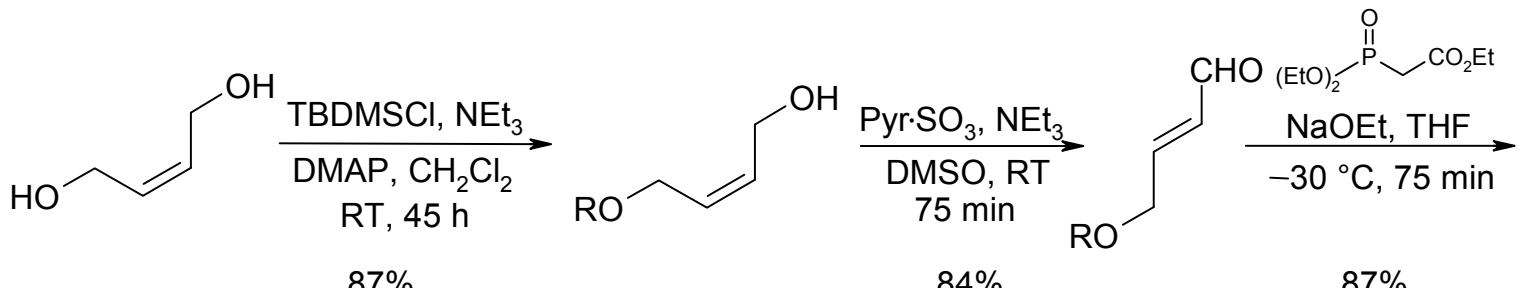

136

$87 \%$

21, $R=$ TBDMS

$84 \% 141, R=$ TBDMS

$87 \%$

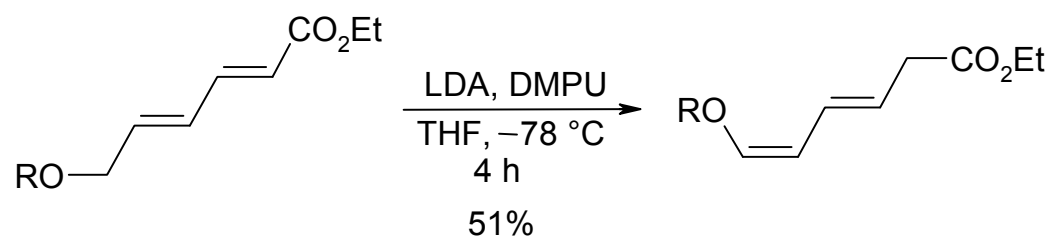

$142, R=$ TBDMS

$22_{Z}, R=T B D M S$

Dieser Zugang sollte einerseits eine leichte Synthese von $\mathbf{2 2}_{Z}$ im Multigramm-Maßstab ermöglichen und andererseits eine höhere Flexibilität hinsichtlich der Substituenten am Dien bieten. Aus diesen Überlegungen ergab sich der in Schema 42 gezeigte Syntheseweg. Schlüsselschritt dieser Synthese ist eine Wittig-Reaktion $\alpha$-substituierter Acetaldehyde mit dem Ylid des bekannten Phosphoniumbromids 148. Dazu wurde zunächst (tert-Butyldimethylsilanyloxy)acetaldehyd (144) analog Literatur, ${ }^{[109]}$ durch Ozonolyse des zweifach geschützten Z-Butendiol 143 hergestellt. Das Phosphoniumsalz 148 erhielt man ebenfalls analog Literatur, ausgehend von Crotonsäureethylester 146, durch radikalische Bromierung $^{[171 b]}$ und anschließendes Erhitzen mit Triphenylphosphan in Xylol. ${ }^{[171 b, 110]}$

Schema 42: Alternativer Zugang zum konjugierten Ester 142.

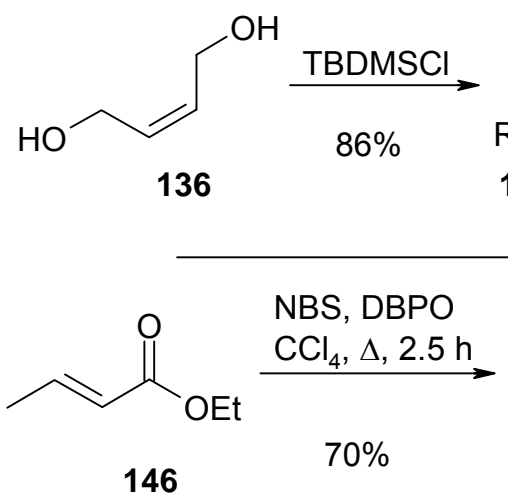

146

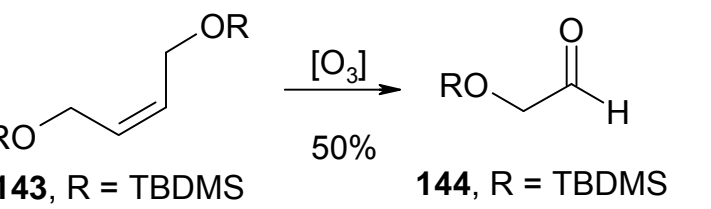

$143, R=T B D M S$<smiles>CCOC(=O)/C=C/CBr</smiles>

147<smiles>CCOC(=O)C=CC[Pb](Br)(Br)c1ccccc1</smiles>

148
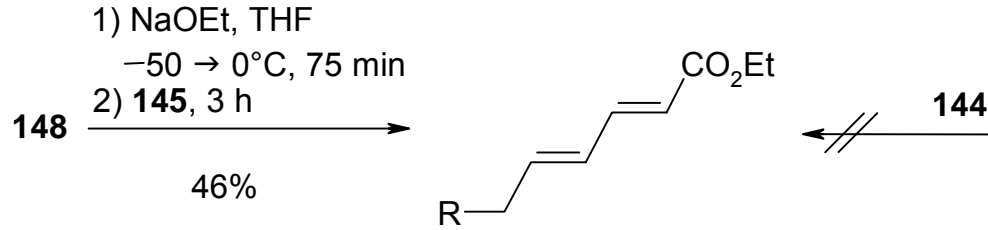

142, $\mathrm{R}=$ OTBDMS

149, $\mathrm{R}=\mathrm{Ph}$ 
Bei der Durchführung der Wittig-Reaktionen zeigte sich jedoch, dass die Umsetzungen mit dem TBDMS-geschützten Aldehyd 144 nur Gemische aus nicht identifizierbaren Zersetzungsprodukten lieferten. Auch nach Variation der Reaktionsbedingungen (Temperatur, Base etc.) konnte kein Zielprodukt isoliert werden. Demgegenüber ergaben die Umsetzungen mit Phenylacetaldehyd (145) das Zielprodukt 149 in 46\% Ausbeute. Wahrscheinlichste Ursache für das Scheitern der Versuche mit Aldehyd 144 ist wohl dessen Instabilität unter den Bedingungen der Wittig-Reaktion. Im Gegensatz zur Dekonjugierung des TBDMS-Ethers 142, welche das entsprechende Produkt $\mathbf{2 2}_{Z}$ mit 3E/5Z-Konfiguration lieferte (Schema 41), erhielt man bei der Umsetzung des Phenyl-substituierten Derivats 149 das dekonjugierte Produkt 150 mit 3E/5E-Konfiguration (Schema 43). Dabei ist insbesondere das Ergebnis mit dem TBDMS-Ether $\mathbf{2 2}_{Z}$ bemerkenswert, da hier aus dem $E / E$ konfigurierten, konjugierten Ester 142 ausschließlich das vermeintlich energetisch ungünstigere $3 E / 5 Z$-konfigurierte Produkt $\mathbf{2 2}_{Z}$ gebildet wird. Die Tatsache, dass das Phenylsubstituierte Edukt ausschließlich das E/E-Produkt ergibt, ist ein Hinweis darauf, dass für die 5Z-Konfiguration in $\mathbf{2 2}_{Z}$ koordinative Effekte zwischen dem Sauerstoff des TBDMS-Ethers und der Carbonylfunktion des Esters verantwortlich sein könnten. Eine genauere Diskussion der Dekonjugierung folgt weiter unten (Kap. II.5.1.5).

Schema 43: Die Dekonjugierung des Esters 149 lieferte das $3 E / 5 E$ Produkt 150.

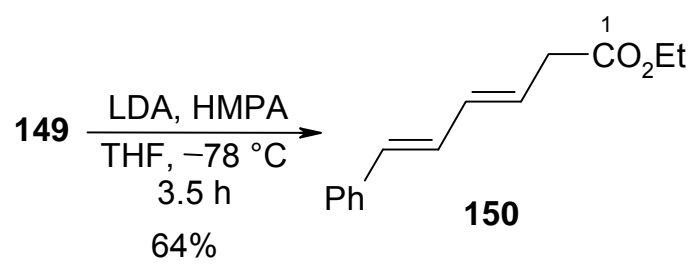

Versuch der Darstellung des Aldehyds 23: Nach erfolgreicher Synthese der Ester $\mathbf{2 2}_{Z}$ und 150 sollten nun die entsprechenden Aldehyde synthetisiert werden. In Tabelle 12 sind die Ergebnisse dieser Versuchsreihe zusammengefasst. In den bereits beschriebenen Vorarbeiten, welche im Rahmen seiner Dissertation von H. SHOJAEI durchgeführt wurden, beschrieb er die erfolgreiche Reduktion des Diensäureesters $\mathbf{2 2}_{Z}$ zum entsprechenden Aldehyd mit Diisobutylaluminiumhydrid. Das Reaktionsgemisch wurde nach abgeschlossener Reaktion mit einer gesättigten ethanolischen Weinsäurelösung hydrolysiert und anschließend mit einem Gemisch aus Natriumsulfat und Celite versetzt. Filtration und Evaporation des Lösungsmittels unter reduziertem Druck ergaben den Aldehyd 23 in 94\% Ausbeute. ${ }^{[38]}$ Nach dieser, erstmals von VIALA et al. ${ }^{[111]}$ beschriebenen Synthesevorschrift konnte der Aldehyd 23 zunächst in 86\% Rohproduktausbeute isoliert werden. Es zeigte sich 
jedoch, dass schon nach Zzurückspülen der NMR-Probe des Aldehyds, Entfernen des Lösungsmittels und erneuter Messung weniger als 60 min nach der ersten Messung sich der Aldehyd fast vollständig zersetzt hatte. Um auszuschließen, dass Säurespuren aus der Weinsäure für diese Beobachtung verantwortlich waren, wurden zunächst andere Hydrolysemethoden untersucht (Einträge 2-4). Bei diesen Versuchen wurden komplexe Gemische erhalten, worin Aldehyd 23 entweder gar nicht oder als Minderkomponente beobachtet wurde. Alle Versuche, den Aldehyd chromatographisch abzutrennen, scheiterten aufgrund der mangelnden Stabilität desselbigen gegenüber Kieselgel und Aluminiumoxid.

Tabelle 12: Versuch der Darstellung der Aldehyde 23 und 150a.

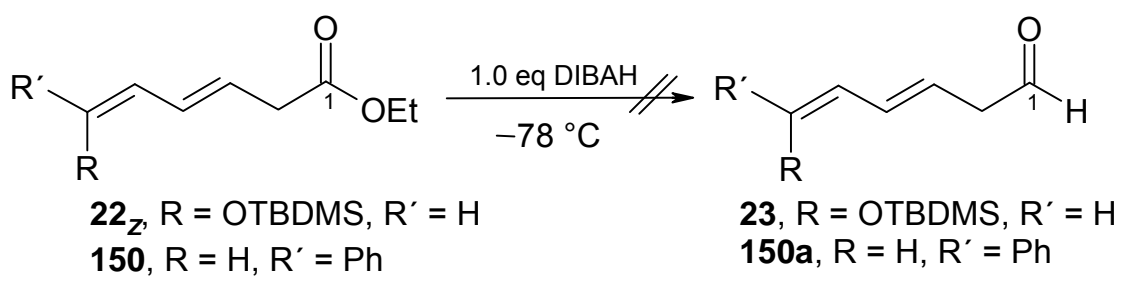

\begin{tabular}{|c|c|c|c|c|c|}
\hline Nr. & Edukt & $\mathbf{L m}$ & $t[h]$ & Hydrolyse & Beobachtung \\
\hline 1 & $22_{Z}$ & $\mathrm{CH}_{2} \mathrm{Cl}_{2}$ & 1.5 & Weinsäure & $86 \%^{\mathrm{a}}$ \\
\hline 2 & $22_{Z}$ & $\mathrm{CH}_{2} \mathrm{Cl}_{2}$ & 2.5 & K,Na-Tartrat & Zersetzung \\
\hline 3 & $22_{Z}$ & $\mathrm{CH}_{2} \mathrm{Cl}_{2}$ & 2.5 & $\mathrm{NH}_{4} \mathrm{Cl}$ & Zersetzung \\
\hline 4 & $22_{Z}$ & $\mathrm{CH}_{2} \mathrm{Cl}_{2}$ & 1.5 & $\mathrm{EtOH}$ & Zersetzung \\
\hline 5 & $22_{Z}$ & THF & 1.5 & Weinsäure & Zersetzung \\
\hline 6 & $22_{Z}$ & $\mathrm{CH}_{2} \mathrm{Cl}_{2} / \mathrm{Et}_{2} \mathrm{O}$ & 1.0 & Weinsäure & Zersetzung \\
\hline 7 & $22_{Z}$ & $\begin{array}{c}\mathrm{CH}_{2} \mathrm{Cl}_{2} / \\
\mathrm{MeOH}\end{array}$ & 2.5 & K,Na-Tartrat & Zersetzung \\
\hline 8 & 150 & $\mathrm{CH}_{2} \mathrm{Cl}_{2}$ & 1.5 & Weinsäure & Zersetzung \\
\hline
\end{tabular}

a) Rohproduktausbeute, schnelle Zersetzung des Aldehydes bei RT.

Auch eine Variation der Lösungsmittel (Einträge 5-7) brachte nicht den gewünschten Erfolg. Die Reduktion des Phenyl-substituierten Esters 150 lieferte ebenfalls ein Gemisch, in welchem der Aldehyd 150a nur als Minderkomponente vorlag. Dies lässt darauf schließen, dass die Instabilität des Aldehyds $\mathbf{2 3}$ zumindest nicht ausschließlich auf eine Zersetzung des TBDMS-Enolethers zurückzuführen ist. Nachdem die direkte Reduktion des Esters $\mathbf{2 2} Z$ mit DIBAH nicht zum gewünschten Produkt führte, wurde versucht über die Reduktion zum Alkohol und anschließende Oxidation das Zielprodukt herzustellen. Die Vielfalt schonender Oxidationsmethoden, welche aus primären Alkoholen die entsprechenden Aldehyde liefern, ist insbesondere für empfindliche Substrate ein Erfolg-versprechender Ansatz. ${ }^{[112]}$ Die Reduktion der Ester $22_{Z}$ und 150 wurde unter Verwendung verschiedener Reduktionsmittel untersucht (Tabelle 13). Die Reduktion mit 2 eq Diisobutylaluminiumhydrid in Anlehnung 
an die von VIALA et al. ${ }^{[11]}$ beschriebene Synthesevorschrift lieferte ein recht unsauberes Rohprodukt, aus dem über Flashchromatographie immerhin 22\% des Alkohols 151 isoliert werden konnten (Eintrag 1). Die Reduktion nach einer von RoQUES et al. ${ }^{[13]}$ publizierten Vorschrift mit Natriumborhydrid als Reduktionsmittel in Gegenwart von Lithiumchlorid lieferte eine auf 39\% gesteigerte Ausbeute. Die Reinigung erfolgte aufgrund der vermuteten mangelnden Stabilität des Alkohols 151 gegenüber Kieselgel an neutralem Aluminiumoxid.

Tabelle 13: Reduktion der Ester $22_{Z}$ und 144 zu den entsprechenden Alkoholen.

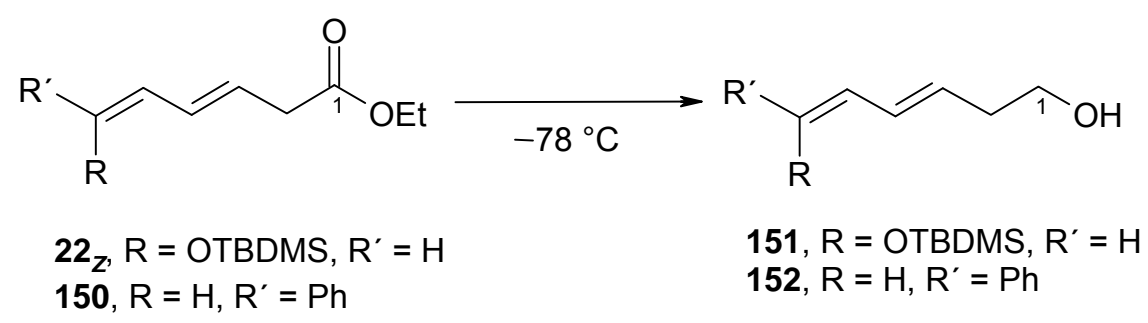

\begin{tabular}{cccccccc}
\hline Nr. & Edukt & $\mathbf{R m}$ & $\mathbf{e q}$ & $\mathbf{L m}$ & $\mathbf{T}\left[{ }^{\circ} \mathbf{C}\right]$ & $\mathbf{t}[\mathbf{h}]$ & $\mathbf{A}[\%]$ \\
\hline 1 & $\mathbf{2 2}_{Z}$ & $\mathrm{DIBAH}$ & 2.1 & $\mathrm{CH}_{2} \mathrm{Cl}_{2}$ & -78 & 3.5 & $22^{\mathrm{c}}$ \\
2 & $\mathbf{2 2}_{Z}$ & $\mathrm{NaBH}_{4}{ }^{\mathrm{a}}$ & 4.0 & $\mathrm{THF} / \mathrm{EtOH}$ & 0 & 16 & $39^{\mathrm{b}}$ \\
3 & $\mathbf{2 2}_{Z}$ & $\mathrm{LiAlH}_{4}$ & 1.3 & $\mathrm{THF}$ & $0 \rightarrow \mathrm{RT}$ & 3.0 & $49^{\mathrm{b}}$ \\
4 & $\mathbf{1 4 9}$ & $\mathrm{LiAlH}_{4}$ & 1.0 & $\mathrm{THF}$ & $-78 \rightarrow 0$ & 3.0 & $99^{\mathrm{d}}$ \\
\hline
\end{tabular}

a) Zusätzlich 4.0 eq LiCl; b) Chromat. mit Alox $\mathrm{pH}=7$; c) Chromat. an Kieselgel; d) Rohprodukt.

Die beste Ausbeute wurde nach TAKEDA ${ }^{[114]}$ et al. mit Lithiumaluminumhydrid als Reduktionsmittel erzielt (Eintrag 3). Die Umsetzung des Phenyl-substituierten Diens 150 lieferte den entsprechenden Alkohol 152 in annähernd quantitativer Ausbeute und ausreichender Reinheit für die folgenden Oxidationsversuche. Die gute Ausbeute ist im Wesentlichen darauf zurückzuführen, dass aufgrund der Reinheit des Rohproduktes auf eine chromatographische Reinigung verzichtet werden konnte, welche beim TBDMS-Enolether 151 auch bei Verwendung von Aluminiumoxid als stationäre Phase immer mit erheblichen Verlusten verbunden war. Die Oxidation der Alkohole 151 und 152 mit verschiedenen Oxidationsmitteln lieferte in Analogie zu den Reduktionsversuchen aus Tabelle 12 größtenteils komplexe Reaktionsgemische, aus denen sich Aldehyd 23 nicht isolieren ließ. Es musste also festgestellt werden, dass 23 für die geplante Syntheseroute nicht die notwendige Stabilität besaß und somit ein neuer Zugang zu Trienon 140 erarbeitet werden. 
Tabelle 14: Versuche zur Darstellung der Aldehyde 23 und 150a durch Oxidation.

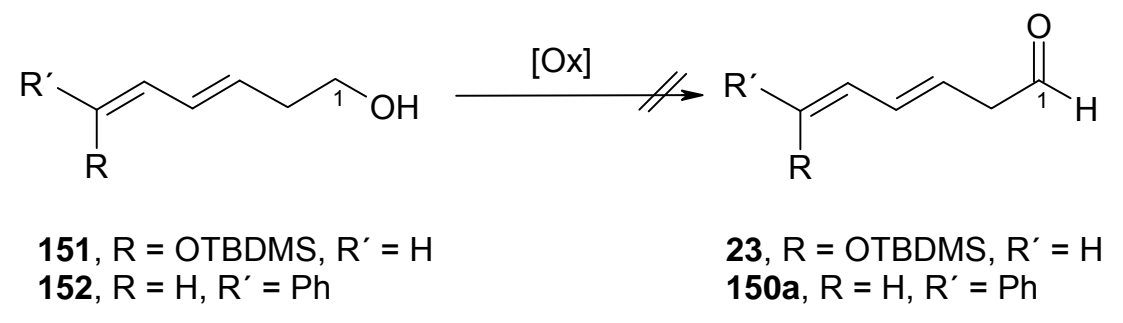

\begin{tabular}{ccccccc}
\hline Nr. & Edukt & $\mathbf{O x m}$ & $\mathbf{L m}$ & $\mathbf{T}\left[{ }^{\circ} \mathbf{C}\right]$ & $\mathbf{t}[\mathbf{m i n}]$ & Beobachtung \\
\hline 1 & $\mathbf{1 5 1}$ & $\mathrm{IBX}$ & $\mathrm{DMSO}$ & $\mathrm{RT}$ & 90 & Zersetzung \\
2 & $\mathbf{1 5 1}$ & $\mathrm{DMP}^{[115]}$ & $\mathrm{CH}_{2} \mathrm{Cl}_{2}$ & $\mathrm{RT}$ & 30 & Zersetzung \\
3 & $\mathbf{1 5 1}$ & $\mathrm{C}_{2} \mathrm{O}_{2} \mathrm{Cl}_{2} / \mathrm{DMSO}^{[171 \mathrm{~b}]}$ & $\mathrm{CH}_{2} \mathrm{Cl}_{2}$ & $-78 \rightarrow \mathrm{RT}$ & 60 & Zersetzung \\
4 & $\mathbf{1 5 1}$ & $\mathrm{Pyr} \cdot \mathrm{SO}_{3}{ }^{[107]}$ & $\mathrm{DMSO}$ & $\mathrm{RT}$ & 25 & Zersetzung \\
5 & $\mathbf{1 5 2}$ & $\mathrm{Pyr} \cdot \mathrm{SO}_{3}{ }^{[107]}$ & $\mathrm{DMSO}$ & $\mathrm{RT}$ & 35 & Zersetzung \\
\hline
\end{tabular}

\section{II.5.2 Synthese des IMDA-Edukts ausgehend von Acetessigsäureethylester}

Nachdem sich die ursprüngliche Synthesestrategie zu Trienon 140 aufgrund der Instabilität des Aldehyds 23 als nicht durchführbar erwiesen hatte, wurde der in Schema 44 gezeigte Zugang entwickelt. Ausgehend von 3-Oxobutansäureethylester (153) sollte durch Bromierung und anschließender Arbuzov-Reaktion der Phosphonoester 154 hergestellt werden, welcher in einer Horner-Wadsworth-Emmons-Reaktion mit dem bereits in der ursprünglichen Synthese verwendeten Aldehyd 141 den konjugierten $\beta$-Ketoester 155 ergeben sollte. Bei der folgenden Dekonjugierung unter den bereits beschriebenen Bedingungen war außerdem eine genauere Untersuchung der Doppelbindungskonfiguration vorgesehen. Die Reduktion des so erhaltenen dekonjugierten $\beta$-Ketoesters 156 zum Alkohol und anschließende Schützung zu 157 sollte zunächst racemisch durchgeführt werden, denn die katalytische Reduktion zum enantiomerenreinen $\beta$-Hydroxyester hätte erst nach erfolgreicher Synthese des Diels-Alder-Produktes 96a untersucht werden sollen. ${ }^{[16]}$ Der Abschluss der Synthese von Trienon 95 verliefe analog der bereits in Schema 40 vorgestellten Sequenz über Weinrebamidbildung zu 158 und anschließender Addition des lithiierten TMSButenins 139. 
Schema 44: Geplante Synthese des Trienons 95 ausgehend von $\beta$-Ketoester 153.

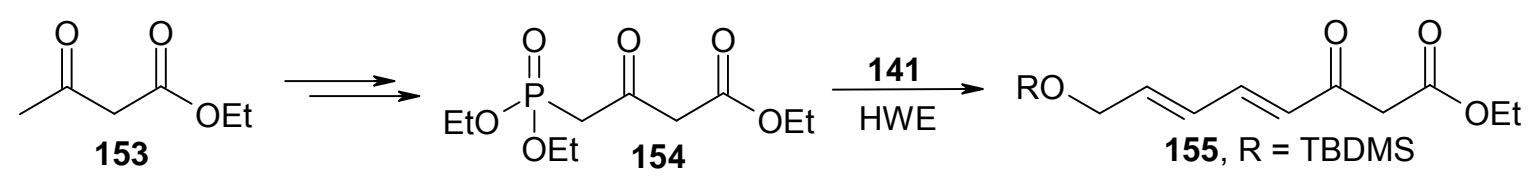<smiles>CC#[R6]/C=C/C=C/CC(=O)CC(=O)OCC</smiles>

156, $R=$ TBDMS

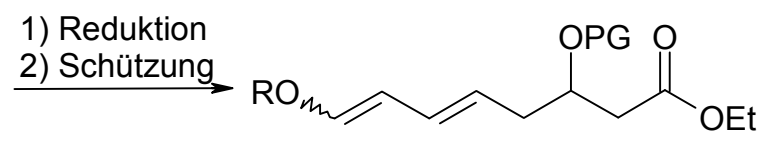
157, $\mathrm{R}=$ TBDMS

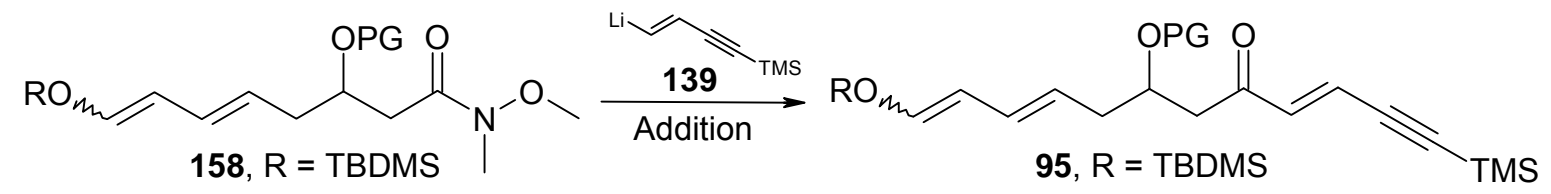

\section{II.5.2.1 Durchführung der Synthese}

Die Synthese des $\beta$-Ketoesters 156 startete mit der Bromierung des 3-Oxobutansäureethylesters (153) nach der von DUTHALER ${ }^{[117]}$ beschriebenen Synthesevorschrift (Schema 45). Dabei erfolgt die Bromierung zunächst an der elektrophilen Position am $\alpha$-C-Atom zwischen den beiden Carbonylfunktionen. ${ }^{[18]}$ Der genaue Mechanismus der sauerstoffkatalysierten Umlagerung des Broms in die $\gamma$-Position des Produktes 159 ist nicht bekannt, es wird jedoch vermutet, dass der Mechanismus über eine Peroxidspezies verläuft. ${ }^{[119]}$ Die Umlagerung wurde sowohl mit reinem Sauerstoff als auch mit Luft erfolgreich durchgeführt. Die Verwendung von Luft erfordert jedoch verlängerte Reaktionszeiten und führt zu einer geringeren isolierten Ausbeute. Bei der destillativen Reinigung des Rohproduktes aus der Bromierung wurde bei einigen Versuchen vollständige Zersetzung zu einem schwarzen Polymer beobachtet. Um dies zu verhindern, ist es entscheidend vor der Destillation die HBr-Rückstände aus der initialen Bromierung vollständig im Wasserstrahlvakuum zu entfernen. Das Edukt für die HWE-Reaktion 154 wurde nach Literatur, durch eine Arbuzow-Reaktion des 4-Brom-3-oxobutansäureethylesters (159) mit Triethylphosphit gewonnen. ${ }^{[120]}$ Die mäßige Ausbeute der Arbuzow-Reaktion von $40 \%$ ist im Wesentlichen auf die Bildung des Regioisomers 3-(Diethoxyphophoryl)but-3ensäureethylester zurückzuführen, dessen destillative Abtrennung sich schwierig gestaltete, was wiederum eine säulenchromatographische Reinigung erforderlich machte. 
Schema 45: Synthese des dekonjugierten Diensäureesters 156.
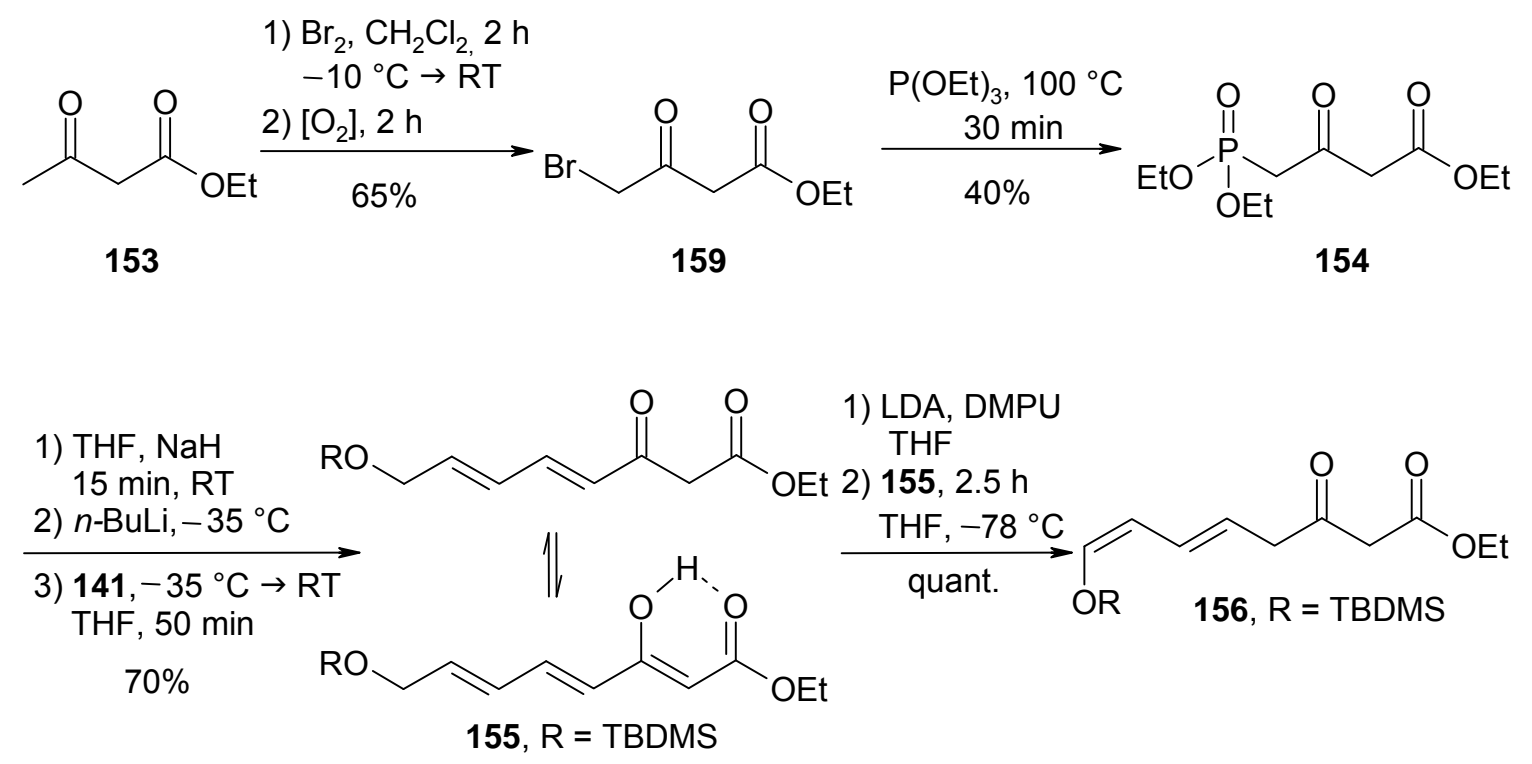

Der nächste Schritt der Synthese war die HWE-Reaktion des Phosphoesters 154 mit dem bereits für die Herstellung des Diensäureesters 22 verwendeten Aldehyd 141 aus Schema 41. Die Reaktion wurde nach einer von DESLONGSCHAMPS beschriebenen Reaktionsvorschrift durchgeführt, welche dieser für die Kupplung des Phosphonoesters 154 mit verschiedenen Aldehyden benutzt hatte. ${ }^{[121]}$ Dabei wurde 154 zunächst mit NaH in $\alpha$-Position deprotoniert und anschließend mit $n$-BuLi versetzt, um das entsprechende Ylid zu erzeugen. Dann wurde das Reaktionsgemisch mit dem Aldehyd 141 versetzt und bei $-20{ }^{\circ} \mathrm{C}$ gerührt. Nach wässriger Aufarbeitung und chromatographischer Reinigung erhielt man so das Produkt 155 mit der abgebildeten $4 E / 6 E$-Konfiguration. Das Isomerenverhältnis zwischen der Keto- und der Enolform betrug etwa 1.5:1. Die anschließende Dekonjugierung wurde auf die bereits beschriebene Weise durchgeführt und lieferte das dekonjugierte Produkt in quantitativer Ausbeute. Auf eine chromatographische Reinigung konnte aufgrund der annehmbaren Reinheit des Rohproduktes verzichtet werden. Außerdem zeigte sich, dass die chromatographische Reinigung an Kieselgel mit recht hohen Verlusten verbunden war. In Übereinstimmung mit den Ergebnissen für den Hexadiensäureester 22 besaß das Produkt der Dekonjugierung ebenfalls die $Z / E$-Konfiguration der beiden Doppelbindungen und lag ausschließlich in der Ketoform vor. Die Reduktion des $\beta$-Ketoesters 156 wurde mit $\mathrm{NaBH}_{4}$ nach einer Reaktionsvorschrift von FLEMING et al. durchgeführt. ${ }^{[122]}$ Der so erhaltene Alkohol 160 erwies sich erneut als instabil gegenüber Kieselgel, was sich durch substantielle Verluste während der chromatographischen Reinigung bemerkbar machte. Um möglichst 
hohe Ausbeuten zu erzielen, wurde der Alkohol 160 deshalb in den späteren Versuchen ohne chromatographische Reinigung möglichst schnell weiter umgesetzt.

Schema 46: Reduktion des $\beta$-Ketoesters 156.<smiles>CCOC(=O)C/C=C\C=C\CC(O)CC(=O)OCC</smiles>

Die Hydroxylgruppe in $\mathbf{1 6 0}$ sollte zunächst als Benzylether geschützt werden. Eine häufig eingesetzte Methode für die Benzylierung von $\beta$-Hydroxyestern ist die durch äquimolare Mengen Silberoxid vermittelte Umsetzung mit Benzylbromid. ${ }^{[123]}$ Das Ergebnis dieses Versuches ist in Tabelle 15 gezeigt (Eintrag 1). Nachdem die Reaktionskontrolle mittels Dünnschichtchromatographie in den ersten Stunden der Reaktion keinen Umsatz anzeigte, hatte sich das Edukt nach 20 h Rühren bei Rautemperatur vollständig zersetzt. Eine weitere Möglichkeit zur schonenden Benzylierung ist das ursprünglich für die Schützung verschiedener Zucker ${ }^{[124]}$ verwendete Trichloracetimidat (TCAI). Es wurde bereits erfolgreich in der Umsetzung verschiedener $\beta$-Hydroxyester eingesetzt. ${ }^{[109 b, 125]}$ Die durch TFA katalysierte Reaktion mit 160 (Eintrag 2) führte jedoch nicht zum gewünschten Produkt, sondern nur zu einem Gemisch aus Edukt und nicht identifizierbaren Zersetzungsprodukten. Dieses Ergebnis ist ein Indiz dafür, dass 160 bzw. das geschützte Produkt im sauren Milieu äußerst instabil sind.

Tabelle 15: Versuche zur Schützung der $\beta$-Hydroxyfunktion in Diensäureester 160.

\begin{tabular}{|c|c|c|c|c|c|c|c|c|}
\hline \multirow{2}{*}{$\frac{\text { Nr. }}{1}$} & \multirow{2}{*}{$\begin{array}{c}\text { Prod. } \\
161\end{array}$} & \multirow{2}{*}{$\frac{\mathbf{R}}{\mathrm{Bn}}$} & \multicolumn{2}{|c|}{ Reagenzien } & \multirow{2}{*}{$\frac{\mathbf{L m}}{\text { Benzol }}$} & \multirow{2}{*}{$\frac{\mathbf{T}}{\mathrm{RT}}$} & \multirow{2}{*}{$\frac{\mathbf{t}[\mathbf{h}]}{20}$} & \multirow{2}{*}{$\begin{array}{c}\text { Beob. } \\
\text { Zers. }\end{array}$} \\
\hline & & & $\mathrm{BnBr}$ & $\mathrm{Ag}_{2} \mathrm{O}$ & & & & \\
\hline 2 & 161 & $\mathrm{Bn}$ & $\mathrm{TCAI}^{\mathrm{a}}$ & TFA & $\mathrm{CH}_{2} \mathrm{Cl}_{2}$ & $0{ }^{\circ} \mathrm{C}$ & 3.0 & Zers. \\
\hline 3 & 162 & $\mathrm{Me}$ & $\mathrm{CH}_{2} \mathrm{~N}_{2}$ & $\mathrm{SiO}_{2}$ & $\mathrm{CH}_{2} \mathrm{Cl}_{2}$ & RT & 3.0 & Edukt $^{\mathrm{b}}$ \\
\hline 4 & 163 & TMS & $\mathrm{TMSCl}$ & $\mathrm{NEt}_{3}$ & $\mathrm{CH}_{2} \mathrm{Cl}_{2}$ & $0{ }^{\circ} \mathrm{C}$ & 1.5 & $86 \%^{\mathrm{c}}$ \\
\hline
\end{tabular}

a) $\mathrm{TCAI}=$ Trichloracetimidat $\left[\mathrm{BnOC}(\mathrm{NH}) \mathrm{CCl}_{3}\right]$; b) Eduktanteil im ${ }^{1} \mathrm{H}-\mathrm{NMR}$ ca. $55 \%$; c) Von konj. Ester 155. 
Da sich Synthese von 161 somit als unerwartet schwierig erwies, sollte eine andere Schutzgruppe verwendet werden. Nachdem der Versuch der Synthese von 162 durch Methylierung mit Diazomethan ${ }^{[126]}$ ebenfalls scheiterte (Eintrag 3), konnte schließlich der TMS-Ether 163 in sehr guter Ausbeute (86\% über drei Stufen) isoliert werden. Dieses Ergebnis konnte jedoch nur erreicht werden, wenn Dekonjugierung, Reduktion und Schützung des $\beta$-Hydroxyesters 160 innerhalb eines Arbeitstages durchgeführt wurden, da sich insbesondere letzterer als äußerst instabil erwies. Die Synthese des IMDA-Vorläufers wurde wie in Schema 47 dargestellt abgeschlossen. Nach Silylierung der Hydroxylfunktion wurde der TMS-geschützte Diensäureester 163 in das Weinreb-Amid 164 überführt. Vergleichbare Umsetzungen wurden häufig unter Verwendung von Trimethylaluminium ${ }^{[127]}$ durchgeführt, welches jedoch aufgrund der pyrophoren Eigenschaften mit besonderer Vorsicht zu handhaben ist. Deshalb wurde die Reaktion mit 163, in Anlehnung an eine Literaturvorschrift von ITO et al. ${ }^{[128]}$, mit iso-Propylmagnesiumbromid durchgeführt und man erhielt das Zielprodukt 164 in quantitativer Ausbeute. Der letzte Schritt dieser Synthesesequenz war die Addition der entsprechenden Lithiumspezies des Bromides 166 an das Weinreb-Amid 164.

Schema 47: Abschluss der Synthese des Trienons 165 und Synthese des Bromides 166 aus Acetylen und IBr.

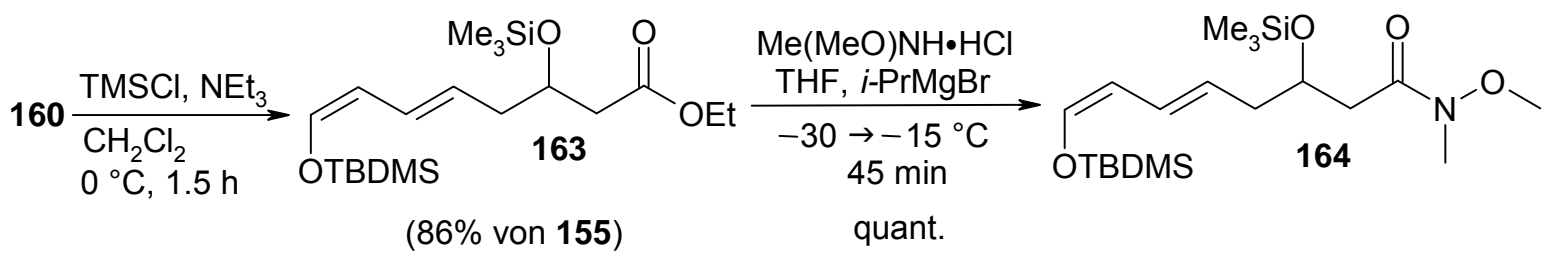

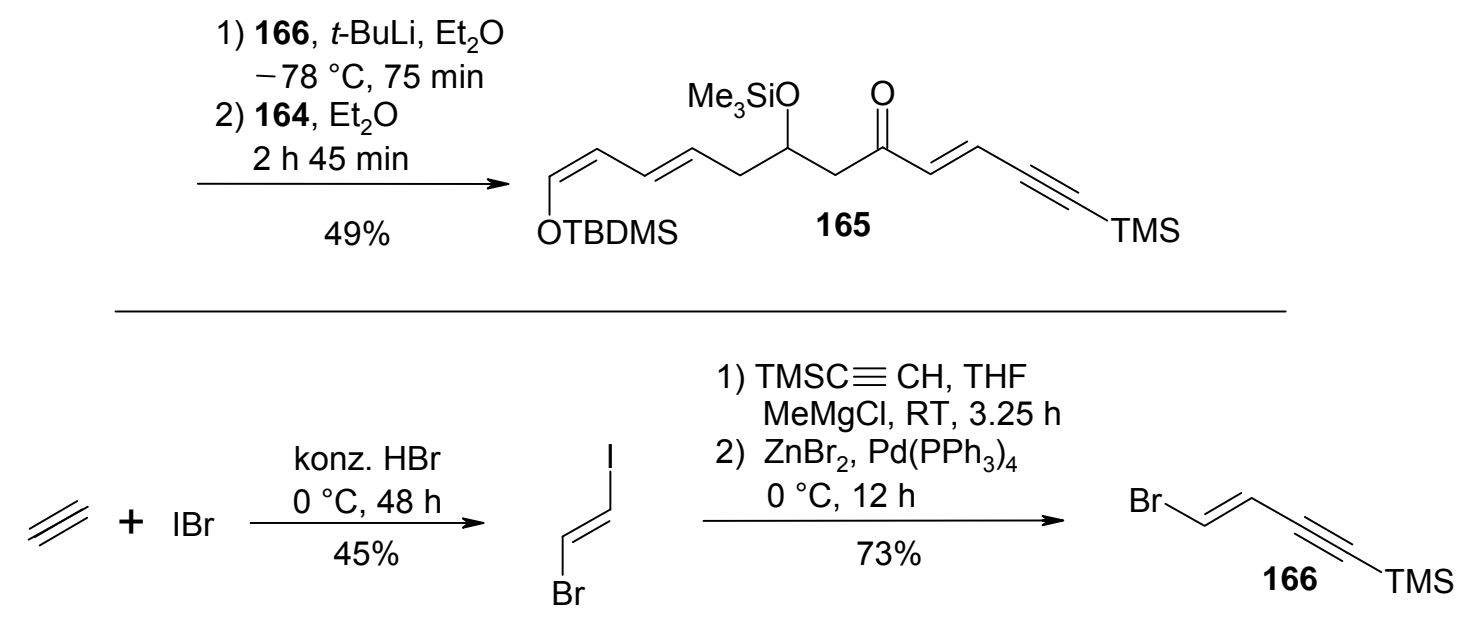

Die Synthese des bekannten Bromides 166 und der dafür benötigten Edukte erfolgte nach literaturbekannten Vorschriften. Dabei wurde aus Acetylen und Iodbromid zunächst Iod- 
bromethylen ${ }^{[129]}$ hergestellt, welches über eine Kreuzkupplung ${ }^{[130]}$ mit Tetrakis(triphenylphosphin)palladium $(0)^{[131]}$ und TMS-Acetylen ${ }^{[132]}$ das Bromid 166 ergab. Dieses wurde wiederum mit tert-Butyllithium lithiiert und anschließend mit Amid 164 versetzt. ${ }^{[133]}$ Nach wässriger Aufarbeitung und chromatographischer Reinigung erhielt man so das Trienon 165 in befriedigender Ausbeute. Dass keine höhere Ausbeute erzielt werden konnte, ist abermals auf Verluste während der Säulenchromatographie zurückzuführen.

\section{II.5.2.2 Synthese weiterer Trienone}

HWE-Reaktion mit verschiedenen Aldehyden: Zur Untersuchung des Einflusses der Substituenten auf die intramolekulare Diels-Alder-Reaktion wurden weitere Trienone synthetisiert. Die Synthese verlief im Wesentlichen wie für den Octadiensäureester 163 bereits beschrieben und startete ebenfalls mit einer HWE-Reaktion eines Aldehydes mit dem Phosphonoester 154. Die dafür verwendeten Aldehyde und deren Synthese sind in Tabelle 16 dargestellt. Crotonaldehyd $\mathbf{1 6 7}$ wurde ohne weitere Reinigung wie gekauft verwendet. Die andern Aldehyde wurden nach Literatur, ausgehend von Z-Buten-1,4-diol, synthetisiert. Um den Einfluss der Doppelbindungskonfiguration der konjugierten HWEProdukte auf die Ergebnisse der Dekonjugierung beurteilen zu können, wurden einige $E$ und Z-Aldehyde hergestellt. Während die E-konfigurierten Aldehyde $\mathbf{1 4 1}^{[109]}$ und $\mathbf{1 6 8}^{[134]}$ in guten Ausbeuten durch Parikh-Doering-Oxidation ${ }^{[107]}$ der einfach geschützten $Z$ Butendiole erhalten wurden, konnten die $Z$-konfigurierten Aldehyde $141 Z^{[135]}$ und $169^{[136]}$ in vergleichbar guten Ausbeuten durch schonende Oxidation mit Braunstein gewonnen werden.

Tabelle 16: Synthese der verschiedenen Aldehyde für die HWE-Reaktionen.

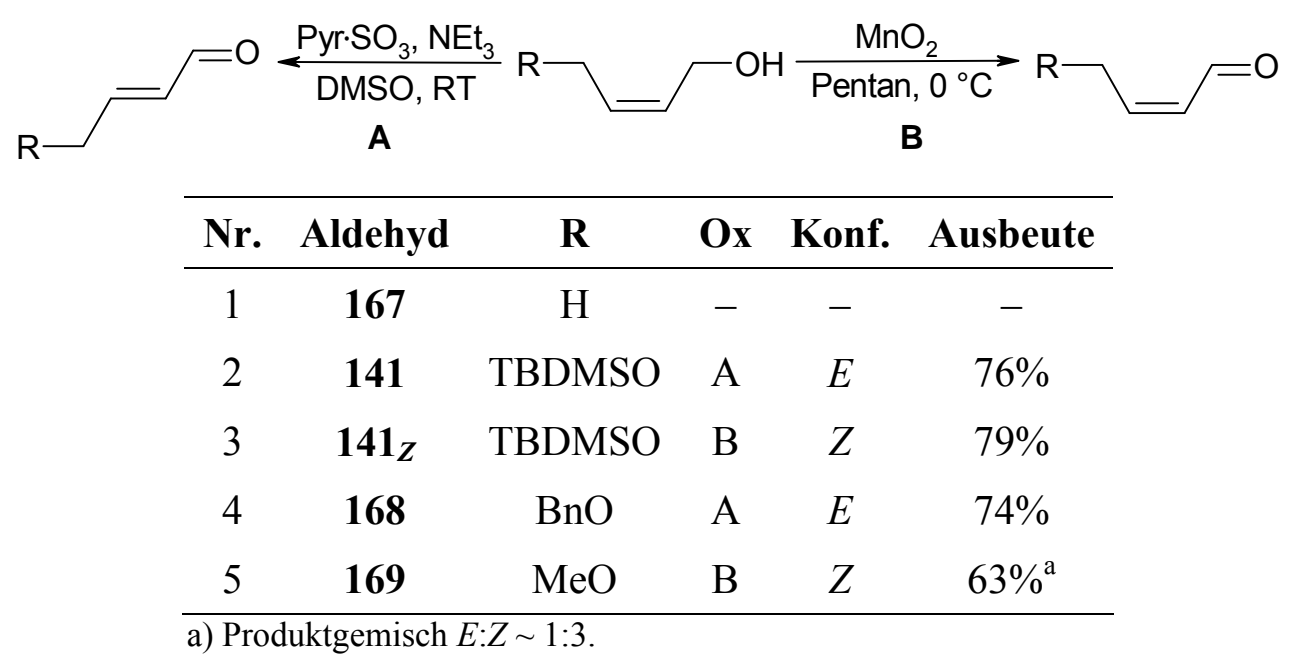


Im Gegensatz zu den anderen Aldehyden, welche alle als isomerenreine Verbindungen isoliert werden konnten, wurde Z-MeO-Butenal 169 als untrennbares $Z / E$-Gemisch in einem Verhältnis von etwa 3:1 erhalten. Die HWE-Reaktion wurde wie oben bereits beschrieben durchgeführt und ergab die konjugierten Octadiensäureester $\mathbf{1 5 5}_{Z}$ und $\mathbf{1 7 0}_{-}$ 172 in guten bis befriedigenden Ausbeuten (Tabelle 17). Wie zu erwarten war, erhielt man bei der Verwendung des MeO-Butenals 169 ein Gemisch aus 4E/6Z und 4E/6E konfiguriertem Produkt, wobei das Verhältnis der beiden Verbindungen dem Isomerenverhältnis im Aldehyd 169 entsprach ( 3:1). Alle konjugierten $\beta$-Oxoester lagen, wie für 155 bereits beschrieben, als Gemisch aus dem Keto- und dem Enol-Tautomer vor.

Tabelle 17: Ergebnisse der HWE-Reaktionen mit den verschiedenen Aldehyden.

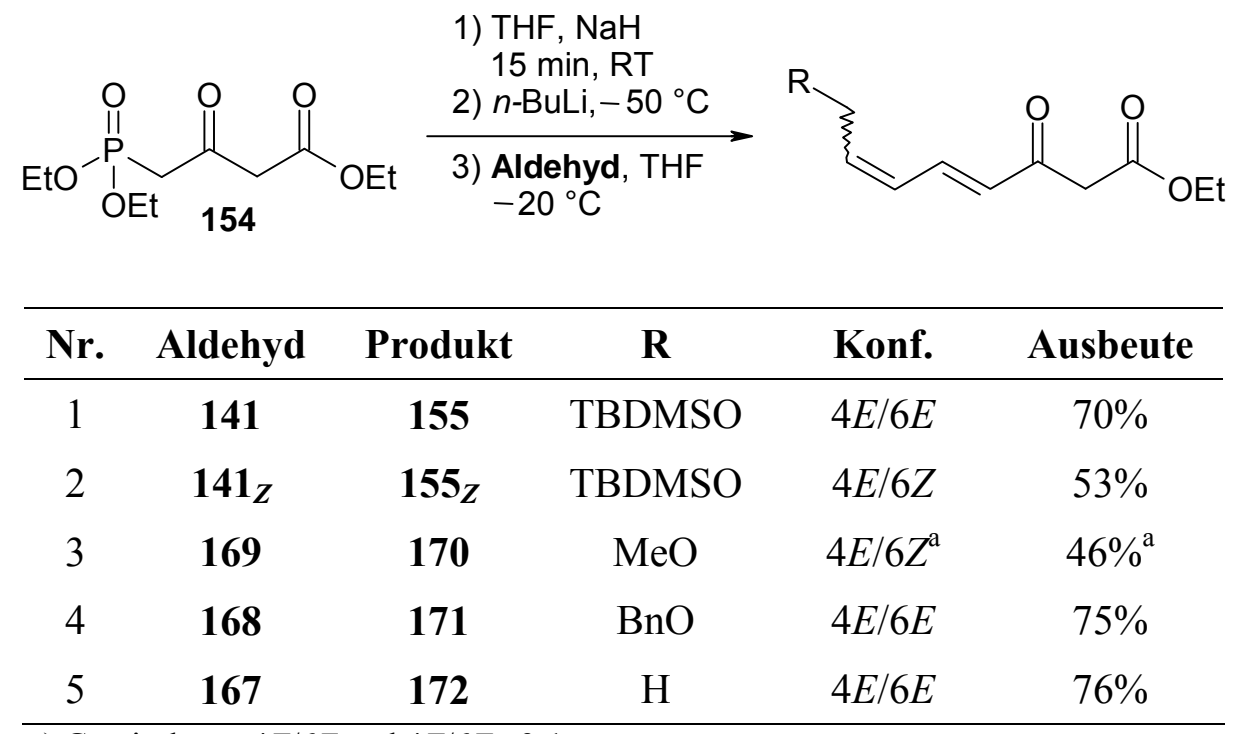

a) Gemisch aus $4 E / 6 Z$ und $4 E / 6 E \sim 3: 1$.

Dekonjugierung, Reduktion und Schützung: Um Reaktionsbedingungen identifizieren zu können, welche eine gezielte Synthese der 5E/7E-konfigurierten, dekonjugierten Produkte ermöglichen sollten, wurde die Dekonjugierung der verschiedenen Diensäureester eingehender untersucht. Die Ergebnisse dieser Untersuchungen werden weiter unten (Kap. II 5.1.5) ausführlicher diskutiert. An dieser Stelle sei kurz zusammengefasst, dass trotz intensiver Bemühungen die 5E/7E-konfigurierten Verbindungen nicht synthetisiert werden konnten. Deshalb wurde die Synthese mit den 5E/7Z-konfigurierten Produkten weitergeführt. Einzig das Methoxy-Derivat 170 lieferte nach der Dekonjugierung zumindest ein Gemisch aus 5E/7E- und 5E/7Z-konfiguriertem Produkt, erwies sich jedoch gleichzeitig als instabilstes Derivat (Tabelle 18). Aufgrund der mäßigen Stabilität der Verbindungen wurden die Produkte der Dekonjugierung und der Reduktion mit $\mathrm{NaBH}_{4}$ nicht isoliert, sondern direkt weiter umgesetzt. So erhielt man die TMS-geschützten, 
dekonjugierten Octadiensäureester 163 und 173-175. Die Verbindungen wurden als Lösungen in Pentan bei $-21^{\circ} \mathrm{C}$ gelagert, da sich diese bei Lagerung in Reinsubstanz, auch bei $-21^{\circ} \mathrm{C}$, innerhalb von Wochen zersetzten.

Tabelle 18: Synthese der dekonjugierten, geschützten $\beta$-Hydroxyester 163 und 173-175.<smiles>[R]C/C=C\C=C\C(=O)CC(=O)OCC</smiles><smiles>[R]C=CC=CCC(=O)CC(=O)OCC</smiles><smiles>[R]C=CC=CCC(O)CC(=O)OCC</smiles><smiles>[R]C=CC=CCC(CC(=O)OCC)O[Si](C)(O)OC</smiles>

\begin{tabular}{cccccc}
\hline Nr. & Edukt & Produkt & $\mathbf{R}$ & Konf. & Ausbeute $^{\mathrm{a}}$ \\
\hline 1 & $\mathbf{1 5 0}$ & $\mathbf{1 5 9}$ & $\mathrm{TBDMSO}$ & $5 E / 7 Z$ & $86 \%$ \\
2 & $\mathbf{1 6 6}$ & $\mathbf{1 6 9}$ & $\mathrm{MeO}$ & $5 E / 7 Z+5 E / 7 E^{\mathrm{b}}$ & $60 \%$ \\
3 & $\mathbf{1 6 7}$ & $\mathbf{1 7 0}$ & $\mathrm{BnO}$ & $5 E / 7 Z$ & $12 \%$ \\
4 & $\mathbf{1 6 8}$ & $\mathbf{1 7 1}$ & $\mathrm{H}$ & $5 E$ & $31 \%$ \\
\hline
\end{tabular}

a) Ausbeute ausgehend vom konj. $\beta$-Oxoester. b) Isomerenverhältnis $\sim 1: 1$.

Amidbildung und Alkinaddtion: Die Synthese der Trienone wurde wie für 165 bereits beschrieben abgeschlossen (Tabelle 19). Dabei erfolgte nach Weinreb-Amidbildung die Addition des mit tert-Butyllithium erzeugten Lithiumorganyls von 166. Die Trienone 176178 konnten so in moderaten Ausbeuten von 11 bis 37\% isoliert werden. Die Ausbeute des Benzyloxyderivates 177 war mit 11\% deutlich geringer als die Ausbeute der anderen Derivate. Dies ist zum Einen darauf zurückzuführen, dass sich dieses Derivat als besonders empfindlich gegenüber den Reaktionsbedingungen der Weinrebamidbildung herausstellte und zum Anderen auf überdurchschnittliche Verluste bei der säulenchromatographischen Reinigung des Trienons 177. Alle Trienone mussten gelöst in Pentan gelagert werden, da sie sich bei Lagerung in Reinsubstanz auch bei $-20^{\circ} \mathrm{C}$ innerhalb weniger Stunden vollständig zersetzten. 
Tabelle 19: Abschluss der Synthese der Trienone 165 und 176-178.

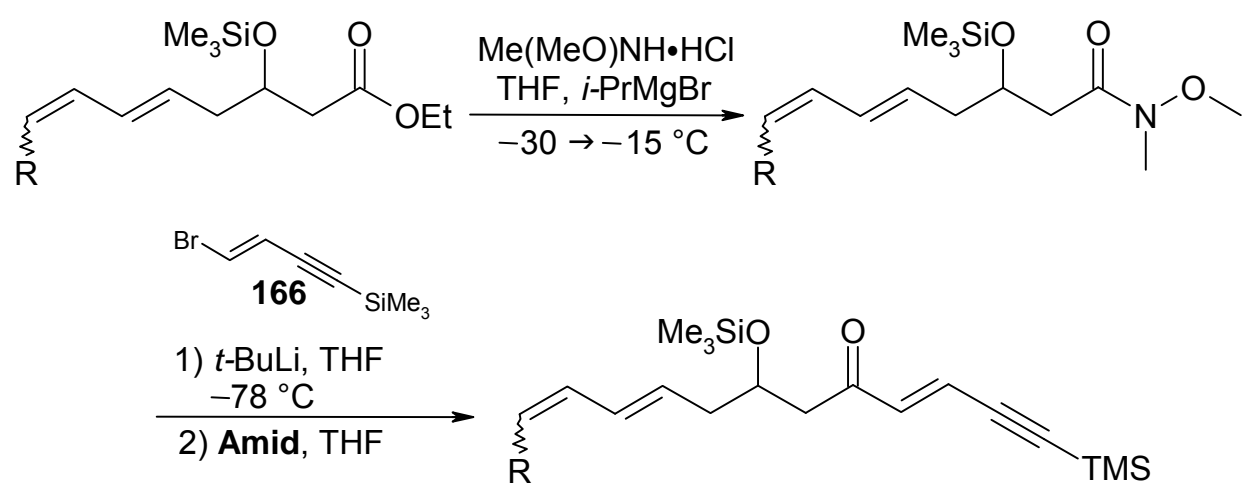

\begin{tabular}{cccccc}
\hline Nr. & Edukt & Trienon & $\mathbf{R}$ & Konf. d. Prod. & Ausbeute $^{\mathrm{b}}$ \\
\hline 1 & $\mathbf{1 5 9}$ & $\mathbf{1 6 1}$ & TBDMSO & $9 E / 11 Z$ & $49 \%$ \\
2 & $\mathbf{1 6 9}$ & $\mathbf{1 7 2}$ & $\mathrm{MeO}$ & $9 E / 11 Z+9 E / 11 E^{\mathrm{a}}$ & $37 \%$ \\
3 & $\mathbf{1 7 0}$ & $\mathbf{1 7 3}$ & $\mathrm{BnO}$ & $9 E / 11 Z$ & $11 \%$ \\
4 & $\mathbf{1 7 1}$ & $\mathbf{1 7 4}$ & $\mathrm{H}$ & $9 E$ & $27 \%$ \\
\hline
\end{tabular}

a) Verhältnis $\sim 1.5: 1$; b) Über zwei Stufen.

Synthese des Trienons 180: Um den Einfluss des Alkinylsubstituenten auf die intramolekulare Diels-Alder-Reaktion beurteilen $\mathrm{zu}$ können, wurde Trienon $\mathbf{1 8 0}$ synthetisiert, in welchem das Dienophil keinen Substituenten trägt. Das Weinreb-Amid 164 wurde in Anlehnung an eine Synthesevorschrift von TASHIRO ${ }^{[137]}$ et al. mit Vinylmagnesiumbromid umgesetzt (Schema 48). Analog der Literatur ließ man im ersten Versuch das Reaktionsgemisch nach der Zugabe des Vinylmagnesiumbromids auf Raumtemperatur erwärmen (A). Dies führte zur Isolierung des Michael-Adduktes 179 und nicht des gewünschten Trienons 180. Diese Nebenreaktion in der Umsetzung von Weinreb-Amiden mit Vinylmagnesiumbromid wurde bereits von anderen Arbeitsgruppen beobachtet und ist auf eine zu hohe Reaktionstemperatur zurückzuführen. ${ }^{[138]}$ Bei einem erneuten Versuch (B) wurde die Reaktionstemperatur bei $-30^{\circ} \mathrm{C}$ gehalten, wodurch die Bildung des MichaelAdduktes effektiv verhindert werden konnte. Nach säulenchromatographischer Aufarbeitung erhielt man das Zielprodukt 180 in 41\% Ausbeute und 29\% des entschützten 5-Hydroxyderivats 181. Beide Verbindungen wurden in den folgenden IMDA-Reaktionen eingesetzt. 
Schema 48: Synthese des Trienons 180 mit unsubstituiertem Dienophil.

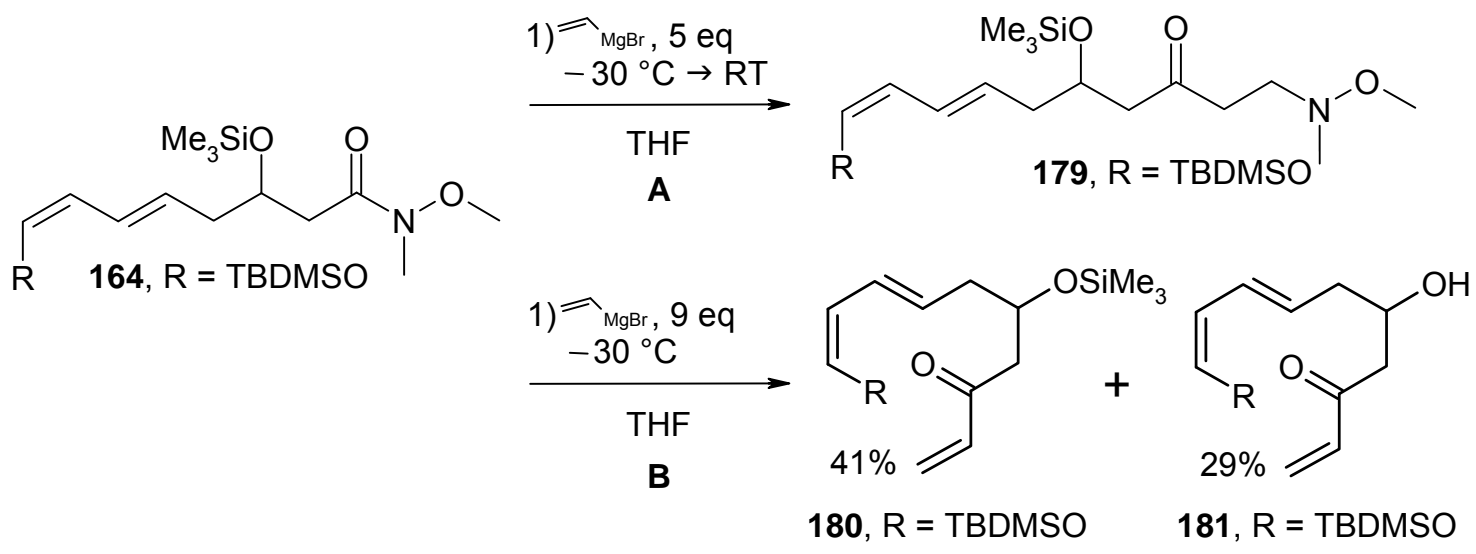

\section{II.5.3 Versuche zur Darstellung der 5E/7E Diensäureester}

Als mögliche Ursache für das Scheitern der Diels-Alder-Reaktionen mit dem $E / Z$ konfigurierten Trienon 165 könnten, neben mangelnder Stabilität des Edukts, vor allem prohibitiv große sterische Wechselwirkungen zwischen der TBDMSO-Gruppe und der TMS-Alkinylfunktion sein (Schema 49). Deshalb wurde mit den hergestellten Octadiensäureestern eine Reihe von Experimenten durchgeführt, welche das Ziel hatten, die entsprechenden 5E/7E-konfigurierten Verbindungen zu synthetisieren. Die Versuche wurden nach der bereits für Hexadiensäureester 155 beschriebenen Vorschrift durchgeführt. Dazu wurden 3.3 eq Diisopropylamin mit einer äquimolaren Menge $n$-Butyllithium deprotoniert und auf $-78{ }^{\circ} \mathrm{C}$ gekühlt. Bei Verwendung von weniger als 3.3 eq LDA wurde kein vollständiger Umsatz erreicht. Danach wurde die Lösung mit Hexamethylphosphorsäuretriamid (HMPA) bzw. 1,3-Dimethyltetrahydropyrimidin-2(1H)-on (DMPU) versetzt. Man ließ bei $-78{ }^{\circ} \mathrm{C}$ rühren und kontrollierte den Reaktionsfortschritt per Dünnschichtchromatographie.

Schema 49: Sterische Wechselwirkungen im ÜZ der IMDA-Reaktion könnten für das Scheitern der IMDA-Versuche mit 165 verantwortlich sein.

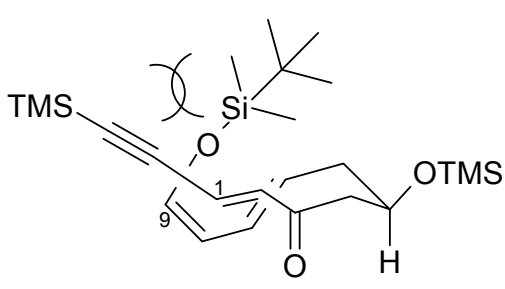

endo-Sessel

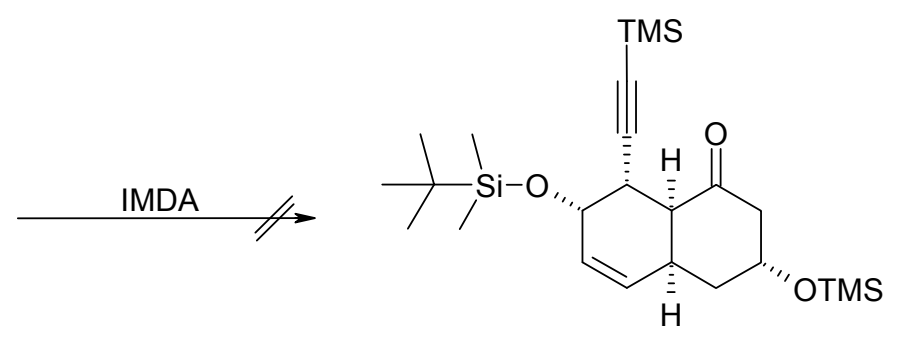

$94 a$ 
Nach beendeter Reaktion wurde durch Zugabe von gesättigter Ammoniumchloridlösung protoniert und nach wässriger Aufarbeitung erhielt man die dekonjugierten Produkte. Bei der Untersuchung der Wirkung der Additive HMPA und DMPU auf die Dekonjugierung zeigte sich, dass diese keinen Einfluss auf die Konfiguration des Produktes haben (Tabelle 20). So konnte bei keinem der Versuche ein dekonjugierter Diensäureester mit gewünschter $5 E / 7 E$-Konfiguration isoliert werden, allerdings wurde die vorher bereits gemachte Beobachtung ${ }^{[38]}$ bestätigt, dass ohne DMPU oder HMPA kein dekonjugiertes Produkt isoliert werden kann (Eintrag 1). GRIECO et al. beschrieb, dass die Doppelbindungskonfiguration des Produkts durch die Konfiguration des Edukts beeinflusst werden kann. ${ }^{[139]}$ So bildete sich bei der Dekonjugierung von 6-Methoxy-2E,4 $\underline{E}$-hexadiensäure mit LDA in THF das entsprechende $3 E, 5 \underline{Z}$ Produkt, während 6-Methoxy-2E,4 $\underline{Z}-$ hexadiensäure die entsprechende $3 E, 5 \underline{E}$-konfigurierte Verbindung lieferte. Mit Blick auf diese Ergebnisse wurden die konjugierten Ester $\mathbf{1 5 5}_{Z}$ und $\mathbf{1 7 0}$ synthetisiert, welche die 4E/6Z-Konfiguration besitzen. Beide Diensäureester wurden den Bedingungen der Dekonjugierung unterzogen. Entgegen den Beobachtungen von GRIECO et al. lieferte Octadiensäurester $\mathbf{1 5 5}_{Z}$ in der Dekonjugierung mit HMPA ausschließlich das 5E/7Zkonfigurierte Produkt 156, welches sich auch bei der Dekonjugierung von 155 bildet. Eine weitere interessante Beobachtung war, dass die Umsetzungen des 4E/6Z-konfigurierten Octadiensäureesters $\mathbf{1 5 5}_{Z}$ mit DMPU als Additiv nicht zum dekonjugierten Produkt führten. Während das 4E/6E-konfigurierte 155 in Gegenwart von entweder DMPU oder HMPA das 5E/7Z-Produkt in vergleichbaren Ausbeuten liefert, lag $\mathbf{1 5 5}_{Z}$ bei der Verwendung von DMPU auch nach der Reaktion noch fast ausschließlich als konjugiertes Edukt vor (Eintrag 3). Mit 170 erhielt man mit DMPU ein sehr unsauberes Rohprodukt, welches neben dem Eduktisomerengemisch nicht identifizierte Zersetzungsprodukte enthielt (Eintrag 4). Dagegen lieferte die Dekonjugierung von $170(4 E / 6 E: 4 E / 6 Z \sim 1: 2.5)$ in Gegenwart von HMPA ein Gemisch der dekonjugierten Ester 182 und $\mathbf{1 8 2}_{Z}$ im Verhältnis $5 E / 7 E: 5 E / 7 Z$ von etwa 1:1.5. In Kombination mit den von $\mathrm{WU}$ et al. gemachten Beobachtungen kann man davon ausgehen, dass die Konfiguration des Eduktes bzw. das Verhältnis zwischen $E / E$ und $E / Z$-konfigurierter Verbindung für das Ergebnis der Reaktion nicht entscheidend ist. ${ }^{[140]}$ So beobachteten WU et al. bei der Dekonjugierung eines Isomerengemisches von 6-Methoxyhexadiensäure $(2 E / 4 E: 2 E / 4 Z \sim 2.5: 1)$ im dekonjugierten Produkt ebenfalls ein Isomeremverhältnis von 1:1. Die Dekonjugierung des Benzyloxyderivates 171 lieferte das dekonjugierte Produkt 183 ebenfalls mit der bei den anderen Derivaten bereits beobachteten 5E/7Z-Konfiguration (Eintrag 5). Wie schon 
mehrfach erwähnt, waren alle dekonjugierten Octadiensäureester nur mäßig stabil, insbesondere gegenüber Kieselgel, was eine sinnvolle Ausbeutenbestimmung unmöglich machte. Demgegnüber konnte der dekonjugierte Octadiensäureester 184, welcher keinen Enolether trägt, problemlos durch Säulenchromatographie in einer guten Ausbeute von $71 \%$ isoliert werden.

Tabelle 20: Zusammenfassung der Dekonjugierungsversuche zur Herstellung der 5E/7Ekonfigurierten Diensäureesters.

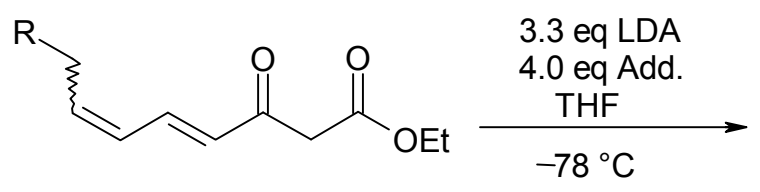<smiles>[R]C=CC=CCC(=O)CC(=O)OCC</smiles>

\begin{tabular}{|c|c|c|c|c|c|c|c|}
\hline \multirow{2}{*}{ Nr. } & \multicolumn{3}{|c|}{ Edukt } & \multirow{2}{*}{ Additiv } & \multirow{2}{*}{ Ausb. } & \multirow{2}{*}{ Prod. } & \multirow{2}{*}{ Bemerkungen } \\
\hline & & $\mathbf{R}$ & Konf. & & & & \\
\hline 1 & 155 & TBDMSO & $4 E / 6 E$ & - & $-{ }^{\mathrm{a}}$ & 156 & Spuren $5 E / 7 Z$ \\
\hline 2 & 155 & TBDMSO & $4 E / 6 E$ & $\begin{array}{l}\text { HMPA } \\
\text { DMPU }\end{array}$ & $-{ }^{a}$ & 156 & $\begin{array}{l}\text { Reines } 5 E / 7 Z \text {-Produkt } \\
\text { Reines } 5 E / 7 Z \text {-Produkt }\end{array}$ \\
\hline 3 & $155_{Z}$ & TBDMSO & $4 E / 6 Z$ & $\begin{array}{l}\text { HMPA } \\
\text { DMPU }\end{array}$ & $-{ }^{\mathrm{a}}$ & 156 & $\begin{array}{l}\text { Reines } 5 E / 7 Z \text {-Produkt } \\
\text { Edukt }+ \text { Spuren } 5 E / 7 Z\end{array}$ \\
\hline 4 & $170^{b}$ & $\mathrm{MeO}$ & $4 E / 6 Z$ & $\begin{array}{l}\text { HMPA } \\
\text { DMPU }\end{array}$ & $-{ }^{a}$ & 182 & $\begin{array}{c}5 E / 7 E: 5 E / 7 Z \sim 1: 1.5 \\
\text { Edukt }^{\mathrm{c}}\end{array}$ \\
\hline 5 & 171 & $\mathrm{BnO}$ & $4 E / 6 E$ & DMPU & $-{ }^{\mathrm{a}}$ & 183 & Reines $5 E / 7 Z$-Produkt \\
\hline 6 & 172 & $\mathrm{H}$ & $4 E / 6 E$ & DMPU & $71 \%$ & 184 & - \\
\hline
\end{tabular}

a) Ausbeuten nicht bestimmt, da die dekonjugierten Produkte instabil waren und deshalb direkt reduziert wurden; b) Isomerengemisch $4 E / 6 E: 4 E / 6 Z, \sim 1: 2.5$; c) Spektrum zeigte Edukt und Zersetzungsprodukte an.

Eine Erklärung für die Bildung der im Vergleich zur E-konfigurierten Doppelbindung eigentlich energetisch ungünstigeren 7Z-Doppelbindung liefert die in Schema 50 gezeigte Darstellung. ${ }^{[141]}$ Wahrscheinlich wird das Ergebnis der Dekonjugierung der E/E-konfigurierten Ester durch sterische Wechselwirkungen zwischen (Silyl)-Etherfunktion und dem $\alpha$-ständigen Proton $\left(\mathrm{A}_{1,2}\right)$ bzw. dem $\beta$-ständigen Proton $\left(\mathrm{A}_{1,3}\right)$ bestimmt. Die Beobachtungen aus den Experimenten zeigen, dass bei $-78{ }^{\circ} \mathrm{C}$ die $A_{1,2}$-Wechselwirkung dominiert und deshalb die Z-Doppelbindung gebildet wird. Das Ergebnis der Dekonjugierung der 4E/6Z-konfigurierten Vorläufer $\mathbf{1 5 5}_{Z}$ und $\mathbf{1 7 0}$ scheint dieser Theorie auf den ersten Blick zu widersprechen. Betrachtet man nämlich die vermeintlichen Vorzugskonformationen aus denen die Deprotonierung erfolgen könnte (Schema 51), so 
erscheint hier eindeutig die $\mathrm{A}_{1,3}$-Wechselwirkung zu dominieren. Dadurch sollte eigentlich das $5 E / 7 E$-Isomer bevorzugt gebildet werden.

Schema 50: Die größeren sterischen Wechselwirkungen zwischen der terminalen Etherfunktion und dem $\alpha$-ständigen Proton sind für die Z-Konfiguration der Doppelbindung im dekonjugierten Produkt verantwortlich.<smiles>[R6]C/C=C\C=C\CC(=O)CC(=O)OCC</smiles>

Die Tatsache, dass dennoch das unerwünschte 5E/7Z-Isomer als Produkt erhalten wurde, lässt sich eventuell auf Chelateffekte zurückführen, wie sie in Schema 51 (rechts) dargestellt sind. Aus diesen mechanistischen Überlegungen ergaben sich zwei Ansatzpunkte für weitere Versuche, die die gewünschten $E / E$-konfigurierten Produkte liefern sollten: 1) Zunächst sollte die Möglichkeit überprüft werden, ob nach der Deprotonierung bei $-78^{\circ} \mathrm{C}$ eine ,nachträgliche“ Isomerisierung in die energetisch günstigere $E / E$-Form erfolgt, wenn das Reaktionsgemisch auf Temperaturen über $-78^{\circ} \mathrm{C}$ erwärmt wird (Schema 52). 2) Danach sollte der Frage nachgegangen werden, ob kleinere und stärkere Basen als LDA das konjugierte Dien aus der in Schema 51 links abgebildeten Konformation deprotonieren zu können (Schema 54).

Schema 51: Die Bildung der 5E/7Z-Diensäurester aus den 4E/6Z-Vorläufern könnte auf Chelateffekte zurückzuführen sein.
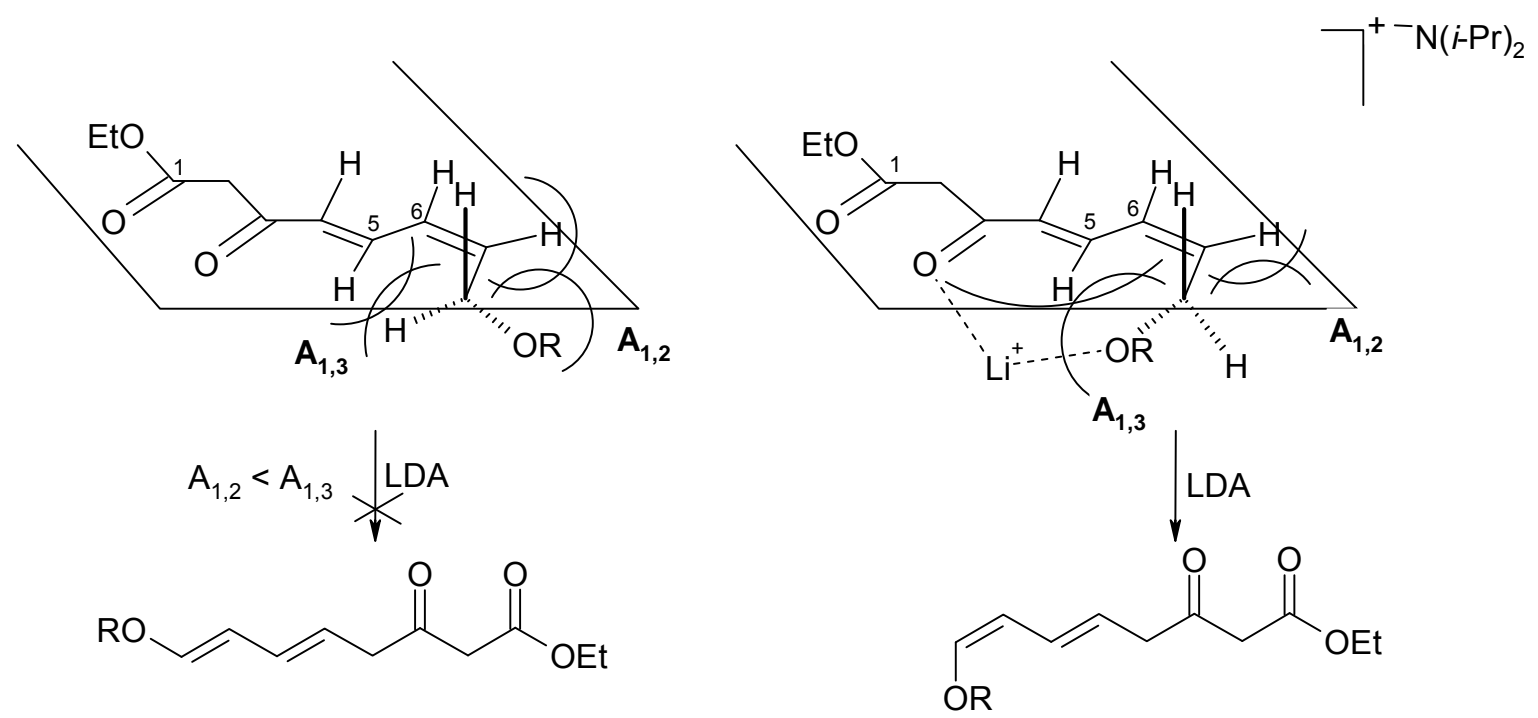
Zur Untersuchung von Punkt 1 wurden vier identische Reaktionsgemische einer Standarddekonjugierung von 155 angesetzt. Drei der Reaktionen wurden für eine Stunde bei -40, $-20 \mathrm{bzw} .0{ }^{\circ} \mathrm{C}$ gerührt, bevor sie wieder auf $-78^{\circ} \mathrm{C}$ gekühlt und gequencht wurden. Alle Reaktionen lieferten ausschließlich das unerwünschte 5E/7Z-Produkt. Damit zeigte sich, dass bei diesen Temperaturen keine Isomerisierung wie in Schema 52 dargestellt stattfand. Da mit steigender Temperatur auch vermehrte Zersetzung beobachtet wurde, war eine Wiederholung der Versuchsreihe mit Temperaturen $>0{ }^{\circ} \mathrm{C}$ nicht sinnvoll.

Schema 52: Bei der Erwärmung des Reaktionsgemisches aus der Dekonjugierung trat keine Isomerisierung ein.

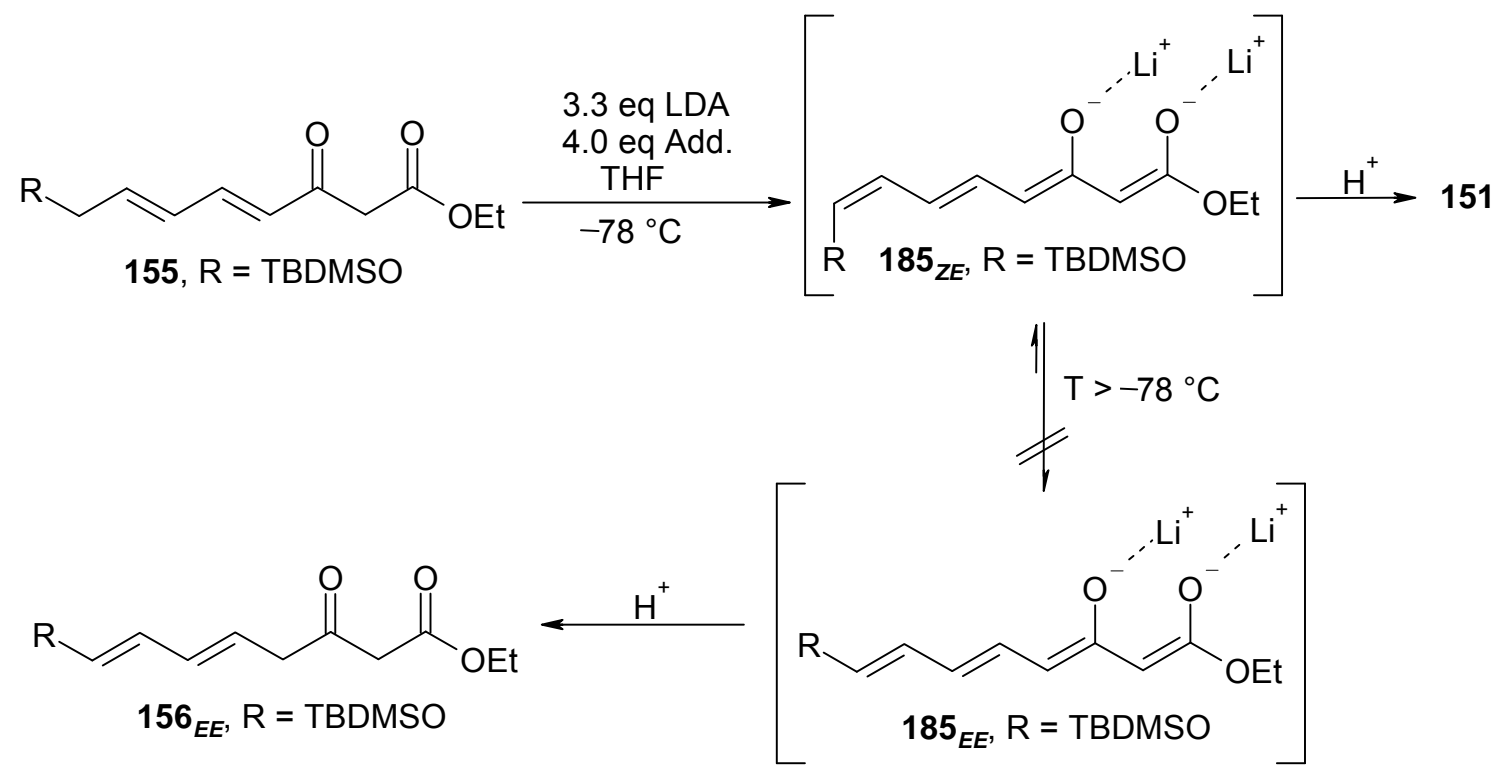

Nachdem sich bei der Erwärmung des Reaktionsgemisches aus der Dekonjugierung auf $0{ }^{\circ} \mathrm{C}$ keine Isomerisierung zum E/E-Produkt jedoch signifikante Zersetzung gezeigt hatte, wurde ein Weg gesucht, das Reaktionsgemisch der Dekonjugierung ohne Zersetzung des deprotonierten Edukts auf Temperaturen größer $0{ }^{\circ} \mathrm{C}$ erwärmen zu können. Dazu sollte der TMS-Enolether 186 synthetisiert und anschließend in situ mit LDA deprotoniert werden (Schema 53). Zugabe von LDA sollte das durchkonjugierte Tetraen 187 ergeben, welches im Idealfall schon in der abgebildeten all-E-Konfiguration vorläge. Durch Protonierung würde man so das gewünschte $E / E$-konfigurierte Produkt $\mathbf{1 5 5}_{E \boldsymbol{E}}$ erhalten. Sollte Tetraen 187 jedoch in der 7-Z-Konfiguration erhalten werden, so wäre es eventuell stabil genug, um es auf Temperaturen $>0{ }^{\circ} \mathrm{C}$ erwärmen zu können, was die energetisch günstigere $E$ Konfiguration ergeben sollte. WHITE et al. beschrieben die Synthese eines $\alpha, \beta / \gamma, \delta$ ungesättigten TMS-Enolethers aus dem entsprechenden $\beta$-Oxoester durch Reaktion mit Triethylamin und TMSCl. ${ }^{[142]}$ Die nach WHITE durchgeführten Versuche mit $\mathbf{1 5 5}$ und $\mathbf{1 5 5}_{\boldsymbol{Z}}$ 
(Tabelle 21, Einträge 1 und 2) führten jedoch nicht zum TMS-Enolether 186. Reaktionskontrolle mittels Dünnschichtchromatographie zeigte unvollständigen Umsatz an; da auch nach Zugabe weiterer Äquivalente Base und TMSCl kein vollständiger Umsatz erzielt werden konnte, wurden die Reaktionen wässrig aufgearbeitet. ${ }^{[143]}$ Nach Aufarbeitung erhielt man das eingesetzte Edukt.

Schema 53: Versuche zur Synthese des $E / E$-konfigurierten Ester 155 über TMS-Enolether 187.

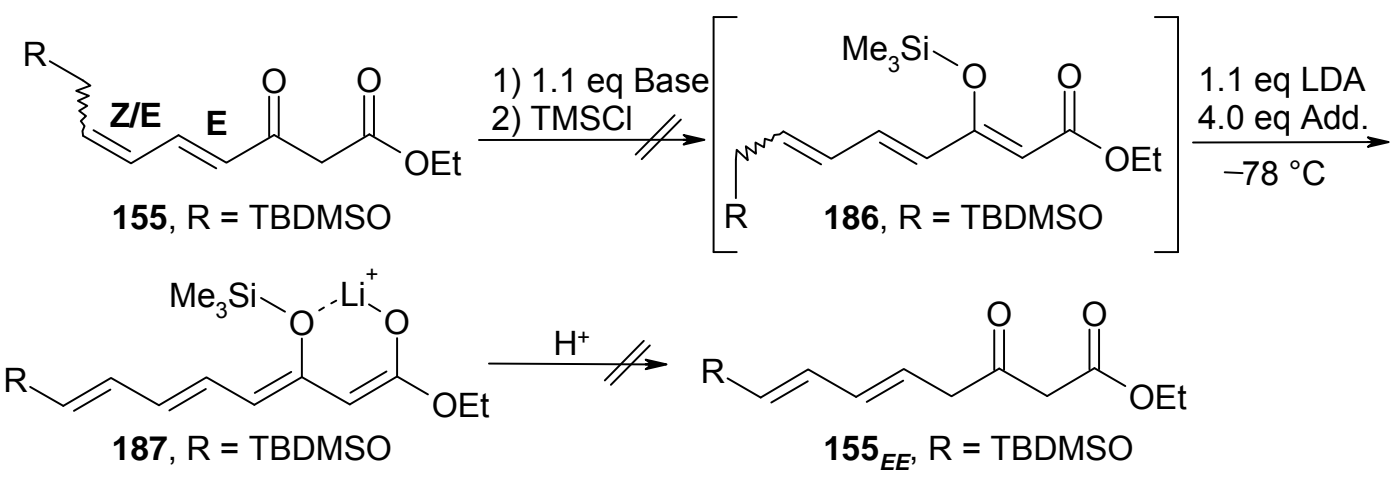

Tabelle 21

\begin{tabular}{ccccccccc}
\hline Nr. & Edukt & Konf. & Base & Reagenz & $\mathbf{T}_{\mathbf{1}}$ & Add & Lm & Beob. $^{\text {a }}$ \\
\hline 1 & $\mathbf{1 5 5}$ & $E / E$ & $\mathrm{NEt}_{3}$ & $\mathrm{TMSCl}$ & $0{ }^{\circ} \mathrm{C} \rightarrow \mathrm{RT}$ & $\mathrm{HMPA}$ & $\mathrm{CH}_{2} \mathrm{Cl}_{2}$ & k.U., E. \\
2 & $\mathbf{1 5 5}_{Z}$ & $Z / E$ & $\mathrm{NEt}_{3}$ & $\mathrm{TMSCl}$ & $0{ }^{\circ} \mathrm{C} \rightarrow \mathrm{RT}$ & $\mathrm{HMPA}$ & $\mathrm{CH}_{2} \mathrm{Cl}_{2}$ & k.U., E. \\
3 & $\mathbf{1 5 5}$ & $E / E$ & $\mathrm{NaH}$ & $\mathrm{TMSCl}$ & $0{ }^{\circ} \mathrm{C}$ & $\mathrm{DMPU}$ & $\mathrm{THF}$ & k.U., E. \\
4 & $\mathbf{1 5 5}$ & $E / E$ & $\mathrm{NaH}$ & $\mathrm{TMSOTf}$ & $0{ }^{\circ} \mathrm{C}$ & $\mathrm{HMPA}$ & $\mathrm{THF}$ & k.U., E. \\
5 & $\mathbf{1 5 5}$ & $E / E$ & $\mathrm{NEt}_{3}$ & $\mathrm{TMSOTf}$ & $\mathrm{RT}$ & $\mathrm{HMPA}$ & $\mathrm{CH}_{2} \mathrm{Cl}_{2}$ & k.U., E. \\
\hline
\end{tabular}

a) k.U. - kein Umsatz, E. - nach Aufarbeitung wurde nur Edukt isoliert.

Auch bei Verwendung einer stärkeren Base (Einträge 3 und 4) bzw. des reaktiveren Silylierungsreagenz TMSOTf konnte kein vollständiger Umsatz erreicht werden. Die Umsetzung der partiell silylierten Gemische mit LDA (Einträge 3 und 4) führte nach wässriger Aufarbeitung ebenfalls nur zur Reisolierung des eingesetzten Edukts. Dekonjugierungsversuche ohne LDA: Nachdem sich gezeigt hatte, dass eine nachträgliche in situ-Isomerisierung des deprotonierten Octadiensäureesters 155 nicht möglich war, sollte nun untersucht werden, ob das $E / E$-konfigurierte Produkt durch Verwendung anderer Basen als LDA synthetisiert werden kann (Schema 54). Dazu wurden der $E / E-$ bzw. $Z / E$ konfigurierte Octadiensäureester 155 bei $0{ }^{\circ} \mathrm{C}$ in THF gelöst und zunächst mit 1.1 Äquivalenten $\mathrm{NaH}$ versetzt. Nach der Deprotonierung an der $\mathrm{CH}_{2}$-Gruppe zwischen beiden Carbonylgruppen wurde das Reaktionsgemisch auf $-78^{\circ} \mathrm{C}$ gekühlt und mit einem weiteren Äquivalent $n$-Butyllithium versetzt. Man ließ 30 min rühren, dann wurde die Reaktion 
wässrig aufgearbeitet. In Gegenwart von HMPA zersetzte sich das Edukt annähernd vollständig und ohne HMPA wurde das eingesetzte, konjugierte Edukt quantitativ zurückgewonnen.

Schema 54: Dekonjugierungsversuche mit kleineren Basen als LDA.

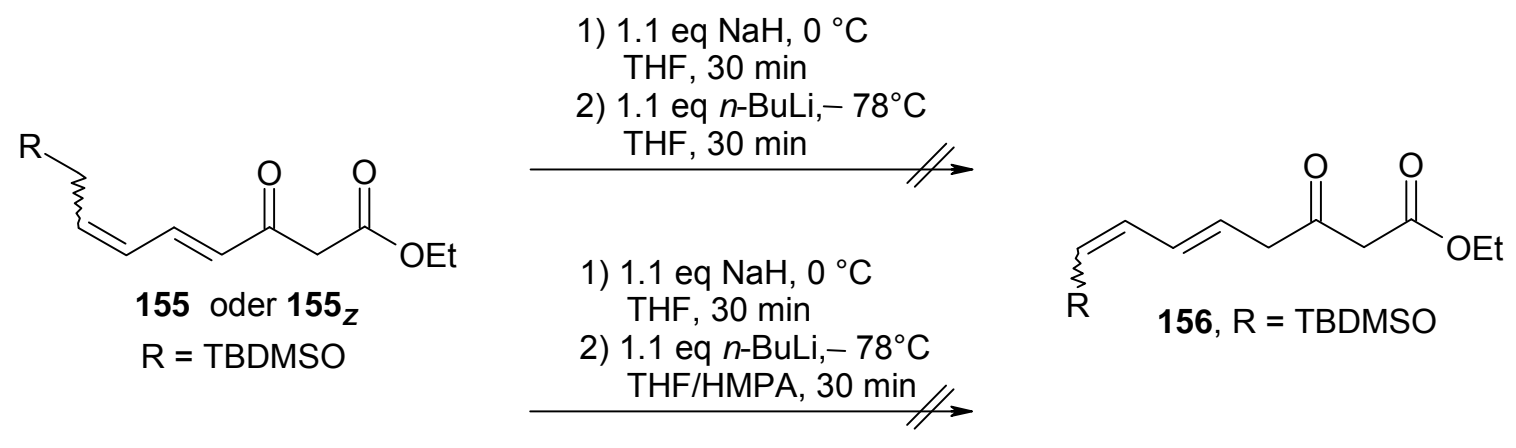

Isomerisierung mit $\mathrm{Cr}(\mathrm{CO})_{3}$ : SHIBASAKI et al. beschrieben die Cr-vermittelte Isomerisierung des Triensäureesters 188 zu Enolether 189 (Schema 55). ${ }^{[135]}$ Der Mechanismus der Isomerisierung von Verbindungen des Typs 190 verläuft vermutlich über eine Uförmige $\eta^{5}$-Zwischenstufe wie 192, aus der durch Abstraktion des cisoiden Protons $\mathrm{H}_{\mathrm{a}} 191$ gebildet wird.

Schema 55: $\mathrm{Cr}(\mathrm{CO})_{3}$-vermittelte Dekonjugierung nach SHIBASAKI et al.<smiles>CC(=O)C=CCCC=CC=CC[OH2+]</smiles>

188

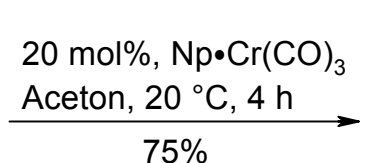
$75 \%$<smiles>COC(=O)C=CCCC/C=C\C=C\[OH2+]</smiles>

189<smiles>[R]C=C=CC=C/C=C\C(C)(C)O[SbH3]</smiles>

190

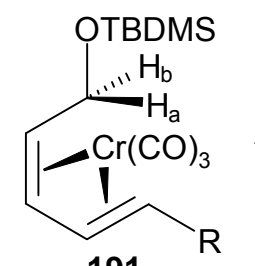

191

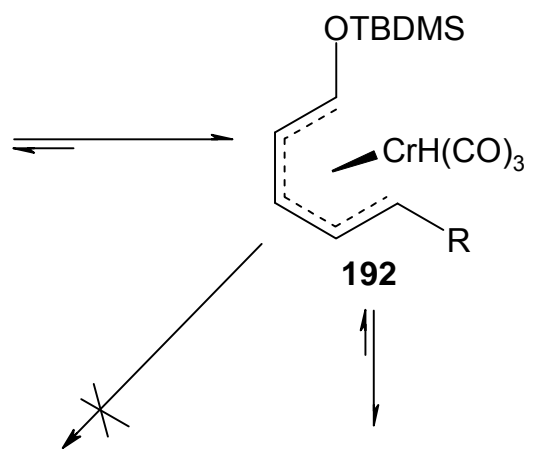

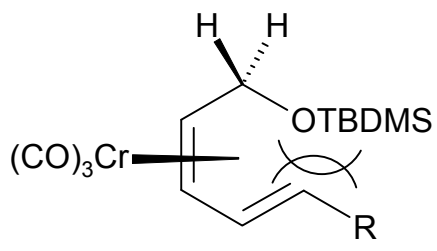

193

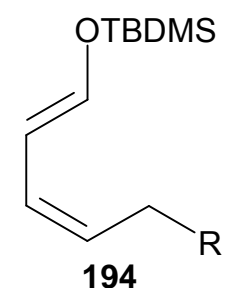

194 
Der Enolether 194 wird exklusiv mit der abgebildeten E-Konfiguration der Enoldoppelbindung gebildet, da eine Konfiguration wie in 193 gezeigt, aufgrund sterischer Wechselwirkungen nicht möglich ist. Bei der Isomerisierung des Octadiensäureesters $\mathbf{1 5 5}_{\boldsymbol{Z}}$ nach dieser Vorschrift erhielte man also das 5Z/7E-Produkt. Das daraus synthetisierte entsprechende Trienonen sollte in die IMDA-Untersuchungen einbezogen werden, um so Erkenntnisse über den Einfluss der Konfiguration der Enoldoppelbindung zu gewinnen. Aus den Versuchen mit den Octadiensäureestern 155 und $\mathbf{1 5 5}_{Z}$ wurde allerdings nur das jeweils eingesetzte Edukt zurückgewonnen (Schema 56). Da eventuell die zum Dien $\alpha$ ständige Carbonylfunktion die Koordination des $\mathrm{Cr}(\mathrm{CO})_{3}$ an die Doppelbindungen verhindert haben könnte, wurde Alkohol 195 synthetisiert und ebenfalls den Isomerisierungsbedingungen unterzogen. Auch bei diesem Versuch wurde ausschließlich das eingesetzte Edukt reisoliert. Ursache für das Scheitern der Versuche könnten sterische Wechselwirkungen zwischen dem Chrom und der Carbonyl- bzw. Hydroxylfunktion an C3 der Octadiensäureester sein.

Schema 56: $\operatorname{Cr}(\mathrm{CO})_{3}$-vermittelte Dekonjugierung der Octadiensäureester 150 und $\mathbf{1 5 0}_{Z}$.

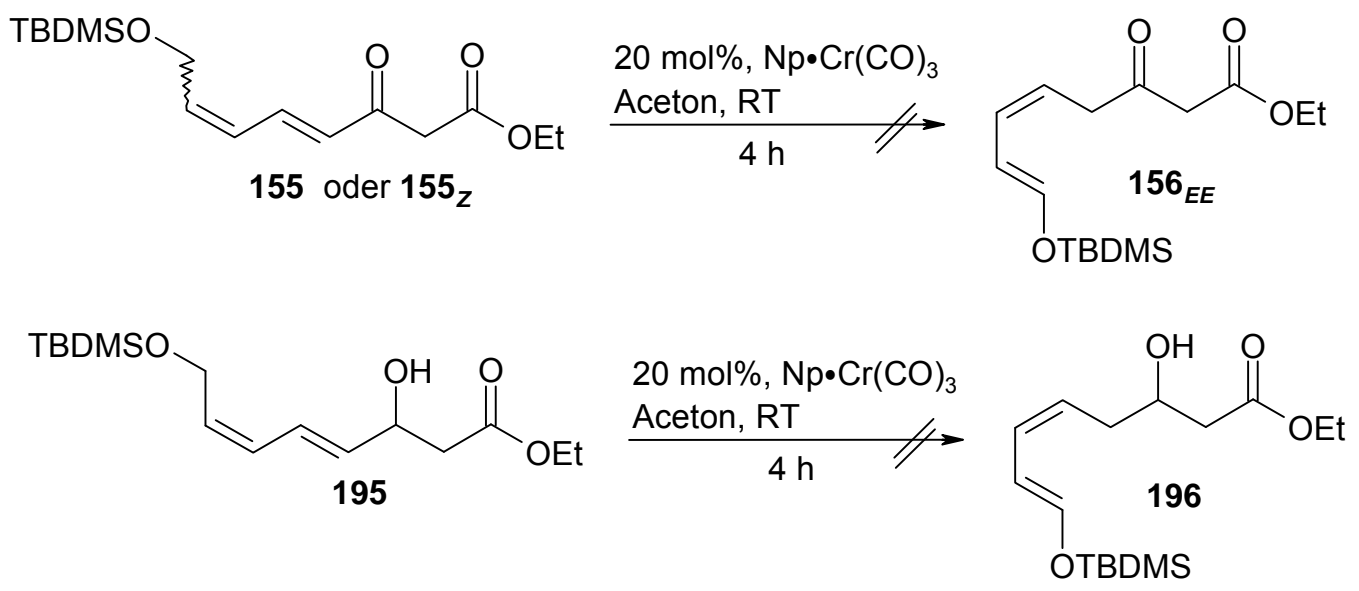

\section{II.5.3.1 Versuche zur Isomerisierung der $5 E / 7 Z$ Diensäureester zu den $5 E / 7 E$ - Verbindungen}

Da die direkte Herstellung der 5E/7E-konfigurierten Octadiensäureester aus deren konjugierten Vorläufern nicht gelungen war, sollte nun überprüft werden, ob eine Isomerisierung der 5E/7Z-konfigurierten Verbindungen möglich wäre. Die Isomerisierung Z-konfigurierter Doppelbindungen kann, unter besonders schonenden Bedingungen, durch 
Licht oder z.B. Iod $^{[80]}$ induziert werden. Die Ergebnisse der Isomerisierungsversuche mit 156 und 174 sind in Tabelle 22 zusammengefasst. Ein zentrales Problem bei den Versuchen mit diesen Octadiensäureestern war die vorher schon mehrfach beobachtete Instabilität der Verbindungen. So konnte bei keinem der Versuche der gewünschte $5 E / 7 E$ konfigurierte Diensäureester isoliert werden. Für die Reaktionen wurden die Ester in Pentan gelöst und in einer Quarzglasapparatur unter Wasserkühlung mit dem Licht einer $250 \mathrm{~W}-$ Quecksilberdampflampe bestrahlt (Einträge 1 und 2). Bei diesen Versuchen wurden schon nach kurzer Reaktionszeit Gemische erhalten, welche keine identifizierbaren Verbindungen mehr enthielten. Deshalb wurde in den folgenden Versuchen eine Lampe mit geringerer Leistung (120 W, Einträge 3 und 4) verwendet und die Versuche in Duranglaskolben durchgeführt. Trotz der somit deutlich geringeren Lichtintensität wurde auch bei diesen Reaktionen entweder nur Edukt oder nach längeren Reaktionszeiten ausschließlich Zersetzung beobachtet. Die Iod-vermittelten Isomerisierungsversuche (Einträge 5 und 6) wurden in Anlehnung an eine von ROUSH und COE beschriebene Vorschrift durchgeführt. ${ }^{[80]}$ Die Octadiensäureester 151 bzw. 174 wurden in Pentan gelöst, bei RT mit katalytischen Mengen Iod versetzt und anschließend bei RT gerührt. Nach wässriger Aufarbeitung erhielt man als Rückstand Gemische, welche sich ${ }^{1} \mathrm{H}-\mathrm{NMR}$ spektroskopisch nicht untersuchen ließen, da sie nicht zu shimmen waren.

Tabelle 22: Isomerisierungsversuche mit den dekonjugierten $\beta$-Hydroxyestern 174 und 156.

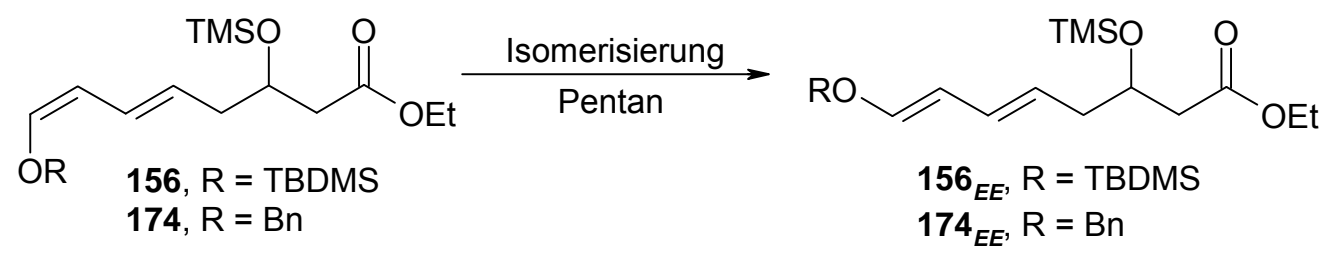

\begin{tabular}{ccccccc}
\hline Nr. & Edukt & T & t & \multicolumn{2}{c}{ Isomerisierung } & Bemerkungen \\
\hline 1 & $\mathbf{1 5 6}$ & Rückfluss & $15 \mathrm{~min}$ & $\mathrm{~h} v$ & $250 \mathrm{~W}^{\mathrm{a}}$ & Zersetzung \\
2 & $\mathbf{1 7 4}$ & Rückfluss & $60 \mathrm{~min}$ & $\mathrm{~h} v$ & $250 \mathrm{~W}^{\mathrm{a}}$ & Zersetzung \\
3 & $\mathbf{1 5 6}$ & Rückfluss & $1 \rightarrow 12 \mathrm{~h}$ & $\mathrm{~h} v$ & $120 \mathrm{~W}^{\mathrm{b}}$ & Zersetzung \\
4 & $\mathbf{1 7 4}$ & Rückfluss & $1 \rightarrow 24 \mathrm{~h}$ & $\mathrm{~h} v$ & $120 \mathrm{~W}^{\mathrm{b}}$ & Zersetzung \\
5 & $\mathbf{1 5 6}$ & RT & $75 \mathrm{~min}$ & $\mathrm{I}_{2}$ & - & n.a. ${ }^{\mathrm{c}}$ \\
6 & $\mathbf{1 7 4}$ & RT & $75 \mathrm{~min}$ & $\mathrm{I}_{2}$ & - & n.a. \\
\hline
\end{tabular}

a) In einer wassergekühlten Quarzglasapparatur; b) Rückfluss in Duranglaskolben; c) NMR nicht auswertbar.

Da die Kontrolle per Dünnschichtchromatographie keine isolierbaren Spots zeigte, wurden die Rückstände verworfen. In der Literatur sind Beispiele für die Isomerisierung verschie- 
dener Enolether mit Quecksilber-(II)-acetat ${ }^{[144]}$ und Natriummethanolat ${ }^{[145]}$ beschrieben. Da bei diesen Versuchen jedoch bestenfalls Gemische aus den $E$ - und Z-konfigurierten Enolethern gewonnen wurden und ein Erfolg aufgrund der Instabilität der Octadiensäureester äußerst fraglich erschien, wurden keine weiteren Isomerisierungsversuche durchgeführt. Trotz intensiver Bemühungen konnten somit die 5E/7Ekonfigurierten Octadiensäureester nicht hergestellt werden. Um dennoch Aussagen über Durchführbarkeit, Stereoselektivität und Substituenteneinflüsse auf die intramolekulare Diels-Alder-Reaktion zu erhalten, wurden die IMDA-Versuche mit den 5E/7Zkonfigurierten Octadiensäureestern weitergeführt.

\section{II.5.4 Versuche zur intramolekularen Diels-Alder-Reaktion der Trienone mit Enoletherfunktion}

Die Ergebnisse der Versuche mit Trienon 165 sind in Tabelle 23 zusammengefasst. In keinem der Versuche konnte das gewünschte Diels-Alder-Produkt isoliert werden. Bei der rein thermischen Umsetzung ${ }^{[82]}$ unter erhöhten Temperaturen wurde nur Zersetzung beobachtet (Eintrag 1), bei niedrigeren Temperaturen konnte, auch nach längerer Reaktionszeit, ausschließlich Edukt nachgewiesen werden (Eintrag 2). Ähnlich unbefriedigende Ergebnisse lieferten die Lewis-Säurekatalysierten Umsetzungen (Einträge $3-6),{ }^{[83]}$ bei denen schon bei niedrigen Temperaturen ausschließlich die Zersetzung des Eduktes beobachtet werden konnte. Die säurekatalysierte Umsetzung von 165 in Benzol (Eintrag 7) lieferte nach nur $1.5 \mathrm{~h}$ bei RT ein komplexes Reaktionsgemisch, welches kein Edukt mehr enthielt. Eine genauere Untersuchung des Gemisches lieferte Hinweise darauf, dass es unter Säurekatalyse zu einer teilweisen Spaltung des TMS-Ethers und anschließender Dehydratisierung gekommen war, wodurch ein Tetraen mit drei konjugierten Doppelbindungen gebildet wurde. Für das Scheitern der IMDA-Versuche mit 161 wurden folgende mögliche Ursachen identifiziert: 1) Sterische Hinderung durch die Z-konfigurierte Doppelbindung im Dien; 2) Instabilität des TBDMS-Enolethers; 3) Instabilität des TMSEthers. Um den Einfluss der einzelnen Komponenten auf das Ergebnis der Diels-AlderReaktion zu untersuchen, wurden die Trienone 176-178 synthetisiert (siehe oben). Die Ergebnisse der Diels-Alder-Reaktionen mit diesen Verbindungen sind in Tabelle 24 zusamengefasst. 
Tabelle 23: IMDA-Versuche mit 165.

\begin{tabular}{cccccccc} 
& & & & & & & \\
\hline
\end{tabular}

a) Nach Chromatographie; b) AV = Aufarbeitungsvariante, siehe Experimenteller II.

Auch für die Trienonderivate 176-178 sowie 180 und 181 konnte in den Reaktionsgemischen kein IMDA-Produkt nachgewiesen werden. Die Reaktionskontrolle, welche per Dünnschichtchromato-graphie und/oder ${ }^{1} \mathrm{H}-\mathrm{NMR}$-Spektroskopie durchgeführt wurde, zeigte entweder Edukt oder nach längerer Reaktionszeit vollständige Zersetzung an. Interessanterweise konnte bei der Umsetzung der Trienone 180 sowie 181 (Einträge 6 bis 8), welche am Dienophil keinen TMS-Alkin-Substituenten tragen, ebenfalls kein DielsAlder-Produkt isoliert werden. Daraus lässt sich ableiten, dass die 7Z-Konfiguration der Edukte zumindest nicht alleinige Ursache für das Scheitern der IMDA-Versuche ist. Dies wird durch die Ergebnisse der Umsetzung des Trienons 176 bekräftigt; da dieses als 1.5:1 Gemisch des 5E/7Z- und des 5E/7E-Isomers eingesetzt wurde, hätte zumindest das 5E/7EIsomer zum Diels-Alder-Produkt reagieren müssen. Die Ergebnisse der Diels-AlderReaktion zeigten, dass die bisher verwendeten Trienone für die Diels-Alder-Umsetzungen deutlich zu instabil waren. 
Tabelle 24: IMDA-Versuche mit den verschiedenen Trienonderivaten.

\begin{tabular}{|c|c|c|c|}
\hline $\mathbf{R}$ & $\mathbf{R}^{1}$ & $\mathbf{R}^{2}$ & \\
\hline $\mathrm{Me}$ & OTMS & $\equiv-\mathrm{TMS}$ & (176) \\
\hline $\mathrm{Bn}$ & OTMS & $\equiv$ TMS & (177) \\
\hline TBDMS & OTMS & $\mathrm{H}$ & (180) \\
\hline TBDMS & $\mathrm{OH}$ & $\mathrm{H}$ & (181) \\
\hline
\end{tabular}

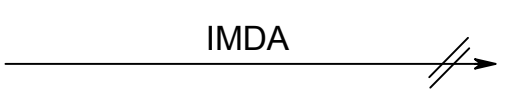<smiles>[R]C1CC(=O)[C@]2(C)C([R])C([R20])C=C[C@H]2C1</smiles>

\begin{tabular}{ccccccccc}
\hline Nr. & Edukt & $\mathbf{T}\left[{ }^{\circ} \mathbf{C}\right]$ & $\mathbf{t}[\mathbf{h}]$ & $\mathbf{L M}$ & Add. & $\mathbf{e q}$ & $\mathbf{A V}^{\mathbf{c}}$ & Beobachtung \\
\hline 1 & $\mathbf{1 7 6}^{\mathrm{a}}$ & 150 & 12 & $\mathrm{Tol}$ & - & & $\mathrm{A}$ & Zersetzung \\
2 & $\mathbf{1 7 6}^{\mathrm{a}}$ & 150 & 12 & $\mathrm{Tol}$ & - & & $\mathrm{A}$ & Zersetzung \\
3 & $\mathbf{1 7 7}$ & 150 & 12 & $\mathrm{Tol}$ & - & & $\mathrm{A}$ & Zersetzung \\
4 & $\mathbf{1 7 7}$ & -78 & 2.5 & $\mathrm{THF}$ & $\mathrm{TiCl}_{4}$ & 2.0 & $\mathrm{C}$ & Zersetzung \\
5 & $\mathbf{1 8 0}$ & 150 & 3.0 & $\mathrm{Tol}$ & - & & $\mathrm{A}$ & Zersetzung \\
6 & $\mathbf{1 8 0}$ & $\mathrm{RT} \rightarrow 40$ & 20 & $\mathrm{CH}_{2} \mathrm{Cl}_{2}$ & $\mathrm{~K}_{2} \mathrm{CO}_{3}$ & 0.5 & $\mathrm{~B}$ & Edukt \\
7 & $\mathbf{1 8 0}$ & -78 & 1.0 & $\mathrm{CH}_{2} \mathrm{Cl}_{2}$ & $\mathrm{SnCl}_{2}$ & 1.5 & $\mathrm{C}$ & Zersetzung \\
8 & $\mathbf{1 8 1}$ & 110 & 2.5 & $\mathrm{Tol}^{\mathrm{n}}$ & - & & $\mathrm{A}$ & Zersetzung \\
\hline
\end{tabular}

a) Isomerengemisch 5E/7Z:5E/7E 1.5:1; b) Nach Chromatographie; c) AV = Aufarbeitungsvariante.

Schema 57: Geplante Synthese des Schlüsselintermediats 200 bzw. 201 aus den Trienonen 178 bzw. 197.

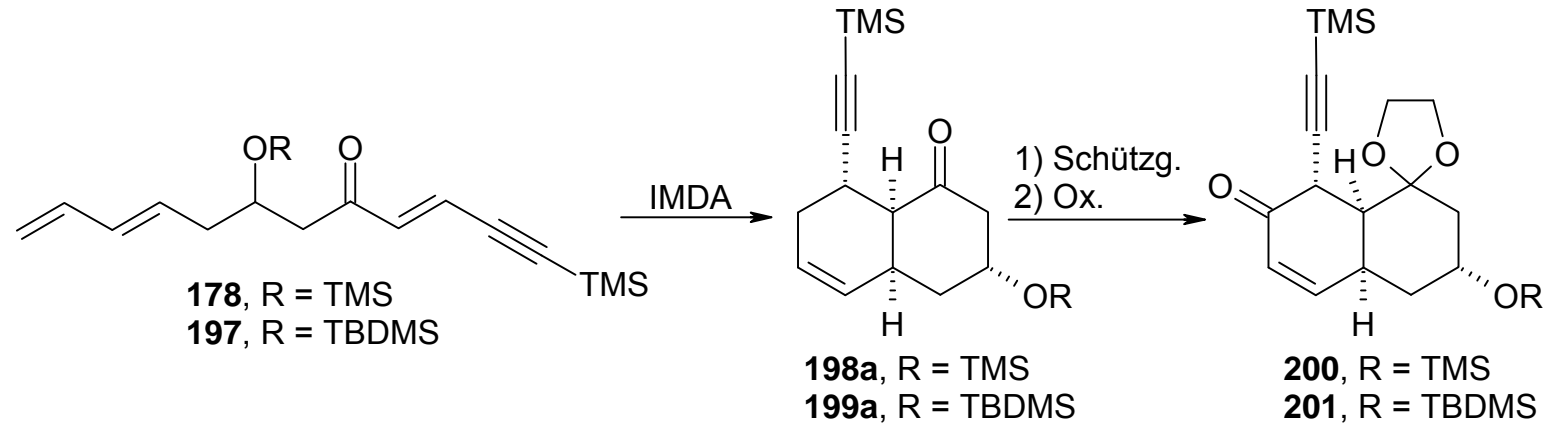

Da wie oben beschrieben außerdem alle Versuche die 5E/7E-konfigurierten Trienone herzustellen, erfolglos verlaufen waren (siehe oben), wurde die Synthese der Trienone 178 und 197 angestrebt. Aus der IMDA-Reaktion dieser beiden Verbindungen sollten zum Einen Erkenntnisse über die generelle Durchführbarkeit der IMDA-Reaktion, die Stereoselektivität und den Einfluss der Hydroxylschutzgruppe auf die Stabilität der Verbindung gewonnen werden. Zum Anderen sollte nach gelungener IMDA-Reaktion versucht werden, ob aus den 
Diels-Alder-Produkten 198 bzw. 199 durch Schützung des Ketons und anschließende allylische Oxidation die Schlüsselintermediate 200 bzw. 201 erhalten werden können.

\section{II.5.5 Synthese und IMDA-Reaktionen der Trienone mit unsubstituiertem Dien}

\section{II.5.5.1 Zusammenfassung der Synthesen der Trienone mit unsubstituiertem Dien}

Die Synthese der IMDA-Vorläufer 178 und 197 verlief analog zu der für die übrigen Trienone bereits beschriebenen Syntheseroute und ist in Schema 58 zusammengefasst. Als Ausgangspunkte der Synthese dienten der bereits mehrfach verwendete Phosphonoester 154 sowie kommerziell erhältlicher Crotonaldehyd. Nach der HWE-Reaktion unter den bereits beschriebenen Reaktionsbedingungen und anschließender chromatographischer Reinigung erhielt man den konjugierten Octadiensäureester 172 in einer guten Ausbeute von 76\%. Nach Dekonjugierung mit LDA erhielt man 184 in 71\% Ausbeute und anschließende Reduktion mit Natriumborhydrid ergab Alkohol 202 mit einer Ausbeute von 87\%. Auffällig ist, dass auch nach chromatographischer Reinigung die Ausbeuten sowohl der Dekonjugierung als auch der anschließenden Reduktion teilweise deutlich über den Ausbeuten der Enoletherderivate (siehe oben) lagen und vor allem zuverlässiger reproduziert werden konnten. Dies lässt auf eine deutlich höhere Stabilität von 202 schließen, was sich auch in den weiteren Versuchen bestätigen sollte. Die Hydroxylfunktion in 202 wurde zunächst, wie bei den anderen Trienonen auch, als TMS-Ether geschützt und man erhielt so den Silylether 175 in $89 \%$ Ausbeute. Bei den weiteren Umsetzungen zum Weinrebamid 204 und Octatrienon 178 konnten jedoch nur mäßige Ausbeuten von 46\% bzw. 58\% erzielt werden. Da die Vermutung nahe lag, dass ein Grund für die nicht zufriedenstellenden Ausbeuten die Labilität der TMS-Schutzgruppe war, wurde der stabilere tert-Butyldimethylsilylether 197 hergestellt. Die höhere Stabilität TBDMS-geschützter $\beta$-Hydroxyester beobachteten auch HEATHCOCK und Mitarbeiter. ${ }^{[146]}$ Die Silylierung mit Imidazol und TBDMSCl erfolgte nach einer Vorschrift von RYCHNOVSKY et al. für die Silylierung von $\beta$-Hydroxyestern. ${ }^{[147]}$ Man erhielt so das gewünschte Produkt 203 in 83\% Ausbeute. Bei den anschließenden Umsetzungen des TBDMS-Ethers 203 konnten die Ausbeuten, wie erwartet, deutlich auf 87\% für 205 bzw. $78 \%$ für 197 gesteigert werden. Bereits bei der ersten säulenchromatographischen Reinigung der Trienone 178 und 197 wurden in den isolierten Produktfraktionen größere Mengen Diels-Alder-Produkt nachgewiesen. Die unkontrollierte Zyklisierung während der Chromatographie konnte effektiv durch Zusatz von 5\% 
Triethylamin zum Laufmittel und eine entsprechend zügig durchgeführte Flashchromatographie unterdrückt werden. Beide Trienone wurden als Lösungen in Pentan (c $\sim 100 \mathrm{mg} / \mathrm{mL}$ ) gelagert, um Zersetzung sowie unkontrollierte intramolekulare DielsAlder-Reaktionen zu verhindern. Unter diesen Lagerungsbedingungen war das TMS-Trienon 178 etwa zwei bis drei Wochen, das TBDMS-Trienon 197 mehr als drei Monate verwendbar.

Schema 58: Zusammenfassung der Synthesen der IMDA-Vorläufer 178 und 197.
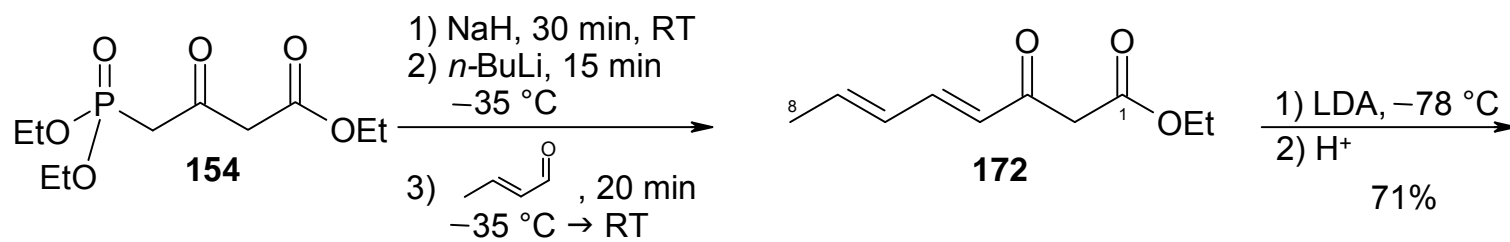

$76 \%$

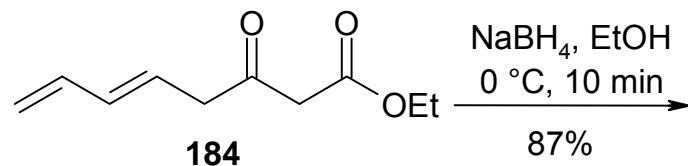<smiles>C=CC=CCC(O)CC(=O)O</smiles>

A) TMSCl, $\mathrm{NEt}_{3}, \mathrm{CH}_{2} \mathrm{Cl}_{2}$

$$
0^{\circ} \mathrm{C}, 1.5 \mathrm{~h}
$$

B) Imidazol, TBDMSCl, $60 \mathrm{~h}$ 202 $\mathrm{DMF} / \mathrm{Toluol}, 0^{\circ} \mathrm{C} \rightarrow \mathrm{RT}$

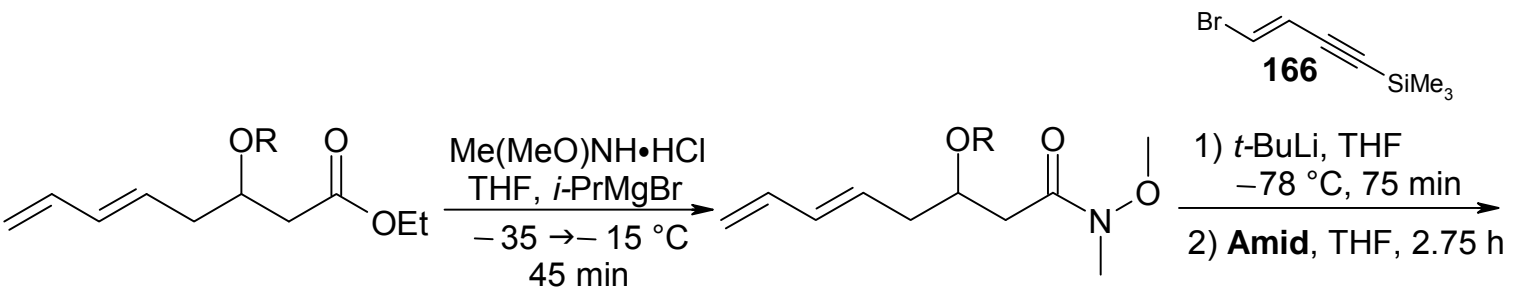

A) $175, R=T M S(89 \%)$

B) $203, R=$ TBDMS (83\% von 172)

204, $R=$ TMS $(46 \%)$

205, $\mathrm{R}=$ TBDMS $(87 \%)$<smiles>[R]C(CC=CC=C)CC(=O)C=CC#CC#N</smiles>

178, $\mathrm{R}=\mathrm{TMS}(58 \%)$

197, $R=$ TBDMS $(84 \%)$

\section{II.5.5.2 Auswertung der IMDA-Reaktionen}

Wie oben schon erwähnt, reagierten die Trienone 178 und 197 schon während der Chromatographie teilweise zu den entsprechenden Diels-Alder-Produkten. Damit konnte mit dem TMS-geschützten Trienon 178 erstmals überhaupt die angestrebte Diels-AlderReaktion beobachtet werden. Das aus der Säulenchromatographie gewonnene 
Produktgemisch, welches etwa 75\% TMS-Trienon 178 und 25\% Diels-Alder-Produkte enthielt wurde in einem ersten Versuch einer thermischen Diels-Alder-Reaktion unterzogen. Dazu wurde das TMS-geschützte Trienon in Toluol gelöst und in einem verschlossenen Druckgefäß aus Glas auf $135^{\circ} \mathrm{C}$ erhitzt (Tabelle 25, Eintrag 1). Die Reaktionskontrolle konnte leicht mittels Dünnschichtchromatographie durchgeführt werden, da die Diels-Alder-Produkte im Gegensatz zu den Trienonen praktisch nicht UVlöschend sind. Nach zwei Stunden war der Umsatz vollständig. Die gelbliche Reaktionslösung wurde in einen Kolben überführt und das Toluol unter vermindertem Druck entfernt. Das NMR-Spektrum des Rückstandes zeigte neben den Diels-AlderProdukten größere Mengen von Zersetzungsprodukten, was sich auch in einer relativ niedrigen isolierten Ausbeute von nur 24\% widerspiegelte. Auch nach Wiederholung des Versuchs mit reinem TMS-Trienon 178 konnten die Ausbeuten nicht gesteigert werden. Als wesentlicher Grund für die unbefriedigenden Ausbeuten wurde die bereits beschriebene mangelnde Stabilität des TMS-Ethers vermutet. Deshalb wurde das TBDMSgeschützte Trienon 197 hergestellt und den gleichen Reaktionsbedingungen unterzogen wie das TMS-geschützte 178. Schon beim ersten Versuch (Eintrag 2) konnte eine kombinierte Ausbeute der Diels-Alder-Produkte von 60\% erzielt werden, was die Vermutungen bezüglich der Labilität des TMS-Ethers nochmals bestätigte. Mit einer etwas kürzeren Reaktionszeit konnte die isolierte Ausbeute auf 69\% gesteigert werden (Eintrag 3). Die Zusammensetzung der Reaktionsgemische wurde per GC-MS überprüft, wodurch vier Signale mit der für die Decalone 199a-199d erwarteten Masse von $m / z=376.69$ identifiziert werden konnten. Durch sorgfältige Säulenchromatographie an Kieselgel ließen sich die beiden Hauptisomere 199a und 199c als Reinsubstanzen isolieren. Die Minderkomponenten 199b und 199d konnten trotz wiederholter Chromatographie nur als Gemische mehrerer Isomere isoliert werden, weshalb eine Bestimmung der Konfiguration für diese Verbindungen nicht möglich war. Die Diastereoselektivität intramolekularer Diels-Alder-Reaktionen kann durch chelatisierende und nichtchelatisierenden LewisSäuren entscheidend beeinflusst werden. ${ }^{[83]} \mathrm{Um} \mathrm{zu}$ untersuchen, ob die Selektivität bezüglich des gewünschten Isomers 199a noch gesteigert werden könnte, wurde der Einfluss verschiedener Lewissäuren auf die IMDA-Reaktion mit 197 untersucht (Tabelle 25, Einträge 4 bis 12). Bei Verwendung schwacher Lewis-Säuren wie Zinkbromid (Eintrag 4), konnte selbst bei Raumtemperatur kaum Umsatz beobachtet werden. Währendessen ergaben starke Lewis-Säuren wie Zinntetrachlorid, Titantetrachlorid oder $\mathrm{BF}_{3}$-Etherat selbst bei $-78{ }^{\circ} \mathrm{C}$ in der Regel nach 30 min vollständigen Umsatz. 
Tabelle 25: Zusammenfassung der IMDA-Reaktionen mit den Trienonen 178 und 197.

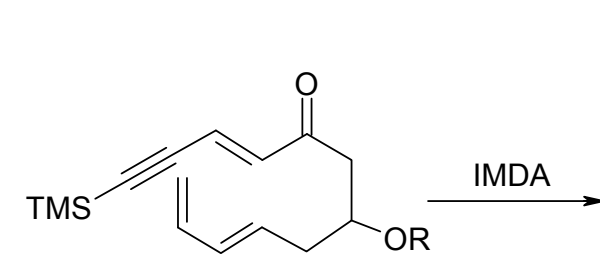

178, $R=$ TMS 197, $\mathrm{R}=\mathrm{TBDMS}$

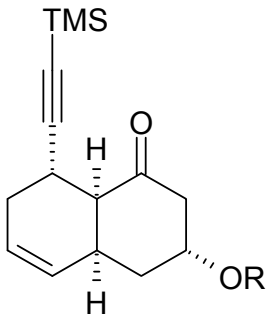

198a, $R=$ TMS 199a, $R=$ TBDMS

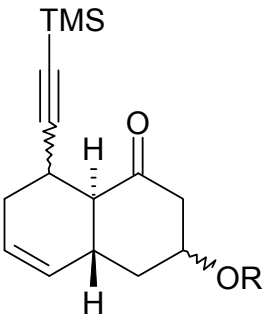

198b, $R=T M S$ 199b, $R=T B D M S$
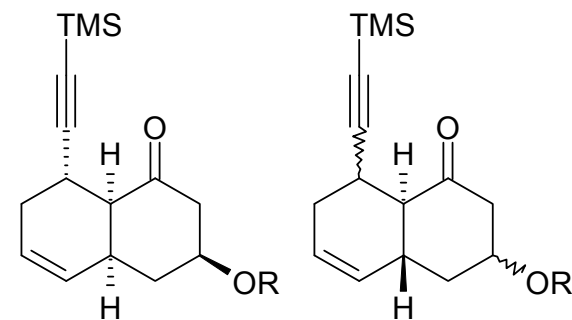

198c, $R=T M S \quad 198 d, R=T M S$

199c, $R=$ TBDMS 199d, $R=$ TBDMS

\begin{tabular}{|c|c|c|c|c|c|c|c|c|c|c|}
\hline \multirow[b]{2}{*}{ Nr. } & \multirow[b]{2}{*}{$\mathbf{E}$} & \multirow[b]{2}{*}{ AAV } & \multirow[b]{2}{*}{$\mathbf{T}\left[{ }^{\circ} \mathbf{C}\right]$} & \multirow[b]{2}{*}{$\mathbf{t}[\mathbf{h}]$} & \multirow[b]{2}{*}{ Lm } & \multicolumn{2}{|c|}{ Add. } & \multirow[b]{2}{*}{$\mathbf{A V} \mathbf{V}^{*}$} & \multicolumn{2}{|c|}{ 199a:199b:199c:199d } \\
\hline & & & & & & LA & eq & & GC-MS & Isol. Ausbeute \\
\hline 1 & 178 & 11 & 135 & 2.0 & Tol & - & - & $\mathrm{A}$ & n.b. & $20: 0: 4: 0$ \\
\hline 2 & 197 & 11 & 135 & 2.0 & Tol & - & - & A & $62: 12: 22: 5$ & $33: 13^{\mathrm{a}}: 14: 0$ \\
\hline 3 & 197 & 11 & 145 & 1.0 & Tol & - & - & A & $62: 12: 21: 5$ & $47: 6^{\mathrm{a}}: 16: 0$ \\
\hline 4 & 197 & 12 & $-78 \rightarrow \mathrm{RT}$ & 70 & $\mathrm{DCM}$ & $\mathrm{ZnBr}_{2}$ & 1.3 & B & n.b. & vorwiegend Edukt \\
\hline 5 & 197 & 12 & -78 & 1.0 & DCM & $\mathrm{SnCl}_{4}$ & 2.0 & $\mathrm{~B}$ & $36: 0: 55: 9$ & $30: 0: 48: 0$ \\
\hline 6 & 197 & 12 & -78 & 0.5 & $\mathrm{DCM}$ & $\mathrm{TiCl}_{4}$ & 0.5 & $\mathrm{C}$ & $85: 1: 12: 2$ & $73: 0: 8^{\mathrm{b}}: 0$ \\
\hline 7 & 197 & 12 & -78 & 0.5 & DCM & $\mathrm{TiCl}_{4}$ & 1.0 & $\mathrm{C}$ & $76: 0: 23: 1$ & $67: 0: 6^{\mathrm{b}}: 0$ \\
\hline 8 & 197 & 12 & -78 & 0.5 & DCM & $\mathrm{TiCl}_{4}$ & 1.5 & $\mathrm{C}$ & $73: 0: 27: 0$ & $66: 0: 8^{\mathrm{b}}: 0$ \\
\hline 9 & 197 & 12 & -78 & 0.5 & $\mathrm{DCM}$ & $\mathrm{BF}_{3} \cdot \mathrm{Et}_{2} \mathrm{O}$ & 1.3 & $\mathrm{D}$ & $72: 4: 21: 3$ & $61^{\mathrm{c}}: 0: 9^{\mathrm{d}}: 0$ \\
\hline 10 & 197 & 12 & $-78 \rightarrow-30$ & 16 & $\mathrm{DCM} / \mathrm{THF}^{\mathrm{e}}$ & $\mathrm{ZrCl}_{2}$ & 1.3 & $\mathrm{D}$ & $77: 1: 19: 3$ & $58: 0: 12^{\mathrm{d}}: 0$ \\
\hline 11 & 197 & 12 & $-78 \rightarrow \mathrm{RT}$ & 13 & $\mathrm{DCM} / \mathrm{THF}^{\mathrm{e}}$ & $\mathrm{Sn}(\mathrm{OTf})_{2}$ & 1.3 & $\mathrm{C}$ & $61: 1: 33: 4$ & $31: 0: 14: 0$ \\
\hline 12 & 197 & 12 & $-78 \rightarrow \mathrm{RT}$ & 13 & $\mathrm{DCM} / \mathrm{THF}^{\mathrm{e}}$ & $\mathrm{Yb}(\mathrm{OTf})_{3}$ & 1.3 & $\mathrm{D}$ & $62: 3: 29: 6$ & 54:0:17:0 \\
\hline
\end{tabular}


Bei allen Lewis-Säurekatalysierten Umsetzungen zeigte sich eine noch deutlichere Bevorzugung der cis-verknüpften Produkte 199a und 199c als in den rein thermischen Umsetzungen. Dieses Ergebnis deckt sich mit Beobachtungen von REIßIG und Mitarbeitern für die Lewis-Säurekatalysierte Umsetzung ähnlicher Decatrienone. ${ }^{[83]}$ Die beste Ausbeute von $73 \%$ des gewünschten Isomers 199a konnte unter Verwendung von 0.5 eq $\mathrm{TiCl}_{4}$ erzielt werden (Eintrag 6) und nur bei der Verwendung von 2.0 eq $\mathrm{SnCl}_{4}$ als Lewis-Säure wurde eine deutliche Abweichung in der Stereoselektivität der Diels-Alder-Reaktion beobachtet (Eintrag 5). Alle Lewis-Säurekatalysierten Diels-Alder-Reaktionen zeigten die erwartete Bevorzugung der endo-Übergangszustände. ${ }^{[83]}$ Die Tatsache, dass chelatisierende LewisSäuren wie Titantetrachlorid annähernd die gleiche Diastereoselektivität zeigen wie das nichtchelatisierende $\mathrm{BF}_{3}$-Etherat, legt die Schlussfolgerung nahe, dass eine Chelatbildung zwischen Carbonylsauerstoff und dem Sauerstoffatom des TBDMS-Ethers aufgrund des sterischen Anspruchs der TBDMS-Gruppe nicht möglich ist.
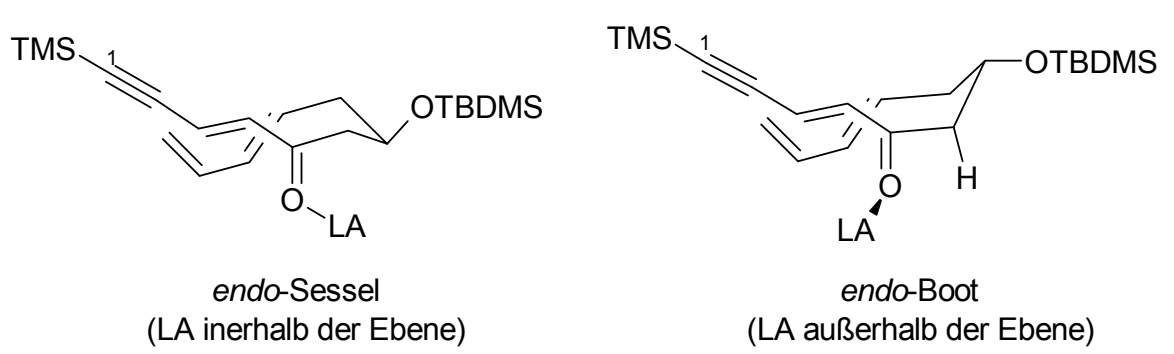

Abbildung 16: Lewis-Säurekatalysierte Bildung der Hauptisomere 199a und 199c.

Dass bei der Verwendung von $\mathrm{SnCl}_{4}$ ein erhöhter Anteil des Stereoisomers 199c gebildet wird bedeutet, dass in Anwesenheit dieser Lewis-Säure mehr Moleküle über den endo-BootÜbergangszustand abreagieren. Um eine mögliche Erklärung für die unterschiedlichen Ergebnisse mit $\mathrm{TiCl}_{4}$ und $\mathrm{SnCl}_{4} \mathrm{zu}$ finden, lohnt es einen Blick auf den Einfluss der LewisSäuren auf die jeweiligen Übergangszustände zu werfen. Es wurde nachgewiesen, dass in Lösung die Koordination von Lewis-Säuren an Carbonylfunktionen bevorzugt coplanar zur Carbonylgruppe erfolgt (Abbildung 1). ${ }^{[148]}$ Beim endo-Boot-ÜZ jedoch sollte durch sterische Wechselwirkungen mit den Protonen der zur Carbonylfunktion $\alpha$-ständigen Methylengruppe die Koordination der Lewissäure außerhalb der Ebene erfolgen. Diese „out of plane“Koordination wurde zwar auch schon für $\mathrm{TiCl}_{4}$ beschrieben. ${ }^{[149]}$ Diese für das Titan gefundenen Ergebnisse sollten jedoch für die deutlich größere und weichere Lewis-Säure $\mathrm{SnCl}_{4}$ eine noch größere Bedeutung haben, womit sich die beobachtete Bevorzugung des endo-Boot-Übergangszustands bei Verwendung von $\mathrm{SnCl}_{4}$ erklären ließe. CoRCORAN et al. postulierten für die $\mathrm{TiCl}_{4}$-vermittelte Diels-Alder-Reaktion verschiedener Enone mit 
Cyclopentadien, dass die Reaktivität höher ist, wenn das Titan außerhalb der Ebene an den Carbonylsauerstoff koordiniert, weil dadurch eventuell eine direkte Wechselwirkung mit den $\pi$-Elektronen des Enons ermöglicht wird. ${ }^{[150]}$ Inwiefern dieser Effekt einen Einfluss auf die beobachteten Diastereoselektivitäten haben könnte, muss in weiteren Versuchen geklärt werden.

\section{II.5.5.3 Aufklärung der relativen Konfiguration der Hauptprodukte der IMDA- Reaktion}

Die Konstitution der IMDA-Isomere 199a und 199c wurde zweifelsfrei mittels ${ }^{1} \mathrm{H}-\mathrm{NMR}$, ${ }^{13} \mathrm{C}-\mathrm{NMR}$, COSY- sowie HSQC-Spektroskopie nachgewiesen. Da alle Versuche, brauchbare Einkristalle für Röntgenstrukturuntersuchungen $\mathrm{zu}$ gewinnen, scheiterten, wurde die relative Konfiguration der racemischen Isomere 199a und 199c mittels eindimensionaler NOE-Spektroskopie untersucht. Bei dieser Variante der NOESYSpektroskopie wird mit der Frequenz eines einzelnen Protons eingestrahlt und die „Antwort“ räumlich benachbarter Protonen aufgenommen (Abbildung 17).

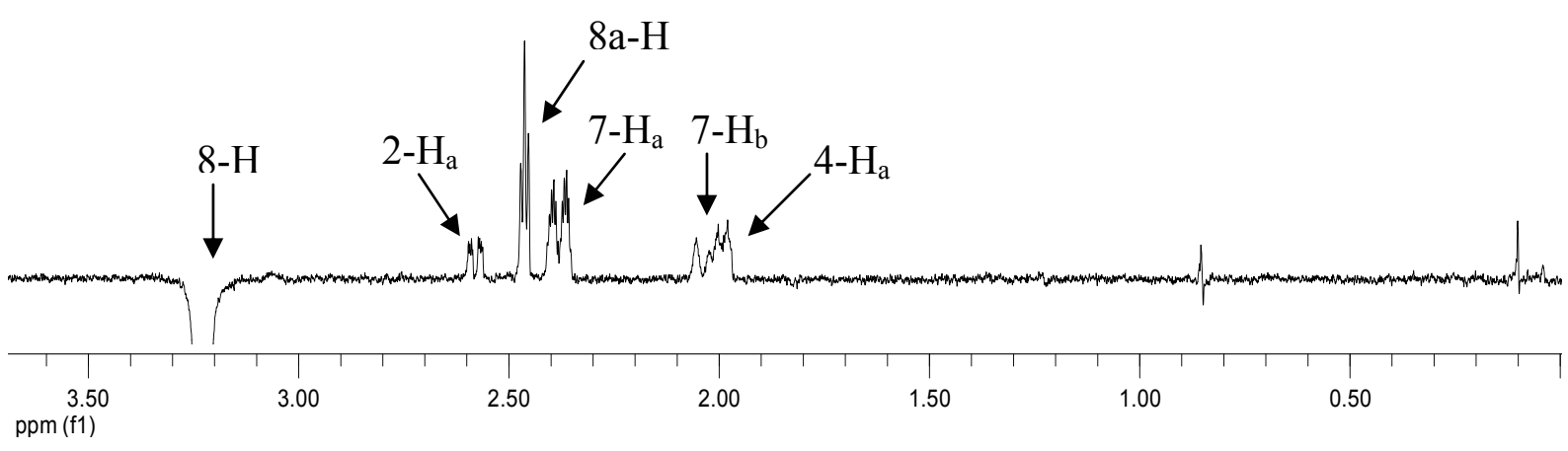

Abbildung 17: Beispiel für ein eindimensionales NOE-Spektrum von 199a; eingestrahlt wurde mit der Frequenz des Protons an C-8.

Die Ergebnisse der NOE-Experimente für die Isomere 199a und 199c sind in Tabelle 26 bzw. Tabelle 27 zusammengefasst. In der ersten Spalte sind die Protonen gezeigt, mit deren Frequenz eingestrahlt wurde; die Anzahl der Kreuze gibt qualitativ die Intensität der beobachteten Wechselwirkung wieder. Da racemische Edukte eingesetzt wurden, erhielt man natürlich auch die IMDA-Produkte als Racemate. Zur besseren Übersichtlichkeit wird im Folgenden aber immer nur ein Enantiomer diskutiert. Aus der Auswertung der NOESpektren ergab sich für 199a die $\left(3 R^{*}, 4 \mathrm{a} S^{*}, 8 S^{*}, 8 \mathrm{a} S^{*}\right)$ - und für 199c die $\left(3 S^{*}, 4 \mathrm{a} S^{*}, 8 S^{*}, 8 \mathrm{a} S^{*}\right)$ Konfiguration, wie sie neben der jeweiligen Tabelle gezeigt ist. In Abbildung 18 sind die 
Strukturen dreidimensional dargestellt. Für die Strukturaufklärung von 199a (Tabelle 26) waren insbesondere die Nachbarschaftsbeziehungen von 8-H und 4a-H entscheidend.

Tabelle 26: Korrelationen für 199a aus 1D-NOESY-Spektren.

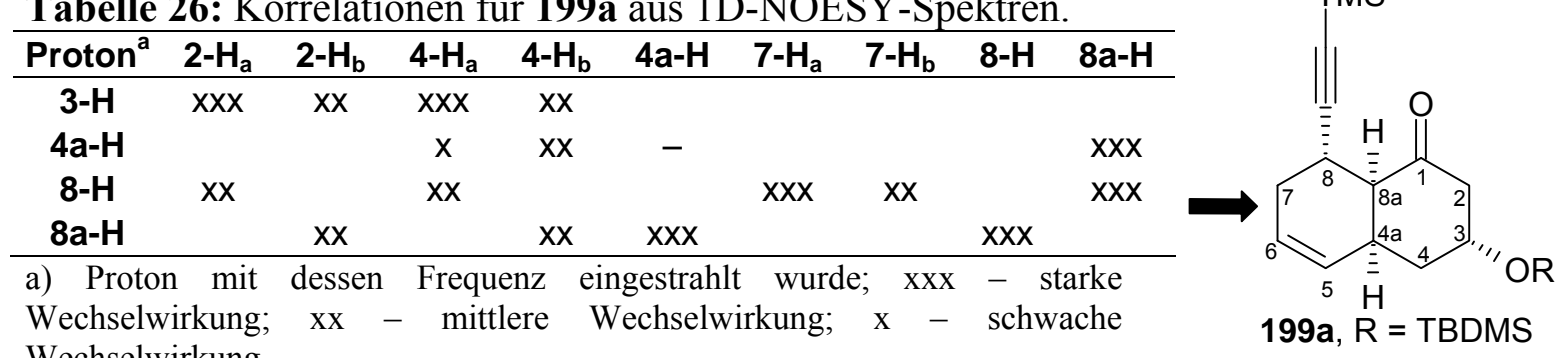

Tabelle 27: Korrelationen für 199c aus 1D-NOESY-Spektren.

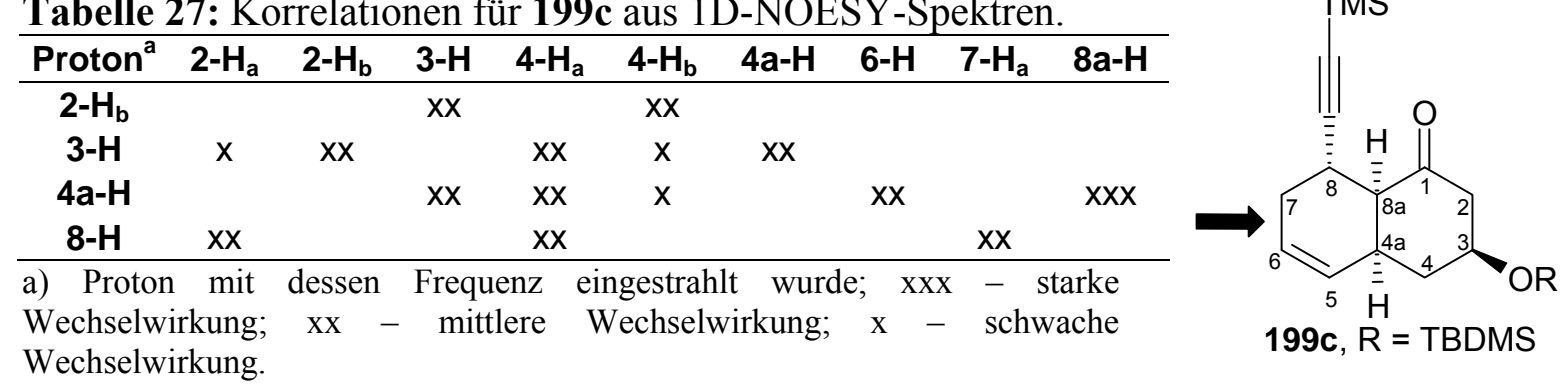

Das Proton an C-8a erscheint im ${ }^{1} \mathrm{H}-\mathrm{NMR}$ als leicht verbreitertes Triplett mit einer Kopplungskonstante von $5.5 \mathrm{~Hz}$, was bereits auf eine cis-Konfiguration des Decalons schließen ließ. Diese Vermutung wurde durch die starke NOE-Wechselwirkung zwischen 8a-H und 4a-H bestätigt. Die Annahme, dass sowohl die OTBDMS-Gruppe als auch 4a-H unterhalb der Molekülebene liegen, wird durch zwei Beobachtungen bekräftigt: 1) 4a-H zeigt kein Kreuzsignal mit dem Proton an C-3, was auf eine trans-Stellung der beiden Protonen schließen lässt; 2) $4 \mathrm{a}-\mathrm{H}$ in $\mathbf{1 9 7 a}$ zeigt im Vergleich $\mathrm{zu} 4 \mathrm{a}-\mathrm{H}$ in 197c eine deutliche Tieffeldverschiebung von ca. $0.3 \mathrm{ppm}$, dies lässt sich durch eine 1,3-diaxiale Stellung zum Sauerstoffatom des TBDMS-Ethers und die daraus resultierenden Wechselwirkungen mit den freien Elektronenpaaren des Sauerstoffs erklären. Ein auf den ersten Blick widersprüchliches Ergebnis ist das NOE-Signal zwischen 8-H und 8a-H, welches eigentlich auf eine cis-Stellung beider Protonen schließen lässt. Da diese Konfiguration jedoch definitiv eine Interaktion von 8-H und Protonen an C-2 oder C-4 ausschließen würde, ist wohl die wahrscheinlichste Erklärung, dass es eine Konformation gibt, in der $8-\mathrm{H}$ und $8 \mathrm{a}-\mathrm{H}$ nahe genug sind, um die beobachteten NOE-Signale hervorzurufen. Ein Vorschlag, wie diese aussehen könnte, ist in Abbildung 18 (rechts) gezeigt. Beide Konformationen sind durch Ringinversion ineinander überführbar. Dabei sollte beachtet werden, dass in der linken Konformation die OTBDMS-Gruppe axial und das TMS-Alkin äquatorial stehen, während in der rechts gezeigten Konformation die 
OTBDMS-Gruppe pseudoäquatorial und das TMS-Alkin pseudoaxial stehen. Es ist also wahrscheinlich, dass es sich hier um zwei Grenzkonformationen handelt, welche im Verhältnis zur NMR-Zeitskala schnell ineinander übergehen und somit das NOE-Signal zwischen 8-H und 8a-H erklärbar machen.
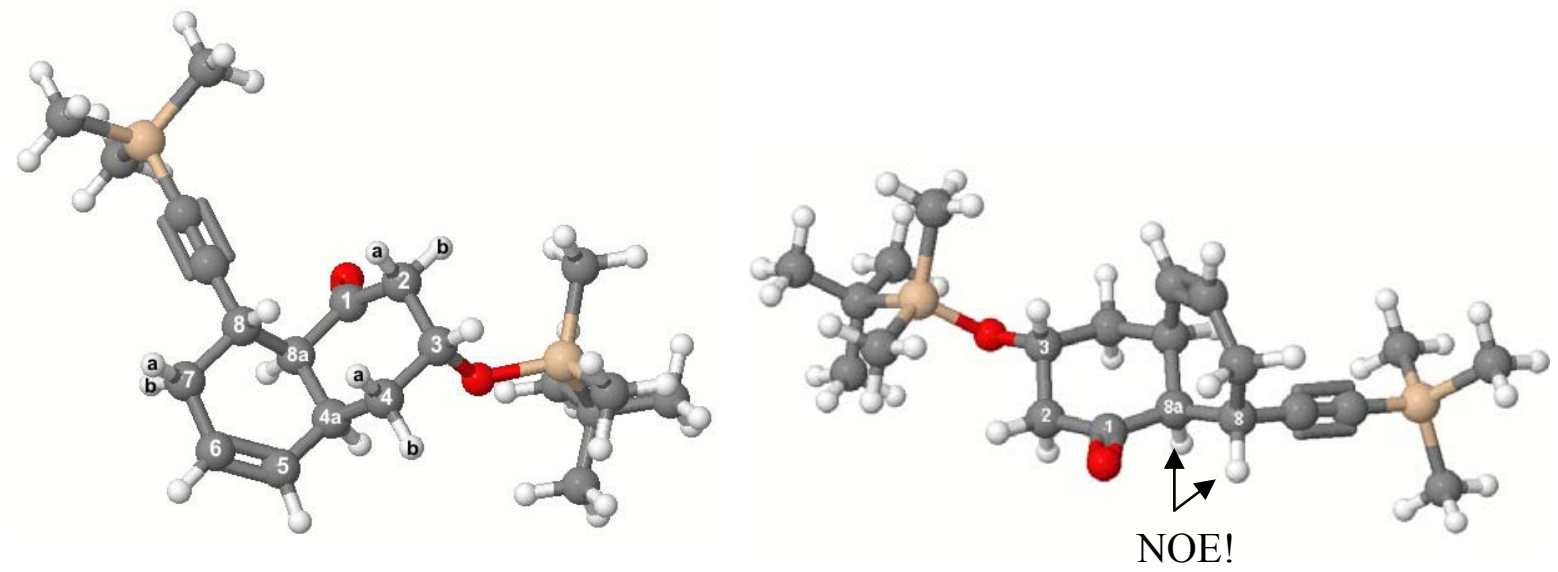

Abbildung 18: Links: Dreidimensionale Darstellung der Konformation von 199a. - Rechts: Eine der möglichen Konformationen in der eine NOE-Wechselwirkung zwischen 8-H und $8 \mathrm{a}-\mathrm{H} \mathrm{zu}$ sehen wäre (Molekülansicht aus Gründen der Übersichtlichkeit von der Rückseite). ${ }^{[\mathrm{i}]}$

Die Ergebnisse der Auswertung der NOE-Spektren von 199c deckten sich mit der für 199c erarbeiteten Struktur. So bestätigte ein starkes Kreuzsignal zwischen 4a-H und 8a-H die erwartete cis-Verknüpfung der beiden Ringe des Decalons. Im Unterschied zu 199a zeigte 199c jedoch ein starkes Signal zwischen $3-\mathrm{H}$ und 4a-H, was auf eine 1,3-diaxiale Stellung der beiden Protonen und eine daraus resultierende äquatoriale Stellung der OTBDMSGruppe schließen lässt (Abbildung 19). In dieser Konfiguration nehmen sowohl der TBDMS-Ether als auch das TMS-Alkin eine energetisch günstige äquatoriale Position ein. Ein Konformationswechsel wie für 199a postuliert würde für 199c zu einer energetisch sehr ungünstigen Struktur mit axialer Stellung der beiden großen Substituenten führen. Es ist deshalb nicht überraschend, dass im Unterschied zu 199a in 199c kein Kreuzsignal zwischen 8-H und 8a-H beobachtet werden konnte. Somit lässt sich festhalten, dass die relative Konfiguration der beiden Hauptdiastereomere der Diels-Alder-Reaktion identifiziert werden konnte. Um die Konfiguration der Minderkomponenten aufzuklären, wird eine Reinigung mittels HPLC notwendig sein. Des Weiteren ist fraglich, ob sich die Minderkomponenten mittels NOE-Spektroskopie aufklären lassen, da die ${ }^{1} \mathrm{H}-\mathrm{NMR}$ -

${ }^{[i]}$ Spartan 04 für Windows (Wavefunction Inc., Irvine, CA); Berechnungsmethode: Semiempirisch auf PM3-Level. 
Spektren der Gemische darauf schließen lassen, dass sich die Signale fast aller der für die Strukturaufklärung interessanten Protonen überlagern.

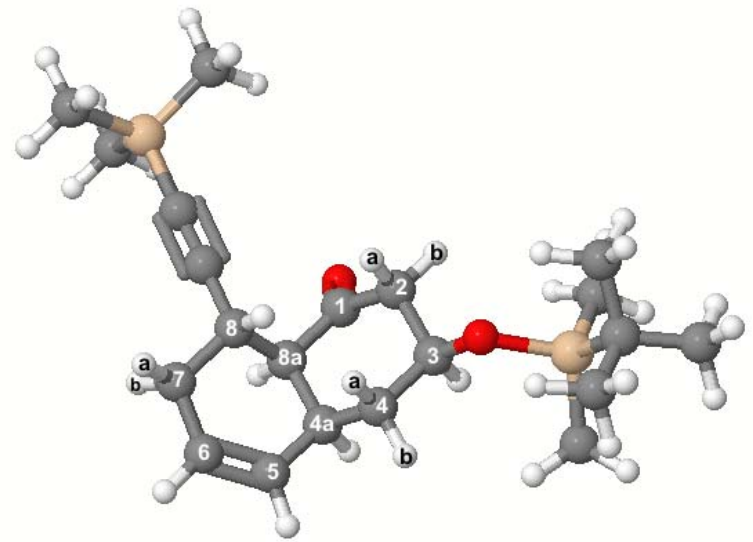

Abbildung 19: Dreidimensionale Darstellung der Konformation von 199c.

\section{II.5.6 Isomerisierungsversuch zur Darstellung des trans-Decalons}

Die freie Standardenthalpie trans-verknüpfter unsubstituierter Decalone liegt bei einer Temperatur von $298 \mathrm{~K}$ um etwa $1.7 \mathrm{~kJ} / \mathrm{mol}$ unterhalb der Enthalpie der cis-verknüpften Struktur. ${ }^{[151]}$ Dies bedeutet, dass unter Gleichgewichtsbedingungen das cis/trans-Verhältnis weit auf der Seite der trans-Decalone liegt. Dieses Verhältnis ist jedoch essentiell vom Substitutionsmuster und der Art der Substituenten am Decalon abhängig. So fanden TATICCI und WENKERT, dass in Decalonen, welche eine zur Carbonylfunktion ,para“ständige tert-Butylgruppe tragen, die cis-Konfiguration energetisch begünstigt ist. ${ }^{[152]}$ Um das Risiko einer ungewollten cis/trans-Isomerisierung des Diels-Alder-Produktes 197a im Verlauf der weiteren Synthese abschätzen zu können, wurde 197a den in Schema 59 gezeigten Isomerisierungsbedingungen unterzogen. Die Reaktion in Anwesenheit von para-Toluolsulfonsäure in Methanol und bei Raumtemperatur führte zu keinen detektierbaren Mengen trans-Produkt $\left({ }^{1} \mathrm{H}-\mathrm{NMR}\right)$. Bei der Isomerisierung mit LDA in THF konnte bei Temperaturen von bis zu $0{ }^{\circ} \mathrm{C}$ kein Umsatz festgestellt werden. Nach 10 Stunden Rühren bei Raumtemperatur waren auf dem Dünnschichtchromatogramm zwei neue deutlich polarere Spots zu erkennen, diese stammten jedoch sehr wahrscheinlich von Zersetzungsprodukten, welche durch TMS-Entschützung und/oder Eliminierung von TBDMSOH entstanden waren. 
Schema 59: Isomerisierungsversuche mit 199a zur Darstellung des trans-Decalons.

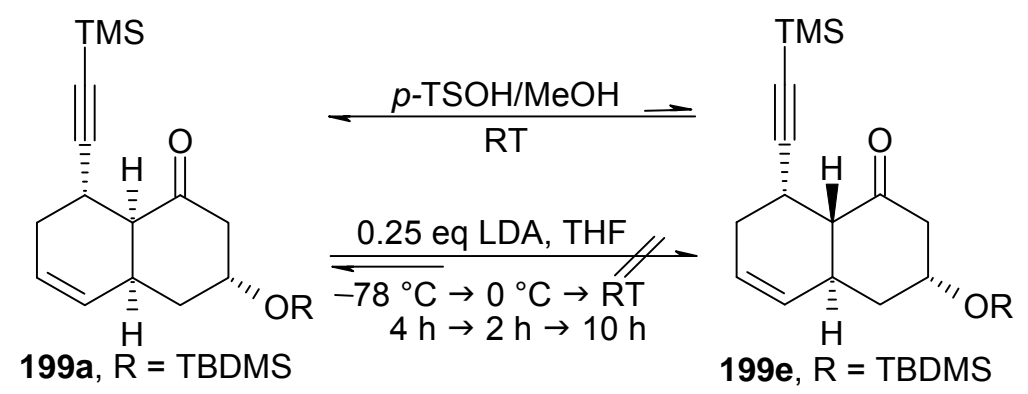

Es ist auffällig, dass bei keinem der Versuche mit 199a (nicht nur Isomerisierung, sondern auch Acetalbildung, TMS-Entschützung, allylische Oxidation) das entsprechende trans-Isomer beobachtet werden konnte. Dies lässt vermuten, dass die cisVerknüpfung der Decalone 199a und 199c gegenüber der trans-Verknüpfung energetisch begünstigt ist. Eine ähnliche Beobachtung machten GRAS und BERTRAND beim Versuch der Isomerisierung des Decalons cis-206 (Schema 60). ${ }^{[153]}$ Unter den abgebildeten Bedingungen war keine Isomerisierung zum trans-206 zu beobachten, was die Autoren mit der energetisch begünstigten äquatorialen Stellung der Isopropylgruppe in cis-205 begründeten.

Schema 60: Das cis-Decalon 206 isomerisiert nicht in die trans-Konfiguration.

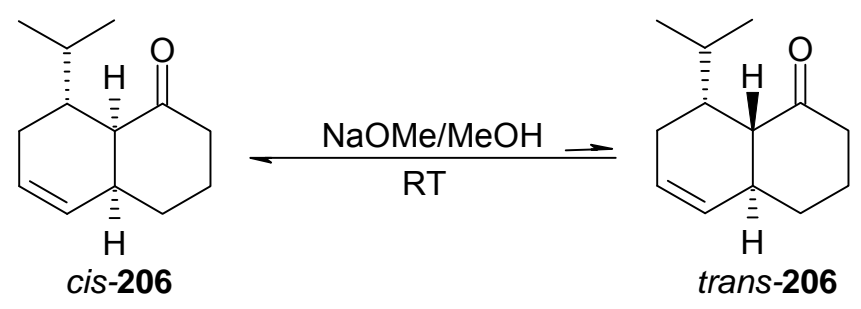

\section{II.5.7 Versuche zur Einführung des $\alpha, \beta$-ungesättigten Ketons an C-6}

\section{II.5.7.1 Entschützung des TMS-Alkins}

Als nächster Schritt wurde die Entschützung des TMS-Alkins in den Decalonen 199a und 199c durchgeführt (Schema 61). In Anlehnung an eine Arbeitsvorschrift von TROST et al. wurde zunächst Decalon 199a in Methanol gelöst und bei $0{ }^{\circ} \mathrm{C}$ mit Kaliumcarbonat versetzt. ${ }^{[154]}$ Nach einer Stunde Rühren bei $0{ }^{\circ} \mathrm{C}$ zeigte die DC-Kontrolle einen Umsatz von ca. $40 \%$, weshalb die Reaktion weitere sieben Stunden bei $0{ }^{\circ} \mathrm{C}$ gerührt wurde. Da die erneute DC-Kontrolle eine ganze Reihe von Zersetzungsprodukten zeigte, wurde die Reaktion abgebrochen und das Reaktionsgemisch verworfen. Die Entschützung konnte 
erfolgreich nach einer von ENGEL publizierten Synthesevorschrift durchgeführt werden. ${ }^{[155]}$ Dazu wurden die Decalone 199a bzw. 199c in einem Ether/Ethanol-Gemisch mit 1.1 eq $\mathrm{AgNO}_{3}$ versetzt und das resultierende Reaktionsgemisch bei RT gerührt. Nach Zusatz eines Überschusses an $\mathrm{KCN}$, anschließender wässriger Aufarbeitung und Chromatographie konnten die entschützten Derivate 207a bzw. 207c problemlos und in guten Ausbeuten von 86\% bzw. 79\% isoliert werden.

Schema 61: Entschützung des Alkins in den Diels-Alder-Produkten.

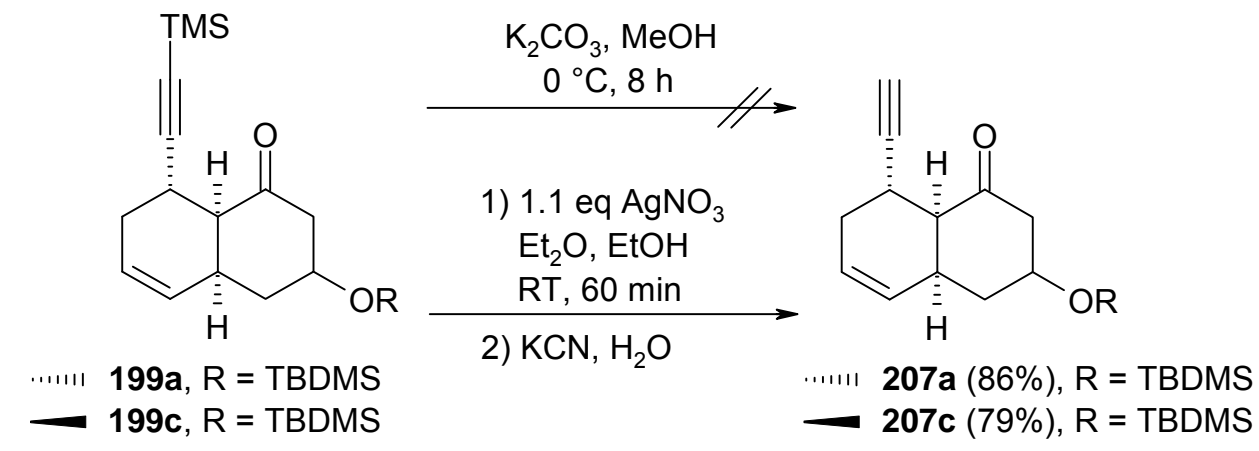

\section{II.5.7.2 Versuche zur Dioxolanbildung}

Das Keton im Diels-Alder-Produkt 199a sollte als Dioxolan geschützt werden. Die Ergebnisse dieser Versuchsreihe sind in Tabelle 28 zusammengefasst. Unter besonders schonenden Bedingungen können Ketone durch die Trimethylsilylmethansulfonat (TMSOTf) vermittelte Reaktion mit 1,2-Bistrimethylsilyloxyethan geschützt werden. ${ }^{[156,157]}$ Die Anwendung dieser Reaktionsbedingungen in der Umsetzung von 199a (Einträge 1 und 2) lieferte jedoch nicht das gewünschte Zielprodukt. Nach Chromatographie konnte bei beiden Reaktionen nur ein Teil des eingesetzten Decalons 199a zurückgewonnen werden. Da bei der ersten Reaktion, welche man nach Zugabe des TMSOTf auf Raumtemperatur hatte erwärmen lassen, signifikante Zersetzung beobachtet worden war, sollte in einem weiteren Versuch geklärt werden, ob sich diese durch eine niedrigere Reaktionstemperatur und eine entsprechend verlängerte Reaktionszeit unterdrücken ließe (Eintrag 2). Bei diesem Versuch konnte neben dem Edukt nur das Eliminierungsprodukt 209 in einer Ausbeute von 20\% isoliert werden. Analog der Umsetzung eine Decalons nach WoLfF et al ${ }^{[158]}$ ließ man ein Gemisch von Ethylenglykol, katalytischen Mengen para-Toluolsulfonsäure ( $p-\mathrm{TsOH})$ und 197a in Rückflussierendem Chloroform am Wasserabscheider sieden. Bei diesem Versuch wurde 209 sogar mit einer auf den Umsatz basierten Ausbeute von 88\% isoliert (Eintrag 3). Um die säurekatalysierte 
Eliminierung der OTBDMS-Gruppe zu verhindern, wurde der folgende Versuch mit weniger aciden Säuren durchgeführt (Einträge 4 und 5). Aus diesen Versuchen konnte nur das unveränderte Edukt zurückgewonnen werden. Die Lewis-Säurekatalysierte Umsetzung mit $\mathrm{BF}_{3}$-Etherat nach HAGIWARA et al. ${ }^{[159]}$ lieferte bei $0{ }^{\circ} \mathrm{C}$ keinen Umsatz des Edukts und bei Raumtemperatur nur zunehmende Zersetzung (Eintrag 6).

Tabelle 28: Zusammenfassung der Versuche zur Acetalisierung der Ketofunktion in 199a und 207a.

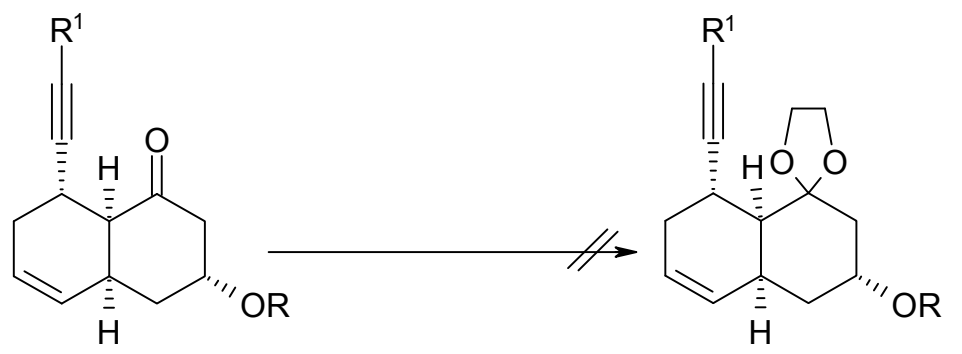

199a, $R^{1}=$ TMS, $R=$ TBDMS

207a, $R^{1}=H, R=$ TBDMS
208, $R^{1}=$ TMS, $R=$ TBDMS

209, $R^{1}=H, R=$ TBDMS<smiles>CS(C)(=O)=CC1CC=C[C@@H]2CC=CC(=O)[C@H]12</smiles>

210

\begin{tabular}{|c|c|c|c|c|c|c|c|c|c|}
\hline \multirow{2}{*}{$\mathbf{N r}$} & \multirow{2}{*}{ Ed. } & \multirow{2}{*}{$\begin{array}{c}\mathbf{n} \\
{[\mu \mathrm{mol}]}\end{array}$} & \multirow{2}{*}{$\mathbf{T}\left[{ }^{\circ} \mathbf{C}\right]$} & \multirow{2}{*}{$\mathbf{t}[\mathbf{h}]$} & \multirow{2}{*}{ Lm } & \multicolumn{2}{|c|}{ Glykol $^{\mathrm{a}}$} & \multirow{2}{*}{ Add. } & \multirow{2}{*}{ Beobachtung } \\
\hline & & & & & & & eq & & \\
\hline 1 & $197 \mathbf{a}$ & 210 & $-30 \rightarrow \mathrm{RT}$ & 3 & $\mathrm{CH}_{2} \mathrm{Cl}_{2}$ & $\mathrm{~A}$ & 3 & TMSOTf & $\begin{array}{l}\text { Edukt }(25 \%)^{\mathrm{b}} \\
+ \text { Zersetzung }\end{array}$ \\
\hline 2 & $197 \mathbf{a}$ & 218 & $-30 \rightarrow 0$ & 24 & $\mathrm{CH}_{2} \mathrm{Cl}_{2}$ & A & 3 & TMSOTf & $\begin{array}{l}\text { Edukt }(18 \%)^{b} \\
+\mathbf{2 1 0}(20 \%)^{b}\end{array}$ \\
\hline 3 & $197 \mathbf{a}$ & 151 & Rückfluss & 16 & $\mathrm{CHCl}_{3}$ & $\mathrm{~B}$ & 5 & $p \mathrm{TsOH}$ & $\begin{array}{l}\text { Edukt }(15 \%)^{\mathrm{b}} \\
+\mathbf{2 1 0}(75 \%)^{\mathrm{b}}\end{array}$ \\
\hline 4 & $197 \mathbf{a}$ & 125 & Rückfluss & 10 & $\mathrm{Ph}-\mathrm{H}$ & $\mathrm{B}$ & 6 & EtOOH & Edukt \\
\hline 5 & $197 \mathbf{a}$ & 63.7 & $\begin{array}{c}\mathrm{RT} \rightarrow \\
\text { Rückfluss }\end{array}$ & 1.5 & $\mathrm{CH}_{2} \mathrm{Cl}_{2}$ & $\mathrm{~B}$ & 5 & $\begin{array}{l}\text { PPTs/ } \\
\text { Alox }\end{array}$ & Edukt \\
\hline 6 & $197 \mathbf{a}$ & 162 & $0 \rightarrow \mathrm{RT}$ & 18 & $\mathrm{CH}_{2} \mathrm{Cl}_{2}$ & A & $\mathrm{Lm}$ & $\mathrm{BF}_{3} \cdot \mathrm{Et}_{2} \mathrm{O}$ & $\begin{array}{c}\text { Edukt } \\
+ \text { Zersetzung }\end{array}$ \\
\hline 7 & $206 a$ & 125 & $0 \rightarrow \mathrm{RT}$ & 18 & $\mathrm{CH}_{2} \mathrm{Cl}_{2}$ & $\mathrm{~A}$ & $\mathrm{Lm}$ & $\mathrm{BF}_{3} \cdot \mathrm{Et}_{2} \mathrm{O}$ & $\begin{array}{c}\text { Edukt } \\
+ \text { Zersetzung }\end{array}$ \\
\hline 8 & $206 a$ & 78.8 & $\begin{array}{l}-30 \rightarrow \\
-20\end{array}$ & 18 & $\mathrm{CH}_{2} \mathrm{Cl}_{2}$ & $\mathrm{~A}$ & 3 & TMSOTf & $\begin{array}{c}\text { Edukt } \\
+ \text { Zersetzung }\end{array}$ \\
\hline
\end{tabular}

a) A = 1,2-Bistrimethylsilanyloxyethan, B = Ethylenglykol; b) Nach Chromatographie.

Da als Grund für das Scheitern der Dioxolanbildung eine sterische Hinderung durch die TMS-Gruppe am Alkin nicht auszuschließen war, wurde das TMS-entschützte 207a den bereits beschriebenen Acetalisierungsbedingungen unterzogen (Einträge 7 und 8). Auch bei diesen Versuchen konnte nur eine mit fortschreitender Reaktionszeit zunehmende 
Zersetzung des Edukts beobachtet werden. Um die Reaktionsbedingungen und insbesondere die verwendeten Reagenzien zu überprüfen, wurde 4-tert-Butylcyclohexanon (210) den Acetalisierungsbedinungen unterzogen (Schema 62). Aus diesem Versuch erhielt man das entsprechend geschützte Produkt 212 in einer isolierten Ausbeute von 83\%. Damit war bewiesen, dass die Umsetzung von 199a und 207a nicht aufgrund mangelhafter Reagenzien erfolglos war. Betrachtet man die dreidimensionalen Darstellungen der IMDAProdukte in Abbildung 18 und Abbildung 19, so ist es wahrscheinlich, dass die Carbonylfunktion sterisch so stark abgeschirmt ist, dass eine Bildung des Dioxolans nicht möglich ist. Eine Möglichkeit, dieses Problem zu umgehen, könnte über eine Reduktion zum Alkohol und anschließende Schützung des entstandenen Alkohols führen. Ein Vorteil dieser Strategie wäre, dass nach der Reduktion der Carbonylfunktion eine unbeabsichtigte Epimerisierung des cis-Decalins zum trans-Decalin praktisch auszuschließen ist.

Schema 62: Testreaktion zur Überprüfung von Reaktionsbedingungen und Reagenzien der Acetalisierung.

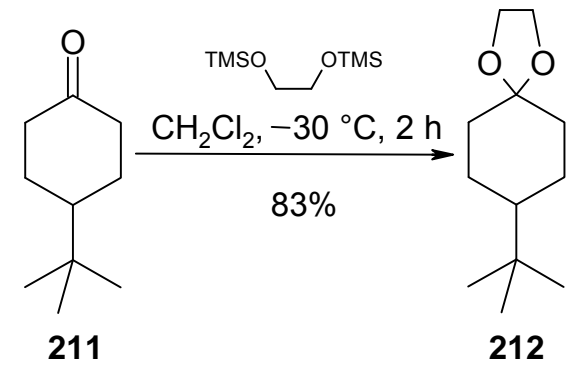

\section{II.5.7.3 Versuche zur allylischen Oxidation}

Zum Schluss der Arbeiten zum Collinolacton wurde untersucht, ob sich die Decalone 199a und 207a durch allylische Oxidation in die $\alpha, \beta$-ungesättigten Diketodecalone 213 bzw. 214 überführen lassen. Die Ergebnisse dieser Versuche sollten zeigen, ob sich das Schlüsselintermediat 199 aus dem Diels-Alder-Produkt 197a herstellen lässt. Eine schonende Methode zur allylischen Oxidation von Cyclohexenen zu Cyclohexenonen ist die durch Palladiumhydroxid katalysierte Oxidation mit tert-Butylhydroperoxid. ${ }^{[160]}$ Die entsprechenden Umsetzungen (Einträge 1-3) ergaben trotz Verwendung verschiedener Chargen $\mathrm{Pd}(\mathrm{OH})_{2}$ und frischen Peroxids (Einträge 2 und 3) nur Edukt bzw. Zersetzung. Aus der durch Chromtrioxid katalysierten Reaktion nach KUMADAKI et al. ${ }^{[161]}$ (Eintrag 4) konnte nach Chromatographie neben 25\% des Edukts erneut das Eliminierungsprodukt 210 
(34\%) isoliert werden. Der Oxidationsversuch mit Selendioxid in Dioxan führte innerhalb von 30 min zur völligen Zersetzung des Edukts.

Tabelle 29: Zusammenfassung der Versuche zur allylischen Oxidation der Decalone 199a und 207a.

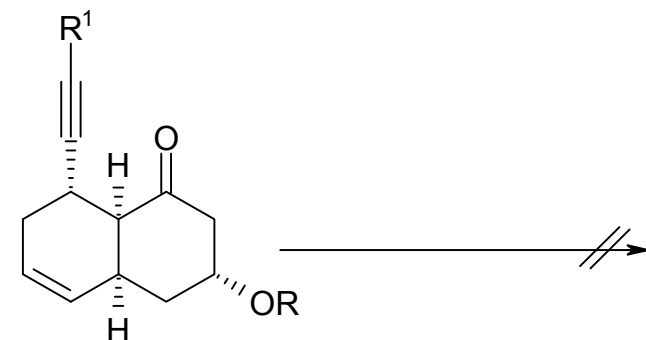

199a, $\mathrm{R}^{1}=\mathrm{TMS}, \mathrm{R}=\mathrm{TBDMS}$

207a, $R^{1}=H, R=T B D M S$

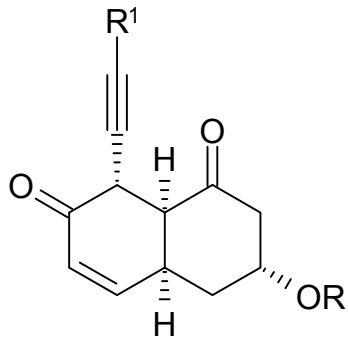

213, $\mathrm{R}^{1}=\mathrm{TMS}, \mathrm{R}=\mathrm{TBDMS}$

214, $R^{1}=H, R=T B D M S$

\begin{tabular}{|c|c|c|c|c|c|c|c|c|c|}
\hline $\mathbf{N r}$ & Ed. & Kat & $\mathrm{mol} \%$ & Oxm & eq & $\begin{array}{c}\mathbf{t} \\
{[\mathbf{h}]}\end{array}$ & $\mathbf{T}$ & $\mathbf{L m}$ & Beob. \\
\hline 1 & $199 a$ & $\mathrm{Pd}(\mathrm{OH})_{2}{ }^{\mathrm{a}}$ & 10 & $t$-BuOOH & 5 & 28 & $\begin{array}{l}\mathrm{RT} \rightarrow \\
40{ }^{\circ} \mathrm{C}\end{array}$ & $\mathrm{CH}_{2} \mathrm{Cl}_{2}$ & Edukt $(30 \%)^{b}$ \\
\hline 2 & $199 a$ & $\mathrm{Pd}(\mathrm{OH})_{2}{ }^{\mathrm{a}}$ & 10 & $t-\mathrm{BuOOH}$ & 5 & 15 & RT & $\mathrm{CH}_{2} \mathrm{Cl}_{2}$ & $\begin{array}{c}\text { Edukt, } \\
\text { Zersetzung }\end{array}$ \\
\hline 3 & $207 a$ & $\mathrm{Pd}(\mathrm{OH})_{2}^{\mathrm{a}}$ & 10 & $t-\mathrm{BuOOH}$ & 5 & 15 & RT & $\mathrm{CH}_{2} \mathrm{Cl}_{2}$ & $\begin{array}{c}\text { Edukt, } \\
\text { Zersetzung }\end{array}$ \\
\hline 4 & $199 a$ & $\mathrm{CrO}_{3}$ & 0.5 & $t$-BuOOH & 10 & 15 & RT & $\mathrm{CH}_{2} \mathrm{Cl}_{2}$ & $\begin{array}{c}\text { Edukt }(25 \%)^{\mathrm{b}} \\
\mathbf{2 1 0}(34 \%)^{\mathrm{b}}\end{array}$ \\
\hline 5 & $199 a$ & - & - & $\mathrm{SeO}_{2}$ & 2 & 0.5 & $180^{\circ} \mathrm{C}$ & Dioxan & Zersetzung \\
\hline
\end{tabular}

a) Zusätzlich Aluminiumoxid; b) nach Chromatographie. 


\section{II.6 Alternative Syntheseroute basierend auf den bisherigen Ergebnissen}

Auf Grundlage der aus den Versuchen zur Totalsynthese des Collinolactons (7) gewonnenen Erkenntnisse wurde der in Schema 63 gezeigte Vorschlag für einen alternativen Zugang zum Decalinintermediat 224 entwickelt, welches als Schlüsselintermediat für die weitere Synthese dienen soll. Bei der Mehrzahl der darin verwendeten Reaktionen handelt es sich um bereits verwendete Synthesen, wodurch die angepasste Route zügig durchführbar sein sollte. Nach DITTMER et al. wird dabei zunächst der $\beta$-Hydroxyester 219 hergestellt. ${ }^{[162]}$ Ausgehend von Essigsäureethylester erhält man durch Kondensation des entsprechenden Enolats mit Acrolein und anschließender Schützung mit TBDMSCl Verbindung 215. Die TBDMS-Schutzgruppe ist hierbei der TMS-Gruppe, wegen der deutlich höheren Stabilität, vorzuziehen. Anschließende Hydroborierung und direkte Oxidation mit PCC ergeben den Aldehyd 216, welcher anschließend in einer Wittig-Reaktion mit dem Ylid 217 zum $\alpha, \beta$ ungesättigten Aldehyd 218 umgesetzt wird. Die Ergebnisse von DITTMER et al. zeigten, dass dabei die Doppelbindung in $\mathbf{2 1 8}$ ausschließlich mit der gewünschten $E$ Konfiguration gebildet wird. Das Diensystem mit der angestrebten E/E-Konfiguration wird schließlich nach QUANG et al. in einer HWE-Reaktion zwischen 218 und dem bekannten Phosphonoester 219 aufgebaut. ${ }^{[163,[164]}$ Die Umsetzung zum Trienon 222 erfolgt auf dem bereits beschriebenen Weg über das Weinreb-Amid 221 und anschließender Addition des entsprechenden Li-Organyls des Bromids 166. Die intramolekulare Diels-Alder-Reaktion unter den oben bereits beschriebenen Bedingungen ergäbe dann das gewünschte Decalon 223. Da die bisherigen Versuche zur Schützung der Ketofunktion im Diels-Alder-Produkt 199a erfolglos waren, wäre es sinnvoll, den Umweg über die Reduktion zum Alkohol und anschließende Schützung als Triphenylsilylether einzuschlagen. Ein Vorteil dieser Strategie wäre es, dass nach der Reduktion der Ketofunktion zum Alkohol eine Isomerisierung zum trans-Decalin praktisch auszuschließen wäre; durch Verwendung der TPS-Schutzgruppe sollte außerdem eine selektive Spaltung der beiden Silylschutzgruppen möglich sein. Abspaltung der TMS-Gruppe mit $\mathrm{AgNO}_{3}$ liefert das Edukt für den Aufbau der nordwestlichen Seitenkette für die anschließende Oxy-Cope-Reaktion. Nach Abschluss dieser Sequenz kann, durch Entschützung des Benzyl-Ethers mit DDQ und Oxidation zum Michael-System, schließlich das Edukt für die Addition des Orthoesters 112 gewonnen werden. Die Modellstudie zur anionischen Oxy-Cope-Reaktion hat deren Durchführbarkeit an einem einfachen Testsystem bereits bewiesen; dennoch muss nun 
getestet werden, wie sich die nun deutlich komplexere Struktur von 225 auf das Ergebnis dieser Reaktionssequenz auswirkt.

Schema 63: Angepasste Synthese für das Diels-Alder-Produkt 223.

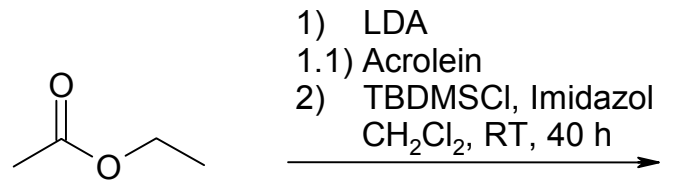

1.1) Acrolein

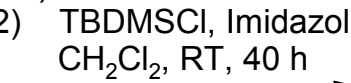<smiles>C=CC(CC(=O)OCC)OCC</smiles>

215
1) $9-\mathrm{BBN}$

2) $\stackrel{\mathrm{PCC}, \mathrm{CH}_{2} \mathrm{Cl}_{2}}{\longrightarrow}$<smiles>CCOP(O)(O)=C[Hg]c1ccccc1</smiles><smiles>CCCCC(CC(=O)[O-])OC(C)(C)C</smiles><smiles>CC(C)C=Cc1ccccc1</smiles><smiles>CCOC(=O)CC(C/C=C/C=O)O[Mg]C(C)(C)C</smiles><smiles>[124IH]</smiles>

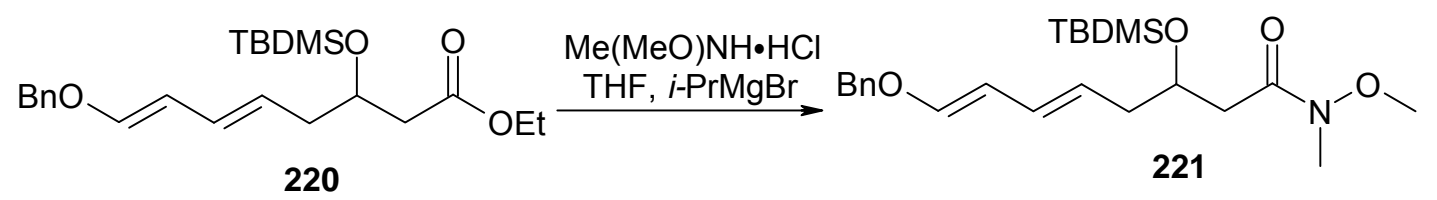<smiles>[Mg][13C]C#C[18Br]</smiles>

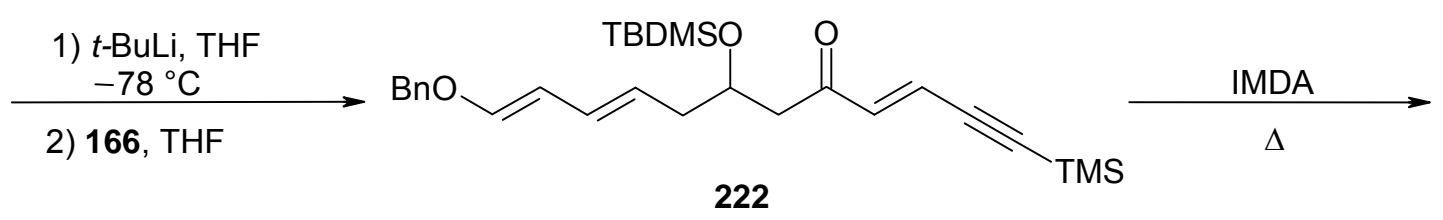

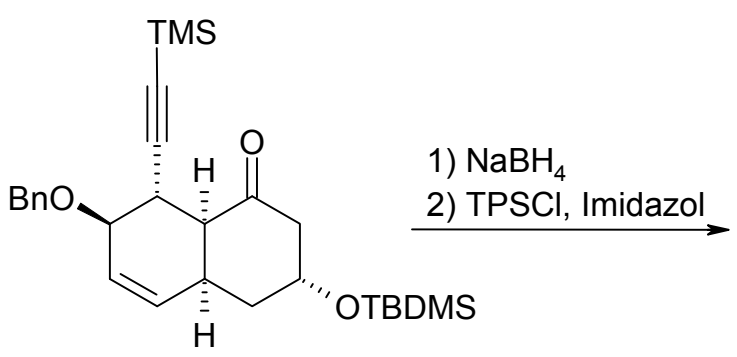

223

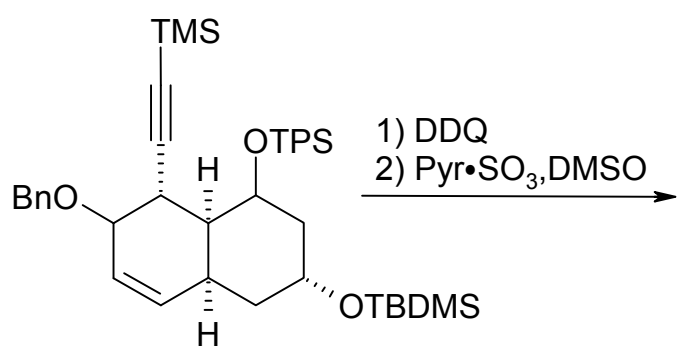

224<smiles>CC(C)(C)C#C[C@H]1C(=O)C=C[C@H]2C[C@H](OC(C)(C)C)CC([OH+])[C@@H]12</smiles>

$\longrightarrow$ Collinolacton (7)

225 


\section{Zusammenfassung}

Im ersten Teil dieser Arbeit konnte erfolgreich ein günstiger und effektiver Zugang zum radioaktiv markierten Diazirinylphotoaffinitätslabel 48a realisiert werden. Durch die Reaktionsführung über das zweifach TMS-geschützte Diaziridinintermediat $\mathbf{6 5}$ ist es außerdem erstmals gelungen, Diaziridine direkt zu lithiieren. Durch die Umsetzung des entsprechenden Li-Organyls mit verschiedenen Elektrophilen konnte bereits die Flexibilität der verwendeten Synthese unter Beweis gestellt werden. Damit wurde ein leichter Zugang zu strukturell diversen Photoaffinitätslabeln geschaffen. Durch die Verwendung von $\mathrm{Ba}^{14} \mathrm{CO}_{3}$, welches eine der günstigsten ${ }^{14} \mathrm{C}$-Quellen überhaupt ist, konnte ausreichend radioaktives Material für die Funktionalisierung mehrerer Naturstoffe gewonnen werden. Dabei waren insbesondere die Stabilität des Photoaffinitätslabels 48 sowie die lange Halbwertzeit des ${ }^{14} \mathrm{C}$ Isotopes von entscheidendem Vorteil. Dadurch konnte noch etwa ein Jahr nach der Synthese des radioaktiven Labels die Markierung des V-ATPase-Inhibitors Apicularen (85) erfolgreich durchgeführt werden. Im Rahmen der vorliegenden Arbeit wurden insgesamt sechs Naturstoffe bzw. -derivate mit dem Photoaffinitätslabel (48) versehen. Bis auf das Apicularen (85), bei dem die entsprechenden Arbeiten noch ausstehen, wurden alle Naturstoffe sowohl radioaktiv als auch „kalt“ funktionalisiert und dem Kooparationspartner Dr. M. Huss für die Wirkortuntersuchungen an den V-ATPasen zur Verfügung gestellt. Bafilomycin und Concanamycin - Während die Markierung des Bafilomycins (2) EDCIvermittelt durchgeführt werden konnte, musste für die Funktionalisierung des Concanolids (4) die Diazirincarbonsäure (48) in das reaktivere Säurechlorid überführt werden, da in Gegenwart von EDCI oder DCC ausschließlich die langsame Zersetzung des Plecomakrolids beobachtetet werden konnte. Die isolierten Ausbeuten der markierten Plecomakrolide lagen zwischen 32 und 42\%, wobei in der Regel zusätzlich etwa ein Drittel des verwendeten Edukts zurückgewonnen werden konnte. In den biologischen Untersuchungen zeigten die Diaziridin-haltigen Derivate eine kaum schwächere Hemmwirkung als die jeweiligen nicht markierten Makrolide. Nach Auswertung der Ergebnisse ergab sich ein sehr genaues Bild, wo die Plecomakrolide $\mathbf{1}$ und $\mathbf{2}$ an die V-ATPasen koordinieren. So zeigte sich, dass die östliche Hälfte der Moleküle um C-21 bzw. C-23 in die Lücke zwischen den Untereinheiten a,c und $\mathrm{F}$ des $\mathrm{V}_{0}$-Komplexes ragt, während die Westhälfte der Moleküle um C-7 bzw. C-9 tief in der c-Untereinheit des $\mathrm{V}_{0}$-Komplexes liegt.

Kendomycine - Da sich der Naturstoff Kendomycin (78) sowohl in der EDCI- bzw. DCCvermittelten Umsetzung mit der Diazirincarbonsäure 48 als auch mit dem Säurechlorid 76 
als instabil erwies, wurden das bekannte Acetonid $\mathbf{8 1}$ und das neue Methylacetal $\mathbf{8 3}$ synthetisiert. Während sich $\mathbf{8 1}$ nur unter Verwendung des aktiveren Säurechlorids $\mathbf{7 6}$ funktionalisieren ließ, konnte das Methylacetal 83 DCC-vermittelt direkt mit der Diazirincarbonsäure 48 umgesetzt werden. Die isolierten Ausbeuten aus diesen Reaktionen lagen bei 48 bzw. 53\%. Die Umsetzung zum radioaktiv markierten Acetonid 82a wurde analog der Vorschrift für die „kalte“ Reaktion durchgeführt und ergab das Zielprodukt mit einer etwas geringeren Ausbeute von 37\%. Die Untersuchung der Hemmwirkung der Kendomycinderivate auf verschiedene ATPasen zeigte, dass die Kendomycine neben der VATPase auch noch die P-ATPase zu hemmen vermögen, wohingegen F-ATPasen in ihrer Wirkung nicht beeinflusst werden. Des Weiteren zeigten die Versuche, dass die Kendomycine anders als die Plecomacrolide 1 und 2 sehr wahrscheinlich nicht im $\mathrm{V}_{0^{-}}$ sondern im $\mathrm{V}_{1}$-Komplex der V-ATPasen wirken. Eine genauere Bestimmung des Wirkortes ist Gegenstand fortlaufender Arbeiten.

Archazolid (70) und Apicularen A (85) - Beide Naturstoffe konnten erfolgreich mit dem Photoaffinätslabel 48 funktionalisiert werden. Die Synthese des ${ }^{14} \mathrm{C}$-markierten Diazirinylapicularens sowie Untersuchungen an den V-ATPasen sind Gegenstand aktueller Arbeiten.

Im zweiten Teil dieser Arbeit wurden Beiträge zur Totalsynthese des Collinolactons (7) erarbeitet. Dabei stand die Synthese der Ausgangssubstanz für die intramolekulare DielsAlder-Reaktion im Mittelpunkt. Die Diels-Alder-Reaktion ist die erste von drei Schlüsselschritten in der Totalsynthese des Collinolactons. Bei den anderen entscheidenden Transformationen handelt es sich um eine anionische Oxy-Cope-Reaktion und die abschließende Baeyer-Villiger-Oxidation des Collinoketons (14) zum Collinolacton (7). Zunächst wurde die Synthese des IMDA-Eduktes ausgehend von Buten-1,4-diol 136 angestrebt. Diese Route, welche über eine Nagao-Aldolreaktion verlaufen sollte, konnte aufgrund der Instabilität des Aldehyds 26 nicht weiter verfolgt werden. Nach diesen Ergebnissen wurde die Synthesestrategie entsprechend angepasst und eine Route ausgehend von Acetessigsäureethylester (153) entwickelt. Durch Bromierung und anschließende Arbuzow-Reaktion erhielt man den Phosphonoester 154, welcher in einer HornerWadsworth-Emmons-Reaktion mit verschiedenen Butenalen umgesetzt wurde. So erhielt man verschiedene konjugierte $\beta$-Ketoester, welche sich durch die Schutzgruppe am Sauerstoffatom des späteren Enolethers unterschieden. Diese Verbindungen wurden anschließend unter kinetischen Bedingungen $\mathrm{zu}$ den entsprechenden Enolethern dekonjugiert. Die Beobachtung, dass die Verbindungen 156 und 183 mit dem TBDMS- 
geschützten bzw. dem Benzylgeschützten Enolether exklusiv als 5E/7Z-Isomere erhalten wurden, ist wohl auf sterische Wechselwirkungen der Schutzgruppen mit dem zur Etherbindung $\alpha$-ständigen Proton zurückzuführen. Die intensiven Bemühungen, eine nachträgliche Isomerisierung zu den 5E/7E-Verbindungen zu erreichen oder entsprechende Dekonjugierungsbedingungen zu identifizieren, verliefen aufgrund der mangelnden Stabilität der betreffenden dekonjugierten Verbindungen erfolglos. Deshalb wurde erfolgreich das Methyletherderivat 178 synthetisiert, welches als 1.5:1 Gemisch der 5E/7Z- und der 5E7Ekonfigurierten Verbindung isoliert werden konnte. Die dekonjugierten $\beta$-Ketoester wurden mit Natriumborhydrid reduziert und als TMS-Ether geschützt. Um gute Ausbeuten zu erhalten, war es dabei notwendig, die Dekonjugierung, Reduktion und TMS-Schützung innerhalb von $12 \mathrm{~h}$ durchzuführen. Trotzdem waren die isolierten Ausbeuten abhängig von der Schutzgruppe am Enolether: Konnte bei dem TBDMS-geschützten Derivat 163 über drei Stufen eine isolierte Ausbeute von 73\% erreicht werden, so waren die Ausbeuten beim Benzylderivat 174 und bei der entsprechenden methylierten Verbindung 173 deutlich geringer. Anschließend wurden die Verbindungen in die jeweiligen Weinreb-Amide überführt und dann mit dem vorher lithiierten Alkenin 166 versetzt. Nach wässriger Aufarbeitung erhielt man so die verschiedenen Trienone 165 und 176-178. Auch bei diesen beiden Reaktionen konnte für das TBDMS-geschützte Derivat 165 mit 49\% die beste Ausbeute erzielt werden. Im Folgenden wurden die synthetisierten Trienone der thermisch oder Lewis-Säure katalysierten intramolekularen Diels-Alder-Reaktion unterzogen. Bei allen Verbindungen konnte bei entsprechend schonender Reaktionsführung (z.B. Rückfluss in $\mathrm{CHCl}_{3}$ ) nur das unveränderte Edukt isoliert werden, unter harscheren Reaktionsbedingungen wurde nur Zersetzung beobachtet. Daraufhin wurde die Synthesestrategie entsprechend angepasst und zunächst der TMS-geschützte $\beta$-Hydroxyester 203 synthetisiert. Dadurch, dass dieser am Dien keinen Substituenten trägt, erwies er sich deutlich stabiler. Anschließend wurde das daraus synthetisierte Trienon 178 den Bedingungen der Diels-Alder-Reaktion unterzogen. Trotz substantieller Zersetzung während der Reaktion oder der anschließenden säulenchromatographischen Reinigung des Decalons 198 konnte erstmals das Produkt der Diels-Alder-Reaktion beobachtet werden. Die mangelnde Ausbeute wurde auf die Labilität des TMS-Ethers zurückgeführt, weshalb in den folgenden Umsetzungen die stabilere TBDMS-Schutzgruppe verwendet wurde. So konnte das Trienon 197 über den bereits beschriebenen Syntheseweg ausgehend von Phosphonoester 154 über sechs Stufen mit einer Gesamtausbeute von 28\% isoliert werden. In der folgenden intramolekularen Diels-AlderReaktion erwies sich das TBDMS-geschützte Trienon 197, im Vergleich zum TMS- 
geschützten Trienon 178, ebenfalls als deutlich stabiler. So konnte die Reaktion problemlos sowohl unter thermischer Reaktionsführung als auch Lewis-Säure katalysiert durchgeführt werden. Erwartungsgemäß wurden die Hauptprodukte über die endo-Übergangszustände gebildet, wobei diese Präferenz in Anwesenheit von Lewis-Säuren noch stärker ausgeprägt war. Das Diastereomer 199a, welches die für die Synthese des Collinolactons benötigte Konfiguration der Stereozentren besitzt, konnte unter Verwendung von Titantetrachlorid als Lewis-Säure in einer Ausbeute von 73\% isoliert werden. An dieser Stelle kann noch nicht abschließend beurteilt werden, was der Grund für das Scheitern der IMDA-Versuche mit den Trienonen 165 und 176-178 war. Als Gründe kommen die Z-Konfiguration der Doppelbindung am Enolether sowie die Instabilität sowohl der TMS-Schutzgruppe als auch der jeweiligen Enolether in Betracht. Mit dem Produkt der Diels-Alder-Reaktion wurden anschließend erste Versuche zur Schützung des Ketons in 199a als Dioxolan durchgeführt. Da das Keton vermutlich sterisch zu stark abgeschirmt ist, konnte keine Bildung des Dioxolans beobachtet werden. Erste Versuche zur allylischen Oxidation des Diels-AlderProduktes zum entsprechenden Diketon führten ebenfalls nicht zum Zielprodukt. Deshalb wurde auf Grundlage der in dieser Arbeit gewonnenen Erkenntnisse eine alternative Synthese konzipiert, welche zu Trienon 222 führt (Schema 63). Da bei dieser Syntheseroute der Enolether im letzten Schritt über eine $E$-selektive HWE-Reaktion aufgebaut wird, sollte so der gezielte Aufbau eines $E / E$-konfigurierten Diens sicher gelingen. Die Problematik der mangelnden Stabilität der Trienone in den Diels-Alder-Reaktionen müsste durch die Verwendung entsprechend stabiler Schutzgruppen in den Griff zu bekommen sein. 


\section{E Experimenteller Teil}

\section{E.1 Material und Methoden}

\section{Spektroskopie}

${ }^{1}$ H-NMR-Spektroskopie: Bruker AM 250 (250 MHz), Varian Unity 300 (300 MHz), Bruker AMX 300 (300 MHz), Varian Inova 500 (500 MHz), Varian AS 600 (600 MHz); Lösungsmittelrestsignal als interne Referenz: $\delta=2.05$ für [ $\left.\mathrm{D}_{5}\right]$-Aceton, 3.31 für $\left[\mathrm{D}_{3}\right]$ Methanol, 7.16 für [D $]$-Benzol, $7.26 \mathrm{ppm}$ für Chloroform. Charakterisierung der Signalaufspaltung: $\mathrm{s}=$ Singulett, $\mathrm{br}=$ breites Singulett, $\mathrm{d}=$ Dublett, $\mathrm{t}=$ Triplett, $\mathrm{q}=$ Quartett, quint $=$ Quintett, $\mathrm{sex}=$ Sextett, $\mathrm{m}=$ Multiplett, $\mathrm{m}_{\mathrm{c}}=$ zentriertes Multiplett, $\mathrm{dd}=$ Dublett vom Dublett, ddd = Dublett vom Dublett vom Dublett, $\mathrm{dt}=$ Dublett vom Triplett, $\mathrm{ddt}=$ Dublett vom Dublett vom Triplett. Die Spektren wurden als Spektren erster Ordnung ausgewertet und die Kopplungskonstanten sind als Betrag angegeben.

${ }^{13}$ C-NMR-Spektroskopie: Bruker AM 250 (62.9 MHz), Varian Unity 300 (75.5 MHz), Bruker AMX $300(75.5 \mathrm{MHz})$, Varian Inova 500 (125.7 MHz), Varian AS 600 (150.8 MHz); Referenzen: $\delta=49.0$ für [D $\left.\mathrm{D}_{4}\right]$-Methanol, 77.0 für Deuterochloroform, 128.0 für [D 6 -Benzol, 206.3 [D $\mathrm{D}_{6}$-Aceton. Die Zuordnung der Kohlenstoffatome erfolgte wenn nötig mit Unterstützung eines HSQC und/oder eines APT-Spektrums.

Massenspektrometrie: Finnigan MAT 95 [Elektronenstoßionisation EI, 70 eV, Direkte Chemische Ionisation DCI, $200 \mathrm{eV}$ und HRMS (High Resolution MS, ESI)], Finnigan LCQ (Electrospray-Ionisation ESI).

GC-MS: Hewlett Packard GCD System G1800C (Säule: 30 m, CP-Sil 5CB) mit EI-MS Quadrupol Massenanalysator.

Infrarot-Spektroskopie: Spektrometer: FT-IR Perkin Elmer Spectrum RX I; Flüssigkeiten und Öle wurden als Film (KBr-Platten) und Feststoffe als KBr-Presslinge gemessen. 


\section{Chromatographische Methoden}

Säulenchromatographie (SC): Chromatographische Trennungen erfolgten an Merck Kieselgel 60 (0.063-0.200 mm, 230-400 mesh) als Schwerkraftchromatographie bzw. an Macherey-Nagel Kieselgel $60 \quad(0.040-0.063 \quad \mathrm{~mm}, \quad 200-400$ mesh) als FlashChromatographie nach Still et al. ${ }^{[165]}$ Alle Lösungsmittel wurden vor Verwendung destilliert.

Dünnschichtchromatographie (DC): Merck DC-Fertigfolien Kieselgel 60 Sil G/UV 254 auf Aluminiumfolie oder Fertigfolien Polyfram Aluminumoxid N/UV $\mathrm{UV}_{254}$. Detektion unter UV-Licht bei $254 \mathrm{~nm}$ oder $366 \mathrm{~nm}$, Entwicklung mit Molybdatophosphat ${ }^{[166]}$ (5\%ige Phosphormolybdänsäure in EtOH) als Tauchreagenz. - Präparative Dünnschichtchromatographie: a) Merck KGaA, KG 60, $20 \times 20 \mathrm{~cm}$ Fertigplatten, Schichtdicke $2 \mathrm{~mm}$. b) Machery Nagel, KG 60 mit Bindemittel, $20 \times 20 \mathrm{~cm}$ Platten, Schichtdicke $\sim 2 \mathrm{~mm}$.

Größenausschlusschromatographie: Stationäre Phase - Sephadex LH 20, Eluent $\mathrm{MeOH}$.

Mitteldruckchromatographie (MPLC): Knauer MaxiStar Pumpe, Säule: RP18-Lobar LiChroprep Säule, Größe B.

Spezifische Radioaktivität: Beckmann bF Betaszint 5000/300 Szintillationsmessgerät.

\section{Elementaranalysen}

Die Elementaranalysen wurden im Mikroanalytischen Laboratorium des Instituts für Organische und Biomolekulare Chemie der Universität Göttingen ausgeführt. Geräte: Leco CHN 2000, Heraeus Verbrennungsapparatur Mikro U/D.

\section{Arbeiten mit radioaktivem Material}

Alle Manipulationen mit radioaktivem Material wurden unter Berücksichtigung der besonderen Sicherheitsmaßnahmen (Handmessgeräte, Schutzkleidung etc.) im Sicherheitsbereich des Isotopenlaboratoriums der Fakultät für Chemie, Georg-AugustUniversität Göttingen durchgeführt. ${ }^{[167]}$ 


\section{Chemikalien und Arbeitstechniken}

Lösungsmittel wurden nach üblichen Laboratoriumsmethoden ${ }^{[168]}$ getrocknet: Lösungsmittel von technischer Qualität wurden unter Stickstoff destilliert (THF, Diethylether, Dioxan, Benzol, Toluol, Hexan und Pentan mit Natrium/Benzophenon, HMPA, DMPU, $\mathrm{NEt}_{3}$, Pyridin, DMF, DMSO und $\mathrm{CH}_{2} \mathrm{Cl}_{2}$ mit $\mathrm{CaH}_{2}$, EtOH und Hexan mit Natrium, $\mathrm{MeOH}$ über $\mathrm{Mg}$ ). Alle Reaktionen wurden, soweit nicht anders erwähnt, unter Sauerstoff- und Feuchtigkeitsausschluss und unter inerter Atmosphäre (Argon oder Stickstoff) durchgeführt. Wenn nicht anders angegeben, sind Lösungen von $\mathrm{NH}_{4} \mathrm{Cl}, \mathrm{NaCl}, \mathrm{Na}_{2} \mathrm{SO}_{4}$, $\mathrm{NaHCO}_{3}, \mathrm{Na}_{2} \mathrm{CO}_{3}$, und $\mathrm{KHSO}_{4}$ gesättigte wässrige Lösungen. Nicht gesondert aufgeführte Chemikalien sind kommerziell erhältlich und wurden ohne weitere Reinigung eingesetzt. Das $\mathrm{Ba}^{14} \mathrm{CO}_{3}$ wurde geliefert von Moravek Biochemicals and Radiochemicals, 577 Mercury Lane, Brea, CA 92821, USA. Folgende Chemikalien wurden nach Literaturvorschrift hergestellt: Zinn(II)triflat ${ }^{[169}$ ]; 1-(4-Bromphenyl)-2,2,2-trifluorethanon $^{[170]}$; Formaldehyd/THF-Lösung ${ }^{[53]}$; Kendomycin-Acetonid $(\mathbf{8 1})^{[62]}$; (tert-Butyldimethyl-silanyloxy)-acetaldehyd (141 $)^{[109]}$; 4-Brom-crotonsäureethylester (147) ${ }^{[171 b]}$; Crotonsäureethylestertriphenylphosphoniumbromid (148) ${ }^{[171]}$; 4-Brom-3-oxobutansäureethylesters (159) ${ }^{[117]}$; 4-(Diethoxyphosphoryl)-3-oxobutansäureethylester (154) ${ }^{[120]}$; 1-Iod2-bromethylen ${ }^{[129]}$; Tetrakis-(triphenylphosphin)palladium $(0)^{[131]}$; TMS-Acetylen ${ }^{[132]} ; Z-(4-$ Methoxy)but-2-en-1-ol ${ }^{[172]} ; Z$-(4-Benzyloxy)but-2-en-1-ol ${ }^{[173]} ;$ Z-(4-tert-Butylsilanyloxy)but-2-en-1-al $\left(\mathbf{1 4 1}_{Z}\right)^{[135]}$; E-(4-Benzyloxy)but-2-en-1-al (169) ${ }^{[134]}$; Z-(4-Methoxy)-but-2-en1-al (168) ${ }^{[136]} ; 1$,2-Bistrimethylsilyloxyethan ${ }^{[156]}$. 


\section{E.2 Arbeitsvorschriften Hauptteil I}

\section{E.2.1 Synthese des Photoaffinitätslabels}

\section{1-(4-Bromphenyl)-2,2,2-trifluorethanon-oxim (56)}

Eine Lösung von 1-(4-Bromphenyl)-2,2,2-trifluorethanon (55) (1.00 g, $3.95 \mathrm{mmol})$ und Hydroxylamin-hydrochlorid (0.270 g, $3.89 \mathrm{mmol})$ in abs. Ethanol $(1 \mathrm{~mL})$ und Pyridin $(2 \mathrm{~mL})$ wurde $2 \mathrm{~h}$ auf $60{ }^{\circ} \mathrm{C}$ erwärmt. Das Lösungsmittel wurde entfernt, Wasser $(20 \mathrm{~mL})$ und Ether $(200 \mathrm{~mL})$ zugegeben, die Phasen getrennt und die organische Phase mit Wasser $(10 \mathrm{~mL})$ und $\mathrm{NaCl}$-Lösung $(10 \mathrm{~mL})$ gewaschen. Trocknen über $\mathrm{Na}_{2} \mathrm{SO}_{4}$ und Entfernen des Lösungsmittels unter reduziertem Druck ergab das

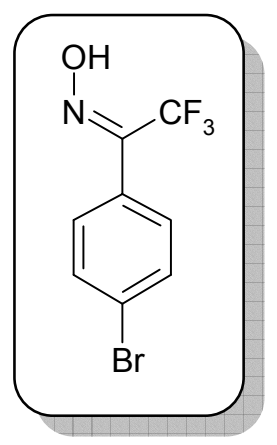
Rohprodukt. Säulenchromatographische Trennung (KG, Hex:EE, 10:1) ergab $0.870 \mathrm{~g}$ (82\%) der Titelverbindung als farblosen Feststoff (1:1 Isomerengemisch). $R_{\mathrm{f}}=0.57$ (Hex:EE, 3:1). - Smp. $=75-80{ }^{\circ} \mathrm{C} .-{ }^{1} \mathrm{H}-\mathrm{NMR}\left(300 \mathrm{MHz}, \mathrm{CDCl}_{3}\right): \delta=7.32-7.42(\mathrm{~m}, 2 \mathrm{H}$, Ar-H), 7.52-7.64 (m, 2 H, Ar-H), 8.89 (s, OH, Isomer 1), 8.92 (s, OH, Isomer 2). $-{ }^{13} \mathrm{C}-$ $\operatorname{NMR}\left(75.5 \mathrm{MHz}, \mathrm{CDCl}_{3}\right): \delta=117.2\left(\mathrm{q},{ }^{1} J_{\mathrm{C}-\mathrm{F}}=175.3 \mathrm{~Hz}, \mathrm{CF}_{3}\right.$, Isomer 1$), 121.0\left(\mathrm{q},{ }^{1} J_{\mathrm{C}-\mathrm{F}}=\right.$ $166.1 \mathrm{~Hz}, \mathrm{CF}_{3}$, Isomer 2), 116.2, 118.5, 120.0, 122.2, 124.6, 125.3, 129.9, 130.3, 131.9, 145.9-148.2 (m, CNOH, beide Isomere). - IR (KBr): $\widetilde{v}=3287(\mathrm{OH}), 1885,1620(\mathrm{C}=\mathrm{N})$, 1587, 1493, 1466, 1000, $806 \mathrm{~cm}^{-1}$. - MS (DCI, $\left.70 \mathrm{eV}\right), \mathrm{m} / z(\%): 266 / 268(50)[\mathrm{M}-\mathrm{H}]^{-}$, 533/535/537 (100) $[2 \mathrm{M}-\mathrm{H}]^{-}$. - Anal. ber. (\%) für $\mathrm{C}_{8} \mathrm{H}_{5} \mathrm{BrF}_{3} \mathrm{NO}$ (268.03): C 35.85, H 1.88, N 5.23; Gef. C 35.87, H 2.00, N 5.12.

\section{1-(4-Bromphenyl)-2,2,2-trifluorethanon-O-(p-tolylsulfonyl)oxim (57)}

$\mathrm{Zu}$ einer Lösung von Oxim 56 (58.1 g, $217 \mathrm{mmol}), \mathrm{NEt}_{3}(26.3 \mathrm{~g}$, $260 \mathrm{mmol})$ und DMAP $(2.65 \mathrm{~g}, 21.7 \mathrm{mmol})$ in $\mathrm{CH}_{2} \mathrm{Cl}_{2}(250 \mathrm{~mL})$ wurde bei $0{ }^{\circ} \mathrm{C}$ portionsweise $p$-Toluolsulfonylchlorid $(47.6 \mathrm{~g}, 250 \mathrm{mmol})$ gegeben. Nach vollständiger Zugabe wurde die Reaktionsmischung $3 \mathrm{~h}$ bei RT gerührt. Nach Entfernen des Lösungsmittels unter reduziertem Druck wurde Ether $(500 \mathrm{~mL})$ zugegeben. Die organische Phase wurde

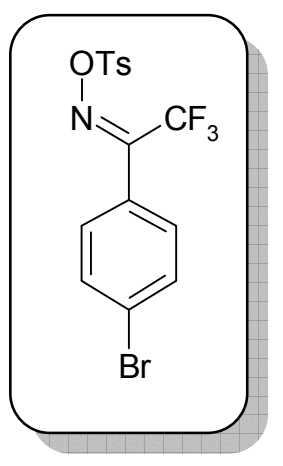
mit Wasser $(3 \times 50 \mathrm{~mL})$ und $\mathrm{NaCl}$-Lösung $(10 \mathrm{~mL})$ gewaschen, über $\mathrm{Na}_{2} \mathrm{SO}_{4}$ getrocknet und das Lösungsmittel unter reduziertem Druck entfernt. Das so erhaltene Rohprodukt (91.0 g, 99\%) war für die weiteren Umsetzungen ausreichend rein. 
Durch Umkristallisation aus $\mathrm{CHCl}_{3}$ wurde eine analytische Probe gewonnen. $R_{\mathrm{f}}=0.43$ (Hex:EE, 2:1). Smp. $=133-135{ }^{\circ} \mathrm{C} .-{ }^{1} \mathrm{H}-\mathrm{NMR}\left(300 \mathrm{MHz}, \mathrm{CDCl}_{3}\right): \delta=2.46\left(\mathrm{~s}, 3 \mathrm{H}, \mathrm{CH}_{3}\right)$, $7.26\left(\mathrm{~d},{ }^{3} J_{\mathrm{H}, \mathrm{H}}=8.6 \mathrm{~Hz}, 2 \mathrm{H}, \mathrm{Ar}-\mathrm{H}\right), 7.37\left(\mathrm{~d},{ }^{3} J_{\mathrm{H}, \mathrm{H}}=8.2 \mathrm{~Hz}, 2 \mathrm{H}, \mathrm{Ar}-\mathrm{H}\right), 7.61\left(\mathrm{~d},{ }^{3} J_{\mathrm{H}, \mathrm{H}}=\right.$ $8.6 \mathrm{~Hz}, 2 \mathrm{H}, \mathrm{Ar}-\mathrm{H}), 7.86\left(\mathrm{~d},{ }^{3} J_{\mathrm{H}, \mathrm{H}}=8.2 \mathrm{~Hz}, 2 \mathrm{H}, \mathrm{Ar}-\mathrm{H}\right) .-{ }^{13} \mathrm{C}-\mathrm{NMR}\left(75.5 \mathrm{MHz}, \mathrm{CDCl}_{3}\right): \delta$ $=21.8\left(\mathrm{CH}_{3}\right), 119.4\left(\mathrm{q},{ }^{1} J_{\mathrm{C}-\mathrm{F}}=277.4 \mathrm{~Hz}, \mathrm{CF}_{3}\right), 123.3(\mathrm{C}-4), 126.6(\mathrm{C}-1), 129.3(\mathrm{C}-\mathrm{Ar})$, 129.9 (C-Ar), 131.0 (C-1'), 132.2 (C-Ar), 146.3 (C-4'), 153.0 (q, $\left.{ }^{2} J_{\mathrm{C}-\mathrm{F}}=33.8 \mathrm{~Hz}, \mathrm{CNO}\right)$. IR $(\mathrm{KBr}): \widetilde{v}=1927,1917,1637(\mathrm{C}=\mathrm{N}), 1589,1489,1447,1390,1179,901,820 \mathrm{~cm}^{-1}$. MS (DCI, $70 \mathrm{eV}), m / z(\%): 439 / 441(100)\left[\mathrm{M}+\mathrm{NH}_{4}\right]^{+}, 456 / 458(20)\left[\mathrm{M}+\mathrm{NH}_{3}+\mathrm{NH}_{4}\right]^{+}$, 860/862/864 (40) $\left[2 \mathrm{M}+\mathrm{NH}_{4}\right]^{+}$. - Anal. ber. (\%) für $\mathrm{C}_{15} \mathrm{H}_{11} \mathrm{BrF}_{3} \mathrm{NO}_{3} \mathrm{~S}$ (422.22): C 42.67, H 2.63, N 3.32; Gef. C 42.63, H 2.73, N 3.25.

\section{3-(4-Bromphenyl)-3-trifluormethyldiaziridin (58)}

Eine Lösung des Tosylats $57(90.9 \mathrm{~g}, 215 \mathrm{mmol})$ und $\mathrm{CH}_{2} \mathrm{Cl}_{2}(200 \mathrm{~mL})$ wurde in einem Autoklaven auf $-78^{\circ} \mathrm{C}$ gekühlt. Innerhalb von $4 \mathrm{~h}$ wurde langsam gasförmiger Ammoniak in den Autoklaven kondensiert. Die resultierende Mischung wurde im Autoklaven für $18 \mathrm{~h}$ gerührt, wobei sie sich auf RT erwärmte. Überschüssiger Ammoniak wurde vorsichtig abgelassen und der Rückstand mit $500 \mathrm{~mL}$ Dichlormethan verdünnt. Die organische Phase wurde mit Wasser $(50 \mathrm{~mL})$ und NaCl-Lösung

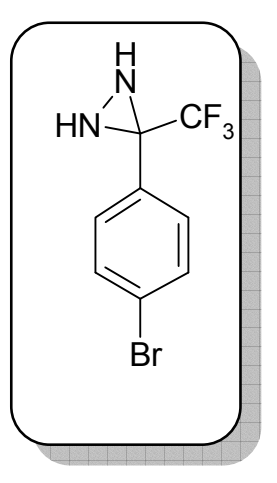
gewaschen, über $\mathrm{Na}_{2} \mathrm{SO}_{4}$ getrocknet und das Lösungsmittel unter reduziertem Druck entfernt. Der Rückstand wurde wiederholt aus einem Pentan $/ \mathrm{CH}_{2} \mathrm{Cl}_{2}$-Gemisch (1:5) umkristallisiert, wodurch $49.4 \mathrm{~g}(86 \%)$ der Titelverbindung als farbloser Feststoff isoliert wurden. $R_{\mathrm{f}}=0.33$ (Hex:EE, 2:1). - Smp. $=47-48{ }^{\circ} \mathrm{C} .-{ }^{1} \mathrm{H}-\mathrm{NMR}\left(300 \mathrm{MHz}, \mathrm{CDCl}_{3}\right): \delta=$ $2.18\left(\mathrm{~d},{ }^{3} J_{\mathrm{H}, \mathrm{H}}=8.4 \mathrm{~Hz}, 1 \mathrm{H}, \mathrm{NH}\right), 2.79\left(\mathrm{~d},{ }^{3} J_{\mathrm{H}, \mathrm{H}}=8.4 \mathrm{~Hz}, 1 \mathrm{H}, \mathrm{NH}\right), 7.48\left(\mathrm{~d},{ }^{3} J_{\mathrm{H}, \mathrm{H}}=8.6\right.$ $\mathrm{Hz}, 2 \mathrm{H}, \mathrm{Ar}-\mathrm{H}), 7.55\left(\mathrm{~d},{ }^{3} J_{\mathrm{H}, \mathrm{H}}=8.6 \mathrm{~Hz}, 2 \mathrm{H}, \mathrm{Ar}-\mathrm{H}\right) .-{ }^{13} \mathrm{C}-\mathrm{NMR}\left(75.5 \mathrm{MHz}, \mathrm{CDCl}_{3}\right): \delta=$ $57.6\left[\mathrm{q},{ }^{2} J_{\mathrm{C}-\mathrm{F}}=36.5 \mathrm{~Hz}, \mathrm{C}(\mathrm{NH})_{2}\right], 123.3\left(\mathrm{q},{ }^{1} J_{\mathrm{C}-\mathrm{F}}=278.2 \mathrm{~Hz}, \mathrm{CF}_{3}\right), 124.6(\mathrm{C}-4), 129.8(\mathrm{C}-$ Ar), 130.7 (C-1), 132.0 (C-Ar). - IR (KBr): $\widetilde{v}=3197(\mathrm{NH}), 1921,1596(\mathrm{NH}), 1495$, 1407, 1398, 1191, 1012, $829 \mathrm{~cm}^{-1}$. - MS (ESI), $m / z(\%): 267 / 269$ (100) [M + H] ; Anal. ber. für $\mathrm{C}_{8} \mathrm{H}_{7} \mathrm{BrF}_{3} \mathrm{~N}_{2}$ : $266.97392[\mathrm{M}+\mathrm{H}]^{+}$Gef. 266.97381. - Anal. ber. (\%) für $\mathrm{C}_{8} \mathrm{H}_{6} \mathrm{BrF}_{3} \mathrm{~N}_{2}$ (267.05): C 35.98, H 2.26, N 10.49; Gef. C 35.98, H 2.19, N 10.42. 


\section{3-(4-Bromphenyl)-3-trifluormethyl-3H-diazirin (59)}

Eine Lösung des Diaziridins 58 (6.01 g, 22.5 mmol), NEt 3 (3.14 mL, 22.5 mmol) und Ethanol (22 mL) wurde auf $0{ }^{\circ} \mathrm{C}$ gekühlt. Danach wurde unter starkem Rühren langsam eine Lösung von tert-Butylhypochlorit (7.34 g, $67.6 \mathrm{mmol})$ und tert-Butanol $(9.5 \mathrm{~mL})$ zugegeben. Das Gemisch wurde $2 \mathrm{~h}$ bei $0{ }^{\circ} \mathrm{C}$ gerührt und dann mit einer $10 \%$ igen, wässrigen $\mathrm{Na}_{2} \mathrm{~S}_{2} \mathrm{O}_{5^{-}}$

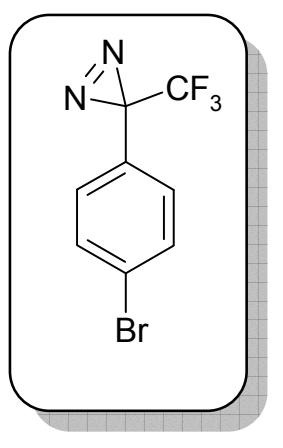
Lösung $(150 \mathrm{~mL})$ gequencht. Die Phasen wurden getrennt und die wässrige Phase mit Ether $(3 \times 50 \mathrm{~mL})$ extrahiert. Die vereinigten organischen Phasen wurden über $\mathrm{Na}_{2} \mathrm{SO}_{4}$ getrocknet und das Lösungsmittel unter reduziertem Druck entfernt. Das Rohprodukt $(5.14 \mathrm{~g}, 86 \%)$ war für die folgenden Umsetzungen von ausreichender Reinheit. ${ }^{1} \mathrm{H}-\mathrm{NMR}$ $\left(300 \mathrm{MHz}, \mathrm{CDCl}_{3}\right): \delta=7.05\left(\mathrm{~d},{ }^{3} J_{\mathrm{H}, \mathrm{H}}=8.4 \mathrm{~Hz}, 2 \mathrm{H}, \mathrm{Ar}-\mathrm{H}\right), 7.51\left(\mathrm{~d},{ }^{3} J_{\mathrm{H}, \mathrm{H}}=8.4 \mathrm{~Hz}, 2 \mathrm{H}\right.$, Ar-H). $-{ }^{13} \mathrm{C}-\mathrm{NMR}\left(75.5 \mathrm{MHz}, \mathrm{CDCl}_{3}\right): \delta=28.2\left(\mathrm{q},{ }^{2} J_{\mathrm{C}-\mathrm{F}}=43.5 \mathrm{~Hz}, \mathrm{CN}_{2}\right), 121.9\left(\mathrm{q},{ }^{1} J_{\mathrm{C}-\mathrm{F}}\right.$ $=274.7 \mathrm{~Hz}, \mathrm{CF}_{3}$ ), 124.2 (C-4), 128.0 (C-Ar), 132.0 (C-Ar), Signale für C-1 nicht sichtbar. - MS (EI, $70 \mathrm{eV}), m / z(\%): 71(82)\left[\mathrm{HCF}_{3}\right]^{+}, 107(20)\left[\mathrm{C}_{7} \mathrm{H}_{9} \mathrm{~N}\right]^{+}, 137$ (40), 155/157 (100) $\left[\mathrm{C}_{6} \mathrm{H}_{4} \mathrm{Br}\right]^{+}, 171(20), 236 / 238(45)\left[\mathrm{M}-\mathrm{N}_{2}\right]^{+}, 500 / 502 / 504(20)\left[\mathrm{C}_{16} \mathrm{H}_{8} \mathrm{Br}_{2} \mathrm{~F}_{6} \mathrm{~N}_{2}\right]^{+}$.

Versuche zur Lithiierung von 3-(4-Bromphenyl)-3-trifluormethyl-3H-diazirin (59)

4-Bromdiazirin 59 (225 mg, $844 \mu \mathrm{mol})$ wurde in THF $(50 \mathrm{~mL})$ gelöst und auf $-95^{\circ} \mathrm{C}$ gekühlt. Nach Zugabe von $n$-BuLi (400 $\mu \mathrm{L}, 928 \mu \mathrm{mol}, 2.32 \mathrm{M}$ in Hexan) ließ man $60 \mathrm{~min}$ bei -95 ${ }^{\circ} \mathrm{C}$ rühren und versetzte dann mit $\mathrm{CO}_{2}$, welches mit konz. $\mathrm{H}_{2} \mathrm{SO}_{4}$ aus Bariumcarbonat $(251 \mathrm{mg}, 1.27 \mathrm{mmol})$ freigesetzt und über einen Teflonschlauch in die Reaktionslösung geleitet wurde. Nach 1 h Rühren bei $-95^{\circ} \mathrm{C}$ ließ man auf $-30{ }^{\circ} \mathrm{C}$ erwärmen und

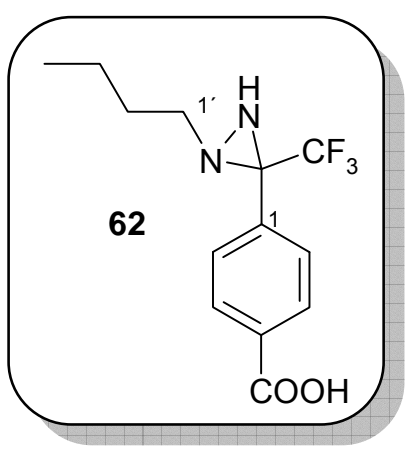
rührte für weitere $10 \mathrm{~h}$. Die Reaktion wurde durch Zugabe von Wasser $(10 \mathrm{~mL})$ beendet. Es wurde mit Ether $(50 \mathrm{~mL})$ verdünnt, und das Reaktionsgemisch mit $\mathrm{NaCO}_{3}$-Lösung extrahiert $(3 \times 20 \mathrm{~mL})$. Der pH-Wert der wässrigen Lösung wurde mit $1 \mathrm{~N}$ HCl-Lösung auf $\mathrm{pH} \sim 2$ eingestellt und es wurde mit Ether $(2 \times 50 \mathrm{~mL})$ extrahiert. Die vereinigten organischen Phasen wurden mit Wasser und NaCl-Lösung gewaschen und über Natriumsulfat getrocknet. Im Rückstand (240 mg) wurde nicht die Titelverbindung, sondern die alkylierte Diaziridinylbenzoesäure 62 als Hauptkomponente identifiziert. Die Ergebnisse der Variation der Reaktionsbedingungen und verwendeten Li-Organyle sind in 
Tabelle 1 (siehe Kap. I.3.2) zusammengefasst. Spektroskopische Daten für 62: ${ }^{1} \mathrm{H}-\mathrm{NMR}$ $\left(250 \mathrm{MHz}, \mathrm{CDCl}_{3}\right): \delta=0.81\left(\mathrm{t},{ }^{3} J_{\mathrm{H}-\mathrm{H}}=7.1 \mathrm{~Hz}, 3 \mathrm{H}, 4^{\prime}-\mathrm{H}\right), 1.28\left(\mathrm{sex},{ }^{3} J_{\mathrm{H}-\mathrm{H}}=7.1 \mathrm{~Hz}, 2 \mathrm{H}\right.$, $\left.3^{\prime}-\mathrm{H}\right), 1.55$ (quint, $\left.{ }^{3} J_{\mathrm{H}-\mathrm{H}}=7.1 \mathrm{~Hz}, 2 \mathrm{H}, 2^{\prime}-\mathrm{H}\right), 1.75-1.85\left(\mathrm{~m}, 1 \mathrm{H}, 1^{\prime}-\mathrm{H}_{\mathrm{a}}\right), 2.37-2.43(\mathrm{~m}, 1 \mathrm{H}$, $\left.1^{\prime}-\mathrm{H}_{\mathrm{b}}\right), 7.70\left(\mathrm{~d},{ }^{3} J_{\mathrm{H}, \mathrm{H}}=8.4 \mathrm{~Hz}, 2 \mathrm{H}, \mathrm{Ar}-\mathrm{H}\right), 8.19\left(\mathrm{~d},{ }^{3} J_{\mathrm{H}, \mathrm{H}}=8.4 \mathrm{~Hz}, 2 \mathrm{H}, \mathrm{Ar}-\mathrm{H}\right) .-{ }^{13} \mathrm{C}-\mathrm{NMR}$ (75.5 MHz, $\left.\mathrm{CDCl}_{3}\right): \delta=13.8\left(\mathrm{C}-4^{\prime}\right), 20.1\left(\mathrm{C}-3^{\prime}\right), 30.6\left(\mathrm{C}-2^{\prime}\right), 54.6\left(\mathrm{C}-1^{\prime}\right),\left(\mathrm{CN}_{2}\right.$, nicht sichtbar), ( $\mathrm{CF}_{3}$, nicht sichtbar), 128.5 (C-Ar), 130.0 (C-Ar), 130.9 (C-Ar), 133.8 (C-Ar), $170.9(\mathrm{COOH}) .-\mathrm{MS}(\mathrm{ESI}), m / z(\%)$ : pos: 423 (100), 531 (70); neg: $287(100)[\mathrm{M}-\mathrm{H}]^{-}$, $597[2 \mathrm{M}-2 \mathrm{H}+\mathrm{Na}]^{-}$.

Versuche zur Grignard-Bildung mit 3-(4-Bromphenyl)-3-trifluormethyl-3H-diazirin (59)

Magnesiumchlorid (112 mg, $1.18 \mathrm{mmol})$ und Kalium (46.0 mg, $1.18 \mathrm{mmol}$ ) wurden in THF $(10 \mathrm{~mL})$ suspendiert und zwei Stunden unter Rückfluss erhitzt. Das schwarze Reaktionsgemisch wurde auf $-78^{\circ} \mathrm{C}$ gekühlt und mit 4-Bromdiazirin 59 (156 mg, $589 \mu \mathrm{mol})$, gelöst in THF $(2 \mathrm{~mL})$ versetzt. Man ließ $1 \mathrm{~h}$ bei $-78^{\circ} \mathrm{C}$ rühren und erwärmte dann auf RT. Nach 16 h Rühren bei RT wurde Wasser $(2 \mathrm{~mL})$ zugegeben, über Aktivkohle filtriert und der Rückstand in Ether aufgenommen. Trocknen über Natriumsulfat und Entfernen des Lösungsmittels unter vermindertem Druck ergab 146 mg Rohprodukt, welches ausschließlich Edukt enthielt. Die Ergebnisse der Variation der Reaktionsbedingungen und verwendeten Methoden zum Halogen-Mg-Austausch sind in Tabelle 2 (siehe Kap. I.3.2) zusammengefasst.

\section{3-(4-Bromphenyl)-3-trifluormethyl-1,2-bistrimethylsilyldiaziridin (64)}

Diaziridin 58 (1.35 g, $5.06 \mathrm{mmol})$ wurde zu einer Lösung von $\mathrm{NEt}_{3}$ (4.2 mL, $30 \mathrm{mmol})$ und $\mathrm{CH}_{2} \mathrm{Cl}_{2}(25 \mathrm{~mL})$ gegeben. Nach $1 \mathrm{~h}$ Rühren bei RT wurde die Lösung auf $-78^{\circ} \mathrm{C}$ gekühlt und langsam mit TMSOTf $(2.0 \mathrm{~mL}, 2.5 \mathrm{~g}, 11 \mathrm{mmol})$ versetzt. Es wurde zunächst für weitere $60 \mathrm{~min}$ bei $-78^{\circ} \mathrm{C}$ gerührt, dann wurde das Kühlbad entfernt und man ließ bei RT rühren. Der Reaktionsverlauf wurde mittels ${ }^{1}$ H-NMR-Spektroskopie verfolgt. Zu diesem Zweck wurden

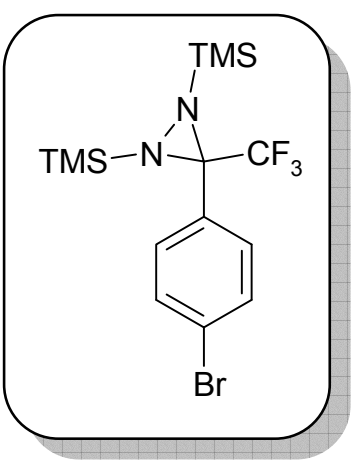
jeweils $0.1 \mathrm{~mL}-$ Proben entnommen und im Vakuum konzentriert. Nach $3 \mathrm{~h}$ war der Umsatz vollständig und alle flüchtigen Bestandteile wurden im Vakuum entfernt. Das so 
erhaltene gelbe Öl wurde mit Benzol extrahiert $(4 \times 10 \mathrm{~mL})$ und die Extrakte im Vakuum konzentriert. Zur Aufreinigung wurde diese Prozedur zweimal mit Hexan (jeweils $3 \times 10$ $\mathrm{mL})$ wiederholt. Man erhielt $1.97 \mathrm{~g}$ (95\%) der Titelverbindung als farblosen, kristallinen Feststoff. ${ }^{1} \mathrm{H}-\mathrm{NMR}\left(300 \mathrm{MHz}, \mathrm{C}_{6} \mathrm{D}_{6}\right): \delta=0.01\left[\mathrm{~s}, 18 \mathrm{H}, \mathrm{Si}\left(\mathrm{CH}_{3}\right)_{3}\right], 7.15\left(\mathrm{~d},{ }^{3} J_{\mathrm{H}, \mathrm{H}}=8.1 \mathrm{~Hz}\right.$, $2 \mathrm{H}, 3-\mathrm{H}, 5-\mathrm{H}), 7.28\left(\mathrm{~d},{ }^{3} J_{\mathrm{H}, \mathrm{H}}=8.1 \mathrm{~Hz}, 2 \mathrm{H}, 2-\mathrm{H}, 6-\mathrm{H}\right) .-{ }^{13} \mathrm{C}-\mathrm{NMR}\left(75.5 \mathrm{MHz}, \mathrm{C}_{6} \mathrm{D}_{6}\right): \delta=$ $-0.75\left[\mathrm{Si}\left(\mathrm{CH}_{3}\right)_{3}\right],-0.76\left[\mathrm{Si}\left(\mathrm{CH}_{3}\right)_{3}\right], 66.4\left[\mathrm{q},{ }^{2} J_{\mathrm{C}-\mathrm{F}}=35.2 \mathrm{~Hz}, \mathrm{C}(\mathrm{NTMS})_{2}\right], 124.0(\mathrm{C}-4)$, $125.3\left(\mathrm{q},{ }^{1} J_{\mathrm{C}-\mathrm{F}}=282.4 \mathrm{~Hz}, \mathrm{CF}_{3}\right), 131.2(\mathrm{C}-\mathrm{Ar}), 131.4$ (C-Ar), 133.1 (C-1).

\section{3-(4-Hydroxymethylphenyl)-3-trifluormethyldiaziridin (68)}

Das geschützte Diaziridin $64(211 \mathrm{mg}, 513 \mu \mathrm{mol})$ wurde in THF $(15 \mathrm{~mL})$ gelöst und auf $-78^{\circ} \mathrm{C}$ gekühlt. Nach Zugabe von $n$-BuLi $(257 \mu \mathrm{L}$, $514 \mu \mathrm{mol}, 2.00 \mathrm{M}$ in Hexan) wurde die orange Lösung $1 \mathrm{~h}$ bei $-78^{\circ} \mathrm{C}$ gerührt. Danach wurde eine frisch hergestellte Formaldehyd-Lösung (2.93 mL, $2.05 \mathrm{mmol}, 0.70 \mathrm{M}$ in THF) zugetropft und anschließend $6 \mathrm{~h}$ gerührt, wobei sich die Lösung auf $-50{ }^{\circ} \mathrm{C}$ erwärmte. Die Reaktion wurde durch Zugabe von $\mathrm{NH}_{4} \mathrm{Cl}$ Lösung $(500 \mu \mathrm{l})$ beendet, das THF

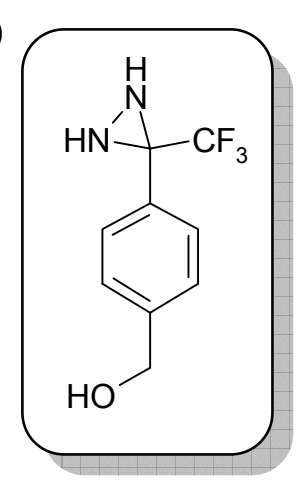
wurde unter reduziertem Druck entfernt und der Rückstand in Diethylether $(250 \mathrm{~mL})$ aufgenommen. Die etherische Phase wurde mit Wasser $(15 \mathrm{~mL}), \mathrm{NaHCO}_{3}(10 \mathrm{~mL})$ und $\mathrm{NaCl}$-Lösung $(10 \mathrm{~mL})$ gewaschen und über $\mathrm{Na}_{2} \mathrm{SO}_{4}$ getrocknet. Das Rohprodukt (302 mg) wurde säulenchromatographisch aufgereinigt: $\left(\mathrm{CH}_{2} \mathrm{Cl}_{2}: \mathrm{MeOH}, 20: 1\right)$ und MPLC $\left(\mathrm{CH}_{3} \mathrm{CN}: \mathrm{H}_{2} \mathrm{O}, 1: 4\right)$. Man erhielt $65.0 \mathrm{mg}(58 \%)$ der Titelverbindung als farbloses Öl. $R_{\mathrm{f}}=$ $0.42\left(\mathrm{CH}_{2} \mathrm{Cl}_{2}: \mathrm{MeOH}, 20: 1\right) .-{ }^{1} \mathrm{H}-\mathrm{NMR}$ (300 MHz, [D 6 -Aceton): $\delta=3.26\left(\mathrm{~d},{ }^{3} J_{\mathrm{H}, \mathrm{H}}=8.2\right.$ $\mathrm{Hz}, 1 \mathrm{H}, \mathrm{NH}), 3.60\left(\mathrm{~d},{ }^{3} \mathrm{~J}_{\mathrm{H}, \mathrm{H}}=8.2 \mathrm{~Hz}, 1 \mathrm{H}, \mathrm{NH}\right), 4.42\left(\mathrm{~s}_{\mathrm{br}}, 1 \mathrm{H}, \mathrm{OH}\right), 4.66\left(\mathrm{~s}, 2 \mathrm{H}, \mathrm{CH}_{2} \mathrm{OH}\right)$, $7.43\left(\mathrm{~d},{ }^{3} J_{\mathrm{H}, \mathrm{H}}=8.2 \mathrm{~Hz}, 2 \mathrm{H}, 3-\mathrm{H}, 5-\mathrm{H}\right), 7.58\left(\mathrm{~d},{ }^{3} J_{\mathrm{H}, \mathrm{H}}=8.2 \mathrm{~Hz}, 2 \mathrm{H}, 2-\mathrm{H}, 6-\mathrm{H}\right) .-{ }^{13} \mathrm{C}-\mathrm{NMR}$ (75.5 MHz, [D 6 -Aceton): $\delta=58.4\left[\mathrm{q},{ }^{2} J_{\mathrm{C}-\mathrm{F}}=35.3 \mathrm{~Hz}, \mathrm{C}(\mathrm{NH})_{2}\right], 64.1\left(\mathrm{CH}_{2} \mathrm{OH}\right), 125.2(\mathrm{q}$, $\left.{ }^{1} J_{\mathrm{C}-\mathrm{F}}=277.4 \mathrm{~Hz}, \mathrm{CF}_{3}\right), 127.2(\mathrm{C}-\mathrm{Ar}), 129.1(\mathrm{C}-\mathrm{Ar}), 131.7$ (C-1), $145.2(\mathrm{C}-4)$. - IR (KBr): $\widetilde{v}=3391,3217,1601,1495,1450,1416,1391,1260,1234,1199,1181,1147,1026,945$, 859, 736, 710, $621 \mathrm{~cm}^{-1}$. - MS (DCI, $\left.70 \mathrm{eV}\right), m / z(\%): 219(100)[\mathrm{M}+\mathrm{H}]^{+}, 236(80)[\mathrm{M}+$ $\left.\mathrm{NH}_{4}\right]^{+}$. 


\section{3-[4-(2-Hydroxyprop-2-yl)phenyl]-3-trifluormethyldiaziridin (69)}

Das geschützte Diaziridin $64(286 \mathrm{mg}, 695 \mu \mathrm{mol})$ wurde in THF $(15 \mathrm{~mL})$ gelöst und auf $-78{ }^{\circ} \mathrm{C}$ gekühlt, $n$-BuLi $(297 \mu \mathrm{L}, 689 \mu \mathrm{mol}, 2.32 \mathrm{M}$ in Hexan) wurde zugetropft und die resultierende Lösung $40 \mathrm{~min}$ bei $-78{ }^{\circ} \mathrm{C}$ gerührt. Dann wurde wasserfreies Aceton $(62 \mu \mathrm{L}, 49 \mathrm{mg}, 0.84 \mathrm{mmol})$ zugegeben und man ließ innerhalb von $4.5 \mathrm{~h}$ auf $-45{ }^{\circ} \mathrm{C}$ erwärmen. Die Reaktion wurde durch Zugabe von $\mathrm{H}_{2} \mathrm{O}(0.5 \mathrm{~mL})$ beendet, das

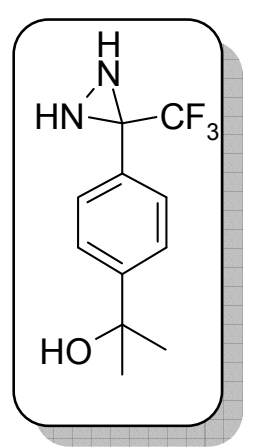
Lösunsmittel in Vacuo entfernt und der Rückstand in Ether $(150 \mathrm{~mL})$ aufgenommen. Die etherische Phase wurde mit $\mathrm{H}_{2} \mathrm{O}(10 \mathrm{~mL})$ and NaCl-Lösung $(2 \times 10 \mathrm{~mL})$ gewaschen, über $\mathrm{Na}_{2} \mathrm{SO}_{4}$ getrocknet und das Lösungsmittel im Vakuum entfernt. Das so erhaltene Rohprodukt (165 mg) wurde säulenchromatographisch gereinigt (Hex:EE, 2:1). Man erhielt $114 \mathrm{mg}$ (67\%) der Titelverbindung als farblosen Feststoff. $R_{\mathrm{f}}=$ 0.26 (Hex:EE, 2:1). - Smp. $=78{ }^{\circ} \mathrm{C} .-{ }^{1} \mathrm{H}-\mathrm{NMR}\left(300 \mathrm{MHz},\left[\mathrm{D}_{6}\right]\right.$-Aceton): $\delta=1.58(\mathrm{~s}, 6 \mathrm{H}$, $\left.\mathrm{CH}_{3}\right), 1.66(\mathrm{~s}, \mathrm{br}, \mathrm{OH}), 2.22\left(\mathrm{~d},{ }^{3} J_{\mathrm{H}, \mathrm{H}}=8.2 \mathrm{~Hz}, 1 \mathrm{H}, \mathrm{NH}\right), 2.80\left(\mathrm{~d},{ }^{3} J_{\mathrm{H}, \mathrm{H}}=8.2 \mathrm{~Hz}, 1 \mathrm{H}, \mathrm{NH}\right)$, 7.44-7.68 (m, $4 \mathrm{H}, \mathrm{Ar}-\mathrm{H}) .-{ }^{13} \mathrm{C}-\mathrm{NMR}\left(75.5 \mathrm{MHz},\left[\mathrm{D}_{6}\right]\right.$-Aceton): $\delta=31.7\left(\mathrm{CH}_{3}\right), 57.8(\mathrm{q}$, $\left.{ }^{2} J_{\mathrm{C}-\mathrm{F}}=35.8 \mathrm{~Hz}, \mathrm{C}(\mathrm{NH})_{2}\right), 72.4(\mathrm{COH}), 125.2\left(\mathrm{q},{ }^{1} J_{\mathrm{C}-\mathrm{F}}=277.4 \mathrm{~Hz}, \mathrm{CF}_{3}\right), 127.2(\mathrm{C}-\mathrm{Ar})$, 129.1 (C-Ar), 131.7 (C-1), 145.2 (C-4). - IR (KBr): $\tilde{v}=3371,3237$, 3214, 2983, 2932, 1615, 1515, 1463, 1403, 1236, 1155, 1118, 1020, 952, 832, $693 \mathrm{~cm}^{-1}$. - MS (DCI, $\left.70 \mathrm{eV}\right)$, $m / z(\%): 229$ (38) $[M-\mathrm{OH}]^{+}, 246(100)\left[\mathrm{M}-\mathrm{H}_{2} \mathrm{O}+\mathrm{NH}_{4}\right]^{+}, 263$ (40) $\left[\mathrm{M}-\mathrm{H}_{2} \mathrm{O}+\mathrm{NH}_{3}+\right.$ $\left.\mathrm{NH}_{4}\right]^{+}$. - Anal. ber. (\%) für $\mathrm{C}_{11} \mathrm{H}_{13} \mathrm{~F}_{3} \mathrm{~N}_{2} \mathrm{O}$ (246.23): C 53.66, H 5.32, N 11.38; Gef. C 53.76, H 5.07, N 11.12.

\section{3-[4-(Hydroxyphenylmethyl)phenyl]-3-trifluormethyldiaziridin (70)}

Das geschützte Diaziridin $64(271 \mathrm{mg}, 659 \mu \mathrm{mol})$ wurde in THF $(15 \mathrm{~mL})$ gelöst und auf $-78{ }^{\circ} \mathrm{C}$ gekühlt. Nach Zugabe von $n$-BuLi $(281 \mu \mathrm{L}$, $652 \mu$ mol, $2.32 \mathrm{M}$ in Hexan) ließ man $40 \mathrm{~min}$ bei $-78^{\circ} \mathrm{C}$ rühren. Danach wurde frisch destillierter Benzaldehyd $(77.0 \mathrm{mg}, 730 \mu \mathrm{mol})$, gelöst in THF ( $2 \mathrm{~mL}$ ), zugetropft. Die Lösung wurde $4.5 \mathrm{~h}$ gerührt, wobei sie sich auf $-40{ }^{\circ} \mathrm{C}$ erwärmte. Durch Zugabe von $\mathrm{H}_{2} \mathrm{O}(0.5 \mathrm{~mL})$ wurde die

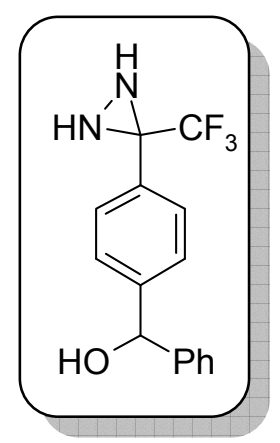
Reaktion beendet. Alle flüchtigen Bestandteile wurden unter reduziertem Druck entfernt und der Rückstand in Ether $(150 \mathrm{~mL})$ aufgenommen. Die etherische Phase wurde mit $\mathrm{H}_{2} \mathrm{O}(10 \mathrm{~mL})$ und $\mathrm{NaCl}$-Lösung $(2 \times 10 \mathrm{~mL})$ gewaschen und über $\mathrm{Na}_{2} \mathrm{SO}_{4}$ 
getrocknet. Das Rohprodukt (205 mg) wurde säulenchromatographisch gereinigt (Hex:EE, 5:1). Man erhielt $130 \mathrm{mg}$ (67\%) der Titelverbindung als farblosen Feststoff. $R_{\mathrm{f}}=0.16$ (Hex:EE, 5:1). - Smp. $=82-84{ }^{\circ} \mathrm{C} .-{ }^{1} \mathrm{H}-\mathrm{NMR}$ (300 MHz, [D 6 -Aceton): $\delta=3.29\left(\mathrm{~d},{ }^{3} J_{\mathrm{H}, \mathrm{H}}\right.$ $=8.1 \mathrm{~Hz}, 1 \mathrm{H}, \mathrm{NH}), 3.59\left(\mathrm{~d},{ }^{3} J_{\mathrm{H}, \mathrm{H}}=8.1 \mathrm{~Hz}, 1 \mathrm{H}, \mathrm{NH}\right), 5.03\left(\mathrm{~d},{ }^{3} J_{\mathrm{H}, \mathrm{H}}=2.7 \mathrm{~Hz}, 1 \mathrm{H}, \mathrm{OH}\right)$, $5.88\left(\mathrm{~d},{ }^{3} J_{\mathrm{H}, \mathrm{H}}=2.7 \mathrm{~Hz}, 1 \mathrm{H}, \mathrm{CHOH}\right), 7.18-7.36$ (m, $\left.3 \mathrm{H}, \mathrm{Ar}-\mathrm{H}\right), 7.40-7.60$ (m, $\left.6 \mathrm{H}, \mathrm{Ar}-\mathrm{H}\right)$. $-{ }^{13} \mathrm{C}-\mathrm{NMR}\left(125.7 \mathrm{~Hz},\left[\mathrm{D}_{6}\right]\right.$-Aceton): $\delta=58.4\left[\mathrm{q},{ }^{2} J_{\mathrm{C}-\mathrm{F}}=35.2 \mathrm{~Hz}, \mathrm{C}(\mathrm{NH})_{2}\right], 75.7(\mathrm{CHOH})$, $125.1\left(\mathrm{q},{ }^{1} J_{\mathrm{C}-\mathrm{F}}=277.7 \mathrm{~Hz}, \mathrm{CF}_{3}\right), 127.1$ (C-Ar), 127.2 (C-Ar), 127.8 (C-Ar), $129.0(\mathrm{C}-\mathrm{Ar})$, 129.2 (C-Ar), 131.8 (C-Ar), 146.0 (C-Ar), 148.2 (C-Ar). - IR (KBr): $\widetilde{v}=3434,3213$, 2984, 1615, 1515, 1403, 1236, 1155, 1118, 951, 831, $693 \mathrm{~cm}^{-1}$. - MS (DCI, $\left.70 \mathrm{eV}\right), \mathrm{m} / \mathrm{z}$ (\%): 229 (100), 246 (90), 264 (10). Anal. ber. (\%) für $\mathrm{C}_{15} \mathrm{H}_{13} \mathrm{~F}_{3} \mathrm{~N}_{2} \mathrm{O}$ (294.28): C 61.22, H 4.45, N 9.52; Gef. C 60.99, H 4.26, N 9.40.

\section{4-(3-Trifluormethyldiaziridin-3-yl)benzoesäure (71)}

Das geschützte Diaziridin 64 (320 mg, $778 \mu \mathrm{mol})$ wurde in THF $(30 \mathrm{~mL})$ gelöst und auf $-78{ }^{\circ} \mathrm{C}$ gekühlt. Nach Zugabe von $n$-BuLi (409 $\mu 1,818$ $\mu \mathrm{mol}, 2.00 \mathrm{M}$ in Hexan) wurde die orange-rote Lösung $30 \mathrm{~min}$ bei $78^{\circ} \mathrm{C}$ gerührt, bevor $\mathrm{CO}_{2}$ (generiert aus ca. $3 \mathrm{~g}$ Trockeneis, getrocknet durch Überleiten über Molekularsieb $3 \AA$ ) durch die Lösung geleitet wurde. Nach 30 min war die Zugabe beendet und man ließ $16 \mathrm{~h}$ bei -78

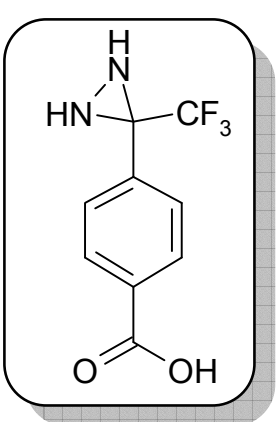
${ }^{\circ} \mathrm{C}$ rühren. Die Reaktion wurde durch Zugabe von $\mathrm{H}_{2} \mathrm{O}(0.5 \mathrm{~mL})$ beendet und die flüchtigen Bestandteile in Vacuo entfernt. Der Rückstand wurde in Ether $(150 \mathrm{~mL})$ aufgenommen und die etherische Phase mit $\mathrm{NaHCO}_{3}$-Lösung $(3 \times 15 \mathrm{~mL})$ extrahiert. Die vereinigten wässrigen Phasen wurden mit halbkonzentrierter $\mathrm{HCl}$ auf $\mathrm{pH} \approx 1$ angesäuert. Dabei bildete sich ein farbloser Niederschlag, welcher mit Ether $(4 \times 50 \mathrm{~mL})$ extrahiert wurde. Die vereinigten organischen Phasen wurden mit Wasser $(3 \times 20 \mathrm{~mL})$ und $\mathrm{NaCl}$ Lösung $(2 \times 10 \mathrm{~mL})$ gewaschen. Entfernen des Lösungsmittels unter reduziertem Druck ergab $161 \mathrm{mg}$ (89\%) der Titelverbindung. Das Produkt zeigte kaum Verunreinigungen und konnte direkt für die weiteren Umsetzungen eingesetzt werden. Eine analytische Probe wurde durch Umkristallisation aus Aceton gewonnen. Smp. $=181{ }^{\circ} \mathrm{C}$ (Zersetzung). $-{ }^{1} \mathrm{H}-$ NMR (300 MHz, [D $]$-Aceton): $\delta=3.50\left(\mathrm{~d},{ }^{3} J_{\mathrm{H}, \mathrm{H}}=7.6 \mathrm{~Hz}, 1 \mathrm{H}, \mathrm{NH}\right), 3.78\left(\mathrm{~d},{ }^{3} J_{\mathrm{H}, \mathrm{H}}=7.6\right.$ $\mathrm{Hz}, 1 \mathrm{H}, \mathrm{NH}), 7.77\left(\mathrm{~d},{ }^{3} J_{\mathrm{H}, \mathrm{H}}=8.3 \mathrm{~Hz}, 2 \mathrm{H}, 2-\mathrm{H}, 6-\mathrm{H}\right), 8.09\left(\mathrm{~d},{ }^{3} J_{\mathrm{H}, \mathrm{H}}=8.3 \mathrm{~Hz}, 2 \mathrm{H}, 3-\mathrm{H}, 5-\right.$ H). $-{ }^{13} \mathrm{C}-\mathrm{NMR}\left(75.5 \mathrm{MHz},\left[\mathrm{D}_{6}\right]\right.$-Aceton): $\delta=58.4\left[\mathrm{q},{ }^{2} J_{\mathrm{C}-\mathrm{F}}=35.7 \mathrm{~Hz}, \mathrm{C}(\mathrm{NH})_{2}\right], 125.0(\mathrm{q}$, $\left.{ }^{1} J_{\mathrm{C}-\mathrm{F}}=277.7 \mathrm{~Hz}, \mathrm{CF}_{3}\right), 129.6$ (C-Ar), 130.5 (C-Ar), 132.8 (C-1), $137.7(\mathrm{C}-4), 167.0$ 
(COOH). - IR (KBr): $\widetilde{v}=3407,3192,3015,2639,2518,1950,1711,1617,1581,1424$, 1386, 1263, 1166, 1110, 1021, 959, 900, 859, $714 \mathrm{~cm}^{-1}$. - MS (ESI), $m / z$ (\%): 231 (100) $[\mathrm{M}-\mathrm{H}]^{-}, 485$ (45) $[2 \mathrm{M}-2 \mathrm{H}+\mathrm{Na}]^{-}$. Anal. ber. (\%) für $\mathrm{C}_{9} \mathrm{H}_{7} \mathrm{~F}_{3} \mathrm{~N}_{2} \mathrm{O}_{2}$ (232.16): C 46.56, H 3.04, N 12.07; Gef. C 46.59, H 2.89, N 12.00.

\section{4-(3-Trifluormethyldiaziridin-3-yl)-[1- $\left.{ }^{14} \mathrm{C}\right]$-benzoesäure (71a)}

An ein $25 \mathrm{~mL}$ Schlenkrohr wurde über ein Septum eine $100 \mathrm{~mL}$ Glasspritze angeschlossen. Diaziridin 64 (1.23 g, $2.99 \mathrm{mmol})$ wurde in THF (20 mL) gelöst und auf $-78^{\circ} \mathrm{C}$ gekühlt. Nach Zugabe von $n$-BuLi (1.23 mL, $2.85 \mathrm{mmol}, 2.32 \mathrm{M}$ in Hexan) wurde die orange-rote Lösung 39 min bei $-78^{\circ} \mathrm{C}$ gerührt. In die Plastikkappe des Glasgefäßes, in welchem das $\mathrm{Ba}^{14} \mathrm{CO}_{3}$ geliefert worden war, wurde ein Loch gebohrt und zwischen Kappe und Flasche ein weiteres Gummiseptum eingefügt. Das

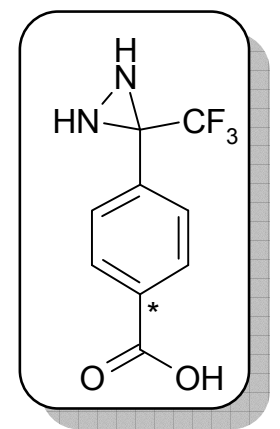
so preparierte Gefäß wurde über einen Teflonschlauch mit dem Schlenkrohr verbunden und die Carboxylierung wurde durch Zugabe von zwei Tropfen konz. $\mathrm{H}_{2} \mathrm{SO}_{4}$ zum $\mathrm{Ba}^{14} \mathrm{CO}_{3}$ $(50 \mathrm{mCi}, 0.91 \mathrm{mmol})$ gestartet. Das entstehende Gas wurde in die THF-Lösung mit der Aryllithiumverbindung eingeleitet. Um eventuelle Säurespuren im ${ }^{14} \mathrm{CO}_{2}$-Gasstrom zu entfernen, wurde ein $10 \mathrm{~cm}$ langes Stück des Teflonschlauches in einem Aceton/Trockeneisbad auf $-78{ }^{\circ} \mathrm{C}$ gekühlt. Nach dem Ende der Gasentwicklung wurde die restliche Schwefelsäure zugetropft ( $2 \mathrm{~mL}$ gesamt). Nachdem sich alles $\mathrm{Ba}^{14} \mathrm{CO}_{3}$ aufgelöst hatte, wurde der Teflonschlauch mit einer Gefäßklemme verschlossen und die resultierende Lösung für $5 \mathrm{~h}$ bei $-78^{\circ} \mathrm{C}$ gerührt. Die dunkelblaue Lösung wurde mit Wasser $(0.5 \mathrm{~mL})$ gequencht und auf RT erwärmt. Die Apparatur wurde mit $\mathrm{N}_{2}$ gespült und der Gasstrom über Natronkalk geleitet, um event. ${ }^{14} \mathrm{CO}_{2}$-Reste zu binden. Das Gemisch wurde mit Ether $(250 \mathrm{~mL})$ verdünnt und mit $\mathrm{NaOH}$-Lösung extrahiert $(1 \mathrm{M}, 3 \times 20 \mathrm{~mL})$. Die vereinigten wässrigen Phasen wurden auf $\mathrm{pH} \approx 1$ angesäuert, worauf sich ein farbloser Feststoff abschied, welcher mit Ether $(4 \times 50 \mathrm{~mL})$ extrahiert wurde. Die vereinigten organischen Phasen wurden mit Wasser $(3 \times 20 \mathrm{~mL})$ und NaCl-Lsg. $(1 \times 10 \mathrm{~mL})$ gewaschen und das Lösungsmittel im Vakuum entfernt. Man erhielt 164 mg (entspricht $78 \%$ des eingesetzten $\mathrm{Ba}^{14} \mathrm{CO}_{3}$ ) der Titelverbindung, welche kaum Verunreinigungen zeigte und direkt weiter oxidiert wurde. 
Allgemeine Arbeitsvorschrift 01: Oxidation zum Diazirin mit Triethylamin/Iod

4-(3-Trifluormethyl-3H-diazirin-3-yl)benzoesäure (48) und 4-(3-Trifluormethyl-3Hdiazirin-3-yl)-[1- $\left.{ }^{14} \mathrm{C}\right]$-benzoesäure (48a)

Diaziridin (71 oder 71a, jeweils $0.59 \mathrm{mmol})$ wurde in $\mathrm{MeOH}(15 \mathrm{~mL})$ und $\mathrm{NEt}_{3}$ (6 eq.) gelöst und bei RT tropfenweise mit einer Iodlösung (30 $\mathrm{mg} / \mathrm{mL}$ in $\mathrm{MeOH}$ ) versetzt. Die Lösung wurde solange zugetropft, bis die orange-rote Farbe des Iods länger als 1 min (ca. 1.2 eq $\mathrm{I}_{2}$ ) bestehen blieb. Die Lösung wurde unter Lichtausschluss 20-30 min weitergerührt, dann wurde das Methanol unter vermindertem Druck entfernt und der Rückstand in Ether aufgenommen. Die organische Phase wurde mit

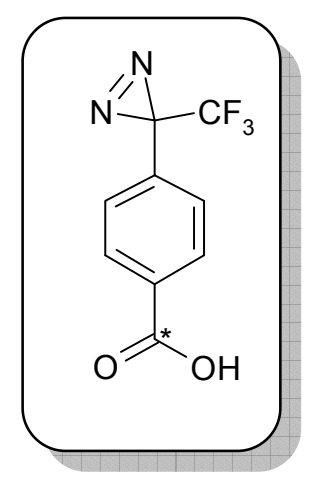
wässriger $\mathrm{NaOH}$-Lösung $(1 \mathrm{M})$ extrahiert. Die vereinte wässrige Phase wurde mit halbkonzentrierter $\mathrm{HCl}$ auf $\mathrm{pH} \approx 1$ angesäuert und das ausgefallene Produkt mit Ether extrahiert. Die vereinigten organischen Phasen wurden mit Wasser und NaCl-Lösung gewaschen und über $\mathrm{NaSO}_{4}$ getrocknet. Entfernen des Lösungsmittels unter reduziertem Druck ergab die Titelverbindung in Ausbeuten von 90 bis 99\%. Bei der Oxidation des ${ }^{14} \mathrm{C}$ markierten Diaziridins 71a wurde das Produkt 48a in $83 \%$ Ausbeute erhalten. Die spezifische Aktivität wurde mit einem Flüssigszintillationsmesser zu $44.11 \mathrm{mCi} / \mathrm{mmol}$ bestimmt. ${ }^{13} \mathrm{C}-\mathrm{NMR}\left(75.5 \mathrm{MHz}, \mathrm{CDCl}_{3}\right): \delta=28.4\left(\mathrm{q},{ }^{2} J_{\mathrm{C}-\mathrm{F}}=39.6 \mathrm{~Hz}, \mathrm{CN}_{2}\right), 121.8\left(\mathrm{q},{ }^{1} J_{\mathrm{C}-\mathrm{F}}\right.$ $\left.=274.7 \mathrm{~Hz}, \mathrm{CF}_{3}\right), 126.4$ (C-Ar), 130.2 (C-1), 130.5 (C-Ar), 134.8 (C-4), 171.3 (COOH). Anal. ber. (\%) für $\mathrm{C}_{9} \mathrm{H}_{5} \mathrm{~F}_{3} \mathrm{~N}_{2} \mathrm{O}_{2}$ (230.15): C 46.97, H 2.19, N 12.17; Gef. C 46.73, H 2.17, $\mathrm{N}$ 12.28. - Alle übrigen analytischen Daten waren konsistent mit den in der Literatur publizierten. ${ }^{[46]}$ 


\section{E.2.2 Kupplung des Photoaffinitätslabels an die Naturstoffe}

\section{3-O-[4-(3-Trifluormethyl-3H-diazirin-3-yl)benzoyl]-21-deoxyconcanolid A (75)}

Diazirin $48 \quad(36.0 \mathrm{mg}$, $156 \mu \mathrm{mol}) \quad$ wurde in Thionylchlorid $\quad(0.50 \mathrm{~mL})$ gelöst und $15 \mathrm{~h}$ bei RT gerührt. Anschließend wurde das Thionylchlorid sorgfältig unter vermindertem Druck (40 mbar) entfernt. Man erhielt so $38.5 \mathrm{mg}(99 \%)$ des

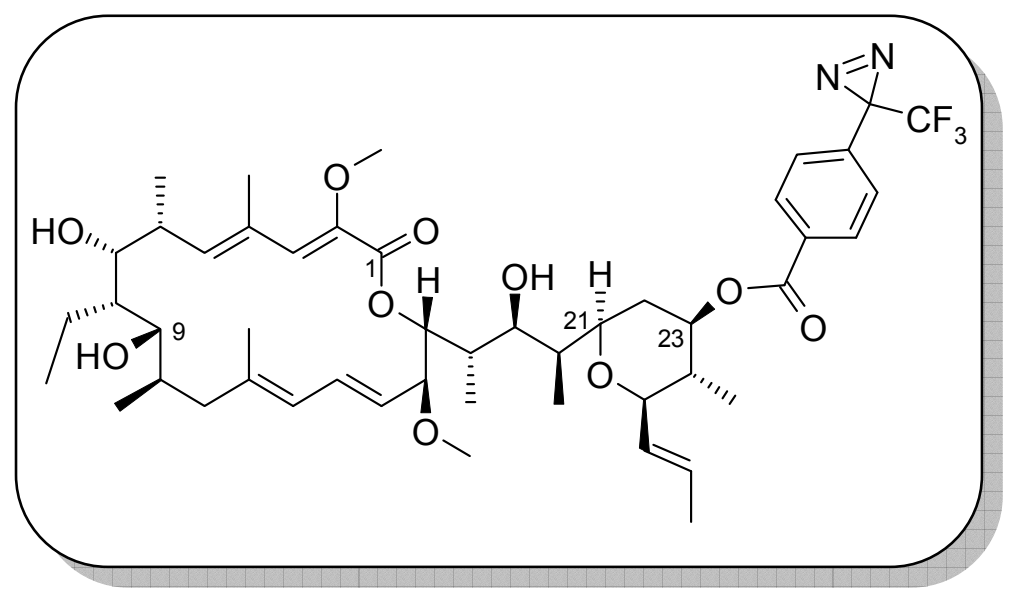
entsprechenden Säurechlorides (4-(3-Trifluormethyl-3H-diazirin-3-yl)benzoylchlorid, 76), welches in $\mathrm{CH}_{2} \mathrm{Cl}_{2}(3 \mathrm{~mL})$ gelöst wurde. 21-Deoxyconcanolid A (4, $\left.12.0 \mathrm{mg}, 17.7 \mu \mathrm{mol}\right)$ und DMAP $(11.5 \mathrm{mg}, 94.1 \mu \mathrm{mol})$ wurden in $\mathrm{CH}_{2} \mathrm{Cl}_{2}(1.5 \mathrm{~mL})$ gelöst und mit $\mathrm{NEt}_{3}(50 \mu \mathrm{L})$ versetzt. Das Säurechlorid $76(5.3 \mathrm{mg}, 21 \mu \mathrm{mol})$ wurde, gelöst in $\mathrm{CH}_{2} \mathrm{Cl}_{2}(0.41 \mathrm{~mL})$, zugetropft und die resultierende Lösung unter Lichtausschluß bei RT gerührt. Der Reaktionsfortschritt wurde per Dünnschichtchromatographie verfolgt. Nach $1.5 \mathrm{~h}$ zeigte sich ein unvollständiger Umsatz, weshalb erneut Benzoylchlorid 76 (2.7 mg, $11 \mu \mathrm{mol})$ in $\mathrm{CH}_{2} \mathrm{Cl}_{2}(0.21 \mathrm{~mL})$ zugesetzt wurde. Nach $4.5 \mathrm{~h}$ wurde außerdem noch weiteres DMAP (11.0 mg, $90.0 \mu \mathrm{mol})$ zugegeben. Die Reaktion wurde nach $7 \mathrm{~h}$ durch Zugabe von Wasser $(1 \mathrm{~mL})$ beendet und das Reaktionsgemisch mit Ether $(150 \mathrm{~mL})$ verdünnt. Die organische Phase wurde mit $\mathrm{NaHCO}_{3}$-Lösung $(1 \mathrm{M}, 5 \mathrm{~mL}), \mathrm{H}_{2} \mathrm{O}(5 \mathrm{~mL})$ und $\mathrm{NaCl}$-Lösung $(5 \mathrm{~mL})$ gewaschen und über $\mathrm{Na}_{2} \mathrm{SO}_{4}$ getrocknet. Man erhielt $51 \mathrm{mg}$ Rohprodukt, welches säulenchromatographisch aufgereinigt wurde (Hex:Aceton, 10:1). Es konnten $5.0 \mathrm{mg}$ (32\%) der Titelverbindung als farbloses Öl und $4.6 \mathrm{mg}$ (38\%) des Startmaterials 4 isoliert werden. $R_{\mathrm{f}}=0.25$ (Hex:Aceton, 6:1). $-{ }^{1} \mathrm{H}-\mathrm{NMR}\left(600 \mathrm{MHz}, \mathrm{CD}_{2} \mathrm{Cl}_{2}\right): \delta=0.80\left(\mathrm{~d},{ }^{3} J_{\mathrm{H}, \mathrm{H}}=7.6 \mathrm{~Hz}\right.$, $\left.3 \mathrm{H}, 18-\mathrm{CH}_{3}\right), 0.81\left(\mathrm{~d},{ }^{3} J_{\mathrm{H}, \mathrm{H}}=6.7 \mathrm{~Hz}, 3 \mathrm{H}, 20-\mathrm{CH}_{3}\right), 0.85\left(\mathrm{~d},{ }^{3} J_{\mathrm{H}, \mathrm{H}}=7.6 \mathrm{~Hz}, 3 \mathrm{H}, 24-\mathrm{CH}_{3}\right)$, $0.85\left(\mathrm{~m}_{\mathrm{c}}, 3 \mathrm{H}, 8-\mathrm{CH}_{2} \mathrm{CH}_{3}\right), 1.00-1.10\left(\mathrm{~m}, 3 \mathrm{H}, 6-\mathrm{CH}_{3}\right), 1.05\left(\mathrm{~d},{ }^{3} J_{\mathrm{H}, \mathrm{H}}=7.0 \mathrm{~Hz}, 3 \mathrm{H}, 10-\right.$ $\left.\mathrm{CH}_{3}\right), 1.13-1.22\left(\mathrm{~m}, 2 \mathrm{H}, 8-\mathrm{CH}_{2} \mathrm{CH}_{3}\right), 1.22-1.30(\mathrm{~m}, 1 \mathrm{H}, 24-\mathrm{H}), 1.48\left(\mathrm{~m}_{\mathrm{c}}, 1 \mathrm{H}, 8-\mathrm{H}\right)$, $1.60\left(\mathrm{~m}_{\mathrm{c}}, 1 \mathrm{H}, 20-\mathrm{H}\right), 1.64\left(\mathrm{dd}, J_{\mathrm{H}, \mathrm{H}}=1.5,6.5 \mathrm{~Hz}, 3 \mathrm{H}, 28-\mathrm{H}_{3}\right), 1.64-1.68\left(\mathrm{~m}, 2 \mathrm{H}, 22-\mathrm{H}_{2}\right)$, 1.84 (s, br, $\left.3 \mathrm{H}, 12-\mathrm{CH}_{3}\right), 1.92-1.99\left(\mathrm{~m}, 2 \mathrm{H}, 11-\mathrm{H}_{2}\right), 1.96\left(\mathrm{~s}, 3 \mathrm{H}, 4-\mathrm{CH}_{3}\right), 2.02\left(\mathrm{~m}_{\mathrm{c}}, 1 \mathrm{H}\right.$, 18-H), $2.23\left(\mathrm{~m}_{\mathrm{c}}, 1 \mathrm{H}, 10-\mathrm{H}\right), 2.72\left(\mathrm{~m}_{\mathrm{c}}, 1 \mathrm{H}, 6-\mathrm{H}\right), 3.13-3.22(\mathrm{~m}, 1 \mathrm{H}, 9-\mathrm{H}), 3.22(\mathrm{~s}, 3 \mathrm{H}$, 
16- $\left.-\mathrm{OCH}_{3}\right), 3.47\left(\mathrm{dd},{ }^{3} J_{\mathrm{H}, \mathrm{H}}=9.7,7.8 \mathrm{~Hz}, 1 \mathrm{H}, 25-\mathrm{H}\right), 3.55\left(\mathrm{~s}, 3 \mathrm{H}, 2-\mathrm{OCH}_{3}\right), 3.56-3.61(\mathrm{~m}$, $2 \mathrm{H}, 7-\mathrm{H}, 19-\mathrm{H}), 3.76\left(\mathrm{~m}_{\mathrm{c}}, 1 \mathrm{H}, 21-\mathrm{H}\right), 3.82\left(\mathrm{t},{ }^{3} J_{\mathrm{H}, \mathrm{H}}=8.9 \mathrm{~Hz}, 1 \mathrm{H}, 16-\mathrm{H}\right), 4.88\left(\mathrm{dt},{ }^{3} J_{\mathrm{H}, \mathrm{H}}=\right.$ 4.8, 10.8 Hz, $1 \mathrm{H}, 23-\mathrm{H}), 5.14\left(\mathrm{~m}_{\mathrm{c}}, 1 \mathrm{H}, 17-\mathrm{H}\right), 5.21\left(\mathrm{~m}_{\mathrm{c}}, 1 \mathrm{H}, 15-\mathrm{H}\right), 5.36\left(\mathrm{ddq}, J_{\mathrm{H}, \mathrm{H}}=1.6\right.$, 7.6, 15.0 Hz, $1 \mathrm{H}, 26-\mathrm{H}), 5.63\left(\mathrm{dq},{ }^{3} J_{\mathrm{H}, \mathrm{H}}=6.4,15.0 \mathrm{~Hz}, 1 \mathrm{H}, 27-\mathrm{H}\right), 5.67\left(\mathrm{~m}_{\mathrm{c}}, 1 \mathrm{H}, 5-\mathrm{H}\right)$, $5.79\left(\mathrm{~d},{ }^{3} J_{\mathrm{H}, \mathrm{H}}=10.5 \mathrm{~Hz}, 1 \mathrm{H}, 13-\mathrm{H}\right), 6.37(\mathrm{~s}, \mathrm{br}, 1 \mathrm{H}, 3-\mathrm{H}), 6.54\left(\mathrm{dd},{ }^{3} J_{\mathrm{H}, \mathrm{H}}=10.5,15.0 \mathrm{~Hz}\right.$, $1 \mathrm{H}, 14-\mathrm{H}), 7.26\left(\mathrm{~d},{ }^{3} J_{\mathrm{H}, \mathrm{H}}=8.3 \mathrm{~Hz}, 2 \mathrm{H}, \mathrm{Ar}-\mathrm{H}\right), 8.05\left(\mathrm{~d},{ }^{3} J_{\mathrm{H}, \mathrm{H}}=8.3 \mathrm{~Hz}, 2 \mathrm{H}, \mathrm{Ar}-\mathrm{H}\right) .-{ }^{13} \mathrm{C}-$ NMR (150.8 MHz, $\left.\mathrm{CD}_{2} \mathrm{Cl}_{2}\right): \delta=8.0\left(20-\mathrm{CH}_{3}\right), 9.4\left(18-\mathrm{CH}_{3}\right), 11.5\left(8-\mathrm{CH}_{2} \mathrm{CH}_{3}\right), 13.3$ (24$\left.\mathrm{CH}_{3}\right), 14.1\left(4-\mathrm{CH}_{3}\right), 16.2\left(12-\mathrm{CH}_{3}\right), 16.7\left(6-\mathrm{CH}_{3}\right), 17.7(\mathrm{C}-28), 21.4\left(10-\mathrm{CH}_{3}\right), 22.8(8-$ $\left.\mathrm{CH}_{2} \mathrm{CH}_{3}\right), 28.4\left(\mathrm{q},{ }^{2} J_{\mathrm{C}-\mathrm{F}}=41.6 \mathrm{~Hz}, \mathrm{CN}_{2}\right), 34.6(\mathrm{C}-6), 35.3(\mathrm{C}-10), 36.4(\mathrm{C}-22), 37.3(\mathrm{C}-18)$, 39.1 (C-20), 40.7 (C-11), $43.4(\mathrm{C}-24), 44.5(\mathrm{C}-8), 55.7\left(16-\mathrm{OCH}_{3}\right), 59.1\left(2-\mathrm{OCH}_{3}\right), 69.1$ (C-19), 74.2 (C-7), 75.7 (C-17), 76.4 (C-21), 77.1 (C-23), 79.4 (C-9), 82.1 (C-16), 82.6 (C25), $121.8\left(\mathrm{q},{ }^{1} J_{\mathrm{C}-\mathrm{F}}=274.8 \mathrm{~Hz}, \mathrm{CF}_{3}\right), 122.9(\mathrm{C}-13), 126.1(\mathrm{C}-\mathrm{Ar}), 127.1(\mathrm{C}-15), 129.1(\mathrm{C}-$ 27), 129.8 (C-Ar), 130.1 (C-Ar), 130.2 (C-26), 131.4 (C-3), 132.0 (C-4), 133.1 (C-Ar), 133.4 (C-14), 139.6 (C-12), 142.0 (C-2), 142.0 (C-5), $164.9\left(\mathrm{CO}_{2}\right), 165.7$ (C-1). - IR (KBr): $\widetilde{v}=3446,2967,2930,1718,1700,1458,1382,1343,1276,1196,1159,1106 \mathrm{~cm}^{-1}$. UV $(\mathrm{MeOH}): \lambda_{\max }(\lg \varepsilon)=240(1.28), 282(0.454) \mathrm{nm}$. - MS (ESI), $m / z(\%): 825$ (20) [M $2 \mathrm{MeOH}+\mathrm{H}]^{+}, 858(100)[\mathrm{M}-\mathrm{MeOH}+\mathrm{H}]^{+}, 912(30)[\mathrm{M}+\mathrm{Na}]^{+}$; HRMS (ESI): Ber. für $\mathrm{C}_{48} \mathrm{H}_{67} \mathrm{~F}_{3} \mathrm{~N}_{2} \mathrm{NaO}_{10}$ : $911.46400[\mathrm{M}+\mathrm{Na}]^{+}$, Gef. 911.46398.

23-O-[4-(3-Trifluormethyl-3H-diazirin-3-yl)-[1- ${ }^{14}$ C]-benzoyl]-21-deoxyconcanolid A (75a)

Die Reaktion wurde wie oben beschrieben durchgeführt. Dabei wurden folgende Mengen Edukt eingesetzt: 21-Deoxyconcanolid A (4, $12.5 \mathrm{mg}, 18.5 \mu \mathrm{mol})$, Diazirinyllabel 76a (7.17 mg, $28.9 \mu \mathrm{mol})$. Es wurden $4.6 \mathrm{mg}$ (36\%) der Titelverbindung isoliert und $3.9 \mathrm{mg}$ (31\%) des 21-Deoxyconcanolid A (4) zurückgewonnen. Das ${ }^{1} \mathrm{H}-\mathrm{NMR}$ Spektrum der ${ }^{14} \mathrm{C}$ Verbindung war konsistent mit dem Spektrum des nichtaktiven Derivates 75. Die spezifische Aktivität wurde mit einem Flüssigszintillationsmesser zu $40.0 \mathrm{mCi} / \mathrm{mmol}$ bestimmt. 
Bafilomycin $\mathrm{A}_{1} \quad(2, \quad 16.4 \mathrm{mg}$, $26.3 \mu \mathrm{mol}), \quad$ DMAP $\quad(7.8 \mathrm{mg}$, $64 \mu \mathrm{mol})$, Diazirin 48 (12.6 mg, $54.7 \mu \mathrm{mol}) \quad$ und Ethyl-(3dimethylamino-

propyl)carbodiimid-Hydro-

chlorid (EDCI, $11.1 \mathrm{mg}$, $57.9 \mu \mathrm{mol}) \quad$ wurden in

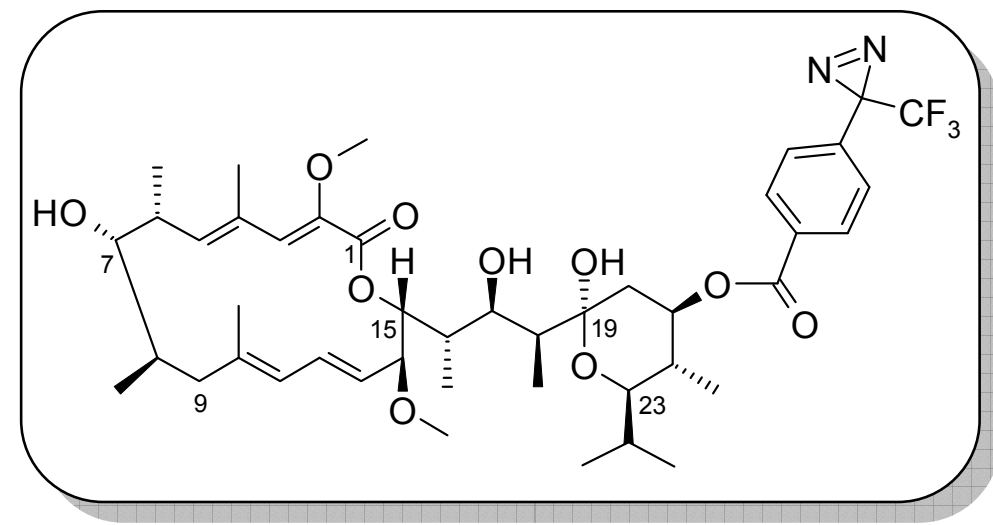
wasserfreiem $\mathrm{CH}_{2} \mathrm{Cl}_{2}(1.5 \mathrm{~mL})$ gelöst und die erhaltene Lösung $16 \mathrm{~h}$ unter Lichtausschluß bei RT gerührt. Das Reaktionsgemisch wurde mit Ether $(150 \mathrm{~mL})$ verdünnt und mit $\mathrm{NaHCO}_{3}$-Lösung $(5 \mathrm{~mL}), \mathrm{H}_{2} \mathrm{O}(5 \mathrm{~mL})$ und $\mathrm{NaCl}$-Lösung $(5 \mathrm{~mL})$ gewaschen. Nach Trocknen über $\mathrm{Na}_{2} \mathrm{SO}_{4}$ wurde das Lösungsmittel unter vermindertem Druck entfernt. Das erhaltene Rohprodukt wurde säulenchromatographisch gereinigt (Hex:Aceton, 6:1). Man erhielt $9.1 \mathrm{mg}$ (41\%) der Titelverbindung als farbloses Öl und reisolierte $3.6 \mathrm{mg}$ (22\%) des Startmaterials 2. $R_{\mathrm{f}}=0.25$ (Hex:Aceton, 6:1). ${ }^{1} \mathrm{H}-\mathrm{NMR}\left(600 \mathrm{MHz}, \mathrm{CD}_{2} \mathrm{Cl}_{2}\right): \delta=0.80(\mathrm{~d}$, $\left.{ }^{3} J_{\mathrm{H}, \mathrm{H}}=7.0 \mathrm{~Hz}, 3 \mathrm{H}, 24-\mathrm{CH}_{3}\right), 0.83\left(\mathrm{~d},{ }^{3} J_{\mathrm{H}, \mathrm{H}}=7.9 \mathrm{~Hz}, 3 \mathrm{H}, 16-\mathrm{CH}_{3}\right), 0.83\left(\mathrm{~d},{ }^{3} J_{\mathrm{H}, \mathrm{H}}=6.7 \mathrm{~Hz}\right.$, $\left.3 \mathrm{H}, 8-\mathrm{CH}_{3}\right), 0.91\left(\mathrm{~d},{ }^{3} J_{\mathrm{H}, \mathrm{H}}=6.1 \mathrm{~Hz}, 3 \mathrm{H}, 24-\mathrm{CH}_{3}\right), 0.92\left(\mathrm{~d},{ }^{3} J_{\mathrm{H}, \mathrm{H}}=6.1 \mathrm{~Hz}, 3 \mathrm{H}, 22-\mathrm{CH}_{3}\right)$, $0.99\left(\mathrm{~d},{ }^{3} J_{\mathrm{H}, \mathrm{H}}=7.2 \mathrm{~Hz}, 3 \mathrm{H}, 18-\mathrm{CH}_{3}\right), 1.03\left(\mathrm{~d},{ }^{3} J_{\mathrm{H}, \mathrm{H}}=7.0 \mathrm{~Hz}, 3 \mathrm{H}, 6-\mathrm{CH}_{3}\right), 1.11\left(\mathrm{dd}, J_{\mathrm{H}, \mathrm{H}}\right.$ $\left.=7.4,13.2 \mathrm{~Hz}, 1 \mathrm{H}, 20-\mathrm{H}_{\mathrm{a}}\right), 1.76-1.81(\mathrm{~m}, 1 \mathrm{H}, 18-\mathrm{H}), 1.82-1.94\left(\mathrm{~m}, 4 \mathrm{H}, 8-\mathrm{H}, 9-\mathrm{H}_{\mathrm{a}}, 22-\mathrm{H}\right.$, 24-H), $1.92\left(\mathrm{~s}, 3 \mathrm{H}, 10-\mathrm{CH}_{3}\right), 1.98\left(\mathrm{~d},{ }^{3} J_{\mathrm{H}, \mathrm{H}}=1.1 \mathrm{~Hz}, 3 \mathrm{H}, 4-\mathrm{CH}_{3}\right), 2.06-2.16\left(\mathrm{~m}, 2 \mathrm{H}, 9-\mathrm{H}_{\mathrm{b}}\right.$, $16-\mathrm{H}), 2.23\left(\mathrm{dd},{ }^{3} J_{\mathrm{H}, \mathrm{H}}=4.8,11.8 \mathrm{~Hz}, 1 \mathrm{H}, 20-\mathrm{H}_{\mathrm{b}}\right), 2.53\left(\mathrm{ddq}, J_{\mathrm{H}, \mathrm{H}}=2.0,7.1,9.1 \mathrm{~Hz}, 1 \mathrm{H}\right.$, 6-H), $3.22\left(\mathrm{~s}, 3 \mathrm{H}, 14-\mathrm{OCH}_{3}\right), 3.27\left(\mathrm{~m}_{\mathrm{c}}, 1 \mathrm{H}, 7-\mathrm{H}\right), 3.61\left(\mathrm{~s}, 3 \mathrm{H}, 2-\mathrm{OCH}_{3}\right), 3.64(\mathrm{dd}$, $\left.J_{\mathrm{H}, \mathrm{H}}=2.2,10.3 \mathrm{~Hz}, 1 \mathrm{H}, 23-\mathrm{H}\right), 3.88\left(\mathrm{t},{ }^{3} J_{\mathrm{H}, \mathrm{H}}=9.0 \mathrm{~Hz}, 1 \mathrm{H}, 14-\mathrm{H}\right), 4.12\left(\mathrm{ddd}, J_{\mathrm{H}, \mathrm{H}}=1.9\right.$, $4.0,10.7 \mathrm{~Hz}, 1 \mathrm{H}, 17-\mathrm{H}), 4.65\left(\mathrm{dd}, J_{\mathrm{H}, \mathrm{H}}=0.7,4.0 \mathrm{~Hz}, 1 \mathrm{H}, 17-\mathrm{OH}\right), 4.88\left(\mathrm{dd},{ }^{3} J_{\mathrm{H}, \mathrm{H}}=1.2\right.$, $8.6 \mathrm{~Hz}, 1 \mathrm{H}, 15-\mathrm{H}), 5.07-5.17$ (m, $2 \mathrm{H}, 13-\mathrm{H}, 21-\mathrm{H}), 5.46\left(\mathrm{~d},{ }^{3} J_{\mathrm{H}, \mathrm{H}}=2.1 \mathrm{~Hz}, 1 \mathrm{H}, 19-\mathrm{OH}\right)$, $5.77\left(\mathrm{~d},{ }^{3} J_{\mathrm{H}, \mathrm{H}}=9.1 \mathrm{~Hz}, 1 \mathrm{H}, 5-\mathrm{H}\right), 5.81\left(\mathrm{~d},{ }^{3} J_{\mathrm{H}, \mathrm{H}}=10.8 \mathrm{~Hz}, 1 \mathrm{H}, 11-\mathrm{H}\right), 6.53(\mathrm{dd}$, $\left.{ }^{3} J_{\mathrm{H}, \mathrm{H}}=10.8,15.0 \mathrm{~Hz}, 1 \mathrm{H}, 12-\mathrm{H}\right), 6.68\left(\mathrm{~d},{ }^{4} J_{\mathrm{H}, \mathrm{H}}=0.6 \mathrm{~Hz}, 1 \mathrm{H}, 3-\mathrm{H}\right), 7.26\left(\mathrm{~d},{ }^{3} J_{\mathrm{H}, \mathrm{H}}=8.2 \mathrm{~Hz}\right.$, $2 \mathrm{H}, \mathrm{Ar}-\mathrm{H}), 8.05\left(\mathrm{~d},{ }^{3} J_{\mathrm{H}, \mathrm{H}}=8.2 \mathrm{~Hz}, 2 \mathrm{H}, \mathrm{Ar}-\mathrm{H}\right) .-{ }^{13} \mathrm{C}-\mathrm{NMR}\left(150.8 \mathrm{MHz}, \mathrm{CD}_{2} \mathrm{Cl}_{2}\right): \delta=7.1$ $\left(18-\mathrm{CH}_{3}\right), 9.8\left(16-\mathrm{CH}_{3}\right), 12.4\left(22-\mathrm{CH}_{3}\right), 14.0\left(4-\mathrm{CH}_{3}\right), 14.3\left(24-\mathrm{CH}_{3}\right), 17.3\left(6-\mathrm{CH}_{3}\right), 20.2$ $\left(10-\mathrm{CH}_{3}\right), 21.2\left(24-\mathrm{CH}_{3}\right), 21.7\left(8-\mathrm{CH}_{3}\right), 28.3(\mathrm{C}-24), 37.0(\mathrm{C}-6), 37.5(\mathrm{C}-16), 40.5(\mathrm{C}-22)$, 41.5 (C-8), 41.5 (C-9), 42.2 (C-18), $53.4(\mathrm{C}-20), 55.7\left(14-\mathrm{OCH}_{3}\right), 60.2\left(2-\mathrm{OCH}_{3}\right), 71.0(\mathrm{C}-$ 17), 75.6 (C-21), 76.0 (C-23), 77.0 (C-15), 81.2 (C-7), 82.6 (C-14), 99.2 (C-19), 125.4 (C11), 126.6 (C-Ar), 127.1 (C-13), 130.1 (C-Ar), 132.3 (C-Ar), 133.2 (C-4), 133.5 (C-12), 
133.6 (C-Ar), 134.0 (C-3), 141.5 (C-2), 143.5 (C-5), 143.6 (C-10), $165.2\left(\mathrm{CO}_{2}\right), 167.6$ (C1). - IR (KBr): $\widetilde{v}=3422,2930,1718,1275,1194,1159,1101 \mathrm{~cm}^{-1} .-\mathrm{UV}(\mathrm{MeOH}): \lambda_{\max }$ $(\lg \varepsilon)=241(0.460), 285(0.170)$ nm. - MS (ESI), $m / z(\%): 853(34)\left[\mathrm{M}+\mathrm{NH}_{4}\right]^{+}, 858(100)$ $[\mathrm{M}+\mathrm{Na}]^{+}$; HRMS (ESI): Ber. für $\mathrm{C}_{44} \mathrm{H}_{61} \mathrm{~F}_{3} \mathrm{~N}_{2} \mathrm{NaO}_{10}$ : $857.41715[\mathrm{M}+\mathrm{Na}]^{+}$, Gef. 857.41705 .

21-O-[4-(3-Trifluormethyl-3H-diazirin-3-yl)-[1- $\left.{ }^{14} \mathrm{C}\right]$-benzoyl]bafilomycin $A_{1}(\mathbf{7 7 a})$

Die Reaktion wurde wie oben beschrieben durchgeführt. Dabei wurden folgende Mengen Edukt eingesetzt: Bafilomycin $A_{1}(2,15.7 \mathrm{mg}, 25.2 \mu \mathrm{mol})$, Diazirinlabel 48a (11.6 mg, $50.4 \mu \mathrm{mol})$. Es wurden $8.6 \mathrm{mg}(41 \%)$ der Titelverbindung isoliert und $4.9 \mathrm{mg}(31 \%) \mathrm{des}$ Bafilomycin $A_{1}$ (2) zurückgewonnen. Das ${ }^{1} \mathrm{H}-\mathrm{NMR}$ Spektrum der ${ }^{14} \mathrm{C}$-Verbindung war konsistent mit dem Spektrum des nichtaktiven Derivates 75. Die spezifische Aktivität wurde mit einem Flüssigszintillationsmesser zu $33.7 \mathrm{mCi} / \mathrm{mmol}$ bestimmt.

8-O-[4-(3-Trifluormethyl-3H-diazirin-3-yl)benzoyl]kendomycin-acetonid (82)

Das Acetonid $81(10.0 \mathrm{mg}, 18.4 \mu \mathrm{mol})$ und DMAP $(30.1 \mathrm{mg}, 247 \mu \mathrm{mol})$ wurden in wasserfreiem Dichlormethan $(1.5 \mathrm{~mL})$ gelöst und mit in Dichlormethan gelöstem Diazirinylbenzoesäurechlorid $\mathbf{7 6}(7.0 \mathrm{mg}$ $28 \mu \mathrm{mol})$ versetzt. Die Lösung wurde unter Lichtausschluß 20 min bei RT gerührt und dann direkt auf eine preparative DC-Platte

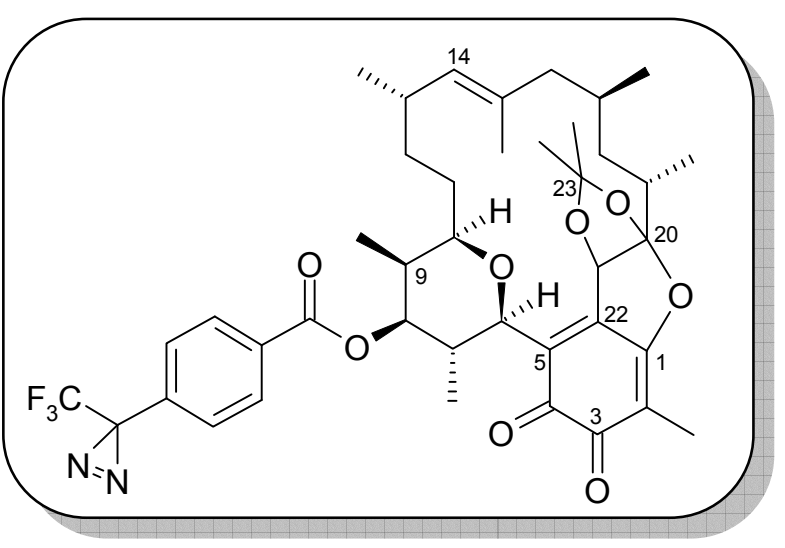
aufgetragen. (KG, Hex:EE, 2:1). Die Titelverbindung konnte als roter amorpher Feststoff isoliert werden: $6.8 \mathrm{mg}$ (49\%). $R_{\mathrm{f}}=0.75$ (Hex:EE, 2:1). $-{ }^{1} \mathrm{H}-\mathrm{NMR}\left(600 \mathrm{MHz},\left[\mathrm{D}_{6}\right]-\right.$ Aceton): $\delta=0.75\left(\mathrm{~d},{ }^{3} J_{\mathrm{H}, \mathrm{H}}=6.6 \mathrm{~Hz}, 3 \mathrm{H}, 7-\mathrm{CH}_{3}\right), 0.90\left(\mathrm{~d},{ }^{3} J_{\mathrm{H}, \mathrm{H}}=6.6 \mathrm{~Hz}, 3 \mathrm{H}, 13-\mathrm{CH}_{3}\right)$, $0.93\left(\mathrm{~d},{ }^{3} J_{\mathrm{H}, \mathrm{H}}=7.0 \mathrm{~Hz}, 3 \mathrm{H}, 19-\mathrm{CH}_{3}\right), 0.97\left(\mathrm{~d},{ }^{3} J_{\mathrm{H}, \mathrm{H}}=6.5 \mathrm{~Hz}, 3 \mathrm{H}, 9-\mathrm{CH}_{3}\right), 1.04\left(\mathrm{~d},{ }^{3} J_{\mathrm{H}, \mathrm{H}}=\right.$ $\left.7.0 \mathrm{~Hz}, 3 \mathrm{H}, 17-\mathrm{CH}_{3}\right), 1.27-1.43\left(\mathrm{~m}, 5 \mathrm{H}, 12-\mathrm{H}_{2}, 18-\mathrm{H}_{2}, 11-\mathrm{H}_{\mathrm{a}}\right), 1.48\left(\mathrm{~s}, 3 \mathrm{H}, 23-\mathrm{CH}_{3}\right)$, $1.52\left(\mathrm{~s}, 3 \mathrm{H}, 23-\mathrm{CH}_{3}\right), 1.57-1.60\left(\mathrm{~m}, 1 \mathrm{H}, 11-\mathrm{H}_{\mathrm{b}}\right), 1.60\left(\mathrm{~s}, 3 \mathrm{H}, 15-\mathrm{CH}_{3}\right), 1.63-1.71(\mathrm{~m}, 2 \mathrm{H}$, 7-H, 16- $\mathrm{H}_{\mathrm{a}}$ ), 1.83 (s, $\left.3 \mathrm{H}, 2-\mathrm{CH}_{3}\right), 2.18-2.26$ (m, $\left.3 \mathrm{H}, 9-\mathrm{H}, 16-\mathrm{H}_{\mathrm{b}}, 17-\mathrm{H}\right), 2.39\left(\mathrm{~m}_{\mathrm{c}}, 1 \mathrm{H}\right.$, $13-\mathrm{H}), 2.46\left(\mathrm{ddq}, J_{\mathrm{H}, \mathrm{H}}=2.7,7.0,13.9 \mathrm{~Hz}, 1 \mathrm{H}, 19-\mathrm{H}\right), 3.71\left(\mathrm{dd},{ }^{3} J_{\mathrm{H}, \mathrm{H}}=2.1,10.3 \mathrm{~Hz}, 1 \mathrm{H}\right.$, 
$10-\mathrm{H}), 4.47\left(\mathrm{~d},{ }^{3} J_{\mathrm{H}, \mathrm{H}}=10.2 \mathrm{~Hz}, 1 \mathrm{H}, 6-\mathrm{H}\right), 4.73\left(\mathrm{~d}, \mathrm{br},{ }^{3} J_{\mathrm{H}, \mathrm{H}}=9.8 \mathrm{~Hz}, 1 \mathrm{H}, 14-\mathrm{H}\right), 5.07$ (dd, $\left.{ }^{3} J_{\mathrm{H}, \mathrm{H}}=4.7,11.2 \mathrm{~Hz}, 1 \mathrm{H}, 8-\mathrm{H}\right), 6.04(\mathrm{~s}, 1 \mathrm{H}, 21-\mathrm{H}), 7.45\left(\mathrm{~d},{ }^{3} J_{\mathrm{H}, \mathrm{H}}=8.5 \mathrm{~Hz}, 2 \mathrm{H}, \mathrm{Ar}-\mathrm{H}\right)$, $8.15\left(\mathrm{~d},{ }^{3} J_{\mathrm{H}, \mathrm{H}}=8.5 \mathrm{~Hz}, 2 \mathrm{H}, \mathrm{Ar}-\mathrm{H}\right) .-{ }^{13} \mathrm{C}-\mathrm{NMR}(150.8 \mathrm{MHz}$, Aceton-D 6$): \delta=7.0\left(9-\mathrm{CH}_{3}\right)$, $8.0\left(2-\mathrm{CH}_{3}\right), 13.2\left(7-\mathrm{CH}_{3}\right), 13.7\left(13-\mathrm{CH}_{3}\right), 19.7\left(15-\mathrm{CH}_{3}\right), 20.3\left(17-\mathrm{CH}_{3}\right), 22.7\left(19-\mathrm{CH}_{3}\right)$, $26.3(\mathrm{C}-17), 27.2\left(23-\mathrm{CH}_{3}\right), 28.3\left(23-\mathrm{CH}_{3}\right), 29.1$ (q, $\left.{ }^{2} J_{\mathrm{C}-\mathrm{F}}=40.5 \mathrm{~Hz}, \mathrm{CN}_{2}\right), 29.9(11), 33.2$ (C-13), 33.5 (C-19), 36.1 (C-18), 37.5 (C-12), 37.9 (C-7), 38.2 (C-9), 45.8 (C-16), 76.2 (C21), 76.5 (C-6), 78.8 (C-10), 79.8 (C-8), 110.4 (C-2), 116.6 (C-22), 122.9 (q, ${ }^{1} J_{\mathrm{C}-\mathrm{F}}=273.2$ $\mathrm{Hz}, \mathrm{CF}_{3}$ ), 124.5 (C-20), 127.6 (C-Ar), 129.8 (C-14), 130.9 (C-Ar), 132.4 (C-15), 132.6 (CAr), 134.0 (C-Ar), 136.9 (C-23), 146.5 (C-4), 164.9 (C-1), 165.2 (COO), 178.0 (C-3), 179.8 (C-5). - IR (KBr): $\widetilde{v}=3447,2928,2373,1718,1627,1458,1382,1342,1278,1196$, $1163,1104,1047,802 \mathrm{~cm}^{-1}$. - UV (MeOH): $\lambda_{\max }(\lg \varepsilon)=233(0.617) \mathrm{nm} .-\mathrm{MS}(\mathrm{ESI}), \mathrm{m} / z$ (\%): $755(30)[\mathrm{M}+\mathrm{H}]^{+}, 777(100)[\mathrm{M}+\mathrm{Na}]^{+}, 793(28), 813(24)\left[\mathrm{M}+2 \mathrm{H}_{2} \mathrm{O}+\mathrm{Na}\right]^{+}, 828$ (10), 856 (11), 990 (25), 1043 (24), 1055 (32), 1168 (15); HRMS (ESI): Ber. für $\mathrm{C}_{41} \mathrm{H}_{49} \mathrm{~F}_{3} \mathrm{~N}_{2} \mathrm{NaO}_{8}: 777.33332[\mathrm{M}+\mathrm{Na}]^{+}$, Gef. 777.33324.

8-O-[4-(3-Trifluormethyl-3H-diazirin-3-yl)-[1- ${ }^{14}$ C]benzoyl]-kendomycinacetonid (82a)

Die Reaktion wurde wie oben beschrieben durchgeführt. Dabei wurden folgende Mengen Edukt eingesetzt: Kendomycin-Acetonid (81, $10.1 \mathrm{mg}, 18.5 \mu \mathrm{mol})$, Diazirinlabel 76a (9.7 mg, $39 \mu \mathrm{mol})$, DMAP (178 $\mu \mathrm{mol})$. Es wurden $5.2 \mathrm{mg}$ (37\%) der Titelverbindung isoliert. Das ${ }^{1} \mathrm{H}$-NMR Spektrum der ${ }^{14} \mathrm{C}$-Verbindung war konsistent mit dem Spektrum des nichtaktiven Derivates 82. Die spezifische Aktivität wurde mit einem Flüssigszintillationsmesser zu $42.3 \mathrm{mCi} / \mathrm{mmol}$ bestimmt.

\section{Kendomycin-methylacetal (83)}

Kendomycin $78 \quad(85.1 \mathrm{mg}, \quad 175 \mu \mathrm{mol})$ und $p$ Toluolsulfonsäure $(29.1 \mathrm{mg}, 153 \mu \mathrm{mol})$ wurden in $\mathrm{MeOH}$ $(5 \mathrm{~mL})$ gelöst und drei Tage bei RT gerührt. Das Reaktionsgemisch wurde anschließend direkt einer Größenausschluss-Chromatographie unterzogen (Sephadex LH-20, Methanol). Die Titelverbindung wurde als gelber

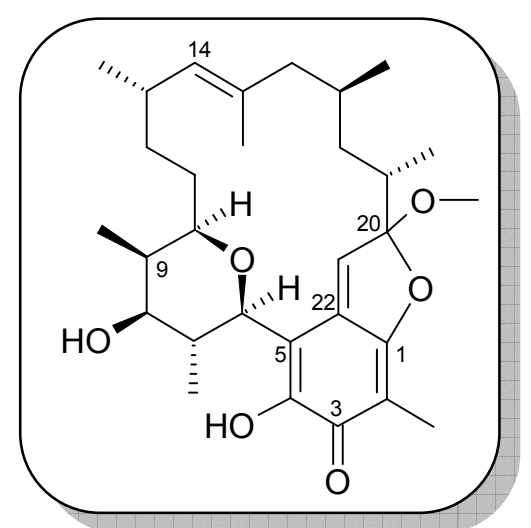


Feststoff isoliert: $87.0 \mathrm{mg}$ (99\%). $\mathrm{R}_{f}=0.56$ (Hex:EE, 2:1). - Smp. $=108-111^{\circ} \mathrm{C}$ (Zersetzung). $-{ }^{1} \mathrm{H}-\mathrm{NMR}\left(600 \mathrm{MHz}, \mathrm{CD}_{2} \mathrm{Cl}_{2}\right): \delta=0.66\left(\mathrm{~d},{ }^{3} J_{\mathrm{H}, \mathrm{H}}=6.8 \mathrm{~Hz}, 3 \mathrm{H}, 19-\mathrm{CH}_{3}\right)$, $0.86\left(\mathrm{~d},{ }^{3} J_{\mathrm{H}, \mathrm{H}}=6.6 \mathrm{~Hz}, 3 \mathrm{H}, 13-\mathrm{CH}_{3}\right), 0.90\left(\mathrm{~d},{ }^{3} J_{\mathrm{H}, \mathrm{H}}=7.0 \mathrm{~Hz}, 3 \mathrm{H}, 7-\mathrm{CH}_{3}\right), 0.91\left(\mathrm{~d},{ }^{3} J_{\mathrm{H}, \mathrm{H}}=\right.$ $\left.6.5 \mathrm{~Hz}, 3 \mathrm{H}, 17-\mathrm{CH}_{3}\right), 0.93\left(\mathrm{~d},{ }^{3} J_{\mathrm{H}, \mathrm{H}}=7.0 \mathrm{~Hz}, 3 \mathrm{H}, 9-\mathrm{CH}_{3}\right), 1.20\left(\mathrm{~m}_{\mathrm{c}}, 1 \mathrm{H}, 12-\mathrm{H}_{\mathrm{a}}\right), 1.23-$ $1.36\left(\mathrm{~m}, 5 \mathrm{H}, 11-\mathrm{H}_{2}, 12-\mathrm{H}_{\mathrm{b}}, 18-\mathrm{H}_{2}\right), 1.50\left(\mathrm{~m}_{\mathrm{c}}, 1 \mathrm{H}, 16-\mathrm{H}_{\mathrm{a}}\right), 1.57\left(\mathrm{~s}, 3 \mathrm{H}, 15-\mathrm{CH}_{3}\right), 1.60-$ $1.70\left(\mathrm{~m}, 2 \mathrm{H}, 7-\mathrm{H}, 16-\mathrm{H}_{\mathrm{b}}\right) 1.87\left(\mathrm{~m}_{\mathrm{c}}, 1 \mathrm{H}, 9-\mathrm{H}\right), 1.91\left(\mathrm{~s}, 3 \mathrm{H}, 2-\mathrm{CH}_{3}\right), 1.94\left(\mathrm{~m}_{\mathrm{c}}, 1 \mathrm{H}, 17-\mathrm{H}\right)$, 2.27-2.39 (m, $2 \mathrm{H}, 19-\mathrm{H}, 13-\mathrm{H}), 3.23$ (s, $\left.3 \mathrm{H}, 20-\mathrm{OCH}_{3}\right), 3.49\left(\mathrm{~d}_{\mathrm{br}},{ }^{3} J_{\mathrm{H}, \mathrm{H}}=11.8 \mathrm{~Hz}, 10-\mathrm{H}\right)$, $3.58\left(\mathrm{~m}_{\mathrm{c}}, 1 \mathrm{H}, 8-\mathrm{H}\right), 4.34\left(\mathrm{~d},{ }^{3} J_{\mathrm{H}, \mathrm{H}}=10.4 \mathrm{~Hz}, 1 \mathrm{H}, 6-\mathrm{H}\right), 4.57\left(\mathrm{~d},{ }^{3} J_{\mathrm{H}, \mathrm{H}}=9.8 \mathrm{~Hz}, 1 \mathrm{H}, 14-\mathrm{H}\right)$, $7.12(\mathrm{~s}, 1 \mathrm{H}, 21-\mathrm{H}), 7.31(\mathrm{~s}, 1 \mathrm{H}, 4-\mathrm{OH}), 7.48$ (s, $1 \mathrm{H}, 8-\mathrm{OH}) .-{ }^{13} \mathrm{C}-\mathrm{NMR}(150.8 \mathrm{MHz}$, $\left.\mathrm{CD}_{2} \mathrm{Cl}_{2}\right): \delta=6.8\left(9-\mathrm{CH}_{3}\right), 7.6\left(2-\mathrm{CH}_{3}\right), 12.2\left(19-\mathrm{CH}_{3}\right), 12.8\left(7-\mathrm{CH}_{3}\right), 19.5\left(17-\mathrm{CH}_{3}\right), 19.7$ (15- $\left.\mathrm{CH}_{3}\right), 22.4$ (13- $\left.\mathrm{CH}_{3}\right), 26.0$ (C-17), 32.9 (C-11), 33.2 (C-13), 35.4 (C-12), 37.8 (C-7), 39.2 (C-18), 40.0 (C-9), 40.4 (C-19), 45.6 (C-16), $52.3\left(20-\mathrm{OCH}_{3}\right), 76.7$ (C-8), 77.1 (C-6), 78.4 (C-10), 104.5 (C-2), 110.0 (C-5), 122.2 (C-20), 129.2 (C-14), 131.5 (C-21), 131.8 (C15), 139.7 (C-22), 146.1 (C-4), 168.1 (C-1), 182.1 (C-3). - IR (KBr): $\tilde{v}=3480,3324$, 2934, 2869, 2351, 2342, 1671, 1616, 1328, 1247, 1100, 1046, 913, 792, $524 \mathrm{~cm}^{-1}$. - UV $(\mathrm{MeOH}): \lambda_{\max }(\lg \varepsilon)=204(0.864), 314(1.12) \mathrm{nm} ;(\mathrm{MeOH} / \mathrm{HCl}): \lambda_{\max }(\lg \varepsilon)=202(0.978)$, $315(1.17) \mathrm{nm} ;(\mathrm{MeOH} / \mathrm{NaOH}): \lambda_{\max }(\lg \varepsilon)=209$ (0.991), $325(0.956) \mathrm{nm} .-\mathrm{MS}(\mathrm{ESI}), \mathrm{m} / z$ (\%): $501(60)[\mathrm{M}+\mathrm{H}]^{+}, 523(100)[\mathrm{M}+\mathrm{Na}]^{+}, 736(30), 1024(70)[2 \mathrm{M}+\mathrm{Na}]^{+}, 1120(30)$, 1148 (30); HRMS (ESI): Ber. für $\mathrm{C}_{30} \mathrm{H}_{44} \mathrm{~F}_{3} \mathrm{~N}_{2} \mathrm{NaO}_{6}$ : $523.30301[\mathrm{M}+\mathrm{Na}]^{+}$, Gef. 523.30321 .

\section{8-O-[4-(3-Trifluormethyl-3H-diazirin-3-yl)benzoyl] kendomycinmethylacetal (84)}

Das Methylacetal $\mathbf{8 3} \quad(19.0 \mathrm{mg}$, $37.9 \mu \mathrm{mol}), \quad$ Dicyclohexylcarbodiimid (DCC, $20.2 \mathrm{mg}, \quad 97.9 \mu \mathrm{mol}$ ) DMAP (10.4 mg, $85.2 \mu \mathrm{mol})$ und Diazirin 48 $(17.5 \mathrm{mg}, \quad 76.1 \mu \mathrm{mol}) \quad$ wurden in Dichlormethan $(1.5 \mathrm{~mL})$ gelöst. Nach $16 \mathrm{~h}$ Rühren unter Lichtausschluß bei RT wurde Methanol (2 mL) zugegeben

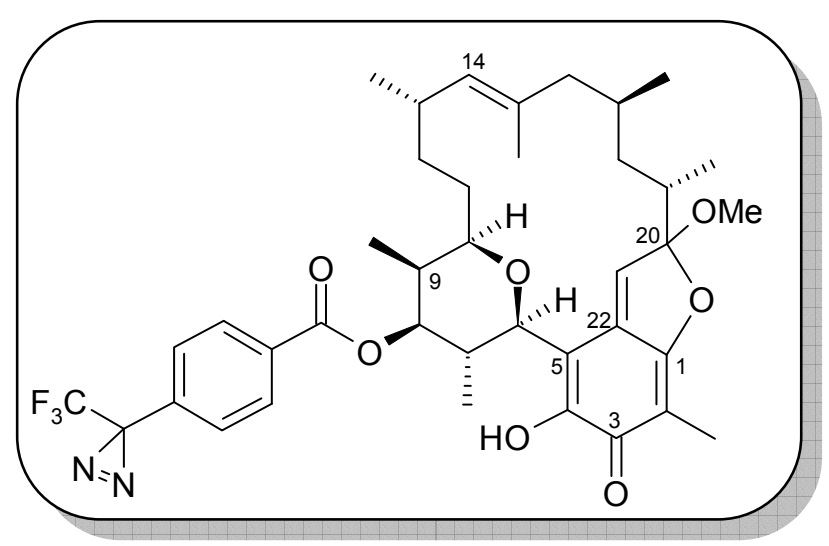
und das Dichlormethan unter vermindertem Druck entfernt. Die methanolische Lösung wurde dann direkt einer Größenausschluss-Chromatographie zugeführt (Sephadex LH-20, Methanol). Man erhielt zwei Fraktionen, welche jeweils größere Mengen des DCC- 
Hydrolyseproduktes ( $N, N$-Dicyclohexylharnstoff) enthielten. Dieses konnte durch wiederholte Extraktion mit Pentan leicht abgetrennt werden. Die erste Fraktion enthielt $13.0 \mathrm{mg}(48 \%)$ der reinen Titelverbindung als gelblichen Feststoff, die zweite Fraktion $(9.0 \mathrm{mg})$ enthielt neben $\sim 6 \mathrm{mg}(32 \%)$ des Edukts 83 etwa $3 \mathrm{mg}$ des Methylesters von Diazirin 48. $\mathrm{R}_{f}=0.16$ (Hex:EE, 5:1). $-{ }^{1} \mathrm{H}-\mathrm{NMR}\left(600 \mathrm{MHz}, \mathrm{CD}_{2} \mathrm{Cl}_{2}\right): \delta=0.69\left(\mathrm{~d},{ }^{3} J_{\mathrm{H}, \mathrm{H}}=\right.$ $\left.6.8 \mathrm{~Hz}, 3 \mathrm{H}, 19-\mathrm{CH}_{3}\right), 0.87\left(\mathrm{~d},{ }^{3} J_{\mathrm{H}, \mathrm{H}}=6.6 \mathrm{~Hz}, 3 \mathrm{H}, 13-\mathrm{CH}_{3}\right), 0.88\left(\mathrm{~d},{ }^{3} J_{\mathrm{H}, \mathrm{H}}=6.6 \mathrm{~Hz}, 3 \mathrm{H}\right.$, 7-CH$), 0.93\left(\mathrm{~d},{ }^{3} J_{\mathrm{H}, \mathrm{H}}=6.5 \mathrm{~Hz}, 3 \mathrm{H}, 9-\mathrm{CH}_{3}\right), 0.99\left(\mathrm{~d},{ }^{3} J_{\mathrm{H}, \mathrm{H}}=7.0 \mathrm{~Hz}, 3 \mathrm{H}, 17-\mathrm{CH}_{3}\right), 1.21$ $\left(\mathrm{m}_{\mathrm{c}}, 1 \mathrm{H}, 12-\mathrm{H}_{\mathrm{a}}\right), 1.25-1.40\left(\mathrm{~m}, 4 \mathrm{H}, 16-\mathrm{H}_{\mathrm{a}}, 12-\mathrm{H}_{\mathrm{b}}, 18-\mathrm{H}_{2}\right), 1.50\left(\mathrm{~m}_{\mathrm{c}}, 2 \mathrm{H}, 11-\mathrm{H}_{2}\right), 1.57(\mathrm{~s}$, $\left.3 \mathrm{H}, 15-\mathrm{CH}_{3}\right), 1.64\left(\mathrm{dd}, J_{\mathrm{H}, \mathrm{H}}=17.3,11.7 \mathrm{~Hz}, 1 \mathrm{H}, 16-\mathrm{H}_{\mathrm{b}}\right), 1.93\left(\mathrm{~s}, 3 \mathrm{H}, 2-\mathrm{CH}_{3}\right), 1.95\left(\mathrm{~m}_{\mathrm{c}}\right.$, $1 \mathrm{H}, 17-\mathrm{H}), 2.14\left(\mathrm{~m}_{\mathrm{c}}, 1 \mathrm{H}, 9-\mathrm{H}\right), 2.22\left(\mathrm{~m}_{\mathrm{c}}, 1 \mathrm{H}, 7-\mathrm{H}\right), 2.32\left(\mathrm{~m}_{\mathrm{c}}, 1 \mathrm{H}, 13-\mathrm{H}\right), 2.39$ (ddq, $J_{\mathrm{H}, \mathrm{H}}$ $=3.1,6.8,13.6 \mathrm{~Hz}, 1 \mathrm{H}, 1 \mathrm{H}, 19-\mathrm{H}), 3.29\left(\mathrm{~s}, 3 \mathrm{H}, 20-\mathrm{OCH}_{3}\right), 3.64\left(\mathrm{~d}_{\mathrm{br}},{ }^{3} J_{\mathrm{H}, \mathrm{H}}=11.8 \mathrm{~Hz}, 10-\right.$ $\mathrm{H}), 4.54\left(\mathrm{~d},{ }^{3} J_{\mathrm{H}, \mathrm{H}}=10.5 \mathrm{~Hz}, 1 \mathrm{H}, 6-\mathrm{H}\right), 4.59\left(\mathrm{~d},{ }^{3} J_{\mathrm{H}, \mathrm{H}}=9.8 \mathrm{~Hz}, 1 \mathrm{H}, 14-\mathrm{H}\right), 5.08\left(\mathrm{dd},{ }^{3} J_{\mathrm{H}, \mathrm{H}}\right.$ $=4.8,11.0 \mathrm{~Hz}, 1 \mathrm{H}, 8-\mathrm{H}), 7.16(\mathrm{~s}, 1 \mathrm{H}, 21-\mathrm{H}), 7.28\left(\mathrm{~d},{ }^{3} J_{\mathrm{H}, \mathrm{H}}=8.3 \mathrm{~Hz}, 2 \mathrm{H}, \mathrm{Ar}-\mathrm{H}\right), 7.50\left(\mathrm{~s}_{\mathrm{br}}\right.$, $1 \mathrm{H}, 4-\mathrm{OH}), 8.09\left(\mathrm{~d},{ }^{3} J_{\mathrm{H}, \mathrm{H}}=8.8 \mathrm{~Hz}, 2 \mathrm{H}, \mathrm{Ar}-\mathrm{H}\right) .-{ }^{13} \mathrm{C}-\mathrm{NMR}\left(150.8 \mathrm{MHz}, \mathrm{CD}_{2} \mathrm{Cl}_{2}\right): \delta=7.6$ $\left(9-\mathrm{CH}_{3}\right), 7.6\left(2-\mathrm{CH}_{3}\right), 12.2\left(19-\mathrm{CH}_{3}\right), 12.9\left(7-\mathrm{CH}_{3}\right), 19.5\left(17-\mathrm{CH}_{3}\right), 19.7\left(15-\mathrm{CH}_{3}\right), 22.4$ $\left(13-\mathrm{CH}_{3}\right), 26.0(\mathrm{C}-17), 28.6\left(\mathrm{q},{ }^{2} J_{\mathrm{C}-\mathrm{F}}=42.2 \mathrm{~Hz}, \mathrm{CN}_{2}\right), 32.7(\mathrm{C}-11), 33.1(\mathrm{C}-13), 35.3(\mathrm{C}-$ 12), 35.8 (C-7), 39.2 (C-18), 37.3 (C-9), 40.4 (C-19), 45.6 (C-16), $52.3\left(20-\mathrm{OCH}_{3}\right), 77.2$ (C-6), 78.0 (C-10), 79.9 (C-8), 104.7 (C-2), 109.3 (C-5), 122.2 (C-20), 122.3 (q, ${ }^{1} J_{\mathrm{C}-\mathrm{F}}=$ $275.3 \mathrm{~Hz}, \mathrm{CF}_{3}$ ), 126.7 (C-Ar), 128.0 (C-Ar), 129.2 (C-14), 130.1 (C-Ar), 131.4 (C-21), 131.8 (C-15), 133.9 (C-Ar), 139.6 (C-22), 146.9 (C-4), 168.1 (C-1), 182.0 (C-3). - IR $(\mathrm{KBr}): \tilde{v}=3420,2932,2363,1723,1616,1383,1328,1277,1195,1159,1103,940$, $767 \mathrm{~cm}^{-1}$. - UV (MeOH): $\lambda_{\max }(\lg \varepsilon)=203(1.04), 236(0.653), 315.6(0.821) \mathrm{nm}$; $(\mathrm{MeOH} / \mathrm{NaOH}): \lambda_{\max }(\lg \varepsilon)=206$ (0.976), 233 (0.857), 329 (0.663) nm. - MS (ESI), m/z (\%): 523 (60) $\left[\mathrm{M}-\mathrm{C}_{9} \mathrm{H}_{3} \mathrm{~F}_{3} \mathrm{~N}_{2} \mathrm{O}+\mathrm{Na}\right]^{+}, 736(20)[\mathrm{M}+\mathrm{Na}]^{+}, 959(20), 1024$ (100) [2 M$\left.\mathrm{C}_{18} \mathrm{H}_{6} \mathrm{~F}_{6} \mathrm{~N}_{4} \mathrm{O}_{2}+\mathrm{Na}\right]^{+}$; HRMS (ESI): Ber. für $\mathrm{C}_{39} \mathrm{H}_{48} \mathrm{~F}_{3} \mathrm{~N}_{2} \mathrm{O}_{7}: 713.34081[\mathrm{M}+\mathrm{H}]^{+}$, Gef. 713.34059 . 
3-O-[4-(3-Trifluormethyl-3H-diazirin-3-yl)benzoyl]-apicularen (86)

Apicularen $\quad(\mathbf{8 5}, \quad 1.5 \mathrm{mg}, \quad 3.4 \mu \mathrm{mol})$ wurde in Dichlormethan $(5 \mathrm{~mL})$ gelöst und bei RT nacheinander mit DMAP (0.9 mg, $7 \mu \mathrm{mol}), \quad$ EDCI $\quad(1.1 \mathrm{mg}$, $6 \mu \mathrm{mol})$ und Diazirinylcarbonsäure 48 (1.1 mg, $4.8 \mu \mathrm{mol})$ versetzt. Nach $2.5 \mathrm{~h}$ Rühren bei RT wurde die Reaktionslösung direkt auf eine KGSäule aufgetragen (Flash, $\mathrm{CHCl}_{3}: \mathrm{MeOH}$, 25:1). Das so erhaltene Produkt enthielt

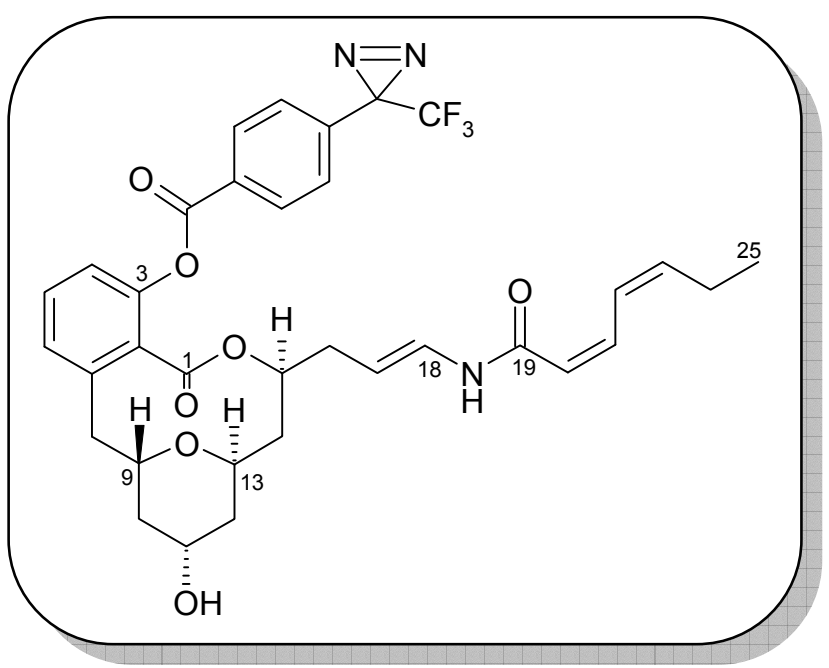
größere Mengen Fett, es wurde in $\mathrm{MeOH}$ aufgenommen und über $3 \mathrm{~cm} \mathrm{Na} \mathrm{SO}_{4}$ filtriert. Man erhielt $1.34 \mathrm{mg}(60 \%)$ der Titelverbindung als farblosen Lack. $R_{\mathrm{f}}=0.17$ $\left(\mathrm{CHCl}_{3}: \mathrm{MeOH}, 50: 1\right)-{ }^{1} \mathrm{H}-\mathrm{NMR}\left(600 \mathrm{MHz},\left[\mathrm{D}_{6}\right]\right.$-Aceton): $\delta=0.99\left(\mathrm{t},{ }^{3} J_{\mathrm{H}-\mathrm{H}}=7.5 \mathrm{~Hz}, 3 \mathrm{H}\right.$, $\left.25-\mathrm{H}_{3}\right), 1.46-1.56\left(\mathrm{~m}, 3 \mathrm{H}, 14-\mathrm{H}_{\mathrm{a}}, 10-\mathrm{H}_{\mathrm{a}}, 12-\mathrm{H}_{\mathrm{a}}\right), 1.65\left(\mathrm{ddd}, J_{\mathrm{H}-\mathrm{H}}=5.3,7.8,13.1 \mathrm{~Hz}, 1 \mathrm{H}\right.$, $\left.12-\mathrm{H}_{\mathrm{b}}\right), 1.78\left(\mathrm{dt}, J_{\mathrm{H}-\mathrm{H}}=10.9,14.7 \mathrm{~Hz}, 1 \mathrm{H}, 14-\mathrm{H}_{\mathrm{b}}\right), 1.95\left(\mathrm{dt}, J_{\mathrm{H}-\mathrm{H}}=4.6,13.0 \mathrm{~Hz}, 1 \mathrm{H}, 10-\right.$ $\left.\mathrm{H}_{\mathrm{b}}\right), 2.00-2.06\left(\mathrm{~m}, 2 \mathrm{H}, 16-\mathrm{H}_{2}\right), 2.27$ (dquint, $\left.J_{\mathrm{H}-\mathrm{H}}=1.6,7.5 \mathrm{~Hz}, 2 \mathrm{H}, 24-\mathrm{H}_{2}\right), 2.60\left(\mathrm{dd}, J_{\mathrm{H}-\mathrm{H}}\right.$ $\left.=1.5,14.6 \mathrm{~Hz}, 1 \mathrm{H}, 8-\mathrm{H}_{\mathrm{a}}\right), 3.44\left(\mathrm{dd},{ }^{3} J_{\mathrm{H}-\mathrm{H}}=10.4,14.6 \mathrm{~Hz}, 1 \mathrm{H}, 8-\mathrm{H}_{\mathrm{b}}\right), 3.83-3.89(\mathrm{~m}, 2 \mathrm{H}$, 9-H, 11-OH), $3.98\left(\mathrm{~m}_{\mathrm{c}}, 1 \mathrm{H}, 11-\mathrm{H}\right), 4.23\left(\mathrm{~m}_{\mathrm{c}}, 1 \mathrm{H}, \mathrm{H}-13\right), 4.97\left(\mathrm{dt},{ }^{3} J_{\mathrm{H}-\mathrm{H}}=7.4,14.5 \mathrm{~Hz}, 17-\right.$ $\mathrm{H}), 5.39\left(\mathrm{~m}_{\mathrm{c}}, 1 \mathrm{H}, 15-\mathrm{H}\right), 5.69\left(\mathrm{~d},{ }^{3} J_{\mathrm{H}-\mathrm{H}}=11.5 \mathrm{~Hz}, 1 \mathrm{H}, 20-\mathrm{H}\right), 5.79\left(\mathrm{~m}_{\mathrm{c}}, 1 \mathrm{H}, 23-\mathrm{H}\right), 6.71$ $\left(\mathrm{dd},{ }^{3} J_{\mathrm{H}-\mathrm{H}}=10.4,14.5 \mathrm{~Hz}, 1 \mathrm{H}, 18-\mathrm{H}\right), 6.85\left(\mathrm{dt}, J_{\mathrm{H}-\mathrm{H}}=1.1,11.5 \mathrm{~Hz}, 1 \mathrm{H}, 21-\mathrm{H}\right), 7.23\left(\mathrm{~m}_{\mathrm{c}}\right.$, $2 \mathrm{H}, 4-\mathrm{H}, 6-\mathrm{H}), 7.44\left(\mathrm{dd},{ }^{3} J_{\mathrm{H}-\mathrm{H}}=7.7,8.2 \mathrm{~Hz}, 1 \mathrm{H}, 5-\mathrm{H}\right), 7.50\left(\mathrm{~d},{ }^{3} J_{\mathrm{H}-\mathrm{H}}=8.5 \mathrm{~Hz}, 2 \mathrm{H}, \mathrm{Ar}-\mathrm{H}\right)$, $7.51\left(\mathrm{~m}_{\mathrm{c}}, 1 \mathrm{H}, 22-\mathrm{H}\right), 8.23\left(\mathrm{~d},{ }^{3} J_{\mathrm{H}-\mathrm{H}}=8.7 \mathrm{~Hz}, 2 \mathrm{H}, \mathrm{Ar}-\mathrm{H}\right), 8.92\left(\mathrm{~d},{ }^{3} J_{\mathrm{H}-\mathrm{H}}=10.4 \mathrm{~Hz}, 1 \mathrm{H}\right.$, $\mathrm{NH})$ ppm. $-{ }^{13} \mathrm{C}-\mathrm{NMR}\left(150.8 \mathrm{MHz},\left[\mathrm{D}_{6}\right]\right.$-Aceton): $\delta=14.3$ (C-25), $21.0(\mathrm{C}-24), 29.9\left(\mathrm{CN}_{2}\right.$, verdeckt), 35.8 (C-16), 38.5 (C-14), 39.0 (C-10), 39.8 (C-8), 40.1 (C-12), 64.7 (C-11), 67.5 (C-13), 73.7 (C-9), 74.6 (C-15), 106.9 (C-17), 120.7 (C-20), 121.6 (C-6), 123.0 (q, ${ }^{1} J_{\mathrm{C}-\mathrm{F}}=$ $275 \mathrm{~Hz}, \mathrm{CF}_{3}$ ), 125.4 (C-22), 126.4 (C-18), 127.8 (C-Ar), 129.0 (C-4), 130.5 (C-5), 130.6 (C-7), 131.4 (C-Ar), 131.7 (C-2) 134.7 (C-Ar), 136.9 (C-21), 140.8 (C-Ar), 141.5 (C-23), 147.7 (C-3), 163.5 (C-19), 164.1 (C-1'), 167.9 (C-1) ppm. 


\section{E.3 Arbeitsvorschriften Hauptteil II}

\section{E.3.1 Versuche zur Synthese des Edukts für die Nagao-Aldol-Reaktion}

\section{(Z)-4-(tert-Butyldimethylsilanyloxy)-but-2-en-1-ol (21)}

$\mathrm{Zu}$ einer Lösung von (Z)-Buten-1,4-diol (136, $132 \mathrm{~g}, 1.50 \mathrm{~mol})$ in Dichlormethan $(500 \mathrm{~mL})$ wurden Triethylamin $(31.9 \mathrm{~g}$, $43.9 \mathrm{~mL}, \quad 315 \mathrm{mmol})$ und DMAP $(915 \mathrm{mg}, \quad 7.50 \mathrm{mmol})$

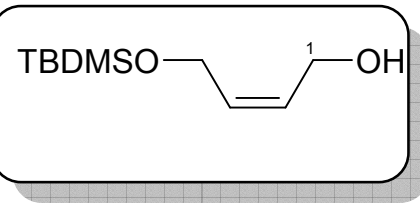
gegeben. Innerhalb von $5 \mathrm{~h}$ wurde per Spritzenpumpe eine TBDMSCl-Lösung (377 mg/mL in Toluol, $120 \mathrm{~mL} 45.2 \mathrm{~g}, 300 \mathrm{mmol}$ ) zugetropft und anschließend $40 \mathrm{~h}$ bei RT gerührt. Die Lösung wurde in einen Scheidetrichter überführt und mit Wasser $(100 \mathrm{~mL}), \mathrm{NH}_{4} \mathrm{Cl}(100 \mathrm{~mL})$, Wasser $(100 \mathrm{~mL})$ und NaCl-Lösung $(2 \times 50 \mathrm{~mL})$ gewaschen. Die vereinigten wässrigen Phasen wurden mit Diethylether $(2 \times 100 \mathrm{~mL})$ reextrahiert und die so erhaltene organische Phase mit NaCl-Lösung gewaschen. Die vereinigten organischen Phasen wurden über Natriumsulfat getrocknet und das Lösungsmittel unter vermindertem Druck entfernt. Das so erhaltene Rohprodukt $\left(\mathrm{m}_{\text {Roh }}=61.0 \mathrm{~g}\right)$ wurde destillativ aufgereinigt. Als Produkt erhielt man $53.1 \mathrm{~g}\left(87 \%, 0.25 \mathrm{mbar}, 75^{\circ} \mathrm{C}\right) \mathrm{der}$ Titelverbindung als farblose Flüssigkeit. ${ }^{1} \mathrm{H}-\mathrm{NMR}\left(250 \mathrm{MHz}, \mathrm{CDCl}_{3}\right): \delta=0.02[\mathrm{~s}, 6 \mathrm{H}$, $\mathrm{Si}\left(\mathrm{CH}_{3}\right)_{2}$ ], 0.87 [s, $\left.9 \mathrm{H}, \mathrm{SiC}\left(\mathrm{CH}_{3}\right)_{3}\right], 2.60(\mathrm{~s} b \mathrm{r}, 1 \mathrm{H}, \mathrm{OH}), 4.20-4.60$ (m, $\left.4 \mathrm{H}, 1-\mathrm{H}_{2}, 4-\mathrm{H}_{2}\right)$, $5.65\left(\mathrm{~m}_{\mathrm{c}}, 2 \mathrm{H}, 2-\mathrm{H}, 3-\mathrm{H}\right) \mathrm{ppm}$. - Alle übrigen analytischen Daten entsprachen den Literaturdaten. ${ }^{[38]}$

\section{(E)-4-(tert-Butyldimethylsilanyloxy)but-2-enal (141)}

Der geschützte Alkohol 21 (9.98 g, $49.3 \mathrm{mmol})$ wurde in DMSO $(150 \mathrm{~mL})$ gelöst und mit Triethylamin $(17.2 \mathrm{~mL}$, $123 \mathrm{mmol}$ ) versetzt. Nach Zugabe einer Lösung von TBDMSO $>0$ Pyridin $\cdot \mathrm{SO}_{3}$-Komplex (10.0 g, $\left.49.4 \mathrm{mmol}\right)$ in DMSO (130 mL) wurde $1 \mathrm{~h} 15 \mathrm{~min}$ bei RT gerührt. Die Reaktionslösung wurde auf Eiswasser $(250 \mathrm{~mL})$ gegossen und das Gemisch mit Diethylether $(4 \times 200 \mathrm{~mL})$ extrahiert. Die vereinigten organischen Phasen wurden mit $\mathrm{NH}_{4} \mathrm{Cl}$-Lösung, Wasser, $\mathrm{NaHCO}_{3}$-Lösung und $\mathrm{NaCl}$-Lösung gewaschen und über Natriumsulfat getrocknet. Entfernen des Lösungsmittels unter vermindertem Druck ergab $9.90 \mathrm{~g}$ einer gelblichen Flüssigkeit, welche neben der Titelverbindung noch Pyridin enthielt. Nach Entfernen des Pyridins im Vakuum erhielt man $8.30 \mathrm{~g}$ (84\%) der 
Titelverbindung als leicht gelbliche Flüssigkeit. ${ }^{1} \mathrm{H}-\mathrm{NMR}\left(250 \mathrm{MHz}, \mathrm{CDCl}_{3}\right): \delta=0.02[\mathrm{~s}$, $\left.6 \mathrm{H}, \mathrm{Si}\left(\mathrm{CH}_{3}\right)_{2}\right], 0.87$ [s, $\left.9 \mathrm{H}, \mathrm{SiC}\left(\mathrm{CH}_{3}\right)_{3}\right], 4.41\left(\mathrm{dd}, J_{\mathrm{H}-\mathrm{H}}=1.0,1.5 \mathrm{~Hz}, 2 \mathrm{H}, 4-\mathrm{H}_{2}\right), 6.39$ (ddt, $\left.J_{\mathrm{H}-\mathrm{H}}=1.0,7.5,14.5 \mathrm{~Hz}, 1 \mathrm{H}, 2-\mathrm{H}\right), 6.90\left(\mathrm{dt},{ }^{3} J_{\mathrm{H}-\mathrm{H}}=1.5 \mathrm{~Hz}, 14.5 \mathrm{~Hz}, 1 \mathrm{H}, 3-\mathrm{H}\right), 9.59$ (d, $\left.{ }^{3} J_{\mathrm{H}-\mathrm{H}}=7.5 \mathrm{~Hz}, 1 \mathrm{H}, 1-\mathrm{H}\right) \mathrm{ppm}$. - Alle übrigen analytischen Daten entsprachen den Literaturdaten. ${ }^{[38]}$

(Z)-4-(tert-Butyldimethylsilanyloxy)but-2-enal (141Z)

(Z)-Butendiol (21, $503 \mathrm{mg}, 2.49 \mathrm{mmol})$ und Pentan (30 mL) wurden auf $0{ }^{\circ} \mathrm{C}$ gekühlt und mit $\mathrm{MnO}_{2}(4.32 \mathrm{~g}, 49.7 \mathrm{mmol})$ versetzt. Der Reaktionsverlauf wurde mittels DC-Kontrolle

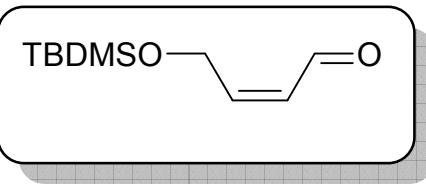
verfolgt, da dieser nach $1.5 \mathrm{~h}$ immer noch unvollständig war, wurden weitere $150 \mathrm{mg}$ Braunstein zugesetzt. Nach $2 \mathrm{~h}$ Rühren bei $0{ }^{\circ} \mathrm{C}$ wurde das Reaktionsgemisch über Celite $(2 \mathrm{~cm})$ filtriert und der Rückstand mit Pentan gewaschen. Entfernen des Lösungsmittels im Vakuum ergab $393 \mathrm{mg}$ (79\%) der Titelverbindung als farbloses Öl. ${ }^{1} \mathrm{H}-\mathrm{NMR}$ (250 MHz, $\left.\mathrm{CDCl}_{3}\right): \delta=0.09$ [s, $\left.6 \mathrm{H}, \mathrm{Si}\left(\mathrm{CH}_{3}\right)_{2}\right], 0.90\left[\mathrm{~s}, 9 \mathrm{H}, \mathrm{SiC}\left(\mathrm{CH}_{3}\right)_{3}\right], 4.67\left(\mathrm{dd}, J_{\mathrm{H}-\mathrm{H}}=2.1,5.1 \mathrm{~Hz}\right.$, $\left.2 \mathrm{H}, 4-\mathrm{H}_{2}\right), 5.98\left(\mathrm{ddt}, J_{\mathrm{H}-\mathrm{H}}=2.0,6.9,11.5 \mathrm{~Hz}, 1 \mathrm{H}, 2-\mathrm{H}\right), 6.56\left(\mathrm{dt},{ }^{3} J_{\mathrm{H}-\mathrm{H}}=5.0 \mathrm{~Hz}, 11.5 \mathrm{~Hz}\right.$, $1 \mathrm{H}, 3-\mathrm{H}), 10.15\left(\mathrm{~d},{ }^{3} J_{\mathrm{H}-\mathrm{H}}=6.8 \mathrm{~Hz}, 1 \mathrm{H}, 1-\mathrm{H}\right) \mathrm{ppm}$. - Alle übrigen analytischen Daten entsprachen den Literaturdaten. ${ }^{[38]}$

(2E,4E)-6-(tert-Butyldimethylsilanyloxy)hexa-2,4-diensäureethylester (142)

Der TBDMS-geschützte Aldehyd $\mathbf{1 4 1}_{Z} \quad(3.21 \mathrm{~g}$, $16.0 \mathrm{mmol})$ wurde in THF $(50 \mathrm{~mL})$ gelöst und auf $-30{ }^{\circ} \mathrm{C}$ gekühlt. Nach Zugabe von Trieethyl-

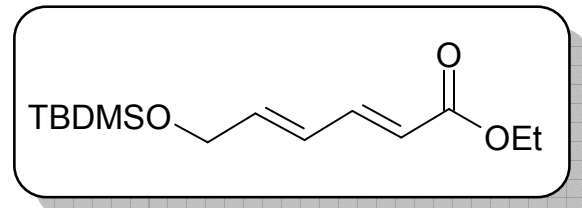
phosphonoacetat $(3.96 \mathrm{~g}, 17.7 \mathrm{mmol})$ wurde langsam eine Lösung von NaOEt (1.31 g, $19.3 \mathrm{mmol})$ in wasserfreiem Ethanol (28.5 mL) zugetropft. Nach 1.25 h zeigte die DC-Kontrolle (Hex:EE, 10:1) vollständigen Umsatz an. Zur Reaktionslösung wurde Wasser $(30 \mathrm{~mL})$ zugegeben und das THF unter reduziertem Druck entfernt. Der Rückstand wurde mit Diethylether $(3 \times 100 \mathrm{~mL})$ extrahiert und die vereinigten organischen Phasen mit Wasser $(2 \times 50 \mathrm{~mL})$ und NaCl-Lösung $(2 \times 25 \mathrm{~mL})$ gewaschen und über Natriumsulfat getrocknet. Das so erhaltene Rohprodukt (4.09 g) wurde mittels Säulenchromatographie gereinigt (Hex:EE, 20:1). Man erhielt 3.75 g (87\%) 
der Titelverbindung als farbloses Ö1. $R_{\mathrm{f}}=0.33$ (Hex:EE, 20:1) - ${ }^{1} \mathrm{H}-\mathrm{NMR}(250 \mathrm{MHz}$, $\left.\mathrm{CDCl}_{3}\right): \delta=0.11\left[\mathrm{~s}, 6 \mathrm{H}, \mathrm{Si}\left(\mathrm{CH}_{3}\right)_{2}\right], 0.89\left[\mathrm{~s}, 9 \mathrm{H}, \mathrm{SiC}\left(\mathrm{CH}_{3}\right)_{3}\right], 1.23\left(\mathrm{t},{ }^{3} J_{\mathrm{H}-\mathrm{H}}=7.1 \mathrm{~Hz}, 3 \mathrm{H}\right.$, $\left.2^{\prime}-\mathrm{H}_{3}\right), 4.18\left(\mathrm{q},{ }^{3} J_{\mathrm{H}-\mathrm{H}}=7.1 \mathrm{~Hz}, 2 \mathrm{H}, 1^{\prime}-\mathrm{H}_{2}\right), 4.23\left(\mathrm{~m}_{\mathrm{c}}, 2 \mathrm{H}, 6-\mathrm{H}_{2}\right), 5.81\left(\mathrm{~d},{ }^{3} J_{\mathrm{H}-\mathrm{H}}=15.0 \mathrm{~Hz}\right.$, $1 \mathrm{H}, 2-\mathrm{H}), 6.16\left(\mathrm{dt},{ }^{3} J_{\mathrm{H}-\mathrm{H}}=4.8,15.5 \mathrm{~Hz}, 1 \mathrm{H}, 5-\mathrm{H}\right), 6.38\left(\mathrm{dd},{ }^{3} J_{\mathrm{H}-\mathrm{H}}=12.0,15.0 \mathrm{~Hz}, 1 \mathrm{H}, 3-\right.$ $\mathrm{H}), 7.23\left(\mathrm{dd},{ }^{3} J_{\mathrm{H}-\mathrm{H}}=12.0,15.5 \mathrm{~Hz}, 1 \mathrm{H}, 4-\mathrm{H}\right) \mathrm{ppm}$. - Alle übrigen analytischen Daten entsprachen den Literaturdaten. ${ }^{[38]}$

(3E,5Z)-6-(tert-Butyldimethylsilanyloxy)hexa-3,5-diensäureethylester $\left(\mathbf{2 2}_{Z}\right)$

Diisopropylamin $(4.93 \mathrm{~g}, 6.82 \mathrm{~mL}, 48.7 \mathrm{mmol})$ wurde in THF (150 mL) gelöst und auf $-78^{\circ} \mathrm{C}$ gekühlt. Nach Zugabe von $n$-BuLi (38.0 mL, $48.7 \mathrm{mmol}, 1.28 \mathrm{M}$ in Hexan) ließ man zunächst 5 min bei $-78^{\circ} \mathrm{C}$ rühren und setzte dann DMPU (9.81 mL, $10.4 \mathrm{~g}, 81.3 \mathrm{mmol})$ hinzu. Nach weiteren

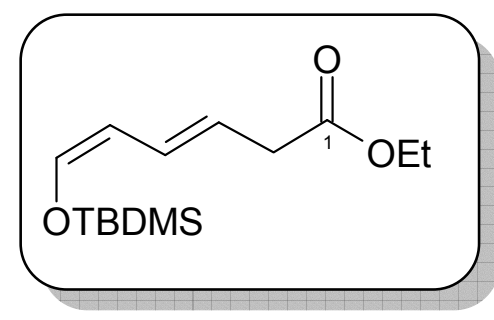
40 min wurde der konjugierte Ester 142 (11.0 g, 40.7 mmol) zugetropft und anschließend ließ man weitere $4 \mathrm{~h}$ bei $-78^{\circ} \mathrm{C}$ rühren. Die Reaktion wurde durch Zugabe von $\mathrm{NH}_{4} \mathrm{Cl}$ Lösung $(5 \mathrm{~mL})$ beendet und man ließ das Gemisch auf RT erwärmen. Nach Zugabe von Wasser $(200 \mathrm{~mL})$ wurde die wässrige Phase mit Diethylether $(4 \times 250 \mathrm{~mL})$ extrahiert. Die vereinigten organischen Phasen wurde mit $\mathrm{NH}_{4} \mathrm{Cl}$-Lösung $(2 \times 100 \mathrm{~mL})$, Wasser und NaCl-Lösung $(2 \times 100 \mathrm{~mL})$ gewaschen. Nach Trocknen über Natriumsulfat erhielt man 11.91 g Rohprodukt, welches durch Säulenchromatographie (Hex:EE, 20:1) gereinigt wurde. Man erhielt $5.61 \mathrm{~g}(51 \%)$ der Titelverbindung als gelbes Öl. $R_{\mathrm{f}}=0.40$ (Hex:EE, 20:1). - ${ }^{1} \mathrm{H}-\mathrm{NMR}\left(250 \mathrm{MHz}, \mathrm{CDCl}_{3}\right): \delta=0.13$ [s, $\left.6 \mathrm{H}, \mathrm{Si}\left(\mathrm{CH}_{3}\right)_{2}\right], 0.91\left[\mathrm{~s}, 9 \mathrm{H}, \mathrm{SiC}\left(\mathrm{CH}_{3}\right)_{3}\right]$, $1.24\left(\mathrm{t},{ }^{3} J_{\mathrm{H}-\mathrm{H}}=7.1 \mathrm{~Hz}, 3 \mathrm{H}, 2^{\prime}-\mathrm{H}_{3}\right), 3.09\left(\mathrm{dd}, J_{\mathrm{H}-\mathrm{H}}=1.3,7.0 \mathrm{~Hz}, 2 \mathrm{H}, 2-\mathrm{H}_{2}\right), 4.11\left(\mathrm{q},{ }^{3} J_{\mathrm{H}-\mathrm{H}}=\right.$ $\left.7.1 \mathrm{~Hz}, 2 \mathrm{H}, 1^{\prime}-\mathrm{H}\right), 5.15\left(\mathrm{dd},{ }^{3} J_{\mathrm{H}-\mathrm{H}}=5.8,10.7 \mathrm{~Hz}, 1 \mathrm{H}, 5-\mathrm{H}\right), 5.60\left(\mathrm{dt},{ }^{3} J_{\mathrm{H}-\mathrm{H}}=7.0,14.6 \mathrm{~Hz}\right.$, $1 \mathrm{H}, 3-\mathrm{H}), 6.14\left(\mathrm{~d},{ }^{3} J_{\mathrm{H}-\mathrm{H}}=5.8 \mathrm{~Hz}, 1 \mathrm{H}, 6-\mathrm{H}\right), 6.50\left(\mathrm{~m}_{\mathrm{c}}, 1 \mathrm{H}, 4-\mathrm{H}\right) \mathrm{ppm}$. - Alle übrigen analytischen Daten entsprachen den Literaturdaten. ${ }^{[38]}$ 


\section{Allgemeine Arbeitsvorschrift 02: Selektive Reduktion zum Aldehyd mit DIBAH}

Versuch der Darstellung von (3E,5Z)-6-(tert-Butyldimethylsilanyloxy)hexa-3,5-dienal (23Z)

Der Ester $22_{Z}(186 \mathrm{mg}, 688 \mu \mathrm{mol})$ wurde in $\mathrm{CH}_{2} \mathrm{Cl}_{2}(10 \mathrm{~mL})$ gelöst und auf $-78^{\circ} \mathrm{C}$ gekühlt. Nach Zugabe von DIBAH $(722 \mu \mathrm{L}$, $0.7 \mathrm{mmol}, 1 \mathrm{M}$ in Heptan) ließ man $1.5 \mathrm{~h}$ bei $-78^{\circ} \mathrm{C}$ rühren. Dann wurde mit einer Lösung aus Weinsäure in Ethanol $(1.44 \mathrm{mmol})$

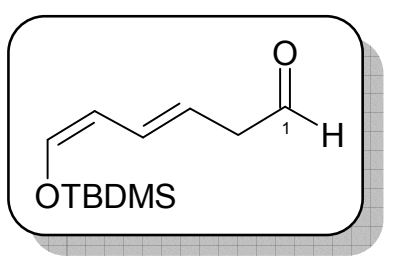
gequencht und man ließ die Lösung auf $-38^{\circ} \mathrm{C}$ erwärmen. Nach Zugabe eines Celite: $\mathrm{Na}_{2} \mathrm{SO}_{4}$-Gemisches (1.00 g, 1:1) wurde noch fünf Minuten gerührt und dann das Kühlbad entfernt. Nachdem das Gemisch RT erreicht hatte, wurde über Celite $(1.5 \mathrm{~cm})$ filtriert und der Rückstand mit Pentan gewaschen. Das ${ }^{1} \mathrm{H}-\mathrm{NMR}-$ Spektrum des Rohproduktes zeigte die Titelverbindung in zufriedenstellender Reinheit. Es zeigte sich jedoch, dass sich das Produkt bereits nach Zurückspülen und anschließender Konzentration im Vakuum zu einem nicht identifizierbaren Substanzgemisch zersetzt hatte. Trotz Variation der Reaktionsbedingungen (siehe Hauptteil) konnte die Titelverbindung nicht isoliert werden. ${ }^{1} \mathrm{H}-\mathrm{NMR}\left(250 \mathrm{MHz}, \mathrm{CDCl}_{3}\right): \delta=0.12\left[\mathrm{~s}, 6 \mathrm{H}, \mathrm{Si}\left(\mathrm{CH}_{3}\right)_{2}\right], 0.90[\mathrm{~s}, 9 \mathrm{H}$, $\left.\mathrm{SiC}\left(\mathrm{CH}_{3}\right)_{3}\right], 3.01\left(\mathrm{dd}, J_{\mathrm{H}-\mathrm{H}}=1.4,6.8 \mathrm{~Hz}, 2 \mathrm{H}, 2-\mathrm{H}\right), 5.16\left(\mathrm{~m}_{\mathrm{c}}, 1 \mathrm{H}, 5-\mathrm{H}\right), 5.52\left(\mathrm{~m}_{\mathrm{c}}, 1 \mathrm{H}, 3-\right.$ $\mathrm{H}), 6.18\left(\mathrm{~d},{ }^{3} J_{\mathrm{H}-\mathrm{H}}=5.9 \mathrm{~Hz}, 1 \mathrm{H}, 6-\mathrm{H}\right), 6.54\left(\mathrm{~m}_{\mathrm{c}}, 1 \mathrm{H}, 4-\mathrm{H}\right), 9.66\left(\mathrm{~m}_{\mathrm{c}}, 1 \mathrm{H}, \mathrm{CHO}\right) \mathrm{ppm}$.

\section{(3Z,5E)-6-(tert-Butyldimethylsilanyloxy)hexa-3,5-dien-1-ol (151)}

$\mathrm{LiAlH}_{4}(19.5 \mathrm{mg}, 514 \mu \mathrm{mol})$ wurde in THF $(10 \mathrm{~mL})$ suspendiert und auf $0{ }^{\circ} \mathrm{C}$ gekühlt. Nach Zugabe des Esters $\mathbf{2 3}_{\boldsymbol{Z}}(111 \mathrm{mg}$, $410 \mu \mathrm{mol})$ ließ man 5 min bei $0{ }^{\circ} \mathrm{C}$ rühren, dann wurde das Kühlbad entfernt und weitere 3 h bei RT gerührt. Nachdem die DC-

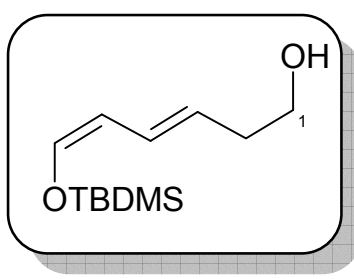
Kontrolle (Hex:EE, 5:1) vollständigen Umsatz, anzeigte wurde die Lösung wieder auf $0{ }^{\circ} \mathrm{C}$ gekühlt und die Reaktion durch Zugabe von 10 Tropfen $\mathrm{Na}_{2} \mathrm{SO}_{4}$-Lösung beendet. Nach 15 min Rühren bei $0{ }^{\circ} \mathrm{C}$ wurde eine Lösung aus Phosphatpuffer (pH 7.0, $10 \mathrm{~mL}$ ) und $\mathrm{K}, \mathrm{Na}$-Tartrat $(1.1 \mathrm{~g})$ zugesetzt. Man ließ $1.5 \mathrm{~h}$ bei RT rühren, dann wurde das Gemisch mit $\mathrm{Et}_{2} \mathrm{O}(4 \times 20 \mathrm{~mL})$ extrahiert und die vereinigten etherischen Phasen mit Wasser $(4 \times 10 \mathrm{~mL})$ und NaCl-Lösung gewaschen. Nach Trocknen über $\mathrm{Na}_{2} \mathrm{SO}_{4}$ erhielt man $109 \mathrm{mg}$ Rohprodukt, welches mittels Säulenchromatographie aufgereinigt wurde (Alox Aktivität 
III, pH 7, Hex:EE, 10:1). Man erhielt 46 mg (49\%) der Titelverbindung als gelbliches Öl. Das Produkt zeichnete sich durch eine hohe Instabilität sowohl auf KG als auch bei standardmäßiger Lagerung als Reinsubstanz bei $-21{ }^{\circ} \mathrm{C}$ aus. $-\mathrm{R}_{f}=0.20$ (Hex:EE, 10:1) ${ }^{1} \mathrm{H}-\mathrm{NMR}\left(250 \mathrm{MHz}, \mathrm{CDCl}_{3}\right): \delta=0.15\left[\mathrm{~s}, 6 \mathrm{H}, \mathrm{Si}\left(\mathrm{CH}_{3}\right)_{2}\right], 0.93\left[\mathrm{~s}, 9 \mathrm{H}, \mathrm{SiC}\left(\mathrm{CH}_{3}\right)_{3}\right], 2.36(\mathrm{dt}$, $\left.J_{\mathrm{H}-\mathrm{H}}=1.3,7.4 \mathrm{~Hz}, 2 \mathrm{H}, 2-\mathrm{H}_{2}\right), 3.65\left(\mathrm{t},{ }^{3} J_{\mathrm{H}-\mathrm{H}}=7.0 \mathrm{~Hz}, 2 \mathrm{H}, 1-\mathrm{H}_{2}\right), 5.15\left(\mathrm{dd},{ }^{3} J_{\mathrm{H}-\mathrm{H}}=5.8\right.$, $10.7 \mathrm{~Hz}, 1 \mathrm{H}, 5-\mathrm{H}), 5.50\left(\mathrm{dt},{ }^{3} J_{\mathrm{H}-\mathrm{H}}=7.4,15.3 \mathrm{~Hz}, 1 \mathrm{H}, 3-\mathrm{H}\right), 6.13\left(\mathrm{~d},{ }^{3} J_{\mathrm{H}-\mathrm{H}}=5.8 \mathrm{~Hz}, 1 \mathrm{H}\right.$, 6-H), 6.50 (dddd, $\left.{ }^{3} J_{\mathrm{H}-\mathrm{H}}=1.3,2.4,10.7,15.3 \mathrm{~Hz}, 1 \mathrm{H}, 4-\mathrm{H}\right) \mathrm{ppm} .-{ }^{13} \mathrm{C}-\mathrm{NMR}(62.9 \mathrm{MHz}$, $\left.\mathrm{CDCl}_{3}\right): \delta=-5.4\left[\mathrm{Si}\left(\mathrm{CH}_{3}\right)_{2}\right], 18.3\left[\mathrm{SiC}\left(\mathrm{CH}_{3}\right)_{3}\right], 25.6\left[\mathrm{SiC}\left(\mathrm{CH}_{3}\right)_{3}\right], 36.3(\mathrm{C}-2), 62.1(\mathrm{C}-1)$, 110.1 (C-5), 125.5 (C-3), 126.1 (C-6), 139.3 (C-4) ppm.

Versuch der Darstellung von (3E,5Z)-6-(tert-Butyldimethylsilanyloxy)hexa-3,5-dienal (23) durch Oxidation

A: IBX (128 mg, $457 \mu \mathrm{mol})$ wurde in DMSO $(2.0 \mathrm{~mL})$ gelöst und mit einer Lösung von Alkohol 151 (52.0 mg, $228 \mu \mathrm{mol})$ in DMSO $(1.0 \mathrm{~mL})$ versetzt. Man ließ bei RT

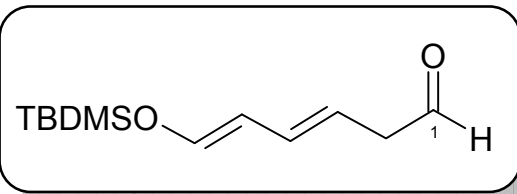
rühren. Nach $1.5 \mathrm{~h}$ wurde Wasser $(20 \mathrm{~mL})$ zugegeben und anschließend mit Ether $(4 \times$ $25 \mathrm{~mL}$ ) extrahiert. Waschen mit $\mathrm{NH}_{4} \mathrm{Cl}$-Lösung, $1 \mathrm{M} \mathrm{NaHCO}_{3}$-Lösung, Wasser, NaClLösung und Entfernen des Lösungsmittels unter reduziertem Druck ergab $46 \mathrm{mg}$ eines gelben Öls. ${ }^{1}$ H-NMR Spektroskopie des Rückstands zeigte ausschließlich nicht identifizierbare Zersetzungsprodukte.

B: Alkohol $151(27.0 \mathrm{mg}, 118 \mu \mathrm{mol})$ wurde in Dichlormethan $(3.0 \mathrm{~mL})$ gelöst und mit $\mathrm{NaHCO}_{3}(49.7 \mathrm{mg}, 591 \mu \mathrm{mol})$ und DMP $(148 \mathrm{mg}, 355 \mu \mathrm{mol})$ versetzt. Nach $30 \mathrm{~min}$ Rühren bei RT zeigte DC-Kontrolle an, dass kein Edukt mehr in der Reaktionslösung vorlag. Es wurde eine Lösung von $\mathrm{NaHCO}_{3}$-Lösung und 10\%iger $\mathrm{Na}_{2} \mathrm{~S}_{2} \mathrm{O}_{3}$-Lösung ( $5 \mathrm{~mL}$, 1:1) zugesetzt und man ließ $15 \mathrm{~min}$ bei RT rühren. Wasser $(10 \mathrm{~mL})$ und Ether $(50 \mathrm{~mL})$ wurden zugegeben, die Phasen getrennt und die organische Phase mit Wasser und NaClLösung gewaschen. Nach Trocknen über Natriumsulfat wurde das Lösungsmittel unter reduziertem Druck entfernt. Im Rückstand (40 mg) wurden ausschließlich nicht identifizierbare Zersetzungsprodukte nachgewiesen.

C: Oxalylchlorid $(28.6 \mu \mathrm{L}, 27.5 \mathrm{mg}, 217 \mu \mathrm{mol})$ wurde in Dichlormethan $(5.0 \mathrm{~mL})$ gelöst und auf $-78{ }^{\circ} \mathrm{C}$ gekühlt. Nach Zugabe von DMSO (29.2 $\left.\mu \mathrm{L}, 32.1 \mathrm{mg}, 433 \mu \mathrm{mol}\right)$ wurde 
15 min gerührt und dann der Alkohol 151 (45 mg, 197 mol) zugegeben. Man ließ 30 min bei $-78^{\circ} \mathrm{C}$ rühren und gab dann Triethylamin $(137 \mu \mathrm{L}, 99.7 \mathrm{mg}, 985 \mu \mathrm{mol})$ hinzu und ließ auf RT erwärmen. Nach 30 min Rühren bei RT wurde Wasser $(10 \mathrm{ml})$ zugegeben und mit Dichlormethan extrahiert. Waschen der vereinigten organischen Phasen mit NaCl-Lösung und Trocknen über Natriumsulfat ergaben $61 \mathrm{mg}$ gelbes Ö1, welches ausschließlich nicht identifizierbare Zersetzungsprodukte enthielt.

\section{Allgemeine Arbeitsvorschrift 03: Oxidation nach Parikh-Doering}

D: Alkohol $146(38.0 \mathrm{mg}, 166 \mu \mathrm{mol})$ wurde in DMSO $(1.0 \mathrm{~mL})$ gelöst und mit $\mathrm{NEt}_{3}$ (58 mL, $42.1 \mathrm{mg}, 0.4 \mathrm{mmol}$ ) versetzt. Nach Zugabe des Pyridin $\cdot \mathrm{SO}_{3}$ Komplexes (79.4 mg, $499 \mu \mathrm{mol})$ wurde 25 min bei RT gerührt. Das Reaktionsgemisch wurde auf Eiswasser $(50 \mathrm{~mL})$ gegossen und die wässrige Phase mit Diethylether $(3 \times 50 \mathrm{~mL})$ extrahiert. Trocknen über Natriumsulfat und Entfernen des Lösungsmittels ergab ein farbloses Öl $(8.9 \mathrm{mg})$ worin ausschließlich nicht identifizierbare Zersetzungsprodukte nachgewiesen wurden.

\section{(2E,4E)-6-Phenylhexa-2,4-diensäureethylester (149)}

(3-Ethoxycarbonylallyl)triphenylphosphoniumbromid (148, $11.4 \mathrm{~g}, 25.0 \mathrm{mmol})$ wurde in THF (40 mL) gelöst und auf $55^{\circ} \mathrm{C}$ gekühlt. Nach Zugabe einer Lösung von

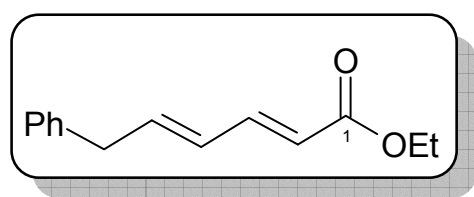
Natriumethanolat in Ethanol $(13.6 \mathrm{~mL}, 126 \mathrm{mg} / \mathrm{mL}, 1.71 \mathrm{~g}, 25.2 \mathrm{mmol})$ wurde $30 \mathrm{~min}$ bei Temperaturen zwischen -55 und $-50{ }^{\circ} \mathrm{C}$ gerührt. Dann wurde das Kühlbad gewechselt und weitere 30 min bei $0{ }^{\circ} \mathrm{C}$ gerührt. Die dunkelbraune Lösung wurde wieder auf $-55^{\circ} \mathrm{C}$ gekühlt und anschließend zügig mit einer Lösung von Phenylacetaldehyd (145, $2.88 \mathrm{~g}$, $24.0 \mathrm{mmol})$ in THF (40 mL) versetzt. Man rührte das dunkelbraune Reaktionsgemisch für $3 \mathrm{~h}$, wobei sich die Lösung auf RT erwärmte. Wasser $(50 \mathrm{~mL})$ wurde zugesetzt und anschließend ein Großteil des THF unter vermindertem Druck entfernt. Der Rückstand wurde mit Essigester $(3 \times 100 \mathrm{~mL})$ extrahiert und die vereinigten organischen Phasen wurden mit NaCl-Lösung gewaschen und über Natriumsulfat getrocknet. Entfernen des Lösungsmittels ergab $12.5 \mathrm{~g}$ Rohprodukt, welches säulenchromatographisch aufgereinigt wurde (Hex:EE, 20:1). Man erhielt 2.38 g (46\%) der Titelverbindung als gelb-braunes Öl. $\mathrm{R}_{f}=0.66$ (Hex:EE, 10:1). $-{ }^{1} \mathrm{H}-\mathrm{NMR}\left(250 \mathrm{MHz}, \mathrm{CDCl}_{3}\right): \delta=1.35\left(\mathrm{t},{ }^{3} J_{\mathrm{H}-\mathrm{H}}=8.3 \mathrm{~Hz}, 3 \mathrm{H}\right.$, 
$\left.\mathrm{CH}_{2} \mathrm{CH}_{3}\right), 3.19\left(\mathrm{~d},{ }^{3} \mathrm{~J}_{\mathrm{H}-\mathrm{H}}=7.5 \mathrm{~Hz}, 2 \mathrm{H}, 6-\mathrm{H}_{2}\right), 4.20\left(\mathrm{q},{ }^{3} \mathrm{~J}_{\mathrm{H}-\mathrm{H}}=8.3 \mathrm{~Hz}, 2 \mathrm{H}, \mathrm{CH}_{2} \mathrm{CH}_{3}\right), 5.90$ $\left(\mathrm{m}_{\mathrm{c}}, 1 \mathrm{H}, 4-\mathrm{H}\right), 6.20-6.40(\mathrm{~m}, 1 \mathrm{H}, 5-\mathrm{H}), 6.55\left(\mathrm{~d},{ }^{3} J_{\mathrm{H}-\mathrm{H}}=15.8 \mathrm{~Hz}, 1 \mathrm{H}, 2-\mathrm{H}\right), 6.81\left(\mathrm{dd},{ }^{3} J_{\mathrm{H}-}\right.$ $\mathrm{H}=9.2,15.8 \mathrm{~Hz}, 1 \mathrm{H}, 3-\mathrm{H}), 7.10-7.50\left(\mathrm{~m}, 5 \mathrm{H}, \mathrm{Ar}-\mathrm{H}_{5}\right) \mathrm{ppm}$.

\section{(3E,5E)-6-Phenylhexa-3,5-diensäureethylester (150)}

Diisopropylamin $(3.42 \mathrm{~mL}, 2.47 \mathrm{~g}, 24.4 \mathrm{mmol})$ wurde in THF gelöst, auf $-78{ }^{\circ} \mathrm{C}$ gekühlt und mit $n$-BuLi $(19.1 \mathrm{~mL}$, $24.4 \mathrm{mmol}, 1.28 \mathrm{M}$ in Hexan) versetzt. Nach 5 min Rühren wurde langsam HMPA (4.25 mL, $4.18 \mathrm{~g}, 24.4 \mathrm{mmol})$

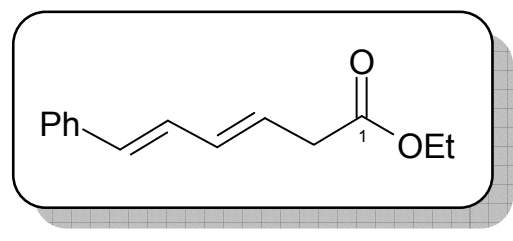
zugegeben und anschließend $30 \mathrm{~min}$ bei $-78^{\circ} \mathrm{C}$ gerührt. Nach langsamer Zugabe einer Lösung des Esters 149 (4.40 g, $20.3 \mathrm{mmol})$ in THF $(15 \mathrm{~mL})$ wurde $3.5 \mathrm{~h}$ bei $-78^{\circ} \mathrm{C}$ gerührt. Das Reaktionsgemisch wurde in eine Mischung aus $\mathrm{NH}_{4} \mathrm{Cl}$-Lösung $(100 \mathrm{~mL})$ und Ether $(150 \mathrm{~mL})$ gegossen und $10 \mathrm{~min}$ bei RT gerührt. Die Phasen wurden getrennt und die $\mathrm{NH}_{4} \mathrm{Cl}$-Phase mit Ether extrahiert $(3 \times 150 \mathrm{~mL})$. Die vereinigten organischen Phasen wurden mit $\mathrm{NH}_{4}$ Cl-Lösung $(100 \mathrm{~mL})$, Wasser $(5 \times 100 \mathrm{~mL})$ und $\mathrm{NaCl}$-Lösung $(3 \times 100 \mathrm{~mL})$ gewaschen, über $\mathrm{Na}_{2} \mathrm{SO}_{4}$ getrocknet und das Lösungsmittel im Vakuum entfernt. Das so erhaltene Rohprodukt (5.32 g) wurde säulenchromatographisch aufgereinigt (Hex:EE, 20:1) und man erhielt $2.83 \mathrm{~g}$ (64\%) der Titelverbindung als rotbraunes Ö1. $\mathrm{R}_{f}=0.66$ (Hex:EE, 10:1). $-{ }^{1} \mathrm{H}-\mathrm{NMR}\left(250 \mathrm{MHz}, \mathrm{CDCl}_{3}\right): \delta=1.29\left(\mathrm{t},{ }^{3} J_{\mathrm{H}-\mathrm{H}}=\right.$ $\left.7.2 \mathrm{~Hz}, 3 \mathrm{H}, 2^{\prime}-\mathrm{H}_{3}\right), 3.19\left(\mathrm{~d},{ }^{3} J_{\mathrm{H}-\mathrm{H}}=7.5 \mathrm{~Hz}, 2 \mathrm{H}, 2-\mathrm{H}_{2}\right), 4.18\left(\mathrm{q},{ }^{3} J_{\mathrm{H}-\mathrm{H}}=7.2 \mathrm{~Hz}, 2 \mathrm{H}, 1^{\prime}-\mathrm{H}_{3}\right)$, $5.92\left(\mathrm{dt},{ }^{3} J_{\mathrm{H}-\mathrm{H}}=7.5,15.0 \mathrm{~Hz}, 1 \mathrm{H}, 3-\mathrm{H}\right), 6.31\left(\mathrm{dd},{ }^{3} J_{\mathrm{H}-\mathrm{H}}=10.3,15.0 \mathrm{~Hz}, 1 \mathrm{H}, 4-\mathrm{H}\right), 6.52(\mathrm{~d}$, $\left.{ }^{3} J_{\mathrm{H}-\mathrm{H}}=15.6 \mathrm{~Hz}, 1 \mathrm{H}, 6-\mathrm{H}\right), 6.80\left(\mathrm{dd},{ }^{3} J_{\mathrm{H}-\mathrm{H}}=10.3,15.6 \mathrm{~Hz}, 1 \mathrm{H}, 5-\mathrm{H}\right), 7.20-7.50(\mathrm{~m}, 5 \mathrm{H}$, Ar- $\left.H_{5}\right)$ ppm. $-{ }^{13} \mathrm{C}-\mathrm{NMR}\left(62.9 \mathrm{MHz}, \mathrm{CDCl}_{3}\right): \delta=14.2\left(\mathrm{C}-2^{\prime}\right), 38.2(\mathrm{C}-2), 60.7\left(\mathrm{C}-1^{\prime}\right)$, 125.7 (C-3), 126.3 (C-Ar), 127.5 (C-Ar), 128.3 (C-4), 128.6 (C-Ar), 132.0 (C-6), 133.9 (CAr), 137.1 (C-5), 171.5 (C-1) ppm.

\section{Allgemeine Arbeitsvorschrift 04: Reduktion des Hexansäureesters mit DIBAH}

Versuch der Darstellung von (3E,5E)-6-Phenylhexa-3,5-dienal (150a)

Ester $144 \quad(0.4-0.5 \mathrm{mmol})$ wurde in wasserfreiem

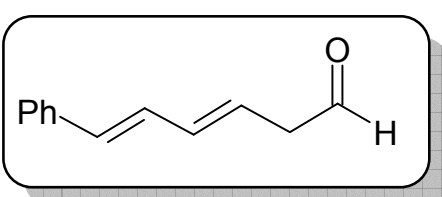


Dichlormethan $(10 \mathrm{~mL})$ gelöst und auf $-78^{\circ} \mathrm{C}$ gekühlt. Nach Zugabe von DIBAH (1.05 eq) wurde 1-3 h gerührt. Man ließ auf $-30{ }^{\circ} \mathrm{C}$ erwärmen und setzte ein $\mathrm{Na}_{2} \mathrm{SO}_{4} / \mathrm{Celite}$ Gemisch $(1: 1,1.0 \mathrm{~g})$ zu und filtrierte über $1 \mathrm{~cm}$ Celite. Der Rückstand wurde mit Pentan gewaschen und das Lösungsmittel im Vakuum entfernt. Das ${ }^{1} \mathrm{H}-\mathrm{NMR}$ Spektrum der Rohprodukte $(\sim 100 \%$ d. Th.) zeigte die Titelverbindung neben sehr starken, nicht identifizierbaren Verunreinigungen. Versuche der säulenchromatographischen Reinigung waren nicht erfolgreich.

(3E,5E)-6-Phenylhexa-3,5-dien-1-ol (152)

Der Ester 150 (225 mg, $1.04 \mathrm{mmol})$ wurde in THF (10 mL) gelöst, auf $-78^{\circ} \mathrm{C}$ gekühlt und mit $\mathrm{LiAlH}_{4}(39.9 \mathrm{mg}$, $1.05 \mathrm{mmol}$ ) versetzt. Man ließ $1 \mathrm{~h}$ bei $-78{ }^{\circ} \mathrm{C}$ und $\mathrm{Ph}_{\mathrm{OH}}^{1}$ anschließend $2 \mathrm{~h}$ bei $0{ }^{\circ} \mathrm{C}$ rühren. Die Aufarbeitung erfolgte analog der Reduktion zum Alkohol 151. Man erhielt $220 \mathrm{mg}$ Rohprodukt, welches eine ausreichende Reinheit für die folgenden Umsetzungen aufwies. Versuche, das Rohprodukt an Kieselgel (Hex:EE, 5:1) oder Aluminiumoxid zu reinigen, waren nicht erfolgreich. $\mathrm{R}_{f}=0.05$ (Hex:EE, 10:1). $-{ }^{1} \mathrm{H}-$ NMR (250 MHz, $\left.\mathrm{CDCl}_{3}\right): \delta=2.40\left(\mathrm{q},{ }^{3} J_{\mathrm{H}-\mathrm{H}}=6.8 \mathrm{~Hz}, 2 \mathrm{H}, 2-\mathrm{H}_{2}\right), 3.70\left(\mathrm{t},{ }^{3} J_{\mathrm{H}-\mathrm{H}}=6.8 \mathrm{~Hz}\right.$, $\left.2 \mathrm{H}, 1-\mathrm{H}_{2}\right), 5.82\left(\mathrm{dt},{ }^{3} J_{\mathrm{H}-\mathrm{H}}=7.5,15.0 \mathrm{~Hz}, 1 \mathrm{H}, 3-\mathrm{H}\right), 6.35\left(\mathrm{dd},{ }^{3} J_{\mathrm{H}-\mathrm{H}}=10.3,15.0 \mathrm{~Hz}, 1 \mathrm{H}\right.$, $4-\mathrm{H}), 6.50\left(\mathrm{~d},{ }^{3} J_{\mathrm{H}-\mathrm{H}}=15.6 \mathrm{~Hz}, 1 \mathrm{H}, 6-\mathrm{H}\right), 6.80\left(\mathrm{dd},{ }^{3} J_{\mathrm{H}-\mathrm{H}}=10.3,15.6 \mathrm{~Hz}, 1 \mathrm{H}, 5-\mathrm{H}\right), 7.15-$ $7.50\left(\mathrm{~m}, 5 \mathrm{H}, \mathrm{Ar}-\mathrm{H}_{5}\right) \mathrm{ppm}$.

Versuche der Darstellung von (3E,5E)-6-Phenylhexa-3,5-dienal (150a) durch Oxidation

Die Oxidationsversuche mit Oxalylchlorid/DMSO, IBX sowie Pyr $\cdot \mathrm{SO}_{3}$ wurden analog den für Alkohol 151 beschriebenen Versuchen durchgeführt und lieferten ebenfalls nur Gemische aus nicht identifizierbaren Zersetzungsprodukten.

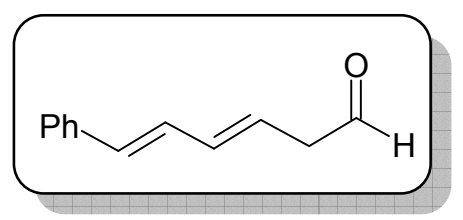




\section{E.3.2 Synthese der IMDA-Edukte ausgehend von Acetessigsäureethylester}

\section{4-Brom-3-oxobutansäureethylester (159)}

$\mathrm{Zu}$ Dichlormethan (1.0 1) wurde Acetessigsäureethylester $(150 \mathrm{~mL}, 1.19 \mathrm{~mol})$ gegeben. Bei $-10^{\circ} \mathrm{C}$ wurde langsam Brom (60.5 mL, $1.19 \mathrm{~mol})$ zugetropft. Anschließend ließ man die Lösung innerhalb von $2 \mathrm{~h}$ auf Raumtemperatur erwärmen.

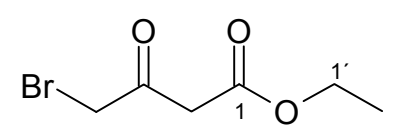
Bei Raumtemperatur wurde $2 \mathrm{~h}$ Sauerstoff durch die Reaktionsmischung geleitet. Das Lösungsmittel wurde abdestilliert und anschließend das Rohprodukt über eine Vigreuxkolonne im Vakuum fraktionierend destilliert. Destillation bei 0.6 mbar lieferte vier Fraktionen. $\mathrm{F}_{1}: 40{ }^{\circ} \mathrm{C}, 11.9 \mathrm{~g}$ (Edukt), $\mathrm{F}_{2}: 59{ }^{\circ} \mathrm{C}, 6.2 \mathrm{~g}$ (Gemisch aus Edukt und Produkt), $\mathrm{F}_{3}: 72{ }^{\circ} \mathrm{C}, 38.9 \mathrm{~g}$ und $\mathrm{F}_{4}: 81^{\circ} \mathrm{C}, 134.9 \mathrm{~g}$. Da die Fraktionen $\mathrm{F}_{3}$ und $\mathrm{F}_{4} \mathrm{zwar}$ die Titelverbindung enthielten, jedoch verunreinigt waren, wurden beide Fraktionen vereinigt und erneut bei 0.4 mbar destilliert. $\mathrm{F}_{1}{ }^{\prime}: 66^{\circ} \mathrm{C}, 11.2 \mathrm{~g}$ (Gemisch aus Edukt und Produkt), $\mathrm{F}_{2}{ }^{\prime}$ : $74{ }^{\circ} \mathrm{C}, 145.5 \mathrm{~g}$ (Produkt, 59\%). ${ }^{1} \mathrm{H}-\mathrm{NMR}\left(250 \mathrm{MHz}, \mathrm{CDCl}_{3}\right)$ : Ketoform: $\delta=1.18\left(\mathrm{t},{ }^{3} J_{\mathrm{H}-\mathrm{H}}\right.$ $\left.=7.2 \mathrm{~Hz}, 3 \mathrm{H}, 2^{\prime}-\mathrm{H}_{3}\right), 3.60\left(\mathrm{~s}, 2 \mathrm{H}, 4-\mathrm{H}_{2}\right), 4.00\left(\mathrm{~s}, 2 \mathrm{H}, 2-\mathrm{H}_{2}\right), 4.09$ (q, ${ }^{3} J_{\mathrm{H}-\mathrm{H}}=7.2 \mathrm{~Hz}, 2 \mathrm{H}$, $\left.1^{\prime}-\mathrm{H}_{2}\right)$ ppm. Enolform (ca. 23\%): $\delta=1.18\left(\mathrm{t},{ }^{3} J_{\mathrm{H}-\mathrm{H}}=7.2 \mathrm{~Hz}, 3 \mathrm{H}, 2^{\prime}-\mathrm{H}_{3}\right), 3.78$ (s, $2 \mathrm{H}, 4-$ $\left.\mathrm{H}_{2}\right), 4.09\left(\mathrm{q},{ }^{3} J_{\mathrm{H}-\mathrm{H}}=7.2 \mathrm{~Hz}, 2 \mathrm{H}, 1^{\prime}-\mathrm{H}_{2}\right), 5.19$ (s, $\left.1 \mathrm{H}, 2-\mathrm{H}\right) 12.1$ (s, $\left.1 \mathrm{H}, 3-\mathrm{OH}\right) \mathrm{ppm}$.

\section{4-(Diethoxyphosphoryl)-3-oxobutansäureethylester (154)}

4-Brom-3-oxobutansäureethylester (159, $41.3 \mathrm{~g}, 198 \mathrm{mmol})$ wurde auf $100^{\circ} \mathrm{C}$ erwärmt und tropfenweise mit Triethylphosphit $(34.6 \mathrm{~mL}, 32.7 \mathrm{~g}, 198 \mathrm{mmol})$ versetzt. Anschließend wurde 30 min bei $100{ }^{\circ} \mathrm{C}$ gerührt. Danach

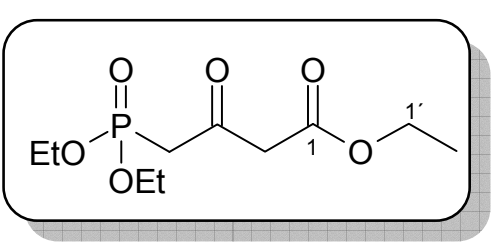
wurde die Reaktionsmischung mit einem Eisbad schnell auf RT gekühlt. Das entstandene Ethylbromid wurde im Vakuum entfernt. Das Rohprodukt (53.3 g) wurde durch Säulenchromatographie (Pent:EE, $1: 5 \rightarrow 1: 9 \rightarrow 1: 15, R_{\mathrm{f}}=0.33$, Pent:EE, 1:5) gereinigt. Man erhielt $21 \mathrm{~g}(40 \%)$ der Titelverbindung als leicht gelbliches Öl. ${ }^{1} \mathrm{H}-\mathrm{NMR}(250 \mathrm{MHz}$, $\left.\mathrm{CDCl}_{3}\right): \delta=1.25\left(\mathrm{t},{ }^{3} J_{\mathrm{H}-\mathrm{H}}=6.8 \mathrm{~Hz}, 6 \mathrm{H}, 22^{\prime \prime}-\mathrm{H}_{3}\right), 1.31\left(\mathrm{t},{ }^{3} J_{\mathrm{H}-\mathrm{H}}=6.8 \mathrm{~Hz}, 3 \mathrm{H}, 2^{\prime}-\mathrm{H}_{3}\right), 3.26$ $\left(\mathrm{d},{ }^{2} J_{\mathrm{P}-\mathrm{H}}=22.7 \mathrm{~Hz}, 2 \mathrm{H}, 4-\mathrm{H}_{2}\right), 3.65\left(\mathrm{~s}, 2 \mathrm{H}, 2-\mathrm{H}_{2}\right), 4.11\left(\mathrm{q},{ }^{3} J_{\mathrm{H}-\mathrm{H}}=6.8 \mathrm{~Hz}, 4 \mathrm{H}, 21^{\prime \prime}-\mathrm{H}_{2}\right)$, $4.16\left(\mathrm{q},{ }^{3} J_{\mathrm{H}-\mathrm{H}}=6.8 \mathrm{~Hz}, 2 \mathrm{H}, 1^{\prime}-\mathrm{H}_{2}\right)$ ppm. - Alle übrigen analytischen Daten entsprachen den Literaturdaten. ${ }^{[174]}$ 


\section{Allgemeine Arbeitsvorschrift 05: HWE-Reaktion}

(4E,6E)-8-(tert-Butyldimethylsilanyloxy)-3-oxoocta-4,6-diensäureethylester (155)

4-(Diethoxyphosphoryl)-3-oxobutansäureethylester $(\mathbf{1 5 4}, 2.88 \mathrm{~g}, 10.8 \mathrm{mmol})$ wurde in $\mathrm{THF}$ $(25 \mathrm{~mL})$ vorgelegt und portionsweise mit $\mathrm{NaH}$

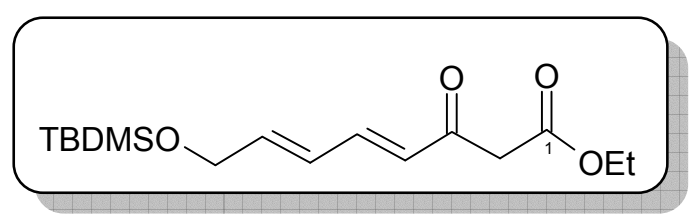
(476 mg, $11.9 \mathrm{mmol}, 60 \%$ Dispersion in Mineralöl) versetzt. Nach 20 min Rühren bei RT wurde auf $-35^{\circ} \mathrm{C}$ abgekühlt und man gab $n$-BuLi (10.8 mL, $10.8 \mathrm{mmol}, 1.00 \mathrm{M}$ in Hexan) zur Reaktionslösung und ließ $10 \mathrm{~min}$ bei $-35{ }^{\circ} \mathrm{C}$ rühren. Der Aldehyd 141 (2.10 g, $10.5 \mathrm{mmol})$ wurde, gelöst in THF $(10 \mathrm{~mL})$, zugegeben und die resultierende Lösung 15 min bei $-35{ }^{\circ} \mathrm{C}$ gerührt. Das Kühlbad wurde entfernt und man ließ innerhalb von 35 min auf RT erwärmen. Die gelb-orange Lösung wurde in ein Gemisch aus Eis, $\mathrm{NH}_{4} \mathrm{Cl}$ Lösung und $\mathrm{Et}_{2} \mathrm{O}(1: 1: 1,300 \mathrm{~mL})$ gegossen. Die Phasen wurden getrennt und die wässrige Phase wurde zweimal mit $\mathrm{Et}_{2} \mathrm{O}$ extrahiert. Die vereinigten organischen Phasen wurden dreimal mit Wasser und mit NaCl-Lsg. gewaschen und über $\mathrm{Na}_{2} \mathrm{SO}_{4}$ getrocknet. Durch Entfernen des Lösungsmittels im Vakuum wurde das Rohprodukt als oranges Öl erhalten (3.53 g). Die Titelverbindung wurde durch säulenchromatographische Reinigung (Hex:EE, 10:1) als gelbes Ö1 isoliert: $2.04 \mathrm{~g}(70 \%) . R_{\mathrm{f}}=0.52$ (Hex:EE, 5:1), 0.28 (Hex:EE, 10:1). ${ }^{1} \mathrm{H}-\mathrm{NMR}\left(250 \mathrm{MHz}, \mathrm{CDCl}_{3}\right)$ : Ketoform: $\delta=0.01$ [s, $\left.6 \mathrm{H}, \mathrm{Si}\left(\mathrm{CH}_{3}\right)_{2}\right], 0.94[\mathrm{~s}, 9 \mathrm{H}$, $\mathrm{Si}\left(\mathrm{C}\left(\mathrm{CH}_{3}\right)_{3}\right], 1.27\left(\mathrm{t},{ }^{3} J_{\mathrm{H}-\mathrm{H}}=7.0 \mathrm{~Hz}, 3 \mathrm{H}, 2^{\prime}-\mathrm{H}_{3}\right), 3.55\left(\mathrm{~s}, 2 \mathrm{H}, 2-\mathrm{H}_{2}\right), 4.17$ (q, ${ }^{3} J_{\mathrm{H}-\mathrm{H}}=7.0 \mathrm{~Hz}$, $\left.2 \mathrm{H}, 1^{\prime}-\mathrm{H}\right), 4.30\left(\mathrm{~m}_{\mathrm{c}}, 2 \mathrm{H}, 8-\mathrm{H}_{2}\right), 5.87\left(\mathrm{~d},{ }^{3} J_{\mathrm{H}-\mathrm{H}}=14.4 \mathrm{~Hz}, 1 \mathrm{H}, 4-\mathrm{H}\right), 6.07\left(\mathrm{dt},{ }^{3} J_{\mathrm{H}-\mathrm{H}}=2.4\right.$, 13.2 Hz, $1 \mathrm{H}, 7-\mathrm{H}), 6.25-6.50(\mathrm{~m}, 1 \mathrm{H}, 6-\mathrm{H}), 7.25\left(\mathrm{dd},{ }^{3} J_{\mathrm{H}-\mathrm{H}}=10.8,15.1 \mathrm{~Hz}, 1 \mathrm{H}, 5-\mathrm{H}\right)$;

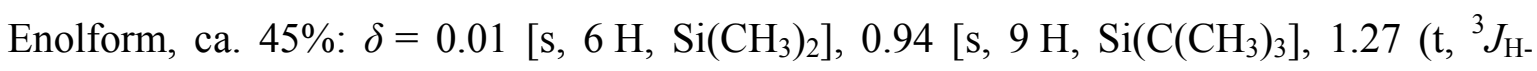
$\left.\mathrm{H}=7.0 \mathrm{~Hz}, 3 \mathrm{H}, 2^{\prime}-\mathrm{H}_{3}\right), 4.17\left(\mathrm{q},{ }^{3} J_{\mathrm{H}-\mathrm{H}}=6.0 \mathrm{~Hz}, 2 \mathrm{H}, 1^{\prime}-\mathrm{H}_{2}\right), 4.30\left(\mathrm{~m}_{\mathrm{c}}, 2 \mathrm{H}, 8-\mathrm{H}_{2}\right), 5.05(\mathrm{~s}$, $1 \mathrm{H}, 2-\mathrm{H}), 6.03\left(\mathrm{~d},{ }^{3} J_{\mathrm{H}-\mathrm{H}}=14.4 \mathrm{~Hz}, 1 \mathrm{H}, 4-\mathrm{H}\right), 6.20-6.60(\mathrm{~m}, 2 \mathrm{H}, 6-\mathrm{H}, 7-\mathrm{H}), 7.05\left(\mathrm{dd},{ }^{3} J_{\mathrm{H}-}\right.$ $\mathrm{H}=10.6,15.6 \mathrm{~Hz}, 1 \mathrm{H}, 5-\mathrm{H}), 11.88(\mathrm{~s}, 1 \mathrm{H}, \mathrm{OH}) \mathrm{ppm} .-{ }^{13} \mathrm{C}-\mathrm{NMR}\left(62.9 \mathrm{MHz}, \mathrm{CDCl}_{3}\right)$ : Ketoform: $\delta=-5.4\left[\mathrm{Si}\left(\mathrm{CH}_{3}\right)_{2}\right], 13.7\left(\mathrm{C}-2^{\prime}\right), 18.3\left[\mathrm{SiC}\left(\mathrm{CH}_{3}\right)_{3}\right], 25.8\left[\mathrm{SiC}\left(\mathrm{CH}_{3}\right)_{3}\right], 47.4(\mathrm{C}-2)$, 61.3 (C-1'), 62.8 (C-8), 126.7 (C-4), 127.9 (C-6), 139.3 (C-7), 144.3 (C-5), 157.0 (C-1), 192.2 (C-3); Enolform: $\delta=-5.4 \quad\left[\mathrm{Si}\left(\mathrm{CH}_{3}\right)_{2}\right], 14.1 \quad\left(\mathrm{C}-2^{\prime}\right), \quad 18.3 \quad\left[\mathrm{SiC}\left(\mathrm{CH}_{3}\right)_{3}\right], 25.8$ [SiC $\left.\left(\mathrm{CH}_{3}\right)_{3}\right], 60.1\left(\mathrm{C}-1^{\prime}\right), 63.0(\mathrm{C}-8), 91.3(\mathrm{C}-2), 124.6$ (C-4), 127.6 (C-6), 136.5 (C-7), 144.0 (C-5), 167.4 (C-1), 169.3 (C-3) ppm. 
(4E,6Z)-8-(tert-Butyldimethylsilanyloxy)-3-oxoocta-4,6-diensäureethylester (155z)

Die Umsetzung wurde analog AAV05 durchgeführt, als Aldehyd wurde jedoch (Z)-4-(tert-Butyldimethylsilanyloxy)but-2-enal $(2.67 \mathrm{~g}, 13.3 \mathrm{mmol})$ verwendet. Nach Chromatographie wurden $2.21 \mathrm{~g}$

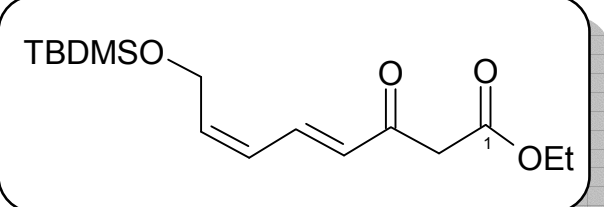
(53\%) der Titelverbindung als leicht gelbes Öl erhalten. $R_{\mathrm{f}}=0.54$ (Hex:EE, 5:1). $-{ }^{1} \mathrm{H}-$

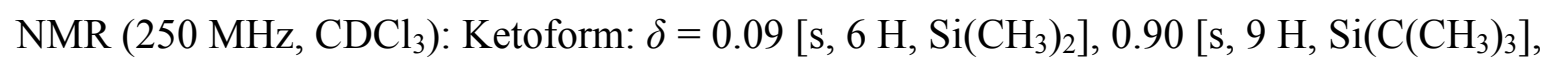
$1.27\left(\mathrm{t},{ }^{3} J_{\mathrm{H}-\mathrm{H}}=7.1 \mathrm{~Hz}, 3 \mathrm{H}, 2^{\prime}-\mathrm{H}_{3}\right), 3.59\left(\mathrm{~s}, 2 \mathrm{H}, 2-\mathrm{H}_{2}\right), 4.17\left(\mathrm{q},{ }^{3} J_{\mathrm{H}-\mathrm{H}}=7.0 \mathrm{~Hz}, 2 \mathrm{H}, 1^{\prime}-\mathrm{H}_{2}\right)$, $4.40-4.50\left(\mathrm{~m}, 2 \mathrm{H}, 8-\mathrm{H}_{2}\right), 5.70-6.30(\mathrm{~m}, 3 \mathrm{H}, 4-\mathrm{H}, 6-\mathrm{H}, 7-\mathrm{H}), 7.55\left(\mathrm{dd},{ }^{3} J_{\mathrm{H}-\mathrm{H}}=11.3\right.$, $15.3 \mathrm{~Hz}, 1 \mathrm{H}, 5-\mathrm{H})$; Enolform, ca. 45\%: $\delta=0.09$ [s, $\left.6 \mathrm{H}, \mathrm{Si}\left(\mathrm{CH}_{3}\right)_{2}\right], 0.90$ [s, $9 \mathrm{H}$,

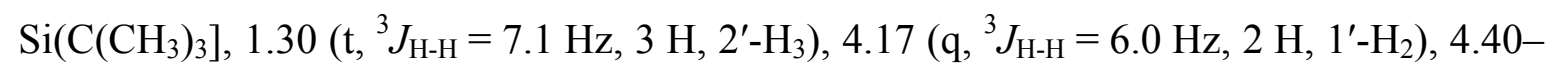
4.50 (m, 2 H, 8- $\mathrm{H}_{2}$ ), 5.05 (s, $\left.1 \mathrm{H}, 2-\mathrm{H}\right), 5.70-6.30$ (m, $\left.3 \mathrm{H}, 4-\mathrm{H}, 6-\mathrm{H}, 7-\mathrm{H}\right), 7.32\left(\mathrm{dd},{ }^{3} J_{\mathrm{H}-\mathrm{H}}\right.$ $=11.6,15.0 \mathrm{~Hz}, 1 \mathrm{H}, 5-\mathrm{H}), 11.88(\mathrm{~s}, 1 \mathrm{H}, \mathrm{OH}) \mathrm{ppm} .-{ }^{13} \mathrm{C}-\mathrm{NMR}\left(75.5 \mathrm{MHz}, \mathrm{CDCl}_{3}\right)$ : Ketoform: $\delta=-5.4\left[\mathrm{Si}\left(\mathrm{CH}_{3}\right)_{2}\right], 14.1\left(\mathrm{C}-2^{\prime}\right), 18.3\left[\mathrm{SiC}\left(\mathrm{CH}_{3}\right)_{3}\right], 25.6\left[\mathrm{SiC}\left(\mathrm{CH}_{3}\right)_{3}\right], 47.7(\mathrm{C}-2)$, 60.1 (C-1'), 61.4 (C-8), 126.5 (C-4), 127.2 (C-6), 139.3 (C-7), 141.5 (C-5), 167.3 (C-1), 192.1 (C-3); Enolform: $\delta=-5.4 \quad\left[\mathrm{Si}\left(\mathrm{CH}_{3}\right)_{2}\right], 14.1 \quad\left(\mathrm{C}-2^{\prime}\right), 18.3 \quad\left[\mathrm{SiC}\left(\mathrm{CH}_{3}\right)_{3}\right], 25.9$ [SiC $\left.\left(\mathrm{CH}_{3}\right)_{3}\right], 60.2\left(\mathrm{C}-1^{\prime}\right), 65.8$ (C-8), 91.9 (C-2), 129.4 (C-4), 131.6 (C-6), 137.0 (C-7), 138.7 (C-5), 169.0 (C-3), 172.7 (C-1) ppm.

\section{Allgemeine Arbeitsvorschrift 06: Dekonjugierung}

(5E,7Z)-8-(tert-Butyldimethylsilanyloxy)-3-oxoocta-5,7-diensäureethylester (156)

Diisopropylamin $(1.55 \mathrm{~mL}, 1.12 \mathrm{~g}, 11.1 \mathrm{mmol})$ wurde in THF $(50 \mathrm{~mL})$ gelöst und auf $0{ }^{\circ} \mathrm{C}$ gekühlt. Nach Zugabe von $n$-BuLi (4.87 mL, $11.5 \mathrm{mmol}, 2.36 \mathrm{M}$ in Hexan) ließ man $30 \mathrm{~min}$ bei $\quad 0^{\circ} \mathrm{C}$ rühren, kühlte auf

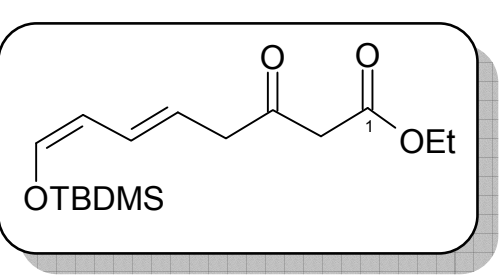
$-78^{\circ} \mathrm{C}$ und versetzte mit DMPU (1.63 mL, $\left.1.73 \mathrm{~g}, 13.5 \mathrm{mmol}\right)$. Nach $5 \mathrm{~min}$ Rühren bei $-78^{\circ} \mathrm{C}$ wurde langsam der konjugierte Ester 155 (1.05 g, $\left.3.36 \mathrm{mmol}\right)$ zugegeben. Die gelborange Lösung wurde $2.5 \mathrm{~h}$ bei $-78^{\circ} \mathrm{C}$ gerührt und anschließend durch langsame Zugabe von konzentrierter Essigsäure $(1.8 \mathrm{~mL})$ hydrolysiert. Nach $10 \mathrm{~min}$ wurde das Kühlbad entfernt und Ether $(100 \mathrm{~mL})$ sowie Wasser $(50 \mathrm{~mL})$ zugegeben. Die Phasen wurden getrennt, die wässrige Phase mit Ether extrahiert $(2 \times 100 \mathrm{~mL})$ und anschließend die 
vereinigten organischen Phasen mit $\mathrm{NH}_{4} \mathrm{Cl}$-Lösung $(20 \mathrm{~mL}), 1 \mathrm{M} \mathrm{NaHCO}_{3}$-Lösung $(30 \mathrm{~mL})$, Wasser $(2 \times 50 \mathrm{~mL})$ und NaCl-Lösung $(25 \mathrm{~mL})$ gewaschen. Nach Trocknen über Natriumsulfat erhielt man 1.07 g Rohprodukt von zufriedenstellender Reinheit, welches aufgrund der limitierten Stabilität (bei Lagerung in Reinsubstanz Zersetzung innerhalb von Tagen, teilweise Zersetzung auf Silicagel) direkt reduziert wurde. $R_{\mathrm{f}}=0.43$ (Hex:EE, 5:1).

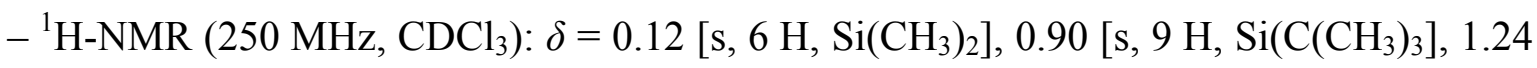
$\left(\mathrm{t},{ }^{3} J_{\mathrm{H}-\mathrm{H}}=7.1 \mathrm{~Hz}, 3 \mathrm{H}, 2^{\prime}-\mathrm{H}_{3}\right), 3.28\left(\mathrm{dd},{ }^{3} J_{\mathrm{H}-\mathrm{H}}=1.0,7.2 \mathrm{~Hz}, 2 \mathrm{H}, 4-\mathrm{H}_{2}\right), 3.44\left(\mathrm{~s}, 2 \mathrm{H}, 2-\mathrm{H}_{2}\right)$, $4.15\left(\mathrm{q},{ }^{3} J_{\mathrm{H}-\mathrm{H}}=7.1 \mathrm{~Hz}, 2 \mathrm{H}, 1^{\prime}-\mathrm{H}_{2}\right), 5.14\left(\mathrm{dd},{ }^{3} J_{\mathrm{H}-\mathrm{H}}=5.8,10.7 \mathrm{~Hz}, 1 \mathrm{H}, 7-\mathrm{H}\right), 5.54\left(\mathrm{dtt}, J_{\mathrm{H}-}\right.$ $\mathrm{H}=1.0,7.3,15.5 \mathrm{~Hz}, 1 \mathrm{H}, 5-\mathrm{H}), 6.15\left(\mathrm{~d},{ }^{3} J_{\mathrm{H}-\mathrm{H}}=5.8 \mathrm{~Hz}, 1 \mathrm{H}, 8-\mathrm{H}\right), 6.50\left(\mathrm{ddt}, J_{\mathrm{H}-\mathrm{H}}=1.0\right.$, 10.7, $15.5 \mathrm{~Hz}, 1 \mathrm{H}, 6-\mathrm{H})$ ppm. $-{ }^{13} \mathrm{C}-\mathrm{NMR}\left(62.9 \mathrm{MHz}, \mathrm{CDCl}_{3}\right): \delta=-5.4\left[\mathrm{Si}\left(\mathrm{CH}_{3}\right)_{2}\right], 14.1$ $\left(\mathrm{C}-2^{\prime}\right), 18.3\left[\mathrm{SiC}\left(\mathrm{CH}_{3}\right)_{3}\right], 25.7$ [ $\left.\mathrm{SiC}\left(\mathrm{CH}_{3}\right)_{3}\right], 47.2(\mathrm{C}-4), 48.5(\mathrm{C}-2), 61.3\left(\mathrm{C}-1^{\prime}\right), 109.6(\mathrm{C}-8)$, 119.7 (C-6), 127.9 (C-7), 140.3 (C-5), 167.1 (C-1), 201.1 (C-3) ppm. - MS (DCI, NH $)_{3}$, $m / z(\%): 314,(55)[\mathrm{M}+\mathrm{H}]^{+}, 326(100)[\mathrm{M}+\mathrm{Na}]^{+}, 676(20)$.

\section{Allgemeine Arbeitsvorschrift 07: Reduktion der $\beta$-Ketofunktion mit Natriumborhydrid}

\section{(5E,7Z)-8-(tert-Butyldimethylsilanyloxy)-3-hydroxyocta-5,7-diensäureethylester (160)}

$\beta$-Ketoester $156 \quad(1.07 \mathrm{~g}, \quad 3.42 \mathrm{mmol}) \quad$ aus vorangegangenem Versuch wurde in p.a. Ethanol $(30 \mathrm{~mL})$ gelöst und auf $0{ }^{\circ} \mathrm{C}$ gekühlt. Nach Zugabe von $\mathrm{NaBH}_{4}$ (64.8 mg, $1.71 \mathrm{mmol})$ wurde bei $0^{\circ} \mathrm{C}$ gerührt. Der Reaktionsverlauf wurde per Dünnschichtchromatographie

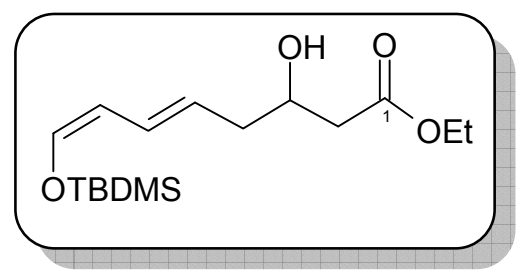
(Hex:EE, 5:1) verfolgt. Nach 10 min war der Umsatz vollständig und die Reaktion wurde durch Zugabe von $1 \mathrm{M} \mathrm{HCl}(2.0 \mathrm{~mL})$ beendet. Es wurden Pentan $(50 \mathrm{~mL})$ und Wasser $(15 \mathrm{~mL})$ zugegeben, die Phasen getrennt und die organische Phase mit Pentan extrahiert $(2 \times 100 \mathrm{~mL})$. Die vereinigten organischen Phasen wurden mit $1 \mathrm{M} \mathrm{NaHCO}_{3}(50 \mathrm{~mL})$, Wasser $(2 \times 100 \mathrm{~mL})$ und NaCl-Lösung $(30 \mathrm{~mL})$ gewaschen, über Natriumsulfat getrocknet und das Lösungsmittel unter vermindertem Druck entfernt. Das so erhaltene Rohprodukt ( $898 \mathrm{mg}$ ) wurde aufgrund der geringen Stabilität direkt weiter umgesetzt. $R_{\mathrm{f}}=$ 0.31 (Hex:EE, 5:1) - ${ }^{1} \mathrm{H}-\mathrm{NMR}\left(250 \mathrm{MHz}, \mathrm{CDCl}_{3}\right): \delta=0.00$ [s, $\left.6 \mathrm{H}, \mathrm{Si}\left(\mathrm{CH}_{3}\right)_{2}\right], 0.78$ [s, $9 \mathrm{H}$,

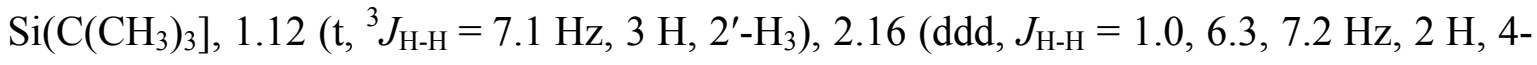
$\left.\mathrm{H}_{2}\right), 2.27\left(\mathrm{dd}, J_{\mathrm{H}-\mathrm{H}}=8.7,16.3 \mathrm{~Hz}, 1 \mathrm{H}, 2-\mathrm{H}_{\mathrm{a}}\right), 2.38\left(\mathrm{dd}, J_{\mathrm{H}-\mathrm{H}}=3.5,16.3 \mathrm{~Hz}, 1 \mathrm{H}, 2-\mathrm{H}_{\mathrm{b}}\right)$, $2.73\left(\mathrm{~d},{ }^{3} J_{\mathrm{H}-\mathrm{H}}=3.5 \mathrm{~Hz}, 1 \mathrm{H}, \mathrm{OH}\right), 3.91$ (dddt, $\left.J_{\mathrm{H}-\mathrm{H}}=3.5,6.3,8.7,10.0 \mathrm{~Hz}, 1 \mathrm{H}, 3-\mathrm{H}\right), 4.02$ 
$\left(\mathrm{q},{ }^{3} J_{\mathrm{H}-\mathrm{H}}=7.1 \mathrm{~Hz}, 2 \mathrm{H}, 1^{\prime}-\mathrm{H}_{2}\right), 5.00\left(\mathrm{dd},{ }^{3} J_{\mathrm{H}-\mathrm{H}}=5.8,10.7 \mathrm{~Hz}, 1 \mathrm{H}, 7-\mathrm{H}\right), 5.37\left(\mathrm{dt},{ }^{3} J_{\mathrm{H}-\mathrm{H}}=\right.$ 7.2, $15.5 \mathrm{~Hz}, 1 \mathrm{H}, 5-\mathrm{H}), 5.98\left(\mathrm{~d},{ }^{3} J_{\mathrm{H}-\mathrm{H}}=5.8 \mathrm{~Hz}, 1 \mathrm{H}, 8-\mathrm{H}\right), 6.35$ (ddd, $J_{\mathrm{H}-\mathrm{H}}=1.0,10.7$, $15.5 \mathrm{~Hz}, 1 \mathrm{H}, 6-\mathrm{H}) \mathrm{ppm} .-{ }^{13} \mathrm{C}-\mathrm{NMR}\left(62.9 \mathrm{MHz}, \mathrm{CDCl}_{3}\right): \delta=-5.5$ [Si( $\left.\left(\mathrm{CH}_{3}\right)_{2}\right], 14.1\left(\mathrm{C}-2^{\prime}\right)$, $18.2\left[\mathrm{SiC}\left(\mathrm{CH}_{3}\right)_{3}\right], 25.5\left[\mathrm{SiC}\left(\mathrm{CH}_{3}\right)_{3}\right], 40.1(\mathrm{C}-4), 40.6(\mathrm{C}-2), 60.6\left(\mathrm{C}-1^{\prime}\right), 67.8(\mathrm{C}-3), 110.1$ (C-8), 124.6 (C-6), 126.4 (C-7), 139.4 (C-5), 172.8 (C-1) ppm. - MS (DCI, NH3), $m / z(\%): 337(65)[\mathrm{M}+\mathrm{Na}]^{+}, 385(100), 667(50)$.

Versuch der Darstellung von (5E,7Z)-8-(tert-Butyldimethylsilanyloxy)-3-benzyloxyocta5,7-diensäureethylester (161)

A: $\mathrm{Ag}_{2} \mathrm{O}(30 \mathrm{mg}, 0.13 \mathrm{mmol}, 1.3 \mathrm{eq})$ wurde in Benzol $(0.5 \mathrm{~mL})$ suspendiert und mit Alkohol 160 (31 mg, $0.10 \mathrm{mmol}$, $1.0 \mathrm{eq}$ ), gelöst in $1.5 \mathrm{~mL}$ Benzol, versetzt.

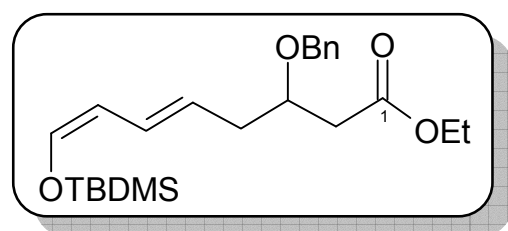

Nach Zugabe von Benzylbromid (41 mg, 0.24 mmol, 2.4 eq) ließ man 20 h bei RT rühren. Das Reaktionsgemisch wurde über Celite filtriert, der Rückstand mit Ether gewaschen und das Lösungsmittel unter vermindertem Druck entfernt. Der Rückstand (46 mg) enthielt nicht die Titelverbindung, sondern ausschließlich nicht identifizierbare Zersetzungsprodukte.

B: Alkohol 160 (60 mg, $0.19 \mathrm{mmol}, 1.0 \mathrm{eq})$ wurde in Dichlormethan (5 mL) gelöst und auf $0{ }^{\circ} \mathrm{C}$ gekühlt. Dann wurden nacheinander das Benzylierungsreagenz Benzyltrichloracetimidat $^{[125]}(0.29 \mathrm{mmol}, 1.5 \mathrm{eq})$ und TFA (46 mg, $\left.40 \mathrm{mmol}, 0.21 \mathrm{eq}\right)$ zugegeben. Man ließ zunächst $3 \mathrm{~h}$ bei $0{ }^{\circ} \mathrm{C}$, dann bei RT rühren. DC-Kontrolle (Hex:EE, 5:1) zeigte auch nach $20 \mathrm{~h}$ bei RT keinen Umsatz an. Das ${ }^{1} \mathrm{H}-\mathrm{NMR}-$ Spektrum nach wässriger Aufarbeitung zeigte nur nicht identifizierbare Zersetzungsprodukte.

Versuch der Darstellung von (5E,7Z)-8-(tert-Butyldimethylsilanyloxy)-3-methoxy-5,7diensäureethylester (162)

Alkohol 160 (30 mg, $0.13 \mathrm{mmol}, \quad 1.0$ eq) wurde in Dichlormethan $(5 \mathrm{~mL})$ gelöst und mit Diazomethanlösung $(1.0 \mathrm{~mL}$, ca. $0.2 \mathrm{M})$ versetzt. Die gelbe Reaktionslösung wurde bei RT gerührt und der Reaktionsfortschritt per DC

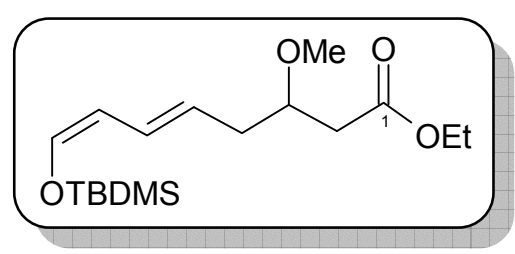
verfolgt (Hex:EE, 5:1). Da nach 2 h Rühren kein Umsatz zu erkennen war, wurde eine Spatelspitze Kieselgel und Diazomethanlösung $(1 \mathrm{~mL})$ zugesetzt. Nach weiteren $60 \mathrm{~min}$ 
wurde das Gemisch filtriert und das Lösungsmittel im Vakuum entfernt. Der Rückstand (44.5 mg) enthielt nur Edukt (50-60\%) und nicht identifizierbare Zersetzungsprodukte.

\section{Allgemeine Arbeitsvorschrift 08: TMS-Schützung der $\beta$-Hydroxyfunktion}

(5E,7Z)-8-(tert-Butyldimethylsilanyloxy)-3-trimethylsilanyloxyocta-5,7-diensäureethylester (163)

Alkohol $160 \quad(898 \mathrm{mg}, \quad 2.86 \mathrm{mmol}) \quad$ wurde in Dichlormethan $(20 \mathrm{~mL})$ gelöst, auf $0{ }^{\circ} \mathrm{C}$ gekühlt und mit Triethylamin (1.98 mL, $1.44 \mathrm{~g}, 14.3 \mathrm{mmol})$ versetzt. Nach Zugabe von TMSCl (1.09 mL, $933 \mathrm{mg}, 8.59 \mathrm{mmol})$ ließ

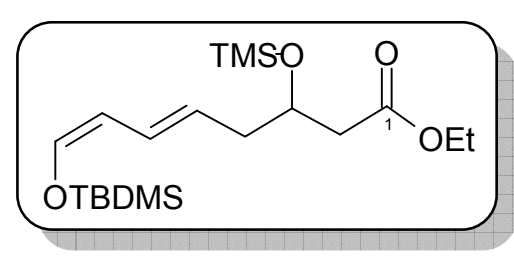
man $1.5 \mathrm{~h}$ bei $0{ }^{\circ} \mathrm{C}$ rühren. Die Reaktion wurde durch Zugabe von Wasser $(25 \mathrm{~mL})$ beendet und das Reaktionsgemisch mit Ether $(100 \mathrm{~mL})$ verdünnt. Die Phasen wurden getrennt und die wässrige Phase mit Ether $(100 \mathrm{~mL})$ und Pentan $(100 \mathrm{~mL})$ extrahiert. Die organische Phase wurde mit verd. $\mathrm{NH}_{4} \mathrm{Cl}$-Lösung $(50 \mathrm{~mL}), 1 \mathrm{M} \mathrm{NaHCO}$-Lösung $(50 \mathrm{~mL})$, Wasser $(50 \mathrm{~mL})$ und $\mathrm{NaCl}-\mathrm{Lösung}(50 \mathrm{~mL})$ gewaschen, über Natriumsulfat getrocknet und das Lösungsmittel im Vakuum entfernt. Das so erhaltene Rohprodukt (1.07 g) wurde säulenchromatographisch aufgereinigt (Hex:EE, 8:1). Man erhielt $952 \mathrm{mg}$ (86\% über drei Stufen, ausgehend von konj. Ester 150) der Titelverbindung als farbloses Öl. $R_{\mathrm{f}}=0.60$ (Hex:EE, 5:1). $-{ }^{1} \mathrm{H}-\mathrm{NMR}\left(300 \mathrm{MHz}, \mathrm{CDCl}_{3}\right): \delta=0.00\left[\mathrm{~s}, 6 \mathrm{H}, \mathrm{Si}\left(\mathrm{CH}_{3}\right)_{2}\right], 0.05[\mathrm{~s}, 9 \mathrm{H}$, $\left.\mathrm{Si}\left(\mathrm{CH}_{3}\right)_{3}\right], 0.84\left[\mathrm{~s}, 9 \mathrm{H}, \mathrm{SiC}\left(\mathrm{CH}_{3}\right)_{3}\right], 1.16\left(\mathrm{t},{ }^{3} J_{\mathrm{H}-\mathrm{H}}=7.1 \mathrm{~Hz}, 3 \mathrm{H}, 2^{\prime}-\mathrm{H}_{3}\right), 2.18\left(\mathrm{ddd}, J_{\mathrm{H}-\mathrm{H}}=\right.$ 1.0, 5.9, 7.3 Hz, $\left.2 \mathrm{H}, 4-\mathrm{H}_{2}\right), 2.34\left(\mathrm{~m}_{\mathrm{c}}, 2 \mathrm{H}, 2-\mathrm{H}_{2}\right), 3.95-4.12\left(\mathrm{~m}, 3 \mathrm{H}, 3-\mathrm{H}, 1^{\prime}-\mathrm{H}_{2}\right), 5.04$ (dd, $\left.{ }^{3} J_{\mathrm{H}-\mathrm{H}}=5.9,10.7 \mathrm{~Hz}, 1 \mathrm{H}, 7-\mathrm{H}\right), 5.39\left(\mathrm{dt},{ }^{3} J_{\mathrm{H}-\mathrm{H}}=7.3,15.5 \mathrm{~Hz}, 1 \mathrm{H}, 5-\mathrm{H}\right), 6.02\left(\mathrm{~d},{ }^{3} J_{\mathrm{H}-}\right.$ $\mathrm{H}=5.8 \mathrm{~Hz}, 1 \mathrm{H}, 8-\mathrm{H}), 6.35\left(\mathrm{ddd}, J_{\mathrm{H}-\mathrm{H}}=1.0,10.7,15.5 \mathrm{~Hz}, 1 \mathrm{H}, 6-\mathrm{H}\right) \mathrm{ppm} .-{ }^{1} \mathrm{H}-\mathrm{NMR}$ $\left(250 \mathrm{MHz}, \mathrm{C}_{6} \mathrm{D}_{6}\right): \delta=0.06\left[\mathrm{~s}, 6 \mathrm{H}, \mathrm{Si}\left(\mathrm{CH}_{3}\right)_{2}\right], 0.18$ [s, $\left.9 \mathrm{H}, \mathrm{Si}\left(\mathrm{CH}_{3}\right)_{3}\right], 0.95$ [s, $9 \mathrm{H}$,

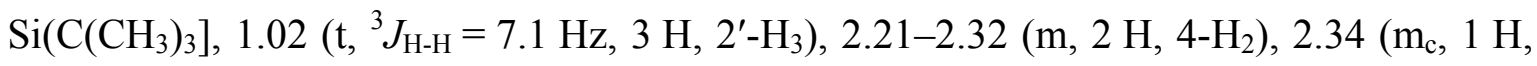
$\left.2-\mathrm{H}_{\mathrm{a}}\right), 2.48\left(\mathrm{dd},{ }^{3} J_{\mathrm{H}-\mathrm{H}}=8.2,15.0 \mathrm{~Hz}, 1 \mathrm{H}, 2-\mathrm{H}_{\mathrm{b}}\right), 4.00\left(\mathrm{q},{ }^{3} J_{\mathrm{H}-\mathrm{H}}=7.1 \mathrm{~Hz}, 2 \mathrm{H}, 1^{\prime}-\mathrm{H}_{2}\right), 4.30$ $\left(\mathrm{m}_{\mathrm{c}}, 1 \mathrm{H}, 3-\mathrm{H}\right), 5.27\left(\mathrm{dd},{ }^{3} J_{\mathrm{H}-\mathrm{H}}=6.0,10.5 \mathrm{~Hz}, 1 \mathrm{H}, 7-\mathrm{H}\right), 5.60\left(\mathrm{~m}_{\mathrm{c}}, 1 \mathrm{H}, 5-\mathrm{H}\right), 6.09\left(\mathrm{~d},{ }^{3} J_{\mathrm{H}-}\right.$ $\mathrm{H}=5.8 \mathrm{~Hz}, 1 \mathrm{H}, 8-\mathrm{H}), 6.81\left(\mathrm{ddd}, J_{\mathrm{H}-\mathrm{H}}=1.0,10.7,15.5 \mathrm{~Hz}, 1 \mathrm{H}, 6-\mathrm{H}\right) \mathrm{ppm} .-{ }^{13} \mathrm{C}-\mathrm{NMR}$ $\left(62.9 \mathrm{MHz}, \mathrm{CDCl}_{3}\right): \delta=-5.7\left[\mathrm{Si}\left(\mathrm{CH}_{3}\right)_{2}\right], 0.1\left[\mathrm{Si}\left(\mathrm{CH}_{3}\right)_{3}\right], 14.0\left(\mathrm{C}-2^{\prime}\right), 18.1\left[\mathrm{SiC}\left(\mathrm{CH}_{3}\right)_{3}\right]$, $25.4\left[\mathrm{SiC}\left(\mathrm{CH}_{3}\right)_{3}\right], 41.4(\mathrm{C}-4), 42.3$ (C-2), 59.7 (C-1'), 69.6 (C-3), 110.9 (C-8), 125.7 (C-6), 126.3 (C-7), 138.8 (C-5), 170.9 (C-1) ppm. 
(4E,6Z)-8-(tert-Butyldimethylsilanyloxy)-3-hydroxyocta-4,6-diensäureethylester (195)

Die Reduktion wurde wie oben für den dekonjugierten $\beta$-Ketoester $\mathbf{1 5 6}$ beschrieben durchgeführt. Ausgehend von $\mathbf{1 5 5}_{Z}$ (579 mg, $1.84 \mathrm{mmol}) \quad$ konnten $\quad 414 \mathrm{mg} \quad(71 \%) \quad$ der

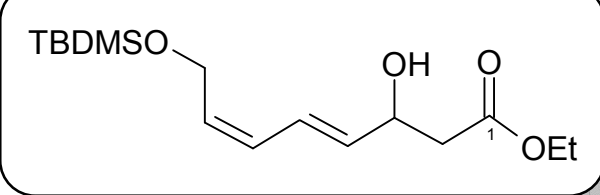

Titelverbindung isoliert werden. $R_{\mathrm{f}}=0.20$ (Hex:EE, 5:1). $-{ }^{1} \mathrm{H}-\mathrm{NMR}\left(300 \mathrm{MHz}, \mathrm{C}_{6} \mathrm{D}_{6}\right)$ : $\delta=0.05\left[\mathrm{~s}, 6 \mathrm{H}, \mathrm{Si}\left(\mathrm{CH}_{3}\right)_{2}\right], 0.84\left[\mathrm{~s}, 9 \mathrm{H}, \mathrm{SiC}\left(\mathrm{CH}_{3}\right)_{3}\right], 1.25\left(\mathrm{t},{ }^{3} J_{\mathrm{H}-\mathrm{H}}=7.1 \mathrm{~Hz}, 3 \mathrm{H}, 2^{\prime}-\mathrm{H}_{3}\right)$, $2.50\left(\mathrm{~m}_{\mathrm{c}}, 2 \mathrm{H}, 2-\mathrm{H}_{2}\right), 3.00\left(\mathrm{~d}_{\mathrm{br}},{ }^{3} J_{\mathrm{H}-\mathrm{H}}=4.5 \mathrm{~Hz}, 1 \mathrm{H}, \mathrm{OH}\right), 4.10-4.20\left(\mathrm{~m}, 2 \mathrm{H}, 8-\mathrm{H}_{2}\right), 4.58$ $\left(\mathrm{m}_{\mathrm{c}}, 1 \mathrm{H}, 3-\mathrm{H}\right), 5.60-5.80$ (m, 2 H, 4-H, 7-H), 6.10-6.30 (m, 2 H, 5-H, 6-H) ppm.

\section{E.3.3 Versuche zur Darstellung des (5E,7E)-Diensäureethylesters}

\section{Variation der Reaktionsbedingungen der Dekonjugierung}

a) Variation der Temperatur

Die Dekonjugierung von 155 wurde wie oben beschrieben durchgeführt. Man ließ jedoch nach der Zugabe des Esters zum frisch generierten LDA auf -40, -20 bzw $0{ }^{\circ} \mathrm{C}$ erwärmen. Nach einer Stunde Rühren bei der jeweiligen Temperatur wurde die Reaktion erneut auf $78^{\circ} \mathrm{C}$ gekühlt und dann wie oben beschrieben hydrolysiert und aufgearbeitet. Die ${ }^{1} \mathrm{H}-$ NMR-Spektren zeigten alle nicht das gewünschte, sondern das dekonjugierte $(5 E, 7 Z)$ Isomer als Hauptprodukt.

\section{b) Variation der Base}

A: Der konjugierte Ester $155(118 \mathrm{mg}, 378 \mu \mathrm{mol})$ wurde in THF (15 mL) gelöst und auf $0{ }^{\circ} \mathrm{C}$ gekühlt. Nach Zugabe von NaH (16 mg, $400 \mu \mathrm{mol}$, 60\% in Mineralöl) wurde 20 min bei $0{ }^{\circ} \mathrm{C}$ gerührt. Anschließend wurde auf $-78^{\circ} \mathrm{C}$ gekühlt und mit $n$-BuLi (418 $\mu$ mol, 1.63 $\mathrm{M}$ in Hexan) versetzt. Man ließ eine Stunde bei $-78{ }^{\circ} \mathrm{C}$ rühren. ${ }^{1} \mathrm{H}-\mathrm{NMR}$ Spektroskopie nach wässriger Aufarbeitung (siehe oben) zeigte nur konjugiertes Edukt. 
B: Die Dekonjugierung wurde wie unter A beschrieben durchgeführt, allerdings wurden zusätzlich 4 eq HMPA zugegeben. ${ }^{1}$ H-NMR Spektroskopie nach wässriger Aufarbeitung (siehe oben) zeigte nur nicht identifizierbare Zersetzungsprodukte.

\section{c) Bestrahlung}

Der dekonjugierte (5E,7Z)-Ester $156(125-160 \mu \mathrm{mol})$ wurde in Pentan $(50 \mathrm{~mL})$ gelöst und unter Wasserkühlung entweder in einer Quarzglasapparatur mit dem Licht einer Quecksilberdampflampe $(250 \mathrm{~W})$ oder unter Rückfluss in einem Kolben mit dem Licht einer Osram Ultra Vitallux (120 W) Lampe bestrahlt. Die Reaktionszeiten wurden von $5 \mathrm{~min}$ bis $24 \mathrm{~h}$ variiert. Bei allen Versuchen zeigten sich in den ${ }^{1} \mathrm{H}-\mathrm{NMR}-$ Spektren entweder nur das $(5 E, 7 Z)$-Edukt oder nicht identifizierbare Zersetzungsprodukte.

d) Reaktion mit $\mathrm{NpCr}(\mathrm{CO})_{3}{ }^{[175]}$

$\beta$-Ketoester 155 $_{Z}(546 \mathrm{mg}, 174 \mu \mathrm{mol})$ bzw. $\beta$-Hydroxyester 195 (575 mg, $\left.182 \mu \mathrm{mol}\right)$ wurden in Aceton $(3.0 \mathrm{ml})$ gelöst und mit $\operatorname{NpCr}(\mathrm{CO})_{3} \quad(0.2 \mathrm{eq})$ versetzt. Die Reaktionslösung wurde entgast (drei freeze-pump-Zyklen) und dann bei RT gerührt. Nach Reaktionszeiten von bis zu $16 \mathrm{~h}$ wurde filtriert und eingeengt. Die ${ }^{1} \mathrm{H}-\mathrm{NMR}-$ Spektren zeigten nur Edukt.

\section{e) Isomerisierung mit Iod}

Der dekonjugierte (5E,7Z)-Ester 156 (502 mg, $159 \mu \mathrm{mol})$ wurde in Pentan gelöst und mit einer Spatelspitze Iod versehen. Man ließ bei RT rühren. DC-Kontrolle (Hex:EE, 5:1) zeigte keinen Umsatz an. Das ${ }^{1}$ H-NMR-Spektrum nach wässriger Aufarbeitung war aufgrund einer sehr starken Linienverbreiterung nicht auswertbar. Eine zusätzliche Kontrolle per DC ließ keine isolierbaren Verbindungen erkennen. 


\section{Versuch der TMS-Enoletherbildung von 155 und anschließende Dekonjugierung}

A: Der konjugierte Ester $155(600-700 \mu \mathrm{mol})$ wurde in Dichlormethan $(5 \mathrm{~mL})$ gelöst und auf $0{ }^{\circ} \mathrm{C}$ gekühlt. Nach Zugabe von Triethylamin (3.00 eq) und TMSCl (1.05 eq) wurde zunächst 20 min bei $0{ }^{\circ} \mathrm{C}$, dann bei RT gerührt. DC-Kontrolle zeigte nur Spuren des TMSgeschützten Produktes. Da auch weitere Zugabe von $\mathrm{NEt}_{3}$ und TMSCl den Umsatz nicht weiter erhöhte, wurde die Reaktion nach 6 h wässrig aufgearbeitet. Sauberes Edukt wurde zu 90\% zurückgewonnen.

B: Der konjugierte Ester $155(678 \mu \mathrm{mol})$ wurde in THF $(5 \mathrm{~mL})$ gelöst und auf $0{ }^{\circ} \mathrm{C}$ gekühlt. Nach Zugabe von $\mathrm{NaH}$ (1.20 eq) wurde 20 min bei $0{ }^{\circ} \mathrm{C}$ gerührt und dann TMSCl (1.05 eq) zugegeben. DC-Kontrolle zeigte nach $1.5 \mathrm{~h}$ Rühren bei $0{ }^{\circ} \mathrm{C}$ unvollständigen Umsatz. Auch durch erneute Zugabe von $\mathrm{NaH}$ (1.20 eq) und TMSCl (0.50 eq) konnte der Umsatz (ca. $60 \%$ laut DC) nicht erhöht werden. Die Reaktionslösung wurde zu einer bei $-78{ }^{\circ} \mathrm{C}$ gerührten Lösung von LDA (2.20 eq) in THF (10 mL) gegeben. Nach 1 h Rühren bei $78^{\circ} \mathrm{C}$ wurde wässrig aufgearbeitet. Der Rückstand (256 mg) enthielt nur das dekonjugierte (5E,7Z)-Isomer sowie das konjugierte Edukt.

\section{Allgemeine Arbeitsvorschrift 09: Weinreb-Amid-Bildung}

(5E,7Z)-8-(tert-Butyldimethylsilanyloxy)-3-trimethylsilanyloxyocta-5,7-diensäure- $N$ methoxy-N-methylamid (164)

Der geschützte $\quad \beta$-Silanoxyester $163 \quad(540 \mathrm{mg}$, $1.40 \mathrm{mmol})$ wurde in THF $(10 \mathrm{~mL})$ gelöst, auf $-30{ }^{\circ} \mathrm{C}$ gekühlt und mit N,O-DimethylhydroxylaminHydrochlorid (272 mg, $279 \mathrm{mmol})$ versetzt. Zur

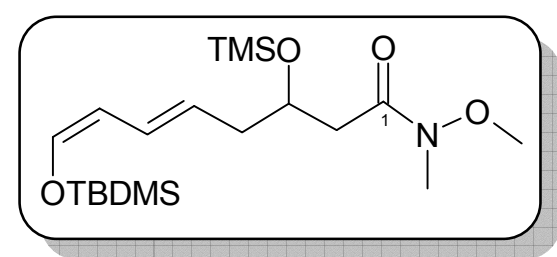
Suspension wurde innerhalb von $15 \mathrm{~min} i$ so-Propylmagnesiumbromid $(3.70 \mathrm{~mL}$, $5.59 \mathrm{mmol}, 1.51 \mathrm{M}$ in THF) gegeben. Man ließ $30 \mathrm{~min}$ rühren, wobei sich die Lösung auf $15{ }^{\circ} \mathrm{C}$ erwärmte. Die Reaktion wurde durch Zugabe von $\mathrm{NH}_{4} \mathrm{Cl}$-Lösung $(5 \mathrm{~mL})$ beendet und das Kühlbad entfernt. Nachdem die Reaktion RT erreicht hatte, wurden Wasser $(20 \mathrm{~mL})$ und Ether $(50 \mathrm{~mL})$ zugegeben und die Phasen getrennt. Die wässrige Phase wurde mit Pentan $(2 \times 50 \mathrm{~mL})$ extrahiert. Die vereinigten organischen Phasen wurden mit $1 \mathrm{M}$ $\mathrm{NaHCO}_{3}$-Lösung $(15 \mathrm{~mL})$, Wasser $(2 \times 20 \mathrm{~mL})$, NaCl-Lösung $(20 \mathrm{~mL})$ gewaschen und über Natriumsulfat getrocknet. Man erhielt $562 \mathrm{mg}(100 \%)$ der Titelverbindung als 
farbloses Ö1, welches aufgrund der mäßigen Stabilität direkt weiter umgesetzt wurde. $R_{\mathrm{f}}=$ 0.31 (Hex:EE, 5:1). - ${ }^{1} \mathrm{H}-\mathrm{NMR}\left(300 \mathrm{MHz}, \mathrm{C}_{6} \mathrm{D}_{6}\right): \delta=0.00$ [s, $\left.6 \mathrm{H}, \mathrm{Si}\left(\mathrm{CH}_{3}\right)_{2}\right], 0.18$ [s, $9 \mathrm{H}$, $\left.\mathrm{Si}\left(\mathrm{CH}_{3}\right)_{3}\right], 0.89\left[\mathrm{~s}, 9 \mathrm{H}, \mathrm{SiC}\left(\mathrm{CH}_{3}\right)_{3}\right], 2.37\left(\mathrm{dd}, J_{\mathrm{H}-\mathrm{H}}=5.7,12.7 \mathrm{~Hz}, 2 \mathrm{H}, 4-\mathrm{H}_{2}\right), 2.38\left(\mathrm{~m}_{\mathrm{c}}\right.$, $\left.1 \mathrm{H}, 2-\mathrm{H}_{\mathrm{a}}\right), 2.79\left(\mathrm{~m}_{\mathrm{c}}, 1 \mathrm{H}, 2-\mathrm{H}_{\mathrm{b}}\right), 2.87\left(\mathrm{~s}, 3 \mathrm{H}, \mathrm{NCH}_{3}\right), 3.10\left(\mathrm{~s}, 3 \mathrm{H}, \mathrm{OCH}_{3}\right) 4.50\left(\mathrm{~m}_{\mathrm{c}}, 1 \mathrm{H}\right.$, $3-\mathrm{H}), 5.25\left(\mathrm{dd},{ }^{3} J_{\mathrm{H}-\mathrm{H}}=5.8,10.7 \mathrm{~Hz}, 1 \mathrm{H}, 7-\mathrm{H}\right), 5.66\left(\mathrm{dt},{ }^{3} J_{\mathrm{H}-\mathrm{H}}=7.3,15.5 \mathrm{~Hz}, 1 \mathrm{H}, 5-\mathrm{H}\right)$, $6.04\left(\mathrm{~d},{ }^{3} J_{\mathrm{H}-\mathrm{H}}=5.8 \mathrm{~Hz}, 1 \mathrm{H}, 8-\mathrm{H}\right), 6.82\left(\mathrm{dd},{ }^{3} J_{\mathrm{H}-\mathrm{H}}=10.7,15.5 \mathrm{~Hz}, 1 \mathrm{H}, 6-\mathrm{H}\right) \mathrm{ppm} .-{ }^{13} \mathrm{C}-$ $\operatorname{NMR}\left(75.5 \mathrm{MHz}, \mathrm{C}_{6} \mathrm{D}_{6}\right): \delta=-5.5\left[\mathrm{Si}\left(\mathrm{CH}_{3}\right)_{2}\right], 0.5 \quad\left[\mathrm{Si}\left(\mathrm{CH}_{3}\right)_{3}\right], 18.4\left(\mathrm{SiC}\left(\mathrm{CH}_{3}\right)_{3}\right), 25.7$ [SiC $\left.\left(\mathrm{CH}_{3}\right)_{3}\right], 31.8\left(\mathrm{NCH}_{3}\right), 39.8(\mathrm{C}-4), 42.0(\mathrm{C}-2), 60.7\left(\mathrm{OCH}_{3}\right), 69.8(\mathrm{C}-3), 111.4(\mathrm{C}-8)$, 126.4 (C-6), 126.7 (C-7), 139.0 (C-5), 172.3 (C-1) ppm. - MS (DCI, NH ),$m / z$ (\%): ) 276 (50), 331 (100) $[\mathrm{M}-\mathrm{TMS}+\mathrm{H}]^{+}, 420(80)\left[\mathrm{M}+\mathrm{NH}_{4}\right]^{+}, 697$ (35).

\section{Allgemeine Arbeitsvorschrift 10 - Alkylierung des Weinreb-Amids}

(3E,9E,11Z)-12-(tert-Butyldimethylsilanyloxy)-1-trimethylsilanyl-7-trimethylsilanyloxydodeca-3,9,11-trien-1-in-5-on (165)

(E)-4-Brom-1-trimethylsilylbut-3-en-1-in (166, $60.3 \mathrm{mg}, 297 \mu \mathrm{mol})$ wurde in Ether $(4.0 \mathrm{~mL})$ gelöst, auf $-78^{\circ} \mathrm{C}$ gekühlt und langsam mit $t$ BuLi $(324 \mu 1,528 \mu \mathrm{mol}, 1.63 \mathrm{M}$ in Pentan)

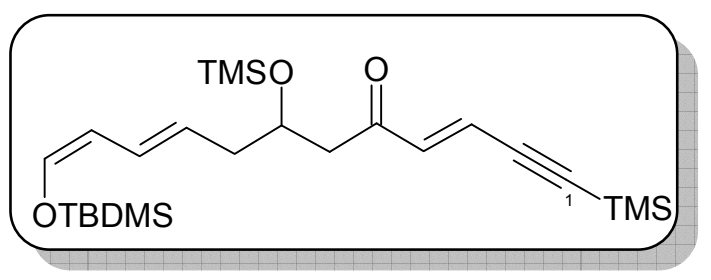
versetzt. Nach 1 h 15 min Rühren bei $-78^{\circ} \mathrm{C}$ wurde das Amid 164 (53.0 mg, $132 \mu \mathrm{mol}$ ), gelöst in Ether $(4.0 \mathrm{~mL})$, zugetropft. Nach $2 \mathrm{~h} 45 \mathrm{~min}$ Rühren bei $-78^{\circ} \mathrm{C}$ war der Umsatz vollständig und das Reaktionsgemisch wurde auf ein Gemisch aus $\mathrm{NH}_{4} \mathrm{Cl}$-Lösung und Ether $(1: 1,100 \mathrm{~mL})$ gegossen. Die Lösung wurde bei RT solange gerührt, bis sie klar war (10 min), dann wurden die Phasen getrennt. Die wässrige Phase wurde mit Ether (2 $\times 30 \mathrm{~mL}$ ) extrahiert. Die vereinigten organischen Phasen wurden mit $1 \mathrm{M} \mathrm{NaHCO}_{3}$ $(15 \mathrm{~mL})$, Wasser $(20 \mathrm{~mL})$ und NaCl-Lösung $(15 \mathrm{~mL})$ gewaschen und über Natriumsulfat getrocknet. Entfernen des Lösungsmittels im Vakuum ergab 72 mg Rohprodukt, welches säulenchromatographisch aufgereinigt wurde (Flash, Hex:EE, 18:1). Man erhielt $30 \mathrm{mg}$ $(49 \%)$ der Titelverbindung als farbloses Öl. $R_{\mathrm{f}}=0.56$ (Hex:EE, 18:1). $-{ }^{1} \mathrm{H}-\mathrm{NMR}$ $\left(300 \mathrm{MHz}, \mathrm{CD}_{2} \mathrm{Cl}_{2}\right): \delta=0.00\left[\mathrm{~s}, 6 \mathrm{H}, \mathrm{Si}\left(\mathrm{CH}_{3}\right)_{2}\right], 0.10$ [s, $\left.9 \mathrm{H}, \mathrm{Si}\left(\mathrm{CH}_{3}\right)_{3}\right], 0.18[\mathrm{~s}, 9 \mathrm{H}$, $\left.\mathrm{Si}\left(\mathrm{CH}_{3}\right)_{3}\right], 0.88\left[\mathrm{~s}, 9 \mathrm{H}, \mathrm{SiC}\left(\mathrm{CH}_{3}\right)_{3}\right], 2.20\left(\mathrm{~m}_{\mathrm{c}}, 2 \mathrm{H}, 8-\mathrm{H}_{2}\right), 2.50\left(\mathrm{dd},{ }^{3} J_{\mathrm{H}-\mathrm{H}}=4.5,15.1 \mathrm{~Hz}\right.$, $\left.1 \mathrm{H}, 6-\mathrm{H}_{\mathrm{a}}\right), 2.65\left(\mathrm{dd},{ }^{3} J_{\mathrm{H}-\mathrm{H}}=7.9,15.1 \mathrm{~Hz}, 1 \mathrm{H}, 6-\mathrm{H}_{\mathrm{b}}\right), 4.14\left(\mathrm{~m}_{\mathrm{c}}, 1 \mathrm{H}, 7-\mathrm{H}\right), 5.09\left(\mathrm{dd},{ }^{3} J_{\mathrm{H}-}\right.$ $\mathrm{H}=5.8,10.7 \mathrm{~Hz}, 1 \mathrm{H}, 11-\mathrm{H}), 5.43\left(\mathrm{dt},{ }^{3} J_{\mathrm{H}-\mathrm{H}}=7.3,15.5 \mathrm{~Hz}, 1 \mathrm{H}, 9-\mathrm{H}\right), 6.09\left(\mathrm{~d},{ }^{3} J_{\mathrm{H}-}\right.$ 
$\mathrm{H}=5.8 \mathrm{~Hz}, 1 \mathrm{H}, 12-\mathrm{H}), 6.38\left(\mathrm{dd},{ }^{3} J_{\mathrm{H}-\mathrm{H}}=10.7,15.5 \mathrm{~Hz}, 1 \mathrm{H}, 10-\mathrm{H}\right), 6.45\left(\mathrm{~d},{ }^{3} J_{\mathrm{H}-}\right.$ $\mathrm{H}=16.0 \mathrm{~Hz}, 1 \mathrm{H}, 3-\mathrm{H}), 6.53\left(\mathrm{~d},{ }^{3} J_{\mathrm{H}-\mathrm{H}}=16.0 \mathrm{~Hz}, 1 \mathrm{H}, 4-\mathrm{H}\right) \mathrm{ppm} .-{ }^{13} \mathrm{C}-\mathrm{NMR}(75.5 \mathrm{MHz}$, $\left.\mathrm{CD}_{2} \mathrm{Cl}_{2}\right): \delta=-5.4\left[\mathrm{Si}\left(\mathrm{CH}_{3}\right)_{2}\right],-0.5\left[\mathrm{Si}\left(\mathrm{CH}_{3}\right)_{3}\right], 0.2\left[\mathrm{Si}\left(\mathrm{CH}_{3}\right)_{3}\right], 18.5\left[\mathrm{SiC}\left(\mathrm{CH}_{3}\right)_{3}\right], 25.7$ [SiC $\left.\left(\mathrm{CH}_{3}\right)_{3}\right], 41.8$ (C-8), 48.4 (C-6), 69.6 (C-7), 102.2 (C-1), 105.8 (C-2), 110.6 (C-12), 122.8 (C-3), 125.6 (C-4), 126.4 (C-10), 139.2 (C-11), 139.4 (C-9), 198.1 (C-5) ppm. - MS (ESI), $m / z(\%): 327(25), 466(100)[\mathrm{M}+\mathrm{H}]^{+}, 489(60)[\mathrm{M}+\mathrm{Na}]^{+}, 687$ (35), 932 (12).

(7E,9Z )-10- (tert-Butyldimethylsilyloxy)-5-(trimethylsilyloxy)deca-1,7,9-trien-3-on (180)

(5E,7Z)-8-( $t$-Butyldimethylsilyloxy)octa-5,7-diensäure$N$-methoxy- $N$-methylamid $\quad(\mathbf{1 6 4}, 218 \mathrm{mg}, 543 \mu \mathrm{mol})$ wurde in THF $(40 \mathrm{ml})$ gelöst und auf $-30^{\circ} \mathrm{C}$ gekühlt. Dann wurde Vinylmagnesiumbromid (1.56 ml, $1.2 \mathrm{mmol}$,

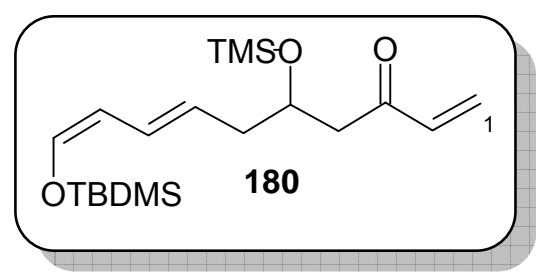
$0.78 \mathrm{M}$ in THF) zugetropft und 30 min gerührt. Da der Umsatz noch unvollständig war, wurde noch viermal Vinylmagnesiumbromid (150 $\mu \mathrm{l}, 120 \mu \mathrm{mol}, 0.78 \mathrm{M}$ in THF) zugegeben und jeweils 30 min gerührt, bis kein Edukt mehr vorhanden war. Die Reaktion wurde durch Zugabe von $\mathrm{NH}_{4} \mathrm{Cl}$-Lösung $(1 \mathrm{~mL})$ beendet und die wässrige Phase mit $\mathrm{CH}_{2} \mathrm{Cl}_{2}(2 \times 40 \mathrm{ml})$ extrahiert. Die kombinierten organischen Phasen wurden mit $\mathrm{NaHCO}_{3}$ Lösung $(40 \mathrm{ml})$, Wasser $(40 \mathrm{ml})$ und $\mathrm{NaCl}$-Lösung $(40 \mathrm{ml})$ gewaschen und über $\mathrm{Na}_{2} \mathrm{SO}_{4}$ getrocknet $\left(\mathrm{m}_{\text {Roh }}=179 \mathrm{mg}\right)$. Durch säulenchromatographische Reinigung (Hex:EE, 5:1) erhielt man $82.5 \mathrm{mg}$ (41\%) der Titelverbindung und $47.4 \mathrm{mg}$ (29\%) des Alkohols 177. 176: $R_{\mathrm{f}}=0.50$ (Hex:EE, 5:1). ${ }^{1} \mathrm{H}-\mathrm{NMR}\left(300 \mathrm{MHz}, \mathrm{CDCl}_{3}\right): \delta=0.04\left[\mathrm{~s}, 9 \mathrm{H}, \mathrm{Si}\left(\mathrm{CH}_{3}\right)_{3}\right]$, $0.14\left[\mathrm{~s}, 6 \mathrm{H}, \mathrm{Si}\left(\mathrm{CH}_{3}\right)_{2}\right], 0.92\left[\mathrm{~s}, 9 \mathrm{H}, \mathrm{SiC}\left(\mathrm{CH}_{3}\right)_{3}\right], 2.26\left(\mathrm{~m}_{\mathrm{c}}, 2 \mathrm{H}, 6-\mathrm{H}_{2}\right), 2.58\left(\mathrm{dd},{ }^{3} J_{\mathrm{H}-\mathrm{H}}=6.0\right.$, $21.0 \mathrm{~Hz}, 1 \mathrm{H}, 4-\mathrm{H}), 2.79\left(\mathrm{dd},{ }^{3} J_{\mathrm{H}-\mathrm{H}}=9.0,21.0 \mathrm{~Hz}, 1 \mathrm{H}, 4-\mathrm{H}\right), 4.22\left(\mathrm{~m}_{\mathrm{c}}, 1 \mathrm{H}, 5-\mathrm{H}\right), 5.12(\mathrm{dd}$, $\left.{ }^{3} J_{\mathrm{H}-\mathrm{H}}=0.6,12.0 \mathrm{~Hz}, 1 \mathrm{H}, 10-\mathrm{H}\right), 5.48\left(\mathrm{dt},{ }^{3} J_{\mathrm{H}-\mathrm{H}}=9.0,15.0 \mathrm{~Hz}, 1 \mathrm{H}, 7-\mathrm{H}\right), 5.82\left(\mathrm{dd},{ }^{3} J_{\mathrm{H}-\mathrm{H}}=\right.$ $3.0,12.0 \mathrm{~Hz}, 1 \mathrm{H}, 1-\mathrm{H}), 6.10\left(\mathrm{~m}_{\mathrm{c}}, 1 \mathrm{H}, 8-\mathrm{H}\right), 6.18\left(\mathrm{dd},{ }^{3} J_{\mathrm{H}-\mathrm{H}}=3.0,18.0 \mathrm{~Hz}, 1 \mathrm{H}, 1-\mathrm{H}\right), 6.33$ $\left(\mathrm{dd},{ }^{3} J_{\mathrm{H}-\mathrm{H}}=12.0,18.0 \mathrm{~Hz}, 1 \mathrm{H}, 2-\mathrm{H}\right), 6.42\left(\mathrm{~m}_{\mathrm{c}}, 1 \mathrm{H}, 9-\mathrm{H}\right) \mathrm{ppm} .-{ }^{13} \mathrm{C}-\mathrm{NMR}(300 \mathrm{MHz}$,

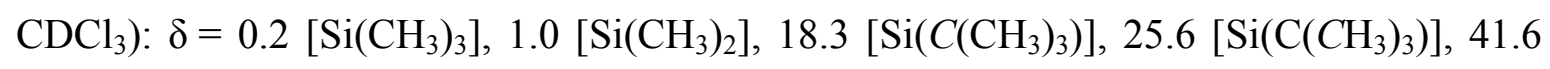
(C-6), 46.7 (C-4), 69.3 (C-5), 110.4 (C-9), 125.4 (C-7), 126.1 (C-8), 128.5 (C-1), 137.4 (C2), 139.1 (C-10), 199.8 (C-3) ppm. - MS (ESI), $m / z$ (\%): 179 (28), $391(100)[\mathrm{M}+\mathrm{Na}]^{+}, 392$ (33), 409 (12), 423 (9), 497 (15), 565 (14), 567 (12), 656 (10). - Daten für 177: ${ }^{1} \mathrm{H}-\mathrm{NMR}\left(300 \mathrm{MHz}, \mathrm{CDCl}_{3}\right): \delta=0.15[\mathrm{~s}, 9 \mathrm{H}$,

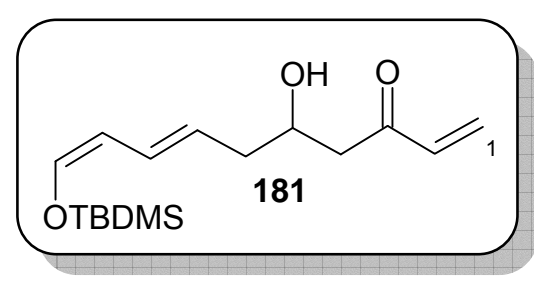

$\left.\mathrm{Si}\left(\mathrm{CH}_{3}\right)_{3}\right], 0.92\left[\mathrm{~s}, 6 \mathrm{H}, \mathrm{Si}\left(\mathrm{CH}_{3}\right)_{2}\right], 2.30\left(\mathrm{~m}_{\mathrm{c}}, 2 \mathrm{H}, 6-\mathrm{H}_{2}\right), 2.67\left(\mathrm{dd},{ }^{3} J_{\mathrm{H}-\mathrm{H}}=9.0,18.0 \mathrm{~Hz}, 1 \mathrm{H}\right.$, 
4-H), $2.78\left(\mathrm{dd},{ }^{3} J_{\mathrm{H}-\mathrm{H}}=6.0,18.0 \mathrm{~Hz}, 1 \mathrm{H}, 4-\mathrm{H}\right), 2.98(\mathrm{~s}, 1 \mathrm{H}, \mathrm{OH}), 4.06-4.20(\mathrm{~m}, 1 \mathrm{H}, 5-\mathrm{H})$, $5.13(\mathrm{dd}, J=0.6,12.0 \mathrm{~Hz}, 1 \mathrm{H}, 10-\mathrm{H}), 5.51\left(\mathrm{dt},{ }^{3} J_{\mathrm{H}-\mathrm{H}}=9.0,15.0 \mathrm{~Hz}, 1 \mathrm{H}, 7-\mathrm{H}\right), 5.86(\mathrm{dd}$, $\left.{ }^{3} J_{\mathrm{H}-\mathrm{H}}=3.0,12.0 \mathrm{~Hz}, 1 \mathrm{H}, 1-\mathrm{H}\right), 6.11\left(\mathrm{~m}_{\mathrm{c}}, 1 \mathrm{H}, 8-\mathrm{H}\right), 6.21\left(\mathrm{dd},{ }^{3} J_{\mathrm{H}-\mathrm{H}}=3.0,18.0 \mathrm{~Hz}, 1 \mathrm{H}, 1-\right.$ $\mathrm{H}), 6.33\left(\mathrm{dd},{ }^{3} J_{\mathrm{H}-\mathrm{H}}=12.0,18.0 \mathrm{~Hz}, 1 \mathrm{H}, 2-\mathrm{H}\right), 6.48\left(\mathrm{~m}_{\mathrm{c}}, 1 \mathrm{H}, 9-\mathrm{H}\right) \mathrm{ppm} .-{ }^{13} \mathrm{C}-\mathrm{NMR}(300$

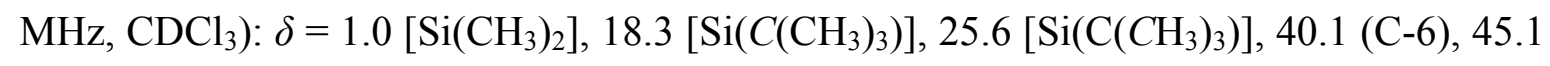
(C-4), 67.5 (C-5), 110.2 (C-9), 124.9 (C-7), 126.4 (C-8), 129.1 (C-1), 136.7 (C-2), 139.4 (C-10), 201.1 (C-3) ppm. - MS (ESI), m/z (\%): 319 (100) [M + Na $]^{+}, 320$ (19), 351 (9), $397(8), 615(36)[2 \mathrm{M}+\mathrm{Na}]^{+}, 616(13)$.

(4E,6E)-8-Benzyloxy-3-oxoocta-4,6-diensäureethylester (171)

Die Umsetzung wurde analog AAV05 durchgeführt, es wurden folgende Edukte eingesetzt: Ethyl 4(Diethoxyphosphoryl)-3-oxobutanoat $(\mathbf{1 5 4}, 10.1 \mathrm{~g}$, $37.8 \mathrm{mmol}), \mathrm{NaH}(1.66 \mathrm{~g}, 41.4 \mathrm{mmol}, 60 \%$ in

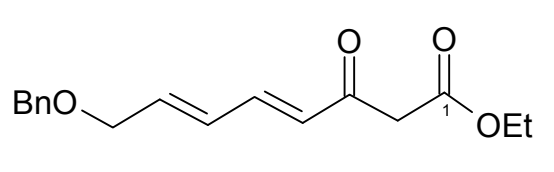

Mineralöl), $n$-BuLi (13.7 mL, 36.0 mmol, 2.63 M in Hexan), E-4-(Benzyloxy)but-2-en-1-al $(6.00 \mathrm{~g}, 34.0 \mathrm{mmol})$. Das Rohprodukt wurde säulenchromatographisch (Säule: $7 \times 24 \mathrm{~cm}$, Hex:EE, 5:1, $\left.R_{\mathrm{f}}=0.36\right)$ gereinigt. Man erhielt $7.35 \mathrm{~g}(75 \%)$ der Titelverbindung als gelbes Ö1. - ${ }^{1} \mathrm{H}-\mathrm{NMR}\left(300 \mathrm{MHz}, \mathrm{CDCl}_{3}\right.$ ) Ketoform: $\delta=1.27$ (t, ${ }^{3} J_{\mathrm{H}-\mathrm{H}}=7.2 \mathrm{~Hz}, 3 \mathrm{H}, 2^{\prime}-\mathrm{H}_{3}$ ), 3.59 $\left(\mathrm{s}, 2 \mathrm{H}, 2-\mathrm{H}_{2}\right), 4.20\left(\mathrm{q},{ }^{3} J_{\mathrm{H}-\mathrm{H}}=7.2 \mathrm{~Hz}, 2 \mathrm{H}, 1^{\prime}-\mathrm{H}_{2}\right), 4.55\left(\mathrm{~d},{ }^{3} J_{\mathrm{H}-\mathrm{H}}=5.5 \mathrm{~Hz}, 2 \mathrm{H}, 8-\mathrm{H}_{2}\right), 5.06$ $\left(\mathrm{s}, 2 \mathrm{H}, \mathrm{CH}_{2} \mathrm{Ph}\right), 5.90\left(\mathrm{~d},{ }^{3} J_{\mathrm{H}-\mathrm{H}}=15.3 \mathrm{~Hz}, 1 \mathrm{H}, 4-\mathrm{H}\right), 6.09\left(\mathrm{dt}, 1 \mathrm{H},{ }^{3} J_{\mathrm{H}-\mathrm{H}}=5.5,15.4 \mathrm{~Hz}, 7-\right.$ $\mathrm{H}), 6.42\left(\mathrm{dd},{ }^{3} J_{\mathrm{H}-\mathrm{H}}=10.5,15.4 \mathrm{~Hz}, 1 \mathrm{H}, 6-\mathrm{H}\right), 7.06\left(\mathrm{dd},{ }^{3} J_{\mathrm{H}-\mathrm{H}}=10.5,15.3 \mathrm{~Hz}, 1 \mathrm{H}, 5-\mathrm{H}\right)$, 7.27-7.40 (m, $5 \mathrm{H}$, Ar-H); Enolform: $\delta=1.29\left(\mathrm{t},{ }^{3} J_{\mathrm{H}-\mathrm{H}}=7.2 \mathrm{~Hz}, 3 \mathrm{H}, 2^{\prime}-\mathrm{H}_{3}\right), 4.21\left(\mathrm{q},{ }^{3} J_{\mathrm{H}-}\right.$ $\left.\mathrm{H}=7.2 \mathrm{~Hz}, 2 \mathrm{H}, 1^{\prime}-\mathrm{H}_{2}\right), 4.55\left(\mathrm{~d},{ }^{3} J_{\mathrm{H}-\mathrm{H}}=4.5 \mathrm{~Hz}, 2 \mathrm{H}, 8-\mathrm{H}_{2}\right), 5.06\left(\mathrm{~s}, 2 \mathrm{H}, \mathrm{CH}_{2} \mathrm{Ph}\right), 6.21(\mathrm{~s}$, $1 \mathrm{H}, 2-\mathrm{H}), 6.23\left(\mathrm{~d},{ }^{3} J_{\mathrm{H}-\mathrm{H}}=15.5 \mathrm{~Hz}, 1 \mathrm{H}, 4-\mathrm{H}\right), 6.28\left(\mathrm{dt},{ }^{3} J_{\mathrm{H}-\mathrm{H}}=4.5,13.6,1 \mathrm{H}, 7-\mathrm{H}\right), 6.45$ $\left(\mathrm{dd},{ }^{3} J_{\mathrm{H}-\mathrm{H}}=10.7,13.6,1 \mathrm{H}, 6-\mathrm{H}\right), 7.20\left(\mathrm{dd},{ }^{3} J_{\mathrm{H}-\mathrm{H}}=10.7,15.5,1 \mathrm{H}, 5-\mathrm{H}\right), 7.27-7.40(\mathrm{~m}$, $5 \mathrm{H}, \mathrm{Ar}-\mathrm{H}), 11.9$ (s, $1 \mathrm{H}, \mathrm{OH})$ ppm. $-{ }^{13} \mathrm{C}-\mathrm{NMR}\left(75.5 \mathrm{MHz}, \mathrm{CDCl}_{3}\right)$ : Ketoform: $\delta=14.1$ (C-2'), 41.5 (C-2), 47.4 (C-1'), 69.5 (C-8), $72.7\left(\mathrm{CH}_{2} \mathrm{Ph}\right), 127.1$ (C-Ar), 128.0 (C-Ar), 129.2 (C-Ar), 128.3 (C-7), 128.4 (C-6), 130.3 (C-4), 141.2 (C-Ar), 144.5 (C-5), 167.7 (C1), 192.4 (C-3); Enolform: $\delta=14.1$ (C-2'), 61.4 (C-1'), 70.0 (C-8), $72.4\left(\mathrm{CH}_{2} \mathrm{Ph}\right), 91.7$ (C2), 125.3 (C-4), 127.3, (C-Ar), 138.5 (C-Ar), 129.6 (C-Ar), 130.2 (C-7), 136.7 (C-6), 138.8 (C-5), 138.2 (C-Ar), 169.6 (C-1), 173.7 (C-3) ppm. - MS (ESI), m/z (\%): pos: 311 (40) [M $+\mathrm{Na}]^{+}, 599$ (100) $[2 \mathrm{M}+\mathrm{Na}]^{+}$; neg: $288(40)[\mathrm{M}-\mathrm{H}]^{-}, 597$ (79) $[2 \mathrm{M}-2 \mathrm{H}+\mathrm{Na}]^{-}, 907$ (100) $[3 \mathrm{M}-4 \mathrm{H}+2 \mathrm{Na}]^{-}$. 
Eine LDA-Lösung in THF wurde hergestellt, indem man bei $0{ }^{\circ} \mathrm{C} n$-BuLi $(2.20 \mathrm{~mL}, 5.79 \mathrm{mmol}, 2.63 \mathrm{M}$ in Hexan) $\mathrm{zu}$ einer Lösung von Diisopropylamin $(800 \mu \mathrm{L}, 580 \mathrm{mg}$, $5.71 \mathrm{mmol})$ und THF $(30 \mathrm{~mL})$ gab und $15 \mathrm{~min}$ unter $\mathrm{N}_{2^{-}}$

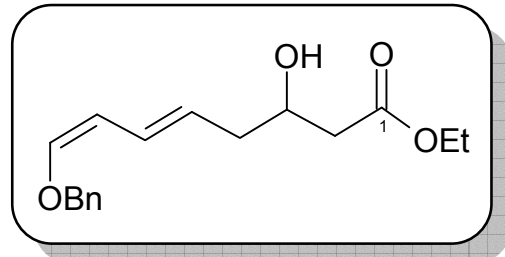
Atmosphäre rührte. Es wurde auf $-78{ }^{\circ} \mathrm{C}$ gekühlt, langsam wasserfreies HMPA (1.20 mL, $1.24 \mathrm{~g}, 6.90 \mathrm{mmol}$ ) zugetropft und $30 \mathrm{~min}$ bei $-78^{\circ} \mathrm{C}$ gerührt. Nach Zugabe von 8Benzyloxy-3-oxoocta-4,6-diensäureethylester (171, $510 \mathrm{mg}, 1.75 \mathrm{mmol})$ wurde $2.5 \mathrm{~h}$ bei $-78{ }^{\circ} \mathrm{C}$ gerührt. Die Reaktionsmischung wurde nach DC-Kontrolle (Hex:EE, 5:1) mit Essigsäure $(1.00 \mathrm{~mL}, 1.05 \mathrm{~g}, 17.5 \mathrm{mmol})$ versetzt und anschließend $5 \mathrm{~min}$ gerührt, das Kühlbad wurde entfernt und man ließ 15 min aufwärmen. Anschließend wurde Wasser $(30 \mathrm{~mL})$ zugegeben und $10 \mathrm{~min}$ gerührt. Die Phasen wurden getrennt und die wässrige Phase mit Pentan $(3 \times 50 \mathrm{~mL})$ extrahiert. Die vereinigten organischen Phasen wurden mit $1 \mathrm{M} \quad \mathrm{NaHCO}_{3}$-Lösung $(100 \mathrm{~mL})$, Wasser $(5 \times 100 \mathrm{~mL})$ und gesättigter Natriumchloridlösung $(100 \mathrm{~mL})$ gewaschen und über Natriumsulfat getrocknet. Das instabile Produkt (460 mg, $1.61 \mathrm{mmol}$ ) wurde direkt weiter umgesetzt und dafür in Ethanol (3 mL) gelöst und auf $0{ }^{\circ} \mathrm{C}$ gekühlt. Natriumborhydrid $(30.8 \mathrm{mg}, 810 \mu \mathrm{mol})$ wurde zugegeben und man rührte 40 min. Die Reaktionsmischung wurde mit AmmoniumchloridLösung $(10 \mathrm{~mL})$ versetzt und man extrahierte mit Diethylether $(3 \times 30 \mathrm{~mL})$. Nach Waschen mit Natriumhydrogencarbonat-Lösung $(50 \mathrm{~mL})$, Wasser $(50 \mathrm{~mL})$ und Natriumchlorid-Lösung $(50 \mathrm{~mL})$ wurde über Natriumsulfat getrocknet und säulenchromatographisch $(2 \times 23 \mathrm{~cm}$, Hex:EE, 5:1) gereinigt. Man erhielt ausgehend vom konj. Ester 171 über zwei Stufen 195 mg (39\%) der Titelverbindung als farbloses Ö1. $R_{\mathrm{f}}=$ 0.19 (Hex:EE, 5:1). $-{ }^{1} \mathrm{H}-\mathrm{NMR}\left(300 \mathrm{MHz}, \mathrm{CDCl}_{3}\right): \delta=1.26\left(\mathrm{t},{ }^{3} J_{\mathrm{H}-\mathrm{H}}=7.2 \mathrm{~Hz}, 3 \mathrm{H}, 2^{\prime}-\mathrm{H}_{3}\right)$, 2.26-2.55 (m, $\left.4 \mathrm{H}, 2-\mathrm{H}_{2}, 4-\mathrm{H}_{2}\right), 2.50(\mathrm{~s}, 1 \mathrm{H}, \mathrm{OH}), 4.06\left(\mathrm{~m}_{\mathrm{c}}, 1 \mathrm{H}, 3-\mathrm{H}\right), 4.16\left(\mathrm{q},{ }^{3} J_{\mathrm{H}-}\right.$ $\left.\mathrm{H}=7.2 \mathrm{~Hz}, 2 \mathrm{H}, 1^{\prime}-\mathrm{H}_{2}\right), 4.85\left(\mathrm{~s}, 2 \mathrm{H}, \mathrm{CH}_{2} \mathrm{Ph}\right), 5.09\left(\mathrm{dd},{ }^{3} J_{\mathrm{H}-\mathrm{H}}=6.3,11.4 \mathrm{~Hz}, 1 \mathrm{H}, 7-\mathrm{H}\right)$, $5.54\left(\mathrm{ddd},{ }^{3} J_{\mathrm{H}-\mathrm{H}}=7.3,7.4,15.5 \mathrm{~Hz}, 1 \mathrm{H}, 5-\mathrm{H}\right), 6.00\left(\mathrm{~d},{ }^{3} J_{\mathrm{H}-\mathrm{H}}=6.3 \mathrm{~Hz}, 1 \mathrm{H}, 8-\mathrm{H}\right), 6.52(\mathrm{dd}$, $\left.{ }^{3} J_{\mathrm{H}-\mathrm{H}}=11.4, \quad 15.5 \mathrm{~Hz}, \quad 1 \mathrm{H}, \quad 6-\mathrm{H}\right), \quad 7.27-7.40 \quad(\mathrm{~m}, \quad 5 \mathrm{H}, \quad \mathrm{Ar}-\mathrm{H}) \quad$ ppm. $\quad-\quad{ }^{13} \mathrm{C}-$ NMR (75.5 MHz, $\left.\mathrm{CDCl}_{3}\right): \delta=14.1$ (C-2'), 40.0 (C-2), 40.6 (C-4), 60.6 (C-1'), 67.8 (C-3), $74.1\left(\mathrm{CH}_{2} \mathrm{Ph}\right), 107.3$ (C-7), 125.4 (C-Ar), 127.1 (C-8), 127.2 (C-6), 128.4 (C-Ar), 128.9 (C-5), 137.3 (C-Ar), 145.3 (C-Ar), 173.7 (C-1) ppm. 
(5E,7Z)-8-Benzyloxy-3-trimethylsilanyloxyocta-5,7-diensäureethylester (174)

Die Umsetzung wurde analog AAV08 durchgeführt, es wurden folgende Edukte eingesetzt: Alkohol 171a (4.50 g, $15.5 \mathrm{mmol})$, Triethylamin (10.8 mL, $7.84 \mathrm{~g}, 77.5 \mathrm{mmol})$, TMSCl (5.90 mL, $5.05 \mathrm{~g}, 46.5 \mathrm{mmol})$. Das Rohprodukt

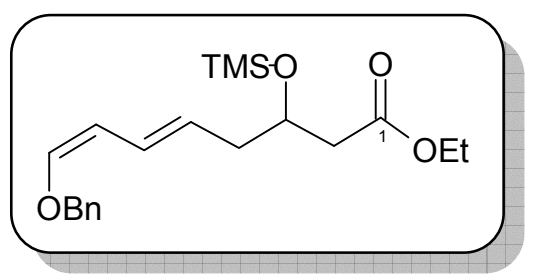
(5.40 g) wurde säulenchromatographisch aufgereinigt. Man erhielt $1.72 \mathrm{~g}$ (31\%) der Titelverbindung als gelbes Öl. $R_{\mathrm{f}}=0.35$ (Hex:EE, 10:1). $-{ }^{1} \mathrm{H}-\mathrm{NMR}\left(250 \mathrm{MHz}, \mathrm{CDCl}_{3}\right)$ : $\delta=0.12\left[\mathrm{~s}, 9 \mathrm{H}, \mathrm{Si}\left(\mathrm{CH}_{3}\right)_{3}\right], 1.26\left(\mathrm{t},{ }^{3} J_{\mathrm{H}-\mathrm{H}}=7.1 \mathrm{~Hz}, 3 \mathrm{H}, 2^{\prime}-\mathrm{H}_{3}\right), 2.20-2.35\left(\mathrm{~m}, 2 \mathrm{H}, 4-\mathrm{H}_{2}\right)$, 2.39-2.50 (m, $\left.2 \mathrm{H}, 2-\mathrm{H}_{2}\right), 4.05-4.25$ (m, $\left.3 \mathrm{H}, 3-\mathrm{H}, 1^{\prime}-\mathrm{H}_{2}\right), 4.84$ (s, $\left.2 \mathrm{H}, \mathrm{CH}_{2} \mathrm{Ph}\right), 5.09$ (dd, $\left.{ }^{3} J_{\mathrm{H}-\mathrm{H}}=6.2,10.9 \mathrm{~Hz}, 1 \mathrm{H}, 7-\mathrm{H}\right), 5.52\left(\mathrm{dt},{ }^{3} J_{\mathrm{H}-\mathrm{H}}=7.4,15.1 \mathrm{~Hz}, 1 \mathrm{H}, 5-\mathrm{H}\right), 5.98\left(\mathrm{~d},{ }^{3} J_{\mathrm{H}-}\right.$ $\mathrm{H}=6.1 \mathrm{~Hz}, 1 \mathrm{H}, 8-\mathrm{H}), 6.48\left(\mathrm{dd},{ }^{3} J_{\mathrm{H}-\mathrm{H}}=10.9,15.4 \mathrm{~Hz}, 1 \mathrm{H}, 6-\mathrm{H}\right), 7.27-7.45(\mathrm{~m}, 5 \mathrm{H}, \mathrm{Ar}-\mathrm{H})$ ppm. $-{ }^{13} \mathrm{C}-\mathrm{NMR}\left(75.6 \mathrm{MHz}, \mathrm{CDCl}_{3}\right): \delta=0.2\left[\mathrm{Si}\left(\mathrm{CH}_{3}\right)_{3}\right], 14.2\left(\mathrm{C}-2^{\prime}\right), 41.3(\mathrm{C}-2), 42.5(\mathrm{C}-$ 4), 60.3 (C-1'), 69.6 (C-3), $74.0\left(\mathrm{CH}_{2} \mathrm{Ph}\right), 107.2$ (C-7), 125.9 (C-8), 126.0 (C-Ar), 127.3 (C-6), 127.9 (C-Ar), 128.5 (C-5), 137.2 (C-Ar), 144.6 (C-Ar), 171.8 (C-1) ppm.

(5E,7Z)-8-Benzyloxy-3-trimethylsilanyloxyocta-5, 7-diensäure- $N$-methoxy-N-methylamid (174a)

Die Umsetzung wurde analog AAV09 durchgeführt, es wurden folgende Edukte eingesetzt: Ester 174 (704 mg, $1.94 \mathrm{mmol})$, N,O-Dimethylhydroxylamin-hydrochlorid (379 mg, $3.88 \mathrm{mmol}), i-\operatorname{PrMgBr}(5.14 \mathrm{~mL}, 7.77 \mathrm{mmol}$,

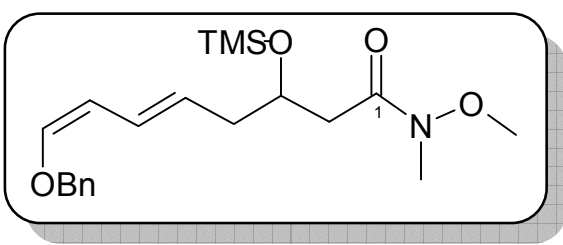
$1.51 \mathrm{M}$ in THF). Das Rohprodukt (709 mg) wurde aufgrund der geringen Stabilität der Titelverbindung direkt weiter umgesetzt. $R_{\mathrm{f}}=0.25$ (Hex:EE, 5:1). $-{ }^{1} \mathrm{H}-\mathrm{NMR}(250 \mathrm{MHz}$, $\left.\mathrm{CDCl}_{3}\right): \delta=0.12\left[\mathrm{~s}, 9 \mathrm{H}, \mathrm{Si}\left(\mathrm{CH}_{3}\right)_{3}\right], 2.20-2.35\left(\mathrm{~m}, 2 \mathrm{H}, 4-\mathrm{H}_{2}\right), 2.39-2.50$ (m, $\left.2 \mathrm{H}, 2-\mathrm{H}_{2}\right)$, 3.15 (s, $\left.3 \mathrm{H}, \mathrm{NCH}_{3}\right), 3.65$ (s, $\left.3 \mathrm{H}, \mathrm{OCH}_{3}\right), 4.20-4.30$ (m, $\left.3 \mathrm{H}, 3-\mathrm{H}, 1^{\prime}-\mathrm{H}_{2}\right), 4.85$ (s, $2 \mathrm{H}$, $\left.\mathrm{CH}_{2} \mathrm{Ph}\right), 5.10\left(\mathrm{dd},{ }^{3} J_{\mathrm{H}-\mathrm{H}}=6.2,10.8 \mathrm{~Hz}, 1 \mathrm{H}, 7-\mathrm{H}\right), 5.52\left(\mathrm{dt},{ }^{3} J_{\mathrm{H}-\mathrm{H}}=7.4,15.1 \mathrm{~Hz}, 1 \mathrm{H}, 5-\mathrm{H}\right)$, $5.98\left(\mathrm{~d},{ }^{3} J_{\mathrm{H}-\mathrm{H}}=6.1 \mathrm{~Hz}, 1 \mathrm{H}, 8-\mathrm{H}\right), 6.48\left(\mathrm{dd},{ }^{3} J_{\mathrm{H}-\mathrm{H}}=10.8,15.4 \mathrm{~Hz}, 1 \mathrm{H}, 6-\mathrm{H}\right), 7.30-7.40(\mathrm{~m}$, $5 \mathrm{H}, \mathrm{Ar}-\mathrm{H})$ ppm. $-{ }^{13} \mathrm{C}-\mathrm{NMR}\left(62.9 \mathrm{MHz}, \mathrm{CDCl}_{3}\right): \delta=0.2\left[\mathrm{Si}\left(\mathrm{CH}_{3}\right)_{3}\right], 31.8\left(\mathrm{NCH}_{3}\right), 39.4$ (C-2), $41.4(\mathrm{C}-4), 61.2\left(\mathrm{NOCH}_{3}\right), 69.6(\mathrm{C}-3), 74.0\left(\mathrm{CH}_{2} \mathrm{Ph}\right), 107.3(\mathrm{C}-7), 126.5(\mathrm{C}-8)$, 127.3 (C-Ar), 127.5 (C-6), 127.9 (C-Ar), 128.4 (C-5), 137.3 (C-Ar), 144.5 (C-Ar), 172.4 (C-1) ppm. 
(3E,9E,11Z)-12-Benzyloxy-1-trimethylsilanyl-7-trimethylsilanyloxydodeca-3,9,11-trien-1in-5-on (177)

Die Umsetzung wurde analog AAV10 durchgeführt, es wurden folgende Edukte eingesetzt: Amid 174a (374 mg, $990 \mu \mathrm{mol})$, Bromid 166 (453 mg, $2.23 \mathrm{mmol}$ ), $t$-BuLi

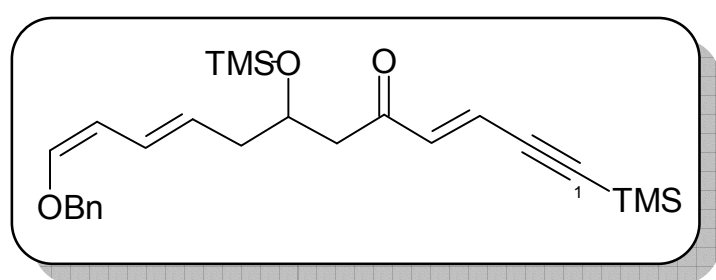
(3.96 mL, $4.00 \mathrm{mmol}, \quad 1.31 \mathrm{M}$ in Pentan). Das Rohprodukt (709 mg) wurde säulenchromatographisch gereinigt und man erhielt $47 \mathrm{mg}(11 \%)$ der Titelverbindung als farbloses Ö1. $R_{\mathrm{f}}=0.36$ (Hex:EE, 10:1). $-{ }^{1} \mathrm{H}-\mathrm{NMR}\left(250 \mathrm{MHz}, \mathrm{CDCl}_{3}\right): \delta=0.07[\mathrm{~s}, 9 \mathrm{H}$, $\left.\mathrm{Si}\left(\mathrm{CH}_{3}\right)_{3}\right], 0.22\left[\mathrm{~s}, 9 \mathrm{H}, \mathrm{Si}\left(\mathrm{CH}_{3}\right)_{3}\right], 2.27\left(\mathrm{~m}_{\mathrm{c}}, 2 \mathrm{H}, 8-\mathrm{H}_{2}\right), 2.54\left(\mathrm{dd},{ }^{3} J_{\mathrm{H}-\mathrm{H}}=4.3,15.1 \mathrm{~Hz}, 1 \mathrm{H}\right.$, $\left.6-\mathrm{H}_{\mathrm{a}}\right), 2.73\left(\mathrm{dd},{ }^{3} J_{\mathrm{H}-\mathrm{H}}=8.0,15.1 \mathrm{~Hz}, 1 \mathrm{H}, 6-\mathrm{H}_{\mathrm{b}}\right), 4.21\left(\mathrm{~m}_{\mathrm{c}}, 1 \mathrm{H}, 7-\mathrm{H}\right), 5.08\left(\mathrm{dd},{ }^{3} J_{\mathrm{H}-\mathrm{H}}=6.2\right.$, $10.8 \mathrm{~Hz}, 1 \mathrm{H}, 11-\mathrm{H}), 5.50\left(\mathrm{dt},{ }^{3} J_{\mathrm{H}-\mathrm{H}}=7.6,15.3 \mathrm{~Hz}, 1 \mathrm{H}, 9-\mathrm{H}\right), 5.98\left(\mathrm{~d},{ }^{3} J_{\mathrm{H}-\mathrm{H}}=6.2 \mathrm{~Hz}, 1 \mathrm{H}\right.$, $12-\mathrm{H}), 6.45\left(\mathrm{ddd}, J_{\mathrm{H}-\mathrm{H}}=0.8,10.8,15.3 \mathrm{~Hz}, 1 \mathrm{H}, 10-\mathrm{H}\right), 6.51\left(\mathrm{~d},{ }^{3} J_{\mathrm{H}-\mathrm{H}}=16.0 \mathrm{~Hz}, 1 \mathrm{H}, 3-\mathrm{H}\right)$, $6.60\left(\mathrm{~d},{ }^{3} J_{\mathrm{H}-\mathrm{H}}=16.0 \mathrm{~Hz}, 1 \mathrm{H}, 4-\mathrm{H}\right), 7.30-7.40(\mathrm{~m}, 5 \mathrm{H}, \mathrm{Ar}-\mathrm{H}) \mathrm{ppm} .-{ }^{13} \mathrm{C}-\mathrm{NMR}(62.9 \mathrm{MHz}$, $\left.\mathrm{CDCl}_{3}\right): \delta=-0.4\left[\mathrm{Si}\left(\mathrm{CH}_{3}\right)_{3}\right], 0.2\left[\mathrm{Si}\left(\mathrm{CH}_{3}\right)_{3}\right], 41.5$ (C-8), 48.1 (C-6), 69.3 (C-7), 74.1 $\left(\mathrm{CH}_{2} \mathrm{Ph}\right), 101.9$ (C-2), 106.0 (C-1), 107.2 (C-12), 122.5 (C-3), 125.9 (C-4), 126.0 (C-Ar), 127.3 (C-Ar), 128.3 (C-Ar), 128.5 (C-10), 137.2 (C-11), 138.9 (C-9), 144.7 (C-Ar), 198.1 (C-5) ppm.

(5E,7E/Z)-8-Methoxy-3-oxoocta-5,7-diensäureethylester (182)

Die Umsetzung wurde analog AAV05 durchgeführt, es wurden folgende Edukte eingesetzt: Aldehyd 168 (3.39 g, 33.2 mmol, Isomerengemisch $E: Z \sim 1: 3)$, 4(Diethoxyphosphoryl)-3-oxobutansäureethylester

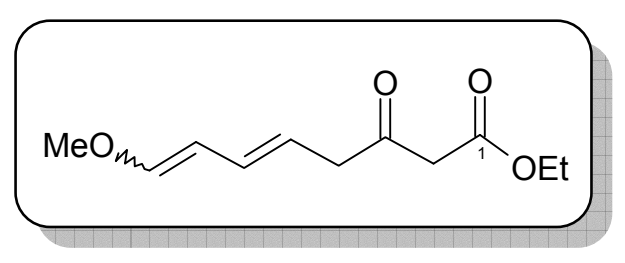
(154, 9.82 g, $36.9 \mathrm{mmol})$, NaH (974 mg, $40.6 \mathrm{mmol}$, 60\% Dispersion in Mineralöl), $n$ BuLi (15.8 mL, 36.9 mmol, $2.33 \mathrm{M}$ in Hexan). Das Produkt (46\%, $3.22 \mathrm{~g}$ ) wurde nach Säulenchromatographie (Hex:EE, 10:1) als komplexes Gemisch der E/Z-Isomere der Doppelbindung aus dem Aldehyd sowie den jeweiligen Keto-Enol-Tautomeren erhalten. Dieses Isomerengemisch wurde direkt in der anschließenden Dekonjugierung nach AAV06 umgesetzt. Man erhielt so das dekonjugierte Produkt 170a (3.15 g) welches aufgrund der mäßigen Stabilität ohne weitere Reinigung in der folgenden Reduktion eingesetzt wurde. $R_{\mathrm{f}}=0.20-0.30$ (Hex:EE, 10:1); $R_{\mathrm{f}}=0.24$ (Hex:EE, 5:1). $-{ }^{1} \mathrm{H}-\mathrm{NMR}\left(250 \mathrm{MHz}, \mathrm{CDCl}_{3}\right)$ : 
(E/Z)-Isomer: $\delta=1.21\left(\mathrm{t},{ }^{3} J_{\mathrm{H}-\mathrm{H}}=7.2 \mathrm{~Hz}, 3 \mathrm{H}, 2^{\prime}-\mathrm{H}_{3}\right), 3.22\left(\mathrm{~m}_{\mathrm{c}}, 2 \mathrm{H}, 4-\mathrm{H}_{2}\right), 3.41(\mathrm{~s}, 2 \mathrm{H}, 2-$ $\left.\mathrm{H}_{2}\right), 3.51\left(\mathrm{~s}, 3 \mathrm{H}, \mathrm{OCH}_{3}\right), 4.12\left(\mathrm{q},{ }^{3} J_{\mathrm{H}-\mathrm{H}}=7.2 \mathrm{~Hz}, 2 \mathrm{H}, 1^{\prime}-\mathrm{H}_{2}\right), 5.00\left(\mathrm{~m}_{\mathrm{c}}, 1 \mathrm{H}, 7-\mathrm{H}\right), 5.40-$ $5.60(\mathrm{~m}, 1 \mathrm{H}, 5-\mathrm{H}), 5.84\left(\mathrm{~d},{ }^{3} J_{\mathrm{H}-\mathrm{H}}=6.1 \mathrm{~Hz}, 1 \mathrm{H}, 8-\mathrm{H}\right), 6.39\left(\mathrm{dd},{ }^{3} J_{\mathrm{H}-\mathrm{H}}=10.6,16.0 \mathrm{~Hz}, 1 \mathrm{H}\right.$, 6-H); (E/E)-Isomer: $\delta=1.21\left(\mathrm{t},{ }^{3} J_{\mathrm{H}-\mathrm{H}}=7.2 \mathrm{~Hz}, 3 \mathrm{H}, 2^{\prime}-\mathrm{H}_{3}\right), 3.22\left(\mathrm{~m}_{\mathrm{c}}, 2 \mathrm{H}, 4-\mathrm{H}_{2}\right), 3.41(\mathrm{~s}$, $\left.2 \mathrm{H}, 2-\mathrm{H}_{2}\right), 3.61\left(\mathrm{~s}, 3 \mathrm{H}, \mathrm{OCH}_{3}\right), 4.12\left(\mathrm{q},{ }^{3} J_{\mathrm{H}-\mathrm{H}}=7.2 \mathrm{~Hz}, 2 \mathrm{H}, 1^{\prime}-\mathrm{H}_{2}\right), 5.40-5.60$ (m, $2 \mathrm{H}, 5-$ $\mathrm{H}, 7-\mathrm{H}), 5.82\left(\mathrm{dd},{ }^{3} J_{\mathrm{H}-\mathrm{H}}=10.7,15.1 \mathrm{~Hz}, 1 \mathrm{H}, 6-\mathrm{H}\right), 6.55\left(\mathrm{~d},{ }^{3} J_{\mathrm{H}-\mathrm{H}}=12.5 \mathrm{~Hz}, 1 \mathrm{H}, 8-\mathrm{H}\right)$ ppm. $-{ }^{13} \mathrm{C}-\mathrm{NMR}\left(62.9 \mathrm{MHz}, \mathrm{CDCl}_{3}\right)$ : (E/Z)-Isomer: $\delta=13.9\left(\mathrm{C}-2^{\prime}\right), 46.7$ (C-4), 48.4, (C2), $59.9\left(\mathrm{OCH}_{3}\right), 61.2\left(\mathrm{C}-1^{\prime}\right), 105.6$ (C-8), 120.1 (C-6), 131.1 (C-7), 151.3 (C-5), 167.0 (C1), 201.1 (C-3); (E/E)-Isomer: $\delta=13.9$ (C-2'), 46.9 (C-4), $48.4(\mathrm{C}-2), 56.2\left(\mathrm{OCH}_{3}\right), 61.2$ (C-1'), 104.7 (C-8), 118.0 (C-6), 127.5 (C-7), 147.3 (C-5), 167.0 (C-1), 201.1 (C-3) ppm.

(5E,7E/Z)-8-Methoxy-3-hydroxyocta-5,7-diensäureethylester (182a)

Die Umsetzung wurde analog AAV07 durchgeführt, es wurden folgende Edukte eingesetzt: $\beta$-Ketoester 166 (1.94 g, $9.14 \mathrm{mmol}), \mathrm{NaBH}_{4}$ (173 mg, $\left.4.57 \mathrm{mmol}\right)$. Das Rohprodukt (1.53 g) zeigte ein Isomerenverhältnis von

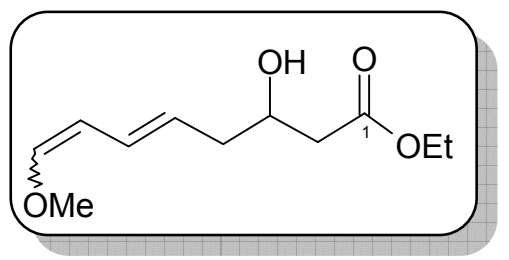
$(E / E):(E / Z) \sim 0.75: 1$ und wurde aufgrund der geringen Stabilität der Titelverbindung ohne weitere Reinigung direkt weiter umgesetzt. $R_{\mathrm{f}}=0.08$ (Hex:EE, 5:1). $-{ }^{1} \mathrm{H}-\mathrm{NMR}(250 \mathrm{MHz}$, $\left.\mathrm{CDCl}_{3}\right)$ : $(E / Z)$-Isomer: $\delta=1.23\left(\mathrm{t},{ }^{3} J_{\mathrm{H}-\mathrm{H}}=7.1 \mathrm{~Hz}, 3 \mathrm{H}, 2^{\prime}-\mathrm{H}_{3}\right), 2.24\left(\mathrm{~m}_{\mathrm{c}}, 2 \mathrm{H}, 4-\mathrm{H}_{2}\right), 2.37$ $\left(\mathrm{dd}, J_{\mathrm{H}-\mathrm{H}}=8.5,16.2 \mathrm{~Hz}, 1 \mathrm{H}, 2-\mathrm{H}_{\mathrm{a}}\right), 2.48\left(\mathrm{dd}, J_{\mathrm{H}-\mathrm{H}}=3.8,16.2 \mathrm{~Hz}, 1 \mathrm{H}, 2-\mathrm{H}_{\mathrm{b}}\right), 3.06\left(\mathrm{~s}_{\mathrm{br}}, 1 \mathrm{H}\right.$, $\mathrm{OH}), 3.54(\mathrm{~s}, 3 \mathrm{H}, 3-\mathrm{H}), 4.12\left(\mathrm{q},{ }^{3} J_{\mathrm{H}-\mathrm{H}}=7.1 \mathrm{~Hz}, 2 \mathrm{H}, 1^{\prime}-\mathrm{H}_{2}\right), 5.01\left(\mathrm{dd},{ }^{3} J_{\mathrm{H}-\mathrm{H}}=6.2,10.9 \mathrm{~Hz}\right.$, $1 \mathrm{H}, 7-\mathrm{H}), 5.35-5.65(\mathrm{~m}, 1 \mathrm{H}, 5-\mathrm{H}), 5.81\left(\mathrm{~d},{ }^{3} J_{\mathrm{H}-\mathrm{H}}=6.2 \mathrm{~Hz}, 1 \mathrm{H}, 8-\mathrm{H}\right), 6.38\left(\mathrm{dd},{ }^{3} J_{\mathrm{H}-\mathrm{H}}=\right.$ 10.9, $15.5 \mathrm{~Hz}, 1 \mathrm{H}, 6-\mathrm{H})$; (E/E)-Isomer: $\delta=1.23\left(\mathrm{t},{ }^{3} J_{\mathrm{H}-\mathrm{H}}=7.2 \mathrm{~Hz}, 3 \mathrm{H}, 2^{\prime}-\mathrm{H}_{3}\right), 2.24\left(\mathrm{~m}_{\mathrm{c}}\right.$, $\left.2 \mathrm{H}, 4-\mathrm{H}_{2}\right), 2.37\left(\mathrm{dd},{ }^{3} J_{\mathrm{H}-\mathrm{H}}=8.5,16.2 \mathrm{~Hz}, 1 \mathrm{H}, 2-\mathrm{H}_{\mathrm{a}}\right), 2.48\left(\mathrm{dd},{ }^{3} J_{\mathrm{H}-\mathrm{H}}=3.8,16.2 \mathrm{~Hz}, 1 \mathrm{H}\right.$, $\left.2-\mathrm{H}_{\mathrm{b}}\right), 3.06$ ( $\mathrm{s}$ r $\left., 1 \mathrm{H}, \mathrm{OH}\right), 3.62(\mathrm{~s}, 3 \mathrm{H}, 3-\mathrm{H}), 4.12$ (q, $\left.{ }^{3} J_{\mathrm{H}-\mathrm{H}}=7.1 \mathrm{~Hz}, 2 \mathrm{H}, 1^{\prime}-\mathrm{H}_{2}\right), 5.31-$ $5.59(\mathrm{~m}, 2 \mathrm{H}, 5-\mathrm{H}, 7-\mathrm{H}), 5.95\left(\mathrm{dd},{ }^{3} J_{\mathrm{H}-\mathrm{H}}=10.2,14.9 \mathrm{~Hz}, 1 \mathrm{H}, 6-\mathrm{H}\right), 6.52\left(\mathrm{~d},{ }^{3} J_{\mathrm{H}-\mathrm{H}}=12.5\right.$ $\mathrm{Hz}, 1 \mathrm{H}, 8-\mathrm{H})$ ppm. $-{ }^{13} \mathrm{C}-\mathrm{NMR}\left(62.9 \mathrm{MHz}, \mathrm{CDCl}_{3}\right)$ : $(E / Z)$-Isomer: $\delta=14.1\left(\mathrm{C}-2^{\prime}\right), 40.0$ (C-4), 40.6 (C-2), $59.9\left(\mathrm{OCH}_{3}\right), 60.6\left(\mathrm{C}-1^{\prime}\right), 67.8$ (C-3), 106.3 (C-8), 125.1 (C-6), 126.1 (C-7), 146.5 (C-5), 172.8 (C-1); (E/E)-Isomer: $\delta=14.1$ (C-2'), 39.9 (C-4), 40.0 (C-2), 56.2 $\left(\mathrm{OCH}_{3}\right), 60.6\left(\mathrm{C}-1^{\prime}\right), 67.8$ (C-3), 105.2 (C-8), 123.0 (C-6), 129.6 (C-7), 150.1 (C-5), 172.8 (C-1) ppm. 
(5E,7E/Z)-8-Methoxy-3-trimethylsilanyloxyocta-5,7-diensäureethylester (173)

Die Umsetzung wurde analog AAV08 durchgeführt, es wurden folgende Edukte eingesetzt: Alkohol 182a (1.49 g, $6.95 \mathrm{mmol})$, Triethylamin $(4.85 \mathrm{~mL}, 3.52 \mathrm{~g}, 34.8 \mathrm{mmol})$, TMSCl (2.65 mL, $2.27 \mathrm{~g}, 20.9 \mathrm{mmol})$. Das Rohprodukt $(2.27 \mathrm{~g})$ zeigte ein Isomerenverhältnis von $(E / E):(E / Z)$

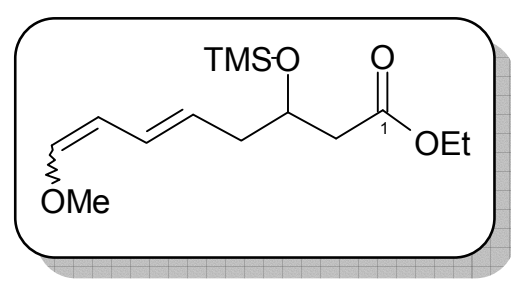
$\sim 0.75: 1$ und wurde säulenchromatographisch (Hex:EE, 5:1) aufgereinigt. Man erhielt die Titelverbindung (1.61 g, 60\% über drei Stufen) als farbloses Ö1. $R_{\mathrm{f}}=0.50$ (Hex:EE, 5:1). ${ }^{1} \mathrm{H}-\mathrm{NMR}\left(250 \mathrm{MHz}, \mathrm{CDCl}_{3}\right):(E / Z)$-Isomer: $\delta=0.07\left[\mathrm{~s}, 9 \mathrm{H}, \mathrm{Si}\left(\mathrm{CH}_{3}\right)_{3}\right], 1.22\left(\mathrm{t},{ }^{3} J_{\mathrm{H}-}\right.$ $\left.\mathrm{H}=7.0 \mathrm{~Hz}, 3 \mathrm{H}, 2^{\prime}-\mathrm{H}_{3}\right), 2.22\left(\mathrm{~m}_{\mathrm{c}}, 2 \mathrm{H}, 4-\mathrm{H}_{2}\right), 2.35-2.50\left(\mathrm{~m}, 1 \mathrm{H}, 2-\mathrm{H}_{\mathrm{a}}\right), 2.40-2.60(\mathrm{~m}, 1 \mathrm{H}$, 2-H $\mathrm{H}_{\mathrm{b}}$, 3.61 (s, $\left.\left.3 \mathrm{H}, \mathrm{OCH}_{3}\right), \mathrm{H}, 3-\mathrm{H}, 1^{\prime}-\mathrm{H}_{2}\right), 4.99$ (dd, $\left.{ }^{3} J_{\mathrm{H}-\mathrm{H}}=6.2,10.9 \mathrm{~Hz}, 1 \mathrm{H}, 7-\mathrm{H}\right), 5.35-$ $5.65(\mathrm{~m}, 1 \mathrm{H}, 5-\mathrm{H}), 5.80\left(\mathrm{~d},{ }^{3} J_{\mathrm{H}-\mathrm{H}}=6.2 \mathrm{~Hz}, 1 \mathrm{H}, 8-\mathrm{H}\right), 6.34\left(\mathrm{dd},{ }^{3} J_{\mathrm{H}-\mathrm{H}}=10.9,15.5 \mathrm{~Hz}, 1 \mathrm{H}\right.$, 6-H); $(E / E)$-Isomer: $\delta=0.07\left[\mathrm{Si}\left(\mathrm{CH}_{3}\right)_{3}\right], 1.22\left(\mathrm{t},{ }^{3} J_{\mathrm{H}-\mathrm{H}}=7.0 \mathrm{~Hz}, 3 \mathrm{H}, 2^{\prime}-\mathrm{H}_{3}\right), 2.22\left(\mathrm{~m}_{\mathrm{c}}, 2 \mathrm{H}\right.$, 4- $\left.\mathrm{H}_{2}\right), 2.35-2.50\left(\mathrm{~m}, 1 \mathrm{H}, 2-\mathrm{H}_{\mathrm{a}}\right), 2.40-2.60\left(\mathrm{~m}, 1 \mathrm{H}, 2-\mathrm{H}_{\mathrm{b}}\right), 3.53\left(\mathrm{~s}, 3 \mathrm{H}, \mathrm{OCH}_{3}\right), 4.10\left(\mathrm{~m}_{\mathrm{c}}\right.$, $\left.3 \mathrm{H}, 3-\mathrm{H}, 1^{\prime}-\mathrm{H}_{2}\right), 5.35-5.65(\mathrm{~m}, 2 \mathrm{H}, 5-\mathrm{H}, 7-\mathrm{H}), 5.89\left(\mathrm{dd},{ }^{3} J_{\mathrm{H}-\mathrm{H}}=10.5,15.2 \mathrm{~Hz}, 1 \mathrm{H}, 6-\mathrm{H}\right)$, $6.51\left(\mathrm{~d},{ }^{3} J_{\mathrm{H}-\mathrm{H}}=12.5 \mathrm{~Hz}, 1 \mathrm{H}, 8-\mathrm{H}\right) \mathrm{ppm} .-{ }^{13} \mathrm{C}-\mathrm{NMR}\left(62.9 \mathrm{MHz}, \mathrm{CDCl}_{3}\right):(E / Z)$-Isomer: $\delta=0.1\left[\mathrm{Si}\left(\mathrm{CH}_{3}\right)_{3}\right], 14.1\left(\mathrm{C}-2^{\prime}\right), 41.3(\mathrm{C}-4), 42.4(\mathrm{C}-2), 59.9\left(\mathrm{OCH}_{3}\right), 60.2\left(\mathrm{C}-1^{\prime}\right), 69.6(\mathrm{C}-$ 3), 106.5 (C-8), 125.7 (C-6), 146.2 (C-5), 150.3 (C-7), 171.8 (C-1); (E/E)-Isomer: $\delta=0.1$ [Si $\left.(\mathrm{CH})_{3}\right], 14.1\left(\mathrm{C}-2^{\prime}\right), 41.2(\mathrm{C}-4), 42.4(\mathrm{C}-2), 56.9\left(\mathrm{OCH}_{3}\right), 60.2\left(\mathrm{C}-1^{\prime}\right), 69.5(\mathrm{C}-3), 105.3$ (C-8), 123.6 (C-6), 129.2 (C-7), 150.3 (C-5), 172.8 (C-1) ppm.

(5E,7Z/E)-8-Methoxy-3-trimethylsilanyloxyocta-5,7-diensäure-N-methoxy-N-methylamid (173a)

Die Umsetzung wurde analog AAV09 durchgeführt, es wurden folgende Edukte eingesetzt: Ester 173 (167 mg, $583 \mu \mathrm{mol}), \quad \mathrm{N}, \mathrm{O}-$ Dimethylhydroxylamin-Hydrochlorid (114 mg, $1.17 \mathrm{mmol})$, i-PrMgBr (1.63 mL, $2.33 \mathrm{mmol}$,

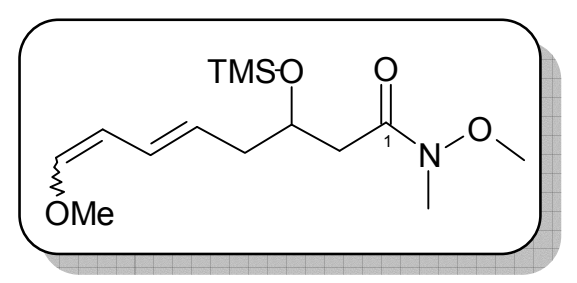
$1.43 \mathrm{M}$ in THF). Das Rohprodukt (167 mg) wurde aufgrund der geringen Stabilität der Titelverbindung ohne weitere Reinigung direkt weiter umgesetzt. $R_{\mathrm{f}}=0.13$ (Hex:EE, 5:1). ${ }^{1} \mathrm{H}-\mathrm{NMR}\left(250 \mathrm{MHz}, \mathrm{CDCl}_{3}\right):(E / Z)$-Isomer: $\delta=0.04$ [s, $\left.9 \mathrm{H}, \mathrm{Si}\left(\mathrm{CH}_{3}\right)_{3}\right], 2.22\left(\mathrm{~m}_{\mathrm{c}}, 2 \mathrm{H}, 4-\mathrm{H}_{2}\right), 2.36\left(\mathrm{~m}_{\mathrm{c}}, 1 \mathrm{H}, 2-\mathrm{H}_{\mathrm{a}}\right), 2.66\left(\mathrm{~m}_{\mathrm{c}}, 1 \mathrm{H}, 2-\mathrm{H}_{\mathrm{b}}\right), 3.13(\mathrm{~s}$, $\left.3 \mathrm{H}, \mathrm{NCH}_{3}\right), 3.58\left(\mathrm{~s}, 33.62\left(\mathrm{~s}, 3 \mathrm{H}, \mathrm{NOCH}_{3}\right), \mathrm{H}, 3-\mathrm{H}\right), 4.98\left(\mathrm{dd},{ }^{3} J_{\mathrm{H}-\mathrm{H}}=6.2,11.0 \mathrm{~Hz}, 1 \mathrm{H}\right.$, 
7-H), 5.31-5.56 (m, $1 \mathrm{H}, 5-\mathrm{H}), 5.77\left(\mathrm{~d},{ }^{3} J_{\mathrm{H}-\mathrm{H}}=6.2 \mathrm{~Hz}, 1 \mathrm{H}, 8-\mathrm{H}\right), 6.32\left(\mathrm{ddd},{ }^{3} J_{\mathrm{H}-\mathrm{H}}=1.0\right.$, 10.9, $15.5 \mathrm{~Hz}, 1 \mathrm{H}, 6-\mathrm{H})$; (E/E)-Isomer: $\delta=0.05$ [s, $\left.9 \mathrm{H}, \mathrm{Si}\left(\mathrm{CH}_{3}\right)_{3}\right], 2.22\left(\mathrm{~m}_{\mathrm{c}}, 2 \mathrm{H}, 4-\mathrm{H}_{2}\right)$, $2.36\left(\mathrm{~m}_{\mathrm{c}}, 1 \mathrm{H}, 2-\mathrm{H}_{\mathrm{a}}\right), 2.66\left(\mathrm{~m}_{\mathrm{c}}, 1 \mathrm{H}, 2-\mathrm{H}_{\mathrm{b}}\right), 3.13\left(\mathrm{~s}, 33.52\left(\mathrm{~s}, 3 \mathrm{H}, \mathrm{OCH}_{3}\right), 3.62(\mathrm{~s}, 3 \mathrm{H}\right.$, $\left.\mathrm{NOCH}_{3}\right), 4.10$ (s, $\left.3 \mathrm{H}, 3-\mathrm{H}\right), 5.35-5.65(\mathrm{~m}, 2 \mathrm{H}, 5-\mathrm{H}, 7-\mathrm{H}), 5.89\left(\mathrm{dd},{ }^{3} J_{\mathrm{H}-\mathrm{H}}=10.5,15.2 \mathrm{~Hz}\right.$, $1 \mathrm{H}, 6-\mathrm{H}), 6.51\left(\mathrm{~d},{ }^{3} J_{\mathrm{H}-\mathrm{H}}=12.5 \mathrm{~Hz}, 1 \mathrm{H}, 8-\mathrm{H}\right) \mathrm{ppm} .-{ }^{13} \mathrm{C}-\mathrm{NMR}\left(62.9 \mathrm{MHz}, \mathrm{CDCl}_{3}\right):(E / Z)-$ Isomer: $\delta=0.1\left[\mathrm{Si}\left(\mathrm{CH}_{3}\right)_{3}\right], 31.8\left(\mathrm{NCH}_{3}\right), 39.3(\mathrm{C}-4), 41.4(\mathrm{C}-2), 59.9\left(\mathrm{OCH}_{3}\right), 61.2$ $\left(\mathrm{NOCH}_{3}\right), 69.5$ (C-3), 106.6 (C-8), 125.5 (C-6), 128.9 (C-5), 146.0 (C-7), 172.4 (C-1); (E/E)-Isomer: $\delta=0.1$ [Si( $\left.\left(\mathrm{CH}_{3}\right)_{3}\right], 31.8\left(\mathrm{NCH}_{3}\right), 39.3(\mathrm{C}-4), 41.3(\mathrm{C}-2), 56.2\left(\mathrm{OCH}_{3}\right), 61.2$ $\left(\mathrm{NOCH}_{3}\right), 69.6$ (C-3), 105.4 (C-8), 124.1 (C-6), 126.3 (C-7), 150.0 (C-5), 172.4 (C-1) ppm.

(3E,9E,11Z/E)-12-Methoxy-1-trimethylsilanyl-7-trimethylsilanyloxydodeca-3,9,11-trien-1in-5-on (176)

Die Umsetzung wurde analog AAV10 durchgeführt, es wurden folgende Edukte eingesetzt: Das aus 173 gewonnene Amid 173a (164 mg, $544 \mu \mathrm{mol})$, Bromid 166

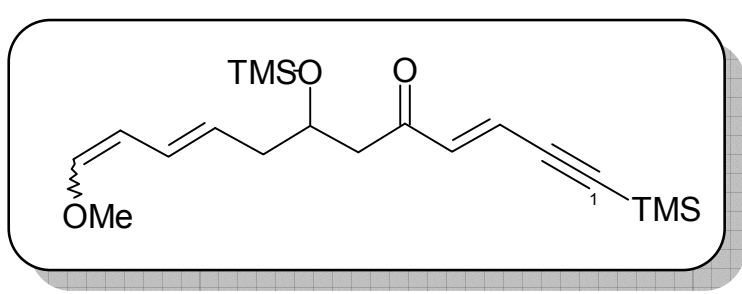
(249 mg, $1.22 \mathrm{mmol}), \quad t$-BuLi $\quad(1.67 \mathrm{~mL}$, $2.18 \mathrm{mmol}, 1.30 \mathrm{M}$ in Pentan). Das Rohprodukt (197 mg) wurde säulenchromatographisch (Hex:EE, 10:1) gereinigt und man erhielt $78 \mathrm{mg}$ (37\%) der Titelverbindung als farbloses Öl. $R_{\mathrm{f}}=0.35$ (Hex:EE, 10:1). ${ }^{1} \mathrm{H}-\mathrm{NMR}\left(600 \mathrm{MHz}, \mathrm{CD}_{2} \mathrm{Cl}_{2}\right)$ : $(E / Z)$-Isomer: $\delta=0.04$ [s, $\left.9 \mathrm{H}, \mathrm{Si}\left(\mathrm{CH}_{3}\right)_{3}\right], 0.20$ [s, $\left.9 \mathrm{H}, \mathrm{Si}\left(\mathrm{CH}_{3}\right)_{3}\right], 2.20\left(\mathrm{~m}_{\mathrm{c}}, 2 \mathrm{H}, 8-\mathrm{H}_{2}\right), 2.55\left(\mathrm{dd}, J_{\mathrm{H}-\mathrm{H}}=4.1,15.0 \mathrm{~Hz}\right.$, $\left.1 \mathrm{H}, 6-\mathrm{H}_{\mathrm{a}}\right), 2.67\left(\mathrm{dd}, J_{\mathrm{H}-\mathrm{H}}=8.0,15.0 \mathrm{~Hz}, 1 \mathrm{H}, 6-\mathrm{H}_{\mathrm{b}}\right), 3.54\left(\mathrm{~s}, 3 \mathrm{H}, \mathrm{OCH}_{3}\right), 4.16\left(\mathrm{~m}_{\mathrm{c}}, 1 \mathrm{H}, 7-\right.$ $\mathrm{H}), 5.01\left(\mathrm{dd},{ }^{3} J_{\mathrm{H}-\mathrm{H}}=6.3,10.8 \mathrm{~Hz}, 1 \mathrm{H}, 11-\mathrm{H}\right), 5.40\left(\mathrm{~m}_{\mathrm{c}}, 1 \mathrm{H}, 9-\mathrm{H}\right), 5.84\left(\mathrm{~d},{ }^{3} J_{\mathrm{H}-\mathrm{H}}=6.3 \mathrm{~Hz}\right.$, $1 \mathrm{H}, 12-\mathrm{H}), 6.32\left(\mathrm{dd}, J_{\mathrm{H}-\mathrm{H}}=10.8,15.5 \mathrm{~Hz}, 1 \mathrm{H}, 10-\mathrm{H}\right), 6.49\left(\mathrm{~d}, J_{\mathrm{H}-\mathrm{H}}=16.0 \mathrm{~Hz}, 1 \mathrm{H}, 3-\mathrm{H}\right)$, $6.56\left(\mathrm{~d}, J_{\mathrm{H}-\mathrm{H}}=16.0 \mathrm{~Hz}, 1 \mathrm{H}, 4-\mathrm{H}\right) ;(E / E)$-Isomer: $\delta=0.04\left[\mathrm{~s}, 9 \mathrm{H}, \mathrm{Si}\left(\mathrm{CH}_{3}\right)_{3}\right], 0.20[\mathrm{~s}, 9 \mathrm{H}$, $\left.\mathrm{Si}\left(\mathrm{CH}_{3}\right)_{3}\right], 2.20\left(\mathrm{~m}_{\mathrm{c}}, 2 \mathrm{H}, 8-\mathrm{H}_{2}\right), 2.53\left(\mathrm{dd},{ }^{3} J_{\mathrm{H}-\mathrm{H}}=4.1,15.0 \mathrm{~Hz}, 1 \mathrm{H}, 6-\mathrm{H}_{\mathrm{a}}\right), 2.66\left(\mathrm{dd},{ }^{3} J_{\mathrm{H}-\mathrm{H}}=\right.$ 8.0, 15.0 Hz, $\left.1 \mathrm{H}, 6-\mathrm{H}_{\mathrm{b}}\right), 3.63$ (s, $\left.3 \mathrm{H}, \mathrm{OCH}_{3}\right), 4.16\left(\mathrm{~m}_{\mathrm{c}}, 1 \mathrm{H}, 7-\mathrm{H}\right), 5.44-5.54$ (m, $2 \mathrm{H}, 11-$ $\mathrm{H}, 9-\mathrm{H}), 5.91\left(\mathrm{dd}, J_{\mathrm{H}-\mathrm{H}}=10.6,15.0 \mathrm{~Hz}, 1 \mathrm{H}, 10-\mathrm{H}\right), 6.49\left(\mathrm{~d}, J_{\mathrm{H}-\mathrm{H}}=16.0 \mathrm{~Hz}, 1 \mathrm{H}, 3-\mathrm{H}\right)$, $6.53\left(\mathrm{~d},{ }^{3} J_{\mathrm{H}-\mathrm{H}}=12.7 \mathrm{~Hz}, 1 \mathrm{H}, 12-\mathrm{H}\right), 6.56\left(\mathrm{~d}, J_{\mathrm{H}-\mathrm{H}}=16.0 \mathrm{~Hz}, 1 \mathrm{H}, 4-\mathrm{H}\right) \mathrm{ppm} .-{ }^{13} \mathrm{C}-\mathrm{NMR}$ (150.8 MHz, $\left.\mathrm{CD}_{2} \mathrm{Cl}_{2}\right)$ : - (E/Z)-Isomer: $\delta=-0.5$ [Si( $\left.\left.\mathrm{CH}_{3}\right)_{3}\right], 0.1\left[\mathrm{Si}\left(\mathrm{CH}_{3}\right)_{3}\right], 41.6(\mathrm{C}-8)$, 48.3 (C-6), $60.2\left(\mathrm{OCH}_{3}\right), 69.5$ (C-7), 102.2 (C-2), 105.9 (C-1), 106.6 (C-12), 122.8 (C-3), 123.9 (C-4), 129.6 (C-11), 139.2 (C-10), 146.7 (C-9), 198.1 (C-5); (E/E)-Isomer: $\delta=-0.5$ 
[Si $\left.\left(\mathrm{CH}_{3}\right)_{3}\right], 0.1$ [Si( $\left.\left.\mathrm{CH}_{3}\right)_{3}\right], 41.8(\mathrm{C}-8), 48.4(\mathrm{C}-6), 56.6\left(\mathrm{OCH}_{3}\right), 69.7(\mathrm{C}-7), 102.2(\mathrm{C}-2)$, 105.8 (C-1), 106.6 (C-12), 123.9 (C-3), 125.5 (C-11), 126.0 (C-4), 128.9 (C-10), 150.7 (C9), 198.1 (C-5) ppm.

(4E,6E)-3-Oxoocta-4,6-diensäureethylester (172)

Die Umsetzung wurde analog AAV05 durchgeführt, es wurden folgende Edukte eingesetzt: Ethyl 4(Diethoxyphosphoryl)-3-oxobutanoat $\quad(\mathbf{1 5 4}, 10.0 \mathrm{~g}$,

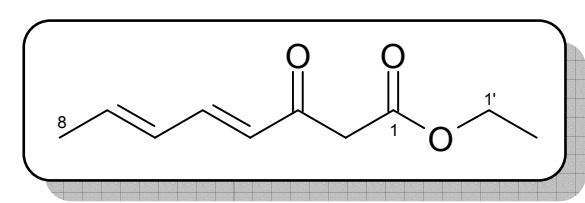
$37.6 \mathrm{mmol}$ ), $\mathrm{NaH}$ (1.66 g, $41 \mathrm{mmol}, 60 \%$ in Mineralöl), $n$-BuLi (14.3 mL, $37.6 \mathrm{mmol}$, $2.63 \mathrm{M}$ in Hexan), Crotonaldehyd $(2.80 \mathrm{~mL}, 33.8 \mathrm{mmol})$. Das Rohprodukt $(7.6 \mathrm{~g})$ wurde durch Säulenchromatographie (Hex:EE, 10:1) gereinigt. Es wurden $4.60 \mathrm{~g}$ der Titelverbindung als farbloses Öl erhalten (76\%). $R_{\mathrm{f}}=0.30$ (Hex:EE, 10:1). ${ }^{1} \mathrm{H}-\mathrm{NMR}$ $\left(300 \mathrm{MHz}, \mathrm{CDCl}_{3}\right)$ : Ketoform: $\delta=1.26\left(\mathrm{t},{ }^{3} J_{\mathrm{H}-\mathrm{H}}=5.0 \mathrm{~Hz}, 3 \mathrm{H}, 2^{\prime}-\mathrm{H}_{3}\right), 1.86\left(\mathrm{~d},{ }^{3} J_{\mathrm{H}-}\right.$ $\left.\mathrm{H}=5.0 \mathrm{~Hz}, 3 \mathrm{H}, 8-\mathrm{H}_{3}\right), 3.56\left(\mathrm{~s}, 2 \mathrm{H}, 2-\mathrm{H}_{2}\right), 4.16\left(\mathrm{q},{ }^{3} J_{\mathrm{H}-\mathrm{H}}=5.0 \mathrm{~Hz}, 2 \mathrm{H}, 1^{\prime}-\mathrm{H}_{2}\right), 5.94-6.32$ (m, $3 \mathrm{H}, 4-\mathrm{H}, 6-\mathrm{H}, 7-\mathrm{H}), 7.15\left(\mathrm{dd},{ }^{3} J_{\mathrm{H}-\mathrm{H}}=9.0,15.0 \mathrm{~Hz}, 1 \mathrm{H}, 5-\mathrm{H}\right) \mathrm{ppm}$. - Enolform (ca. 45\%): $\delta=1.29\left(\mathrm{t},{ }^{3} J_{\mathrm{H}-\mathrm{H}}=5.0 \mathrm{~Hz}, 3 \mathrm{H}, 2^{\prime}-\mathrm{H}_{3}\right), 1.82\left(\mathrm{~d},{ }^{3} J_{\mathrm{H}-\mathrm{H}}=5.0 \mathrm{~Hz}, 3 \mathrm{H}, 8-\mathrm{H}_{3}\right), 4.20(\mathrm{q}$, $\left.{ }^{3} J_{\mathrm{H}-\mathrm{H}}=5.0 \mathrm{~Hz}, 2 \mathrm{H}, 1^{\prime}-\mathrm{H}_{2}\right), 4.99(\mathrm{~s}, 1 \mathrm{H}, 2-\mathrm{H}), 5.76\left(\mathrm{~d},{ }^{3} J_{\mathrm{H}-\mathrm{H}}=9.0 \mathrm{~Hz}, 1 \mathrm{H}, 4-\mathrm{H}\right), 5.94-6.32$ (m, 2 H, 6-H, 7-H), $7.00\left(\mathrm{dd},{ }^{3} J_{\mathrm{H}-\mathrm{H}}=9.0,15.0 \mathrm{~Hz}, 1 \mathrm{H}, 5-\mathrm{H}\right), 11.86$ (s, $\left.1 \mathrm{H}, \mathrm{OH}\right) \mathrm{ppm}$. ${ }^{13} \mathrm{C}-\mathrm{NMR}$ (75.5 MHz, $\mathrm{CDCl}_{3}$ ): Ketoform: $\delta=14.1$ (C-2'), $18.9(\mathrm{C}-8), 47.3(\mathrm{C}-2), 61.3(\mathrm{C}-$ 1'), 126.6 (C-4), 130.1 (C-6), 141.9 (C-7), 145.0 (C-5), 167.5 (C-1), 192.3 (C-3) ppm. Enolform: $\delta=14.3\left(\mathrm{C}-2^{\circ}\right), 18.6$ (C-8), 60.1 (C-1'), 90.9 (C-2), 122.0 (C-4), 130.5 (C-6), 136.7 (C-7), 137.4 (C-5), 169.9 (C-1), 172.3 (C-3) ppm. - IR: $\widetilde{v}=2984,2938,1743,1647$, 1422, 1384, 1319, 1234, 1141, 1096, 1069, 1038, 993, 952, 868, 805, $729 \mathrm{~cm}^{-1}$. - MS (ESI), $m / z(\%): 205(100)[\mathrm{M}+\mathrm{Na}]^{+}, 236(14), 289(12), 386(46)[2 \mathrm{M}+\mathrm{Na}]^{+}, 453$ (10).

\section{(E)-3-Oxooct-5,7-diensäureethylester (184)}

Die Umsetzung wurde analog AAV06 durchgeführt, es wurden folgende Edukte eingesetzt: $(4 E, 6 E)$-3-Oxoocta4,6-diensäureethylester (172, $3.00 \mathrm{~g}, 16.5 \mathrm{mmol})$, DIPA

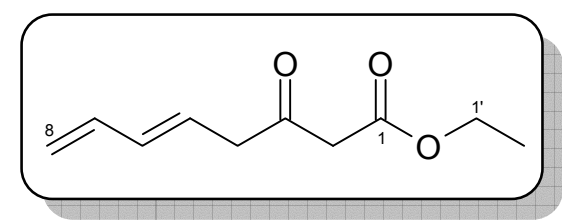
(7.63 mL, $5.51 \mathrm{~g}, 54.5 \mathrm{mmol}), n$-BuLi $(20.7 \mathrm{~mL}, 54.5 \mathrm{mmol}, 3.28 \mathrm{M}$ in Hexan), DMPU (7.98 mL, $66.0 \mathrm{mmol}$ ). Das Rohprodukt (3.98 g) wurde ohne weitere Reinigung direkt 
weiter umgesetzt. Eine analytische Probe von 479 mg wurde durch Säulenchromatographie aufgereinigt, (Hex:EE, 10:1). Die Titelverbindung wurde als gelbliches Öl erhalten, $342 \mathrm{mg}(71 \%) . R_{\mathrm{f}}=0.30$ (Hex:EE, 10:1). $-{ }^{1} \mathrm{H}-\mathrm{NMR}\left(300 \mathrm{MHz}, \mathrm{CDCl}_{3}\right): \delta=1.24\left(\mathrm{t},{ }^{3} J_{\mathrm{H}-}\right.$ $\left.\mathrm{H}=7.0 \mathrm{~Hz}, 3 \mathrm{H}, 2^{\prime}-\mathrm{H}_{3}\right), 3.30\left(\mathrm{~d},{ }^{3} J_{\mathrm{H}-\mathrm{H}}=7.0 \mathrm{~Hz}, 2 \mathrm{H}, 4-\mathrm{H}_{2}\right), 3.44\left(\mathrm{~s}, 2 \mathrm{H}, 2-\mathrm{H}_{2}\right), 4.16\left(\mathrm{q},{ }^{3} J_{\mathrm{H}-}\right.$ $\left.\mathrm{H}=7.0 \mathrm{~Hz}, 2 \mathrm{H}, 1^{\prime}-\mathrm{H}_{2}\right), 5.04\left(\mathrm{~d},{ }^{3} J_{\mathrm{H}-\mathrm{H}}=12.0 \mathrm{~Hz}, 1 \mathrm{H}, 8-\mathrm{H}\right), 5.16\left(\mathrm{~d},{ }^{3} J_{\mathrm{H}-\mathrm{H}}=18.0 \mathrm{~Hz}, 1 \mathrm{H}\right.$, $8-\mathrm{H}), 5.73\left(\mathrm{dt},{ }^{3} J_{\mathrm{H}-\mathrm{H}}=7.0,18.0 \mathrm{~Hz}, 1 \mathrm{H}, 5-\mathrm{H}\right), 6.12\left(\mathrm{dd},{ }^{3} J_{\mathrm{H}-\mathrm{H}}=12.0,18.0 \mathrm{~Hz}, 1 \mathrm{H}, 6-\mathrm{H}\right)$, $6.30\left(\mathrm{dt},{ }^{3} J_{\mathrm{H}-\mathrm{H}}=12.0,18.0 \mathrm{~Hz}, 1 \mathrm{H}, 7-\mathrm{H}\right) \mathrm{ppm} .-{ }^{13} \mathrm{C}-\mathrm{NMR}\left(75.5 \mathrm{MHz}, \mathrm{CDCl}_{3}\right): \delta=14.0$ (C-2'), 46.4 (C-4), 48.7 (C-2), 61.4 (C-1'), 117.4 (C-8), 124.7 (C-5), 135.4 (C-6), 136.2 (C7), 167.0 (C-1), 200.6 (C-3) ppm. - IR: $\widetilde{v}=3431$, 3089, 2985, 1717, 1651, 1467, 1412, 1368, 1317, 1240, 1094, 1028, 1007, 954, 907, 861, $804 \mathrm{~cm}^{-1}$. - MS (ESI), $m / z(\%): 167$ (16) $\left[\mathrm{M}-\mathrm{CH}_{3}\right]^{+}, 200(98)\left[\mathrm{M}+\mathrm{NH}_{4}\right]^{+}, 217(100)\left[\mathrm{M}+\mathrm{NH}_{3}+\mathrm{NH}_{4}\right]^{+}, 231$ (44), 232 (42), 249 (16), 382 (8) $\left[2 \mathrm{M}+\mathrm{NH}_{4}\right]^{+}$. - Anal. ber. (\%) für $\mathrm{C}_{10} \mathrm{H}_{14} \mathrm{O}_{3}$ (182.22): $\mathrm{C} 65.92, \mathrm{H} 7.74$, N 5.23; Gef. C 66.04, H 7.81.

\section{(E)-3-Hydroxyocta-5, 7-diensäureethylester (202)}

Die Umsetzung wurde analog AAV07 durchgeführt, es wurden folgende Edukte eingesetzt: $(E)$-3-Oxooct-5,7diensäureethylester $(\mathbf{1 8 4}, 2.40 \mathrm{~g}, 13.1 \mathrm{mmol}), \mathrm{NaBH}_{4}$ (252 mg, $6.06 \mathrm{mmol})$. Vom Rohprodukt (1.38 g, 57\%)

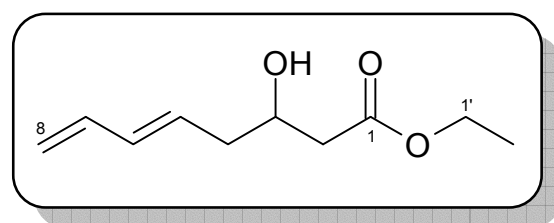
wurden $1.17 \mathrm{~g}$ direkt weiter umgesetzt und eine analytische Probe (209 mg) durch Säulenchromatographie (Hex:EE, 5:1) gereinigt, $181 \mathrm{mg}, 87 \% . R_{\mathrm{f}}=0.20$ (Hex:EE, 5:1). ${ }^{1} \mathrm{H}-\mathrm{NMR}\left(300 \mathrm{MHz}, \mathrm{CDCl}_{3}\right): \delta=1.26\left(\mathrm{t},{ }^{3} \mathrm{~J}_{\mathrm{H}-\mathrm{H}}=6.5 \mathrm{~Hz}, 3 \mathrm{H}, 2^{\prime}-\mathrm{H}_{3}\right), 2.18-3.00(\mathrm{~m}, 5 \mathrm{H}, 2-$ $\left.\mathrm{H}_{2}, 4-\mathrm{H}_{2}, \mathrm{OH}\right), 4.00-4.10(\mathrm{~m}, 1 \mathrm{H}, 3-\mathrm{H}), 4.14$ (q, $\left.{ }^{3} J_{\mathrm{H}-\mathrm{H}}=6.5 \mathrm{~Hz}, 2 \mathrm{H}, 1^{\prime}-\mathrm{H}_{2}\right), 4.99\left(\mathrm{~d},{ }^{3} J_{\mathrm{H}-}\right.$ $\mathrm{H}=12.0 \mathrm{~Hz}, 1 \mathrm{H}, 8-\mathrm{H}), 5.12\left(\mathrm{~d},{ }^{3} J_{\mathrm{H}-\mathrm{H}}=18.0 \mathrm{~Hz}, 1 \mathrm{H}, 8-\mathrm{H}\right), 5.68\left(\mathrm{dt},{ }^{3} J_{\mathrm{H}-\mathrm{H}}=6.5,15.0 \mathrm{~Hz}\right.$, $1 \mathrm{H}, 5-\mathrm{H}), 6.11\left(\mathrm{dd},{ }^{3} J_{\mathrm{H}-\mathrm{H}}=12.0,15.0 \mathrm{~Hz}, 1 \mathrm{H}, 6-\mathrm{H}\right), 6.30\left(\mathrm{dt},{ }^{3} J_{\mathrm{H}-\mathrm{H}}=12.0,18.0 \mathrm{~Hz}, 1 \mathrm{H}\right.$, 7-H) ppm. $-{ }^{13} \mathrm{C}-\mathrm{NMR}\left(75.5 \mathrm{MHz}, \mathrm{CDCl}_{3}\right): \delta=14.1$ (C-2'), $39.6(\mathrm{C}-4), 40.6(\mathrm{C}-2), 60.7$ (C-1'), 67.6 (C-3), 116.1 (C-8), 129.6 (C-6), 134.2 (C-5), 136.7 (C-7), 172.8 (C-1) ppm. IR: $\widetilde{v}=3438,2921,2361,1733,1636,1376,1180,1030,1668 \mathrm{~cm}^{-1}$. - MS (ESI), $m / z$ (\%): 201 (12) $\left[\mathrm{M}+\mathrm{NH}_{3}\right]^{+}, 204$ (48), 234 (100), 251 (12), 420 (16), 450 (34). 


\section{(E)-3-Trimethylsilyloxyocta-5,7-diensäureethylester (175)}

Die Umsetzung wurde analog AAV08 durchgeführt, es wurden folgende Edukte eingesetzt: $(E)$-3Hydroxyoct-5,7-diensäureethylester (202, $1.00 \mathrm{~g}$, $5.43 \mathrm{mmol})$, Triethylamin $\quad(4.40 \mathrm{ml}, \quad 3.19 \mathrm{~g}$, $31.6 \mathrm{mmol})$, TMSCl (2.40 ml, $2.05 \mathrm{~g}, 18.9 \mathrm{mmol})$. Das

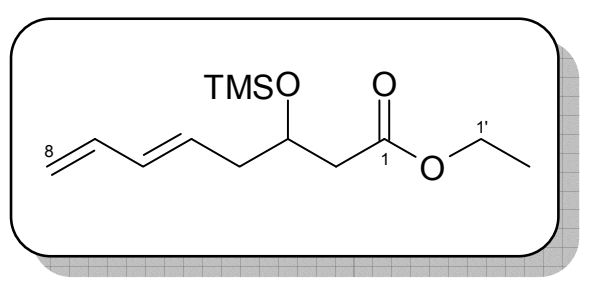

Rohprodukt (1.92 g) wurde säulenchromatographisch gereinigt, (Hex:EE 8:1, $\left.R_{\mathrm{f}}=0.4\right)$. Man erhielt 1.24 g (89\%) der Titelverbindung als farbloses Öl. $R_{\mathrm{f}}=0.35$ (Hex:EE, 10:1). ${ }^{1} \mathrm{H}-\mathrm{NMR}\left(300 \mathrm{MHz}, \mathrm{CDCl}_{3}\right): \delta=0.06\left[\mathrm{~s}, 9 \mathrm{H}, \mathrm{Si}\left(\mathrm{CH}_{3}\right)_{3}\right], 1.23\left(\mathrm{t},{ }^{3} J_{\mathrm{H}-\mathrm{H}}=6.5 \mathrm{~Hz}, 3 \mathrm{H}, 2^{\prime}-\right.$ $\left.\mathrm{H}_{3}\right), 2.24\left(\mathrm{t},{ }^{3} J_{\mathrm{H}-\mathrm{H}}=6.5 \mathrm{~Hz}, 2 \mathrm{H}, 4-\mathrm{H}_{2}\right), 2.40\left(\mathrm{~d},{ }^{3} J_{\mathrm{H}-\mathrm{H}}=6.5 \mathrm{~Hz}, 2 \mathrm{H}, 2-\mathrm{H}_{2}\right), 4.09\left(\mathrm{q},{ }^{3} J_{\mathrm{H}-\mathrm{H}}=\right.$ $\left.6.5 \mathrm{~Hz}, 2 \mathrm{H}, 1^{\prime}-\mathrm{H}_{2}\right), 4.16$ (quint, ${ }^{3} J_{\mathrm{H}-\mathrm{H}}=6.5 \mathrm{~Hz}, 1 \mathrm{H}, 3-\mathrm{H}$ ), $4.97\left(\mathrm{~d},{ }^{3} J_{\mathrm{H}-\mathrm{H}}=12.0 \mathrm{~Hz}, 1 \mathrm{H}, 8-\right.$ H), $5.09\left(\mathrm{~d},{ }^{3} J_{\mathrm{H}-\mathrm{H}}=18.0 \mathrm{~Hz}, 1 \mathrm{H}, 8-\mathrm{H}\right), 5.64\left(\mathrm{dt},{ }^{3} J_{\mathrm{H}-\mathrm{H}}=6.5,15.0 \mathrm{~Hz}, 1 \mathrm{H}, 5-\mathrm{H}\right), 6.06(\mathrm{dd}$, $\left.{ }^{3} J_{\mathrm{H}-\mathrm{H}}=12.0,15.0 \mathrm{~Hz}, 1 \mathrm{H}, 6-\mathrm{H}\right), 6.28\left(\mathrm{dt},{ }^{3} J_{\mathrm{H}-\mathrm{H}}=12.0,18.0 \mathrm{~Hz}, 1 . \mathrm{H}, 7-\mathrm{H}\right) \mathrm{ppm} .-{ }^{13} \mathrm{C}-$ NMR (75.5 MHz, $\left.\mathrm{CDCl}_{3}\right): \delta=0.4\left[\mathrm{Si}\left(\mathrm{CH}_{3}\right)_{3}\right], 14.5$ (2'-C), 41.1 (4-C), 42.8 (2-C), 60.9 (1'C), 69.5 (3-C), 116.0 (8-C), 130.4 (6-C), 134.2 (5-C), 137.1 (7-C), 171.9 (1-C) ppm. - IR: $\widetilde{v}=3433,2960,1737,1635,1377,1251,1180,1097,1005,843,754 \mathrm{~cm}^{-1}$. - MS (ESI), $m / z(\%): 167$ (36), 185 (20), 207 (100) [M - TMS + Na] $]^{+}, 292(10), 419$ (16).

\section{(E)-3-Trimethylsilyloxyocta-5,7-diensäure-N-methoxy-N-methylamid (204)}

Die Umsetzung wurde analog AAV09 durchgeführt, es wurden folgende Edukte eingesetzt: $(E)-3-$ Trimethylsilyloxyokta-5,7-diensäureethylester

(175, $1.08 \mathrm{~g}, \quad 4.21 \mathrm{mmol}), \quad$ N,O-Dimethylhydroxylamin

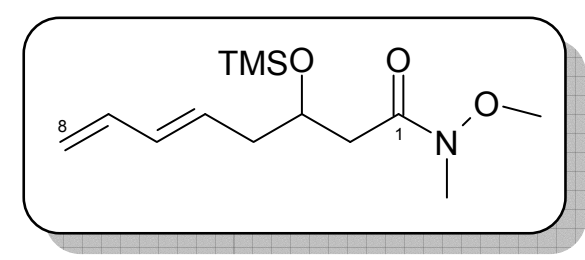
Hydrochlorid (821 mg, $8.42 \mathrm{mmol}), i$-PrMgBr (11.0 ml, $16.6 \mathrm{mmol}, 1.51 \mathrm{M}$ in $\mathrm{Et}_{2} \mathrm{O}$ ). Das Rohprodukt (1.10 g), welches laut ${ }^{1}$ H-NMR Spektrum eine Reinheit von $>90 \%$ aufwies, wurde säulenchromatographisch gereinigt (Hex:EE, 5:1). Man erhielt $530 \mathrm{mg}$ (46\%) der Titelverbindung als farbloses Öl. $R_{\mathrm{f}}=0.20$ (Hex:EE, 5:1) $-{ }^{1} \mathrm{H}-\mathrm{NMR}\left(300 \mathrm{MHz}, \mathrm{CDCl}_{3}\right)$ : $\delta=0.06\left[\mathrm{~s}, 9 \mathrm{H}, \mathrm{Si}\left(\mathrm{CH}_{3}\right)_{3}\right], 2.12-2.76\left(\mathrm{~m}, 4 \mathrm{H}, 2-\mathrm{H}_{2}, 4-\mathrm{H}_{2}\right), 3.14\left(\mathrm{~s}, 3 \mathrm{H}, \mathrm{NCH}_{3}\right), 3.64(\mathrm{~s}$, $\left.3 \mathrm{H}, \mathrm{NOCH}_{3}\right), 4.20-4.29(\mathrm{~m}, 1 \mathrm{H}, 3-\mathrm{H}), 4.96\left(\mathrm{~d},{ }^{3} J_{\mathrm{H}-\mathrm{H}}=12.0 \mathrm{~Hz}, 1 \mathrm{H}, 8-\mathrm{H}\right), 5.09\left(\mathrm{~d},{ }^{3} J_{\mathrm{H}-\mathrm{H}}\right.$ $=18.0 \mathrm{~Hz}, 1 \mathrm{H}, 8-\mathrm{H}), 5.66\left(\mathrm{dt},{ }^{3} J_{\mathrm{H}-\mathrm{H}}=6.5,15.0 \mathrm{~Hz}, 1 \mathrm{H}, 5-\mathrm{H}\right), 6.06\left(\mathrm{dd},{ }^{3} J_{\mathrm{H}-\mathrm{H}}=12.0\right.$, $15.0 \mathrm{~Hz}, 1 \mathrm{H}, 6-\mathrm{H}), 6.28\left(\mathrm{dt},{ }^{3} \mathrm{~J}_{\mathrm{H}-\mathrm{H}}=12.0,18.0 \mathrm{~Hz}, 1 \mathrm{H}, 7-\mathrm{H}\right) \mathrm{ppm} .-{ }^{13} \mathrm{C}-\mathrm{NMR}(75.5 \mathrm{MHz}$, $\left.\mathrm{CDCl}_{3}\right): \delta=0.16\left[\mathrm{Si}\left(\mathrm{CH}_{3}\right)_{3}\right], 39.5(\mathrm{C}-2), 41.0(\mathrm{C}-4), 51.6\left(\mathrm{NCH}_{3}\right), 61.3\left(\mathrm{NOCH}_{3}\right), 69.3(\mathrm{C}-$ 
3), 115.5 (C-8), 130.7 (C-6), 133.7 (C-5), 137.0 (C-7), 173.3 (C-1) ppm. - IR: $\widetilde{v}=3448$, 2960, 2362, 1654, 1419, 1386, 1250, 1180, 1094, 1005, 900, 843, $752 \mathrm{~cm}^{-1}$. - MS (ESI), $m / z(\%): 200(16)[\mathrm{M}-\mathrm{TMS}+\mathrm{H}]^{+}, 222(12)[\mathrm{M}-\mathrm{TMS}+\mathrm{Na}]^{+}, 260(35)[\mathrm{M}-\mathrm{OMe}+$ $\left.\mathrm{NH}_{4}\right]^{+}, 262$ (42), $310(27), 318$ (100), 418 (25), 423 (19).

(3E,9E)-1-(Trimethylsilyl)-7-(trimethylsilyloxy)dodeca-3,9,11-trien-1-in-5-on (178)

Die Umsetzung wurde analog AAV10 durchgeführt, es wurden folgende Edukte eingesetzt: (E)-3-(Trimethylsilyloxy)octa-5,7diensäure- $N$-methoxy- $N$-methylamid (204,

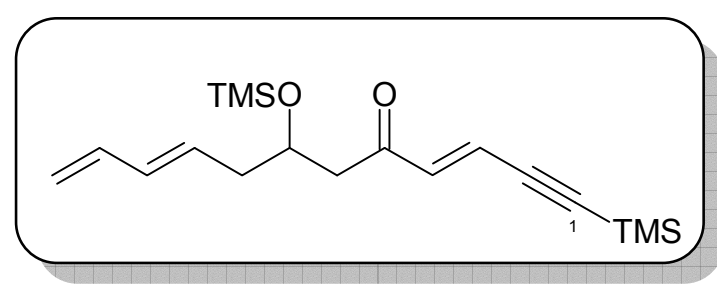
$250 \mathrm{mg}, 921 \mu \mathrm{mol}), 1$-Brom-4-(trimethylsilyl)prop-1-en-3-in (166, $421 \mathrm{mg}, 2.07 \mathrm{mmol}), t$ BuLi (2.25 ml, $3.68 \mathrm{mmol}, 1.63 \mathrm{M}$ in Pentan). Das Rohprodukt (469 mg) wurde durch Säulenchromatographie (Hex:EE, 15:1 $\rightarrow$ 5:1) gereinigt. Man erhielt $178 \mathrm{mg}(58 \%)$ der Titelverbindung als farbloses Öl, außerdem wurden $35.9 \mathrm{mg}(9 \%)$ Edukt zurückgewonnen. $-R_{\mathrm{f}}=0.60$ (Hex:EE, 15:1) $-{ }^{1} \mathrm{H}-\mathrm{NMR}\left(300 \mathrm{MHz}, \mathrm{CDCl}_{3}\right): \delta=0.04\left[\mathrm{~s}, 9 \mathrm{H}, \mathrm{Si}\left(\mathrm{CH}_{3}\right)_{3}\right]$, $0.10\left[\mathrm{~s}, 9 \mathrm{H}, \mathrm{OSi}\left(\mathrm{CH}_{3}\right)_{3}\right], 2.23\left(\mathrm{~m}_{\mathrm{c}}, 2 \mathrm{H}, 8-\mathrm{H}_{2}\right), 2.54\left(\mathrm{dd}, J_{\mathrm{H}-\mathrm{H}}=8.0,18.0 . \mathrm{Hz}, 1 \mathrm{H}, 6-\mathrm{H}\right)$, $2.70\left(\mathrm{dd},{ }^{3} J_{\mathrm{H}-\mathrm{H}}=9.0,18.0 \mathrm{~Hz}, 1 \mathrm{H}, 6-\mathrm{H}\right), 4.22\left(\mathrm{~m}_{\mathrm{c}}, 1 \mathrm{H}, 7-\mathrm{H}\right), 4.98\left(\mathrm{~d},{ }^{3} J_{\mathrm{H}-\mathrm{H}}=12.0 \mathrm{~Hz}, 1 \mathrm{H}\right.$, $12-\mathrm{H}), 5.10\left(\mathrm{~d},{ }^{3} J_{\mathrm{H}-\mathrm{H}}=18.0 \mathrm{~Hz}, 1 \mathrm{H}, 12-\mathrm{H}\right), 5.64\left(\mathrm{dt},{ }^{3} J_{\mathrm{H}-\mathrm{H}}=9.0,15.0 \mathrm{~Hz}, 1 \mathrm{H}, 9-\mathrm{H}\right), 6.04$ $\left(\mathrm{dd},{ }^{3} J_{\mathrm{H}-\mathrm{H}}=12.0,15.0 \mathrm{~Hz}, 1 \mathrm{H}, 10-\mathrm{H}\right), 6.28\left(\mathrm{dt},{ }^{3} J_{\mathrm{H}-\mathrm{H}}=12.0,18.0 \mathrm{~Hz}, 1 \mathrm{H}, 11-\mathrm{H}\right), 6.49(\mathrm{~d}$, $\left.{ }^{3} J_{\mathrm{H}-\mathrm{H}}=18.0 \mathrm{~Hz}, 1 \mathrm{H}, 3-\mathrm{H}\right), 6.58\left(\mathrm{~d},{ }^{3} J_{\mathrm{H}-\mathrm{H}}=18.0 \mathrm{~Hz}, 1 \mathrm{H}, 4-\mathrm{H}\right) \mathrm{ppm} .-{ }^{13} \mathrm{C}-\mathrm{NMR}$ $\left(75.5 \mathrm{MHz}, \mathrm{CDCl}_{3}\right): \delta=-0.4\left[\mathrm{Si}\left(\mathrm{CH}_{3}\right)_{3}\right], 0.2\left[\mathrm{OSi}\left(\mathrm{CH}_{3}\right)_{3}\right], 41.1(\mathrm{C}-8), 48.1(\mathrm{C}-6), 69.0(\mathrm{C}-$ 7), 101.9 (C-1), 106.2 (C-2), 115.9 (C-12), 123.1 (C-3), 130.1 (C-10), 134.0 (C-9), 136.9 (C-11), 138.8 (C-4), 197.9 (C-5) ppm. - IR: $\widetilde{v}=3436,2961,2362,1634,1252,1097,844$, $668 \mathrm{~cm}^{-1}$. - MS (ESI), m/z (\%): 357 (36) [M + Na $]^{+}, 358$ (12), 415 (12), 607 (10), 619 (14), $690(100)[2 \mathrm{M}+\mathrm{Na}]^{+}, 691$ (52), 749 (40), 750 (12), 845 (20). 
(E)-3-(tert-Butyldimethylsilanyloxy)octa-5,7-diensäureethylester (203)

Alkohol 202 (7.04 g, 38.2 mmol) und Imidazol (9.36 g, $137 \mathrm{mmol})$ wurde in wasserfreiem DMF $(40 \mathrm{~mL})$ gelöst und unter Eisbadkühlung wurde tertButyldimethylsilylchlorid $(8.63 \mathrm{~g}, 57.3 \mathrm{mmol})$ gelöst in Toluol (22.9 mL) zugegeben. Man ließ $60 \mathrm{~h}$ rühren,

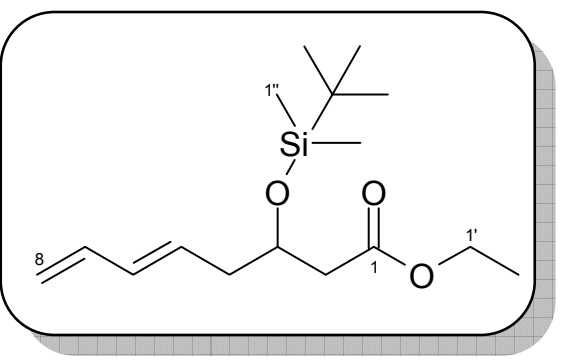
währenddessen sich die Lösung auf Raumtemperatur erwärmte. Die Lösung wurde mit Pentan $(250 \mathrm{~mL})$ und Wasser $(150 \mathrm{~mL})$ versetzt und die Phasen getrennt. Die organsiche Phase wurde mit Pentan $(2 \times 150 \mathrm{~mL})$ extrahiert. Die vereinigten organischen Phasen wurden mit Wasser $(2 \times 150 \mathrm{~mL})$ und gesättigter NaCl-Lösung $(100 \mathrm{~mL})$ gewaschen. Trocknen über $\mathrm{Na}_{2} \mathrm{SO}_{4}$ und Entfernen des Lösungsmittels ergab $14.9 \mathrm{~g}$ Rohprodukt, welches säulenchromatographisch aufgereinigt wurde (Hex:EE, 10:1). Die Titelverbindung wurde als leicht gelbliches Öl erhalten $(11.4 \mathrm{~g}, 83 \%) . R_{\mathrm{f}}=0.42$ (Hex:EE, 10:1). $-{ }^{1} \mathrm{H}-\mathrm{NMR}$ (300 MHz, $\left.\mathrm{CDCl}_{3}\right): \delta=0.00$ (s, $\left.3 \mathrm{H}, \mathrm{SiCH}_{3}\right), 0.03$ (s, $\left.3 \mathrm{H}, \mathrm{SiCH}_{3}\right), 0.83$ [s, $9 \mathrm{H}, \mathrm{SiC}\left(\mathrm{CH}_{3}\right)$ ], $1.22\left(\mathrm{t},{ }^{3} J_{\mathrm{H}-\mathrm{H}}=7.1 \mathrm{~Hz}, 3 \mathrm{H}, 2^{\prime}-\mathrm{H}_{3}\right), 2.26\left(\mathrm{t},{ }^{3} J_{\mathrm{H}-\mathrm{H}}=6.5 \mathrm{~Hz}, 2 \mathrm{H}, 4-\mathrm{H}_{2}\right), 2.38\left(\mathrm{~d},{ }^{3} J_{\mathrm{H}-\mathrm{H}}=\right.$ $6.4 \mathrm{~Hz}, 2 \mathrm{H}, 2-\mathrm{H}_{2}$ ), 4.07 (q, ${ }^{3} J_{\mathrm{H}-\mathrm{H}}=7.1 \mathrm{~Hz}, 2 \mathrm{H}, 1^{\prime}-\mathrm{H}_{2}$ ), 4.16 (quint, ${ }^{3} J_{\mathrm{H}-\mathrm{H}}=6.5 \mathrm{~Hz}, 1 \mathrm{H}, 3-$ $\mathrm{H}), 4.96\left(\mathrm{dd}, J_{\mathrm{H}-\mathrm{H}}=1.2,10.1 \mathrm{~Hz}, 1 \mathrm{H}, 8-\mathrm{H}_{\mathrm{a}}\right), 5.07\left(\mathrm{dd}, J_{\mathrm{H}-\mathrm{H}}=1.2,16.9 \mathrm{~Hz}, 1 \mathrm{H}, 8-\mathrm{H}_{\mathrm{b}}\right), 5.63$ $\left(\mathrm{dt},{ }^{3} J_{\mathrm{H}-\mathrm{H}}=6.6,15.1 \mathrm{~Hz}, 1 \mathrm{H}, 5-\mathrm{H}\right), 6.04\left(\mathrm{dd},{ }^{3} J_{\mathrm{H}-\mathrm{H}}=10.1,15.1 \mathrm{~Hz}, 1 \mathrm{H}, 6-\mathrm{H}\right), 6.27(\mathrm{dt}$, $\left.{ }^{3} J_{\mathrm{H}-\mathrm{H}}=10.1,16.9 \mathrm{~Hz}, 1 \mathrm{H}, 7-\mathrm{H}\right) \mathrm{ppm} .-{ }^{13} \mathrm{C}-\mathrm{NMR}\left(75.5 \mathrm{MHz}, \mathrm{CDCl}_{3}\right): \delta=-5.0\left(\mathrm{SiCH}_{3}\right)$, - $4.6\left(\mathrm{SiCH}_{3}\right), 14.1\left(\mathrm{C}-2^{\prime}\right), 17.9$ [SiC( $\left.\left.\mathrm{CH}_{3}\right)\right], 25.7$ [SiC $\left.\left(\mathrm{CH}_{3}\right)\right], 40.8(\mathrm{C}-4), 42.3(\mathrm{C}-2), 60.3$ (C-1'), 69.1 (C-3), 115.7 (C-8), 130.0 (C-5), 133.9 (C-6), 135.9 (C-7), 171.7 (C-1) ppm. IR: $\widetilde{v}=3088,2957,2931,2858,2342,1736,1653,1603,1587,1473,1464,1375,1311$, 1255, 1178, 1140, 1083, 836, $777 \mathrm{~cm}^{-1}$. - MS (ESI), m/z (\%): 167 (10), 251 (50), 299 (100) $[\mathrm{M}+\mathrm{H}]^{+}, 321(30)[\mathrm{M}+\mathrm{Na}]^{+}, 617(8), 777$ (7). HRMS (ESI): Ber. für $\mathrm{C}_{16} \mathrm{H}_{31} \mathrm{O}_{3} \mathrm{Si}$ 299.20370, Gef. 299.20391.

\section{(E)-3-(tert-Butyldimethylsilanyloxy)-octa-5,7-diensäure-N-methoxy-N-methylamid (205)}

Die Umsetzung wurde analog AAV09 durchgeführt, es wurden folgende Edukte eingesetzt: Ester 203 (7.50 g, $25.1 \mathrm{mmol}$ ), N,O-Dimethylhydroxylamin-Hydrochlorid (4.90 g, $50.2 \mathrm{mmol}), i$-PrMgBr $(68.8 \mathrm{~mL}, 100 \mathrm{mmol}$, $1.46 \mathrm{M}$ in THF). Durch Säulenchromatographie

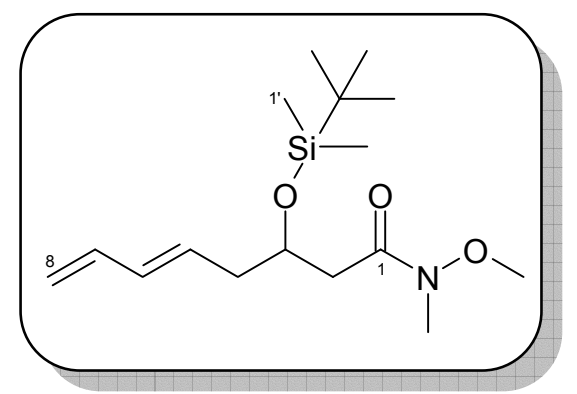


(Pent:EE, 7:1) erhielt man $6.86 \mathrm{~g}$ (87\%) der Titelverbindung als leicht gelbliches Öl. $R_{\mathrm{f}}=0.20$ (Pent:EE, 7:1) - ${ }^{1} \mathrm{H}-\mathrm{NMR}\left(300 \mathrm{MHz}, \mathrm{CDCl}_{3}\right): \delta=0.00\left(\mathrm{~s}, 3 \mathrm{H}, \mathrm{SiCH}_{3}\right), 0.03(\mathrm{~s}$, $\left.3 \mathrm{H}, \mathrm{SiCH}_{3}\right), 0.83\left[\mathrm{~s}, 9 \mathrm{H}, \mathrm{SiC}\left(\mathrm{CH}_{3}\right)_{3}\right], 2.28\left(\mathrm{~m}_{\mathrm{c}}, 2 \mathrm{H}, 4-\mathrm{H}_{2}\right), 2.38\left(\mathrm{dd}, J_{\mathrm{H}-\mathrm{H}}=5.3,14.9 \mathrm{~Hz}\right.$, $\left.1 \mathrm{H}, 2-\mathrm{H}_{\mathrm{a}}\right), 2.66\left(\mathrm{dd}, J_{\mathrm{H}-\mathrm{H}}=7.5,14.9 \mathrm{~Hz}, 1 \mathrm{H}, 2-\mathrm{H}_{\mathrm{b}}\right), 3.13\left(\mathrm{~s}, 3 \mathrm{H}, \mathrm{NCH}_{3}\right), 3.64(\mathrm{~s}, 3 \mathrm{H}$, $\left.\mathrm{NOCH}_{3}\right), 4.27\left(\mathrm{dq},{ }^{3} J_{\mathrm{H}-\mathrm{H}}=5.3,7.5 \mathrm{~Hz}, 1 \mathrm{H}, 3-\mathrm{H}\right), 4.95\left(\mathrm{dd}, J_{\mathrm{H}-\mathrm{H}}=1.5,10.1 \mathrm{~Hz}, 1 \mathrm{H}, 8-\mathrm{H}_{\mathrm{a}}\right)$, $5.07\left(\mathrm{dd}, J_{\mathrm{H}-\mathrm{H}}=1.5,16.9 \mathrm{~Hz}, 1 \mathrm{H}, 8-\mathrm{H}_{\mathrm{b}}\right), 5.67\left(\mathrm{dt},{ }^{3} J_{\mathrm{H}-\mathrm{H}}=7.5,15.0 \mathrm{~Hz}, 1 \mathrm{H}, 5-\mathrm{H}\right), 6.05(\mathrm{dd}$, $\left.{ }^{3} J_{\mathrm{H}-\mathrm{H}}=10.1,15.0 \mathrm{~Hz}, 1 \mathrm{H}, 6-\mathrm{H}\right), 6.28\left(\mathrm{dt},{ }^{3} J_{\mathrm{H}-\mathrm{H}}=10.1,16.9 \mathrm{~Hz}, 1 \mathrm{H}, 7-\mathrm{H}\right) \mathrm{ppm} .-{ }^{13} \mathrm{C}-$ NMR (75.5 MHz, $\left.\mathrm{CDCl}_{3}\right): \delta=-4.8\left(\mathrm{SiCH}_{3}\right),-4.7 \mathrm{Si}\left(\mathrm{CH}_{3}\right), 18.0\left[\mathrm{SiC}\left(\mathrm{CH}_{3}\right)\right], 25.8$ [SiC $\left.\left(\mathrm{CH}_{3}\right)\right], 31.9\left(\mathrm{NCH}_{3}\right), 39.15(\mathrm{C}-2), 41.0(\mathrm{C}-4), 61.3\left(\mathrm{NOCH}_{3}\right), 69.1(\mathrm{C}-3), 115.4(\mathrm{C}-8)$, 130.6 (C-5), 133.7 (C-6), 137.0 (C-7), (C-1 nicht sichtbar) ppm. - IR: $\widetilde{v}=2957,2930$, 2898, 2857, 1802, 1665, 1604, 1472, 1464, 1414, 1386, 1255, 1179, 1091, 1004, 836, 777 $\mathrm{cm}^{-1}$. - MS (DCI, $\left.\mathrm{NH}_{3}\right), m / z(\%): 314(100)[\mathrm{M}+\mathrm{H}]^{+}$. - HRMS (ESI): Ber. für $\mathrm{C}_{16} \mathrm{H}_{31} \mathrm{NO}_{3} \mathrm{SiNa} 336.19654$, Gef. 336.19678.

(3E,9E)-7-(tert-Butyldimethylsilanyloxy)-1-trimethylsilanyldodeca-3,9,11-trien-1-in-5-on (197)

Die Umsetzung wurde analog AAV10 durchgeführt, es wurden folgende Edukte eingesetzt: Amid 205 (4.31 g, $13.7 \mathrm{mmol})$, Bromid $166(6.00 \mathrm{~g}, 29.5 \mathrm{mmol}), \quad t$-BuLi (40.4 mL, $54.9 \mathrm{mmol}, 1.36 \mathrm{M}$ in Pentan). Das Rohprodukt (5.7 g) wurde säulenchro-

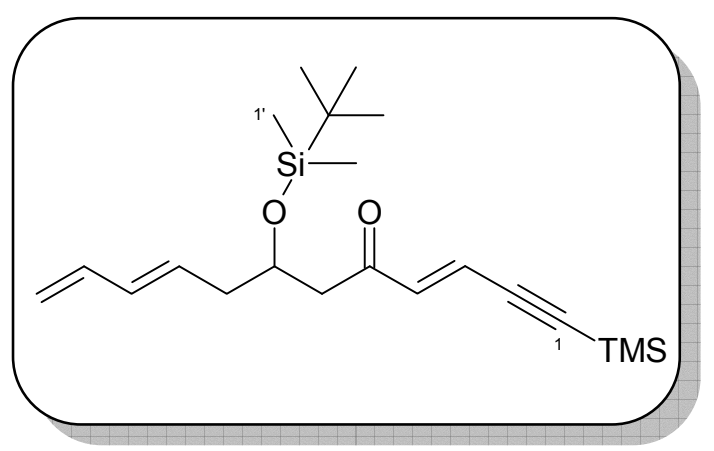
matographisch gereinigt (Pent:EE, 15:1 + 5\% $\left.\mathrm{NEt}_{3}\right)$. Man erhielt $4.36 \mathrm{~g}(84 \%)$ der Titelverbindung als leicht gelbliches Öl. $R_{\mathrm{f}}=0.54$ (Hex:EE, 10:1). $-{ }^{1} \mathrm{H}-\mathrm{NMR}\left(300 \mathrm{MHz}, \mathrm{CDCl}_{3}\right.$ ): $\delta=-0.03$ (s, $3 \mathrm{H}, \mathrm{SiCH}_{3}$ ), 0.03 (s, $3 \mathrm{H}$, $\left.\mathrm{SiCH}_{3}\right), 0.19$ [s, $\left.9 \mathrm{H}, \mathrm{Si}\left(\mathrm{CH}_{3}\right)_{3}\right], 0.83$ [s, $\left.9 \mathrm{H}, \mathrm{SiC}\left(\mathrm{CH}_{3}\right)_{3}\right], 2.25\left(\mathrm{~m}_{\mathrm{c}}, 2 \mathrm{H}, 8-\mathrm{H}_{2}\right), 2.53$ (dd, $\left.{ }^{3} J_{\mathrm{H}-\mathrm{H}}=5.0,15.1 \mathrm{~Hz}, 1 \mathrm{H}, 6-\mathrm{H}_{\mathrm{a}}\right), 2.69\left(\mathrm{dd}, J_{\mathrm{H}-\mathrm{H}}=7.2,15.1 \mathrm{~Hz}, 1 \mathrm{H}, 6-\mathrm{H}_{\mathrm{b}}\right), 4.22\left(\mathrm{~m}_{\mathrm{c}}, 1 \mathrm{H}, 7-\right.$ $\mathrm{H}), 4.98\left(\mathrm{~d},{ }^{3} J_{\mathrm{H}-\mathrm{H}}=10.1 \mathrm{~Hz}, 1 \mathrm{H}, 12-\mathrm{H}_{\mathrm{a}}\right), 5.09\left(\mathrm{~d},{ }^{3} J_{\mathrm{H}-\mathrm{H}}=16.8 \mathrm{~Hz}, 1 \mathrm{H}, 12-\mathrm{H}_{\mathrm{b}}\right), 5.64(\mathrm{dt}$, $\left.{ }^{3} J_{\mathrm{H}-\mathrm{H}}=7.4,15.1 \mathrm{~Hz}, 1 \mathrm{H}, 9-\mathrm{H}\right), 6.04\left(\mathrm{dd}, J_{\mathrm{H}-\mathrm{H}}=10.1,15.1 \mathrm{~Hz}, 1 \mathrm{H}, 10-\mathrm{H}\right), 6.28\left(\mathrm{dt},{ }^{3} J_{\mathrm{H}-\mathrm{H}}\right.$ $=10.1,16.8 \mathrm{~Hz}, 1 \mathrm{H}, 11-\mathrm{H}), 6.49\left(\mathrm{~d},{ }^{3} J_{\mathrm{H}-\mathrm{H}}=16.1 \mathrm{~Hz}, 1 \mathrm{H}, 3-\mathrm{H}\right), 6.57\left(\mathrm{~d},{ }^{3} J_{\mathrm{H}-\mathrm{H}}=16.1 \mathrm{~Hz}\right.$, $1 \mathrm{H}, 4-\mathrm{H})$ ppm. $-{ }^{13} \mathrm{C}-\mathrm{NMR}\left(75.5 \mathrm{MHz}, \mathrm{CDCl}_{3}\right): \delta=-4.8\left(\mathrm{SiCH}_{3}\right),-4.6\left(\mathrm{SiCH}_{3}\right),-0.43$ [Si( $\left.\left(\mathrm{CH}_{3}\right)_{3}\right], 18.0\left[\mathrm{SiC}\left(\mathrm{CH}_{3}\right)_{3}\right], 25.8\left[\mathrm{SiC}\left(\mathrm{CH}_{3}\right)_{3}\right], 41.0(\mathrm{C}-8), 48.0(\mathrm{C}-6), 69.0(\mathrm{C}-7), 101.9$ (C-1), 106.1 (C-2), 115.8 (C-12), 123.1 (C-3), 130.0 (C-9), 134.0 (C-10), 136.9 (C-11), 
138.9 (C-4), 198.0 (C-5) ppm. - MS (DCI, NH $\mathrm{NH}_{3}, m / z$ (\%): 377 (100) [M + H] $]^{+}, 394$ (65) $\left[\mathrm{M}+\mathrm{NH}_{4}\right]^{+}, 770\left[2 \mathrm{M}+\mathrm{NH}_{4}\right]^{+}$. HRMS (ESI): Ber. für $\mathrm{C}_{21} \mathrm{H}_{37} \mathrm{O}_{2} \mathrm{Si}_{2}$ 377.23266, Gef. 377.23266 .

\section{E.3.4 Intramolekularen Diels-Alder Reaktion}

\section{Allgemeine Arbeitsvorschrift 11 - Thermisch induzierte IMDA Reaktionen}

Das jeweilige Trienon (100-250 $\mu \mathrm{mol})$ wurde im betreffenden Lösungsmittel (Toluol oder DCM, 10-20 mL) gelöst. Reaktionen bei Temperaturen oberhalb der Siedetemperatur des Lösungsmittels wurden in einem abgeschlossenen Reaktionsgefäß ('Bombenrohr') durchgeführt. Die übrigen Reaktionen wurden bei Rückfluss unter Schutzgasatmosphäre gerührt. Ergebnisse und Details für die Versuche zur Umsetzung der Verbindungen 165 bzw. 177-181 sind in Tabelle 23 bzw. 24 und für Trienone 178 und 197 in Tabelle 25 zusammengefasst.

\section{Allgemeine Arbeitsvorschrift 12 - IMDA Reaktionen katalysiert durch Zusatz von Säuren}

$L A$ : Das Trien $(100-250 \mu \mathrm{mol})$ wurde im betreffenden Lösungsmittel $(10-20 \mathrm{~mL})$ gelöst und bei $-78^{\circ} \mathrm{C}$ mit der LA versetzt. Der Reaktionsfortschritt wurde per Dünnschichtchromatographie verfolgt. Ergebnisse und Details für die Versuche zur Umsetzung der Verbindungen 165 bzw. 177-181 sind in Tabelle 23 bzw. 24 und für Trienone 178 und 197 in Tabelle 25 zusammengefasst.

pTsOH: Das Trien $(25.0 \mathrm{mg}, 53.8 \mu \mathrm{mol})$ wurde in Benzol $(6.0 \mathrm{~mL})$ gelöst und mit einigen Kristallen $p$ TsOH versetzt. Nach 3 h Rühren bei RT konnte per DC-Kontrolle kein Edukt mehr nachgewiesen werden. Ergebnisse und Details für die Versuche zu dieser Umsetzung der Verbindungen 165 sind in Tabelle 23 zusammengefasst. 
Aufarbeitungsvarianten (AV):

A: Das Lösungsmittel wurde unter vermindertem Druck entfernt.

B: Es wurde $\mathrm{NaHCO}_{3}$-Lösung $(10 \mathrm{~mL} / \mathrm{mmol})$ zugesetzt und man ließ auf RT erwärmen. Die Reaktionslösung wurde mit Ether verdünnt, die Phasen getrennt und die wässrige Phase mit Ether extrahiert. Anschließend wurde mit NaCl-Lösung gewaschen, über $\mathrm{NaSO}_{4}$ getrocknet und das Lösungsmittel unter vermindertem Druck entfernt. Die Rohproduktausbeuten lagen zwischen 75 und 120\% der Theorie.

C: Triethylamin $(1.5 \mathrm{~mL} / \mathrm{mmol})$ wurde zugegeben und man ließ auf RT erwärmen. Nach Zugabe von Wasser, $\mathrm{NH}_{4} \mathrm{Cl}$-Lösung und Verdünnen mit Ether wurden die Phasen getrennt und die organische Phase mit Ether extrahiert. Die vereinigten organischen Phasen wurden mit Wasser und NaCl-Lösung gewaschen, über Natriumsulfat getrocknet und das Lösungsmittel im Vakuum entfernt.

D: Pyridin (1.5 mL/mmol) wurde zugegeben und man ließ auf RT erwärmen. Nach Zugabe von Wasser, $\mathrm{NH}_{4} \mathrm{Cl}$-Lösung und Verdünnen mit Ether wurden die Phasen getrennt und die organische Phase mit Ether extrahiert. Die vereinigten organischen Phasen wurden mit Wasser und NaCl-Lösung gewaschen, über Natriumsulfat getrocknet und das Lösungsmittel im Vakuum entfernt.

$\left(3 R^{*}, 4 a S^{*}, 8 S^{*}, 8 a S^{*}\right)-3-($ Trimethylsilanyloxy)-8-trimethylsilanylethinyl-3,4,4a, 7,8,8ahexahydro-2H-naphthalin-1-on (198a)

${ }^{1} \mathrm{H}-\mathrm{NMR}\left(600 \mathrm{MHz}, \mathrm{CDCl}_{3}\right): \delta=0.10\left[\mathrm{~s}, 9 \mathrm{H}, \mathrm{Si}\left(\mathrm{CH}_{3}\right)_{3}\right], 0.11[\mathrm{~s}$, $\left.9 \mathrm{H}, \mathrm{Si}\left(\mathrm{CH}_{3}\right)_{3}\right], 1.85\left(\mathrm{ddd},{ }^{3} J_{\mathrm{H}-\mathrm{H}}=4.7,9.9,13.5 \mathrm{~Hz}, 1 \mathrm{H}, 4-\mathrm{H}_{\mathrm{a}}\right)$, $2.04\left(\mathrm{~m}_{\mathrm{c}}, 2 \mathrm{H}, 4-\mathrm{H}_{\mathrm{b}}, 7-\mathrm{H}_{\mathrm{a}}\right), 2.30\left(\mathrm{ddd}, J_{\mathrm{H}-\mathrm{H}}=1.0,9.8,14.2 \mathrm{~Hz}\right.$, $\left.1 \mathrm{H}, 2-\mathrm{H}_{\mathrm{a}}\right), 2.39\left(\mathrm{~m}_{\mathrm{c}}, 1 \mathrm{H}, 7-\mathrm{H}_{\mathrm{b}}\right), 2.47\left(\mathrm{~m}_{\mathrm{c}}, 1 \mathrm{H}, 8 \mathrm{a}-\mathrm{H}\right), 2.59$ (ddd, $\left.J_{\mathrm{H}-\mathrm{H}}=2.1,4.4,14.2 \mathrm{~Hz}, 1 \mathrm{H}, 2-\mathrm{H}_{\mathrm{b}}\right), 3.10\left(\mathrm{~m}_{\mathrm{c}}, 1 \mathrm{H}, 4 \mathrm{a}-\mathrm{H}\right), 3.28$ $\left(\mathrm{m}_{\mathrm{c}}, 1 \mathrm{H}, 8-\mathrm{H}\right), 3.90\left(\mathrm{~m}_{\mathrm{c}}, 1 \mathrm{H}, 3-\mathrm{H}\right), 5.50\left(\mathrm{dd}, J_{\mathrm{H}-\mathrm{H}}=2.1,10.0 \mathrm{~Hz}\right.$, $1 \mathrm{H}, 5-\mathrm{H}), 5.62\left(\mathrm{ddd}, J_{\mathrm{H}-\mathrm{H}}=2.8,6.7,10.0 \mathrm{~Hz}, 1 \mathrm{H}, 6-\mathrm{H}\right) \mathrm{ppm}$. -

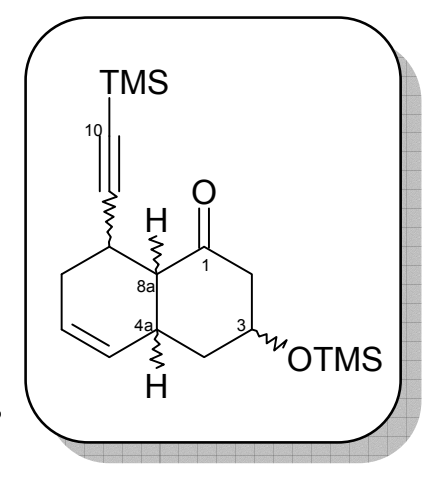

${ }^{13} \mathrm{C}-\mathrm{NMR}\left(150.8 \mathrm{MHz}, \mathrm{CDCl}_{3}\right): \delta=0.0\left[\mathrm{Si}\left(\mathrm{CH}_{3}\right)_{3}\right], 0.1$ [Si( $\left.\left.\mathrm{CH}_{3}\right)_{3}\right], 25.0(\mathrm{C}-8), 28.1(\mathrm{C}-7)$, 30.5 (C-4), 39.1 (C-4a), 50.1 (C-8a), 50.8 (C-2), 67.2 (C-3), 85.1 (C-10), 109.1 (C-9), 26.5 
(C-5), 128.9 (C-6), 207.3 (C-1) ppm. - IR: $\widetilde{v}=3438,2922,1631,1377 \mathrm{~cm}^{-1}$. - MS (ESI), $m / z(\%): 357(36)[\mathrm{M}+\mathrm{Na}]^{+}, 413$ (12), 691 (100), [2 M + Na] $]^{+}, 692$ (38), 746 (32).

$\left(3 S^{*}, 4 a S^{*}, 8 S^{*}, 8 a S^{*}\right)-3-($ Trimethylsilanyloxy)-8-trimethylsilanylethinyl-3, 4, 4a, 7,8,8ahexahydro-2H-naphthalin-1-on (198c)

${ }^{1} \mathrm{H}-\mathrm{NMR}\left(600 \mathrm{MHz}, \mathrm{CDCl}_{3}\right): \delta=0.10\left[\mathrm{~s}, 9 \mathrm{H}, \mathrm{Si}\left(\mathrm{CH}_{3}\right)_{3}\right], 0.11[\mathrm{~s}$, $\left.9 \mathrm{H}, \mathrm{Si}\left(\mathrm{CH}_{3}\right)_{3}\right], 1.63\left(\mathrm{~m}_{\mathrm{c}}, 1 \mathrm{H}, 4-\mathrm{H}_{\mathrm{a}}\right), 2.01\left(\mathrm{dt},{ }^{3} J_{\mathrm{H}-\mathrm{H}}=4.2\right.$, $13.4 \mathrm{~Hz}, 1 \mathrm{H}, 7-\mathrm{H}_{\mathrm{a}}$ ), 2.09 (dddd, $J_{\mathrm{H}-\mathrm{H}}=2.4,4.6,9.9,17.9 \mathrm{~Hz}$, $\left.1 \mathrm{H}, 4-\mathrm{H}_{\mathrm{b}}\right), 2.37-2.52$ (m, $\left.3 \mathrm{H}, 2-\mathrm{Ha}, 7-\mathrm{H}_{\mathrm{b}}, 8 \mathrm{a}-\mathrm{H}\right), 2.54\left(\mathrm{~m}_{\mathrm{c}}, 1 \mathrm{H}\right.$, $\left.2-\mathrm{H}_{\mathrm{b}}\right), 2.58-2.72(\mathrm{~m}, 1 \mathrm{H}, 4 \mathrm{a}-\mathrm{H}), 3.00\left(\mathrm{dd},{ }^{3} J_{\mathrm{H}-\mathrm{H}}=5.3,9.9 \mathrm{~Hz}\right.$, $1 \mathrm{H}, 8-\mathrm{H}), 3.87\left(\mathrm{~m}_{\mathrm{c}}, 1 \mathrm{H}, 3-\mathrm{H}\right), 5.57\left(\mathrm{~m}_{\mathrm{c}}, 1 \mathrm{H}, 5-\mathrm{H}\right), 5.64\left(\mathrm{~m}_{\mathrm{c}}\right.$, $1 \mathrm{H}, 6-\mathrm{H})$ ppm. $-{ }^{13} \mathrm{C}-\mathrm{NMR}\left(150.8 \mathrm{MHz}, \mathrm{CDCl}_{3}\right): \delta=0.0$

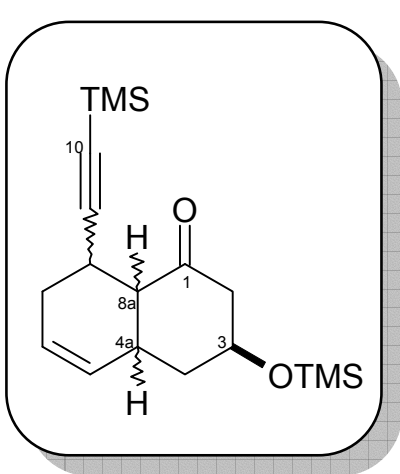
[Si $\left.\left(\mathrm{CH}_{3}\right)_{3}\right], 0.1\left[\mathrm{Si}\left(\mathrm{CH}_{3}\right)_{3}\right], 25.5(\mathrm{C}-8), 31.4(\mathrm{C}-7), 32.9(\mathrm{C}-4), 38.2(\mathrm{C}-4 \mathrm{a}), 49.0(\mathrm{C}-2), 53.4$ (C-8a), 69.2 (C-3), 87.3 (C-10), 106.4 (C-9), 124.7 (C-5), 129.1 (C-6), 208.5 (C-1) ppm.

$\left(3 R^{*}, 4 a S^{*}, 8 S^{*}, 8 a S^{*}\right)-3-(t e r t-B u t y l d i m e t h y l$ silanyloxy)-8-trimethylsilanylethinyl3,4,4a,7,8,8a-hexahydro-2H-naphthalin-1-on (199a)

$R_{\mathrm{f}}=0.56$ (Hex:EE, 10:1), $R_{\mathrm{f}}=0.31$ (Hex:EE, 20:1). - Smp. $=$ $35{ }^{\circ} \mathrm{C}$. $-{ }^{1} \mathrm{H}-\mathrm{NMR}\left(600 \mathrm{MHz}, \mathrm{CDCl}_{3}\right): \delta=0.03[\mathrm{~s}, 3 \mathrm{H}$, $\mathrm{Si}\left(\mathrm{CH}_{3}\right)_{2}$ ], 0.04 [s, $\left.3 \mathrm{H}, \mathrm{Si}\left(\mathrm{CH}_{3}\right)_{2}\right] 0.10$ [s, $\left.9 \mathrm{H}, \mathrm{Si}\left(\mathrm{CH}_{3}\right)_{3}\right]$, $0.85\left[\mathrm{~s}, 3 \mathrm{H}, \mathrm{SiC}\left(\mathrm{CH}_{3}\right)_{3}\right], 1.84\left(\mathrm{ddd}, J_{\mathrm{H}-\mathrm{H}}=4.7,9.3,13.5 \mathrm{~Hz}\right.$, $\left.1 \mathrm{H}, 4-\mathrm{H}_{\mathrm{a}}\right), 1.99\left(\mathrm{~m}_{\mathrm{c}}, 1 \mathrm{H}, 4-\mathrm{H}_{\mathrm{b}}\right), 2.04\left(\mathrm{~m}_{\mathrm{c}}, 1 \mathrm{H}, 7-\mathrm{H}_{\mathrm{a}}\right), 2.29$ $\left(\mathrm{ddd}, J_{\mathrm{H}-\mathrm{H}}=1.1,9.1,14.2 \mathrm{~Hz}, 1 \mathrm{H}, 2-\mathrm{H}_{\mathrm{a}}\right), 2.38\left(\mathrm{ddt}, J_{\mathrm{H}-\mathrm{H}}=\right.$ $\left.3.1,6.1,18.3 \mathrm{~Hz}, 1 \mathrm{H}, 7-\mathrm{H}_{\mathrm{b}}\right), 2.46\left(\mathrm{t},{ }^{3} J_{\mathrm{H}-\mathrm{H}}=5.5 \mathrm{~Hz}, 1 \mathrm{H}, 8 \mathrm{a}-\right.$

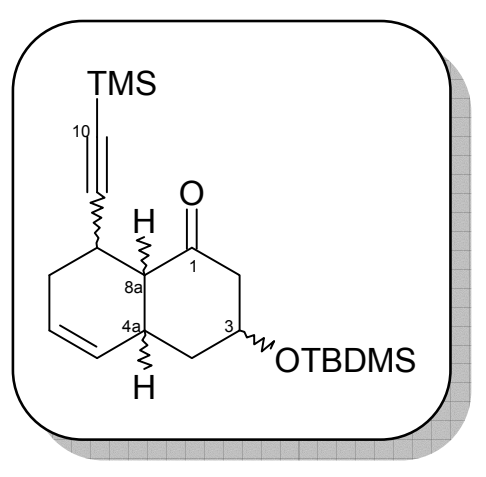
H), $2.58\left(\mathrm{ddd}, J_{\mathrm{H}-\mathrm{H}}=1.9,4.3,14.2 \mathrm{~Hz}, 1 \mathrm{H}, 2-\mathrm{H}_{\mathrm{b}}\right), 3.07\left(\mathrm{~m}_{\mathrm{c}}, 1 \mathrm{H}, 4 \mathrm{a}-\mathrm{H}\right), 3.23\left(\mathrm{ddd},{ }^{3} J_{\mathrm{H}-\mathrm{H}}=\right.$ 4.0, 5.1, $6.1 \mathrm{~Hz}, 1 \mathrm{H}, 8-\mathrm{H}), 3.95\left(\mathrm{ddt},{ }^{3} J_{\mathrm{H}-\mathrm{H}}=3.9,9.0,13.0 \mathrm{~Hz}, 1 \mathrm{H}, 3-\mathrm{H}\right), 5.50\left(\mathrm{ddd}, J_{\mathrm{H}-\mathrm{H}}=\right.$ 2.2, 4.4, 10.1 Hz, $1 \mathrm{H}, 5-\mathrm{H}), 5.59$ (ddd, $\left.1 \mathrm{H},{ }^{3} J_{\mathrm{H}-\mathrm{H}}=3.1,6.4,9.8 \mathrm{~Hz}, 1 \mathrm{H}, 6-\mathrm{H}\right) \mathrm{ppm} .-{ }^{13} \mathrm{C}-$ NMR (150.8 MHz, $\left.\mathrm{CDCl}_{3}\right): \delta=-4.8\left[\mathrm{Si}\left(\mathrm{CH}_{3}\right)_{2}\right],-4.7\left[\mathrm{Si}\left(\mathrm{CH}_{3}\right)_{2}\right], 0.12\left[\mathrm{Si}\left(\mathrm{CH}_{3}\right)_{3}\right], 18.0$ [SiC $\left.\left(\mathrm{CH}_{3}\right)_{3}\right], 24.9(\mathrm{C}-8), 25.7$ [ $\left.\mathrm{SiC}\left(\mathrm{CH}_{3}\right)_{3}\right], 28.6(\mathrm{C}-7), 30.8(\mathrm{C}-4 \mathrm{a}), 38.9(\mathrm{C}-4), 50.5(\mathrm{C}-8 \mathrm{a})$, 50.7 (C-2), 67.7 (C-3), 85.4 (C-10), 108.9 (C-9), 126.4 (C-6), 129.0 (C-5), 207.6 (C-1) ppm. - IR: $\widetilde{v}=2957,2937,2905,2863,2361,2342,2173$, 1709, 1472, 1250, 1155, 1093, 
1077, 842, $778 \mathrm{~cm}^{-1}$. - MS (ESI), m/z (\%): 305 (27) [M - TMS + H], $400(100)[\mathrm{M}+$ $\mathrm{Na}]^{+}, 777(30)[2 \mathrm{M}+\mathrm{Na}]^{+}$. - Anal. ber. (\%) für $\mathrm{C}_{21} \mathrm{H}_{36} \mathrm{O}_{2} \mathrm{Si}_{2}$ (376.69): C 66.96, H 9.63, O 8.49, Si 14.91; Gef. C 66.88, H 9.54, O 8.60, Si 14.81.

3-(tert-Butyldimethylsilanyloxy)-8-trimethylsilanylethinyl-3,4,4a, 7,8,8a-hexahydro-2Hnaphthalin-1-on (199b)

$R_{\mathrm{f}}=0.55$ (Hex:EE, 10:1), $R_{\mathrm{f}}=0.30$ (Hex:EE, 20:1). $-{ }^{1} \mathrm{H}-$ NMR (600 MHz, $\left.\mathrm{CD}_{3} \mathrm{OD}\right): \delta=0.08\left[\mathrm{~s}, 9 \mathrm{H}, \mathrm{Si}\left(\mathrm{CH}_{3}\right)_{3}\right], 0.09$ [s, $\left.3 \mathrm{H}, \mathrm{Si}\left(\mathrm{CH}_{3}\right)_{2}\right], 0.09$ [s, $\left.3 \mathrm{H}, \mathrm{Si}\left(\mathrm{CH}_{3}\right)_{2}\right], 0.90$ [s, $9 \mathrm{H}$, $\left.\mathrm{Si}\left(\mathrm{CH}_{3}\right)_{3}\right], 1.58\left(\mathrm{dt},{ }^{3} J_{\mathrm{H}-\mathrm{H}}=10.6,12.9 \mathrm{~Hz}, 1 \mathrm{H}, 4-\mathrm{H}_{\mathrm{a}}\right), 1.95$ (ddt, $\left.J_{\mathrm{H}-\mathrm{H}}=3.0,7.5,12.9 \mathrm{~Hz}, 1 \mathrm{H}, 4 \mathrm{a}-\mathrm{H}\right), 2.10-2.20(\mathrm{~m}, 2 \mathrm{H}$, $\left.4-\mathrm{H}_{\mathrm{b}}, 7-\mathrm{H}_{\mathrm{a}}\right), 2.44\left(\mathrm{~m}_{\mathrm{c}}, 1 \mathrm{H}, 7-\mathrm{H}_{\mathrm{b}}\right), 2.48\left(\mathrm{~m}_{\mathrm{c}}, 1 \mathrm{H}, 8 \mathrm{a}-\mathrm{H}\right)$, 2.54-2.62 (m, $\left.2 \mathrm{H}, 2-\mathrm{H}_{\mathrm{a}}, 8-\mathrm{H}\right), 2.62\left(\mathrm{~m}_{\mathrm{c}}, 1 \mathrm{H}, 2-\mathrm{H}_{\mathrm{b}}\right), 3.95$ (tt,

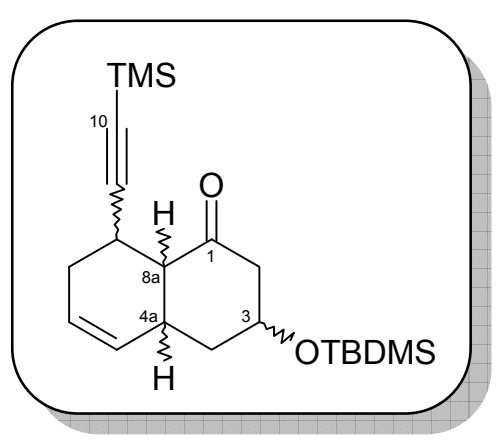
$\left.{ }^{3} J_{\mathrm{H}-\mathrm{H}}=5.1,10.5 \mathrm{~Hz}, 1 \mathrm{H}, 3-\mathrm{H}\right), 5.53\left(\mathrm{ddd}, J_{\mathrm{H}-\mathrm{H}}=1.7,4.1,9.9 \mathrm{~Hz}, 1 \mathrm{H}, 5-\mathrm{H}\right), 5.67\left(\mathrm{ddt}, J_{\mathrm{H}-\mathrm{H}}\right.$ $=2.5,4.9,9.9 \mathrm{~Hz}, 1 \mathrm{H}, 6-\mathrm{H}) \mathrm{ppm} .-{ }^{13} \mathrm{C}-\mathrm{NMR}\left(150.8 \mathrm{MHz}, \mathrm{CDCl}_{3}\right): \delta=-4.6\left[\mathrm{Si}\left(\mathrm{CH}_{3}\right)_{2}\right],-$ $4.7\left[\mathrm{Si}\left(\mathrm{CH}_{3}\right)_{2}\right], 0.2\left[\mathrm{Si}\left(\mathrm{CH}_{3}\right)_{3}\right], 18.8\left[\mathrm{SiC}\left(\mathrm{CH}_{3}\right)_{3}\right], 26.2\left[\mathrm{SiC}\left(\mathrm{CH}_{3}\right)_{3}\right], 27.0(\mathrm{C}-8), 33.5(\mathrm{C}-7)$, 37.7 (C-4a), 43.1 (C-4), 53.4 (C-2), 56.5 (C-8a), 72.1 (C-3), 85.4 (C-10), 110.7 (C-9), 126.8 (C-6), 130.1 (C-5), 208.2 ( C-1) ppm. - MS (ESI), m/z (\%): 305 (15) [M - TMS + $\mathrm{H}]^{+}, 400(67)[\mathrm{M}+\mathrm{Na}]^{+}$.

$\left(3 S^{*}, 4 a S^{*}, 8 S^{*}, 8 a S^{*}\right)-3-($ tert-Butyldimethylsilanyloxy)-8-trimethylsilanylethinyl3,4,4a, 7,8,8a-hexahydro-2H-naphthalin-1-on (199c)

$R_{\mathrm{f}}=0.54$ (Hex:EE, 10:1), $R_{\mathrm{f}}=0.29$ (Hex:EE, 20:1). $-{ }^{1} \mathrm{H}-$ NMR (600 MHz, $\left.\mathrm{CD}_{3} \mathrm{OD}\right): \delta=0.06\left[\mathrm{~s}, 3 \mathrm{H}, \mathrm{Si}\left(\mathrm{CH}_{3}\right)_{2}\right], 0.07$ [s, $\left.3 \mathrm{H}, \mathrm{Si}\left(\mathrm{CH}_{3}\right)_{2}\right], 0.09\left[\mathrm{~s}, 9 \mathrm{H}, \mathrm{Si}\left(\mathrm{CH}_{3}\right)_{3}\right], 0.87$ [s, $9 \mathrm{H}$, $\left.\mathrm{SiC}\left(\mathrm{CH}_{3}\right)_{3}\right], 1.77\left(\mathrm{dt},{ }^{3} J_{\mathrm{H}-\mathrm{H}}=8.3,13.8 \mathrm{~Hz}, 1 \mathrm{H}, 4-\mathrm{H}_{\mathrm{a}}\right), 2.03$ (dddd, $\left.J_{\mathrm{H}-\mathrm{H}}=2.3,5.5,7.5,17.8 \mathrm{~Hz}, 1 \mathrm{H}, 7-\mathrm{H}_{\mathrm{a}}\right), 2.10$ (dddd, $\left.J_{\mathrm{H}-\mathrm{H}}=1.3,3.8,4.9,13.8 \mathrm{~Hz}, 1 \mathrm{H}, 4-\mathrm{H}_{\mathrm{b}}\right), 2.41-2.50(\mathrm{~m}, 3 \mathrm{H}$, $\left.2-\mathrm{H}_{\mathrm{a}}, 7-\mathrm{H}_{\mathrm{b}}, 8 \mathrm{a}-\mathrm{H}\right), 2.54\left(\mathrm{ddt}, J_{\mathrm{H}-\mathrm{H}}=1.3,4.1,13.4 \mathrm{~Hz}, 1 \mathrm{H}, 2-\right.$

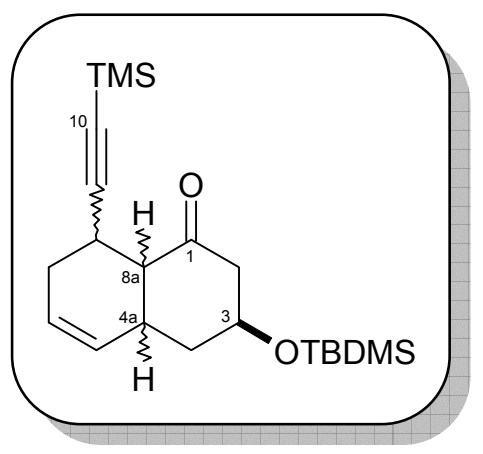
$\left.\mathrm{H}_{\mathrm{b}}\right), 2.75\left(\mathrm{~m}_{\mathrm{c}}, 1 \mathrm{H}, 4 \mathrm{a}-\mathrm{H}\right), 3.14\left(\mathrm{dt},{ }^{3} J_{\mathrm{H}-\mathrm{H}}=5.8,7.5 \mathrm{~Hz}, 1 \mathrm{H}, 8-\mathrm{H}\right), 4.12\left(\mathrm{qd},{ }^{3} J_{\mathrm{H}-\mathrm{H}}=3.9\right.$, $11.9 \mathrm{~Hz}, 3-\mathrm{H}), 5.53\left(\mathrm{~m}_{\mathrm{c}}, 1 \mathrm{H}, 6-\mathrm{H}\right), 5.68\left(\mathrm{ddt}, J_{\mathrm{H}-\mathrm{H}}=2.1,4.1,10.1 \mathrm{~Hz}, 5-\mathrm{H}\right) \mathrm{ppm} .-{ }^{13} \mathrm{C}-$ 
$\operatorname{NMR}\left(150.8 \mathrm{MHz}, \mathrm{CDCl}_{3}\right): \delta=-4.8\left[\mathrm{Si}\left(\mathrm{CH}_{3}\right)_{2}\right],-4.7\left[\mathrm{Si}\left(\mathrm{CH}_{3}\right)_{2}\right], 0.1\left[\mathrm{Si}\left(\mathrm{CH}_{3}\right)_{3}\right], 18.8$ [SiC $\left.\left(\mathrm{CH}_{3}\right)_{3}\right], 26.2$ [SiC $\left.\left(\mathrm{CH}_{3}\right)_{3}\right], 26.6(\mathrm{C}-8), 31.2(\mathrm{C}-7), 34.2(\mathrm{C}-4 \mathrm{a}), 39.0(\mathrm{C}-4), 50.2(\mathrm{C}-2)$, 53.8 (C-8a), 71.3 (C-3), 87.0 (C-10), 109.1 (C-9), 124.4 (C-6), 131.5 (C-5), 210.8 (C-1) ppm. - IR: $\widetilde{v}=3027,2957,2930,2858,2173,1719,1472,1463,1373,1250,1099$, 1058, 1006, 840, 776, 760, 716, $643 \mathrm{~cm}^{-1}-\mathrm{MS}\left(\mathrm{DCI}, \mathrm{NH}_{3}\right), m / z(\%): 400(100)[\mathrm{M}+\mathrm{Na}]^{+}$.

\section{E.3.5 Versuche zur Einführung des $\alpha, \beta$-ungesättigten Ketons an C-6}

\section{E.3.5.1 Abspaltung der TMS-Schutzgruppe am Alkin}

$\left(3 R^{*}, 4 a S^{*}, 8 S^{*}, 8 a S^{*}\right)-3-($ tert-Butyldimethylsilanyloxy)-8-ethinyl-3,4,4a, 7,8,8a-hexahydro2H-naphthalin-1-on (207a)

Das TMS-geschützte Decalon 199a $(25.0 \mathrm{mg}, 66.4 \mu \mathrm{mol})$ wurde in Diethylether $(1.0 \mathrm{~mL})$ und Ethanol $(0.1 \mathrm{~mL})$ gelöst und mit einer Lösung von $\mathrm{AgNO}_{3}(12.4 \mathrm{mg}, 73.0 \mu \mathrm{mol})$ in einem Ethanol/Wasser-Gemisch $(0.3 \mathrm{~mL}, 2: 1)$ versetzt. Es bildete sich ein weißer Niederschlag. DC-Kontrolle

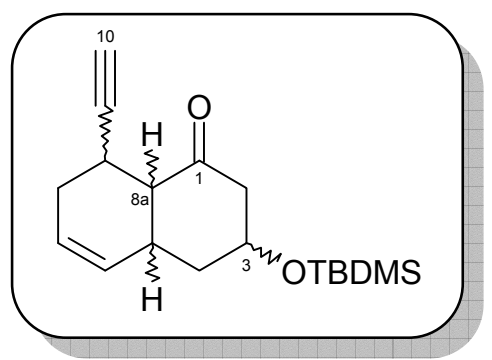
(Hex:EE, 10:1) nach $1 \mathrm{~h}$ Rühren bei RT zeigte vollständigen Umsatz an. Es wurde eine Lösung von $\mathrm{KCN}(21.6 \mathrm{mg}, 332 \mu \mathrm{mol})$ gelöst in $\mathrm{H}_{2} \mathrm{O}(1.0 \mathrm{~mL})$ zugegeben, worauf sich der Niederschlag auflöste. Nach Zugabe von $\mathrm{H}_{2} \mathrm{O}(10 \mathrm{~mL})$ und $\mathrm{Et}_{2} \mathrm{O}$ wurden die Phasen getrennt und die wässrige Phase zweimal mit $\mathrm{Et}_{2} \mathrm{O}(20 \mathrm{~mL})$ extrahiert. Die vereinigten organischen Phasen wurden mit NaCl-Lösung gewaschen, über $\mathrm{Na}_{2} \mathrm{SO}_{4}$ getrocknet und das Lösungsmittel unter reduziertem Druck entfernt. Das erhaltene Rohprodukt (26.6 mg) wurde säulenchromatographisch aufgereinigt (15 g KG, Hex:EE, 20:1). Man erhielt $17.3 \mathrm{mg}(86 \%)$ der Titelverbindung als farbloser Feststoff. $R_{\mathrm{f}}=0.27$ (Hex:EE, 20:1). Smp. $=51{ }^{\circ} \mathrm{C} .-{ }^{1} \mathrm{H}-\mathrm{NMR}\left(600 \mathrm{MHz}, \mathrm{CDCl}_{3}\right): \delta=0.03\left[\mathrm{~s}, 3 \mathrm{H}, \mathrm{Si}\left(\mathrm{CH}_{3}\right)_{2}\right], 0.04[\mathrm{~s}, 3 \mathrm{H}$, $\mathrm{Si}\left(\mathrm{CH}_{3}\right)_{2}$ ], 0.85 [s, $\left.9 \mathrm{H}, \mathrm{SiC}\left(\mathrm{CH}_{3}\right)_{3}\right], 1.84\left(\mathrm{ddd}, J_{\mathrm{H}-\mathrm{H}}=4.7,9.3,13.4 \mathrm{~Hz}, 1 \mathrm{H}, 4-\mathrm{H}_{\mathrm{a}}\right), 2.00$ (dddd, $\left.J_{\mathrm{H}-\mathrm{H}}=2.1,3.7,5.5,13.4 \mathrm{~Hz}, 1 \mathrm{H}, 4-\mathrm{H}_{\mathrm{b}}\right), 2.03\left(\mathrm{~d},{ }^{4} J_{\mathrm{H}-\mathrm{H}}=2.5 \mathrm{~Hz}, 1 \mathrm{H}, 10-\mathrm{H}\right), 2.06$ $\left(\right.$ dddd, $\left.J_{\mathrm{H}-\mathrm{H}}=2.1,4.1,6.1,9.8 \mathrm{~Hz}, 1 \mathrm{H}, 7-\mathrm{H}_{\mathrm{a}}\right), 2.31\left(\mathrm{ddd}, J_{\mathrm{H}-\mathrm{H}}=1.2,9.3,14.3 \mathrm{~Hz}, 1 \mathrm{H}, 2-\right.$ $\left.\mathrm{H}_{\mathrm{a}}\right), 2.41\left(\mathrm{ddt}, J_{\mathrm{H}-\mathrm{H}}=3.1,6.1,18.3 \mathrm{~Hz}, 1 \mathrm{H}, 7-\mathrm{H}_{\mathrm{b}}\right), 2.48\left(\mathrm{t},{ }^{3} J_{\mathrm{H}-\mathrm{H}}=5.4 \mathrm{~Hz}, 1 \mathrm{H}, 8 \mathrm{a}-\mathrm{H}\right), 2.59$ (ddd, $\left.J_{\mathrm{H}-\mathrm{H}}=2.0,4.1,14.3 \mathrm{~Hz}, 1 \mathrm{H}, 2-\mathrm{H}_{\mathrm{b}}\right), 3.11\left(\mathrm{~m}_{\mathrm{c}}, 1 \mathrm{H}, 4 \mathrm{a}-\mathrm{H}\right), 3.24\left(\mathrm{~m}_{\mathrm{c}}, 1 \mathrm{H}, 8-\mathrm{H}\right), 3.94$ $\left(\mathrm{ddt},{ }^{3} J_{\mathrm{H}-\mathrm{H}}=4.1,9.3,13.4 \mathrm{~Hz}, 1-\mathrm{H}, 3-\mathrm{H}\right), 5.53\left(\mathrm{ddd}, J_{\mathrm{H}-\mathrm{H}}=2.1,4.4,9.9 \mathrm{~Hz}, 1 \mathrm{H}, 5-\mathrm{H}\right), 5.61$ (ddd, $\left.1 \mathrm{H},{ }^{3} J_{\mathrm{H}-\mathrm{H}}=3.0,6.6,9.9 \mathrm{~Hz}, 1 \mathrm{H}, 6-\mathrm{H}\right) \mathrm{ppm} .-{ }^{13} \mathrm{C}-\mathrm{NMR}\left(150.8 \mathrm{MHz}, \mathrm{CDCl}_{3}\right): \delta=-$ 
$4.8\left[\mathrm{Si}\left(\mathrm{CH}_{3}\right)_{2}\right],-4.7\left[\mathrm{Si}\left(\mathrm{CH}_{3}\right)_{2}\right], 18.0\left[\mathrm{SiC}\left(\mathrm{CH}_{3}\right)_{3}\right], 23.8(\mathrm{C}-8), 25.7\left[\mathrm{SiC}\left(\mathrm{CH}_{3}\right)_{3}\right], 28.4(\mathrm{C}-$ 7), 30.7 (C-4a), 38.8 (C-4), 50.3 (C-8a), 50.5 (C-2), 67.6 (C-3), 69.2 (C-10), 86.4 (C-9), 126.3 (C-6), 129.2 (C-5), 207.6 (C-1) ppm. - MS (DCI, NH N $_{3}, m / z(\%): 322$ (100) [M + $\left.\mathrm{NH}_{4}\right]^{+}$. HRMS (ESI): Ber. für $\mathrm{C}_{18} \mathrm{H}_{29} \mathrm{O}_{2} \mathrm{Si} 305.19313$, Gef. 305.19331.

$\left(3 S^{*}, 4 a S^{*}, 8 S^{*}, 8 a S^{*}\right)-3$-(tert-Butyldimethylsilanyloxy)-8-ethinyl-3,4,4a, 7, 8, 8a-hexahydro2H-naphthalin-1-on (207c)

Die Umsetzung des Isomers 199c erfolgte analog der oben beschriebenen Reaktion für 199a. Als Edukt diente ein Gemisch der Isomere 199a und 199c (78.6 mg, $209 \mu \mathrm{mol}$, ca. 1:1). Als Produkt erhielt man $26.0 \mathrm{mg}$ des bekannten Isomers 207a und $24.0 \mathrm{mg}$ der Titelverbindung 207c (79\% kombinierte Ausbeute). $R_{\mathrm{f}}=0.40$ (Hex:EE, 10:1), $R_{\mathrm{f}}=0.16$

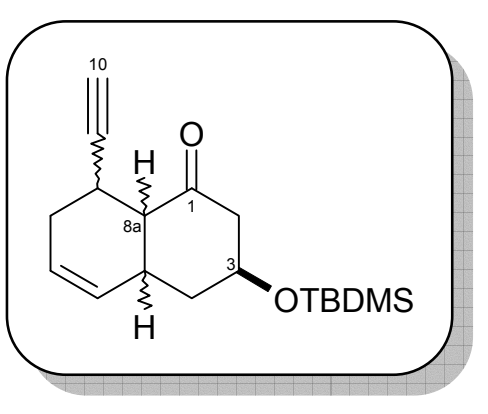
(Hex:EE, 20:1). $-{ }^{1} \mathrm{H}-\mathrm{NMR}\left(600 \mathrm{MHz}, \mathrm{CDCl}_{3}\right): \delta=0.06$ [s, $\left.3 \mathrm{H}, \mathrm{Si}\left(\mathrm{CH}_{3}\right)_{2}\right], 0.07$ [s, $3 \mathrm{H}$, $\left.\mathrm{Si}\left(\mathrm{CH}_{3}\right)_{2}\right], 0.87\left[\mathrm{~s}, 9 \mathrm{H}, \mathrm{SiC}\left(\mathrm{CH}_{3}\right)_{3}\right], 1.79\left(\mathrm{dt},{ }^{3} J_{\mathrm{H}-\mathrm{H}}=8.0,13.9 \mathrm{~Hz}, 1 \mathrm{H}, 4-\mathrm{H}_{\mathrm{a}}\right), 2.05$ (dddd, $\left.J_{\mathrm{H}-\mathrm{H}}=2.3,3.1,7.0,17.8 \mathrm{~Hz}, 1 \mathrm{H}, 7-\mathrm{H}_{\mathrm{a}}\right), 2.11$ (dddd, $J_{\mathrm{H}-\mathrm{H}}=1.4,3.7,4.9,13.8 \mathrm{~Hz}, 1 \mathrm{H}, 4-$ $\left.\mathrm{H}_{\mathrm{b}}\right), 2.38\left(\mathrm{~d},{ }^{4} J_{\mathrm{H}-\mathrm{H}}=2.5 \mathrm{~Hz}, 1 \mathrm{H}, 10-\mathrm{H}\right), 2.45\left(\mathrm{ddt}, J_{\mathrm{H}-\mathrm{H}}=0.7,7.9,13.5 \mathrm{~Hz}, 1 \mathrm{H}, 2-\mathrm{H}_{\mathrm{a}}\right)$, 2.44-2.54 (m, $\left.2 \mathrm{H}, 7-\mathrm{H}_{\mathrm{b}}, 8 \mathrm{a}-\mathrm{H}\right), 2.55$ (ddt, $\left.J_{\mathrm{H}-\mathrm{H}}=1.4,4.1,13.4 \mathrm{~Hz}, 1 \mathrm{H}, 2-\mathrm{H}_{\mathrm{b}}\right), 2.78\left(\mathrm{~m}_{\mathrm{c}}, 1\right.$ $\mathrm{H}, 4 \mathrm{a}-\mathrm{H}), 3.14$ (ddt, $\left.J_{\mathrm{H}-\mathrm{H}}=2.5,5.8,7.5 \mathrm{~Hz}, 1 \mathrm{H}, 8-\mathrm{H}\right), 4.14\left(\mathrm{qd},{ }^{3} J_{\mathrm{H}-\mathrm{H}}=3.9,11.6 \mathrm{~Hz}, 1 \mathrm{H}\right.$, 3-H), 5.53 (ddd, $\left.J_{\mathrm{H}-\mathrm{H}}=3.8,5.9,9.9 \mathrm{~Hz}, 1 \mathrm{H}, 6-\mathrm{H}\right), 5.69\left(\mathrm{~m}_{\mathrm{c}}, 1 \mathrm{H}, 5-\mathrm{H}\right) \mathrm{ppm} .-{ }^{13} \mathrm{C}-\mathrm{NMR}$ $\left(150.8 \mathrm{MHz}, \mathrm{CDCl}_{3}\right): \delta=-4.8 \quad\left[\mathrm{Si}\left(\mathrm{CH}_{3}\right)_{2}\right],-4.7 \quad\left[\mathrm{Si}\left(\mathrm{CH}_{3}\right)_{2}\right], \quad 18.8 \quad\left[\mathrm{SiC}\left(\mathrm{CH}_{3}\right)_{3}\right], 25.4$ [SiC $\left(\mathrm{CH}_{3}\right)_{3}$ ], $26.2(\mathrm{C}-8), 31.1(\mathrm{C}-7), 34.2(\mathrm{C}-4 \mathrm{a}), 38.9$ (C-4), 50.3 (C-2), $53.6(\mathrm{C}-8 \mathrm{a}), 71.3$ (C-3), 71.4 .0 (C-10), 86.1 (C-9), 124.3 (C-6), 131.6 (C-5), 210.9 ( C-1) ppm. - MS (DCI, $\left.\mathrm{NH}_{3}\right), m / z(\%): 322(100)\left[\mathrm{M}+\mathrm{NH}_{4}\right]^{+}$. HRMS (ESI): Ber. für $\mathrm{C}_{18} \mathrm{H}_{28} \mathrm{O}_{2} \mathrm{SiNa}$ 327.17508, Gef. 327.17511 . 


\section{E.3.5.2 Versuche zur Dioxolanbildung}

Versuch der Darstellung von 1-(1,2-Dioxyethyl)-3-(tert-butyldimethylsilanyloxy)-8trimethylsilanylethinyl-3,4,4a,7,8,8a-hexahydro-2H-naphthalin-1-on (208)

AAV11: IMDA-Produkt 199a bzw. 207a wurden im betreffenden Lösungsmittel gelöst, mit Ethylenglykol oder 1,2-Bistrimethylsilanyloxyethan versetzt und ggf. auf die in Tabelle 28 genannte Temperatur gekühlt. Es wurde die betreffenden Säure oder Lewis-Säure zugegeben und der Reaktionsfortschritt per Dünnnschichtchromatographie verfolgt. Nach beendeter Reaktion wurde $\mathrm{NaHCO}_{3}$-Lösung

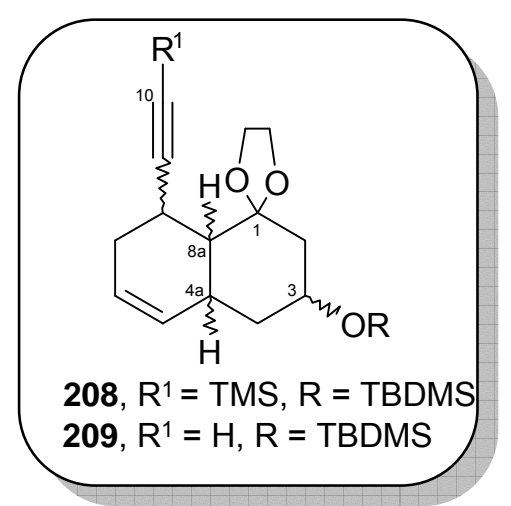
$(10 \mathrm{~mL} / \mathrm{mmol})$ zugesetzt und mit Ether extrahiert. Die vereinigten organischen Phasen wurden mit Wasser und NaCl-Lösung gewaschen, über Natriumsulfat getrocknet und das Lösungsmittel unter reduziertem Druck entfernt. Teilweise wurden die Rückstände an Kieselgel chromatographiert. Bei keinem der Versuche konnte die Titelverbindung isoliert werden. Bei Reaktionen Nr 2 und 3 wurde das Eliminierungsprodukt 210 als Hauptprodukt isoliert. Die Ergebnisse und Reaktionsbedingungen sind in Tabelle 28 zusammengefasst. $R_{\mathrm{f}}=0.24$ (Hex:EE, 10:1). $-{ }^{1} \mathrm{H}-\mathrm{NMR}\left(300 \mathrm{MHz}, \mathrm{CDCl}_{3}\right): \delta=0.12$ [s, $\left.9 \mathrm{H}, \mathrm{Si}\left(\mathrm{CH}_{3}\right)_{3}\right], 2.04$ $\left(\mathrm{ddt}, J_{\mathrm{H}-\mathrm{H}}=2.1,4.2,18.4 \mathrm{~Hz}, 1 \mathrm{H}, 7-\mathrm{H}_{\mathrm{a}}\right), 2.33\left(\mathrm{~m}_{\mathrm{c}}, 1 \mathrm{H}, 4-\mathrm{H}_{\mathrm{a}}\right), 2.37\left(\mathrm{~m}_{\mathrm{c}}\right.$, $\left.1 \mathrm{H}, 7-\mathrm{H}_{\mathrm{b}}\right), 2.63\left(\mathrm{t},{ }^{3} J_{\mathrm{H}-\mathrm{H}}=4.4 \mathrm{~Hz}, 1 \mathrm{H}, 8 \mathrm{a}-\mathrm{H}\right), 2.71\left(\mathrm{~m}_{\mathrm{c}}, 1 \mathrm{H}, 4-\mathrm{H}_{\mathrm{b}}\right), 3.25$ (dddd, $\left.J_{\mathrm{H}-\mathrm{H}}=1.4,2.5,3.5,5.9 \mathrm{~Hz}, 1 \mathrm{H}, 4 \mathrm{a}-\mathrm{H}\right), 3.52\left(\mathrm{ddd}, J_{\mathrm{H}-\mathrm{H}}=2.1,3.9\right.$, $6.3 \mathrm{~Hz}, 1 \mathrm{H}, 8-\mathrm{H}), 5.43\left(\mathrm{dd},{ }^{3} J_{\mathrm{H}-\mathrm{H}}=1.9,10.2 \mathrm{~Hz}, 6-\mathrm{H}\right), 5.64\left(\mathrm{~m}_{\mathrm{c}}, 1 \mathrm{H}, 5-\right.$ H), $5.93\left(\mathrm{ddd}, J_{\mathrm{H}-\mathrm{H}}=1.1,2.9,10.0 \mathrm{~Hz}, 1 \mathrm{H}, 2-\mathrm{H}\right), 6.76\left(\mathrm{dddd}, J_{\mathrm{H}-\mathrm{H}}=0.9\right.$, 2.5, 5.6, $10.0 \mathrm{~Hz}, 1 \mathrm{H}, 3-\mathrm{H}) \mathrm{ppm} .-{ }^{13} \mathrm{C}-\mathrm{NMR}\left(75.5 \mathrm{MHz}, \mathrm{CDCl}_{3}\right): \delta=$ $0.2\left[\mathrm{Si}\left(\mathrm{CH}_{3}\right)\right], 25.1(\mathrm{C}-4), 27.9(\mathrm{C}-8), 30.8$ (C-7), 31.5 (C-4a), 47.9 (C-

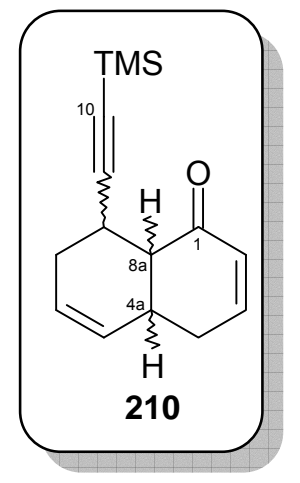
8a), 85.0 (C-10), 109.6 (C-9), 127.7 (C-5), 128.9 (C-6), 129.2 (C-2), 147.0 (C-3), 197.8 ( C-1) ppm.

8-tert-Butyl-1,4-dioxaspiro[4.5]decan (212)

4-tert-Butylcyclohexanon (211, $104 \mathrm{mg}, 674 \mathrm{mmol})$ wurde in Dichlormethan $(5 \mathrm{~mL})$ gelöst und auf $-30^{\circ} \mathrm{C}$ gekühlt. Nach Zugabe von 1,2-Bistrimethylsilanyloxyethan $(563 \mu \mathrm{L}, 418 \mathrm{mg}, 2.02 \mathrm{mmol})$

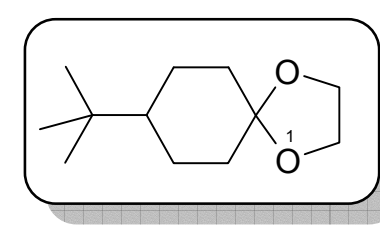
und TMSOTf $(13.0 \mu \mathrm{L}, 16.0 \mathrm{mg}, 72.0 \mu \mathrm{mol})$ wurde $2 \mathrm{~h}$ bei $-30{ }^{\circ} \mathrm{C}$ gerührt. Nach Zugabe von Pyridin $(0.5 \mathrm{~mL})$ ließ man auf RT erwärmen und setzte Ether $(50 \mathrm{~mL})$ sowie Wasser 
(10 mL) hinzu. Die Phasen wurden getrennt und die wässrige Phase mit Ether $(2 \times 25 \mathrm{~mL})$ extrahiert. Die vereinigte organische Phase wurde mit $\mathrm{NaHCO}_{3}$-Lösung, Wasser und $\mathrm{NaCl}$ Lösung gewaschen und über Natriumsulfat getrocknet. Das so erhaltene Rohprodukt (350 mg) wurde säulenchromatographisch gereinigt (Flash, Hex:EE, 20:1). Man erhielt $111 \mathrm{mg}(83 \%)$ der Titelverbindung als farblosen Feststoff. Smp.: 26-28 ${ }^{\circ} \mathrm{C}$. - Alle übrigen analytischen Daten waren konsistent mit den in der Literatur publizierten. ${ }^{[176]}$

\section{E.3.5.3 Zur allylischen Oxidation}

Versuche der Darstellung von 3-(tert-Butyldimethylsilanyloxy)-8-trimethylsilanylethinyl2,3,4,4a,8,8a-hexahydronaphthalin-1,7-dion (213) sowie 3-(tert-Butyldimethylsilanyloxy)8-ethinyl-2,3,4,4a,8,8a-hexahydronaphthalin-1,7-dion (214)

A: IMDA-Produkt 199a $(55.0 \mathrm{mg}, 146 \mu \mathrm{mol})$ wurde in Dichlormethan $(1.5 \mathrm{~mL})$ gelöst und mit $\mathrm{Pd}(\mathrm{OH})_{2}(8.1 \mathrm{mg}$, $15 \mu \mathrm{mol}, 20 \%$ Pd auf Aktivkohle) versetzt. Nach Zugabe von TBHP $(133 \mu \mathrm{L}, 730 \mu \mathrm{mol}, 5.5 \mathrm{M}$ in Decan) ließ man bei RT rühren. Nach $15 \mathrm{~h}$ wurde das Reaktionsgemisch über Kieselgel $(2.5 \mathrm{~cm})$ filtriert, der Rückstand mit Dichlormethan $(10 \mathrm{~mL})$ gewaschen und das Lösungsmittel

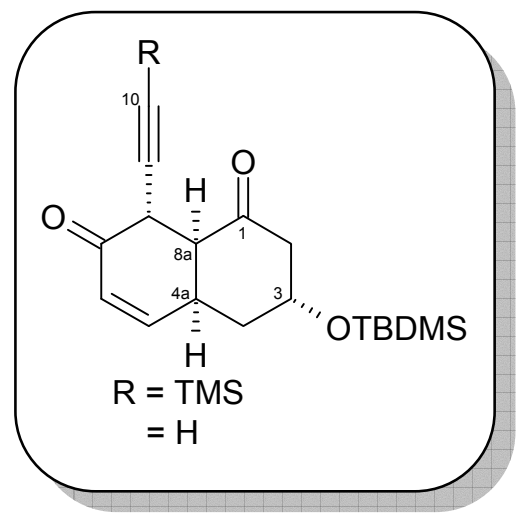
im Vakuum entfernt. Im ${ }^{1}$ H-NMR-Spektrum konnten nur Edukt und nicht identifizierbare Zersetzungsprodukte nachgewiesen werden.

B: Das TMS entschützte IMDA-Produkt $207 \mathbf{a}(43.0 \mathrm{mg}, 141 \mu \mathrm{mol})$ wurde in Dichlormethan $(1.5 \mathrm{~mL})$ gelöst und mit $\mathrm{Pd}(\mathrm{OH})_{2}(8.1 \mathrm{mg}, 15 \mu \mathrm{mol}, 20 \% \mathrm{Pd}$ auf Aktivkohle) versetzt. Nach Zugabe von TBHP (128 $\mu \mathrm{L}, 706 \mu \mathrm{mol}, 5.5 \mathrm{M}$ in Decan) ließ man bei RT rühren. Nach $15 \mathrm{~h}$ wurde das Reaktionsgemisch über Kieselgel $(2.5 \mathrm{~cm})$ filtriert, der Rückstand mit Dichlormethan $(10 \mathrm{~mL})$ gewaschen und das Lösungsmittel im Vakuum entfernt. Im ${ }^{1} \mathrm{H}-\mathrm{NMR}$-Spektrum konnten nur Edukt und nicht identifizierbare Zersetzungsprodukte nachgewiesen werden.

C: IMDA-Produkt 197a $(68.0 \mathrm{mg}, 181 \mu \mathrm{mol})$ wurde in Dichlormethan $(1.0 \mathrm{~mL})$ gelöst und mit einer Lösung von $\mathrm{CrO}_{3}(1.0 \mathrm{mg}, 0.9 \mu \mathrm{mol})$ und TBHP $(328 \mu \mathrm{L}, 1.81 \mathrm{mmol}, 5.5 \mathrm{M}$ in Decan) in Dichlormethan $(2.0 \mathrm{ml})$ versetzt. Nach $15 \mathrm{~h}$ Rühren bei RT wurde das 
Reaktionsgemisch über Kieselgel $(2.5 \mathrm{~cm})$ filtriert, der Rückstand mit Dichlormethan $(10 \mathrm{~mL})$ gewaschen und das Lösungsmittel im Vakuum entfernt. Im ${ }^{1} \mathrm{H}-\mathrm{NMR}-$ Spektrum konnten nur Edukt und nicht identifizierbare Zersetzungsprodukte nachgewiesen werden.

D: IMDA-Produkt 197a (40.0 mg, $106 \mu \mathrm{mol})$ wurde in Dichlormethan $(2.0 \mathrm{~mL})$ gelöst und mit $\mathrm{SeO}_{2}(24.0 \mathrm{mg}, 212 \mu \mathrm{mol})$ versetzt. Man erwärmte auf $180^{\circ} \mathrm{C}$. Kurz vor Erreichen der Zieltemperatur wurde die Reaktion wegen starker Dunkelfärbung der Reaktionslösung abgebrochen. Das Reaktionsgemisch wurde über Celite $(2.5 \mathrm{~cm})$ filtriert, mit DCM gewaschen und das Lösungsmittel im Vakuum entfernt. Im ${ }^{1}$ H-NMR-Spektrum konnten nur nicht identifizierbare Zersetzungsprodukte nachgewiesen werden. 


\section{F Literaturverzeichnis}

${ }^{[1]}$ D. J. Payne, M. N. Gwynn, D. J. Holmes, D. L. Pompliano, Nat. Rev. Drug Discovery 2007, 6, 29-40.

${ }^{[2]}$ S. D. Mills, Biochem. Pharm. 2006, 71, 1096-1102.

[3] J. H. Van Drie, J. Comput. Aided Mol. Des. 2007, 21, 591-601.

${ }^{[4]}$ M. Hashimoto, Y. Hatanaka, Eur. J. Org. Chem. 2008, 2513-2523.

${ }^{[5]}$ A. Singh, E. R. Thornton, F. H. Westheimer, J. Biol. Chem. 1962, PC3006-PC3008.

[6] Allgemeine Übersichten über Photoaffinitätsmarkierung: a) J. Brunner, Annu. Rev. Biochem. 1993, 62, 483-514; b) F. Kotzyba-Hibert, I. Kapfer, M. Goeldner, Angew. Chem. 1995, 107, 1391-1408; Angew. Chem. Int. Ed. Engl. 1995, 34, 1296-1312; c) S. A. Fleming, Tetrahedron 1995, 51, 12479-12520. Eher anwendungsorientierte Übersichtsartikel: d) Y. Hatanaka, Y. Sadakane, Curr. Top. Med. Chem. 2002, 2, 271288; e) T. Tomohiro, M. Hashimoto, Y. Hatanaka, Chemical Record 2005, 5, 385-395; f) Y. Sadakane, Y. Hatanaka, Analytical Sciences 2006, 22, 209-218; g) N. K. Tyagi, R. K. H. Kinne, Analytical Biochemistry 2003, 323, 74-83; h) A. Blencowe, W. Hayes, Soft Matter 2005, 1, 178-205. Zur Stabilität verschiedener photoaktivierbarer Gruppen: M. Wiegand, T. K. Lindhorst, Eur. J. Org. Chem. 2006, 4841-4851.

${ }^{[7]}$ R. E. Galardy, L. C. Craig, M. P. Printz, Nat. New. Biol. 1973, 242, 127-128.

${ }^{[8]}$ a) G. Dormán, G. D. Prestwich, Biochemistry 1994, 33, 5661-5673; b) J. J. Tate, J. Persinger, B. Bartholomew, Nucleic Acids Res. 1998, 26, 1421-1426.

[9] a) J. Zotzmann, L. Hennig, P. Welzel, D. Müller, C. Schäfer, S. Zillikens, H. Pusch, H. G. Glitsch, R. Regenthal, Tetrahedron 2000, 56, 9625-9632; b) D. Fillion, M. Deraët, B. J. Holleran, E. Escher, J. Med. Chem. 2006, 49, 2200-2209.

${ }^{[10]}$ P. L. Pedersen, E. Carafoli, Trends Biochem Sci. 1987, 12, 146-150.

${ }^{[11]}$ M. Huss, Dissertation, Universität Osnabrück 2001.

${ }^{[12]}$ Anonymus, Wikipedia, http://en.wikipedia.org/wiki/V-ATPase, 15.07.2008.

${ }^{[13]}$ T. Manabe, T. Yoshimori, N. Henomatsu, Y. Tashiro, J. Cell. Physiol. 1993, 157, 445452.

${ }^{[14]}$ P. H. Schlesinger, H. C. Blair, S. L. Teitelbaum, J. C. Edwards, J. Biol. Chem. 1997, 272, 18636-18643.

${ }^{[15]}$ S. R. Sennoune, D. Luo, R. Martinez-Zaguilan, Cell. Biochem. Biophys. 2004, 40, 185206. 
${ }^{[16]}$ K. Beyenbach, H. Wieczorek, J. Exp. Biol. 2006, 209, 577-589.

${ }^{[17]}$ a) C. Farina, S. Gagliardi, Curr. Pharm. Des. 2002, 8, 2033-2048; b) H. Izumi, T. Torigoe, H. Ishiguchi, H. Uramoto, Y. Yoshidy, M. Tanabe, T. Ise, T. Murakami, T. Yoshida, M. Nomoto, K. Kohno, Cancer Treat. Rev. 2003, 29, 541-549.

${ }^{[18]}$ K. U. Bindseil, A. Zeeck, Liebigs Ann. Chem. 1994, 305-312.

${ }^{[19]}$ G. Werner, H. Hagenmaier, H. Drautz, A. Baumgartner, H. Zähner, J. Antibiot. 1984, $37,110-117$.

${ }^{[20]}$ V. V. Teplova, A. A. Tonshin, P. A. Grigoriev, N. E. L. Saris, M. S. Salkinoja-Salonen, J. Bioenerg. Biomembr. 2007, 39, 321-329.

${ }^{[21]}$ S. M. Eswarappa, N. Basu, O. Joy, D. Chakravortty, Innate Immunity 2008, 14, 13-24.

${ }^{[22]}$ E. J. Bowman, A. Siebers, K. Altendorf, Proc. Natl. Acad. Sci. USA 1988, 85, 79727976.

${ }^{[23]}$ S. Dröse, K. U. Bindseil, E. J. Bowman, A. Siebers, A. Zeeck, K. Altendorf, Biochemistry 1993, 32, 3902-3906.

${ }^{[24]}$ J. A. Beutler, T. C. McKee, Curr. Med. Chem. 2003, 10, 787-796.

${ }^{[25]}$ S. Dröse, K. Altendorf, J. Exp. Biol. 1997, 200, 1-8.

${ }^{[26]}$ E. J. Bowman, B. J. Bowman, J. Bioenerg. Biomemb. 2005, 37, 431-435

${ }^{[27]}$ B. J. Bowman, M. E. McCall, R. Baertsch, E. J. Bowman, J. Biol. Chem. 2006, 281, 31885-31893.

${ }^{[28]}$ C. Boddien, Dissertation, Universität Göttingen, 1995.

${ }^{[29]}$ K. U. Bindseil, A. Zeeck, J. Org. Chem. 1993, 58, 5487-5492.

${ }^{[30]}$ G. Ingenhorst, K. U. Bindseil, C. Boddien, S. Dröse, M. Gaßel, K. Altendorf, A. Zeeck, Eur. J. Org. Chem. 2001, 4525-4532.

${ }^{[31]}$ M. Huss, G. Ingenhorst, S. König, M. Gaßel, S. Dröse, A. Zeeck, K. Altendorf, H. Wieczorek, J. Biol. Chem. 2002, 277, 40544-40548.

${ }^{[32]}$ E. J. Bowman, L. A. Grahams, T. H. Stevens, B. J. Bowman, J. Biol. Chem. 2004, 279, 33131-33138.

${ }^{[33]}$ S. Dröse, C. Boddien, M. Gassel, G. Ingenhorst, A. Zeeck, K. Altendorf, Biochemistry 2001, 40, 2816-2825.

${ }^{[34]}$ T. Schuhmann, Dissertation, Universität Göttingen, 2005.

${ }^{[35]}$ H.-J. Schiewe, Dissertation, Universität Göttingen, 1997.

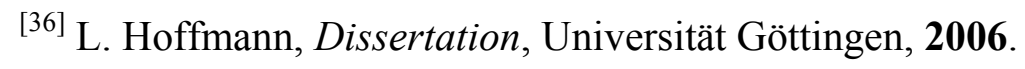


[37] P. v. Zezschwitz, S. Grond, DFG-Forschungsantrag im Rahmen des SFB 416, Universität Göttingen 2006.

${ }^{[38]}$ H. Shojaei, Dissertation, Universität Göttingen, 2007.

${ }^{[39]}$ a) R. A. G. Smith, J. R. Knowles, J. Am. Chem. Soc. 1973, 95, 5072-5073; b) R. A. G. Smith, J. R. Knowles, J. Chem. Soc. 1975, 2, 686-694.

${ }^{[40]}$ J. Brunner, H. Senn, F. M. Richards, J. Biol. Chem. 1980, 255, 3313-3318.

${ }^{[41]}$ T. Weber, J. Brunner, J. Am. Chem. Soc. 1995, 117, 3084-3095.

${ }^{[42]}$ Y. Ambroise, F. Pillon, C. Mioskowski, A. Valleix, B. Rousseau, Eur. J. Org. Chem. 2001, 3961-3964.

${ }^{[43]}$ Y. Hatanaka, M. Hashimoto, H. Kurihara, H. Nakayama, Y. Kanaoka, J. Org. Chem. 1994, 59, 383-387.

${ }^{[44]}$ K. Altendorf, M. Gassel, W. Puppe, T. Möllenkamp, A. Zeeck, C. Boddien, K. Fendler, E. Bamberg, S. Dröse, Acta Physiol. Scand. 1998, 643, 137-146.

${ }^{[45]}{ }^{14} \mathrm{CH}_{3} \mathrm{I}$ (50 mCi) ist etwa 40 -mal teurer als $\mathrm{Ba}^{14} \mathrm{CO}_{3}$ gemäß den Katalogpreisen des hiesigen Lieferantens für radioaktive Chemikalien.

${ }^{[46]}$ a) M. Nassal, Liebigs Ann. Chem. 1983, 1510-1523; b) Y. Hatanaka, H. Nakayama, Y. Kanaoka, Heterocycles 1993, 35, 997-1004.

${ }^{[47]}$ H. Nakashima, M. Hashimoto, Y. Sadakane, T. Tomohiro, Y. Hatanaka, J. Am. Chem. Soc. 2006, 128, 15092-15093.

${ }^{[48]}$ X. Creary, J. Org. Chem. 1987, 52, 5026-5030.

${ }^{[49]}$ A. A. Kogon, D. E. Bochkariov, B. P. Baskunov, A. V. Cheprakov, Liebigs Ann. Chem. 1992, 879-881.

${ }^{[50]}$ J. E. Baldwin, C. D. Jesudason, M. G. Moloney, D. R. Morgan, A. J. Pratt, Tetrahedron 1991, 47, 5603-5614.

${ }^{[51]}$ R. E. Sammelson, J. E. Casida, J. Org. Chem. 2003, 68, 8075-8079.

${ }^{[52]}$ a) R. D. Rieke, S. E. Bales, J. Am. Chem. Soc. 1974, 96, 1775-1781; b) R. D. Rieke, M. V. Hanson, Tetrahedron 1997, 53, 1925-1956.

${ }^{[53]}$ P. Knochel, W. Dohle, N. Gommermann, F. F. Kneisel, F. Kopp, T. Korn, I. Sapountzis, V. Anh Vu, Angew. Chem. 2003, 115, 4438-4456; Angew. Chem. Int. Ed. 2003, 42, 4302-4320.

${ }^{[54]}$ M. Schlosser, D. Coffinet, Synthesis 1971, 380-381.

${ }^{[55]}$ D. H. R. Barton, J. C. Jaszberenyi, E. A. Theodorakis, J. Reibenspies, J. Am. Chem. Soc. 1993, $115,8050-8059$. 
${ }^{[56]}$ A. Rühmann, C. Wentrup, Tetrahedron 1994, 50, 3785-3798.

[57] J. Hassfeld, C. Fare`s, H. Steinmetz, T. Carlomagno, D. Menche, Org. Lett. 2007, 8, $4751-4754$.

${ }^{[58]}$ S. Rudolf, T. Bender, D. Menche, S. Grond, P. von Zezschwitz, unveröffentlichte Ergebnisse, 2007.

${ }^{[59]}$ J. Terpinski, D. Z. Denney, R. Beveridge, D. P. Cox, R. A. Moss, Magnetic Resonance in Chemistry 1987, 25, 923-927.

${ }^{[60]}$ a) H. B. Bode, A. Zeeck, J. Chem. Soc. Perkin Trans. 1 2000, 323-328; b) H. B. Bode, A. Zeeck, J. Chem. Soc. Perkin Trans. 1 2000, 2665-2670.

${ }^{[61]}$ M. Huss, S. Grond, unveröffentlichte Ergebnisse, 2006.

${ }^{[62]}$ H. B. Bode, Dissertation, Göttingen, 2000.

${ }^{[63]}$ M. Mure, S. X. Wang, J. P. Klinman, J. Am. Chem. Soc. 2003, 125, 6113-6125.

${ }^{[64]}$ R. Jansen, B. Kunze, H. Reichenbach, G. Höfle, Eur. J. Org. Chem. 2000, 913-919.

${ }^{[65]}$ L. Yet, Chem. Rev 2003, 103, 4286-4306.

${ }^{[66]}$ M. Huss, F. Sasse, B. Kunze, R. Jansen, H. Steinmetz, G. Ingenhorst, A. Zeeck, H. Wieczorek, BMC Biochemistry, 2005, 6, 13-23.

${ }^{[67]}$ a) R. Hoffmann, R. B. Woodward, J. Am. Chem. Soc. 1965, 87, 2046-2048; b) A. G. Leach, E. Goldstein, K. N. Houk, J. Am. Chem. Soc. 2003, 125, 8330-8339.

${ }^{[68]}$ a) K. N. Houk, R. B. Woodward, J. Am. Chem. Soc. 1970, 92, 4143-4145; b) K. N. Houk, R. B. Woodward, J. Am. Chem. Soc. 1970, 92, 4145-4147; c) N. S. Bhacca, L. J. Luskus, K. N. Houk, Chem. Commun. 1971, 109-111.

${ }^{[69]}$ A. M. Moiseev, E. S. Balenkova, V. G. Nenajdenko, Russ. Chem. Bul., International Edition 2006, 55, 141-146.

${ }^{[70]}$ J. H. Rigby, K. R. Fales, Tetrahedron Lett. 1998, 39, 5717-5720.

${ }^{[71]}$ So zum Beispiel: a) A. Textor, I. Papastavrou, J. Siewert, J. Magull, A. Kulik, H.-P. Fiedler, P. von Zezschwitz, S. Grond, Chem. Eur. J. 2007, 13, 7416-7423; b) H. Oikawa, Y. Suzuki, K. Katayama, A. Naya, C. Sakano, A. Ichihara, J. Chem. Soc. Perkin Trans. 1 1999, 1225-1232.

${ }^{[72]}$ a) S. Marti, J. Andrés, V. Moliner, E. Silla, I. Tunon, J. Bertrán, J. Am. Chem. Soc. 2004, 126, 311-319; b) A. Aemissegger, B. Jaun, D. Hilvert, J. Org. Chem. 2002, 67, 6725-6730. 
${ }^{[73]}$ a) H. S. Sutherland, F. E. S. Souza, R. G. A. Rodrigo, J. Org. Chem. 2001, 66, 36393641; b) R. Carlini, K. Higgs, C. Older, S. Randhawa, R. G. A. Rodrigo, J. Org. Chem. 1997, 62, 2330-2331.

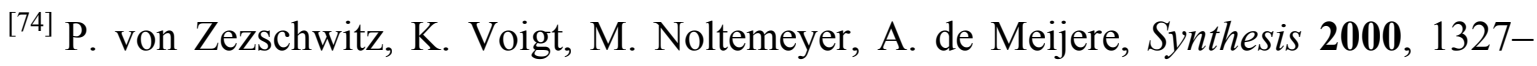
1340.

${ }^{[75]}$ O. Diels, K. Alder, Justus Liebigs Ann. Chem. 1928, 460, 98-122.

${ }^{[76]}$ a) K. C. Nicolaou, S. A. Snyder, T. Montagnon, G. Vassilikogiannakis, Angew. Chem. 2002, 114, 1742-1773; Angew. Chem. Int. Ed. 2002, 41, 1668-1698; b) K. Takao, R. Munakata, K. Tadano, Chem. Rev. 2005, 105, 4779-4807.

${ }^{[77]}$ a) M. N. Paddon-Row, D. Moran, G. A. Jones, M. S. Sherburn, J. Org. Chem. 2005, 70, 10841-10853; b) M. N. Paddon-Row, M. S. Sherburn, Chem. Commun. 2000, 22152216; c) L. Raimondi, F. K. Brown, J. Gonzalez, K. N. Houk, J. Am. Chem. Soc. 1992, $114,4796-4806$.

${ }^{[78]}$ E. L. Pearson, L. C. H. Kwan, C. I. Turner, G. A. Jones, A. C. Willis, M. N. PaddonRow, M. S. Sherburn, J. Org. Chem. 2006, 71, 6099-6109.

${ }^{[79]}$ Y. Araki, T. Konoike, J. Org. Chem. 1997, 62, 5299-5309.

${ }^{[80]}$ J. W. Coe, W. R. Roush, J. Org. Chem. 1989, 54, 915-930.

${ }^{[81]}$ T. A. Dineen, W. R. Roush, Org. Lett. 2005, 7, 1355-1358.

${ }^{[82]}$ B. Frey, J. Schnaubelt, H. U. Reißig, Eur. J. Org. Chem. 1999, 1377-1384.

${ }^{[83]}$ B. Frey, J. Schnaubelt, H. U. Reißig, Eur. J. Org. Chem. 1999, 1385-1393.

${ }^{\left[{ }^{84]}\right.}$ L. Raimondi, F. K. Brown, J. Gonzalez, K. N. Houk, J. Am. Chem. Soc. 1992, 114, 4796-4804.

${ }^{[85]}$ O. Mitsunobu, Bull. Chem. Soc. Jpn. 1967, 40, 4235-4238.

${ }^{[86]}$ Übersichten: a) L. A. Paquette, Tetrahedron 1997, 53, 13971-14020; b) K. Duraiaj, Current Science 1994, 66, 917-922; c) L. A. Paquette, Angew. Chem. 1990, 102, 642660; Angew. Chem. Int. Ed. 1990, 29, 609-626.

${ }^{[87]}$ M. Urion, Ann. Chim. (Paris) 1934, 1, 5.

${ }^{[88]}$ D. A. Evans, A. M. Golob, J. Am. Chem. Soc. 1975, 97, 4765-4766.

${ }^{[89]}$ a) S. Swaminathan, J. P. John, S. Ramachandran, Tetrahedron Lett. 1962, 3, 729-734; b)

R. Uma, K. Rajagopalan, S. Swaminathan, Tetrahedron 1986, 42, 2757-2769.

${ }^{\left[{ }^{90]}\right.}$ H. Y. Yoo, K. N. Houk, J. Am. Chem. Soc. 1998, 120, 205-206.

${ }^{[91]}$ A. Viola, E. J. Iorio, J. Org. Chem. 1970, 35, 856-857. 
${ }^{[92]}$ P. von Zezschwitz, K. Voigt, M. Noltemeyer, A. de Meijere, Synthesis 2000, 13271340.

${ }^{[93]}$ D. L. J. Clive, C. G. Russell, S. C. Curi, J. Org. Chem. 1982, 47, 1632-1641.

${ }^{[94]}$ B. White, M. L. Snapper, J. Am. Chem. Soc. 2003, 125, 14901-14904.

${ }^{[95]}$ E. Zeiler, Diplomarbeit, Göttingen, 2008.

${ }^{[96]}$ a) N. Okukado, E. Negishi, Tetrahedron Lett. 1978, 19, 2357-2360; b) E. Negishi, L. F. Valente, M. Kobayashi, J. Am. Chem. Soc. 1980, 102, 3298-3299.

${ }^{\left[{ }^{97]}\right.}$ M. Kobayashi, L. F. Valente, E. Negishi, W. Patterson, A. Silveira Jr, Synthesis 1980, 1034-1035.

${ }^{[98]}$ P. Wipf, S. Lim, Angew. Chem. 1993, 105, 1095-1097; Angew. Chem. Int. Ed. 1993, 32, 1068-1071.

${ }^{[99]}$ W. C. Still, J. Am. Chem. Soc. 1977, 99, 4186-4187.

${ }^{[100]}$ S. L. Schreiber, R. C. Hawley, Tetrahedron Lett. 1985, 26, 5971-5974.

${ }^{[101]}$ M. T. Reetz, B. Brunner, T. Schneider, F. Schulz, C. M. Clouthier, M. M. Kayser, Angew. Chem. 2004, 116, 4167-4170; Angew. Chem. Int. Ed. 2004, 43, 4075-4078.

${ }^{[102]}$ R. Göttlich, K. Yamakoshi, H. Sasai, M. Shibasaki, Synlett 1997, 971-973.

${ }^{[103]}$ J. Clayden, N. Greeves, S. Warren, P. Wothers, Organic Chemistry, Oxford University Press, Oxford New York, 2007.

${ }^{[104]}$ C. L. Sann, D. M. Munoz, N. Saunders, T. J. Simpson, D. I. Smith, F. Soulas, P. Watts, C. L. Willis, Org. Biomol. Chem. 2005, 3, 1719-1728.

${ }^{[105]}$ Y. Nagao, W. M. Dai, M. Ochiai, M. Shiro, J. Org. Chem. 1989, 54, 5211-5217.

${ }^{[106]}$ D. A. Evans, S. L. Bender, J. Morris, J. Am. Chem. Soc. 1988, 110, 2506-2526.

${ }^{[107]}$ a) J. Parikh, W. Doering, J. Am. Chem. Soc. 1967, 89, 5505-5507; b) S. Dei, C. Bellucci, M. Buccioni, M. Ferraroni, F. Gualtieri, L. Guandalini, D. Manetti, R. Matucci, M. N. Romanelli, S. Scapecchia, E. Teodoria, Bioorg. Med. Chem. 2003, 11, 3153-3164.

${ }^{[108]}$ Y. Ishii, C. G. Wen-Xiang Xu, M. Iwasaki, M. Hidai, J. Org. Chem. 1993, 58, 68186825.

${ }^{[109]}$ a) Pollex, A. Millet, J. Müller, M. Hiersemann, L. Abraham, J. Org. Chem. 2005, 70, 5579-5591; b) J. A. Lafontaine, D. P. Provencal, C. Gardelli, J. W. Leahy, J. Org.

Chem. 2003, 68, 4215-4234.

${ }^{[110]}$ E. Buchta, F. Andree, Chem. Ber. 1959, 92, 3111-3116.

${ }^{[111]}$ J. Sandri, J. Viala, Synthesis 1995, 3, 271-275. 
${ }^{[112]}$ J. D. Surmatis, A. Walser, J. Gibas, U. Schwieter, R. Thommen, Helv. Chim. Acta 1970, 53, 974-990.

${ }^{[113]}$ E. N. Chauvel, P. Ceric, C. Llorens-Cort, S. Wilk, B. P. Roques, M. C. Fournie-Zaluski, J. Med. Chem. 1994, 37, 1339-1346.

${ }^{[114]}$ N. Okamoto, M. Sasaki, M. Kawahata, K. Yamaguchi, K. Takeda, Org. Lett. 2006, 8, 1889-1891.

${ }^{[115]}$ P. A. Wender, S. G. Hegde, R. D. Hubbard, L. Zhang, J. Am. Chem. Soc. 2002, 124, 4956-4957.

${ }^{[116]}$ Enantioselektive katalytische Reduktion von $\beta$-Ketoestern: a) J. P. Genet, C. Pinel, V. Ratovelomanana-Vidals, S. Mallart, X. Pfisters, L. Bischoff, M.C. Cano De Andrades, S. Darses, C. Galopin, J. A. Laffitte, Tetrahedron Asymmetry 1994, 5, 675-690; b) R. ter Halle, B. Colasson, E. Schulz, M. Spagnol, Marc Lemaire, Tetrahedron Lett. 2000, $41,643-646$.

${ }^{[117]}$ R. O. Duthaler, Helv. Chim. Acta 1983, 66, 1484-1485.

${ }^{[118]}$ A. Svendsen, P. M. Boll, Tetrahedron 1973, 29, 4251-4258.

${ }^{[119]}$ M. S. Kharasch, E. Sternfeld, F. R. Mayo, J. Am. Chem. Soc. 1937, 59, 1655-1657.

${ }^{[120]}$ C. Yuan, K. Wang, J. Li, Z. Li, Heteroatom Chemistry 2002, 13, 153-156.

${ }^{[121]}$ J. F. Lavallée, C. Spino, R. Ruel, K. T. Hogan, P. Deslongchamps, Can. J. Chem. 1992, 70, 1406-1418.

${ }^{[122]}$ F. F. Fleming, J. Guo, Q. Wang, D. Weaver, J. Org. Chem. 1999, 64, 8568-8575.

${ }^{[123]}$ a) F. P. Marmsäter, J. A. Vanecko, F. G. West, Org. Lett. 2004, 6, 1657-1660; b) E. A. Bercot, T. Rovis, J. Am. Chem. Soc. 2005, 127, 247-254.

${ }^{[124]}$ H. P. Wessel, T. Iversen, D. R. Bundle, J. Chem. Soc. Perkin Trans. 1 1985, 22472250.

${ }^{[125]}$ A. V. Demchenko, M. A. Wolfert, B. Santhanam, J. N. Moore, G. J. Boons, J. Am. Chem. Soc. 2003, 125, 6103-6112.

${ }^{[126]}$ T. Bender, Diplomarbeit, Universität Göttingen, 2005.

${ }^{[127]}$ a) F. Colobert, R. Des Mazery, G. Solladié, M. C. Carreno, Org. Lett. 2002, 4, $1723-$ 1725 ; b) Y. Y. Yang, W. D. Meng, F. L. Qing, Org. Lett. 2004, 6, 4257-4259; c) B. M. Trost, M. R. Machacek, B. D. Faulk, J. Am. Chem. Soc. 2006, 128, 6745-6754.

${ }^{[128]}$ A. Wada, N. Fujioka, M. Ito, Chem. Pharm. Bull. 1999, 47, 171-176.

${ }^{[129]}$ E. Negishi, A. Alimardanov, C. Xu, Org. Lett. 2000, 2, 65-67. 
${ }^{[130]}$ a) F. Zeng, E. Negishi, Org. Lett 2001, 3, 719-722; b) Z. Huang, E. Negishi, Org. Lett. 2006, 8, 3675-3678.

${ }^{[131]}$ T. Kruck, K. Baur, Angew. Chem. 1965, 77, 505-506.

${ }^{[132]}$ C. R. Cambie, D. Chambers, P. S. Rutledge, P. D. Woodgate, J. Chem. Soc. Perkin Trans 1 1981, 40-51.

${ }^{[133]}$ J. Schuppan, H. Wehlan, S. Keiper, U. Koert, Chem. Eur. J. 2006, 12, 7364-7377.

${ }^{[134]}$ P. A. Clarke, G. A. Gabriele, A. P. Cridland, A. A. Gill, Tetrahedron 2007, 63, 9124 9128.

${ }^{[135]}$ M. Sodeoka, H. Yamada, M. Shibasaki, J. Am. Chem. Soc. 1990, 112, 4906-4911. ${ }^{[136]}$ A. Arai, I. Ichikizaki, Bull. Chem. Soc. 1961, 34, 1571-1583.

${ }^{[137]}$ T. Tashiro, M. Bando, K. Mori, Synthesis 2000, 1852-1862. ${ }^{[138]}$ a) I. Abrunhosa-Thomas, O. Roy, M. Barra, T. Besset, P. Chalard, Y. Troin, Synlett 2007, 10, 1613-1615; b) F. A. Davis, M. B. Nolt, Y. Wu, K. R. Prasad, D. Li, B. Yang, K. Bowen, S. H. Lee, J. H. Eardley, J. Org. Chem. 2005, 70, 2184-2190; c) A. Gomtsyan, Org. Lett. 2000, 2, 11-14.

${ }^{[139]}$ P. A. Grieco, K. Yoshida, Z. He, Tetrahedron Lett. 1984, 25, 5715-5718. ${ }^{[140]}$ P. Wu, T. Chung, Y. Chou, J. Org. Chem. 2001, 66, 6585-6594.

${ }^{[141]}$ Y. Ikeda, J. Ukai, N. Ikeda, H. Yamamoto, Tetrahedron 1987, 43, 743-753.

${ }^{[142]}$ J. D. White, R. W. Skeean, G. L. Trammell, J. Org. Chem. 1985, 50, 1939-1948.

${ }^{[143]}$ K. Takasu, H. Ohsato, J. Kuroyanagi, M. Ihara, J. Org. Chem. 2002, 67, 6001-6007.

${ }^{[144]}$ T. Okuyama, T. Fueno, J. Furukawa, Tetrahedron 1969, 25, 5409-5414.

${ }^{[145]}$ S. J. Rhoads, J. K. Chattopadhyay, E. E. Waali, J. Org. Chem. 1970, 35, 3352-3358.

${ }^{[146]}$ T. Rosen, M. Watanabe, C. H. Heathcock, J. Org. Chem. 1984, 49, 3657-3659.

${ }^{[147]}$ D. J. Kopecky, S. D. Rychnovsky, J. Am. Chem. Soc. 2001, 123, 8420-8421.

${ }^{[148]}$ S. E. Denmark, N. G. Almstead, J. Am. Chem. Soc. 1993, 115, 3133-3139.

${ }^{[149]}$ a) D. K. Singh, J. B. Springer, P. A. Goodson, R. C. Corcoran, J. Org. Chem. 1996, 61, 1436-1442; b) J. B. Springer, R. C. Corcoran, J. Org. Chem. 1996, 61, 1443-1448.

${ }^{[150]}$ R. C. Corcoran, J. Ma, J. Am. Chem. Soc. 1991, 113, 8973-8975.

${ }^{[151]}$ H. W. Thompson, D. J. Long, J. Org. Chem. 1988, 53, 4201-4209.

${ }^{[152]}$ a) E. C. Angell, F. Fringuelli, T. D. J. Halls, F. Pizzo, B. Porter, A. Taticchi, A. P. Tourris, E. Wenkert, J. Org. Chem. 1985, 50, 4686-4690; b) E. C. Angell, F. Fringuelli, T. D. J. Halls, F. Pizzo, B. Porter, A. Taticchi, A. P. Tourris, E. Wenkert, J. Org. Chem. 1985, 50, 4691-4696. 
${ }^{[153]}$ J. L. Gras, M. Bertrand, Tetrahedron Lett. 1979, 47, 4549-4552.

${ }^{[154]}$ B. M. Trost, Z. T. Ball, J. Am. Chem. Soc. 2005, 127, 17644-17655.

${ }^{[155]}$ M. A. Schexnayder, P. S. Engel, J. Am. Chem. Soc. 1975, 97, 4825-4836.

${ }^{[156]}$ A. Pollex, A. Millet, J. Müller, M. Hiersemann, L. Abraham, J. Org. Chem. 2005, 70, 5579-5591.

${ }^{[157]}$ a) J. Siewert, Dissertation, Göttingen 2007; b) W. G. Dauben, J. M. Gerdes, G. C. Look, J. Org. Chem. 1986, 51, 4964-4970.

${ }^{[158]}$ J. J. Wolff, G. Frenking, K. Harms, Chemische Berichte 1991, 124, 551-561.

${ }^{[159]}$ H. Hagiwara, T. Akama, A. Okano, H. Uda, J. Chem. Soc. Perkin Trans. 1 1993, 2173-2184.

[160] a) J. Yu, E. J. Corey, J. Am. Chem. Soc. 2003, 125, 3232-3233; b) J. Beerlink, Dissertation, Göttingen, 2008.

${ }^{[161]}$ T. Nagai, K. Ogawa, M. Morita, M. Koyama, A. Ando, T. Miki, I. Kumadaki, Chem. Pharm. Bull. 1989, 37, 1751-1754.

${ }^{[162]}$ A. Kumar, D. C. Dittmer, J. Org. Chem. 1994, 59, 4760-4764.

${ }^{[163]}$ Y. Vo-Quang, D. Carniato, L. Vo-Quang, F. Le Goffic, Chem. Commun. 1983, 15051506.

${ }^{[164]}$ A. Flohr, A. Aemissegger, D. Hilvert, J. Org. Chem. 1999, 42, 2633-2640.

${ }^{[165]}$ W. C. Still, M. Kahn, A. Mitra, J. Org. Chem. 1978, 43, 2923-2925.

${ }^{[166]}$ E. Merck, Anfärbereagenzien für Dünnschicht- und Papier-Chromatographie, E. Merck, Darmstadt, 1984.

${ }^{[167]}$ Wiss. Leiter: Prof. Dr. Götz Eckold, Institut für Physikalische Chemie, Tammannstr. 6, 37077 Göttingen.

${ }^{168}$ Autorenkollektiv, Organikum, 20. Aufl., Johann Ambrosius Barth Verlag, Heidelberg, Leipzig, 1996.

${ }^{[169]}$ D. E. Evans, A. E. Weber, J. Am. Chem. Soc. 1986, 108, 6757-6761.

${ }^{[170]}$ Y. Hatanaka, M. Hashimoto, H. Kurihara, H. Nakayama, Y. Kanaoka, J. Org. Chem. 1994, 59, 383-387.

${ }^{[171]}$ a) D. D. Perrin, W. L. F. Armarego, Purification of Laboratory Chemicals, Pergamon Press, New York, 1987; b) K. Sato, S. Mizuno, M. Hirayama, J. Org. Chem. 1967, 32 , 177-180; c) L. F. Tietze, T. Eicher, Reaktionen und Synthesen, 2. Aufl., Georg Thieme Verlag, Stuttgart, New York, 1991.

${ }^{[172]}$ E. Tayama, W. Isaka, Org. Lett. 2006, 8, 5437-5439. 
${ }^{[173]}$ A. Chattopadhyay, B. Dhotare, S. Hassarajani, J. Org. Chem. 1999, 64, 6874-6878.

${ }^{[174]}$ C. Yuan, K. Wang, J. Li, Z. Li, Heteroatom Chemistry 2002, 13, 153-156.

${ }^{[175]} \mathrm{NpCr}(\mathrm{CO})_{3}$ : M. Uemura, T. Minami, K. Hirotsu, Y. Hayashi, J. Org. Chem. 1989, 54, 469-477.

${ }^{[176]}$ D. S. Torok, J .J. Figueroa, J. W. Scott, J. Org. Chem. 1993, 58, 7274-7276. 
1. ${ }^{1} \mathrm{H}-\mathrm{NMR}$ (300 MHz) von 1-(4-Bromphenyl)-2,2,2-trifluorethanon-oxim (56)

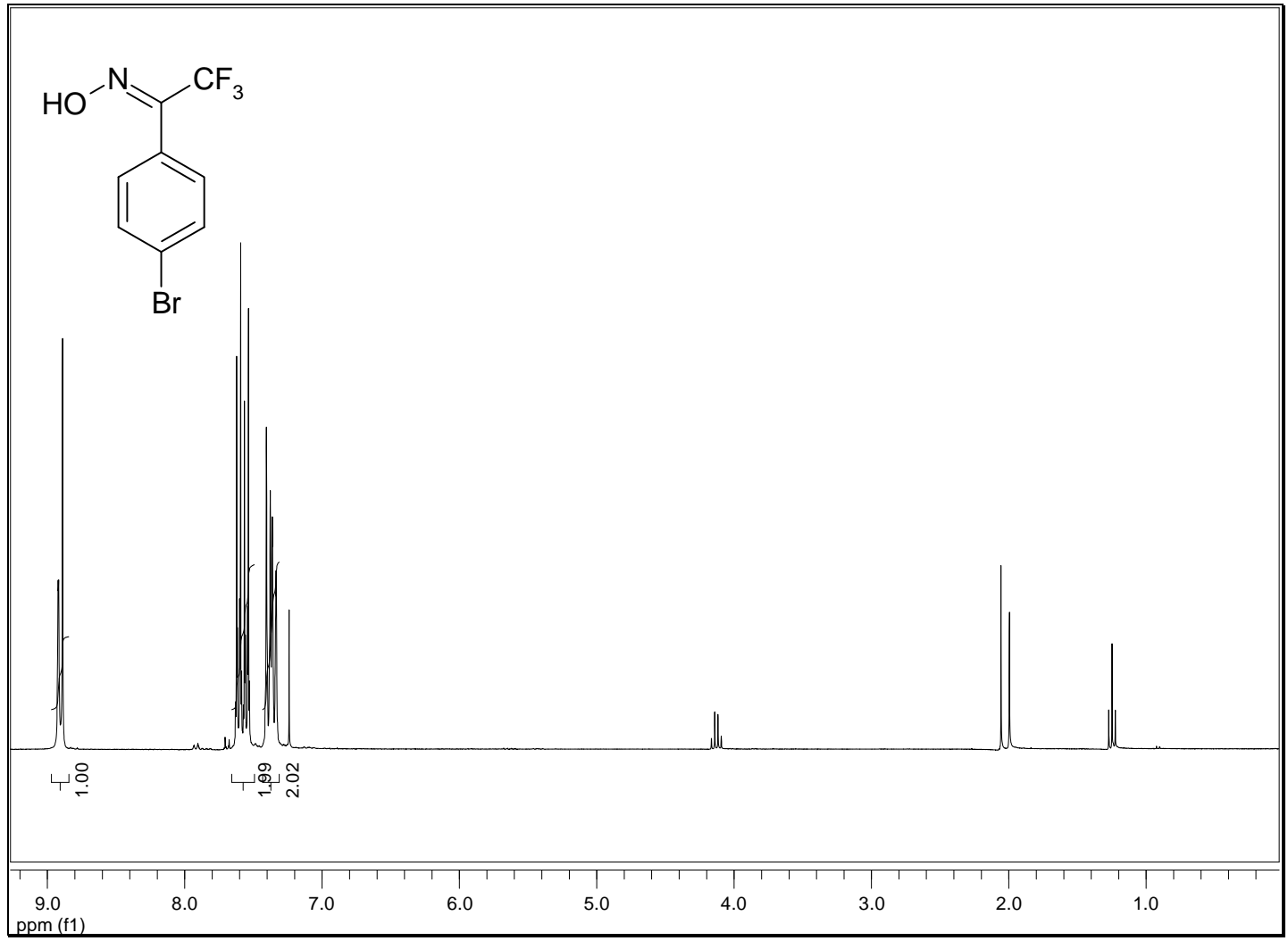

2. ${ }^{1} \mathrm{H}-\mathrm{NMR}$ (300 MHz) von 1-(4-Bromphenyl)-2,2,2-trifluorethanon-O-(ptolylsulfonyl)oxim (57)

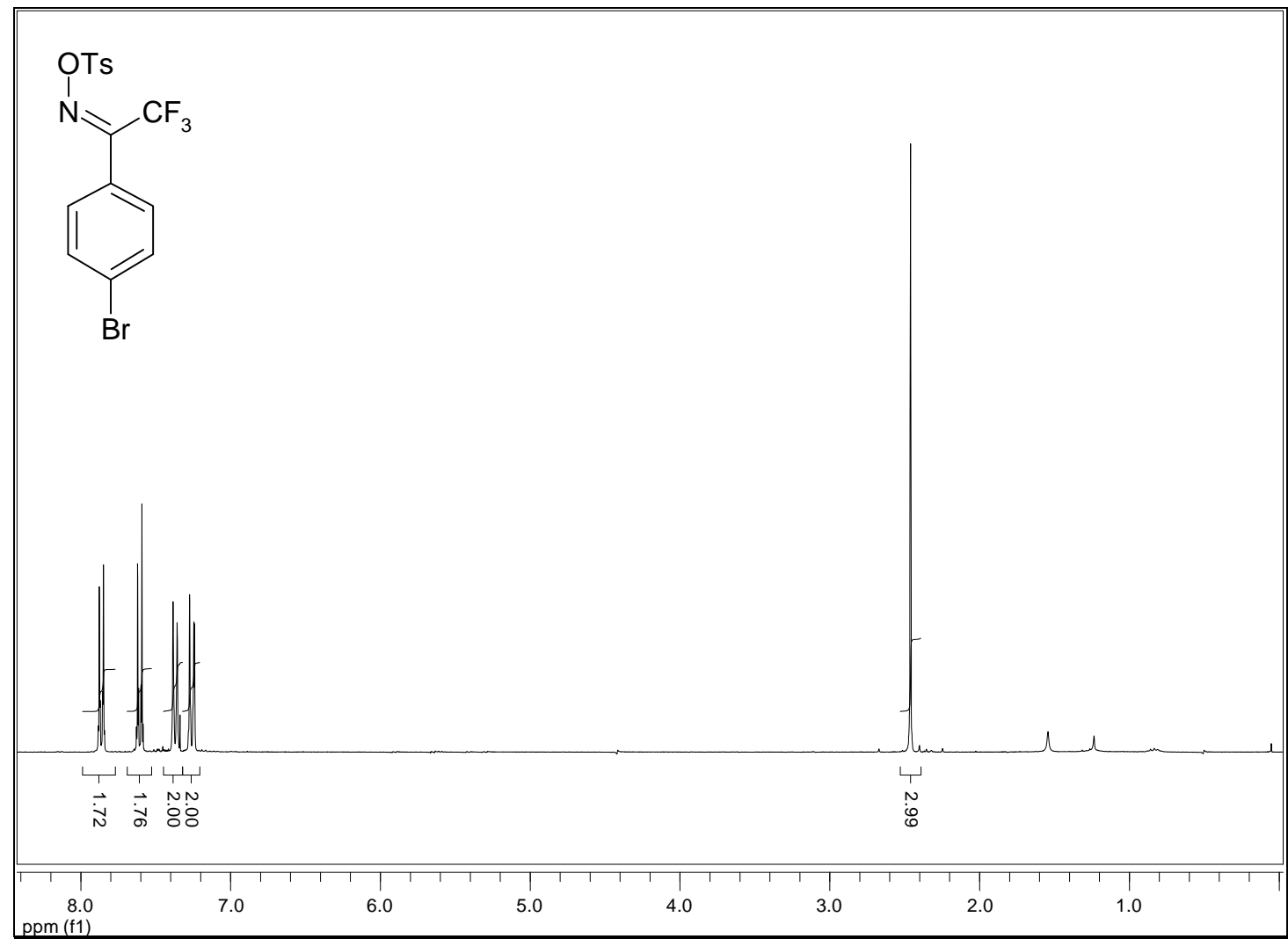


3. ${ }^{1}$ H-NMR (300 MHz) von 3-(4-Bromphenyl)-3-trifluormethyldiaziridin (58)

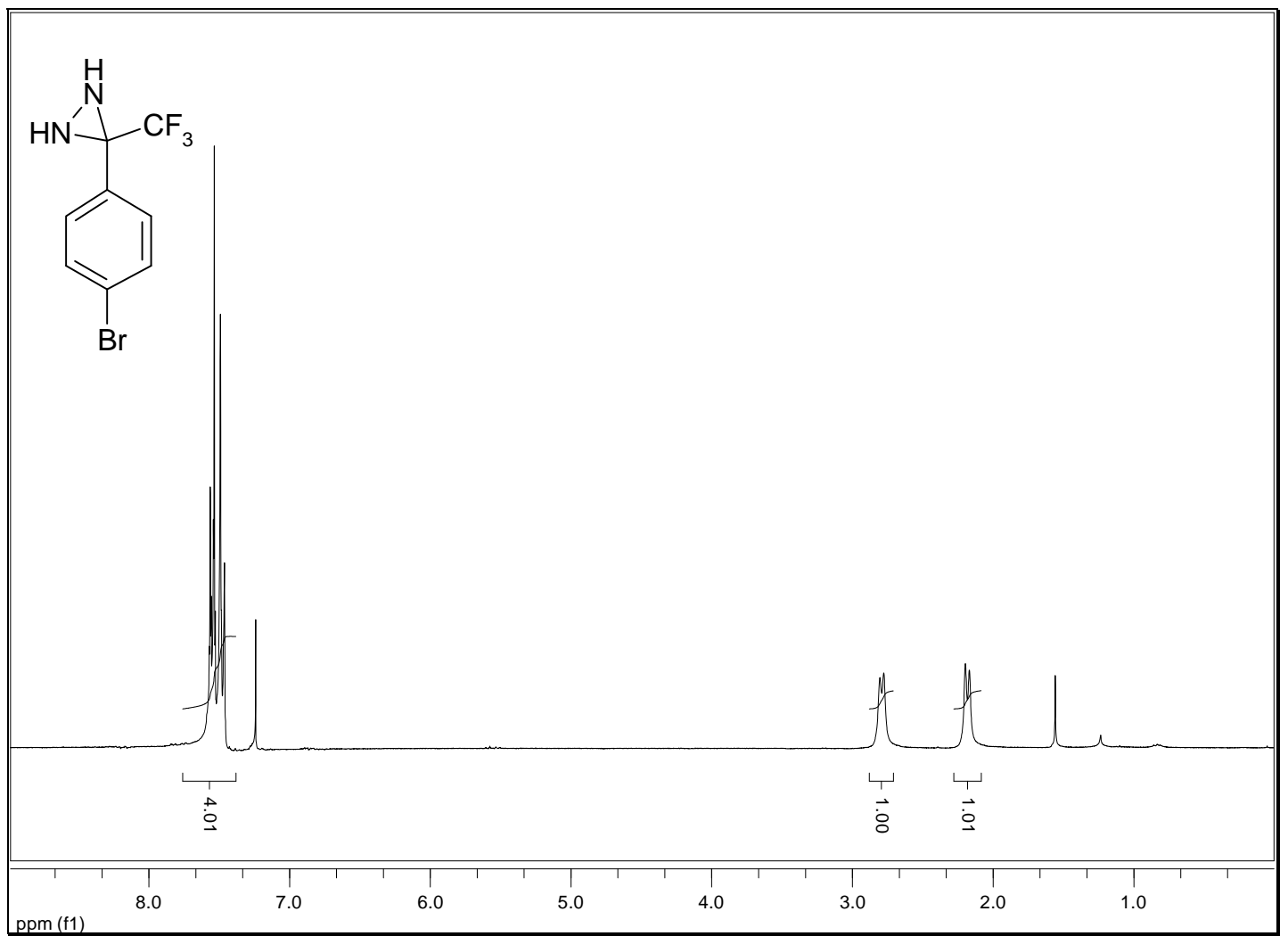

4. ${ }^{1} \mathrm{H}-\mathrm{NMR}$ (300 MHz) von 3-(4-Bromphenyl)-3-trifluormethyl-3H-diazirin (59)

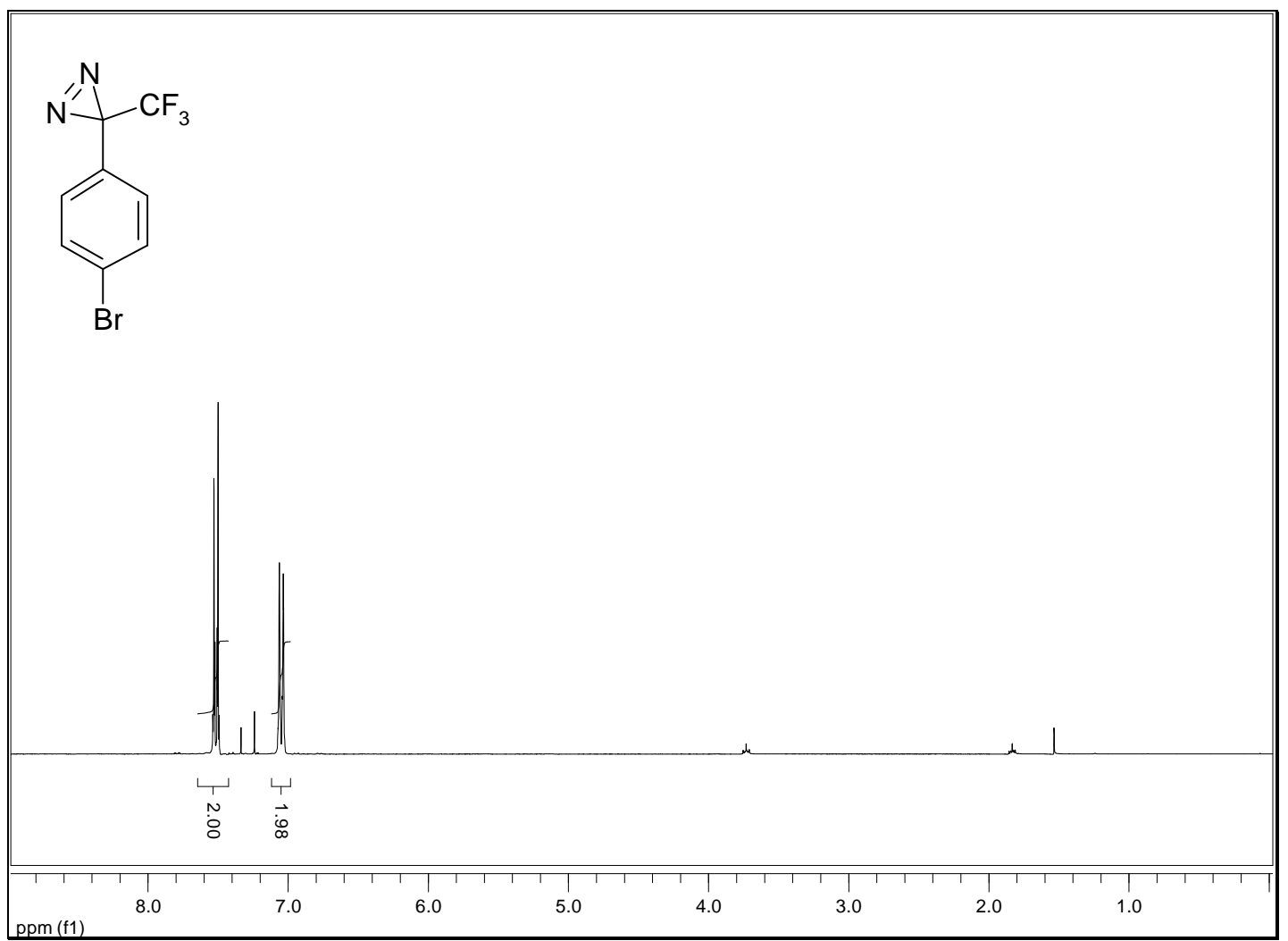


5. ${ }^{1} \mathrm{H}-\mathrm{NMR}$ (250 MHz) von 3-(4-Bromphenyl)-3-trifluormethyl-1,2-bistrimethylsilyldiaziridin (64)

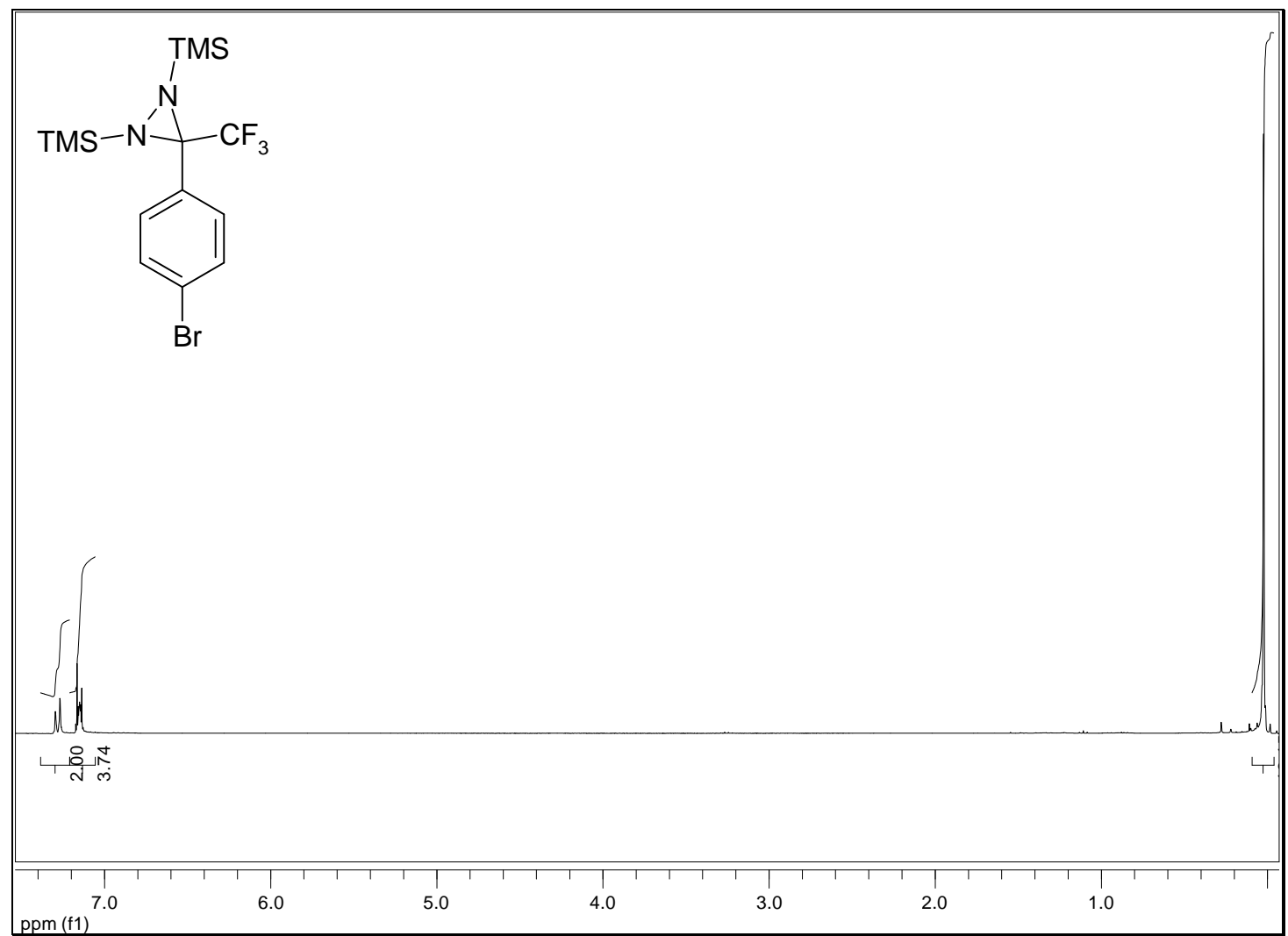

6. ${ }^{1} \mathrm{H}$ NMR (300 MHz) von 3-(4-Hydroxymethylphenyl)-3-trifluormethyldiaziridin (68)

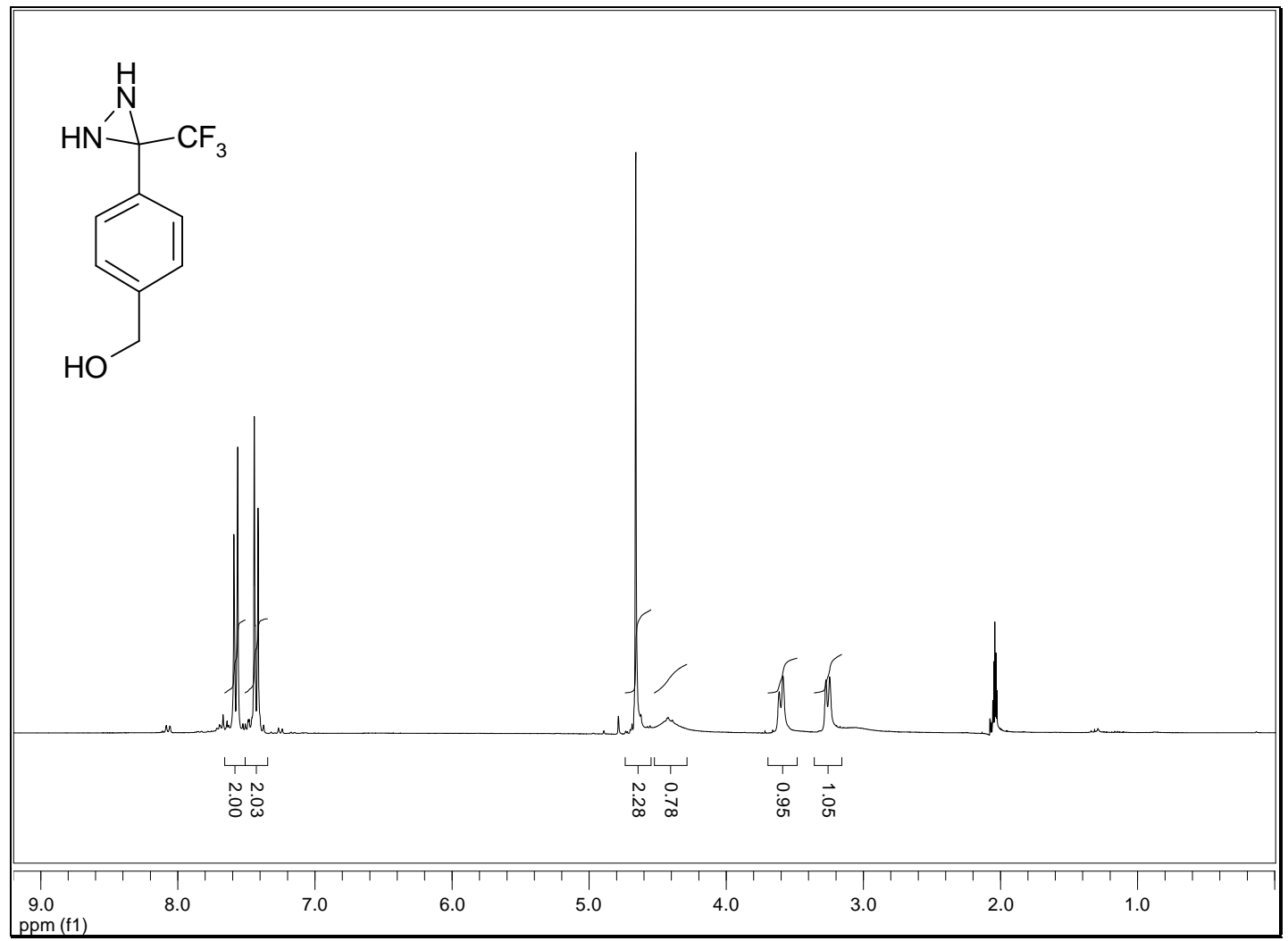


7. ${ }^{1}$ H-NMR (300 MHz) von 3-[4-(2-Hydroxyprop-2-yl)phenyl]-3-trifluormethyldiaziridin (69)

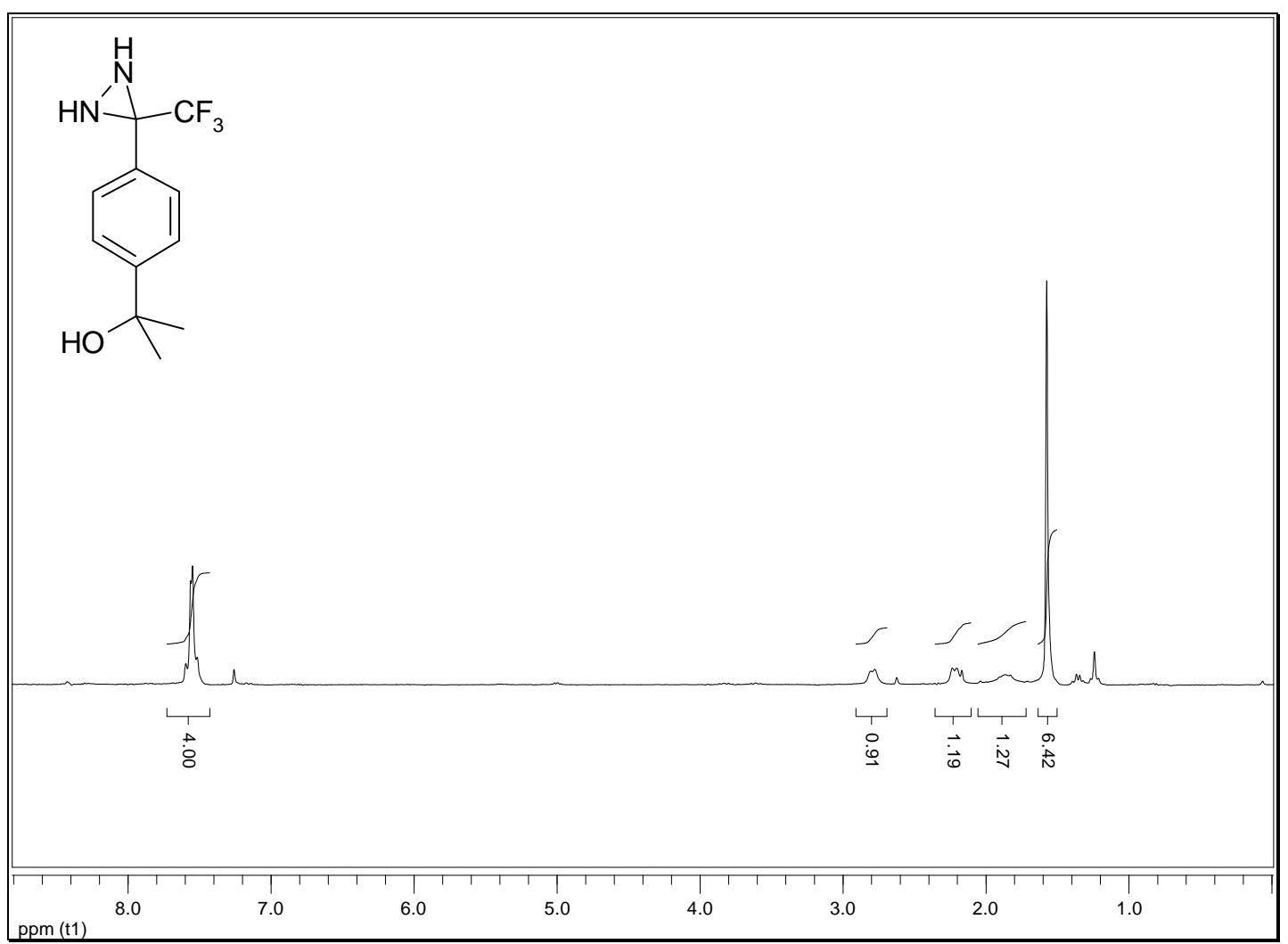

8. ${ }^{1}$ H-NMR (300 MHz) von 3-[4-(Hydroxyphenylmethyl)phenyl]-3-trifluormethyldiaziridin (70)

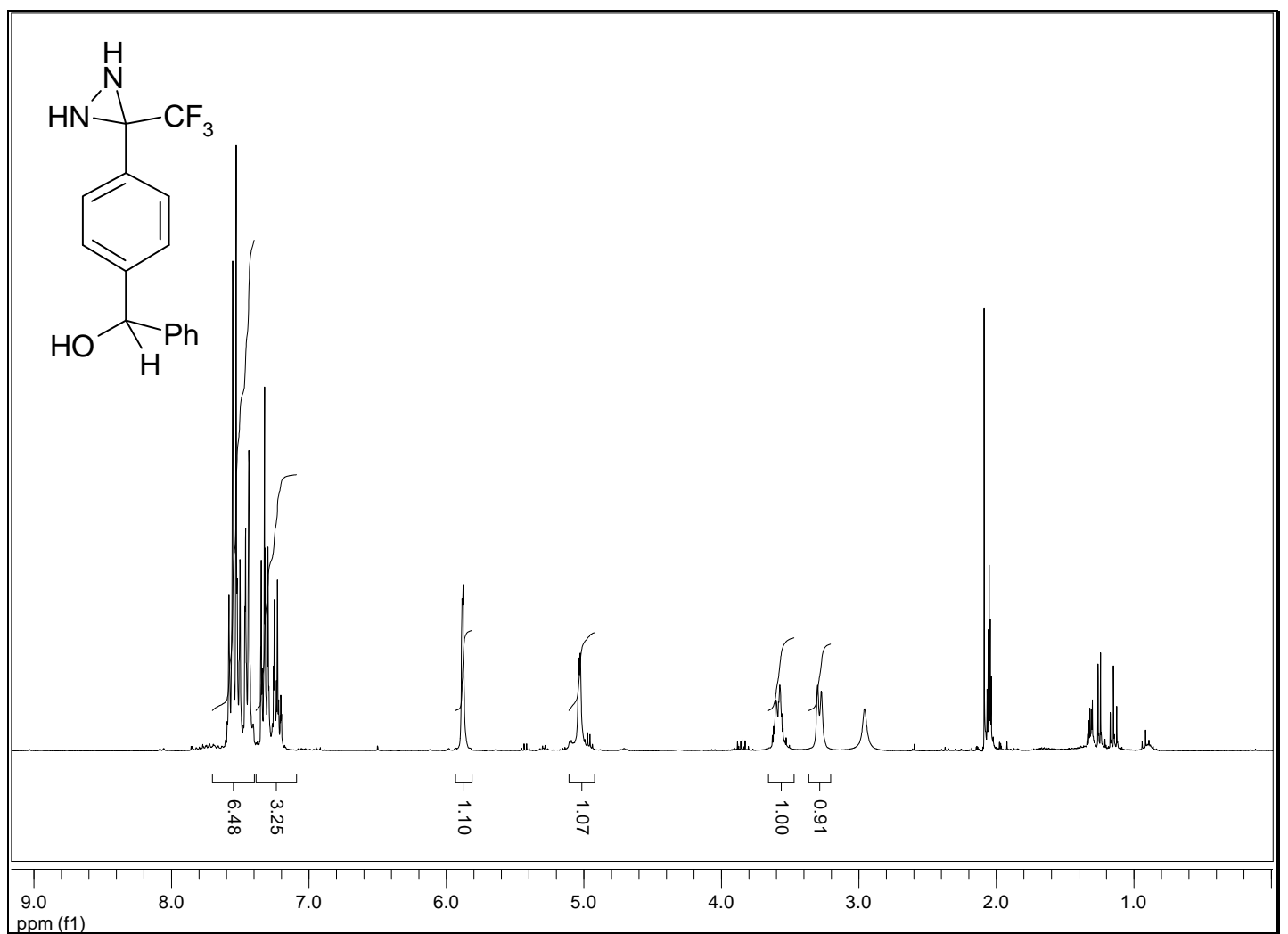


9. $\quad{ }^{1} \mathrm{H}-\mathrm{NMR}$ (300 MHz) von 4-(3-Trifluormethyldiaziridin-3-yl)benzoesäure (71)

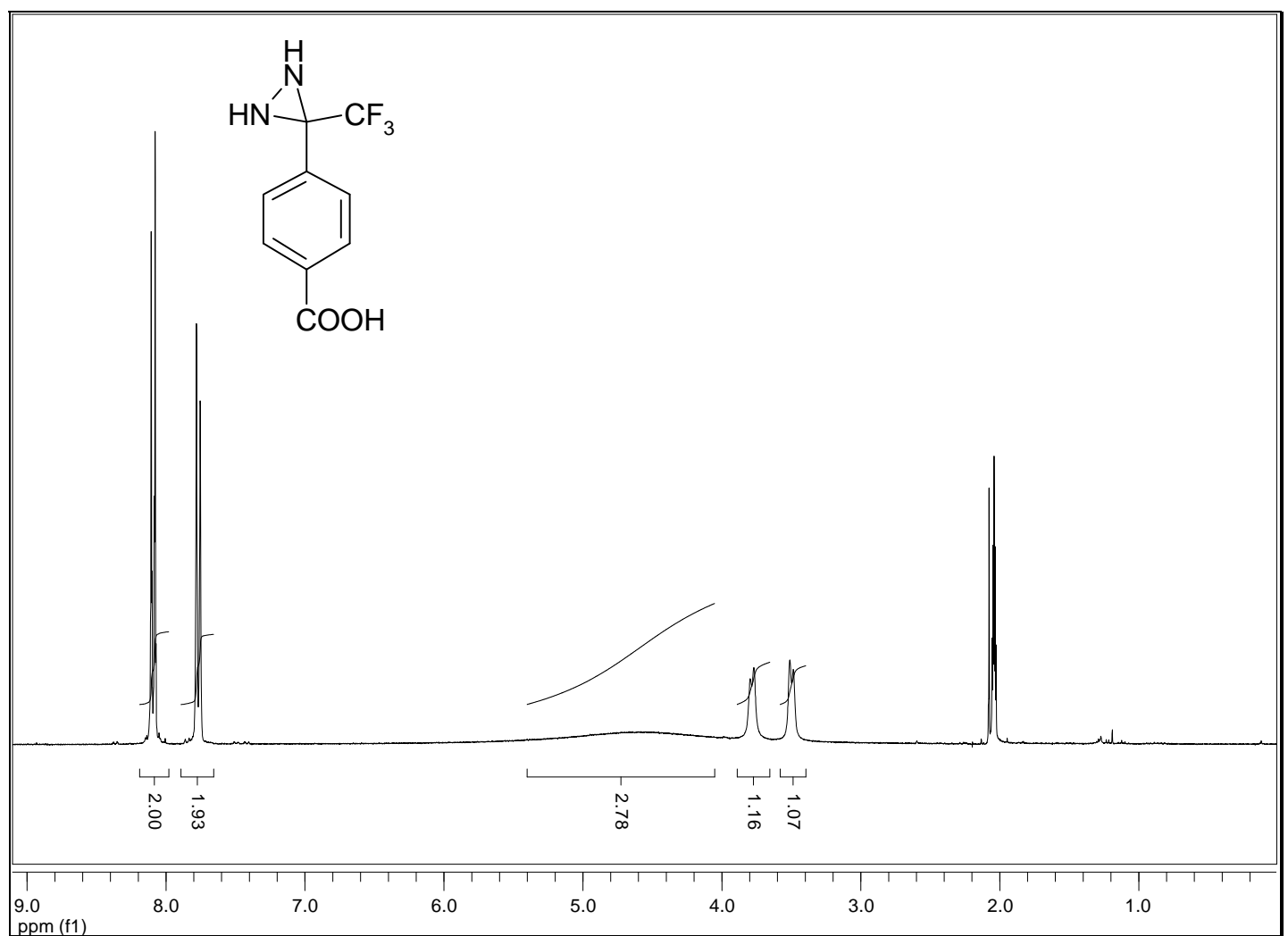

10. ${ }^{1}$ H-NMR (300 MHz) von 4-(3-Trifluormethyl-3H-diazirin-3-yl)benzoesäure (48)

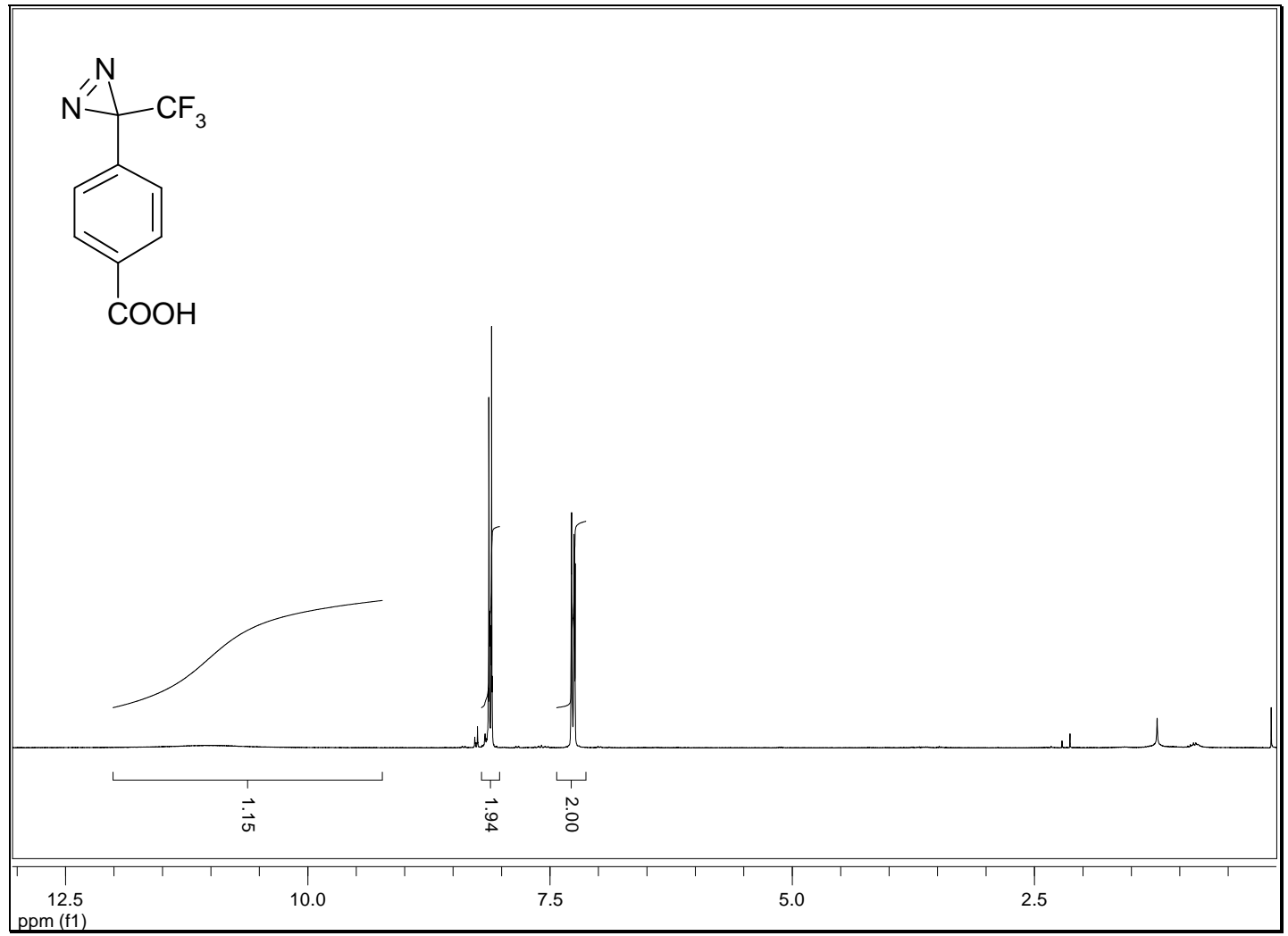


11. ${ }^{1}$ H-NMR (600 MHz) von 23-O-[4-(3-Trifluormethyl-3H-diazirin-3-yl)benzoyl]21-deoxyconcanolid A (75)

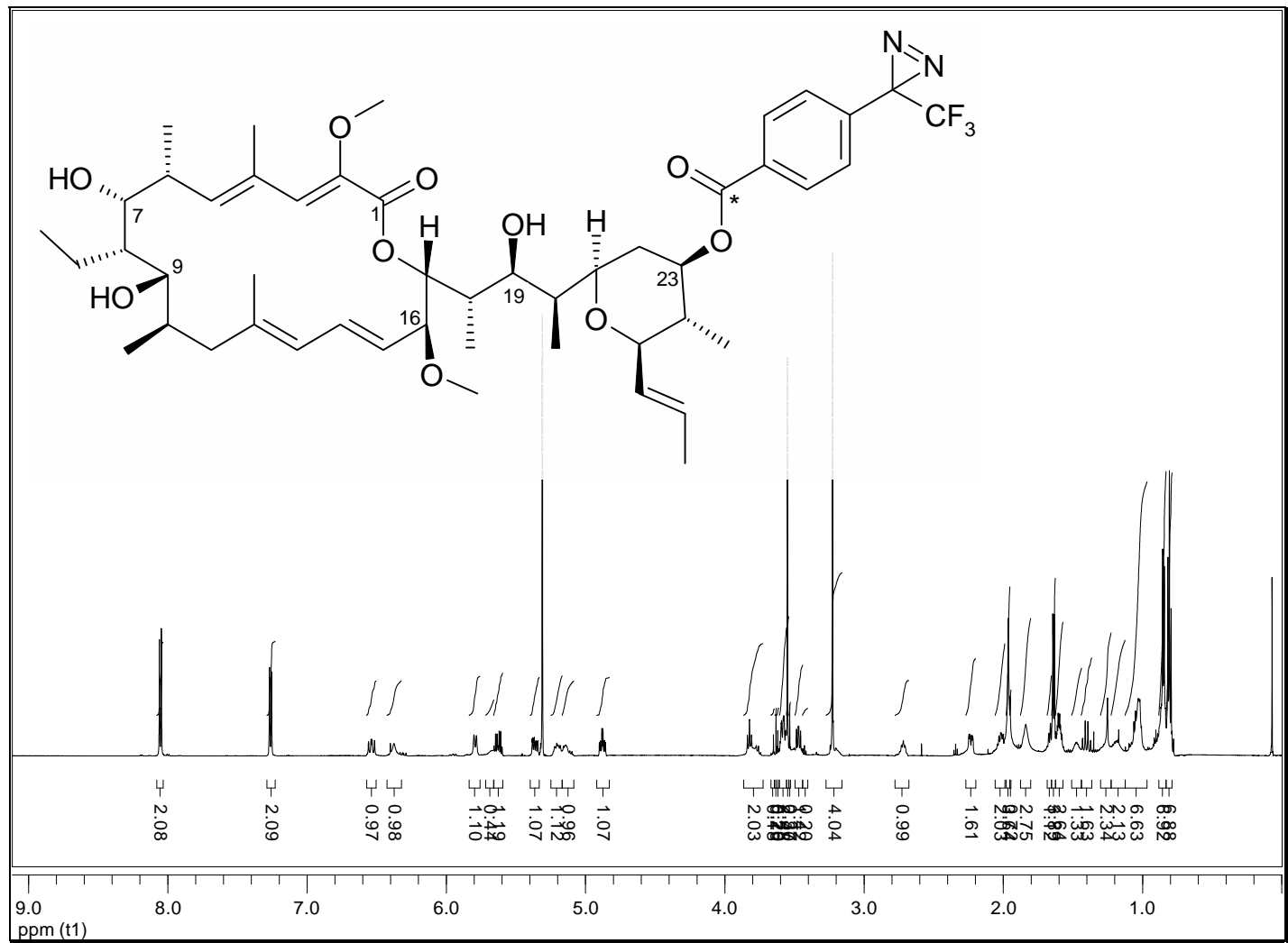

\section{${ }^{13}$ C-NMR (151 MHz)}

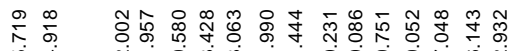

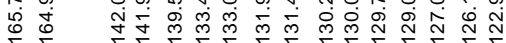

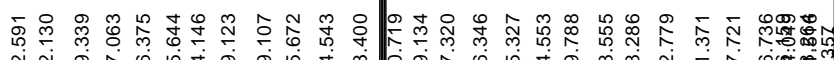
施
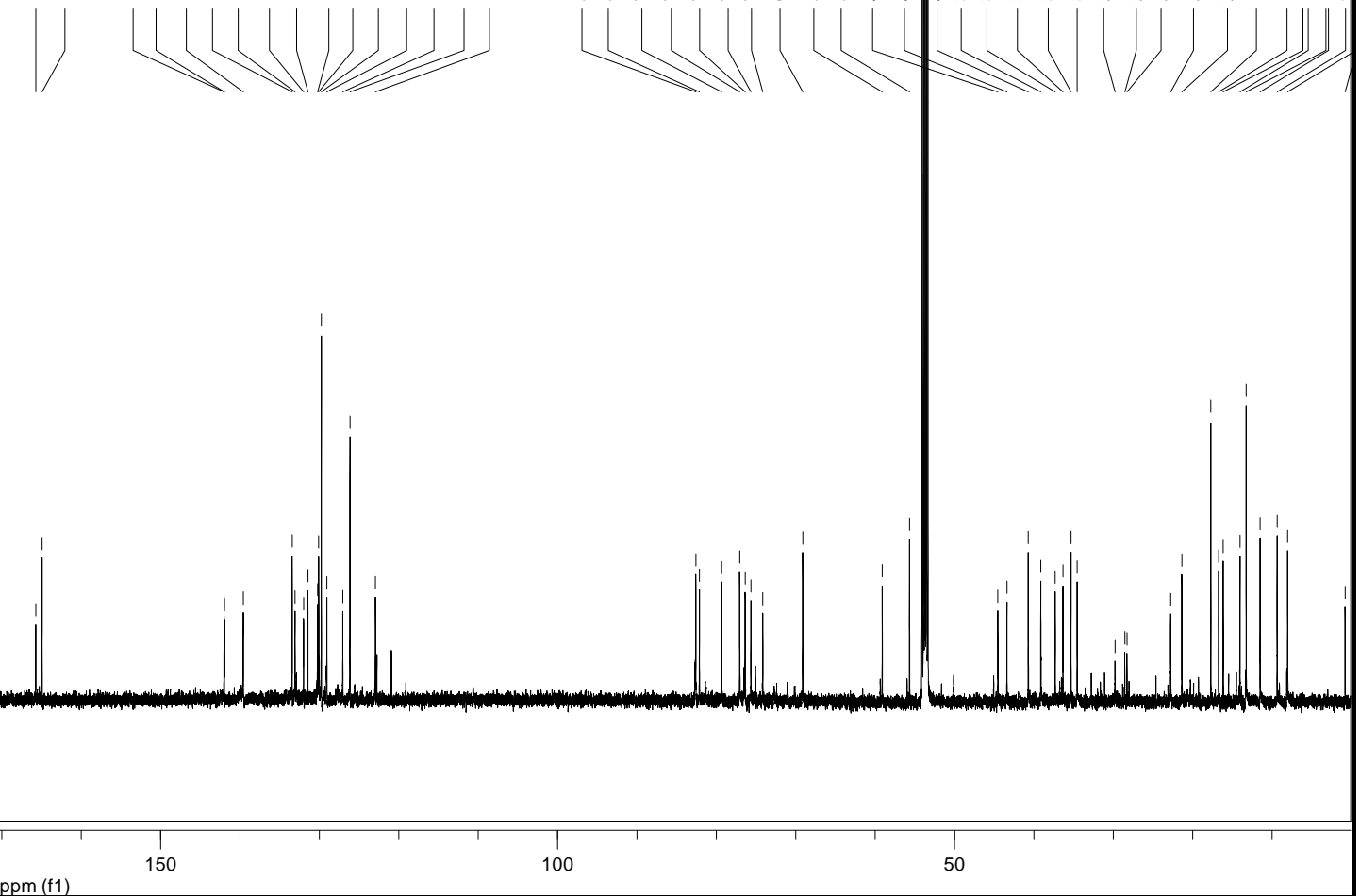
12. ${ }^{1} \mathrm{H}-\mathrm{NMR}$ (600 MHz) von 21-O-[4-(3-Trifluormethyl-3H-diazirin-3-yl)benzoyl]bafilomycin $A_{1}$ (77)

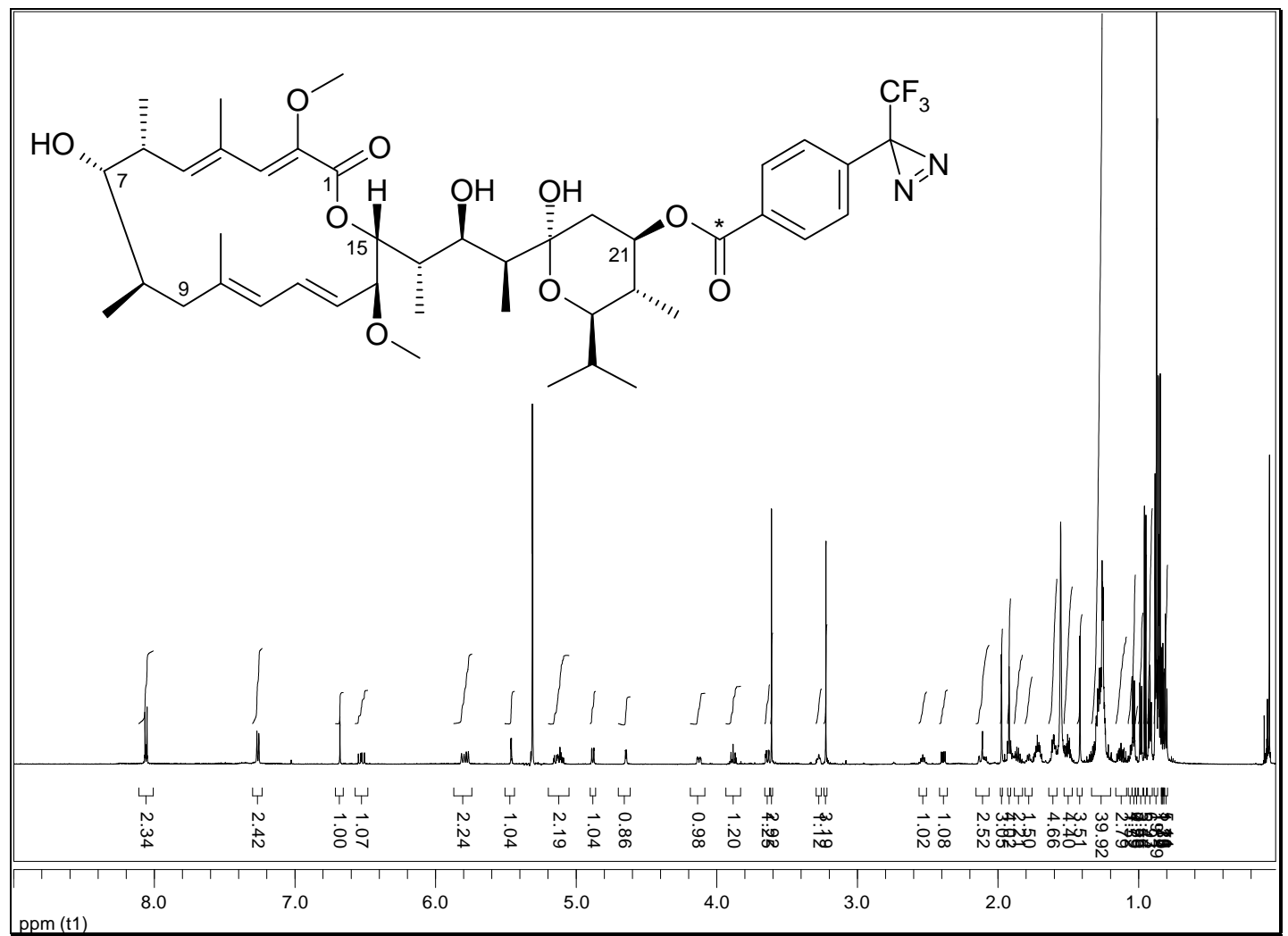

\section{${ }^{13}$ C-NMR (151 MHz)}

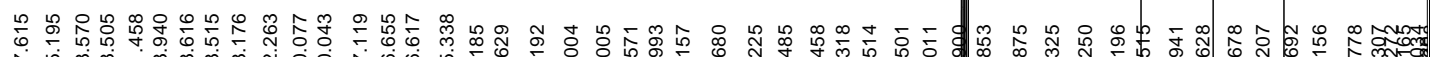

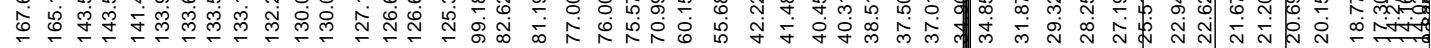

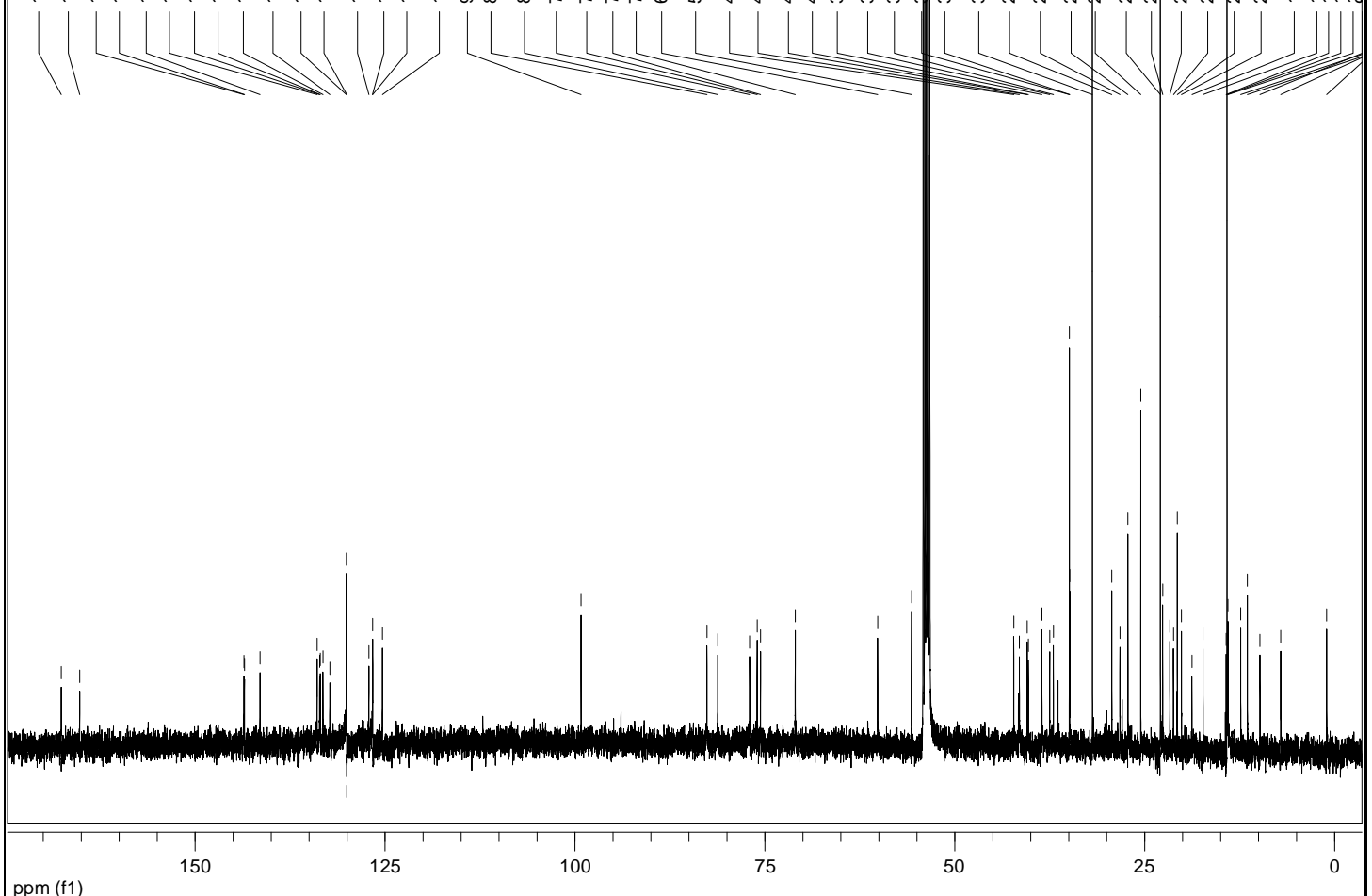


13. ${ }^{1} \mathrm{H}-\mathrm{NMR}(600 \mathrm{MHz})$ von 8-O-[4-(3-Trifluormethyl-3H-diazirin-3-yl)benzoyl]kendomycin-acetonid (82)

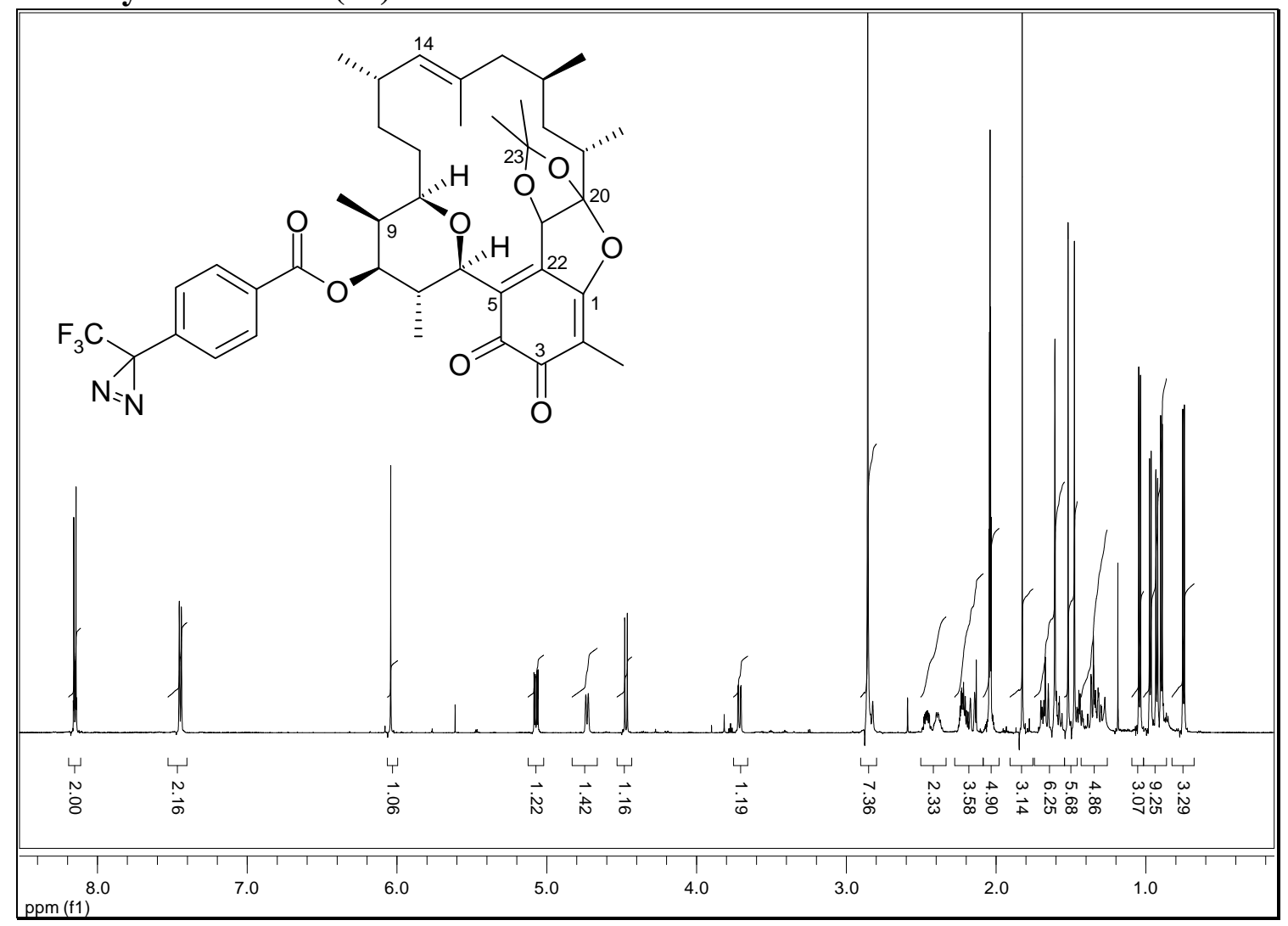

\section{${ }^{13}$ C-NMR (151 MHz)}

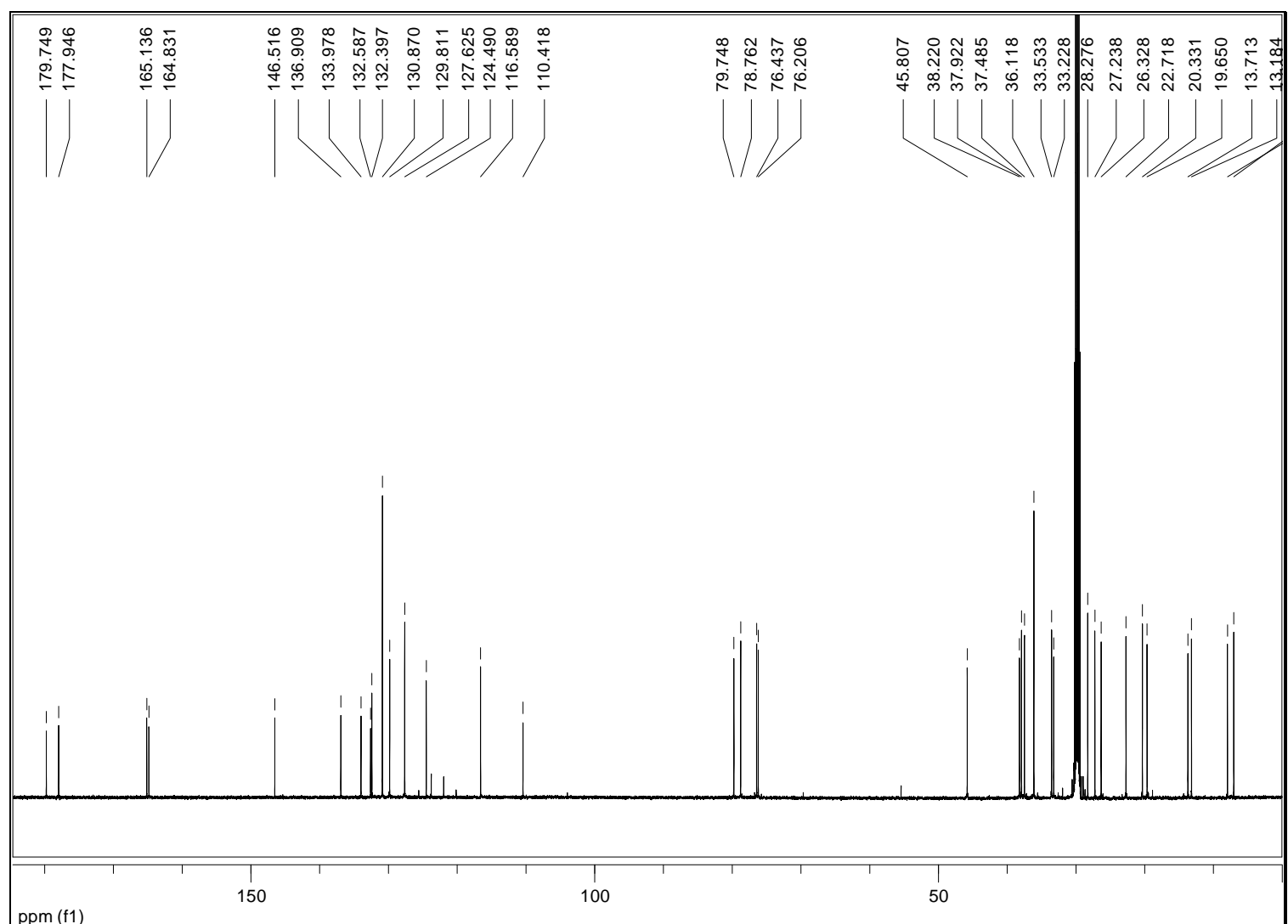


14. ${ }^{1}$ H-NMR (600 MHz) vom Kendomycin-methylacetal (83)

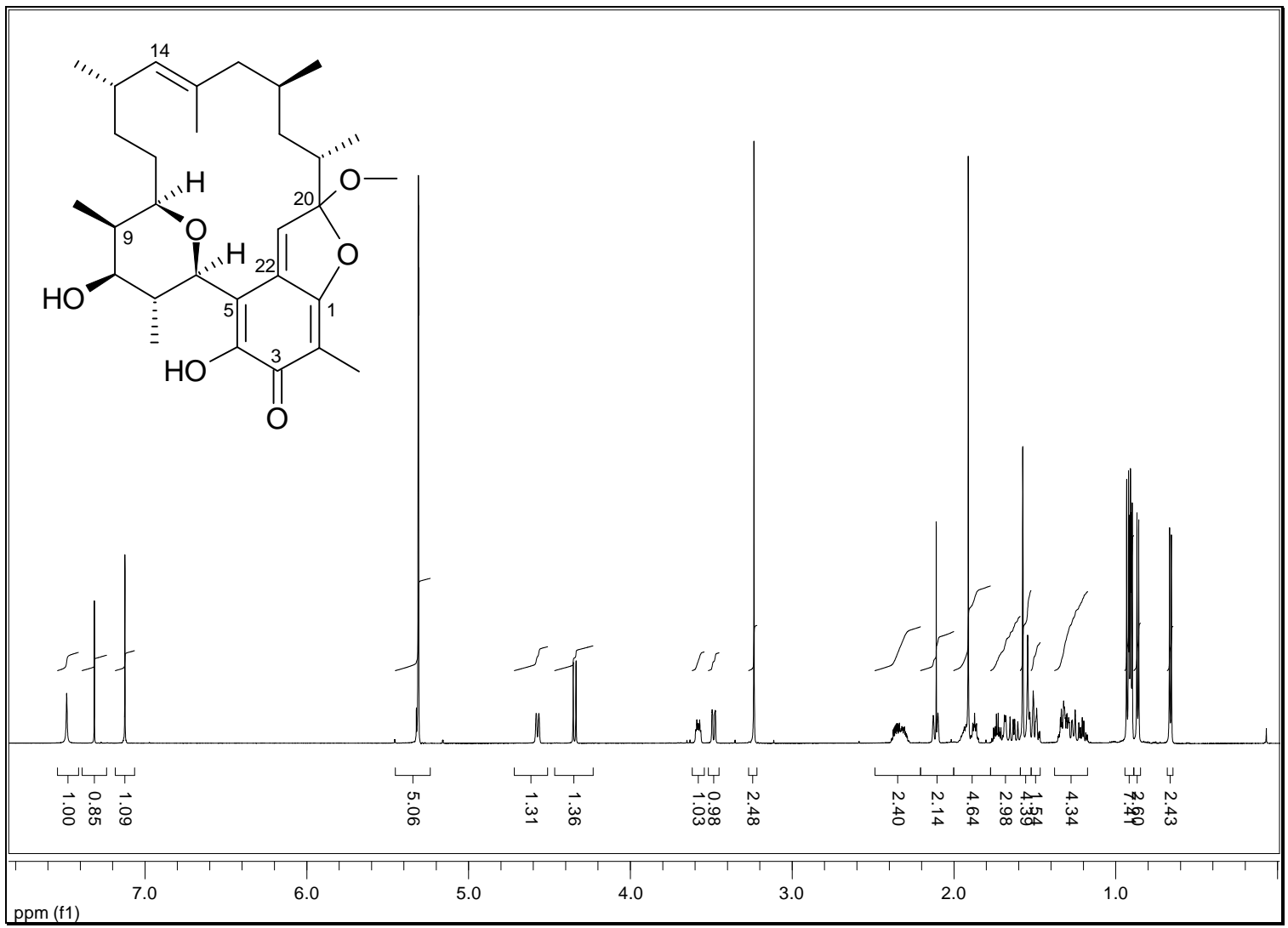

${ }^{13} \mathrm{C}-\mathrm{NMR}(151 \mathrm{MHz})$

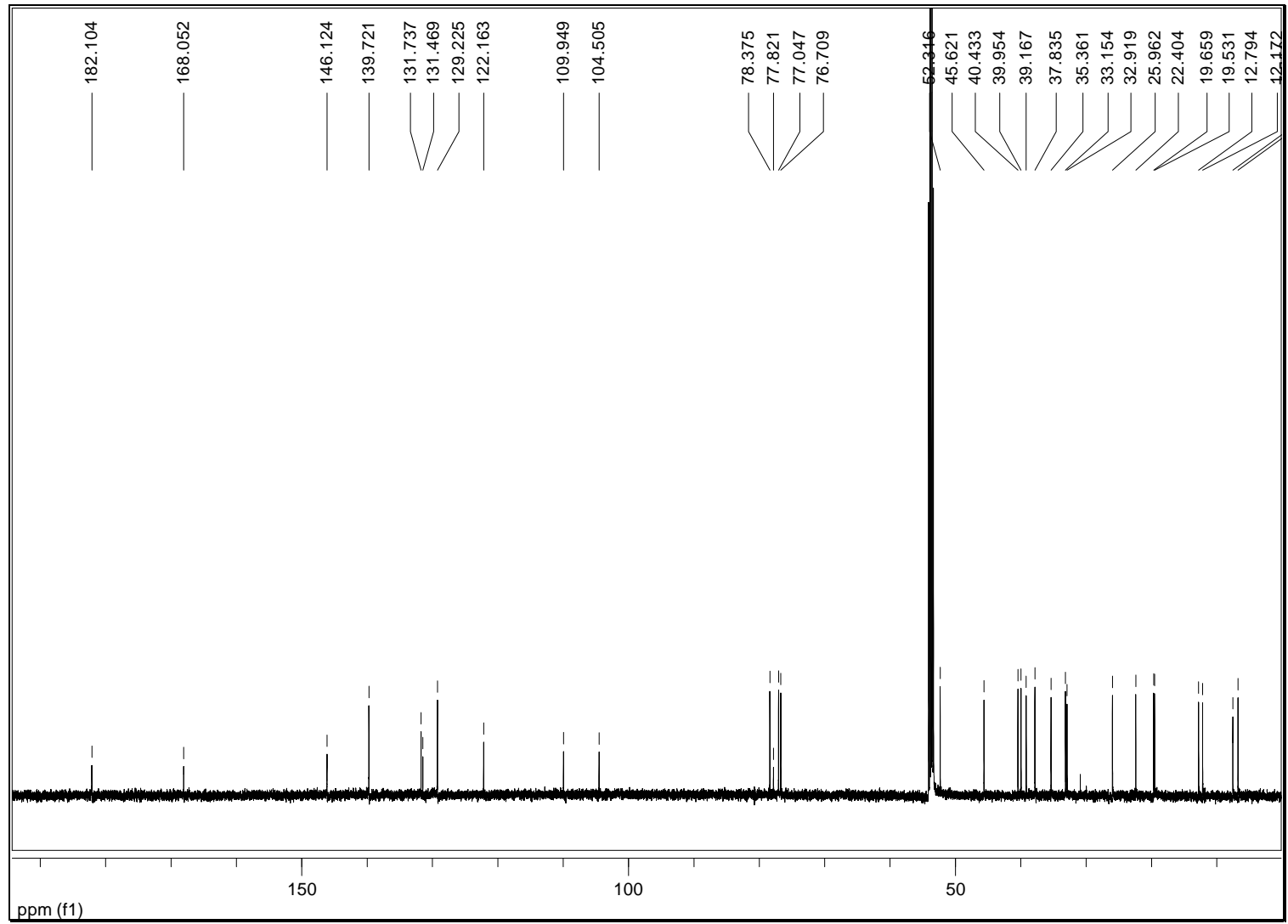


15. ${ }^{1} \mathrm{H}-\mathrm{NMR}$ (600 MHz) von 8-O-[4-(3-Trifluormethyl-3H-diazirin-3-yl)benzoyl]kendomycinmethylacetal (84)

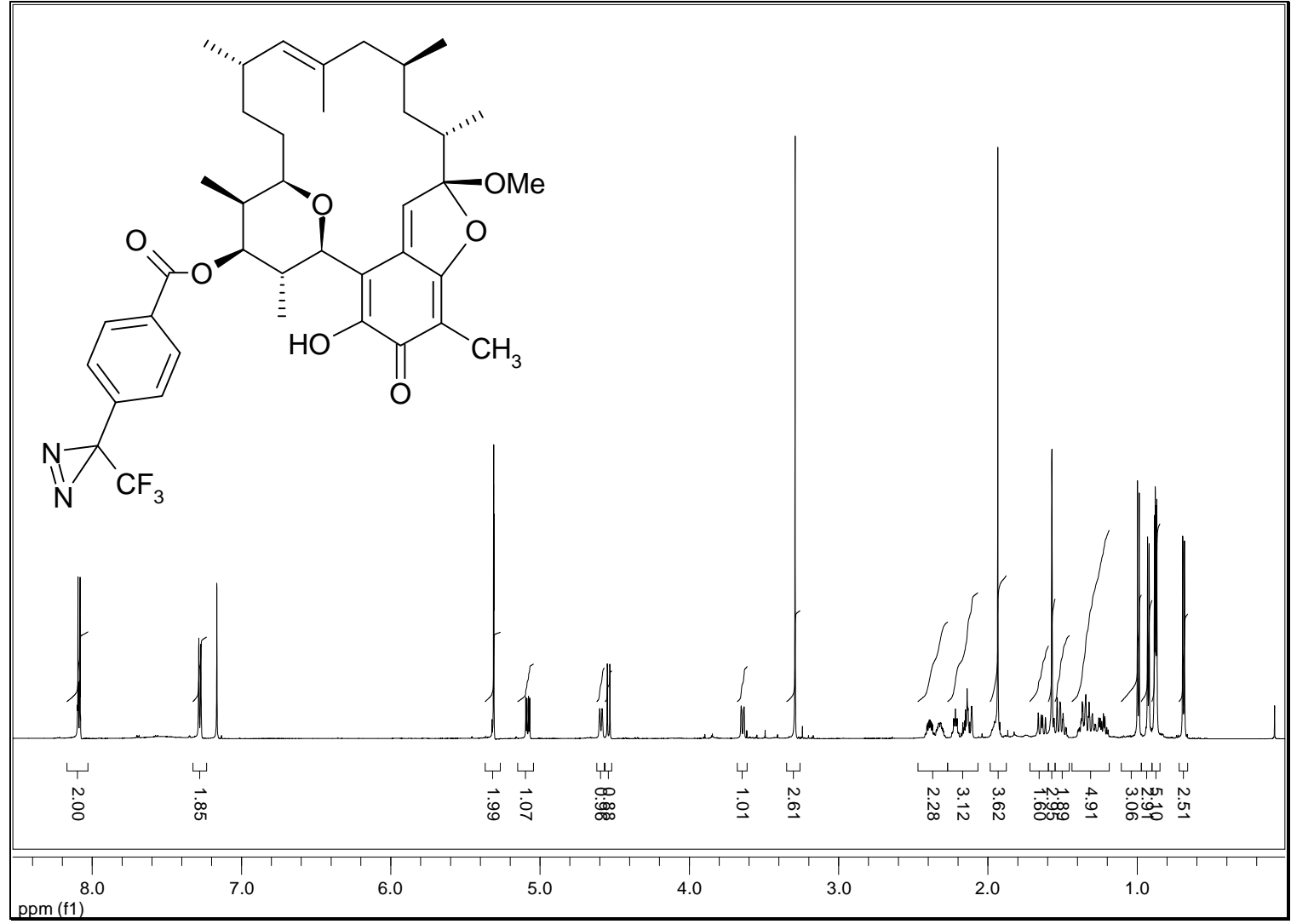

\section{${ }^{13}$ C-NMR (151 MHz)}

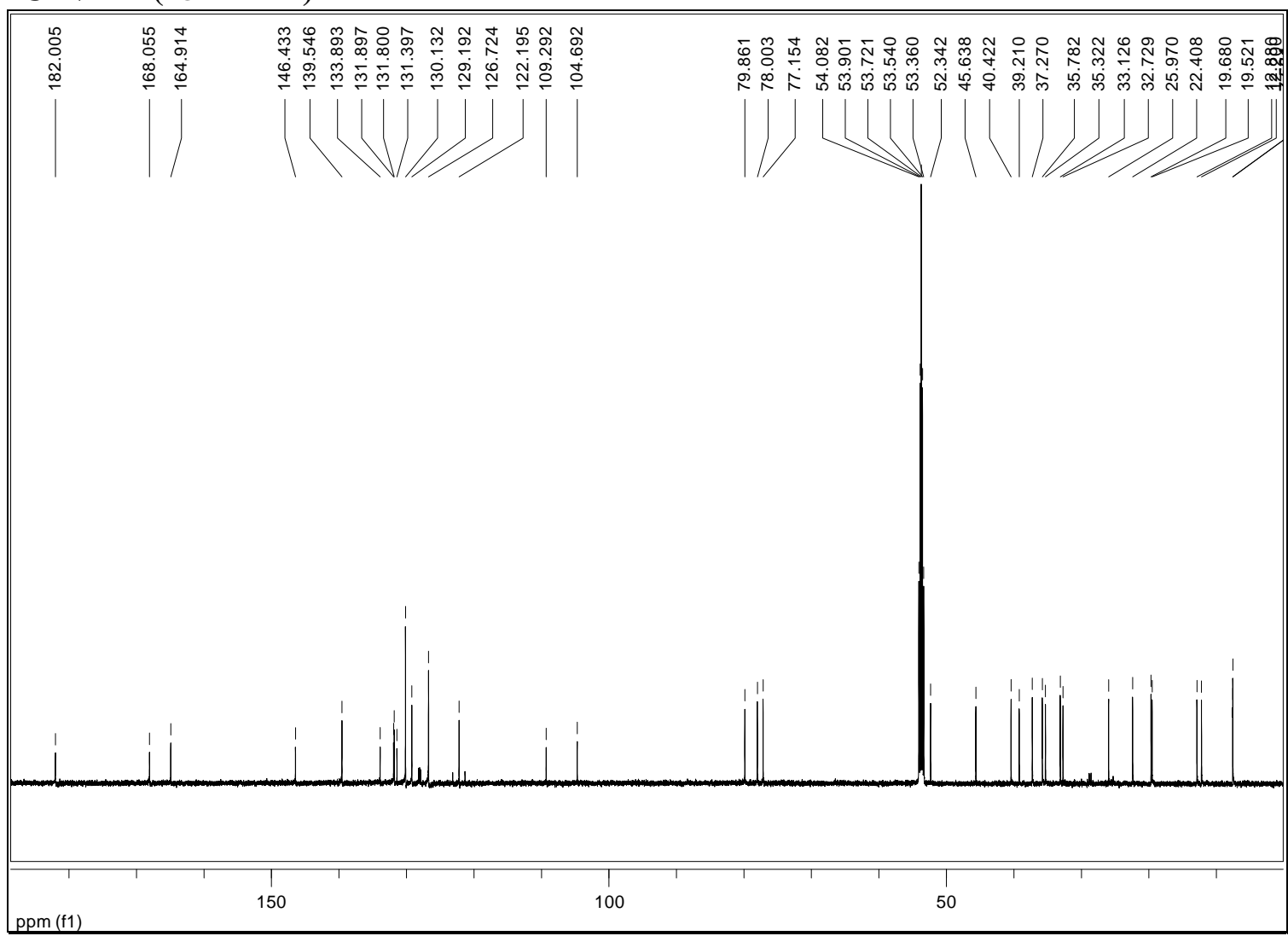


16. ${ }^{1}$ H-NMR von 3-O-[4-(3-Trifluormethyl-3H-diazirin-3-yl)benzoyl]-apicularen (86)

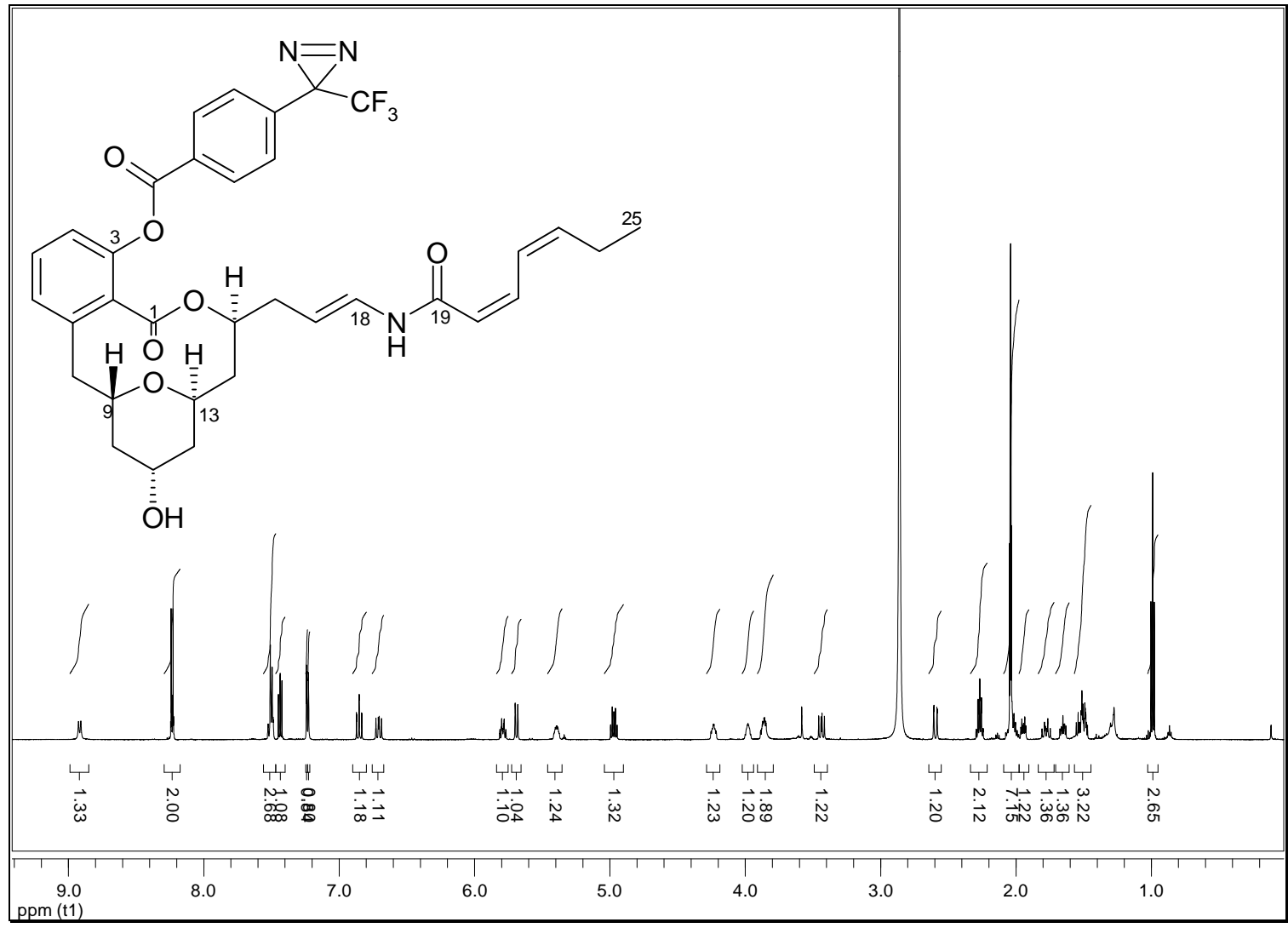

\section{${ }^{13}$ C-NMR (151 MHz)}

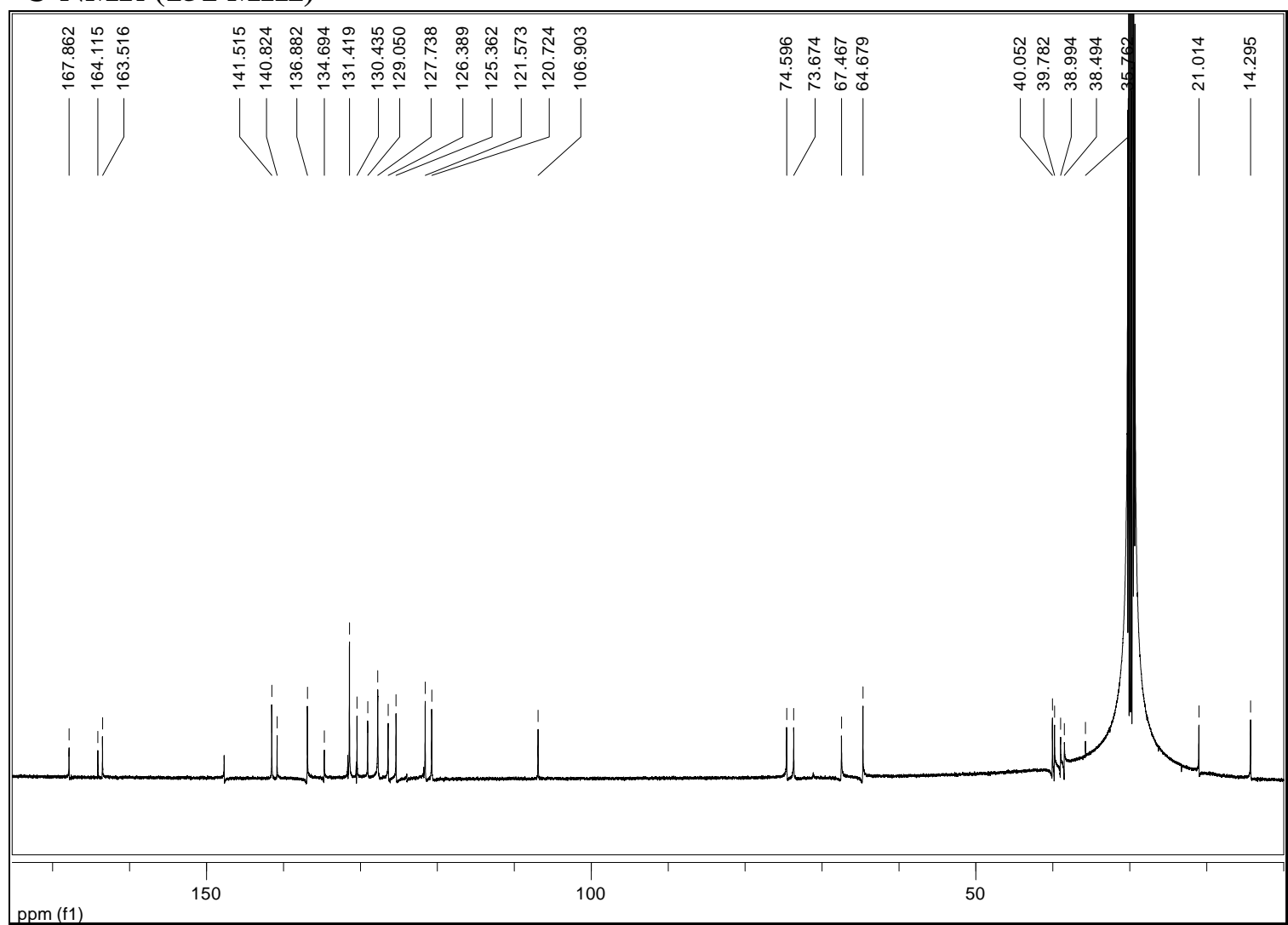


17. ${ }^{1} \mathrm{H}-\mathrm{NMR}$ (250 MHz) von (4E,6Z)-8-(tert-Butyldimethylsilanyloxy)-3-oxoocta-4,6diensäureethylester $\left(155_{Z}\right)$

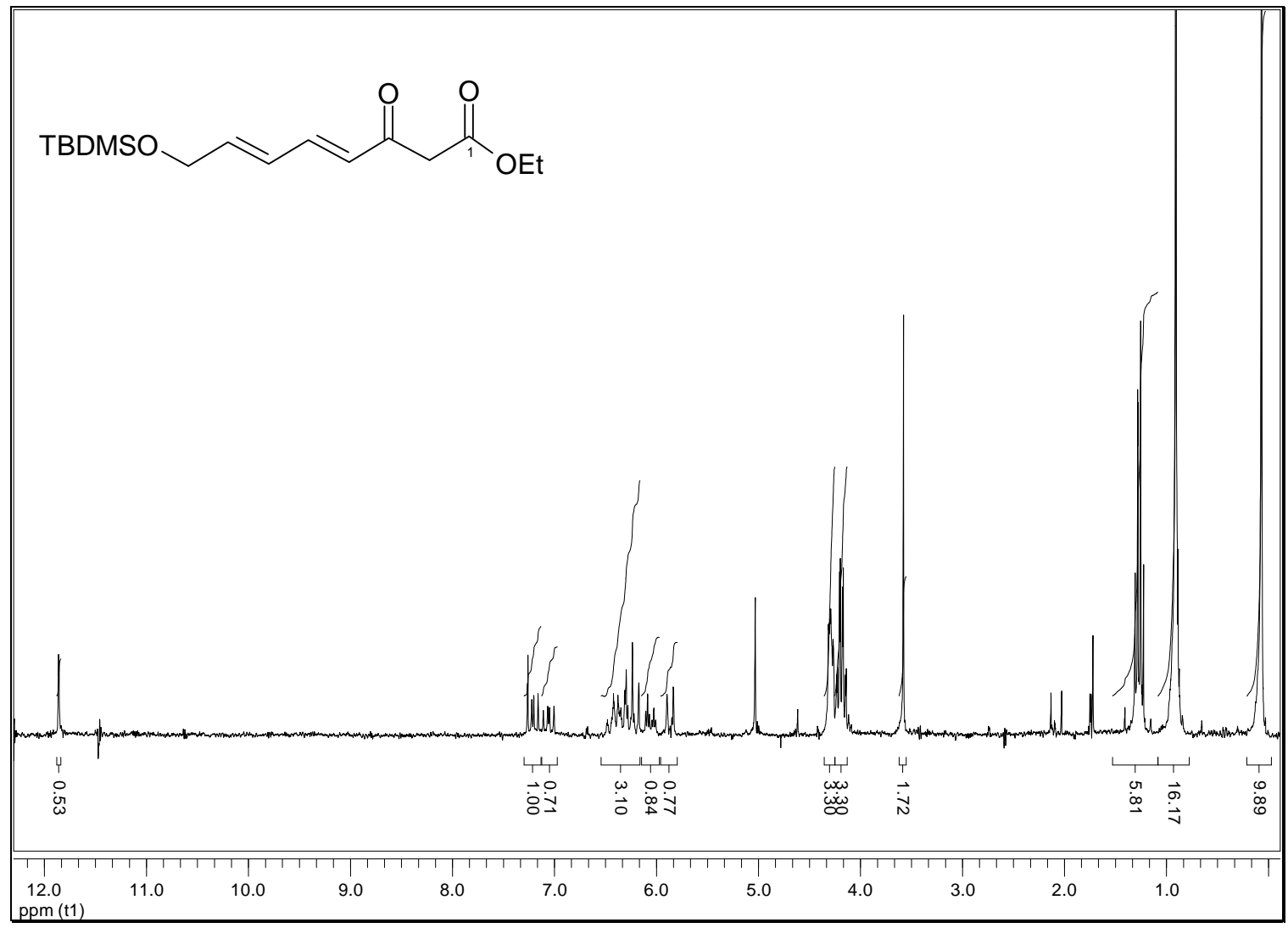

18. ${ }^{1} \mathrm{H}$-NMR (250 MHz) von (4E,6Z)-8-(tert-Butyldimethylsilanyloxy)-3-oxoocta-4,6diensäureethylester $\left(155_{Z}\right)$

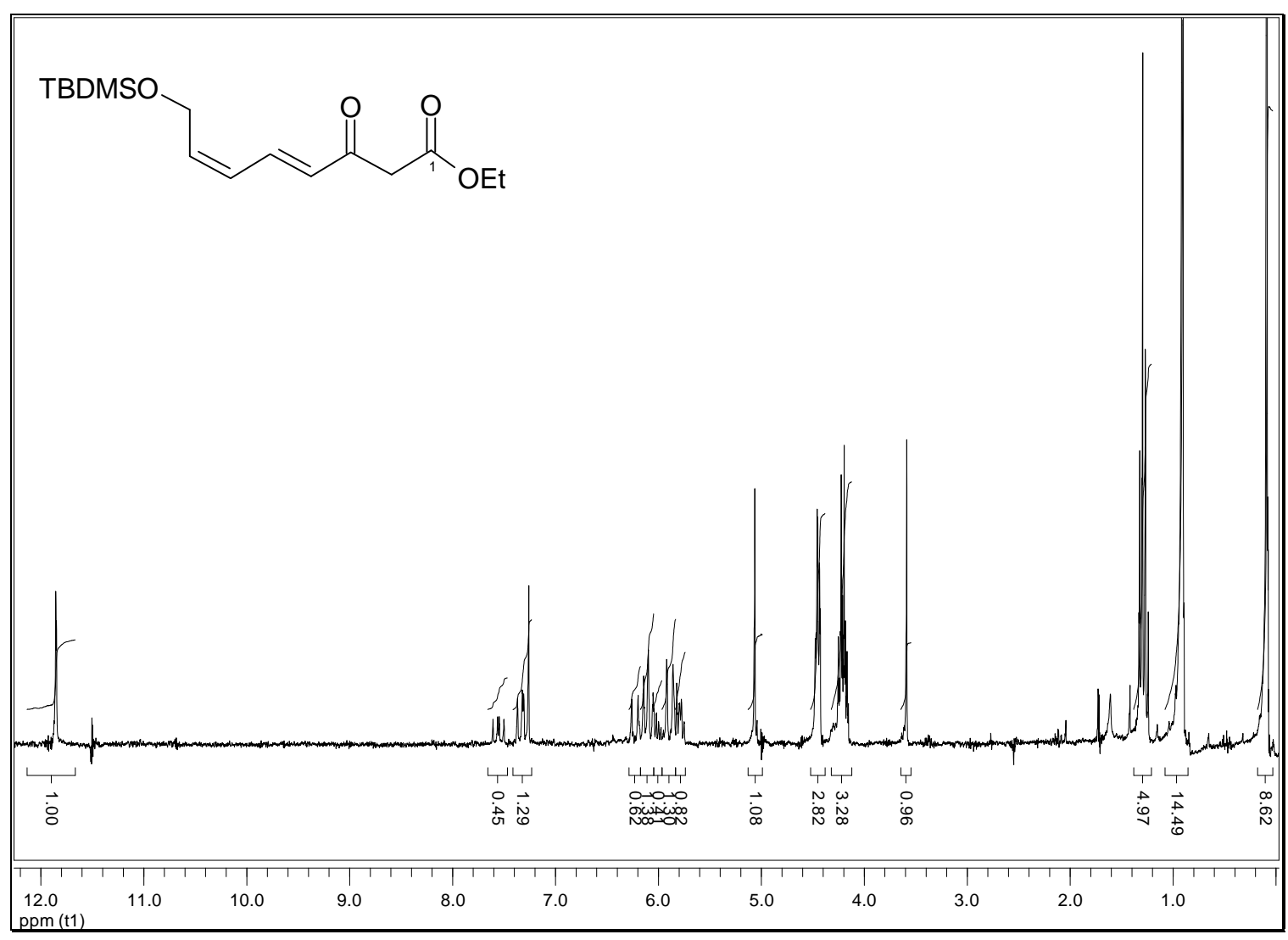


19. ${ }^{1} \mathrm{H}-\mathrm{NMR}(250 \mathrm{MHz})$ von (5E,7Z)-8-(tert-Butyldimethylsilanyloxy)-3-oxoocta-5,7diensäureethylester (156)

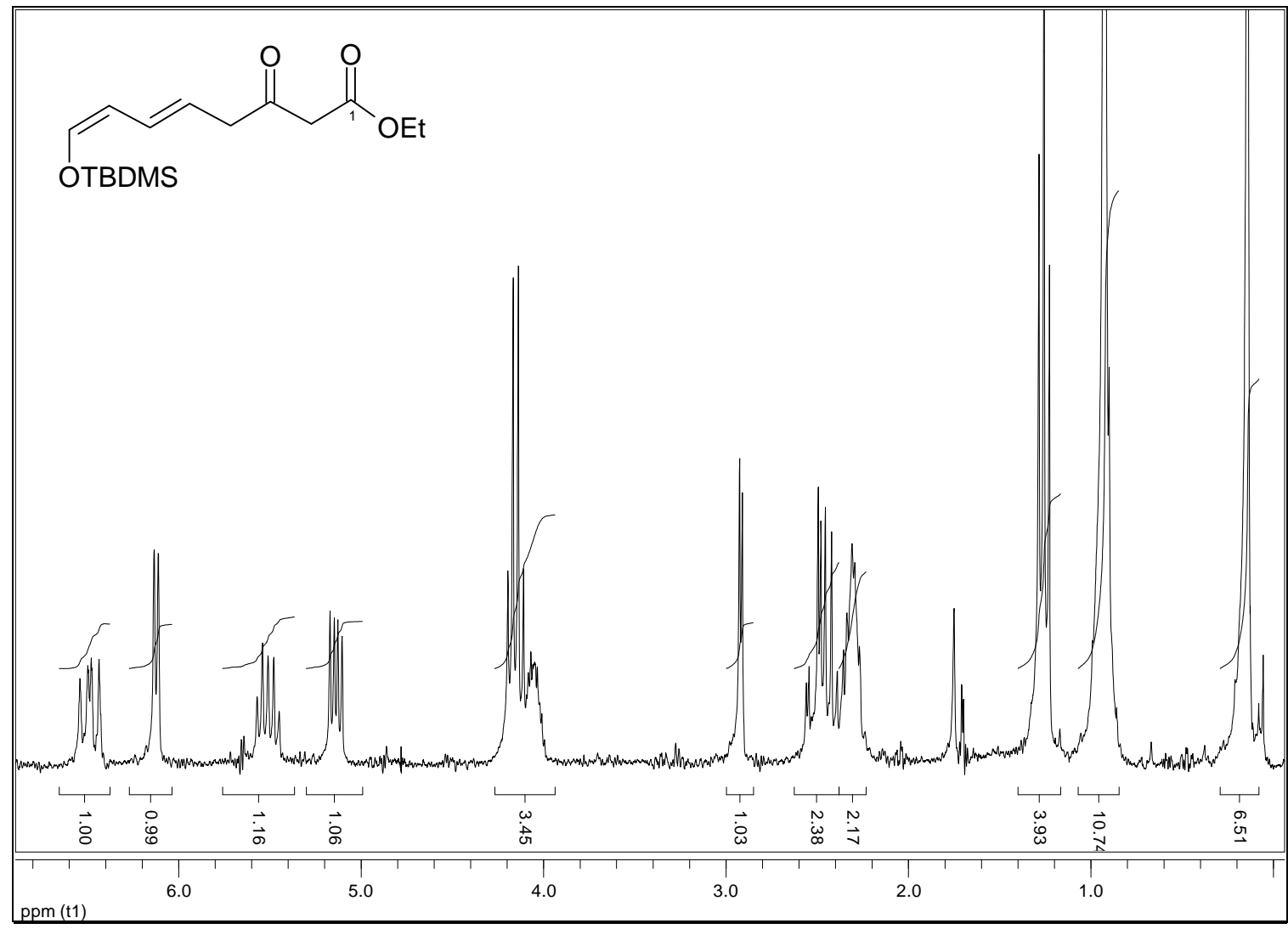

20. ${ }^{1} \mathrm{H}$-NMR (300 MHz) von (5E,7Z)-8-(tert-Butyldimethylsilanyloxy)-3-hydroxyocta-5,7-diensäureethylester (160)

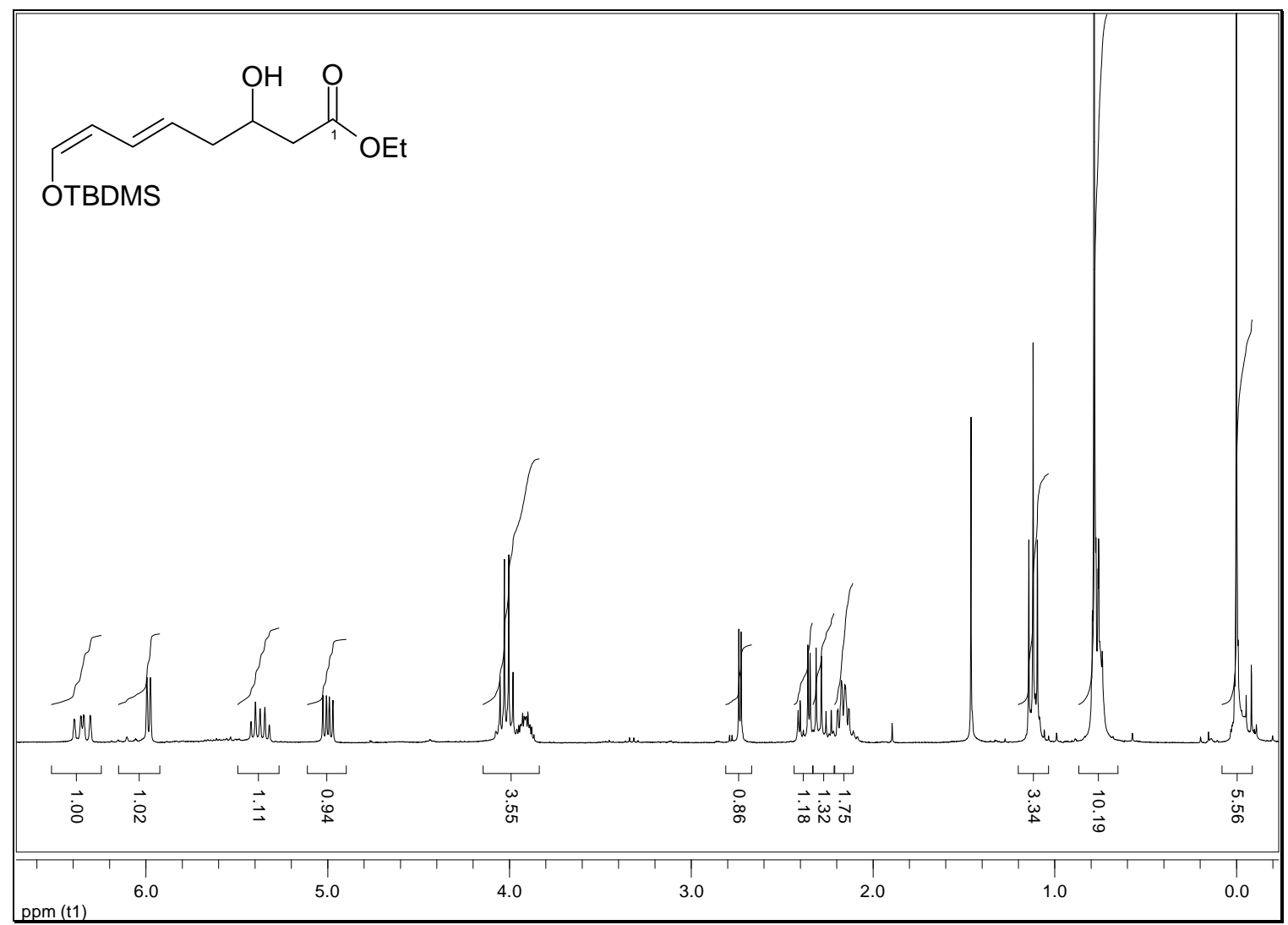


21. ${ }^{1}$ H-NMR (300 MHz) von (5E,7Z)-8-(tert-Butyldimethylsilanyloxy)-3-trimethylsilanyloxyocta-5,7-diensäureethyl-ester (163)

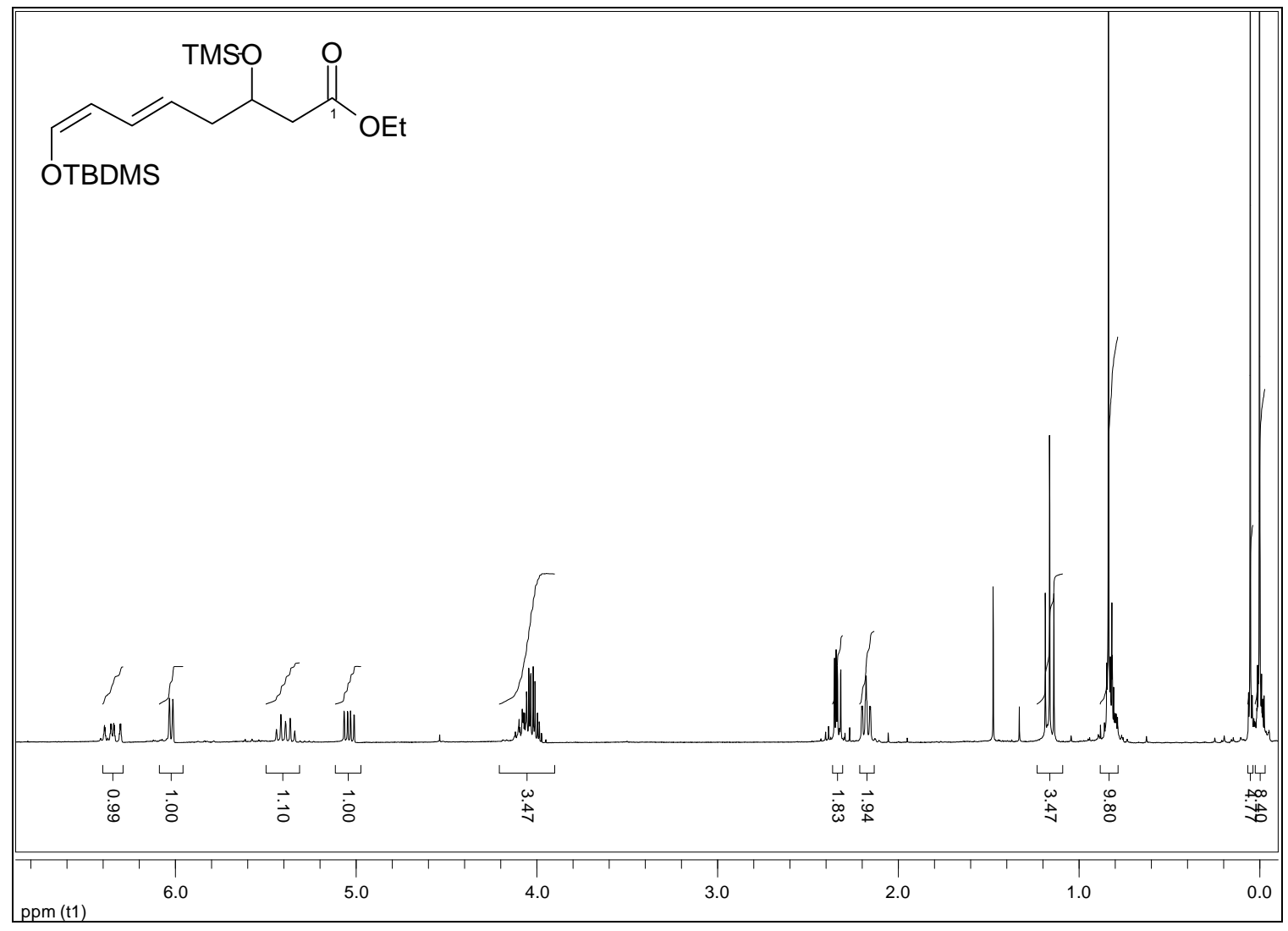

22. ${ }^{1} \mathrm{H}-\mathrm{NMR}$ (250 MHz) von (5E,7Z)-8-(tert-Butyldimethylsilanyloxy)-3-trimethylsilanyloxyocta-5,7-diensäure- $N$-methoxy- $N$-methylamid (164)

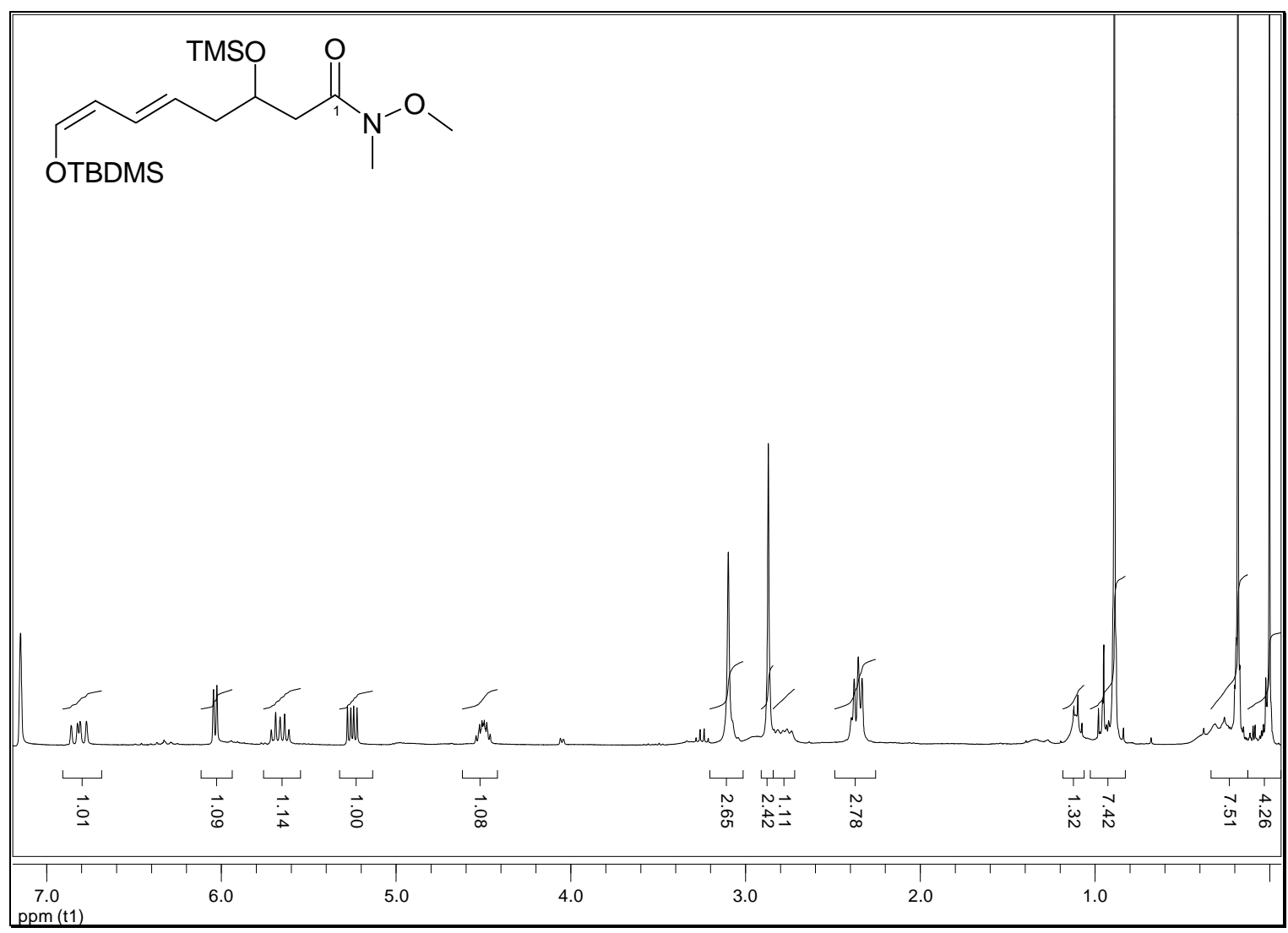


23. ${ }^{1} \mathrm{H}-\mathrm{NMR}$ (300 MHz) von (3E,9E,11Z)-12-(tert-Butyldimethylsilanyloxy)-1trimethylsilanyl-7-trimethylsilanyloxy-dodeca-3,9,11-trien-1-in-5-on (165)

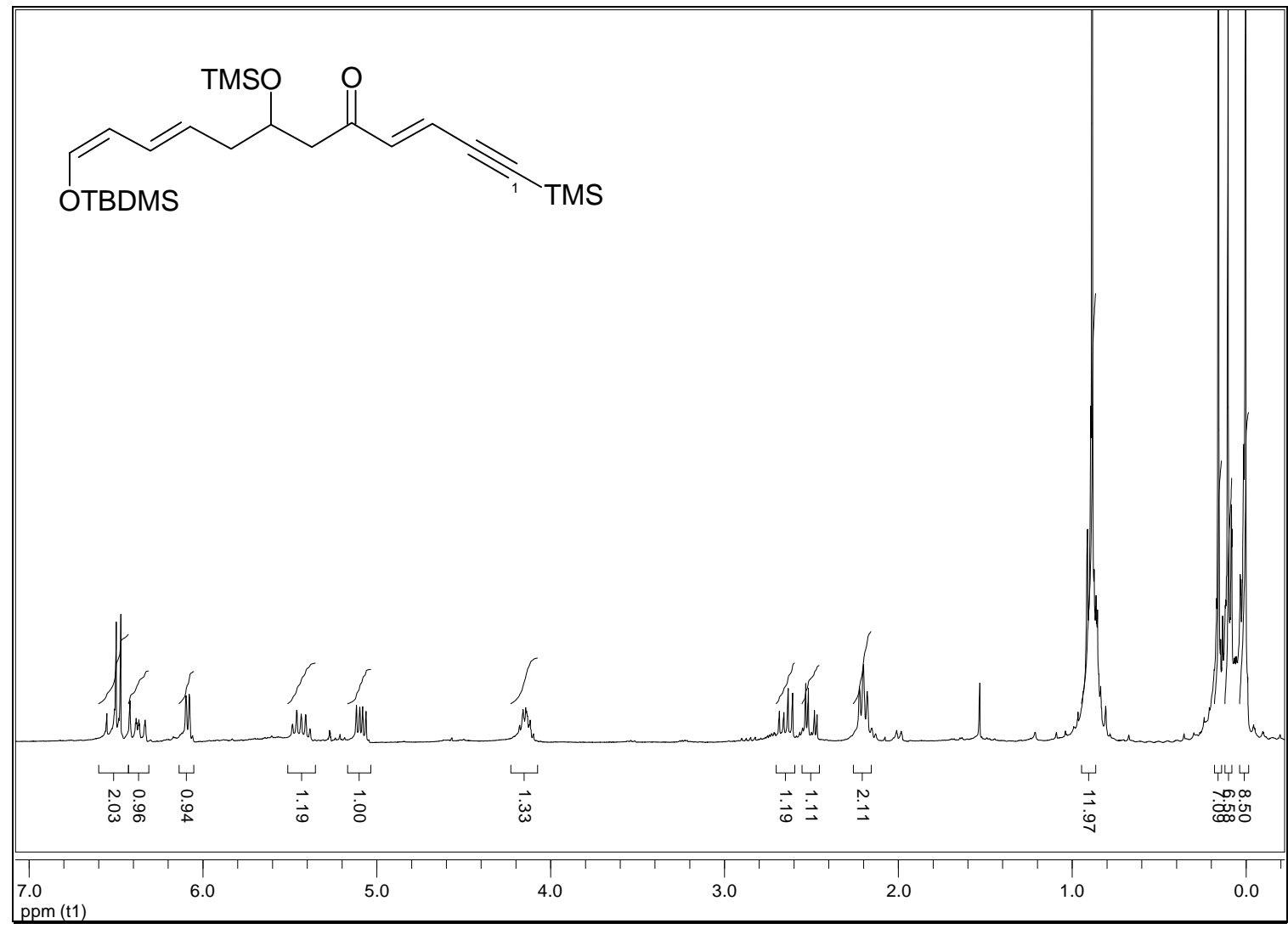

24. ${ }^{1} \mathrm{H}-\mathrm{NMR}$ (250 MHz) von (3E,9E,11Z)-12-Benzyloxy-1-trimethylsilanyl-7trimethylsilanyloxydodeca-3,9,11-trien-1-in-5-on (177)

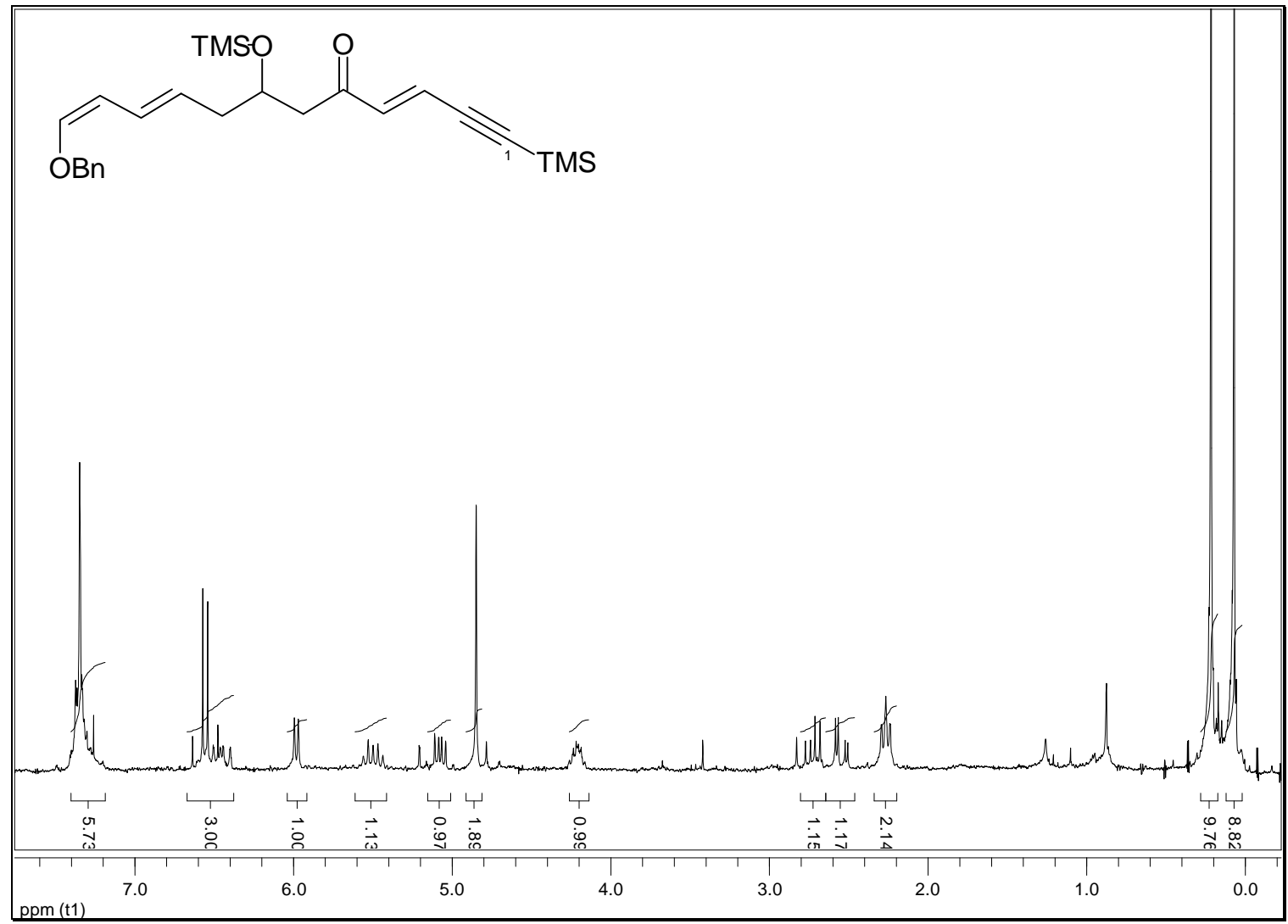


25. ${ }^{1} \mathrm{H}-\mathrm{NMR}(250 \mathrm{MHz})$ von (3E,9E,11Z/E)-12-Methoxy-1-trimethylsilanyl-7trimethylsilanyloxydodeca-3,9,11-trien-1-in-5-on (176)

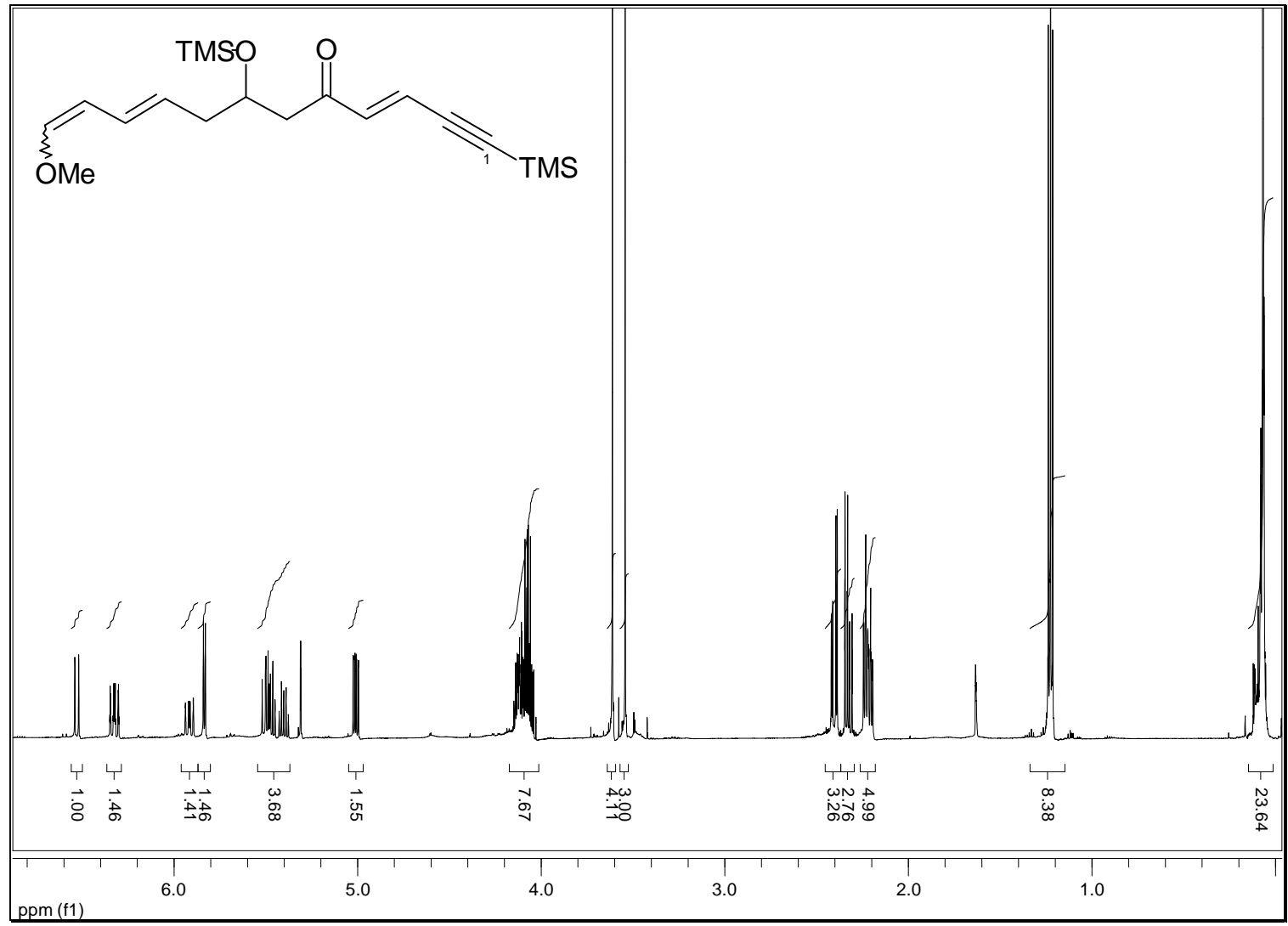

26. ${ }^{1} \mathrm{H}-\mathrm{NMR}$ (250 MHz) von (4E,6E)-3-Oxoocta-4,6-diensäureethylester (172)

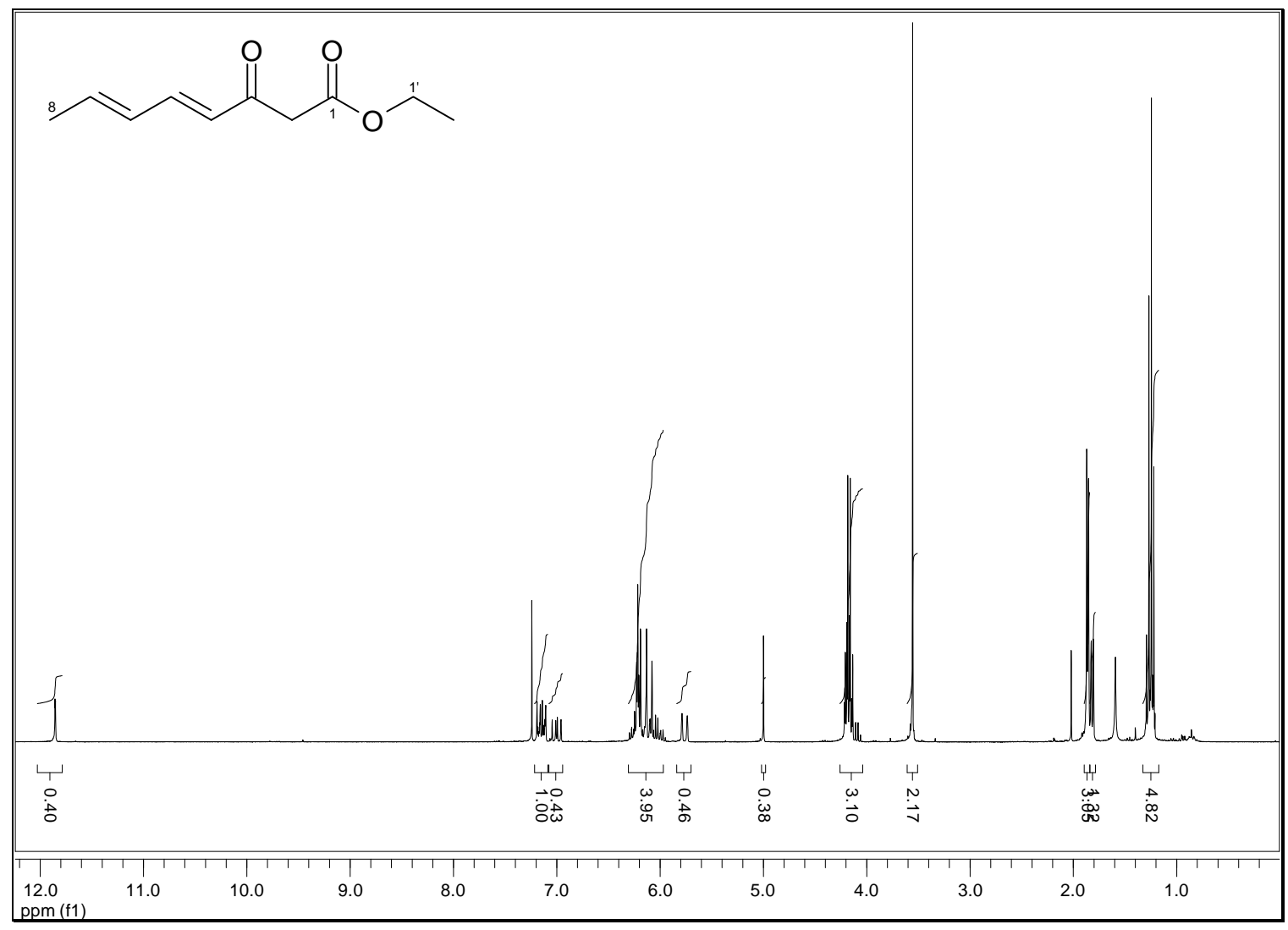


27. ${ }^{1}$ H-NMR (300 MHz) von (E)-3-Oxooct-5,7-diensäureethylester (184)

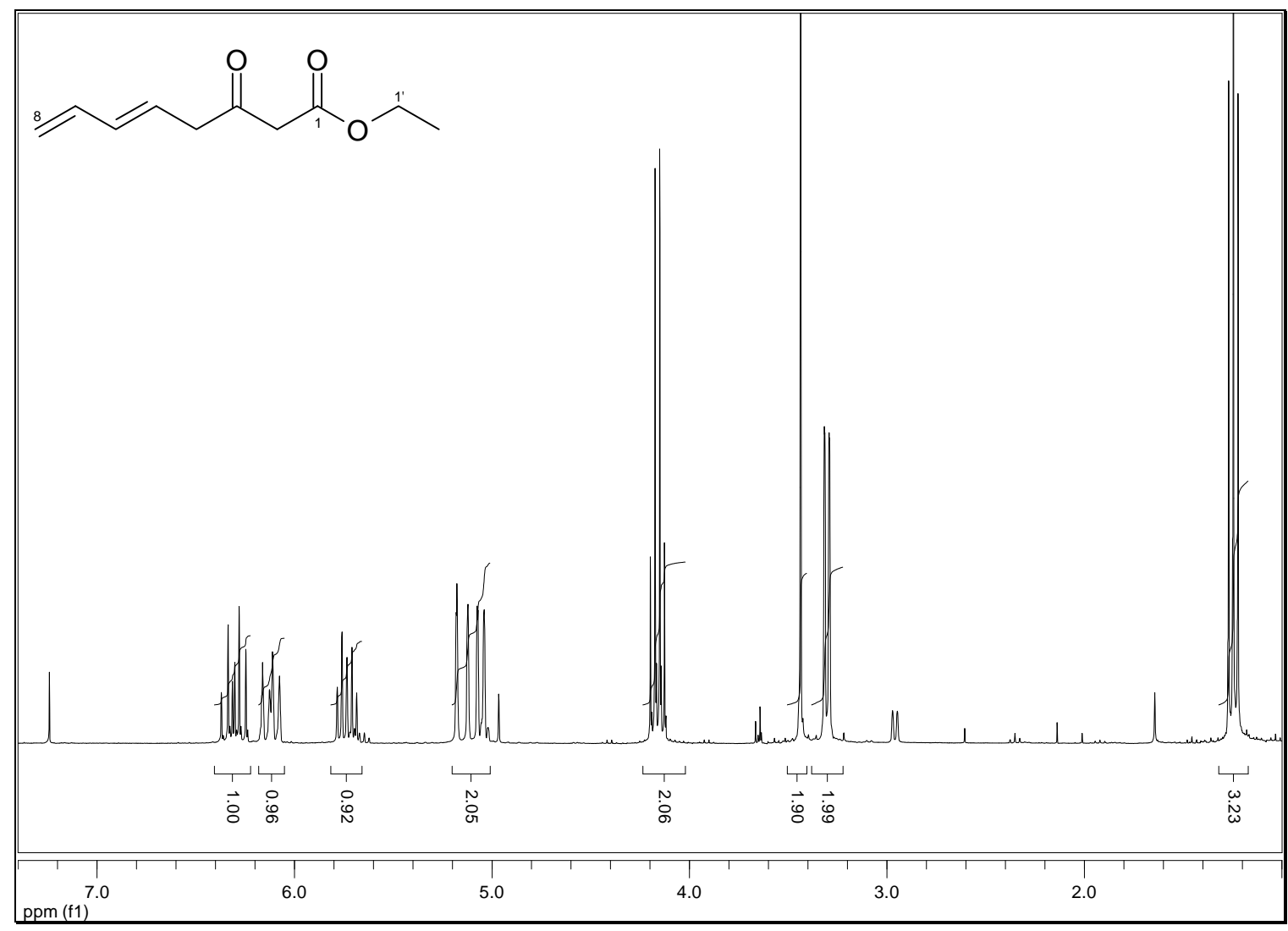

28. ${ }^{1} \mathrm{H}-\mathrm{NMR}$ (300 MHz) von (E)-3-Hydroxyocta-5,7-diensäureethylester (202)

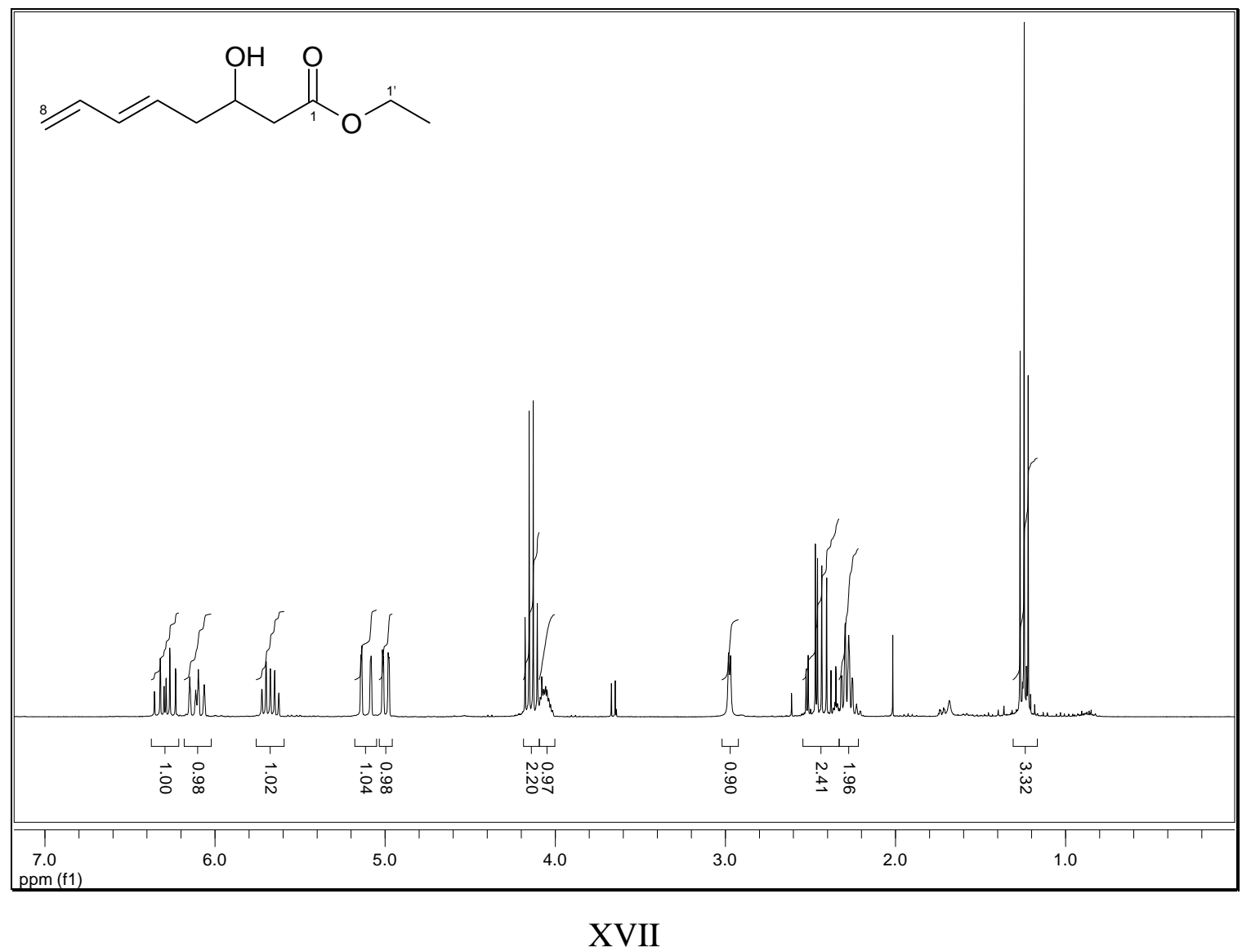


29. ${ }^{1} \mathrm{H}-\mathrm{NMR}$ (300 MHz) von (E)-3-(tert-Butyldimethylsilanyloxy)octa-5,7diensäureethylester (203)

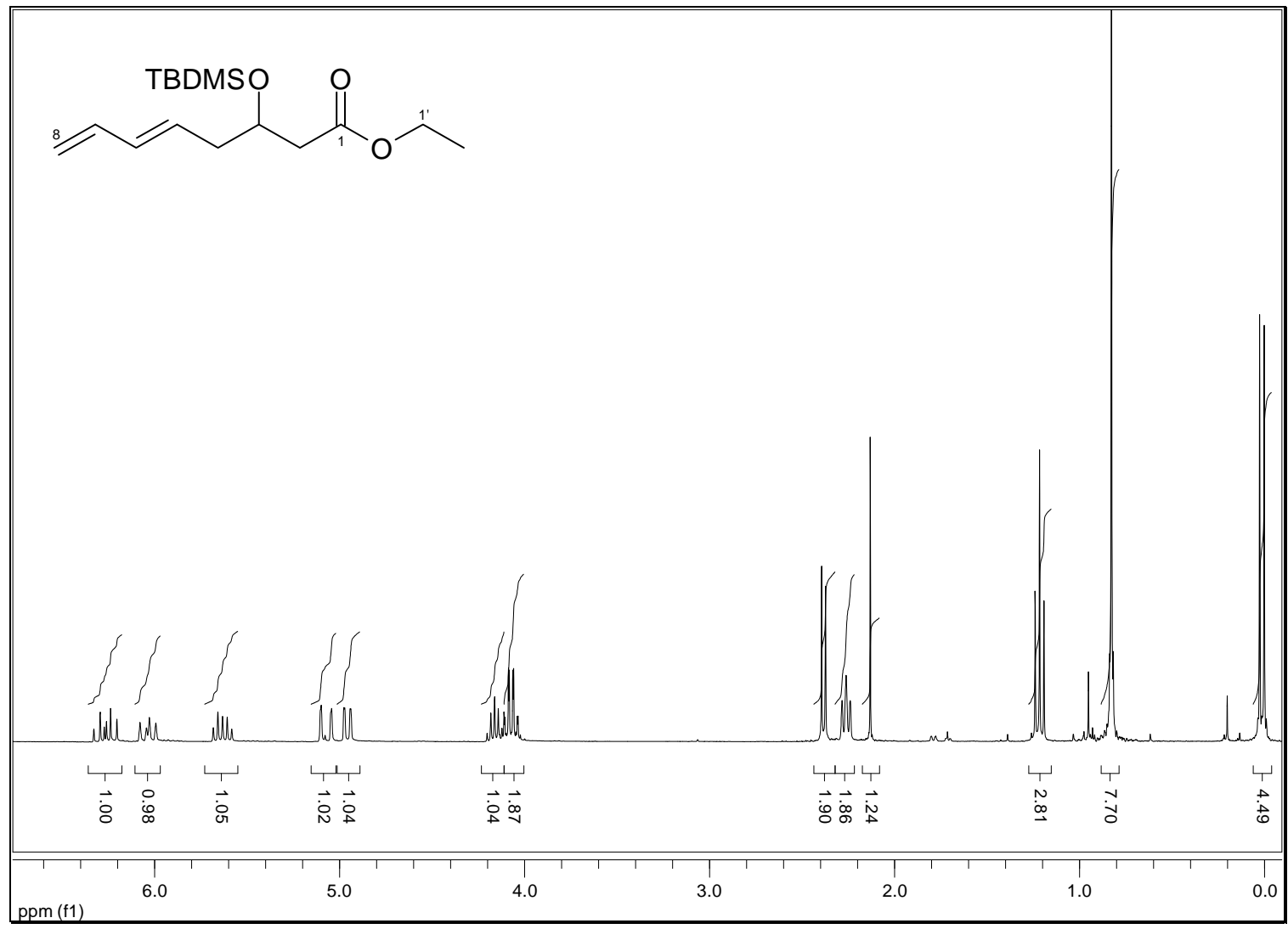

30. ${ }^{1} \mathrm{H}-\mathrm{NMR}$ (300 MHz) von (E)-3-(tert-Butyldimethylsilanyloxy)-octa-5,7-diensäure-N-methoxy-N-methylamid (205)

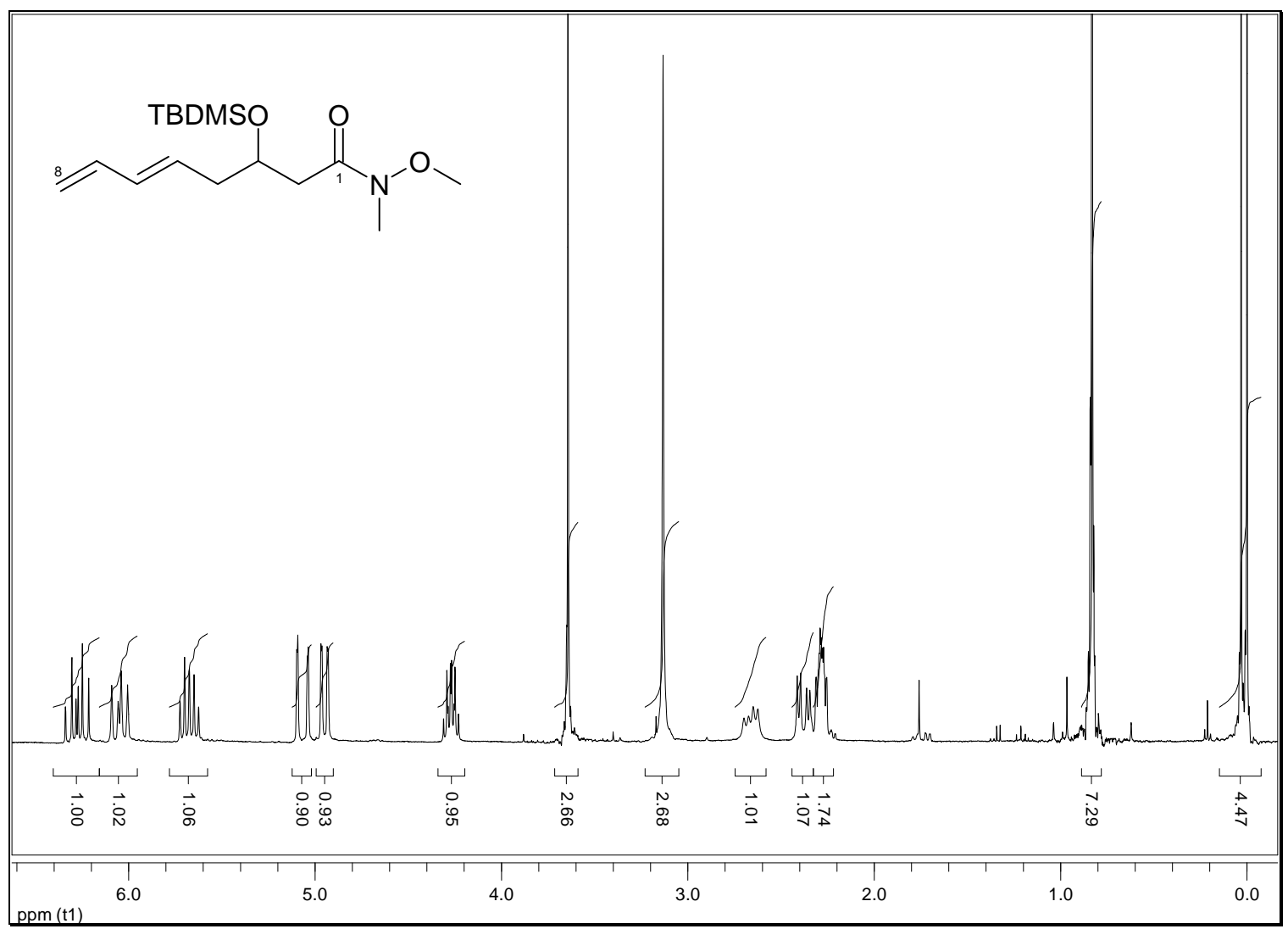


31. ${ }^{1} \mathrm{H}-\mathrm{NMR}$ (600 MHz) von (3E,9E)-7-(tert-Butyldimethylsilanyloxy)-1-trimethylsilanyldodeca-3,9,11-trien-1-in-5-on (197)

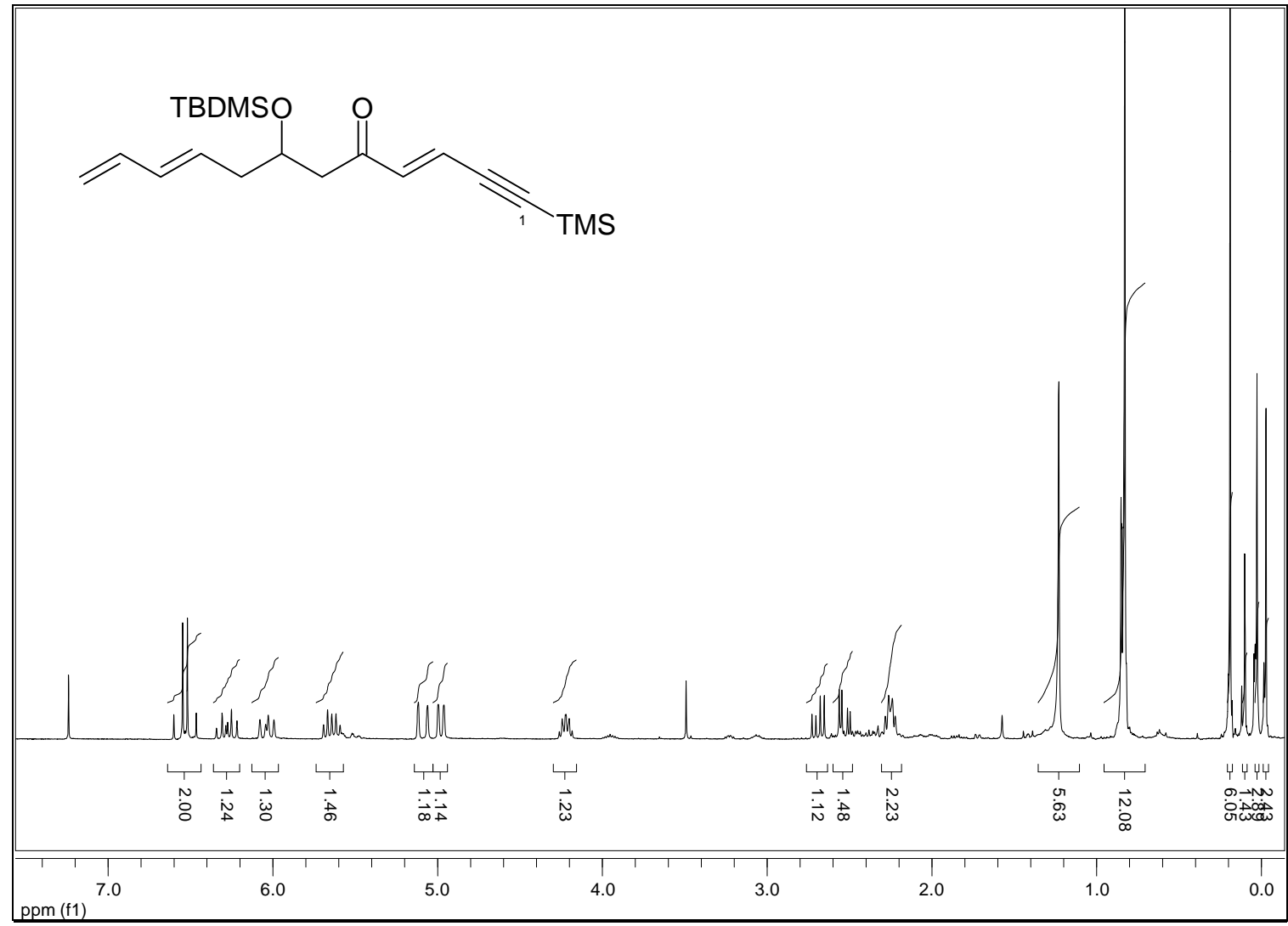

\section{${ }^{13} \mathrm{C}-\mathrm{NMR}(151 \mathrm{MHz})$}

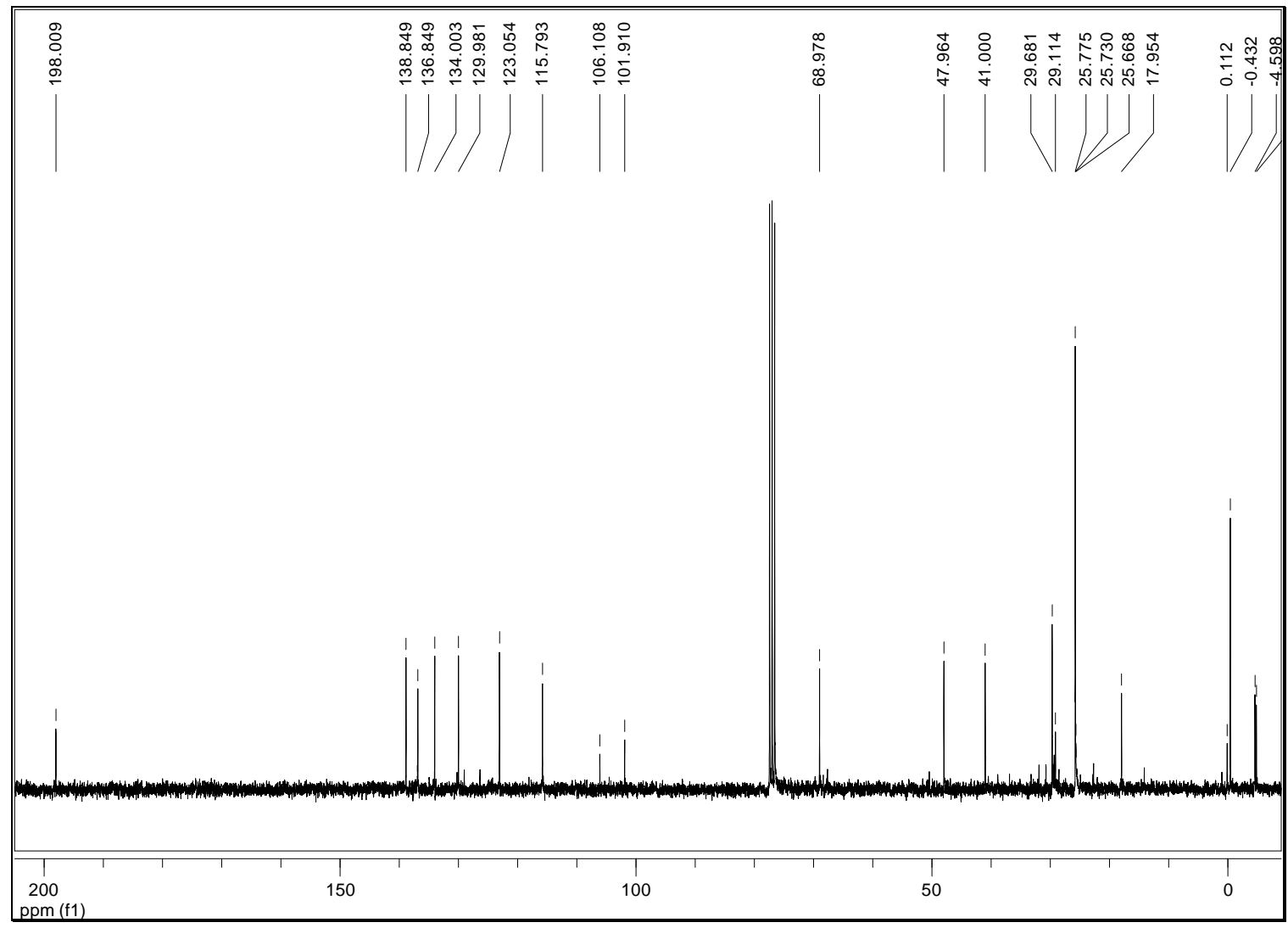


32. ${ }^{1} \mathrm{H}-\mathrm{NMR}(600 \mathrm{MHz})$ von $\left(3 R^{*}, 4 \mathrm{a} S^{*}, 8 S^{*}, 8 \mathrm{a} S^{*}\right)$-3-(tert-Butyldimethylsilanyloxy)-8trimethylsilanylethinyl-3,4,4a,7,8,8a-hexahydro-2H-naphthalin-1-on (199a)

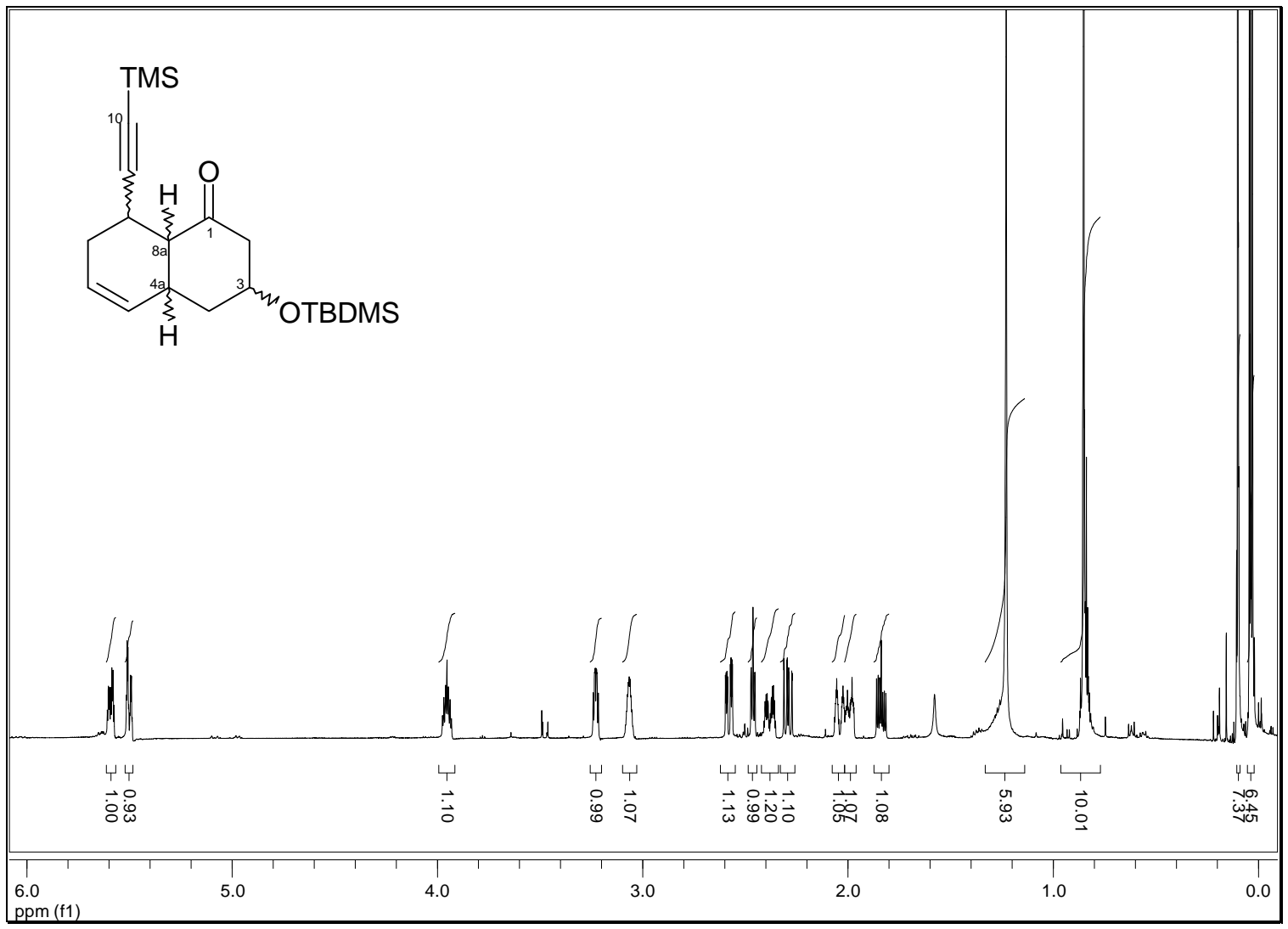

\section{${ }^{13}$ C-NMR (151 MHz)}

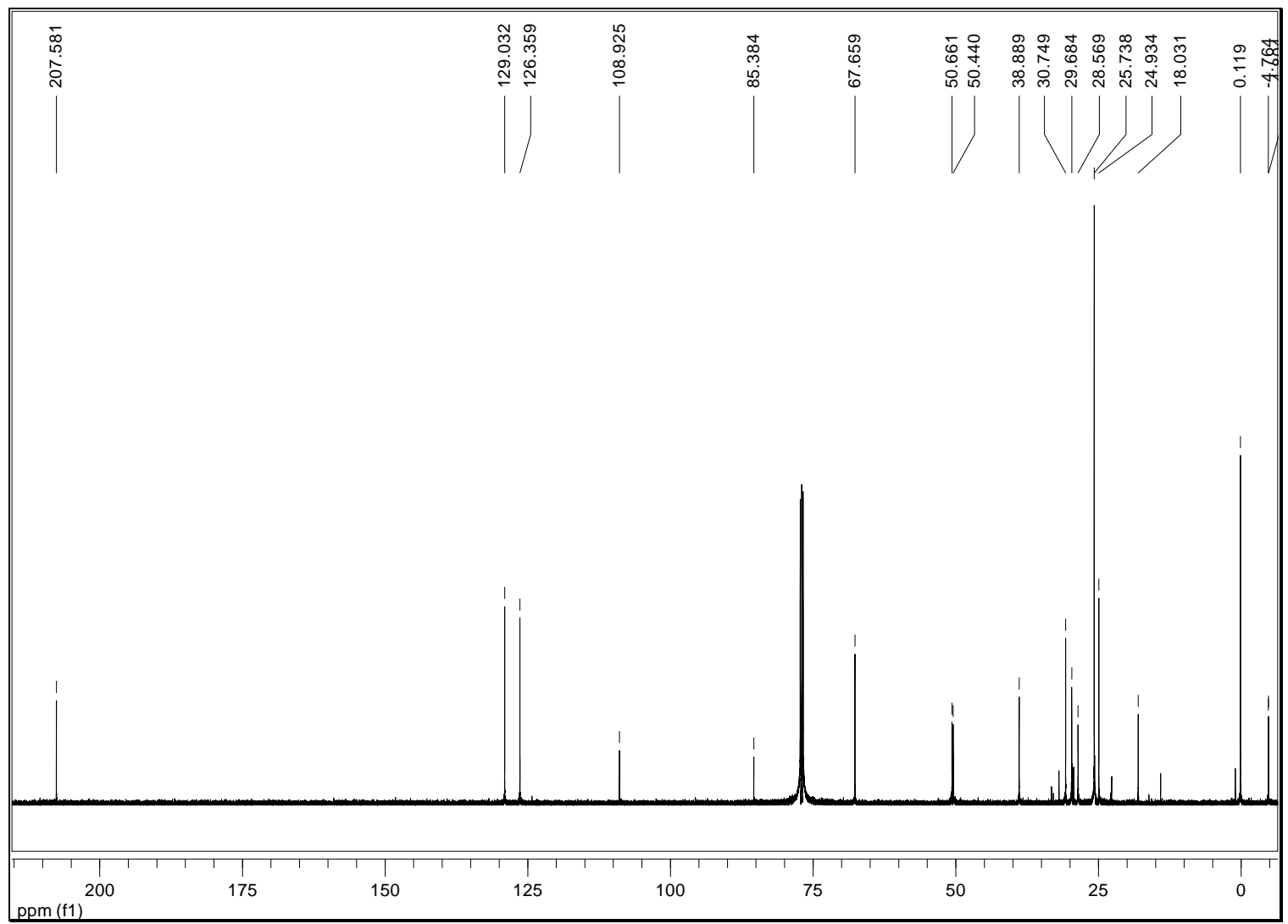


33. ${ }^{1} \mathrm{H}-\mathrm{NMR}(600 \mathrm{MHz})$ von 3-(tert-Butyldimethylsilanyloxy)-8-trimethylsilanylethinyl-3,4,4a,7,8,8a-hexahydro-2H-naphthalin-1-on (199b)

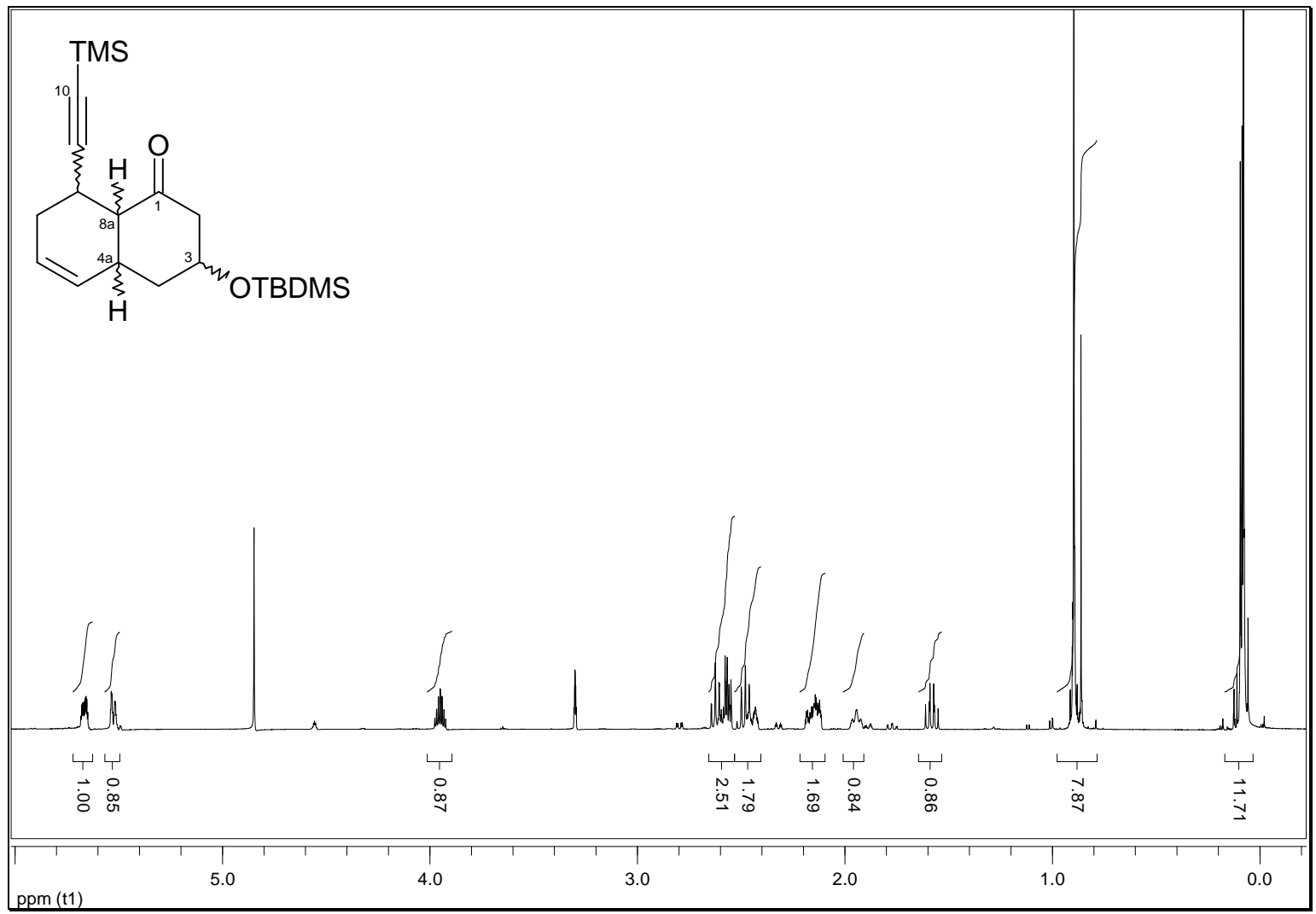

${ }^{13}$ C-NMR (151 MHz)

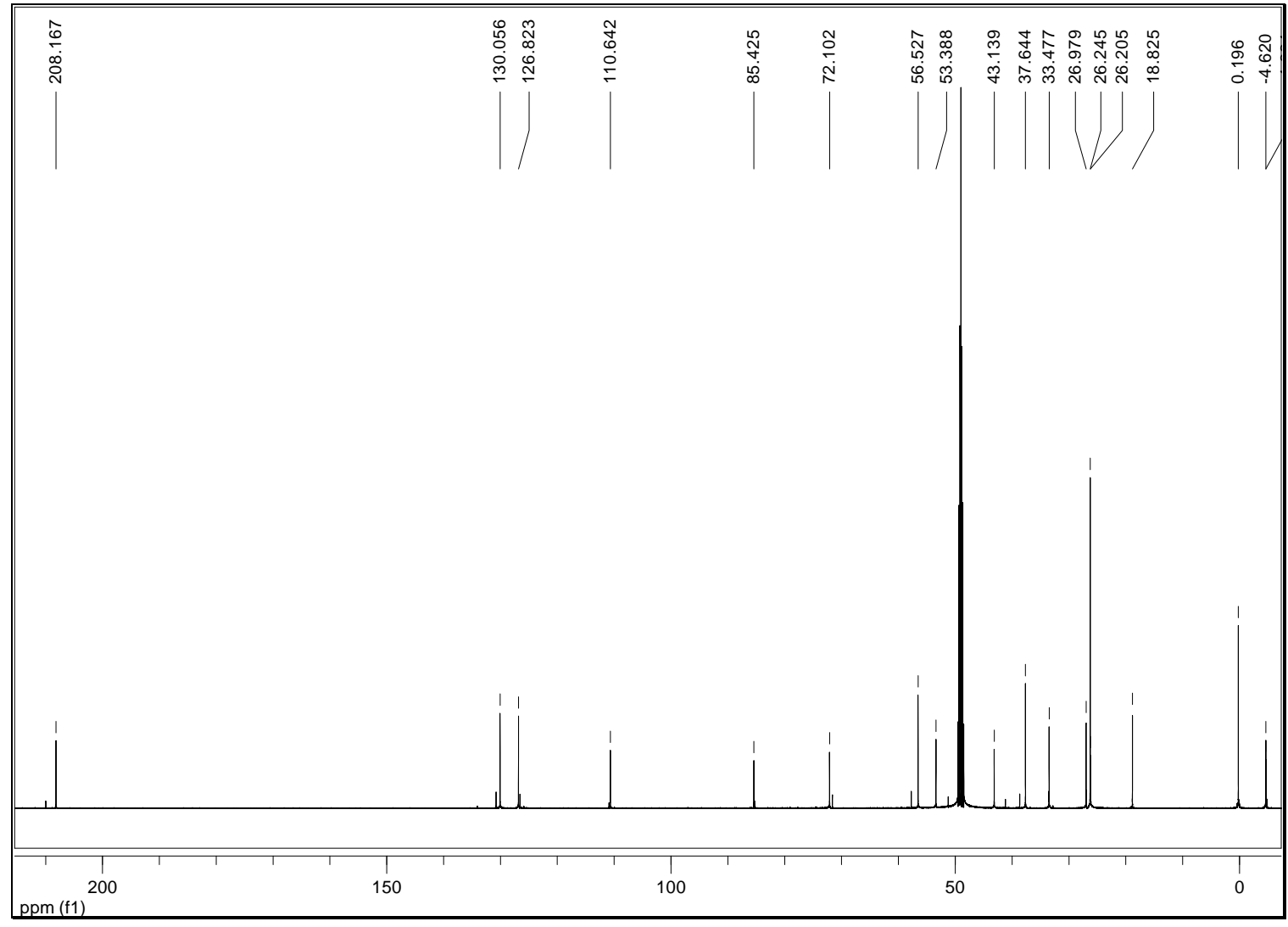


34. ${ }^{1} \mathrm{H}-\mathrm{NMR}(600 \mathrm{MHz})$ von $\left(3 S^{*}, 4 \mathrm{a} S^{*}, 8 S^{*}, 8 \mathrm{a} S^{*}\right)$-3-(tert-Butyldimethylsilanyloxy)-8trimethylsilanylethinyl-3,4,4a,7,8,8a-hexahydro-2H-naphthalin-1-on (199c)

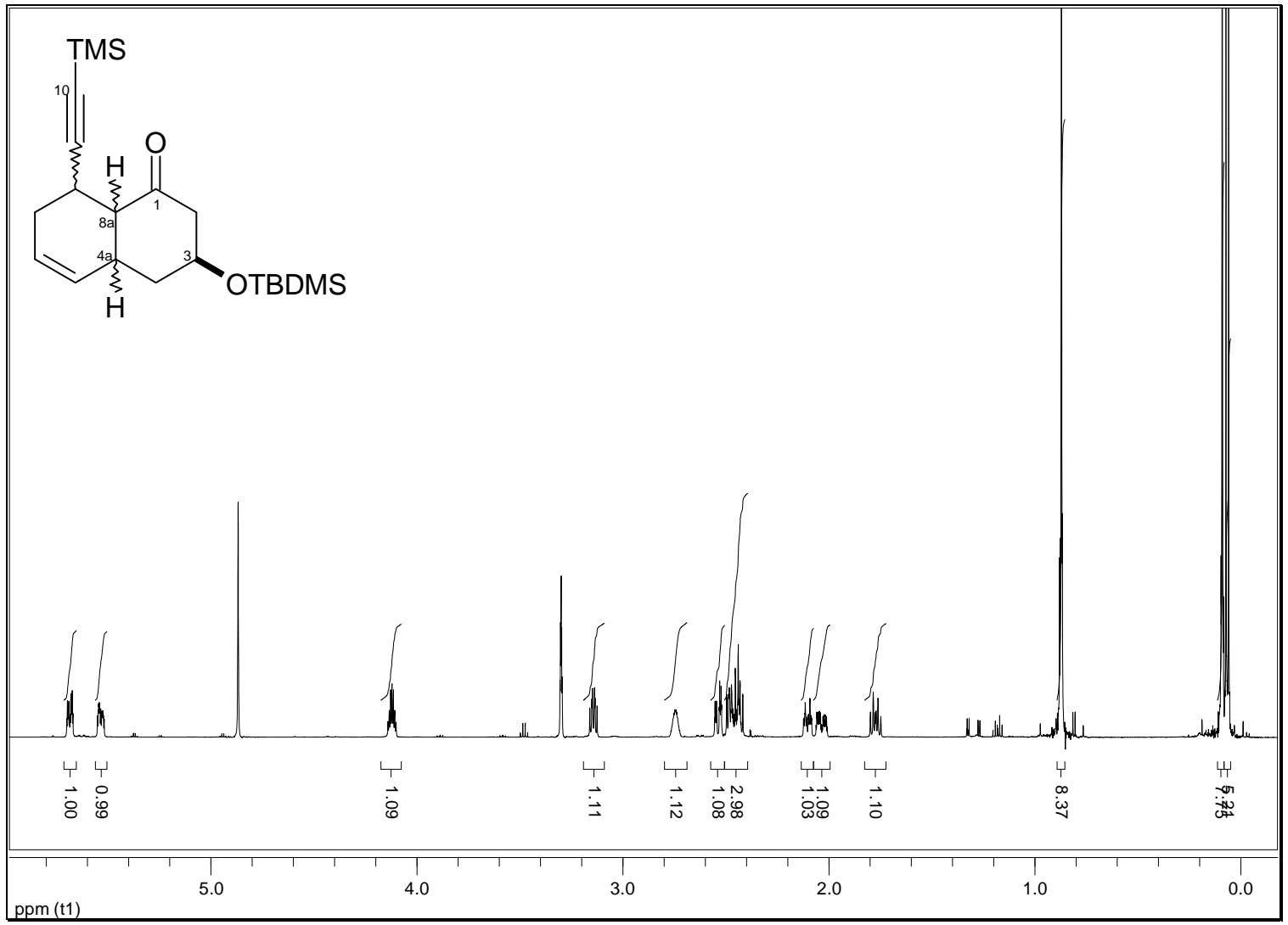

${ }^{13}$ C-NMR (151 MHz)

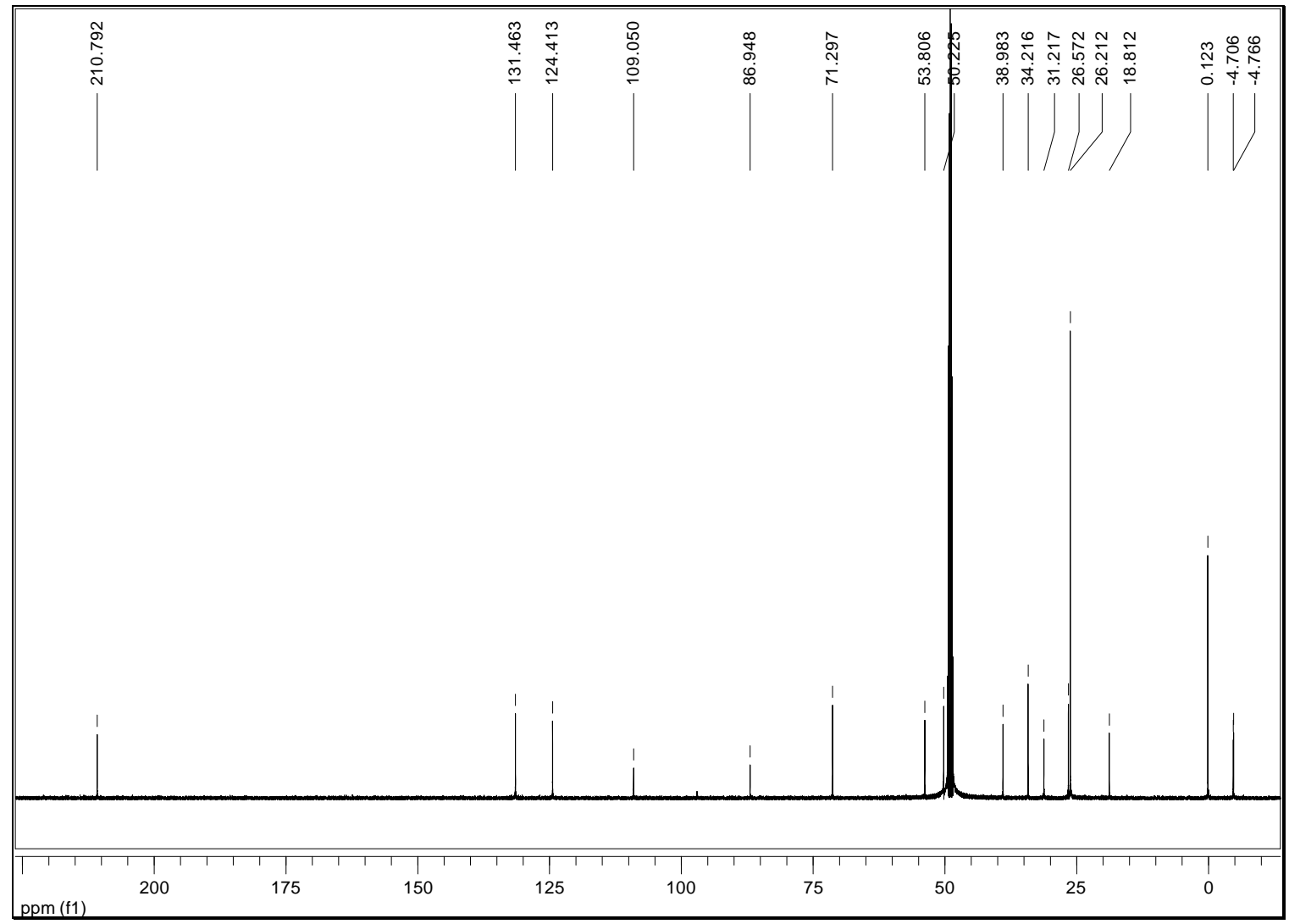


35. ${ }^{1} \mathrm{H}-\mathrm{NMR}(600 \mathrm{MHz})$ von $\left(4 \mathrm{a} S^{*}, 8 S^{*}, 8 \mathrm{a} S^{*}\right)-8$-Trimethylsilanylethynyl-4a,7,8,8atetrahydro-4H-naphthalin-1-on (210)

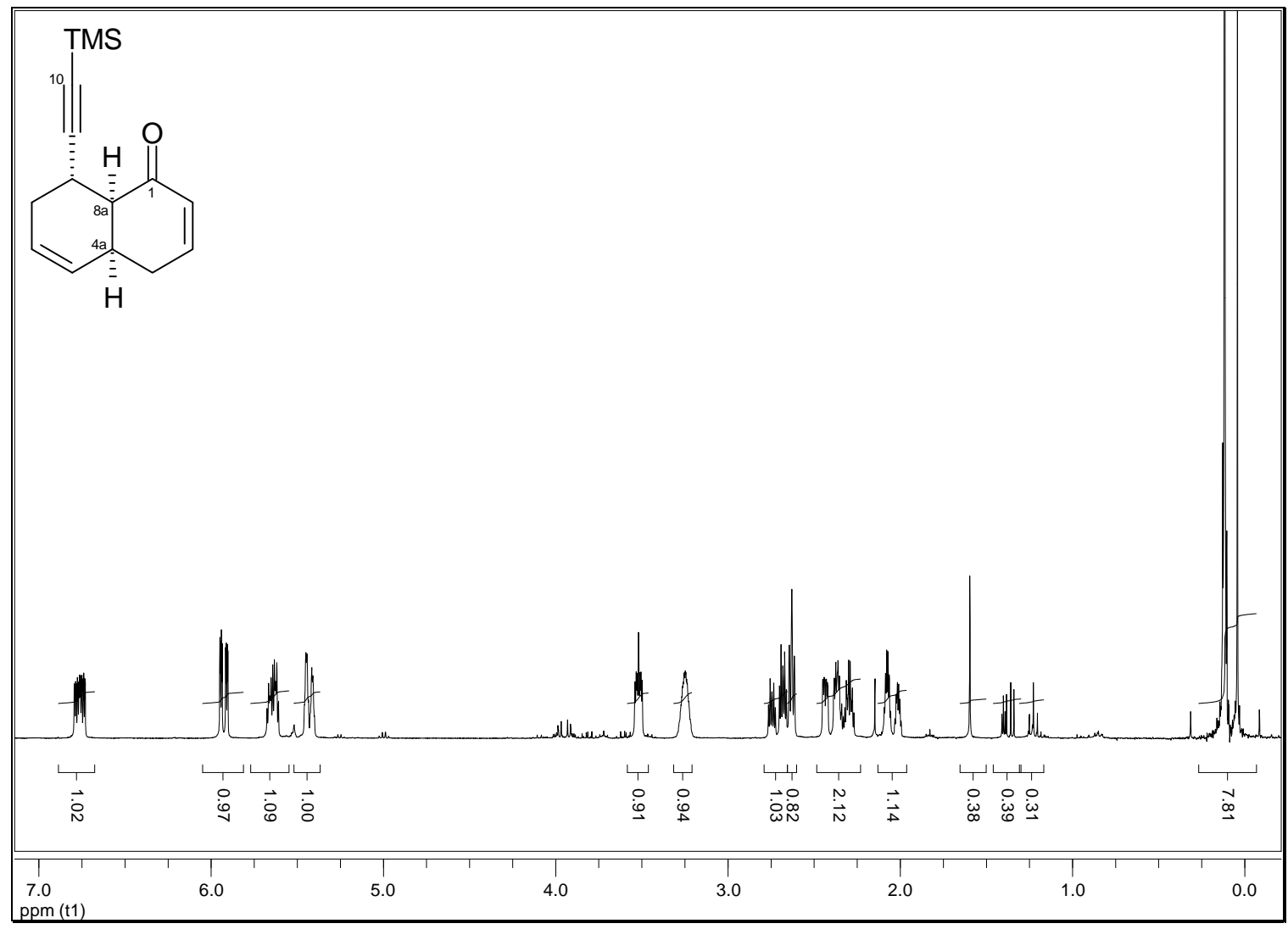

\section{${ }^{13} \mathrm{C}-\mathrm{NMR}(151 \mathrm{MHz})$}

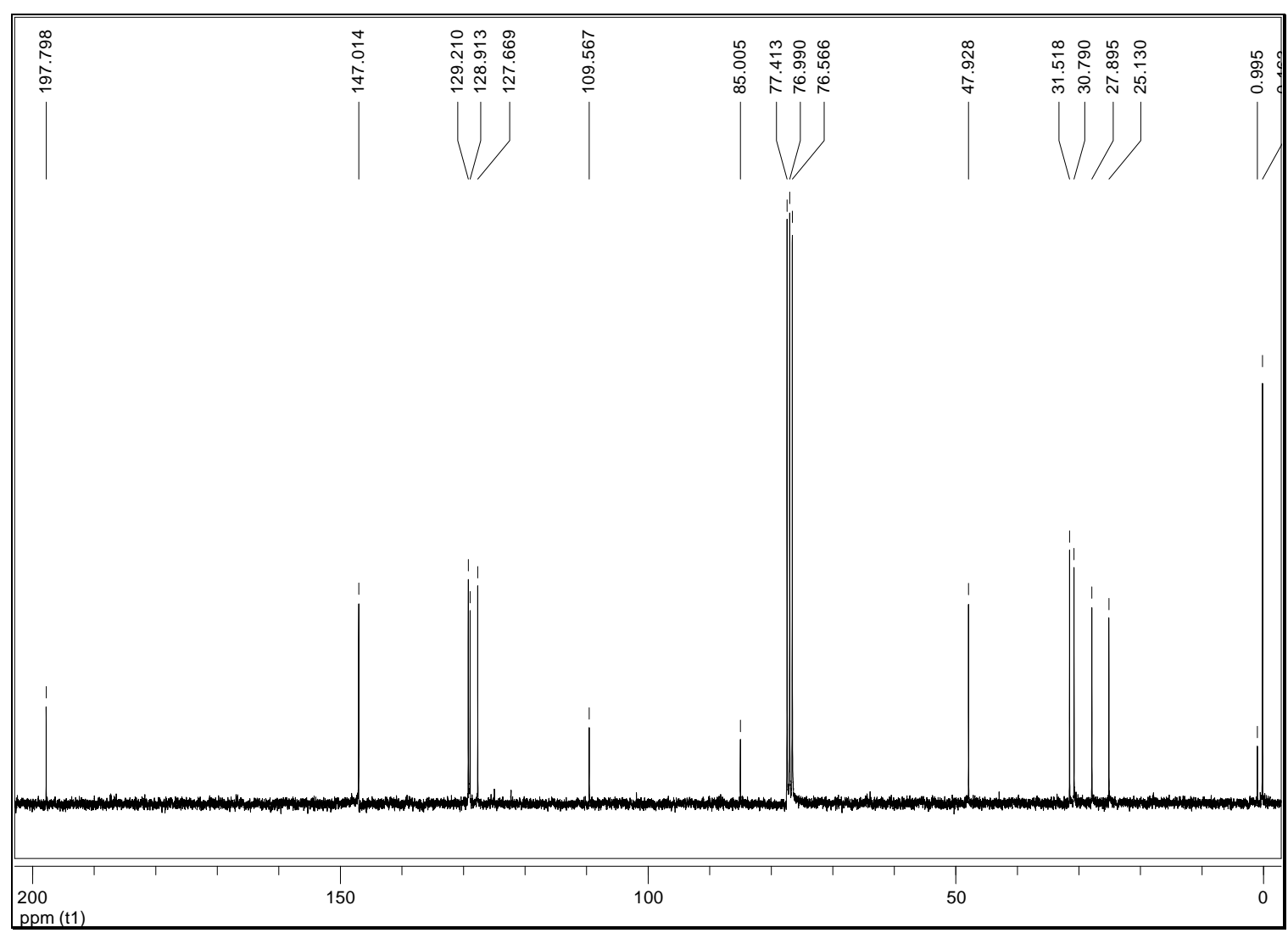


36. ${ }^{1} \mathrm{H}-\mathrm{NMR}(600 \mathrm{MHz})$ von $\left(3 R^{*}, 4 \mathrm{a} S^{*}, 8 S^{*}, 8 \mathrm{a} S^{*}\right)$-3-(tert-Butyldimethylsilanyloxy)-8ethinyl-3,4,4a,7,8,8a-hexahydro-2H-naphthalin-1-on (207a)

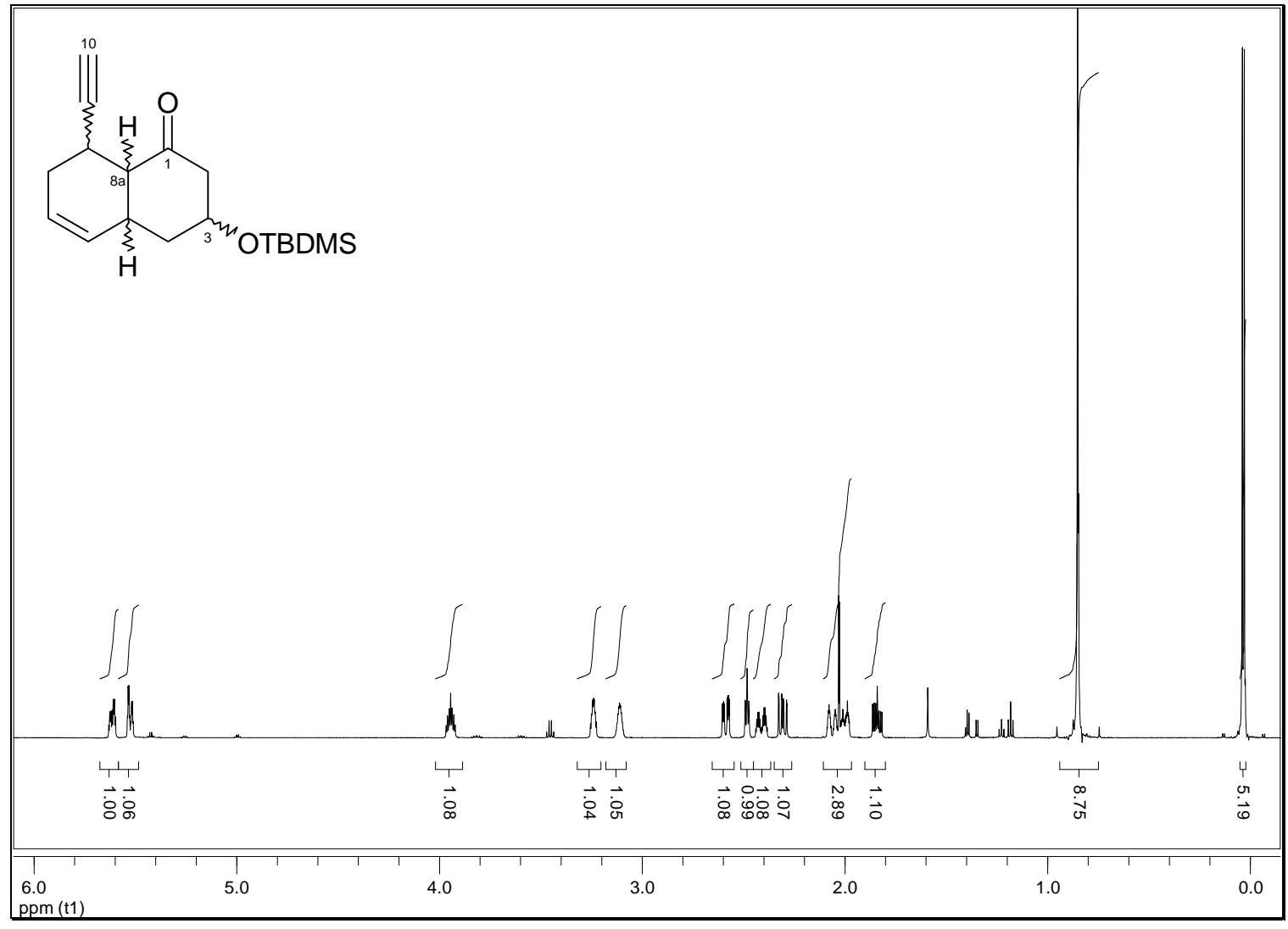

37. ${ }^{1} \mathrm{H}-\mathrm{NMR}(600 \mathrm{MHz})$ von $\left(3 S^{*}, 4 \mathrm{a} S^{*}, 8 S^{*}, 8 \mathrm{a} S^{*}\right)$-3-(tert-Butyldimethylsilanyloxy)-8ethinyl-3,4,4a,7,8,8a-hexahydro-2H-naphthalin-1-on (207c)

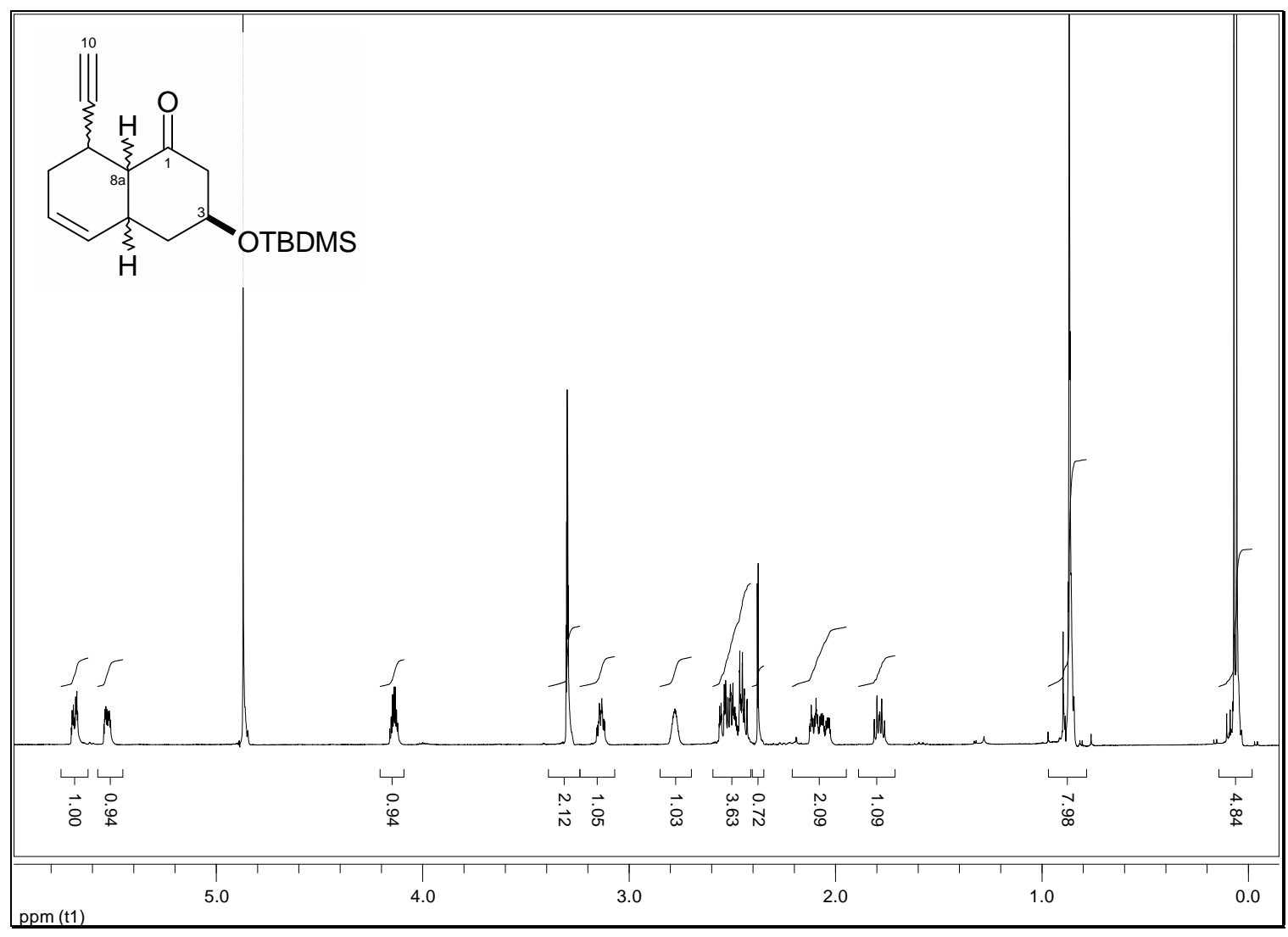






\section{Danksagung}

Ohne die fachliche und moralische Unterstützung eines ganzen Heeres großartiger Menschen wäre die vorliegende Arbeit sicher nie zustande gekommen. Insbesondere Prof. Dr. Paultheo v. Zezschwitz möchte ich dafür danken, dass er mich in seine Arbeitsgruppe aufgenommen hat und er mir immer mit Rat und Tat zur Seite stand, wenn es an irgendeiner Ecke klemmte. Außerdem möchte ich ihm für die angenehme Arbeitsatmosphäre danken, die er durch seine geduldige, kollegiale und offene Art maßgeblich mitgestaltet hat. Als nächstes möchte ich Prof. Dr. A. de Meijere und Prof. Dr. A. Zeeck für die Unterstützung danken, die es mir ermöglicht hat, unter optimalen Bedingungen zu forschen. PD Dr. Stephanie Grond möchte ich für die gute und vor allem fruchtbare Zusammenarbeit beim Okaspirodiol und natürlich dem Photoaffinitätslabel danken.

Meiner ganzen Familie, meinem Bruder und ganz besonders meiner Mutter und meinen Großeltern möchte ich für die uneingeschränkte Unterstützung, Geduld und Liebe danken, ohne die ich diese Arbeit niemals hätte anfertigen können. An dieser Stelle möchte ich auch meiner langjährigen Lebensgefährtin Annett danken, die mir mit ihrer Stärke und Geradlinigkeit oft den richtigen Weg gewiesen hat und in unserer gemeinsamen Zeit immer den Ruhepunkt meines Lebens darstellte. Danke.

Allen meinen Kollegen möchte ich für die entspannte Arbeitsatmosphäre danken, in der man immer offen und ehrlich miteinander diskutieren, streiten und natürlich auch mal feiern konnte. Jürgen Sievert danke ich für die anregenden Diskussionen und dafür, dass er mit seinem sonnigen Gemüt die Welt für uns alle etwas heller erscheinen ließ. René Sandmann muss ich gleich mehrfach danken, zum Einen für die Messung der GC-MSSpektren der Diels-Alder-Reaktionen und zum Anderen für das gewissenhafte Korrekturlesen dieser Arbeit. In diesem Zusammenhang möchte ich auch H. Shojaei für seinen Einsatz danken. Für Einblicke in mir bis dahin unbekannte Seiten des Metals und der moralischen Unterstützung in der Endphase dieser Arbeit möchte ich Michael Richter danken und wünsche ihm hiermit alles Gute und viel Erfolg in Hannover. Für seine Unterstützung und die geselligen Abende danke ich Johannes Beerlink und wünsche ihm allzeit artige Schüler. Evelyn Zeiler möchte ich für die angenehme Zusammenarbeit und ihren Beitrag zur Totalsynthese des Collinolactons danken. 
Den vielen fleißigen Helfern „hinter den Kulissen“ des Institutes möchte ich natürlich auch meinen Dank aussprechen. Für die Aufnahme der NMR-Spektren danke ich: R. Machinek, C. Siebert, C. Zolke, M. Weitemeyer; Massenspektren: H. Frauendorf, G. Krökel, G. Sommer-Udvarnoki und für LC-MS und Elementaranalysen: F. Hambloch und O. Senge. Herrn Hans-Jörg Langer möchte ich für die geduldige Einführung in die spannende Durchführung der Diazomethansynthese und die ausgedehnten Fachsimpeleien über jegliche Aspekte des Profifußballs danken.

Für die Mitgestaltung der außeruniversitären „Arbeitsfreien Zeit“ möchte ich zunächst Philipp Wagener danken. In unzähligen Championsleague Spielen des FC Bayern und Werder Bremens haben wir gemeinsam gelitten und gefeiert. Trotz vier gemeinsamer internationaler Turniere und immerhin zwei Endspielen konnten wir leider keinen internationalen Titel feiern. Aber die WM 2010 ist ja nicht mehr lange hin...

Danke auch an meine „alten“ Freunde Martin, Schupi, Hübel und natürlich Tim für die Unterstützung und dass ihr immer für mich da seit, wenn ich euch brauche!

Last, but not least, möchte ich meinen WG-Mitbewohnern danken: Wilhelm Hüttner, Elena Penkhus und Sarah Berger. Insbesondere in der Endphase meiner Arbeit war ich sicherlich nicht immer der Vorzeigemitbewohner und deshalb noch einmal ein ganz großes Danke für Geduld, Aufmunterungen und Zuspruch.

Bei allen Anderen, die bis hier aus Zeit- und/oder Gedächtnismangel nicht genannt worden sind, möchte ich mich an dieser Stelle entschuldigen und noch mal ausdrücklich und aufrichtig danke sagen. Ohne Euch wäre dies alles nicht möglich gewesen...

\section{DANKE!}




\section{LEBENSLAUF}

Name Tobias Bender

Geboren am $\quad 01.03 .1980 \quad$ in Halberstadt

Schule

1986-1991

Käthe-Kollwitz Oberschule Halberstadt

1991-1992

J. W. L. Gleim Realschule Halberstadt

1992-1998

Käthe-Kollwitz Gymnasium Halberstadt, Abitur

Wehrdienst

1998-1999

Grundwehrdienst in der Bundeswehr, Bremen

Studienverlauf 1999-

2003

Diplom Chemie an der Georg-August-Universität

Göttingen

Erasmusaufenthalt an der Königlich Tech. Hochschule Stockholm/Schweden

(Arbeitskreis O. Ramström)

$-2005$

Diplom: "Okaspirodiol: Biosynthese und Totalsynthese eines neuen Polyketids” Note: Sehr gut. (Arbeitskreise A. Zeeck und A. de Meijere, Göttingen)

Doktorarbeit 2005-10.2008 "Synthese und Anwendung eines radioaktiven Photoaffinitätslabels zur Wirkortbestimmung von Naturstoffen sowie Beiträge zur Totalsynthese des Collinolactons" (AK A. de Meijere und P. v. Zezschwitz, Göttingen)

$\begin{array}{lll}\text { Weiterbildung } & 2007-2008 & \text { Projektmanager Wirtschaftschemie GDCh } \\ 2007 & \text { Fachkunde im Strahlenschutz }\end{array}$

Göttingen, 17.09.2008. 
